\title{
BIOPHYSICAL STUDIES OF SPERMATOZOA
}

\section{David Andrew Pistenma}

(Ph.D. Thesis)

December 1970

AEC Contract No. W $-7405-$ eng -48

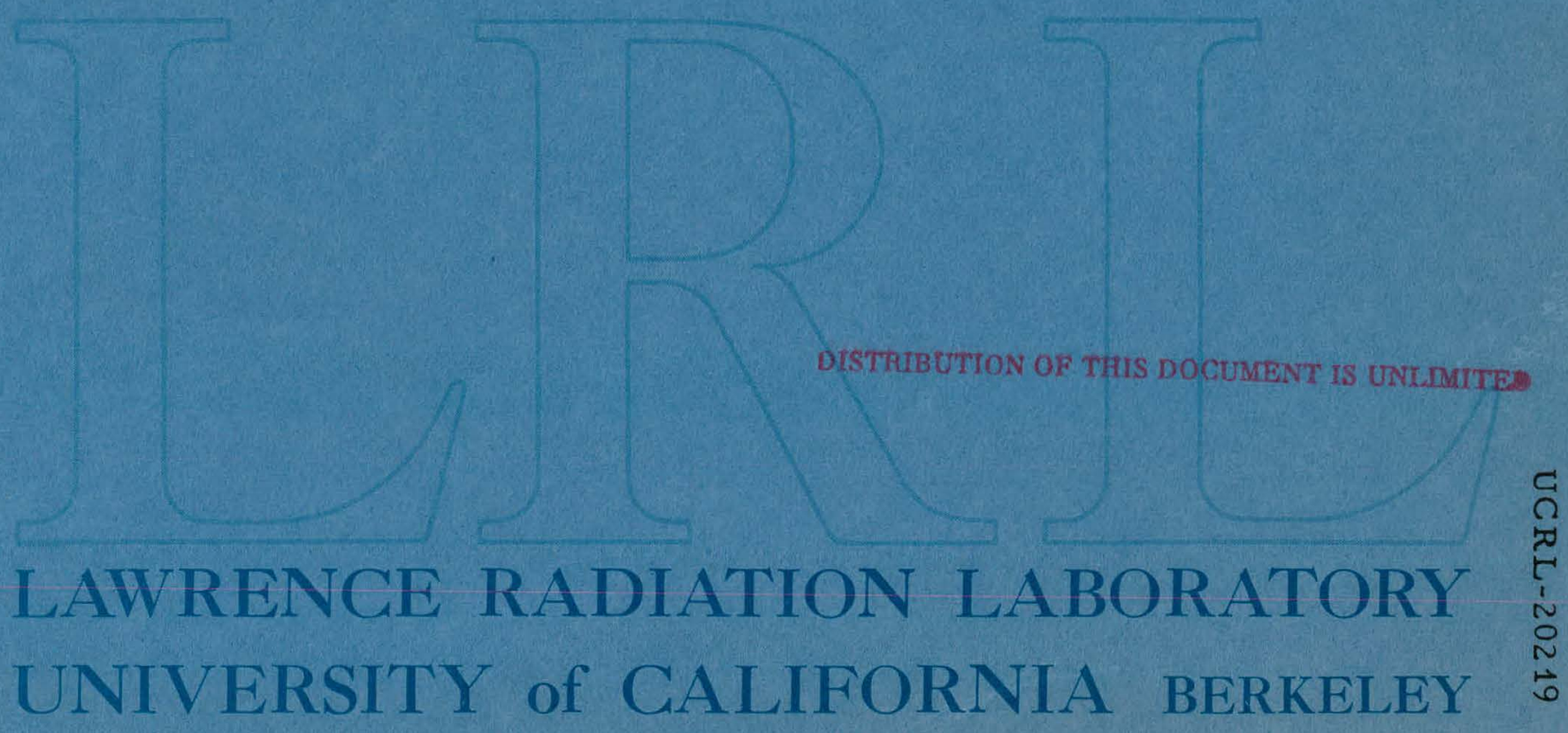




\section{DISCLAIMER}

This report was prepared as an account of work sponsored by an agency of the United States Government. Neither the United States Government nor any agency Thereof, nor any of their employees, makes any warranty, express or implied, or assumes any legal liability or responsibility for the accuracy, completeness, or usefulness of any information, apparatus, product, or process disclosed, or represents that its use would not infringe privately owned rights. Reference herein to any specific commercial product, process, or service by trade name, trademark, manufacturer, or otherwise does not necessarily constitute or imply its endorsement, recommendation, or favoring by the United States Government or any agency thereof. The views and opinions of authors expressed herein do not necessarily state or reflect those of the United States Government or any agency thereof. 


\section{DISCLAIMER}

Portions of this document may be illegible in electronic image products. Images are produced from the best available original document. 


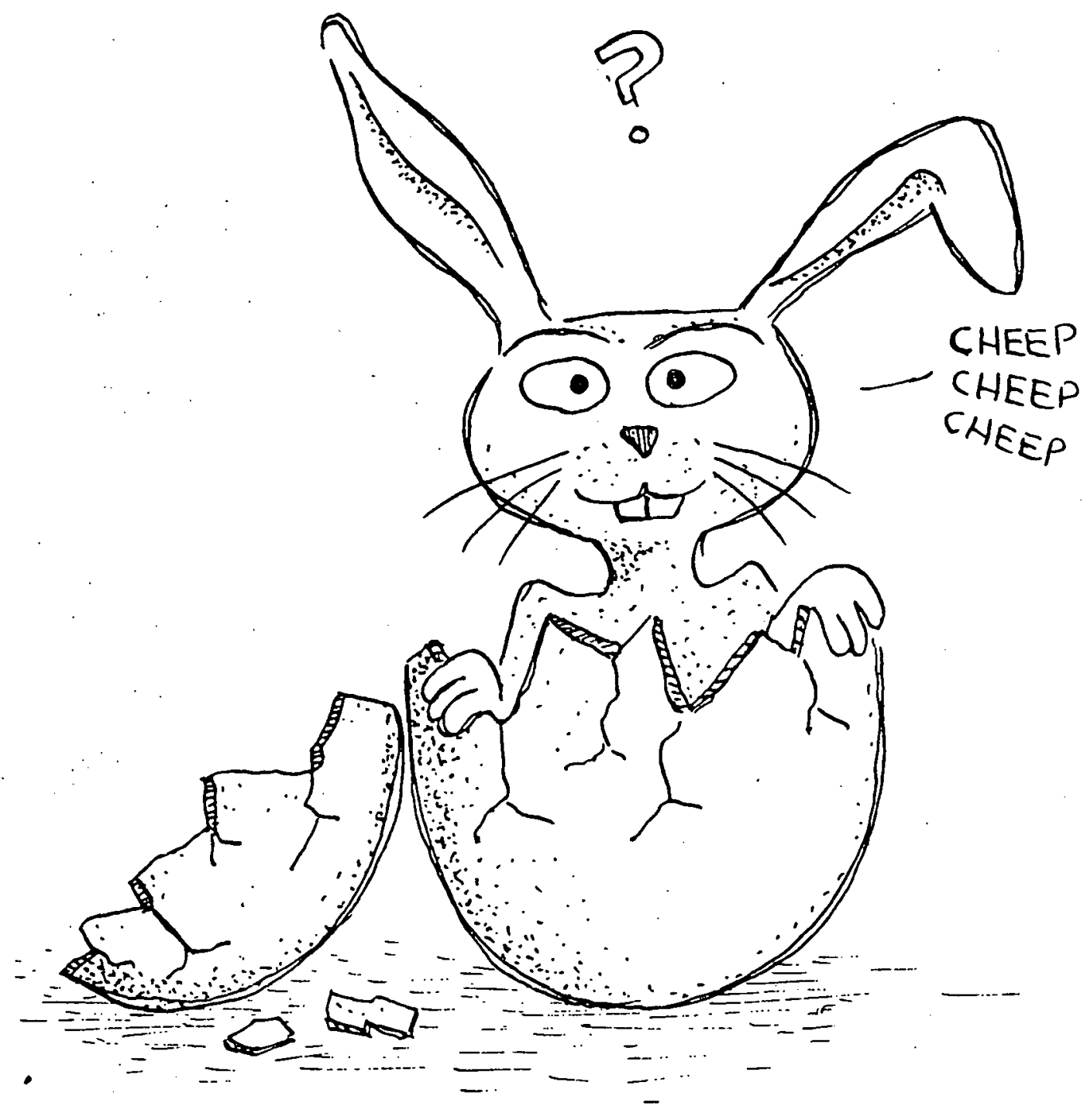

I guess I should have kept a record of which sperm sample was which. 
BIOPHYSICAL STUDIES

OF SPERMATOZOA

\section{Contents}

List of Figures. . . . . . . . . . . . . . . . . . . iv

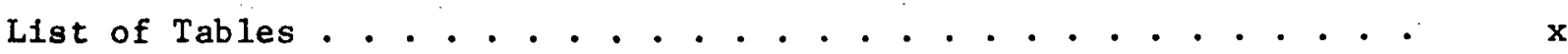

Abstract . . . . . . . . . . . . . . . . . . . xv

I. Introduction . . . . . . . . . . . . . . . . . 1

II. Interrelationships among Physical and Biological Properties of Spermatozoa and Important Experimental Variables . . . 5

III. The Staflo Method and Fractionation Principles . . . . . 53

IV. Early Staflo Sedimentation Experiments: Rabbit Spermatozoa 106

V. Early Staflo Electrophoresis Experiments: Rabbit and Fowl Spermatozoa . . . . . . . . . . . . . . 142

VI. Staflo Electrophoresis: Fowl Spermatozoa. . . . . . . . 179

VII. Equilibrium Dens1ty Gradient Centrifugation: Rabbit and

Fow1 Spermatozoa. . . . . . . . . . . . . . 208

VIII. Mult1-Characterization Staflo Studies: Rabbit Spermatozoa 228

IX. The Quality of Rabbit Spermatozoa Populations: A Critical

Discussion. . . . . . . . . . . . . . 282

X. Sumary. . . . . . . . . . . . . . 315

Acknowledgments. . . . . . . . . . . . . . . . . . . 320

Glossary . . . . . . . . . . . . . . . . . . . 322

Appendices . . . . . . . . . . . . . . . . . . . . 326

A. Considerations in Selecting the Rabbit as a Sperm Donor 326

B. Supplemental Information on Work with Germ Cells . . . 327

C. A New Artificlal Vagina for Collecting Rabbit Spermatozoa 355

D. Evaluation of Media. . . . . . . . . . . . . 358 
E. Expressions for Minimum, Maximum and Average Migration Distances for Particles In Staflo Collection Fractions

F. Sample Calculation of Sedimentation Velocities and Rates for Staflo-Sedimentation Experiment 12-26-67. . . . . 381

G. Sample Calculation of Electrophoretic Mobilities for

Staflo Sedimentation-Upward-Electrophoresis Ex-

periment 7-29-67................. 383

References . . . . . . . . . . . . . . . . . . 385 


\section{LIST OF FIGURES}

Fig. 1. Structure of the rabbit spermatozoon.

FIg. 2. Structure of the fowl spermatozoon.

Fig. 3. Photograph of $3 \times 16$ Staflo apparatus.

Fig. 4. Schematic diagram of Staflo apparatus.

Fig. 5. Photograph of Staflo flow-cell and mount with microscope in place.

Fig. 6. Photograph of flow-cell and mount with microscope removed. 61

Fig. 7. Closeup of flow-cell.

Fig. 8. Schematic diagram of flow-cell showing spermatozoa migration pattern.

Fig. 9. Closeup of Staflo microscope mounted on Staflo apparatus.

Fig. 10. Photograph of Staflo microscope showing component parts.

Fig. 11. Schematic diagram of sedimentation or sedimentationdownward electrophoresis migration pattern; two layer spread. 76

Fig. 12. Schematic diagram of sedimentation or sedimentationdownward electrophoresis migration pattern; three-1ayer spread. 76a

Fig. 13. Range and distribution diagram for migration distances in sedimentation and sedimentation-downward-electrophoresis experiments; two-layer spread. 
Fig. 14. Range and distribution diagram for migration distances in sedimentation and sedimentation-downward-electrophoresis experiments; three-layer spread.

Fig. 15. Alternative range and distribution diagram for migration distances in sedimentation and sedimentation-downwardelectrophoresis experiments; two- and three-layer spread.

Fig. 16. Schematic diagram of sedimentation-downward-electrophoresis migration pattern showing separate components.

Fig. 17. Schematic diagram of sedimentation-upward-electrophoresis migration patterns; two-layer spread.

Fig. 18. Schematic diagram of sedimentation-upward-electrophoresis migration pattern; three-layer spread.

Fig. 19. Schematic diagram of aedimentation-upward-electrophoresis migration pattern showing separate components.

Fig. 20. Range and distribution diagram for sedimentation-upwardelectrophoresis migration distances; two-layer spread.

Fig. 21. Range and distribution diagram for sedimentation-upwardelectrophoresis migration distances; three-layer spread.

Fig. 22. Alternative range and distribution diagram for migration distances in sedimentation-upward-electrophoresis experiments; two-1ayer spread.

Fig. 23. Alternative range and distribution diagram for migration distances in sedimentation-upward-electrophoresis experiments; three-1ayer spread. 
Fig. 24. Schematic diagram of a sedimentation migration pattern showing the effect of intrinsic motility on migration.

Fig. 25. Schematic diagram of a sedimentation-downward-electrophoresis migration pattern showing the interrelationships among the sedimentation, electrophoresis and intrinsic motility components.

Fig. 26. Schematic diagram of a sedimentation-upward-electrophoresis migration pattern showing the interrelationships among the sedimentation, electrophoresis and intrinsic motility components.

Fig. 27. Schematic diagram of Staflo flow-cell showing typical rabbit spermatozoa sedimentation migration pattern at $7^{\circ} \mathrm{C}$; sucrose density gradient.

Fig. 28. Characteristics of dextran and of sucrose density gradients, for early sedimentation experiments.

Fig. 29. Results of sedimentation Experiment 10-21 with rabbit spermatozoa at $7^{\circ} \mathrm{C}$; sucrose density gradient.

Fig. 30. Individual results of five sedimentation experiments with rabbit spermatozoa at $7^{\circ} \mathrm{C}$; sucrose density gradient.

F1g. 31. Average results of five sedimentation experiments with rabbit spermatozoa at $7^{\circ} \mathrm{C}$; sucrose density gradient.

Fig. 32. Average results of two sedimentation experiments at $15^{\circ} \mathrm{C}$ and a comparison of the average total rabbit spermatozoa distribution at $15^{\circ} \mathrm{C}$ with that from five experiments at $7^{\circ} \mathrm{C}$; sucrose density gradient. 
Fig. 33. Results of one sedimentation experiment with rabbit spermatozoa at $4^{\circ} \mathrm{C}$; sucrose density gradient.

Fig. 34. Individual results of three sedimentation experiments with rabbit spermatozoa at $2.5^{\circ} \mathrm{C}$; sucrose density gradient.

Fig. 35. Average results of three sedimentation experiments with rabbit spermatozoa at $2.5^{\circ} \mathrm{C}$; sucrose density gradient.

Fig. 36. Individual results of two sedimentation experiments with rabbit spermatozoa at $7^{\circ} \mathrm{C}$; dextran density gradient.

Fig. 37. Average distribution of rabbit spermatozoa in sucrose gradient sedimentation experiments at $2.5^{\circ} \mathrm{C}, 4^{\circ} \mathrm{C}, 7^{\circ} \mathrm{C}$ and $15^{\circ} \mathrm{C}$.

Fig. 38. Comparison of expected and actual distributions of rabbit spermatozoa in sucrose gradient sedimentation experiments at various temperatures.

Fig. 39. Comparison of expected and actual distributions of rabbit spermatozoa in dextran density gradient sedimentation experiments at various temperatures.

Fig. 40. Individual results of four upward-electrophoresis experiments with rabbit spermatozoa at $5 \mathrm{~V} / \mathrm{cm}$ in $1 \times 12 \mathrm{staflo}$ apparalus.

Fig. 41. Individual results of two upward-electrophoresis experiments with rabbit spermatozoa at $6.25 \mathrm{~V} / \mathrm{cm}$ in $1 \times 12 \mathrm{staflo}$ apparatus .

Fig. 42. Individual results of four upward-electrophoresis experiments with rabbit spermatozoa at $7.5 \mathrm{~V} / \mathrm{cm}$ in $1 \times 12$ 
Staflo apparatus.

Fig. 43. Comparison of results of upward-electrophoresis experiments with rabbit spermatozoa at 6.25 and $7.5 \mathrm{~V} / \mathrm{cm}$, at $2.5^{\circ} \mathrm{C}$, in $1 \times 12$ Staflo apparatus.

Fig. 44. Average results for four upward-electrophoresis experiments with rabbit spermatozoa at $5 \mathrm{~V} / \mathrm{cm}$ in $1 \times 12$ Staflo apparatus.

Fig. 45. Average results for four upward-electrophoresis experiments with rabbit spermatozoa at $6.25 \mathrm{~V} / \mathrm{cm}$ in $1 \times 12 \mathrm{Staf1o}$ apparatus.

Fig. 46. Average results for four upward-electrophoresis experiments with rabbit spermatozoa at $7.5 \mathrm{~V} / \mathrm{cm}$ in $1 \times 12 \mathrm{staflo}$ apparatus.

Fig. 47. Average results for two upward-electrophoresis experiments with rabbit spermatozoa at $8 \mathrm{~V} / \mathrm{cm}$ and $3.5^{\circ} \mathrm{C}$ in $3 \times 16$ Staflo apparatus.

Fig. 48. Schematic diagram of Staflo flow-cell illustrating fowl spermatozoa upward-electrophoresis migration pattern.

Fig. 49. Schematic diagram showing ideal three-dimensional Staflo upward-electrophoresis migration pattern of fowl spermatozoa. 197b

Fig. 50. Photograph of upward-electrophoresis migration pattern with fowl spermatozoa in an enrichment experiment at $5.5^{\circ} \mathrm{C} . \quad 200 \mathrm{a}$

Fig. 51. Ranges of apparent specific gravities of fowl spermatozoa 
in sodium iothalamate density gradients.

Fig. 52. Ranges of apparent specific gravities of rabbit spermatozoa in a sodium iothalamate density gradient.

Fig. 53. Characteristics of the glycine-phosphate buffer and sucrose density gradient for multi-characterization studies of rabbit spermatozoa.

Fig. 54. Photograph of the migration pattern in a sedimentationupward-electrophoresis experiment with rabbit spermatozoa.

Fig. 55. Distributions of rabbit spermatozoa among collection fracLiuns for actual, and three hypothetical distributions of ocdimentation velocities.

Fig. 56. Orientation of rabbit spermatozoa in Staflo sedimentation and sedimentation-electrophoresis experiments at $3-5^{\circ} \mathrm{C}$.

Fig. 57. Effects of environmental temperature on the motility of rabbit spermatozoa.

Fig. 58. Effects of environmental temperature on the occurrence of capless spermatozoa in rabbit ejaculates.

Fig. 59. Lifespan of rabbit spermatogenetic cells.

Fig. 60. Schematic diagram showing categories of rabbit spermatozoa observed with light microscopy scoring of nigrosin-eosin preparations.

Fig. 61. Photograph of Teflon artificial vagina. 
Table I. Interrelationships among physical and biological properties of spermatozoa and important experimental variables.

Table II. Characteristics of Staflo flow-cells.

Table III. Intrinsic motility-sedimentation velocities of rabbit spermatozoa; sucrose density gradient; $2.5,4,7$ and $15^{\circ} \mathrm{C}$.

Table IV. Intrinsic motility-sedimentation velocities of rabbit spermatozoa; dextran density gradient; $2.5,4$ and $7^{\circ} \mathrm{C}$.

Table V. Spermatozoa sample and Staflo operating parameters for $1 \times 12$ flow-ce11 upward-electrophoresis experiments.

Table VI. Differential distribution of mature sperm, sperm with cytoplasmic droplets (CPD), and sperm heads and tails in upward-electrophoresis Experiment 2-28-66, $3.5^{\circ} \mathrm{C}$.

Table VII. Two-dimensional distribution of spermatozoa in upwardelectrophoresis Experiment 2-28-66 in the $3 \times 16$ Staflo flow$\operatorname{ce} 11,3.5^{\circ} \mathrm{C}$.

Table VIII. Comparison of distributions of rabbit spermatozoa in centerline collection fractions in downward-electrophoresis experiments; dextran density gradient, $6^{\circ} \mathrm{C}$.

Table IX. Distribution of tail-wagging and capped rabbit spermatozoa in upward-electrophoresis Experiment 5-14-67, 5.4 ${ }^{\circ} \mathrm{C}$.

Table $x . \quad$ Average electrophoretic mobilities of rabbit and fowl sperma tozoa. 
Table XI. Preservation of fertilizing capacity of fowl spermatozoa diluted 1:2 and stored in "high" Ionic strength media at $4^{\circ} \mathrm{C}$.

Table XII. Preservation of fertilizing capacity of fowl spermatozoa diluted 1:30 and stored in "high" and "1ow" ionic strength media at $3^{\circ} \mathrm{C}$.

Table XIII. Effects of dilution and media on the preservation of the fertilizing capacity of fowl spermatozoa stored at $3^{u} \mathrm{C}$.

Table XIV. Apparent electrophoretic mublillies of fuwl speritaluzua at $4^{\circ} \mathrm{C}$.

Table XV. Enrichment-fractionation and artificial insemination results of electrophoresis experiments with fowl spermatozoa at $5.5^{\circ} \mathrm{C}$.

Table XVI. Ranges of apparent specific gravities of fowl spermatozoa

in sodium lothalamate density gradients.

Table XVII. Ranges of apparent specific gravities of rabbit spermatozoa in a sodium iothalamate density gradient.

Table XVIII. Viability and morphology of rabbit spermatozoa in different reglons of a IInear sodlum lothalamate denslty gradlent. 218

Table XIX. Comparison of specific gravity measurements on rabbit and bu11 epermatozoa.

Table XX. Spermatozoa sample and Staflo operating parameters for multi-characterlzation sedimentation experiments. 
Table XXI. Phase contrast observations and nigrosin-eosin staining of spermatozoa; sedimentation experiments.

Table XXII. Sedimentation velocities for spermatozoa in collection fractions; sedimentation Experiment $12-26-67,3^{\circ} \mathrm{C}$.

Table XXIII. Summary of normalized calculated sedimentation velocities.

Table XXIV. Spermatozoa sample and Staflo operating parameters for sedimentation-upward-electrophoresis experiments using high electric field strengths.

Table XXV. Summary of distribution, phase contrast observations and nigrosin-eosin staining of spermatozoa in sedimentationupward-electrophoresis experiments using high electric field strengths.

Table XXVI. Sumary of artificial Inscmination results.

Table XXVII. Observed (uncorrected) and theoretical electrophoretic mobilities of rabbit spermatozoa; sedimentation-upwardelectrophoresis experiments using high electric field strengths.

Table XXVIII. Spermatozoa sample and Staflo operating parameters for sedimentation-electrophoresis experiments using low electric field strengths.

Table XXIX. Sumary of distribution, phase contrast observations and nigrosin-eosin staining of spermatozoa; sedimentationelectrophoresis experiments using low electric field strengths. 
Table XXX. Summary of artificial insemination results according to spermatozoa sample source.

Table XXXI. Comparison of Staflo operating parameters and results of sedimentation-upward-electrophoresis experiments using low electric field strengths.

Table XXXII. Comparison of light and phase contrast microscopy scoring of nigrosin-eosin stained rabbit spermatozoa.

Table XXXIII. Comparison of phase contrast observations of unstained spermatozoa and nigrosin-eosin stained preparations.

Table XXXIV. Correlation of scoring of caplessness by phase contrast observations of unstained and nigrosin-eosin stalned spermatozoa.

Table XXXV. Variations in the quality of consecutive ejaculates from the same rabbit.

Table XXXVI. Effect of low temperature centrifugation on the morphology of rabbit spermatozoa.

Table XXXVII. Estimated lifespan of rabbit spermatogenetic cells. 306

Table XXXVIII. "Best values" of physical properties of rabbit and fowl spermatozoa.

Table XXXIX. Preservation of motility of rabbit spermatozoa in various media.

Table XL. Radiographic contrast solutions evaluated for use as density gradient constituents in equilibrium centrifugation studies. 
Table XII. Effect of sodium iothalamate in egg yolk medium on the motility of rabbit spermatozoa. 
ABSTRACT

The objectives of this thesis include characterization of spermatozoa according to several physical properties (morphology, size, electrophoretic mobility, sedimentation rate and specific gravity), correlation of these properties with several biological properties (viability, intrinsic motility, fertilizing capacity, antigenicity and genetic composition) and an evaluation of interrelationships among these properties and with selected experimental variables. The major effort was devoted to stable-flow free-boundary (Staflo) sedimentation and electrophoresis studies and the use of the intrinsic motility of the spermatozoa to achieve relative or absolute enrichment of sperm sub-populations. A special microscopic optical system was developed to facilitate these studies.

Analytical (theoretical) methods were developed to clarify the requirements for optimum fractionation of cell populations and to allow separate determination of two or more of the properties sedimentation rate, electrophoretic mobility and intrinsic motility when they may all contribute simultaneously to a given migration proces3.

Temperatures of $3^{\circ} \mathrm{C}$ or lower were found necessary to eliminate the effects of intrinsic motility. At $3^{\circ} \mathrm{C}$ in low density-low viscosity buffers, the best measured values of physical properties (normalized to conditions of $1 \%$ sucrose) are as follows: Average sedimentation rate, rabbit: $0.53 \times 10^{-4} \mathrm{~cm} / \mathrm{sec} / \mathrm{cm} / \mathrm{sec}^{2}$. Average electrophoretic mobility, rabbit: $0.85 \pm 0.10 \times 10^{-4} \mathrm{~cm} / \mathrm{sec} / \mathrm{V} / \mathrm{cm}$; fowl: $1.65 \pm$ $0.10 \times 10^{-4} \mathrm{~cm} / \mathrm{sec} / \mathrm{V} / \mathrm{cm}$. At these low temperatures no significant differences were found among sedimentation or electrophores is collection 
fractions with respect to morphology, motility or viability of spermatozoa.

Above $4^{\circ} \mathrm{C}$, the relative contribution of intrinsic motility became significant in both sedimentation and electrophoretic studies, and depended upon both the temperature and the initial quality of the ejaculate. The relative (fowl) and absolute (rabbit) enrichment of sperm subpopulations with regard to motility, viability and fertilizing capacity achieved above $4^{\circ} \mathrm{C}$ was attributed to the intrinsic motilicy vector.

A separate study was made on the quality of rabbit ejaculates. This revealed an apparent differential sensitivity of cells in various stages of spermatogenesis to elevated environmental temperatures, with some deleterious effects seen as long as 7-8 weeks after particularly hot days.

In equilibrium density gradient centrifugation experiments using sodium iothalamate gradients, rabbit sperm formed three or more bands ranging from specific gravity 1.19 to 1.37. Absolute enrichment was obtained in the low density band (1.19-1.21) with regard to both motility and viability. Fowl spermatozoa formed a single band ranging from specific gravity 1.19 to 1.21 , within which there was a differential distribution of motile sperm (greatest motility at low density border).

In the limited fertility trials, the "low resolution" fractionation of rabbit ejaculates did not give a clear separation of $\mathrm{X}$ - and $\mathrm{Y}$ chromosome-bearing spermatozoa. Perhaps more significant, however, the physical properties measured in these experiments are inconsistent with the explanations given in the literature for the partial separation 
of rabbit and bu1l spermatozoa using electrophoretic and sedimentation methods. The present work does show that high resolution experiments are feasible and would give a much better test of the ability to separate $X$ - and Y-sperm according to one or more of the properties enumerated above. 


\section{INTRODUCTION}

Although man's interest in spermatozoa as cells dates from the initial report by Hamm and Leeuwenhoek in 1677, his interest in the processes controlling the sex of offspring far precedes their discovery. Gordon ${ }^{92}$ presents an excellent review of the literature of folklore on the subject. That the folklore is not just ancient history was evident from the Ann Landers column of the June 17, 1969 Berkeley (California) Gazette in which a reader recommended a method used successfully to control the sex of her children - "sleeping on the left side as soon as pregnancy is suspected yields a boy and vice versa."

The possibility of sex control has generated interest among sociologists, scientists and others concerned with genetics, animal husbandry and reproductive biology in general. Etzioni, in an article entitled "Sex, Science and Society", suggests that sex control is only one of many social problems arising from scientific investigation that should be of concern to everyone ${ }^{253}$. More recently in Life magazine, Rosenfeld focused on the subject ${ }^{272}$. For the more interested reader, Rorvik and Shettles have recently published a book presenting their ideas on sex control 274 . An important obvious consequence of the ability to control sex would be the possibility of eliminating sex-linked genetic disorders such as color blindness, diabetes insipidus, and hemophilia $A$ and $B$ from the population. A simple means of selecting the sex of offspring might also encourage the voluntary reduction of family size thereby assisting in the limitation of population growth. Many of the widespread social concerns related to the latter problem are summarized by Kiefer ${ }^{255}$.

Spermatozoa per se are of great scientific interest. Reasons 
include their dramatic morphological changes during spermatogenesis, their property of intrinsic motility and its relationship to fertilization and their significance in genetics.

The purpose of this project was to study the physical properties of spermatozoa, and their relationships to biological features, including possible sex control, utilizing some newer experimental methods of characterizing and fractionating cell populations. The stable-flow free-boundary (Staflo) method proved particularly useful in this regard. The rabbit was selected as the donor of mammalian spermatozoa for reasons summarized in Appendix A. For comparison, fowl spermatozoa were later added to the study.

Despite the reputation of rabbits for fecundity, a persistent and perplexing problem encountered was the variable initial quality and durability of ejaculate spermatozoa. Attempts to understand the dependence of this initial quality of spermatozoa on environmental variables, and to interpret the subsequent deterioration of spermatozoa in terms of experimental factors, became an important part of the study and illustrate many of the difficulties encountered in work with spermatozoa. It was eventually possible to develop conditions for media and manipulative procedures such that the "ultimate" biological assay system (obtaining offspring) worked with a high degree of reliability, provided that the initial spermatozoa sample was of high quality.

\section{Organization of Chapters}

Chapter II reviews the physical and biological properties of spermatozoa and the sex control literature, and discusses the 
Interrelationships among these properties and selected experimental variables.

Chapter III describes the basic Staflo method and additional ancillary equipment developed during this study. It also includes a detailed analysis of Staflo migration-fractionation principles which significantly extends the limits of analytical precision for this method and for other related fractionation methods.

Chapters IV through VII describe the evolution of the experimental work. Chapter IV presents the results of early Staflo-sedimentation experiments during which procedures for handing spermatozoa were developed. The temperature-dependent effects of motility on the sedimentation of rabbit spermatozoa are dramatically illustrated. Chapter V reports on the electrophoretic properties of rabbit and fowl spermatozoa as well as on the effects of temperature and sperm quality on electrophoretic migration. Chapter VI presents the evaluation of media for use with fowl spermatozoa and the results of electrophoresis and electrophoresis-enrichment experiments with these media. Chapter VII 18 devoted to equilibrlum density gradient centrifugation of fowl and rabbit spermatozoa and shows both similarities and differences in the blophysical propertles of the two cell types. Chapter VIII presents a series of milti-characterization experiments with rabbit spermatozoa, exploring their separate and combined sedimentation and electrophoretic properties. This work also explicitly demonstrates the importance of the initial sperm quality for eventual fertilization. Chapter IX discusses differences in measured sperm quality according to the method used for evaluation, and also shows significant prolonged and intermittent effects of elevated environmental tempera- 
tures on the initial quality of ejaculate spermatozoa.

The final Chapter $X$ presents overall conclusions and recommendations derived from this study. 
II. INTERRELATIONSHIPS AMONG PHYSICAL AND BIOLOGICAL PROPERTIES OF SPERMATOZOA AND IMPORTANT EXPERIMENTAL VARIABLES

\section{Contents}

A. Introduction. . . . . . . . . . . . . . . 6

B. Physical Properties... . . . . . . . . . . . 6

1. Morphology and Size . . . . . . . . . . . . . 8

2. Electrophoretic Mobility. . . . . . . . . . . . 15

3. Sedimentation Rate. . . . . . . . . . . . . . . 21

4. Specific Gravity. . . . . . . . . . . . . . . 23

C. Biological Properties . . . . . . . . . . . . . 28

1. Vlability .................... . . 28

a. Vital Staining. ................. 28

b. Metabolism. . . . . . . . . . . . . . 30

c. Factors Affecting Spermatozoa Production and Quality. . 31

d. Seasonal Variations in Spermatozoa Quality. . . . . 35

2. Intrinsic Mot1lity. . . . . . . . . . . . . . 36

a. Spermatozoa Concentration ............ 36

ь. pH. . . . . . . . . . . . . . . . . 38

c. Ionic Strength and Osmolarity of the Medium . . . . . 39

d. Temperature ................ . . . 40

e. Stage of Maturation ................. 41

f. Fluctuation in the Swimming Rates of Individual 42

Spermatozoa . . . . . . . . . . . . . 42

3. Fertilizing Capacity. . . . . . . . . . . . . 43

4. Antigenicity. . . . . . . . . . . . . . . . 45

5. Genetic Composition .................. 19

D. Sumary. . . . . . . . . . . . . . . . . 50 


\section{A. Introduction}

The great interest in studying mammalian spermatozoa undoubtedly arises from the active role of that cell type in reproduction, especially in the control of sex by virtue of the presence of elther an X- or Y-chromosome in the Individual spermatozoon. In addition, 1ts property of intrinsic mot111ty makes the spermatozoon an exciting object to study and provides a practical biological: assay system.

In reviewing the literature on work with spermatozoa, it is convenient to consider the physical and blological properties separately, even though the purpose of this thesis is to integrate them. The various categories of physical and blological properties, Iisted in Table I, are discussed in Sections B and C, below. An early attempt to discuss, in a separate section, the interrelationshipo among these properties and the wany experlmental vartabies (also listed in Table I) was abandoned in favor of emphasizing the interrelationships when approprlate in Sections $B$ and $C$. The importance of these interrelationships is illustrated in many of the subsequent chapters.

Appendix B contains additional information on work with germ cells Including 1) the collection and handling of spermatozoa, 2) the normal fertility of the female rabbit, 3) fertilization IN VIVO and IN VITRO, 4) technical considerations in artificial insemination and 5) methods for sexing offspring.

\section{B. Physical Properties}

Physical methods used to study spermatozoa include 1) electrophoresis, 
Table I. Interrelattonships Among Physical and Biological Properties of Spermatozoa and Important Experimental Variables

\section{Physical Properzies}

Morphology and size

Electrophoretic Mobility

Sedimentation Rate

Specific Gravity

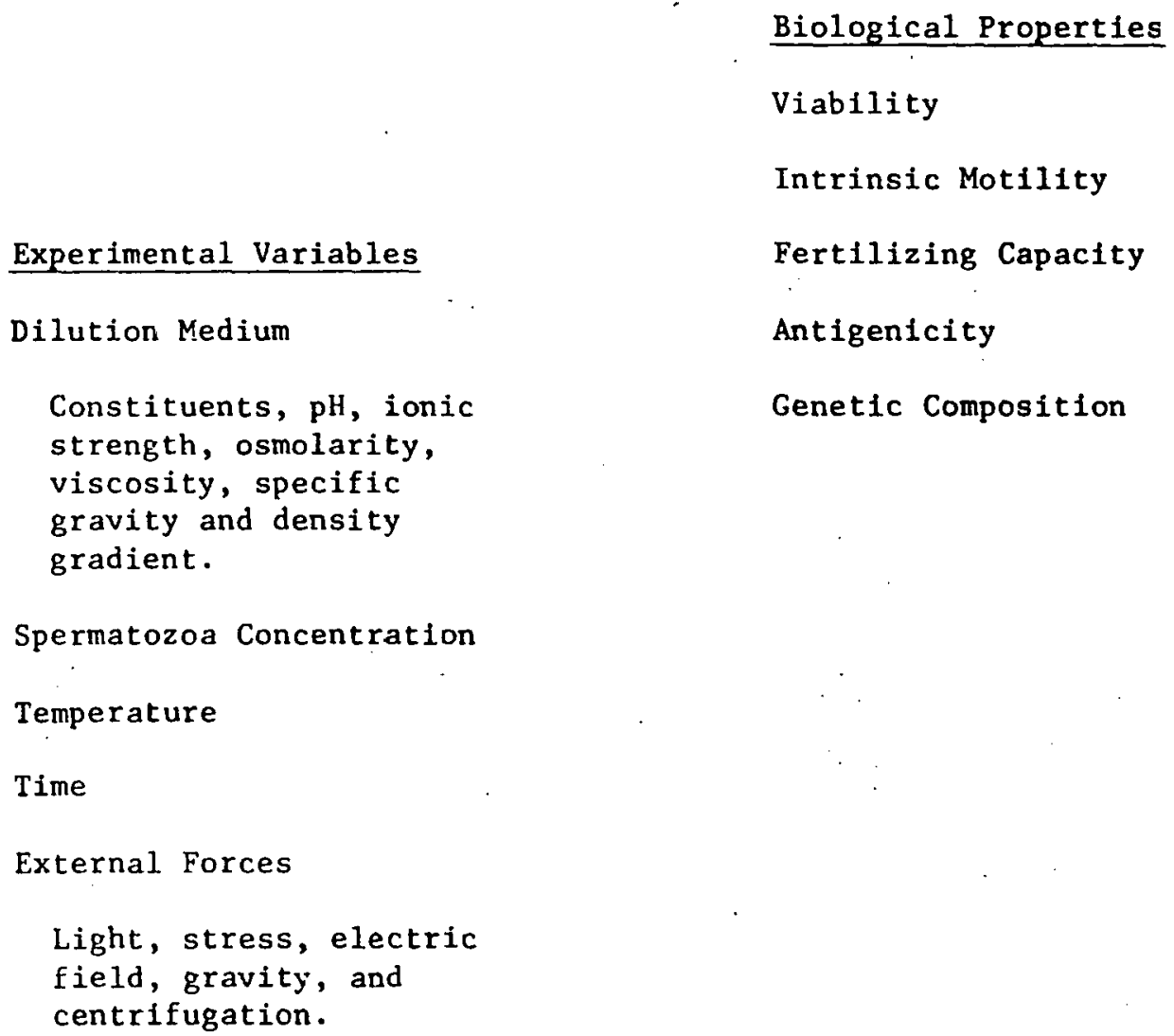

Experimental Variables

Dilution Medium

Constituents, $\mathrm{pH}$, ionic strength, osmolarity,

viscosity, specific gravity and density gradient.

Spermatozoa Concentration

Temperature

Time

External Forces

Light, stress, electric

field, gravity, and

centrifugation.

Fertilizing Capacity

Antigenicity

Genetic Composition 
2) sedimentation and froth flotation and 3) differential and equilibrium density gradient centrifugation. The physical properties upon which these techntques depend include morphology and size, electrophoretic mobility, sedimentation rate, and specific gravity. Each of these properties is considered separately below.

1. Morphology and Size.

Information on spermatogenesis and the morphology of mamalian spermatozoa can be found in the works by Bishop and Walton ${ }^{42}$, by Ortavant ${ }^{183}$ and by Austin ${ }^{13}$, and on fowl spermatozoa in the works by Lorenz ${ }^{140}$ and by Sturkie 220 . A detailed discussion of the fine structure of the rabbit spermatozoon is given by Bedford ${ }^{25}$. The discussion in this section is restricted to considerations of certain aspects of the morphology and size of fowl and rabbit spermatozoa primarily, and to the relationships of these features to the physical and biological properties of spermatozoa and experimental parameters.

Rabb1r Spermátózoa. Figure 1 is a composite drawing of a rabbit spermatozoon prepared trom both light and electron microscopy observations. The overall length of the rabbit spermatozoon varies from 60-70 microns with head and midplece lengths of approximately 8-10 and 10 microns, respectively. The head is flat with a width of 4-5 microns and a thickness of approximately 1 micron. The average head lengths of spermatozoa from the vas deterens and the efaculate are abuut the same 22 . The breadth and area measurements of heads of spermatozoa vary little among males of the same litter, but vary markedly among the means of litters ${ }^{22}$. The weight of the rabbit and the environment have 11ttle influence on these parameters ${ }^{170}$. Among males of a litter, the head length, percent capless, and percent stalned spermatozoa all vary markedly, whlle the variations among the litter 


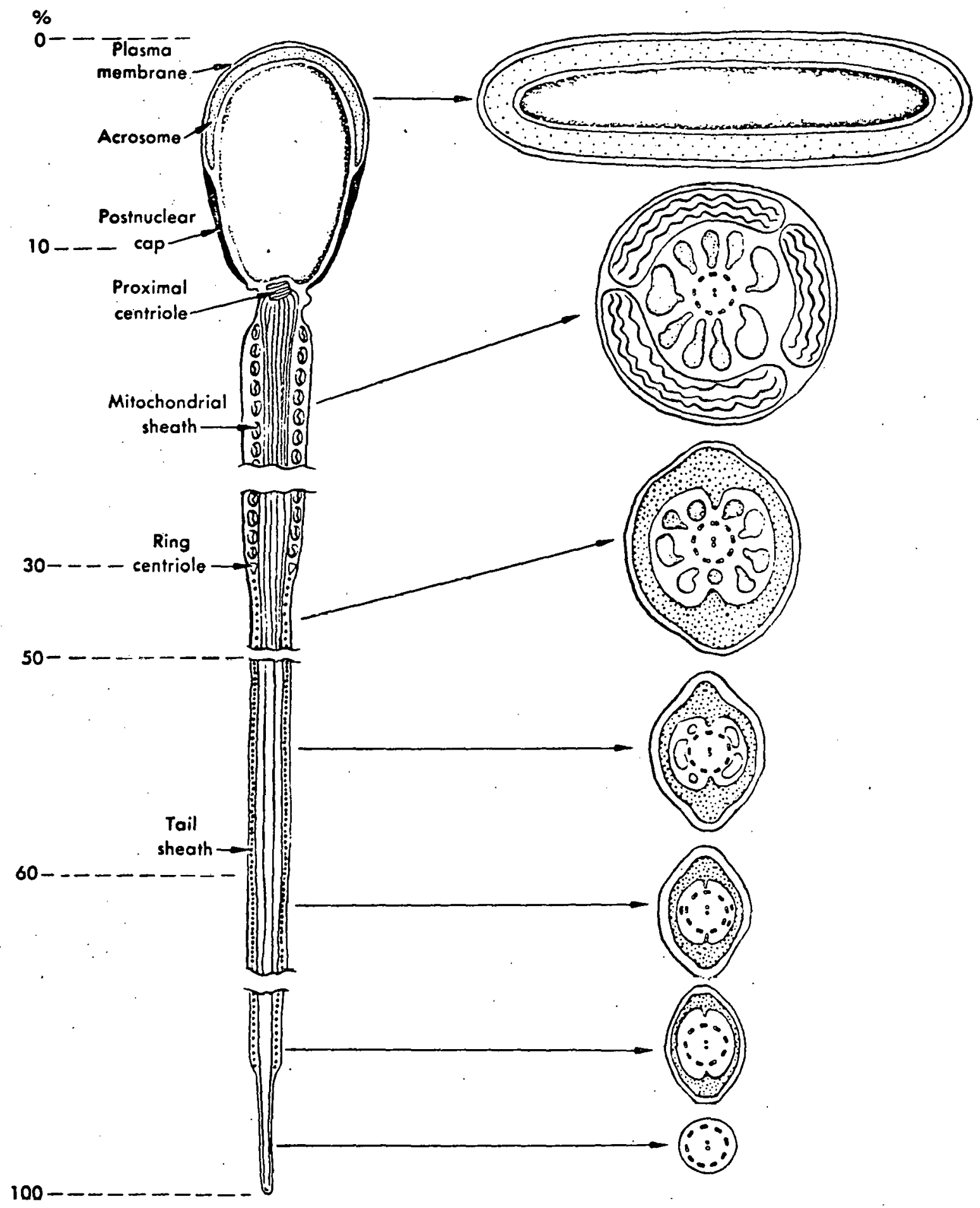

DBL 70115966

Fig. 1. Structure of the rabbit spermatozoon. 
means is smal1 22 . There is a positive association between the head length of normal, unstained spermatozoa and fertility ${ }^{170}$.

Shettles ${ }^{213}$ describes 2 populations of human spermatozoa distinguished by elliptical and concentric configurations of light diffraction patterns in small and large heads, respectively, under phase contrast microscopy. He hypothesizes that the Y-chromosome is found in the smaller head and the $\mathrm{X}$-chromosome in the larger head. This observation is questioned by Rothschild 197 and by Bishop 41 .

Gordon observed head length dimorphism of the rabbit spermatozoa populations he studied but found no correlation of the size dimorphism with the sex ratio of offspring from the insemination of 2 groups of spermatozoa obtained by electrophoretic fractionation ${ }^{92}$. Bhattacharya reports that the length and breadth measurements of the heads are less for spermatozoa obtained from the top than from the bottom tractions of his gravity sedimentation device. 35

Iversen describes a surface reflection interference microscopy technique which may provide more accurate measurements of the size of spermatozoa ${ }^{121}$. Bahr and Zeftler describe a method of quantitative electron microscopy permitting the recording of the mass cross-section (total mass per unit length) of an object, making possible determinations of the distribution of the total mass of very long and narrow structures ${ }^{17}$. They estimate that the distribution of mass in the bull spermatozoon among the head, tail and midpiece are $63.7 \pm 3.1 \%, 36.3 \pm 1.0 \%$ and $17.3 \pm .05 \%$, respectively. Table 4 of their article presents a sumary of measurements of bull spermatozoa structures from the literature ${ }^{17}$.

Referring to Figure 1, the spermatozoon head is surrounded by a plasma 
membrane which appears to be continuous over the midpiece and over most of the length of the tail. The plasma membrane appears to be applied more loosely and to have greater stickiness over the acrosomal cap region than over the post-nuclear cap region ${ }^{25}$. The continuity of the plasma membrane over most of the spermatozoon is an important consideration with regard to surface charge, which is considered in another section. The anterior portion of the nuclear material is surrounded by a dense acrosomal cap which has its own Inner and outer cytoplasmlc membranes. The integrity of the acrosomal cap appears to be essential for the maintenance of fertilizing capactty and is a key morphological feature in assessing viability of spermatozoa. Hereditary abnormalities of the acrosomal cap are associated with sterility in bulls 273 .

Bedford has shown that during passage of spermatozoa through the epididymis, there is a decrease in acrosome width and length, which is correlated with the movement of the cytoplasmic droplet from the base of the sperm head to the center or distal portion of the midpiece ${ }^{26}$. This reduction in acrosome size appears to be related to the acquisition of fertilizIng ability, as shown by a significantly higher conception rate with the Insemination of spermatozoa from the cauda epididymis than trom the caput epldidymis $^{26}$. The histology of the epididymis is described very well by Nicander ${ }^{175^{\circ}}$.

Austin found no morphological difference between epididymal spermatozoa and sperm recovered from the fallopian tube ${ }^{13}$. Bedford suggests that the plasma membrane may be separated more easily from the anterior and lateral edges of the underlying acrosomal cap in uterine sperm than in spermatozoa from the ejaculate ${ }^{25}$. Uterine spermatozoa also showed the presence of small particles apposed to, and probably adherent to, the plasma membrane over the 
acrosome. He suggest that this adhesiveness may help to keep the spermatozoa attached to the ovum ${ }^{25}$. In disintegrating spermatozoa, the inner acrosomal membrane and the apical body always remained intact. The apical body was observed to persist in spermatozoa penetrating the zona pellucida at which time the material of the acrosomal cap had almost disappeared ${ }^{13}$.

The molecular structure of spermatoroa is being elucidated by studies such as those concerning (1) the distribution of metabolic activity, phuspholipid and hyaluronidase between the heads and tails of bull spermatozoa ${ }^{147}$, (2) the distribution of ATP artivity in ram and bull opcrmatosoa ${ }^{191}$, the phospholipid composition of rat and ram epididymal spermatozoa 62 , the chemlcal composition of the acrosomes of ram opcrmatoroall, (5) tlie chemical composition of bull spermatozoa ${ }^{33}$, (6) free amino acids in the semen and spermatozoa of the fowl and turkey ${ }^{6}$, and (7) free carbohydrates In the seminal plasma and sperm of fow ${ }^{7}$. Microspectrophotometric techniques have been used to determine the DNA composition of rabbit ${ }^{45}$ and bul1 241, 242 spermatozoa and blochemical techniques have been used tn study the DNA composition of fowl spermatozoa ${ }^{1 U i}$.

Welch, Hanly and Guest found a significant correlation between the DNA content of spermatozoa measured microspectrophotometrically and the motility and sperm count, and, to a lesser extent, the morphology of the semen spermatozoa from bulls ${ }^{242}$. Compared to bulls showing the greatest varlation in DNA content, bulls showing the least variation had higher quality spermatozoa samples judged by the degree of motility, morphology and sperm count ${ }^{242}$. Baker and Salisbury found that cytophotometrically determined Feulgen DNA values in fresh bovine spermatozoa were distributed normally ${ }^{268}$. Spermatozoa stored for 11 days at $5^{\circ} \mathrm{C}$ in an egg yolk-citrate buffer had a normal distribution of DNA values, but had a lower mean value 
and a larger standard deviation than the fresh spermatozoa. Assuming an equal contribution of Individual chromosomes to the Feulgen stained materfal and an equal probability of nondisjunction among all chromosomal pairs, $10 \%$ nondisjunction is needed to explain all of the observed withinsample Feulgen DNA biological variance. Because $10 \%$ nondisjunction is unacceptably high, they concluded that aneuploidy contributes little to normal Feulgen-DNA variance in spermatozoa and that the major sources of biological DNA variance among spermatozoa in single, normal ejaculates are related to variations in sperm age and to the extent of autolysis of dead and dy1ng cel1s 268 .

Bouters and coworkers attributed the variable Feulgen-DNA content of Individual ejaculated spermatozoa to varying percentages of older sperm cells in the ejaculates 45 . They found no significant differences among 3 consecutive ejaculates, with respect to Feulgen-DNA content, in 15 experimental rabbits. Aging appeared to have no influence on the UV-DNA of spermatozoa. They suggest that the decrease in Feulgen-positive material which occurs in spermatozoa during the passage along the male tract results from structural changes in $\mathrm{DNA}^{45}$.

Fowl Spermatozoa. Fowl spermatozoa, illustrated in Figure 2, are approximately $140 \mathrm{microns}$ long. The cylindrical sperm head is approximately 14 microns in length and 0.5 microns in diameter, while the midpiece is approximately 4 microns long.

According to Lorenz, the cytoplasmic membrane over the head is continuous over about two-thirds of the midpiece ${ }^{140}$. The acrosomal cap is present but less conspicuous in fowl than in rabbit spermatozoa. The tail is covered by an amorphous sheath which gradually becomes thinner distally and ends about $2 \mathrm{microns}$ from the tip of the tail ${ }^{140}$. As shown in Figure 2, 


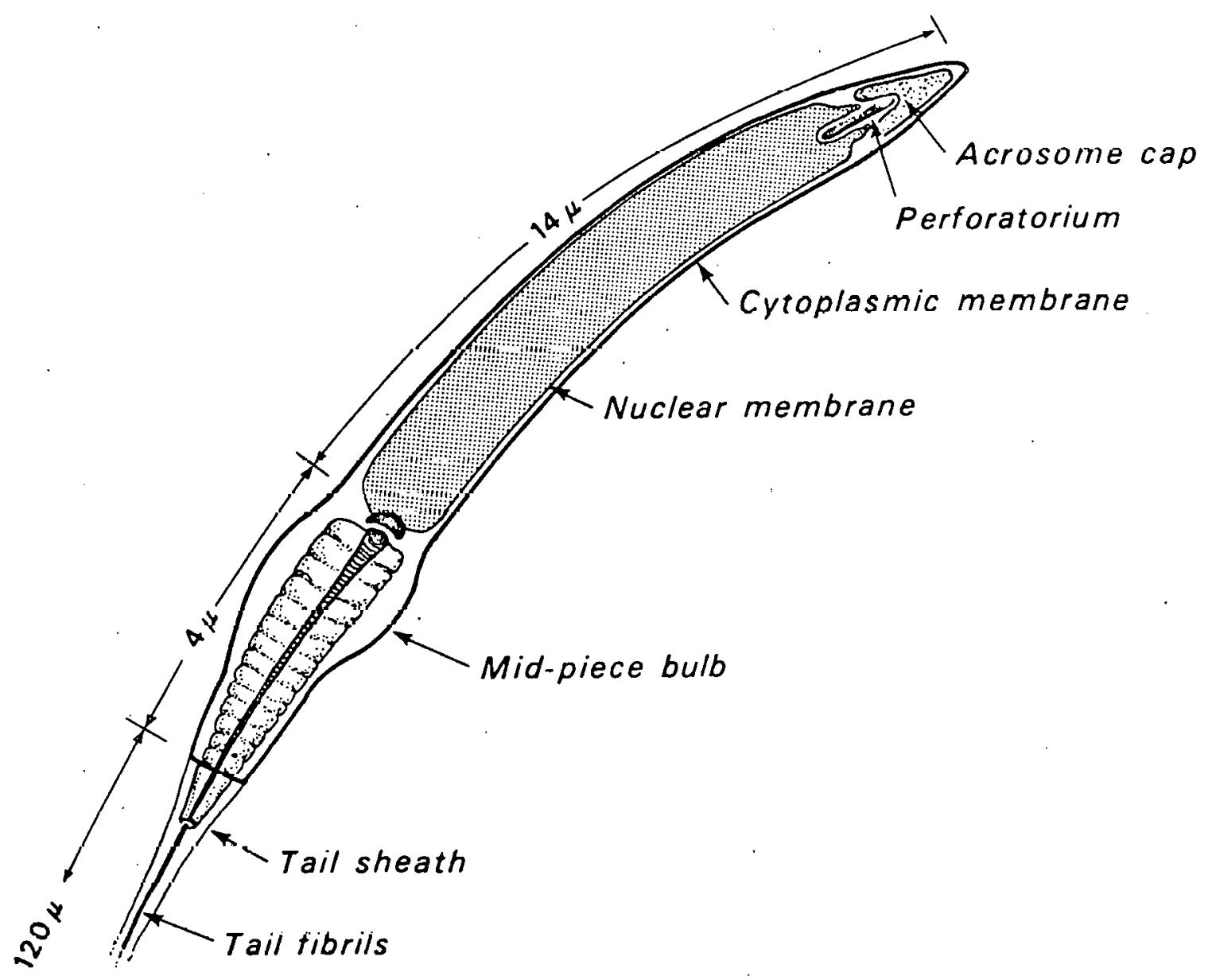

DBL 70115967

Fig. 2. Structure of the fowl spermatozoon. 
fowl spermatozoa have a small acrosomal cap. Trypsin completely removes the cytoplasmic membrane around the head, permitting disorganization and at least partial digestion of the contents, while the head is little affected by pepsin treatment 140 .

\section{Electrophoretic Mobility.}

Schröder reported electrophoretic separation of rabbit spermatozoa Into two populations using a sperm dilution of $1: 15$ in a glycine-Ringer buffer at $\mathrm{pH} 7.10$ in a U-shaped electrophoresis cel1 ${ }^{206}$. Insemination of spermatozoa which migrated toward the anode gave predominantly female offopring, whereas cathode spermatozoa gave predominantly male offopring. The number of $\mathrm{Y}$-chromosome bearing spermatozoa obtained from the cathode was a maximum at $10^{\circ} \mathrm{C}^{206}$. Blochemical and Immunological studies tended to confirm the blological results 207 .

Gordon tested Schröder's experiments, using a similar U-shaped electrophoresis cell w1th a glycine-Ringer buffer at $\mathrm{pH} 7.10$ and $15^{\circ} \mathrm{C}^{91,92}$. The electrophoresis time was 45-50 minutes, and, for any one experiment, spermatozoa could be obtained only from one electrode. Insemination of spermatozos recovered from the anode and cathode gave predominantly female and male offspring, respectively. Upon applying the electric field, he observed immediate and passive orientation of previously active and randomly orlented spermatozoa such that they moved tail first toward the anode 92 . Lewin also reported two-way electrophoretic migration of rabbit spermato$20 \mathrm{a}^{135}$, but other Invest1gators have shown that, at $\mathrm{pH} 5-8$, all bull, ram and rabbit spermatozoa have a net negative charge $18,174,233$.

Vesselinovitch reported that Imotile bovine spermatozoa, when suspended in Krebs-Henselelt-Ringer buffer or modifled Schröder buffer always migrated electrophoretically towards the anode ${ }^{233}$. He concluded 
that bovine semen does not represent a mixture of spermatozoa of two distinct electrical densities which would migrate to opposite poles when suspended in buffer solution of a $\mathrm{pH}$ intermediate to their isoelectric points. He observed also that with an electric field of the proper magnitude, spermatozoa are recovered from both the anode and the cathode due to: 1) electrophoretic movement of immotile spermatozoa toward the anode, 2) active swimming of spermatozoa of medium motility toward the cathode after undergoing galvanotaxis and 3) active swimming of highly motile spermatozoa toward both electrodes at random. Variations in the strength of the electric field would be reflected primarily in the distribution of the second group of spermatozoa. Because of the random contribution of highly motile spermatozoa to the sperm population obtained from both electrodes, and the equal probability of their being X- or Y-chromosome bearing spermatozoa, Vesselinovitch belleves that the sex ratio will depend upon the ratio of $\mathrm{X}$ - to $\mathrm{Y}$-chromosome bearing spermatozoa in the inftial sample 233 . MacPherson and Vesselinovitch obtained no significant difterence in the sex ratio of offspring from cattle inseminated with spermatozoa from anodic and cathodic electrophoretic fractions ${ }^{146}$.

Nevo, Michaell and Schindler stressed the importance of immohilizing spermatozoa prior to making electrophoretic measurements ${ }^{174}$. They inactivated spermatozoa by the use of low temperature $\left(4-5^{\circ} \mathrm{C}\right)$ or by the use of $5 \% \mathrm{CO}_{2}$ at room temperature. Neither method had adverse effects on the spermatozoa. Using a Northrop-Kunitz microelectrophoresis apparatus, they found that the electrophoretic mobilities of bul.j spermatnzna were essentially the same whether the spermatozoa were in seminal plasma, egg yolkcitrate-glycine buffer with diluent or had been washed up to four times in diluent. They concluded that no substances which contributed to the 
surface charge were removed from the surface of the cells by the washings or were adsorbed from the egg yolk-glycine-citrate buffer. Their observations showed that both before and after dilution and washing, originally immotile spermatozoa and those inactivated by the low temperature were all negatively charged and that they had essentially the same electrophoretfc properties. The isoelectric point was approximately $\mathrm{pH} 3.5$ for both immotile and reversibly 1mnobilized spermatozoa. Additional work with rabbit spermatozoa showed an 1soelectric point of approximately $\mathrm{pH} 3.5$. They saw no two-way migration with either species between $\mathrm{pH} 2.5$ and $\mathrm{pH}$ 8.5 in any of the buffers used. They obtained electrophoretic mobilities for bull spermatozoa suspended in seminal plasma and in diluent, after 4 washings, of 0.89 and $0.88 \times 10^{-4}(\mathrm{~cm} / \mathrm{sec}) /(\mathrm{volt} / \mathrm{cm})$, respectively, with an electric field strength of $5.4 \mathrm{~V} / \mathrm{cm}$ at $4-5^{\circ} \mathrm{C}^{174}$.

Bangham studied the electrophoretic characteristics of ram and rabbit spermatozoa over a wide range of $\mathrm{pH}^{\text {and }}$ ionic strength ${ }^{18}$. In a solution consisting of $1 \mathrm{mM} \mathrm{NaCl}$ and $277 \mathrm{mM}$ fructose at an electric field strength of approximately $1.2 \mathrm{~V} / \mathrm{cm}$, he observed (1) that motionless (dead) ram spermatozoa showed no particular orientation in the electric field and moved as though they had a uniform negative charge over the1r entire surface, (2). that certain spermatozoa had a greater negative charge density on their talls than on their heads (which oriented their tails toward the anode), and, depending upon the magnitude of the electrophoretic component relative to their intrinsic forward velocity, the spermatozoa swam toward the cathode or were pulled toward the anode, and (3) that head anode spermatozoa had a greater negative charge density on their heads than on their tails and, therefore, moved toward the anode at a rate determined by their intrinsic forward velocity and the electrophoretic 
component in the same direction. In this $1 \mathrm{mM} \mathrm{NaCl}$ medium from $\mathrm{pH} 4.8-7.5$; the percentage of head anode spermatozoa was $75 \%$ or greater, whereas an increase in $\mathrm{NaCl}$ concentration to $10 \mathrm{mM}$ led to a reduction in the percentage of head anode spermatozoa from $70 \%$ at $\mathrm{pH} 4.6$ to less than $10 \%$ at $\mathrm{pH}$ 5.8 In the $10 \mathrm{mM} \mathrm{NaCl}$ solution at $\mathrm{pH} \mathrm{4.9,} \mathrm{approximately} \mathrm{equal} \mathrm{numbers} \mathrm{of}$ head anode and tail anode spermatozoa were present. In $145 \mathrm{mM} \mathrm{NaCl}$ all spermatozoa were tail anode and the net electrophoretic charge on the spermatozoa varled only slightly over the range $\mathrm{pH} 4$ to 7 . In a glycineRinger buffer or In $145 \mathrm{mM} \mathrm{NaCl}$ over this $\mathrm{pH}$ range, no tail cathode spermatozoa were observed with samples of ram or rabbit spermatozoa at intermediate temperatures. At $8^{\circ} \mathrm{C}$ when spermatozoa are reversibly immobilized, all spermatozoa were tail anode type. At $25^{\circ} \mathrm{C}$, the sperm appeared to be of two types, but Bangham explains this by the relative effects of the electric field and the intrinstc movement of the spermatozoa ${ }^{18}$.

Bangham concluded that separation of cooled, motionless spermatozoa would be impractical because of the small difference in the magnitude of the negative charge on the tails and the heads, but that separation would be enhanced if swimming spermatozoa were used and the contrasting effect of the electrophoretic component on the tail and head exploited. Thus, by a suitable cholce of electric fleld strength, those spermatozoa with a net negative charge on their tails could be held stationary or allowed to progress toward the cathode, while those with the net negative charge on thelr heads would move rapidly toward the anode ${ }^{18}$.

Work by Joel and coworkers showed no significant difference in electrophoretic mobility of spermatozoa from normal and pathological human semen. The electrophoretic mobility of normal human spermatozoa ranged from $0.61-$ $0.86 \times 10^{-4}(\mathrm{~cm} / \mathrm{sec}) /(\mathrm{V} / \mathrm{cm})$ at $20^{\circ} \mathrm{C}$ and $\mathrm{pH} 7.8^{122}$. 
Laird studied the electrophoretic properties of bull spermatozoa using a phosphate diluent and buffer at $\mathrm{pH} 7.0$ with a microelectrophoresis chamber similiar to that used by Gordon ${ }^{129}$. At the end of an 8-hour electrophoresis run, he observed the distribution of spermatozoa characterized morphologically by a collar at the base of the head to be approximately $100 \%$ at the cathode and only $5 \%$ at the anode. The initial percentage of spermatozoa with collars was approximately $50 \%$. The viability of the spermatozoa at the end of the experiment was extremely poor at both poles of the electrophoresis chamber. Spermatozoa recovered from the anode were longer than those recovered from the cathode, but both categories of separated spermatozoa were shorter than the unseparated sperm. He concluded that the spermatozoa migrated in two different directions in the electric field ${ }^{129}$.

The change in electrophoretic mobility of spermatozoa when passing through the epididymis was measured by Bedford with a microelectrophoresis apparatus using a spermatozoa concentration of $2 \times 10^{6} / \mathrm{ml}$ in a $0.145 \mathrm{M}$ NaCl solution ( $\mathrm{pH}$ not given) at $4^{\circ} \mathrm{C}^{24}$. He obtained mobilities of 0.3468 $\times 10^{-4}$ and $0.5363 \times 10^{-4}(\mathrm{~cm} / \mathrm{sec}) /(\mathrm{V} / \mathrm{cm})$ for spermatozoa from the caput and corpus eptdddymis, respectively, and $0.5371 \times 10^{-4}(\mathrm{~cm} / \mathrm{sec}) /(\mathrm{V} / \mathrm{cm})$ for spermatozoa from the cauda epididymis, vas deferens or washed, mature sperm from an ejaculate. Spermatozoa from the caput epldidymis were primarily head anode with some random orlentation and less than $10 \%$ tail anode. Spermatozoa from the corpus epididymis were primarily tail anode; however, there was some random orlentation and, occasionally, head anode spermatozoa. The orfentation of spermatozoa from the cauda epididymis and the ejaculate was primarily tall anode. With reversal of the direction of the electric fleld, the reorientation of spermatozoa from the caput epididymis took 3-4 minutes for Individual spermatozoa and from 5-6 minutes for the entire 
fleld of spermatozoa. The reorientation of spermatozoa from the corpus epididymis took 2-4 minutes for individuals and 3-4 minutes for the entire fleld. Both individual and the entire field of opermatozoa from the cauda epididymis, vas deferens and ejaculate took only 1-1.5 minutes to reorient ${ }^{24}$.

In a solution of $6 \%$ fructose, cytoplasmic droplets appear to possess electrophoretic properties similar to those of spermatozoa heads, both of which are more strongly attracted to the anode than is the distal part of the $\operatorname{tail}^{24}$.

Bey reports that, after washing fresh spermatozoa 3 times with 1sotonic $\mathrm{NaCl}$ at $4^{\circ} \mathrm{C}$, most bull, boar, rabbit and cock spermatozoa had lost their intrinsic motility ${ }^{251}$. In a low ionic strength buffer in a rectangular electrophoresis chamber, she obtained electrophoretic mobilities as follows: (units of $10^{-4} \mathrm{~cm} / \mathrm{sec} / \mathrm{V} / \mathrm{cm}$ ) bull, $1.302 \pm 0.031$; rabbit, $1.121 \pm 0.022$ and cock, $1.545 \pm 0.020^{251}$.

Sevinç (1968) observed two-way migration of rabbit spermatozoa using an intermittent electric fleld and motile spermatozoa at temperatures of 29 aind $30^{\circ} \mathrm{C}$ at pH 7.1 la 3 und 7 chambered electrophoretic cells ${ }^{210}$. In one experiment using 3 different buffers, the insemination of spermatozoa recovered from the anode gave 19 offopring, 15 (79\%) of which were male, while insemination of spermatozoa recovered from the cathode gave 68 offspring, $26(38 \%)$ of which were males. The difference between the expected normal sex ratio and the sex ratios of the offspring obtained in thic experiment was highly bignificant. However, in 3 subsequent experiments, only a slightly higher percentage of male offspring was born following the insemination of spermatozoa which migrated both to the anode and to the cathode 210 . In view of the conflicting results of the several experiments, no conclusions could be drawn with regard to the possibility of separating the $\mathrm{X}$ - and $\mathrm{Y}$-chromosome bearing spermatozoa by this technique. 
From the description of the apparatus given, it does not appear that observations of the spermatozoa in the electric field could be made. With the media and conditions he used, he achieved excellent fertility and a high offspring per litter ratio.

\section{Sedimentation Rate.}

Using a sedimentation apparatus consisting of a $10 \mathrm{ml}$ burette with a $1.1 \mathrm{~cm}$ diameter, Bhattacharya observed that nearly $30 \%$ of the spermatozoa placed at the top of the burette sedimented a distance of $10 \mathrm{~cm}$ in 24 hours at $0{ }^{\circ} \mathrm{C}$ through a medium of spectfic gravity 1.0012 and viscosity 17.956

centipolses (cp) ${ }^{36}$. Approximately $70 \%$ of the spermatozoa sedimented the same distance in 24 hours at $0^{\circ} \mathrm{C}$ through a medium of specific gravity 1.0025 and viscosity $13.436 \mathrm{cp}$. The specific gravities and viscosities of the media were determined at $21^{\circ} \mathrm{C}$. The "1ight" and "heavy" media contained 5\% glycine solution plus egg yolk in ratios of $1: 2$ and $1.75: 2$; respectively. At the end of a 24-hour sedimentation time, the bottom 1-2 ml of the column of high density medium contained from $9.7 \%$ to $14.4 \%$ and the top $2 \mathrm{ml}$ contalned $5 \%$ to $16.5 \%$ of the total number of spermatozoa. Insemination of the spermatozoa from the bottom $2 \mathrm{ml}$ resulted in 22 offspring, 17 (77.3\%) of which were females and spermatozoa from the top $2 \mathrm{ml}$ gave a total of 14 offspring, $12(85.7 \%)$ of which were males. Insemination of spermatozoa recovered near the middle of the sedimentation column gave approximately $50 \%$ of each sex ${ }^{36}$.

Bhattacharya repeated his sedimentation experiment, using a medium consisting of $42.5 \%$ egg yolk in a glycine-citrate buffer with a specific gravity of 1.0304 and a viscosity of $75 \mathrm{cp}^{35}$. Approximately $200 \times 10^{6}$ spermatozoa were placed upon a column of medium $10 \mathrm{~cm}$ high, and, after 12 hours at $0^{\circ} \mathrm{C}$, spermatozoa were distributed over the top $6 \mathrm{~cm}$ of the column. 
Insemination of $1-20 \times 10^{6}$ spermatozoa from the top 2 fractions gave $77.4 \%$ male offspring, and spermatozoa from the bottom 2 fractions gave $28.2 \%$ male offspring. Insemination of spermatozoa from the intermediate fractions gave near normal sex ratios. All inseminations resulted in a low percenage fertilization. The dimorphism observed by Bhattacharya suggested that the spermatozoa with the greater volume sedimented at a faster rate and gave a predominance of female offspring ${ }^{35}$.

Bhattacharya ${ }^{3}$ reported a partial bimodal distribution of rabbit and bull spermatozoa and a unimodal distribution of fowl spermatozoa in the sedimentation apparatus ${ }^{35}$. The sedimentation rate of fowl spermatomoa was significantly less than that of the rabbit or bull spermatozoa. He had not yet investigated the relationship of sedimentation rate to specific gravity or to the DNA content of the spermatozoa 35 .

Bhattacharya, Bangham, Cro, Keynes and Rowson applied Bhattacharya's sedimentation technique to the separation of bull spermatozoa. Eighty-four of 173 (48.6\%) oftspring obtained in 3 groups of inseminations were males, or nearly a $1: 1$ sex ratio 37 .

Schilling fractionated bull spermatozoa using equipment similar to that used by Bhattacharya and a medium consisting of skimmed milk, salt solutions and egg yolk with a specific gravity ranging from 1.0.037-1.044 and a viscosity ranging from $7-10 \mathrm{cp}^{205}$. Sixty minutes after being layered on top of the column at $0^{\circ} \mathrm{C}$, the spermatozoa appeared to be distributed in a single peak over 10-12 fractions. No additional migration was observed after 60 minutes. The spermatozoa in the different fractions were concentrated by centrifuging at 4,000 rpm for 15 minutes to make possible insemination of a high number of spermatozoa in a small volume. Insemination 
of spermatiozoa from the lowest fractions resulted in 86 calves, 60 (69.8\%) of which were females compared to the normal ratio of cattle of 123 males to 100 females $(44.8 \%)$ in artificial insemination ${ }^{205}$.

Bedford and Bibeau found no difference in the sex ratio of rabbits resulting from the insemination of spermatozoa fractionated by gravity sedimentation using a device and medium similar to those used by Schilling ${ }^{29}$ The specific gravity and viscosity of the medium at $2^{\circ} \mathrm{C}$ were 1.048 and $6.7 \mathrm{cp}$, respectively. The total height of the medium in the burette was $19 \mathrm{~cm}$. Spermatozoa in $1.0 \mathrm{ml}$ of the medium were layered on top of the column and twenty fractions of $1 \mathrm{ml}$ each were recovered from the column at the conclusion of the sedimentation time. In one series of experiments with a 2-hour sedimentation time, spermatozoa inseminated from the bottom 4 fractions of the sedimentation column resulted in 35 male and 27 female offspring. In another series of experiments with a 1-hour sedimentation time, insemination of the bottom 2 fractions resulted in 22 male and 21 female offspring. Insemination of the spermatozoa from the top 2 fractions recovered after 2 hours of sedimentation resulted in 15 male and 19 female offspring ${ }^{29}$. In a figure showing the distribution of spermatozoa throughout the sedimentation column in $\underline{8}$ trials, one profile suggested a unimodal distribution, 1 or 2 a bimodal distribution and the remainder were more representative of uniform distributions.

\section{Specifle Gravity.}

In 1925, Lush Investigated the possibility of sex control by artificial Insemination of centrifuged spermatozoa employing a differential centrifugation technique ${ }^{143}$. His observations showed no significant difference in the sex of offspring obtained by insemination of spermatozoa samples fractionated by this technique. His results suggested a possible 
dimorphism in measurements of head lengths. However, the difference was very small and he could not show a relationship between differential migration rate and head length ${ }^{143}$.

Lindahl and Kihlström studied alterations in the specific gravity of bull spermatozoa during ripening ${ }^{138}$. Spermatozoa from 3 ejaculates collected from each bull in immediate succession were centrifuged for 10 minutes at $2,300 \mathrm{~g}$ In hematocrit tubes over solutions of different concentrations of the methylglucamine salt of umbradil (umbradilic acid). The percentage of unripe spermatozoa increased while that of overripe spermatozoa decreased from the first to the third ejaculate in each series. The percentage of ripe spermatozoa was nearly equal in each of the 3 ejaculates constituting a series. The specific gravity of the spermatozoa ranged from about 1.24-1.35 and the mean specific gravities of the first, second and third ejaculates were $1.2867 \pm 0.0022,1.2897 \pm 0.0040$, and $1.2668 \pm 0.0048$, respectively. The distribution of speciflc gravities appeared to be unimodal around the mean. From the distribution of the unripe, ripe and overripe spermatozoa, the authors concluded that the density of spermatozoa increases during ripening ${ }^{138}$.

Using test solutions of umbradilic acid in water, Kihlstrom found the specific gravity of the tall and middle pieces of bull spermatozoa to be from $1.035-1.056$ and that of the heads to be greater than $1.276^{124}$.

Lindahl attempted to separate the X- and Y-chromosome bearing spermatozoa in bull ejaculates by counter-streaming centrifugation ${ }^{137}$. Insemination of "heavy" spermatozoa gave a total of 63 offspring, 27 (42.9\%) of which were male. The fertility from spermatozoa subjected to a rotatlonal speed of $1,000 \mathrm{rpm}$ was $53.6 \%$ compared to $35.6 \%$ at $1,100 \mathrm{rpm}$. He obtained $24.3 \%$ males from pooled samples giving greater than $50 \%$ fertility 
compared to $69.2 \%$ males from those giving less than $50 \%$ fertility. From these data, he suggested that it may be possible to select viable female determining spermatozoa by running the counter-streaming centrifuge at moderate speed $(1,000 \mathrm{rpm})^{137}$.

Insemination of the "1ight" spermatozoa gave 27 males (58.7\%) out of a total of 46 offspring. This series of inseminations was divided into pooled samples with more or less than $40 \%$ fertility for analysis. The group of inseminations resulting in an average fertility rate of $47.4 \%$ gave $40.7 \%$ males, while the group with an average fertility rate of $29.7 \%$ gave $84.2 \%$ male offspring. The average sex ratio of 58.7 for the 2 groups did not differ significantly from the control sex ratio of $59.8^{137}$.

Lindahl and Thunqvist centrifuged intact epididymal and ejaculate spermatozoa and their component parts for 12-15 m1nutes at $1,000 \mathrm{~g}$ in spectfic weight gradients composed of the synthetic polysaccharide Ficoll ${ }^{139}$. They observed spectfic gravities of 1.10-1.125 for unfragmented epididymal spermatozoa, 1.240-1.334 for ejaculate spermatozoa, 1.045 for midpieces with tails and 1.276 for heads from ejaculate spermatozoa not treated with ultrasonics. After ultrasonic treatment, speciffc gravities observed were as follows: unfragmented epididymal and ejaculate spermatozoa, 1.100-1.120 and 1.210-1.330, respectively; midpieces with tails from both type of spermatozoa, 1.040-1.070; heads of epididymal spermatozoa, 1.12-1.140; and heads of ejaculate spermatozoa, 1.250-1.350. The authors concluded that the marked difference in speciflc gravity of epididymal and ejaculate spermatozoa appears to be localized to the head ${ }^{139}$.

Beatty evaluated the use of bovine plasma albumin, colloidal silica and dextran as major constituents in media for equilibrium density gradient centrifugation of mammalian spermatozoa ${ }^{20}$. Rabbit spermatozoa remained 
fertile after exposure for up to 2 hours in bovine plasma albumin (25\% in elther 0.45 or $0.90 \% \mathrm{NaCl}$ ) and in dextran (F1coll, $37.9 \%$ ) but not in colloldal silica. A unimodal distribution of specific gravities of rabbit spermatozoa was obtalned with a colloldal silica gradient. About $95 \%$ of the spermatozoa were in the range $1.09-1.18$, and $50 \%$ were in the range 1.11-1.14. The mean was $1.132^{20}$.

Benedict, Schumaker and Davies ${ }^{32}$ evaluated sodium chloride, sodium nitrate, potassium tartrate, potassium lodide, cesium chloride, glycerol, sucrose, Ficoll, bovine serum albumin, a colloidal silica solution (Ludox), and the methyl-glucamine salt of 3,5-di-iodo-4-pyridone-N-acetic acid (MGU) as gradient materials for equilibrium centrifugation studies. With all solutions except Ficoll, glycerol and potassium iodide, they observed a low density class of bull spermatozoe with a buoyant density of 1.16-1.19 $\mathrm{g} / \mathrm{ml}$ and, depending upon the solute, a high density group ranging from 1.25-1.32 $\mathrm{g} / \mathrm{ml}$, which, on many occasions, divided into subclasses over a narrow range. Motility was preserved in the bovine serum albumin, Fico11, Ludox and MGU solutions. Because of the undesirable effects of the high viscosity of albumin and Ficoll solutions, and because of gelation and lowered motility in the Ludox solution, they primarily used MGU sol.utions to prepare the linear density gradients. They used a Beckman Model L UItra-centrifuge with a SW 39 swinging-bucket rotor ${ }^{32}$.

Benedict, et al, centrlfuged spermatozoa from the same bull sample for 5,15 and 30 minutes at rotor speeds of $5,000,15,000$ and $30,000 \mathrm{rpm}$ at temperatures of 5,25 and $37^{\circ} \mathrm{C}$. Under all conditions of time, centrifugal force and temperature, apparent buoyant densities were $1.170 \pm 0.025$ and $1.290 \pm 0.040 \mathrm{~g} / \mathrm{ml}$ for the upper and lower bands, respectively. They noted increased numbers of spermatozoa in the lower band with increasing temperature and time of exposure in the medium. Recentrifugation of the 
upper band gave both an upper band and a lower band, the development of which appeared to be related to time. Recentrifugation of the lower bands produced diffuse bands in the corresponding density range. They observed a splitting of the low density band into 2 sub-bands only at $5^{\circ} \mathrm{C}$ and a rotor speed of $30,000 \mathrm{rpm}$. No significant changes in the size or shape of the spermatozoa were observed. Similar results were obtained with rabbit and bull spermatozoa ${ }^{32}$.

Using a nigrosin-eosin staining technique, they observed a lower percentage of eosin-positive cells in the low density fraction than in the high density fraction in all experiments ${ }^{32}$. With fresh spermatozoa, the low density (top) fraction had a slightly higher percentage of eosinpositive cells than did uncentrifuged spermatozoa from the same sample. However, when using aged and mixed (aged and fresh) spermatozoa, the low density (top) fraction had a lower percentage of eosin-positive cells than did the uncentrifuged sample ${ }^{32}$.

Insemination of rabbit spermatozoa from the different bands gave fert1lity rates of $36 \%$ from the upper band, $11 \%$ from the middle band, $4 \%$ from each of 2 bottom bands, and $6 \%$ from combined bottom bands compared to $58 \%$ fertility with uncentrifuged (control) spermatozoa ${ }^{32}$. However, no conclusions could be drawn with regard to the sex ratio of the offspring.

Lavon and co-workers determined the specific gravity of bull spermatozoa from different parts of the reproductive tract using non-watermiscible phthalate ester mixtures of predetermined specific gravity in micro-hematocrit tubes ${ }^{133}$. The average specific gravities of spermatozoa from the mediastinum testis, caput epididymis and cauda epididymis were estimated to be $1.0440 \pm 0.002,1.0583 \pm .002$ and $1.0817 \pm .002$, respectively ${ }^{133}$. Thus, the specific gravity increases during maturation as suggested by Lindah1. In another report, the spectfic gravity of bull 
spermatozoa in the first ejaculate was $1.0760 \pm 0.003^{132}$. These values are considerably lower than those reported by Lindahl and Thunquist.

Morton used a colloid mill to disrupt bovine epididymal spermatozoa and used a sucrose linear density gradient to determine the buoyant density of parts of spermatozoa ${ }^{164}$. The apparent buoyant densities of components ranged from approximately $1.25 \mathrm{~g} / \mathrm{ml}$ for tails to greater than $1.347 \mathrm{~g} / \mathrm{ml}$ for heads only. Midpieces were slightly more dense than tails and the apparent buoyant density of combinations of heads with midpieces were less for the fragments with the larger midpieces ${ }^{164}$.

\section{Biological Properties}

Because of the close relationships among the biological properties of spermatozoa, any arbitrary division of them is artificial. However, for convenience of discussion, they will be divided into 5 main categories as follows: (1) viability, (2) intrinsic motility, (3) fertilizing capacity, (4) antigenicity and (5) genetic composition.

\section{Viability.}

Among the criteria by which the viability of spermatozoa is judged are the stalning properties and their relationship to morphology, motility, fertilfing capacity, and metabolic rate. In addition, one is interested in the effects of the age and breed of the rabbit, the frequency of ejaculation and the season of the year on the quality of the spermatozoa.

a. Vital Staining. Beatty and Napier found a great difference between the percent capless and the percent stained spermatozoa in ejaculates 22 . For Instance, in efaculates with $25.4,34.2$ and 32.6 percent stained spermatozoa, the percentages of capless spermatozoa were $13.3,18.6$ and 16.9 percent, respectively. They stated that ocoring of stained spermatozoa Is difficult ${ }^{22}$. Napler suggests that the percentage of stained spermatozoa 
using a nigrosin-eosin technique is one of the best guides for predicting fertilizing capacity, which is inversely proportional to the percentage of stained spermatozoa ${ }^{169}$. He found the mean litter size to be related to the mean spermatozoan head length of the sire ${ }^{171}$. Among strains the incidence of stained spermatozoa varies markedly, indicating the possibility of hereditary differences in fertility; however, the variation in the percentage of capless spermatozoa is not significant ${ }^{23}$.

In studies with bull spermatozoa, Munro saw no difference among samples with visual observation of motility, but found a $10 \%$ difference with vital staining ${ }^{165}$. Conception rates were approximately $65 \%$ for samples with over $70 \%$ 11ve spermatozoa by stalning. Fertility was reduced an unusual degree when efther the incldence of live spermatozoa fell below $70 \%$ or the incidence of primary and secondary abnormal spermatozoa rose above $23 \%$. Head defects appeared to be the most important abnormalities ${ }^{165}$. Napier reports that, in samples containing a high percentage of stained or capless spermatozoa, the unstained spermatozoa are of low viability ${ }^{169}$.

The percentages of stained and capless spermatozoa increased significantly after incubation at $38.5^{\circ} \mathrm{C}$ for 5 hours in $0.9 \%$ saline, while incubation for the same perlod of time in a suspension of $50 \%$ seminal fluid and $50 \%$ saline did not signiflcantly change the mean percentage of stained or capless spermatozoa ${ }^{169}$. Ne1ther suspending a sample from 1 male in the seminal plasma from a different male nor mixing of spermatozoa from 2 different males affects the viability of the spermatozoa ${ }^{169}$.

Stress, such as centrifugation, causes the same absolute change in the percontage of stalned or rap.less spermatozoa in all samples, regardless of the initial viability. Thus, with inftially low viability, the percentage change is greater than with high vlability spermatozoa, indicating 
that the apparently normal spermatozod in a large population of abnormal "low quality" sperm are less viable 169 .

b. Metabolism. Work by Murdoch and White has shown that rabbit spermatozoa oxidize glucose faster than acetate and oxidize lactate at a faster rate than glucose, fructose, pyruvate or acetate ${ }^{166}$. Fructose is the predominant sugar found in the semen of most mammalian species, including the rabbit, while glucose is the sugar found in the semen of tho domcatic cock $^{166}$. Salisbury reported that the optimum oxygen uptake hy ejarulated bull spermatozoa in semen occurred on.1.y when $\mathrm{Cn}_{2}$ in amounte normally rcspired (about 5\%) was present ${ }^{201}$. Wales and 0 'Shea reported that the presence of respired $\mathrm{CO}_{2}$ during tncubation of bull epormatozoa had no effect on oxygen uptake or substrate oxidation, but increased lactate accumulation 237 . Hamner and Williams reported that $2.36 \times 10^{-3} \mathrm{M} \mathrm{NaHCO}_{3}$ in equilibrium with $2 \% \mathrm{CO}_{2}$ at $\mathrm{pH} 7.0$ is the optimum $\mathrm{CO}_{2}$ level for maximum metabolic activity of rabbit spermatozoa 107.

Salishnry states that, during a 3 hour periud of Illsubition at $37^{\circ} \mathrm{C}$, both optimum oxygen consumption and optimum aerobic glycolysis by bull spermatozoa occurred in a $\mathrm{NaCl}$ solution of approximately 280 mosm $^{201}$. (The osmolarity of semina.1. p.lasma is approximately $285 \mathrm{mosm}$ ). The effect of osmolarity was less obvious when a potassium chloride solution was used. He suggests that the optimum osmolarity for the preservation of spermatozoa and conservation of Intracellular reserves would be provided by a medium with an osmolarity of $350 \mathrm{mosm}$, which is approximately that found in the epididymis 201 .

Wales and Wallace studied the effects of potassium, magnesium, calcium and phosphate ions on the metabolism of bull, dog, rabbit and fowl spermatozoa 236. Fowl spermatozoa had the lowest rate of metabolism, and 
dog spermatozoa had the highest respiratory activity. Washing bull, dog and rabbit spermatozoa greatly reduced the oxidation of substrates other than fructose, while washing had little effect on fowl spermatozoa. Phosphate lons generally depressed the respiration of bull spermatozoa and stimulated their aerobic fructolysis. It stimulated the respiration of unwashed dog spermatozoa, but had no important influence on the metabolism of rabbit and fowl spermatozoa. Potassium stimulated some aspects of metabolism of sperm of all species, but had its greatest effect upon the respiration of dog spermatozoa. It stimulated the oxidation of fructose by rabbit spermatozoa. Magnesium depressed the respiration of bull and fowl spermatozoa. Calcium apparently had no effect on any of the spermatozoa. They observed few significant interactions among the ions tested 236 . The Injurlous effects of high concentrations of phosphate on the oxidative metabolism of bull spermatozoa has also been reported by other investigators $^{193}$.

Nevo studied the dependence of motility and respiration of bull, ram and cock spermatozoa on oxygen concentration ${ }^{173}$. Both motility and respiratory rate were constant in the range of oxygen concentration from that in air to that at a $\mathrm{pO}_{2}$ of $4-5 \mathrm{~mm} \mathrm{Hg}$, below which there was a marked reduction in respiratory rate and thactivation of most of the spermatozoa. Motility and oxygen consumption ceased below an oxygen partial pressure of $1 \mathrm{~mm} . \mathrm{Hg}$. The critical $\mathrm{O}_{2}$ values were similar for all 3 species 173 . They are considerably below the $\mathrm{pO}_{2}$ of $60 \mathrm{~mm} \mathrm{Hg}$ in the fallopian tube of the unanesthesized rabblt before, and for 3 days after, the induction of ovulation with $\mathrm{HCG}^{149}$. If the minimum $\mathrm{O}_{2}$ partial pressure requirement is similar in other species, a significant safety factor is present.

$$
\text { c. Factors Affecting Spermatozoa Production and Quality. The volume }
$$

and number of spermatozoa in an ejaculate depend upon the age and breed 
of the rabbit and upon characteristics of Individual males. For mature rabbits volumes range from $0.4-0.7 \mathrm{ml}$ and concentrations range from $1 \times 10^{8}-2 \times 10^{9}$ spermatozoa/ml ${ }^{1}$. The variation in the fertility of semen samples is attributed to (1) the inherent quality of the spermatozoa, (2) the composition of the seminal fluid and (3) the interaction of seminal fluid and spermatozoa ${ }^{169}$.

Amann and Lambiase studied the changes in semen characteristics and sperm output between puberty and one year of age in New Zealand White bucks $^{9}$. Daily sperm output doubled between 20 and 32 weeks of age, reachIng an average of $148 \pm 11 \times 10^{6}$ spermatozoa per day and did not increase after that time, suggesting that these rabbits reach sexual maturity at about 32 weeks of age. The mean ejaculate volume was $0.56 \mathrm{ml}$ with a spermatozoa concentration of $301 \times 10^{6} / \mathrm{ml}{ }^{9}$.

The effect of the frequency of ejaculation upon the viability of spermatozóa was evaluated by Gregoire, Bratton and Foote who compared the spermatozoa from 2 groups of 4 Dutch Belt rabbits each, ejaculated at dally or weekly intervals 94 . The average number of motile spermatozoa decreased from $243 \times 10^{6}$ to $55 \times 10^{6}$ per ejaculate for the bucks ejaculated dally, and from $138 \times 10^{6}$ to $97 \times 10^{6}$ for the bucks ejaculated at weekly intervals. The weekly output of motile spermatozoa was $385 \times 10^{6}$ compared to $97 \times 10^{6}$ for the bucks ejaculated at daily and weekly intervals, respectively. There was no significant difference in the size of the 11tters or in the percentage of does kindling following artificial insemination with semen from the 2 groups of bucks after they had been ejaculated for a period of 37 to 40 weeks 94 .

Amann evaluated the effect of ejaculation frequency and breed on semen charaçteristics and sperm output of Dutch and New Zealand White 
rabbits using ejaculation frequencies of $1 \times 12$ hours, $1 \times 24$ hours, and $2 \times 48$ hours with the 2 collections separated by about 15 minutes ${ }^{8}$. Each ejaculation frequency was imposed for 36 days, the last 30 of which constituted the experimental period. No signiflcant differences were observed among the daily sperm outputs at the 3 different collection frequencies. The averages were $88 \times 10^{6}$ and $123 \times 10^{6}$ spermatozoa/day for the Dutch and New Zealand White rabbits, respectively ${ }^{8}$. Amann did not discuss the quality of spermatozoa from the different rabbits at the different collection frequencies:

Another study of the sperm output of rabbits at various ejaculation frequencies was conducted by Desjardins, Kirton and Hafs using 9 Dutch Belt and 3 New Zealand White rabbits, approximately 12 months of age ${ }^{64}$. Each of the rabbits was subjected to 4 ejaculation frequencies imposed for 5 week periods, consisting of frequencies of once a week ( $1 \times \mathrm{F}$ ), 4 times on Friday ( $4 \times \mathrm{F})$, twice on Monday, Wednesday and Friday ( $2 \times \mathrm{MWF})$ and once daily, except Sunday $(1 \times M-S)$. They observed no significant differences among the first ejaculates at all 4 frequencies. Despite a decrease in volume in the second ejaculate of the $4 \times \mathrm{F}$ and $2 \times$ MWF groups, an Increase in the spermatozoa concentration gave a higher total sperm output in those efaculates. In the third and fourth ejaculates of the $4 \times F$ group, both the volumes and concentrations decreased. There were no significant differences in the total number of spermatozoa per week from the $4 \times$ F, $2 \times$ MWF and $1 \times$ M-S groups ${ }^{64}$. No observations on the quality of the spermatozoa in the different ejaculates were reported. An analysis of the protein, fructose and citric acid content of the ejaculates showed both an absolute decrease in successive ejaculates in the $4 \times F$ and $2 \times$ MWF groups, and a decrease in concentration of those sub- 
strates, which was most prominent in the third and fourth ejaculates in the $4 \times$ F group 64 .

Orgebin-Crist observed that, when rabblts were ejaculated only twice weekly, the daily sperm output was approximately $50 \%$ of the estimated testicular sperm production, Indicating that a high percentage of the spermatozoa produced were absorbed ${ }^{181}$.

Doggett showed that the total sperm count of rabbits ejaculated daily for prolonged periods rose to a definite peak every 2-7 days with an average of 3.16 days $^{68}$. The low values usually were about $50 \%$ of the preceding peaks. In mature animals; the peaks were fairly consistent and similar among individual animals. Sperm motility and the volume of liquid semen rose and fell with the total daily sperm production 68 .

Degerman and Kihlström have shown a cyclic variation in the body temperature of the male rabbit which correlates statistically with the volume of semen produced dally. Cycles vary in length from 3-9 days with a mean value of 7 days ${ }^{63}$. The authors did not repurt the quildty of the spermatozoa during the cycles.

El Jack and Lake evaluated the effect of resting roosters from ejaculation on the quality of spermatozoa by subdividing the roosters into 5 groups $^{77}$. One group was kept as a control, while the other 4 groups were rested for $2,4,6$ and 8 weeks, respectively, between ejaculations. Semen was collected from the control group throughout the 10 week experimental period at intervals of 2,3 or 7 days. Even though there was a tendency for the number of degenerating spermatozoa in an ejaculate to rise after roosters had been rested for more than 2 weeks, there were Individual variations in the degree of deterioration of the ejaculates. Some roosters did not appear to be affected appreciably by the change in 
collection schedule. When the experimental males were milked frequently after a rest period, the percentage of normal spermatozoa in the semen returned towards the pre-rest value. It was suggested that the rooster should be ejaculated regularly to insure the avallability of uniformly good samples ${ }^{77}$.

Eliasson showed that frequent efaculations reduced the volume of human seminal plasma, but did not significantly change the relative amounts of constituents from the seminal vesicles and prostate gland. Ejaculates were obtained from individuals at 24,12 and 8 hour intervals ${ }^{76}$.

Freund and Wiederman evaluated the influence of spermatozoa quality on storage and fertility ${ }^{88}$. They found that compared to high quality spermatozoa, the motillty of spermatozoa of poor quality ejaculates was good immediately after cooling to $5^{\circ} \mathrm{C}$ and after 1 day of storage, but showed a greater loss of motility with longer storage than did ejaculates with high quality spermatozoa 88 .

\section{d. Seasonal Variations in Spermatozoa Quality. The seasonal variation} in the reproductive traits of New Zealand white rabbits from 1943-1960 was evaluated by Sittman and co-workers at the United States Rabbit Experiment Station, Fontana, California ${ }^{216}$. The maximum average daily temperature increased from 63 to $75^{\circ} \mathrm{F}$ from January through May, increased to above $80^{\circ} \mathrm{F}$ in June, was approximately $90^{\circ} \mathrm{F}$ from July through September, decreased to about $80^{\circ} \mathrm{F}$ in October, and was about $75^{\circ} \mathrm{F}$ and $70^{\circ} \mathrm{F}$ in November and December, respectively. The animals were housed in sheds cooled by sprinklers during the hot, dry summer months. The monthly conception rate was $77.4 \%$ from January through May, $62 \%$ from June through November, with a low of $52 \%$ in September, and was $72 \%$ in December. In addition to the decrease in the conception rate during the hot months, the following 
also were observed at that time: (1) a decrease in the number of litters born per. month, (2) a decrease in the average number of offspring born alive per litter per month, (3) a decrease in the average total litter size per. month as a percent of the overall average, and (4) a decrease in the number of rabbits born per month as percentages of the overall total. This variation in reproductive capacity appeared to be more dependent upon changes in temperature than upon changes in light conditions ${ }^{216}$.

Orgebin-Crist evaluated the effect of environmental light on the gonadal and epididymal sperm reserve and the daily sperm production in rabbits. Rabbits exposed to 14 hours of light daily for $26.3 \pm 1.7$ weeks had an average sperm production of $115.8 \times 10^{6} /$ day and a testes yield of $21.4 \times 10^{6}$ sperm/day/gm compared to an average sperm output of 147.4 $\times 10^{6} /$ day and a testes yield of $26.5 \times 10^{6}$ sperm/day/gm for bucks exposed to 14 hours of light for only $6.9 \pm 8$ weeks. This suggests that a conscanc photo-period is deleterious for sperm production ${ }^{181}$.

\section{Intrinsic Motility.}

This section is concerned primarily with factors influencing the preservation of motility (cell concentration; and the $\mathrm{pH}$, ionic strength and osmolarity of the medium) and factors modifying the degree of motility (temperature and stage of maturation of the spermatozoa). In addition, observations on the fluctuations in swimming rates of individual bull epermatozoa are presented.

\section{a. Spermatozoa Concentration. Emmens and Swyer observed that rabbit} spermatozoa from most ejaculates, when suspended in Baker's solution at a concentration of $0.4 \times 10^{6} / \mathrm{ml}$, became completely 1motile wi.thin 2 or 3 hours ${ }^{80}$. The same phenomenon occurred with chloride-free diluents and is, therefore, not due to a toxic effect of chloride. They reported that 
the rapid immobilization may be prevented by suspension in cell-free supermatants from other more concentrated suspensions of rabbit semen, by accessory secretions from a vasectomized buck, and by the addition of agents such as gum arabic, starch, glycogen, and serum proteins to Baker's solution. In no case was the action of the additives as effectIve in preserving motility as more concentrated suspensions of spermatozoa In Baker's solution. Washing with Baker's solution did not cause immobilization of spermatozoa at concentrations of $20 \times 10^{6} / \mathrm{ml}$ at a stage when the concentration of seminal plasma had been reduced to the equivalent of dilution to $0.4 \times 10^{6} / \mathrm{ml}$. However, 6 washings did immobilize the spermatozoa and was interpreted by the authors as evidence that the dilution phenomenon is caused by the loss of intracellular or paracellular material 80 .

Swyer (as noted in the discussion following Kok's report) observed a significant decrease in the motility of rabbit spermatozoa when the concentration fell below approximately $4 \times 10^{6}$ spermatozoa/m1 ${ }^{125}$. Emmens and Swyer ${ }^{80}$. observed that a concentration of $20 \times 10^{6}$ spermatozoa/ml maintained as good, or nearly as good, a degree of motility as higher concentrations.

Freund and Wiederman, using human spermatozoa, observed that with dilutions of $1: 1,1: 5,1: 25$ and $1: 125$ in Norman-Johnson Solution, seminal plasma and White's phosphate buffer, there was an initial loss of motility at the greater dilutions 88 . Over a period of 5 hours at $25^{\circ} \mathrm{C}$ the decrease in the percentage of motile sperm at all dilutions appeared to be approximately the same in the Norman-Johnson-1 (NJ-1) Solution. The decrease in the percentage of motile sperm in raw semen appeared to be greater than in semen diluted $1: 1$ and $1: 5$ in the $\mathrm{NJ}-1$ Solution ${ }^{88}$. The actual spermatozoa 
concentration at the different dilutions was not given.

Quinn, White and Wirrick studied the effect of dilution on the concentration of sodium, potassium, calcium and magnesium in ram and bull spermatozoa using an atomic absorption spectrophotometer ${ }^{193}$. They observed an intracellular accumulation of sodium and a loss of potassium, calcium and magnesium upon dilutions as low as 1:8. Equilibrium was reached soon after dilution and little change in the concentration of those cations was observed over a 3 hour period. At dilutions of $1: 100$ and after washing 4 times, they ohserved a similar but morc severe influx of sodium. This was accompanied by a marked intracellular depletion of potassium whlch Lended to warsen over the 3 hour poriod. This exchange of sodium and potassium increased with increasing $\mathrm{pH}^{193}$.

b. $\mathrm{pH}$. Emmens studied the effect of $\mathrm{pH}$ on rabbit spermatozoa using a diluent containing $3 \%$ dextrose, $0.2-0.4 \%$ sodium chloride and sufficient butfer to bring the solutions to a tonicity equivalent to $0.9-1.0 \%$ sodium chlortde ${ }^{79}$. Suspensions of $10-60 \times 10^{6}$ spermatozoa/ml were prepared only from active ejaculates with high counts. All observations were made at room temperature. Baker's solution at approximately. $\mathrm{pH} 7.3$ was used as a control. Observations included the following: (1) Rabbit semen had a pH between 7.0 and 8.2 with a mean of 24 samples being $\mathrm{pH} 7.6$. Spermatozoa were much more sensitive to acidity than to alkalinity:

A pH range of 7.2-7.9 was optlmum for maintaining motility IN VITRO. (4) At pH's slightly below 5.8, spermatozoa were immotile. After exposure for 1.25 hours at this $\mathrm{pH}$, the addition of Baker's solution restored the motility to a reasonable degree. With exposure to the low $\mathrm{pH}$ for less than 5 hours, a slight degree of motllity was restored upon addition of Baker's solution but after 5 hours, the loss of motility was irreversible. 
(5) For very short exposures to $\mathrm{pH}^{\prime} \mathrm{s}$ between 4.4 and 5.4 , motility could be restored by the addition of Baker's solution to the medium. In these cases, the duration of exposure was limited to 1 hour or less. (6) At $\mathrm{pH}$ 9.5-10.0, spermatozoa remalned motile and survived for several hours, but with a depressed degree of motility. (7) At all pH's as death occurred, the sperm talls tended to stain before the heads ${ }^{79}$.

Spermatozoa tolerate well a pH range of $6.5-8.0$ in most media IN VITRO. The reversiblilty of the effects of extreme pH's on viability and Intrinsic motility is time dependent and therefore of great significance in designing experiments using spermatozoa.

The relationship of these IN VITRO observations to the natural environment of spermatozoa in the female genttal tract is illustrated by the following observations. Moghissi observed that human spermatozoa were Immobilized in acid mucus while alkaline mucus enhanced motility ${ }^{161}$. According to Stevens, Hafs and Kirton, the uterine fluld of rabbits is $\mathrm{pH} 7.64^{219}$. They stress the importance of $\mathrm{CO}_{2}$ in buffering uterine fluids for they observed a rapid rise in $\mathrm{pH}$ upon removal of the fluids from the uterus $^{219}$.

In addition to 1ts influence on the preservation of motility, $\mathrm{pH}$ also has a significant effect on the electrophoretic mobility of spermatozoa as noted previously.

\section{c. Ionic Strength and Osmolarity of the Medium. Items related to ionic} strength which have been mentioned previously include the net surface charge, the relative surface charge on the heads and tails of the spermatozoa, and the effects of lonic strength on the type of agglutination of spermatozoa populations.

Observations by Emmens on the effects of osmotic pressure and electro- 
lyte concentration on the motility of rabbit spermatozoa at different hydrogen ion concentrations included the following: (1) At pH 5.86.6, spermatozoa were less motile than at $\mathrm{pH} 7-8$, were very sensitive to $\mathrm{pH}$ changes, and were more sensitive to hypotonicity than to hypertonicity. Motility was affected little or not at all by substitution of dextrose for part or all of a sodium chloride solution. (2) At $\mathrm{pH} 7-8.7$, spermatozoa were relatively insensitive to changes in $\mathrm{pH}$, but hypo- and hypertonfcity, whether caused by alterations in sodium chloride or in dextrose concentration, were about equally deleterious to motility. The replacement of sodium chloride by dextrose in hypertonic diluents tended to lower initial motility but this level of motllity was maintalned with little fall over the next 6 hours. (3) At pH 8.6-9.8, spermatozoa were much less motile than at $\mathrm{pH} 7-8$, but still were not very sensitive to minor changes in $\mathrm{pH}$. Hypertonicity was more deleterious than hypotonicity. The latter may even have been favorable to motility under certain conditions. (4) The replacement of sodium chloride by dextrose enhanced preservation of motility in isotonic media, but made little difference in hypo- or hypertonic solutions. The favorable effect of hypotonicity was expressed if it involved a reduction in the sodium chloride content of the diluent, but not if it involved a reduction in the dextrose content. The characterIstlc action of dextrose was a lowering of the rate of decline of motility, not the maintenance of a high initial motility. (5) In alkaline suspen slons, rabbit spermatozoa were affected adversely by a sodium chloride concentration greater than approximately $0.2 \%$. Motility was better in a hypotonic medium of less than $0.2 \%$ sodium chloride content than in an isotonic medium containing more than $0.2 \%$ sodium chlorlde ${ }^{78}$.

d. Temperature. As noted earlier, the use of low temperatures is a 
convenient way to reversibly immobilize spermatozoa. Bhattacharya observed that the degree of motility of rabbit spermatozoa at low temperatures depended upon the medium in which the sperm were suspended ${ }^{35}$.

The deleterious effect of sudden exposure of mamalian spermatozoa to low temperatures (cold shock) has been known for some time. Epididymal spermatozoa from ram, boar and bull apparently are more resistant to cold shock than are spermatozoa from the ejaculatory ducts ${ }^{43}$. Quinn and White recently have shown that cold shocking and deep freezing bull and ram semen caused an influx of sodium and an efflux of potassium and magnesium from the spermatozoa. They did not observe any significant changes in the cation content of human, dog, rabbit or fowl spermatozoa after cold shocking 192 .

At the other extreme, a temperature of $48^{\circ} \mathrm{C}$ for 30 seconds caused. a 1088 of motility of rabbit spermatozoa IN VITRO ${ }^{265}$.

e. Stage of Maturation. Blandau and Rumery have also shown that rat spermatozoa suspensions prepared from the cauda epididymis and inseminated Into the left uterine horn gave a $93 \%$ fertilization rate, whereas only $8 \%$ of ova were fertilized by suspensions of spermatozoa from the caput epididymis when injected into the right uterine horn of the same animals ${ }^{43}$. Most spermatozoa from the caput epididymis were actively motile but swam In circles because of retroflexion of the head and stiffness in the neck plece of the flagellum. They attributed the lower fertilization rate by these spermatozoa to the circular swimning pattern and suggested that 1t interfered with their ability to ascend to the ampulla of the oviduct ${ }^{43}$.

Barack has shown that the amount of fluid produced within the testis of the mouse is suffleient to flush the tubal system at least once a day and that this fluid production is the major factor in the transport 
of spermatozoa from the seminiferous tubules to the ductuli efferentes ${ }^{19}$. From their observations, Blandau and Rumery concluded that rat spermatozoa pass through the epldidymis passively because of peristaltic waves that sweep over the tubules at a rate of approximately 8 per minute 43 .

Other changes during maturation are considered in Section 4, Antigenicity.

f. Fluctuations in the Swiming Rates of Individual Spermatozoa. In a study of fluctuations in the swimming rates of individual bull spermatozoa at $37^{\circ} \mathrm{C}$, Tampion reported that spermatozna from some bullo owam at a rate greater than $150 \mu / \mathrm{sec}$ in sperm extract ${ }^{226}$. The mean swimming rate of straight and circling spermatozoa in 4 ejaculates were estimated to be $133.4 \mu / \mathrm{sec}$ and $134.0 \mu / \mathrm{sec}$, respectively. In a series of 4 experiments, $56-90 \%$ of the spermatozoa swam straight over a distance of more than 310 microns, while $4-10 \%$ of the spermatinzna swemed to the right or left. The remainder of the spermatozoa swam in polygonal patterns. Spermatozoa dilutcd to a concentration of $5,000 / \mu 1$ with saline had swimming rates ranging from 57.4 to $103.3 \mu / \mathrm{sec}$. Variations within individual groups ranged from $15-40 \mu / \mathrm{sec}$. In addition to differences in swimming rates among spermatozoa, Individual spermatozoa changed velocity considerably from time to time 226 .

l'ampion studied the effects of drugs and hormones on the swimming performance of bull spermatozoa and showed that the most satisfactory medium for achieving and maintaining a high rate of motility was $135 \mathrm{mM}$ $\mathrm{NaCl}^{227}$. The addition of fructose in concentrations of $5.0,0.5$ and 0.05 $\mathrm{mg} / \mathrm{ml}$ to $135 \mathrm{mM} \mathrm{NaCl}$ had no significant effect on the swimming rate or on its maintenance for 1 hour. In $135 \mathrm{mM} \mathrm{NaCl}$, the mean swimming rates ranged from $130.8-158.0 \mu / \mathrm{sec}$. Despite the greater viscosity of both 
fresh and frozen seminal plasma, the swiming rates after 5 minutes of Incubation in these media were st111 $110.2 \pm 4.6$ and $99.0 \pm 12.6 \mu / \mathrm{sec}$, respectively, compared to $139.2 \pm 4.7$ and $136.2 \pm 7.2 \mathrm{\mu} / \mathrm{sec}$ in $115 \mathrm{mM} \mathrm{NaCl}$ controls. Thus, there does not appear to be a significant inhibitory effect of fresh seminal plasma on motility.

\section{Fertilizing Capacity}

The fertilizing capacity of spermatozoa is acquired during passage through the epididymis. Orgebin-Crist found that spermatozoa from the caput and the proximal corpus epldidymis were infertile when inseminated surglcally into the uterus of does ${ }^{180}$. Insemination of does in a similar manner with spermatozoa from the distal corpus epldidymis, and from the proximal and the distal cauda epididymis, gave fertilization rates of $57 \%, 92.7 \%$ and $95 \%$, respectively. Compared to control ova fertilized with surgically Inseminated efaculated opermatozoa, the fertilization of ova was delayed significantly when distal corpus spermatozoa were Inseminated. Another Interesting observation was that spermatozoa 1solated surgically in the proximal caput epldidymis for 3-8 days developed thelr capacity for motility but did not develop fertilizing ability ${ }^{180}$.

In another paper, Orgebin-Crist reported that, in does inseminated with spermatozoa from the lower corpus epididymis, only $52 \%$ of the ova that ultimately would be fertilized had been penetrated 16 hours after the Infection of $\mathrm{HCG}^{182}$. In about half of these ova, the fertilizing spermatozoon was just penetrating the ovum at that time. Approximately $10 \%$ of ova fertilized with epididymal spermatozoa were polyploid. This was attributed to the delayed fertilization because the fertilization of aged rabbit ova 18 known to lead to polyploidy 182,212 . 
of rabbit spermatozoa lasted for 8-10 days, while motility laṣted for 14-38 days, indicating that mature sperm can remain in the epididymis for 10 days without loss of fertilizing capacity ${ }^{54}$. Tesh and Glover observed that the fertilizing capacity of rabbit spermatozoa, which had been isolated in the epididymis for periods of up to 2 weeks, was apparently normal ${ }^{228}$. However, it was reduced greatly after 4 weeks and was completely lost after 7 weeks. Increased embryo mortality was observed before a significant fallure in fertilization was detected ${ }^{228}$.

Bedford compared the interaction between ova and spermatozod taken from the vas deferens and the cauda epididymis by inseminating spermatozoa of each group into opposite Fallopian tubes in the same rabbits ${ }^{28}$. He found a significantly greater number of spermatozoa from the vas deferens than from the epididymis in association with ova. He suggested that, In the rabbit, capacitation invests spermatozoa with the competence Lu establish coñact with the surface of the zona pellucida and to penetrate ova during the fertile life of the nvim ${ }^{28}$.

Burfening and Ujlberg incubated portions of split ejaculates at $38^{\circ} \mathrm{C}$ or $40^{\circ} \mathrm{C}$ for 3 hours and found no difference in the fertilizing capactity of rabbit spermatozoa Incubated at the 2 temperatures $4 \%$. However, the embryonic survival rate was higher in the uterine horns inseminated with semen incubated at $38^{\circ} \mathrm{C}$ than in the opposite horns inseminated with semen incubated at $40^{\circ} \mathrm{C}^{47}$. Asdell and Salisbury reported that the morphology of spermatozoa from rabbits in whom the testes had been placed in the abdomen surgically for varying perlods of time showed increasing deterioration with time due to the higher temperature in the abdomen ${ }^{12}$. Thus, the short incubation perfod used by Burfening and U1berg, although not changing the morphology or fertilizing capacity of the spermatozoa, had caused a more subtle change which showed up in the embryonic survival 
rate.

Spermatozoa of normal rabbits retained their full fertilizing capacity in the female genftal tract for only about 25 hours before ovulation and had lost fertilizing capacity after 32 hours ${ }^{54}$. McGaughey, Marston and Chang found the fertilizing life of mouse spermatozoa in the female genital tract to be approximately 12 hours ${ }^{154}$. According to the authors, a review of literature gives survival times of spermatozoa of other species in the respective females as follows: 14 hours in the rat, 22 hours in the guinea pig, 30 hours in the rabbit, 126 hours in the ferret, and 144 hours in the horse. ${ }^{154}$. Fowl spermatozoa remain fertile in the genttal tract of the female for several weeks and turkey spermatozoa retain their fertilizing capacity for at least 30 days 140,220

The number of spermatozoa required for fertilization varies from one species to another. Even though it is estimated that only $1,000^{14}$ to $2,000^{3}$ spermatozoa reach the site of fertilization after normal mating in rabbits, it appears that with vaginal insemination, $0.5-1 \times 10^{6}$ spermatozoa are needed to achieve optimum fertilization results. In poultry husbandry, the usual minimum number of spermatozoa inseminated is $1 \times 10^{8}$ in a volume of approximately $0.1 \mathrm{ml}^{140}$. With both species, high percentages of fertilization have been achieved with lower numbers of spermatozoa $56,140,262$. Additional considerations concerning artificlal lusemfualion in the rabit are given in Appendix B.

\section{Antigenicity.}

The antigenictty of spermatozoa has been studied with regard to the slgniflcance of auto-antibodies against spermatozoa in the blood of men with obstructed vas deferens ${ }^{186}$; the presence of sperm agglutinins in human semen and blood ${ }^{244}$; a comparison of the specificity of antigens 
In semen on spermatozoa and in blood of humans ${ }^{160}$ and bul1s ${ }^{117}$; the fallure of the control of fertility in the rabbit by isoimmunization with seminal plasma 240 , and success in the mouse ${ }^{155,250}$; and the induction of antibodies against spermatozoa in female rabbits by intravaginal or intramuscular injections of rabbit semen and bull semen ${ }^{73}$. Edwards, Ferguson and Coombs found human A and B blood group isoantigens on the spermatozoa of secretors but not on the spermatozoa of non-secretors 75 . The presence of the Isoantigens $M, N$ and $T j a$ was established. However, the sex-linked antigen Xga could not be shown on the spermatozoa ${ }^{75}$. Boettcher found $A B O$ antigens on seminal spermatozoa from secretors but not on spermatozoa from non-secretors, and concluded that the ABO antigens are adsorbed from the seminal plasma ${ }^{14}$.

Although studies such as those mentioned in the above paragraph are of great interest and may eventually be incorporated into the experimental program in progress in this laboratory, the problem of antigenicity is of importance in current work only with regard to agglutinatton in experimental procedures. Because clumps of spermatuzua behave quite differently from individual spermatozoa during sedimentation and electrophoresis, it is desirable to avoid agglutination ${ }^{158}$.

In a study of the agglutination of mammalian spermatozoa, Bedford found a tendency for washed ejaculate or epididymal spermatozoa of several mammalian species to agglutinate by the head region in physiological media such as Ringer's and Tyrode's solutions, $0.9 \%$ sodium chloride, and especially in either normal heated isologous, homologous, or heterologous sera ${ }^{27}$. In these aggregates, the sperm tails remained quite free and showed persistent, vigorous activity. He stated that tail-tail agglutination was never observed between mature rabbit spermatozoa in electrolyte solutions or in normal homologous or non-speciflc hetero- 
logous sera. Tall-tall agglutination did occur readily when rabbit or other spermatozoa were suspended in specific heterologous antisera. He attributed this to antibodies against tall and head specific antigens giving posstble comblnations of head-head, head-tail and tail-tail agglutination. Thus, tall-tall agglutination in homologous and, especially, 1sologous sera was considered to be due to specific antibodies against tal1 antigens 27 .

When mature rabbit spermatozoa were suspended in isotonic solutions of sucrose, glucose or fructose at $37^{\circ} \mathrm{C}$, the aggregation of motile spermatozoa by the tail region similar to tall-tail agglutination seen in spectfic antisera occurred rapidly ${ }^{27}$. In lsotonic media of low electrolyte concentration, the surfaces of the sperm tails became attracted not only to other tall surfaces, but also to particulate matter in the medium. Materials that adhered to the surface of the tail never did so to the surface of the sperm head. Addition of increasing amounts of Ringer's solution to a suspension of motile spermatozoa in an isotonic solution of sucrose or glucose caused a transition from the tail-tail agglutination to head-head agglutination 27 .

The agglutination properties of rabbit spermatozoa changed during passage through the epididymis ${ }^{27}$. There was little tendency for headhead agglutination of spermatozoa from the caput epididymis, but there was for tall-tall agglutination in $0.145 \mathrm{M} \mathrm{NaCl}$. Spermatozoa from the corpus epididymis showed some tendency for head-head agglutination, but much less so than spermatozoa from the cauda epididymis. Spermatozoa from the cauda epididymis, vas deferens and mature, washed spermatozoa from the ejaculate tended to agglutinate by the head region in homologous heated serum or in $0.145 \mathrm{M} \mathrm{NaCl}$ but showed no tail-tail agglutination ${ }^{27}$. 
Bedford concluded that the orientation of many caput spermatozoa in an electric fleld with heads pointing towards the anode indicated the existence of a relatively high net surface charge over the head region ${ }^{24}$. The change to tail anode orientation and the relatively higher electrophoret1c mobility and speed of re-orientation of cauda spermatozoa under the same conditions reflected an absolute increase in the net negative surface charge over the tails during maturation of the spermatozoa, He raised the question of whether or not an absolute reduction in the negative surface charge density of the head region occurred during epididymal passage of the sperm. Spermatozoa failed to exhibit head-head agglutination in media giving head anode orientation in a mirroelectrophoresis cell using media of low ionic strength. This suggested that the increased net surface charge over the head region in all epididymal spermatozoa might exert a repulsive force great enough to negate any inherent attractive forces on the sperm surface ${ }^{24}$. However, the failure of caput spermatozoa to agglutinate and their weak head anode orientation response hit high lunle slrenglh media suggested that the failure to agglutinate also may be due equally to the absence of attractive forces over the immature sperm surface 24 . Bangham showed that with isotonic reduction in ionic strength of the surrounding medium, the increase in sperm surface potential was distinctly non-uniform in character and that the ortentation of spermatozoa in the electric ficld changed from predominantly tail-anode to head-anode ${ }^{18}$. Bedford suggested that this change in surface property observed by Bangham reflected the interaction of free radicals, such as the amino or imino groups at the sperm surface, with solute molecules in the medium ${ }^{27}$. This occurred to a greater extent over the head region emphasizing the different characteristics of the sperm surface over the two regions 27 . 


\section{Genetic Composition.}

Somatic cells of the rabbit, bull and domestic cock normally contain 44, 60 and 78 chromosomes, respectively. Chiarelli, De Carli and Nuzzo present excellent pictures of the chromosomes of the rabbit in which 1 appears that the $\mathrm{Y}$-chromosome is approximately one-quarter to one-third of the length of the $x$-chromosome ${ }^{57}$. Gustavsson presents photographs of chromosomes from cattle showing the normal patterns for both male and female with 60 chromosomes $^{96}$. In addition, abnormal patterns with 59 and 58 chromosomes, show the replacement of 2 telocentric chromosomes by a long, sub-telocentric chromosome, and the replacement of 2 telocentric chromosome pairs by a sub-telocentric chromosome pair, respectively ${ }^{96}$. Thus, even though the organization of the chromosomes has changed, the total genetic composition is unchanged.

In regard to this paper, the interesting question is whether or not differences in the genetic composition of spermatozoa are reflected in differences in physical properties which could be detected by experimental techniques. The most obvious and most interesting difference in genetic composition is that between the $\mathrm{X}$ - and $\mathrm{Y}$-chromosome bearing spermatozoa of marmals. Many attempts to segregate these 2 types of spermatozoa have been mentioned in Section $B$. As noted above, the sex-1inked antigen Xga has not been shown to be present on spermatozoa.

Because all spermatozoa in the domestic fowl normally bear X-chromosomes, there is no opportunity to effect a fractionation of fowl spermatozoa based on sex chromosomes. Work with fowl spermatozoa was motivated by the availability of a line of cocks with spermatozoa, some of which have a translocation which was produced by $\mathrm{X}$-irradiation with 4,000 $\mathrm{r}$ at the Department of Poultry Husbandry in Berkeley, California. The trans- 
location, TN IV, has been demonstrated genetically and cytologically ${ }^{120}$. The semi-sterility of the translocation heterzygotes furnishes a simple and practical means of detecting the translocation in spermatozoa. The spermatozoa carrying the translocation apparently are motile and capable of fertilizing ova, which, however, will give non-viable zygotes.

\section{Summary}

The interrelationships among the physical and blological properties of spermatozoa and the important experimental variables, listed in Table I, are too numerous to recapitulate in detail. It is sufficient to say that the characteristics of the dilution medium affect the electrophoretic mobility, sedimentation rate and specific gravity as well as the viability, intrinsic motility, fertilizing capacity and agglutination of spermatozoa. In certain experimental systems, the spermatozoa concentration can jeopardize electrophoretic mobility and sedimentation rate measurements and is also a critical factor in preserving the viability, intrinsic motility and fertilizing capacity of spermatozoa. The Ionic strength, viscosity, and, to a lesser extent, the specific gravity of the medium are affected by temperature and consequentiy influence the results of electrophoresis and sedimentation experiments. Temperature also influences the preservation of the vital functions of spermatozoa IN VIVO and IN VITRO.

The time-dependence of the preservation of viability, intrinsic motility and fertilizing capacity of spermatozoa is a primary consideration in the design of experiments. If one is trying to exploit small differences in sedimentation rate, electrophoretic mobility or specific gravity among spermatozoa, relatively more time for experiments might be necessary to achieve satisfactory resolution of the components. Thus, 
the selection of media, spermatozoa concentration, temperature and experimental operating conditions are all related by time.

Finally, the external forces are determined primarily by the physical properties of the spermatozoa, and, in turn, influence the preservation of the vital functions of spermatozoa generally in a deleterious manner. Thus, as will become evident in the subsequent chapters, these interrelationships remind the author of one's attempting to put one too many marbles into a dish: "for every marble put in, one pops out".

In the review of the physical and biological properties in Sections $B$ and C above, many possible methods were presented by which spermatozoa populations might be fractionated into groups with interesting biological differences. Gordon reported a partial dimorphism in the rabbit spermatozoa populations he studied, but found no correlation with the sex ratio of offspring from the insemination of electrophoretically fractionated samples 92 . Shettles reported differences in the size of human spermatozoa and speculated that the larger contained the $X$-chromosome ${ }^{214}$. Lalrd reported a correlation between the morphological features of bull spermatozoa with two-way electrophoretic migration in an apparatus similar to that used by Gordon ${ }^{129}$. However, only Bhattacharya has reported. a correlation of the size of spermatozoa with the sex of offspring therefrom (large sperm give female offspring) ${ }^{35}$.

The electrophoretic separation of $\mathrm{X}$ - and Y-chromosome bearing rabbit. spermatozoa reported by Schröder 206,207 and by Gordon 91,92 has not been confirmed by other investigators. In fact, Bangham and others have shown that above $\mathrm{pH} 4$, all spermatozoa have a net negative charge $18,174,233$. Bangham has shown slight differences in the electrophoretic mobility of head anode and tail anode ram and rabblt spermatozoa under extreme conditions 
of $\mathrm{pH}$ and ionic strength ${ }^{18}$. The latter differences have not been studied with regard to sex ratio of offspring. There are changes in the electrophoretic properties of rabbit spermatozoa during maturation ${ }^{24}$, but these, again, do not appear related to the properties of spermatozoa in the ejaculate.

Bhattacharya showed that the faster sedimenting rabbit spermatozoa gave predominantly female offspring after insemination ${ }^{35}$. Schilling, working with bull spermatozoa, obtained slightly more female than male offspring from the insemination of the faster sedimenting group of spermatozoa 205 , lending support to Bhattacharya's hypothesis. However, Bhattacharya and co-workers, in a study similar to that by schilling, found no difference in the sex ratio from the insemination of fast and slow sedimenting groups of bull spermatozoa ${ }^{37}$. Recently, Bedford and Bibeau found no correlation between the sedimentation rate of rabbit spermatozoa and the sex ratio of offspring ${ }^{29}$. Thus, Bhattacharya's results with rabbit spermatozoa and Schilling's work with bull speimatozoa have yct to be duplicated.

Lindahl and Thunquist have shown a difference between the specific gravities of epididymal and efaculate speruatozoa that does not have much relationship to the fractionation of mature spermatozoa in the ejaçulate ${ }^{139}$. Benedict and co-workers have found light and heavy groups of mature rabbit spermatozoa using a hypertonic equilibrium denefty gradient ${ }^{32}$. There is a suggestion that dead spermatozon have a higher apparent buoyant density in this hypertonic medium than do live spermatozoa ${ }^{32}$. Thus, the heavy group may be an artifact due to uptake of the density gradient material. The use of small, heavy molecules to prepare high specific gravity density gradients may account for the great discrepancies in the apparent buoyant densities of rabbit and bull spermatozoa mentioned above. 
III. THE STAFLO METHOD AND FRACTIONATION PRINCIPLES

Contents

A. Introduction. . . . . . . . . . . . . . 54

B. The Staflo Method ............... 55

1. The Staflo Apparatus. ............. 55

a. Flow-Cells.................. 55

b. Pumping System and Sample Introduction...... 58

c. Collection Systems. ............. 65

d. Electrode/Electrical Systems. . . . . . . . 66

e. Optical Equipment ............ 67

2. Modes of Operation. .............. 68

a. Types of Experiments. ........... 71

b. Experimental Varlables. ........... 71

c. Interrelationships among Varlables. . . . . 73

c. Staflo Migration-Fractionation Principles ....... 74

1. Sedimentation Experiments . . . . . . . . 74

a. Migration Distances.............. 75

b. Calculation of Sedimentation Velocity . . . . 84

2. Sedimentation-Electrophoresis Experiments . . . . 85

a. Sedimentation-Downward-Electrophoresis Migration Distances ................ 85

b. Sedimentation-Upward-Electrophoresis Migration. . . 86

Distances : . . . . . . . . . . . .

c. Comparison of Sedimentation-Downward-and-UpwardElectrophoresis ............... 95

d. Calculation of Electrophoretic Mobility . . . . 96

3. Effects of Motility on Migration and Fractionation. . 97 
a. Sedimentation Experiments. . . . . . . . . 98

b. Sedimentation-Electrophoresis Experiments. . . . 98

4. Signiflcance of This Method of Analysis. . . . . . . 103

D. Summary and Conclusions. . . . . . . . . . . . 105

\section{A. Introduction}

In the previous chapter, electrophoretic mobility, sedimentation rate and Intrinsic motility were considered among the physical and biological properties by which semen spermatozoa might be fractionated. One purpose of this section is to describe the stable-flow free-boundary (Stafio) apparatus and ancillary equipment used in this study. In so doing, the flexibility of use of the Staflo apparatus, which makes it possible to investigate the properties of spermatozoa mentioned above, either individually or in various combinations, will be illustrated. The other objective of this section is to present a new approach to quantitate the design and interpretation of Staflo experiments, including equations for determining migration distances and rates for particles in each collection fraction in Staflo-sedimentation and -sedimentation-electrophoresis experiments.

Except for the interesting ramifications of migration patterns caused by the Intrinsic motility of spermatozoa, and concelvably by other cells or organisms with significant motility, the discussion of the Staflo method and fractionation principles applies to all migrating species. In addition, the fractionation principles, with slight modification, may serve as a model for the analysis of component parts of migration patterns in other fractionation devices. 


\section{B. The Staflo Method}

The theory of the Staflo method has been described by Mel $1^{156-158}$ and amplified recently with regard to the three dimensional aspects of flow by Tippetts ${ }^{263}$ and by Tippetts, Mel and Nichols ${ }^{229}$. This section describes the different models of the Staflo apparatus and ancillary equipment used in the studies reported in subsequent chapters. In addition, a partial listing of the types of experiments which can be conducted with the Staflo apparatus is presented to demonstrate the versatility of the apparatus.

\section{The Staflo Apparatus.}

Figure 3 is a photograph of the $3 \times 16$ Staflo apparatus in a refrigerated room. The letters on Figure 3 correspond to those in Figure 4 which is a schematic drawing of the Staflo apparatus. The essential features of the Staflo apparatus are as follows: (A) A multi-syringe pumping system for introducing solutions into the flow-cell, (B) the flow-cell proper and (C) a multiple-tube collection system for solutions exiting the flow-cell. Ancillary equipment includes the electrical system consisting of electrode compartments, an electrode wash flow system (D), an electric power supply unit (E) and the Staflo optical system, (F) shown only in.Figure 3. Each of these features and its modifications during the course of the study is discussed below.

a. Flow-Cells. The key feature of the Staflo apparatus is the flow-cell proper, 3 types of which were used in the experiments discussed in subsequent chapters. A flow-cell consists of a flow (migration)-chamber and upper and lower electrode chambers separated by cellophane membranes (dialysis tubing). They are distinguished by the design of the inlet and outlet flow dividers. Greater importance is attached to the latter because it determines the extent of fractionation of the migration profile. The 


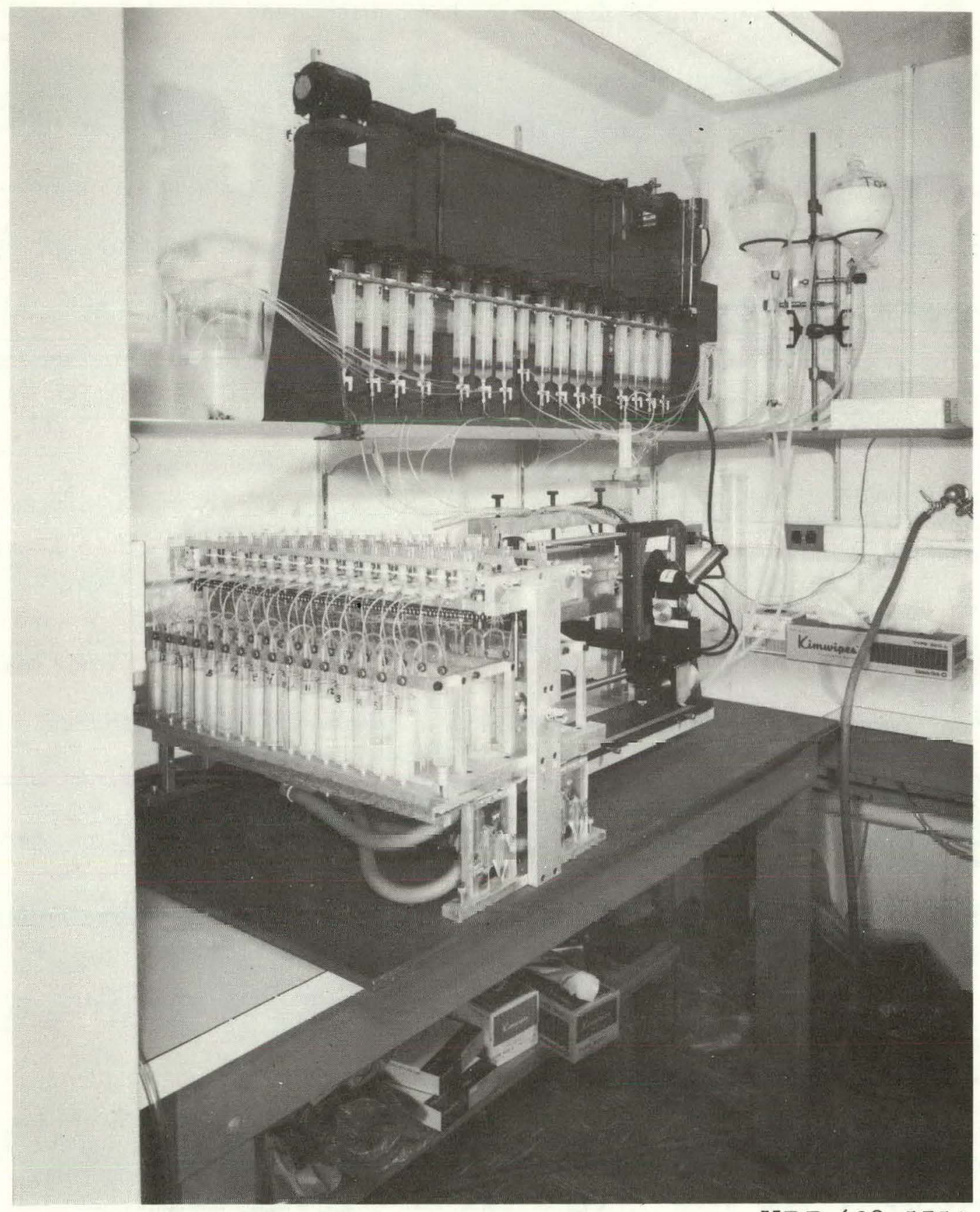

XBB 689-5751

Fig. 3. Photograph of $3 \times 16$ Staflo apparatus. Letters correspond to components similarly designated in Figure 4. 


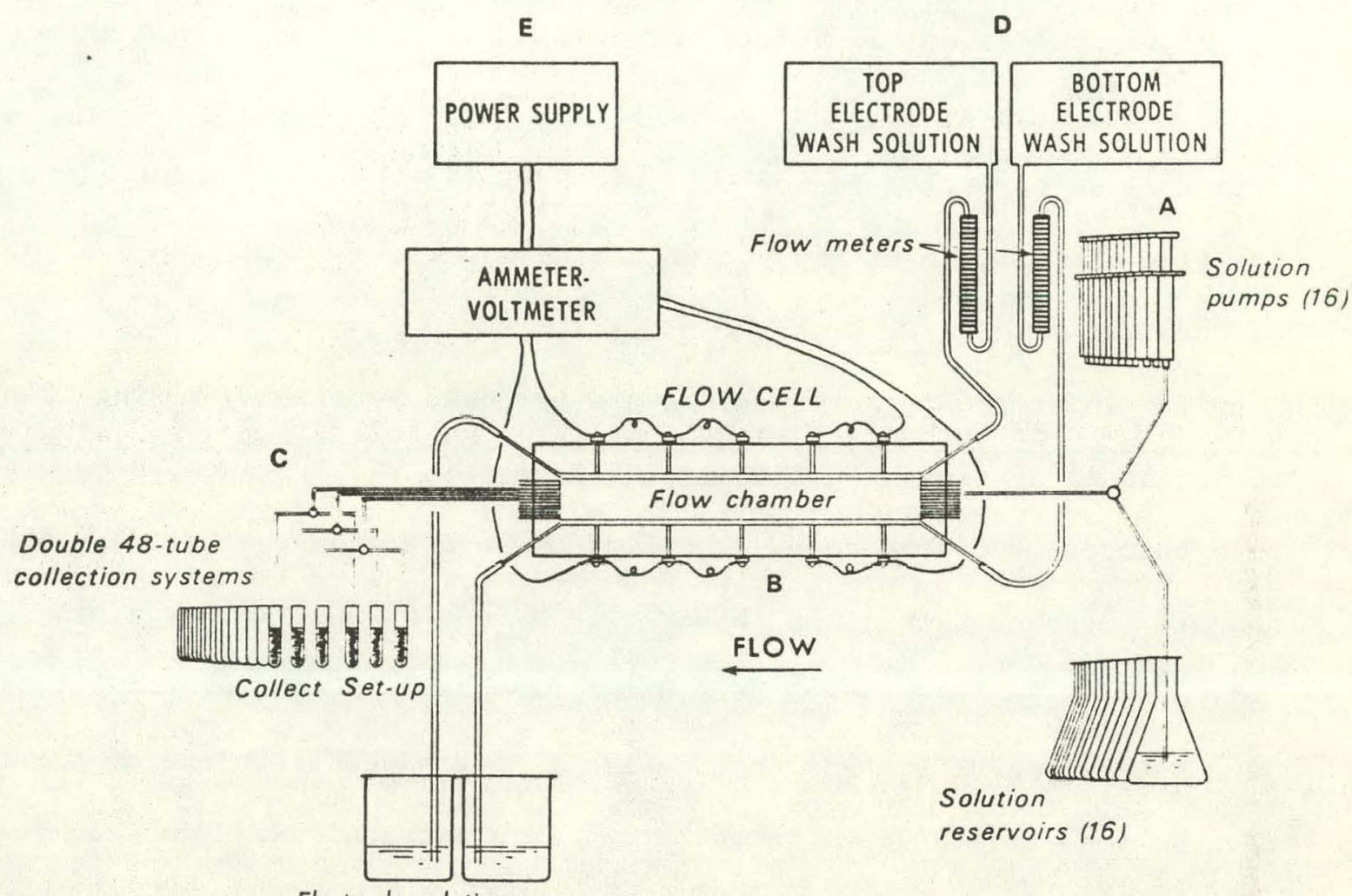

Electrode solution sumps

$M U .36571$

Fig. 4. Schematic diagram of Staflo apparatus showing main components. 
characteristics of the $3 \mathrm{flow}$-cells used in this study (designated numbers VII C, XI and XIII), are presented in Table II. The flow-cells, including the flow dividers, were machined from lucite. On the newer flow-cells, a wall thickness of $0.8 \mathrm{~mm}$ was used to provide better observation with the microscope described below. Figures 5 and 6 show flow-cell number $\mathrm{XI}$ in place with and without the Staflo microscope, respectively. Figure 7 is a close-up photograph of the $3 \times 16$ flow-cell on its mount showing the flow-chamber, inlet and outlet tubing and wires to the top and bottom electrodes.

The inlet and outlet openings are arranged vertically giving 12 or 16 horizontally flowing $0.125 \mathrm{~cm}$ high layers in the 1.5 and $2.0 \mathrm{~cm}$ high flow-chambers, respectively. The flowing layers are stabilized by hydrodynamic feedback principles rather than by use of a solid supporting medium 156,157 . The average horizontal flow rate is the same in all layers. If a heterogeneous suspension of cells is introduced into one of the upper layers, the gravitational force acting in a vertical direction can be expected to cause a differential downward migration of the cells ${ }^{158}$. This is illustrated in Figure 8 where a cell suspension introduced into layer 8 of the $3 \times 16$ Staflo apparatus gives a downward migration pattern over 3 layers.

The $1 \times 12$ flow-cell (No. VII C) was used in all of the early sedimentation cxperiments (Chapter IV) and some of the early electrophoresis experiments (Chapter V). The $3 \times 12$ flow-cell (No. XIII) was used only in 2 sedimentation experiments discussed in Chapter VIII. In all other Staflo experiments, the $3 \times 16$ flow-cell (No. XI) was used.

b. Pumping System and Sample Introduction. Different pumping systems were used with the 3 types of flow-cells described above. Although differing in design, the principle of operation is the same for all pumping 
Table II. Characteristics of Staflo Flow-Cells

\begin{tabular}{|c|c|c|c|}
\hline \multirow[t]{2}{*}{ Design Features } & \multicolumn{3}{|c|}{$\begin{array}{c}\text { Flow-Cell Type } \\
\text { (Number) }\end{array}$} \\
\hline & $\begin{array}{l}1 \times 12 \\
\left(\begin{array}{lll}\text { VII } & C\end{array}\right)\end{array}$ & $\begin{array}{l}3 \times x \quad 12 \\
\left(\begin{array}{lll}X I I\end{array}\right)\end{array}$ & $\begin{array}{l}3 \times 16 \\
(\mathrm{XI})\end{array}$ \\
\hline \multicolumn{4}{|l|}{ Flow-Chamber: } \\
\hline Length $(\mathrm{cm})$ & 30 & 30 & 30 \\
\hline Width $(\mathrm{cm})$ & 0.7 & 0.7 & 0.8 \\
\hline Height $(\mathrm{cm})$ & 1.5 & 1.5 & 2.0 \\
\hline \multicolumn{4}{|l|}{ Inlet array: } \\
\hline Horizontal (no.) & 1 & 3 & $3^{a}$ \\
\hline Vertical layers (no.) & 12 & 12 & 16 \\
\hline Layer height (cm) & 0.125 & 0.125 & 0.125 \\
\hline \multicolumn{4}{|l|}{ Outlet array: } \\
\hline Horizontal (no.) & 1 & 3 & 3 \\
\hline Vertical layers (no.) & 12 & 12 & 16 \\
\hline Wall thickness (cm) & 1.33 & 0.8 & 0.8 \\
\hline \multicolumn{4}{|l|}{ Electrode chamber: } \\
\hline Height $(\mathrm{cm})$ & 0.25 & 0.25 & 0.25 \\
\hline Number of electrodes & 1 & 6 & 6 \\
\hline Electrode length $(\mathrm{cm})$ & 29.5 & 4.95 & 4.95 \\
\hline Electrode spacing $(\mathrm{cm})$ & 0 & 0.05 & 0.05 \\
\hline
\end{tabular}

a. In practice, solutions were introduced only in the center inlet in each of the 16 layers. 


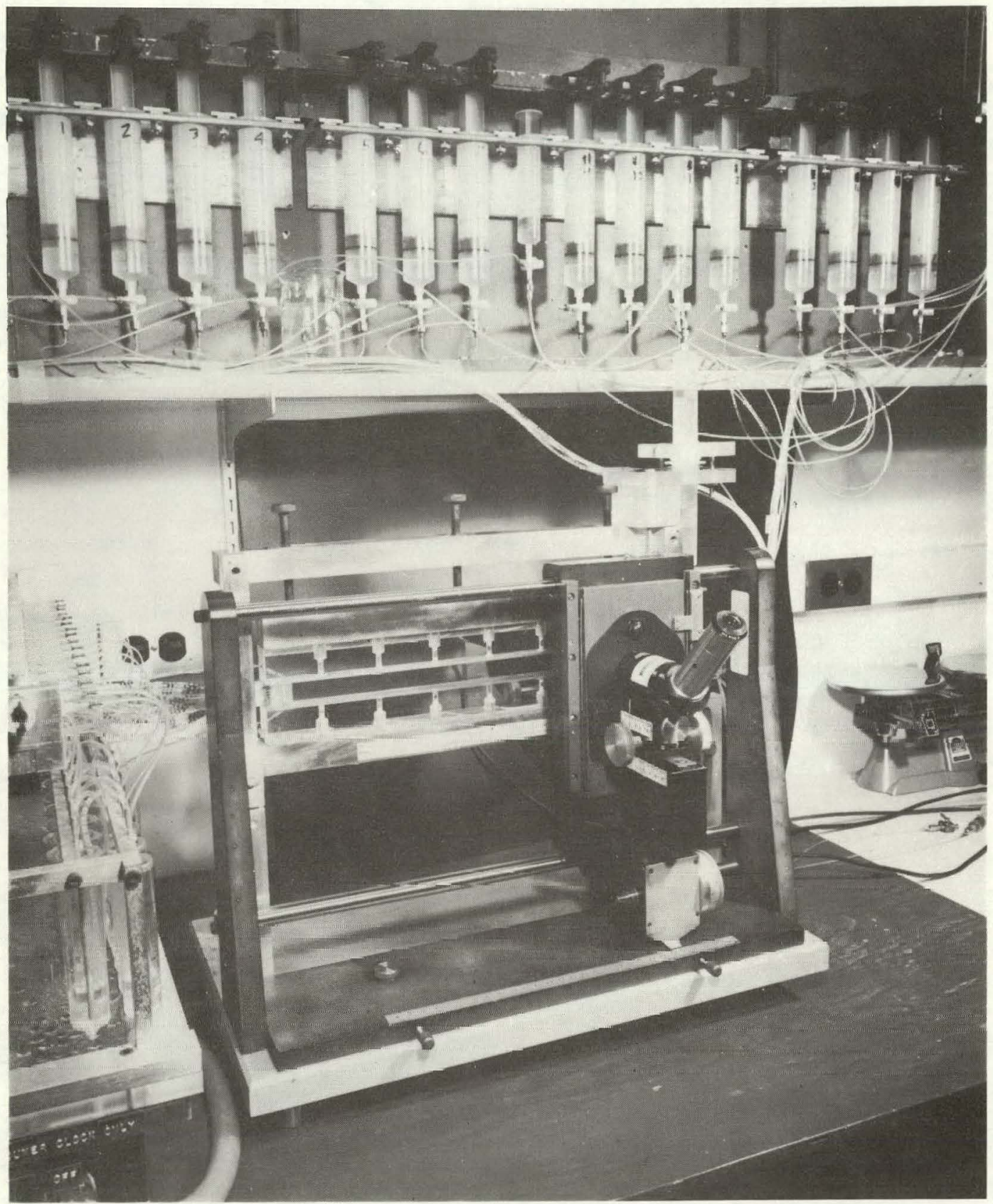

CBB $6812-7720$

Fig. 5. Photograph of Staflo flow-cell and mount with microscope in place. 


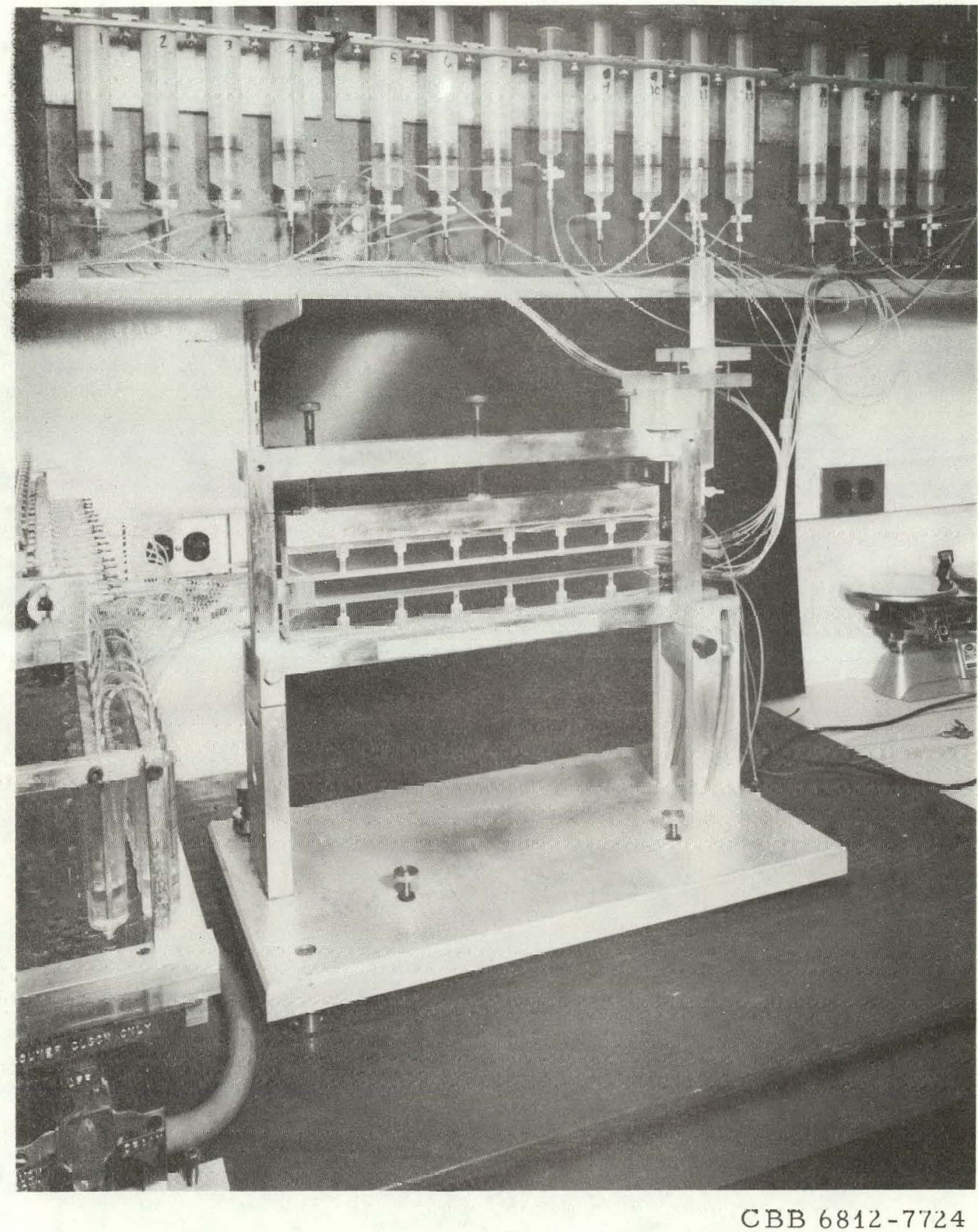

Fig. 6. Photograph of flow-cell and mount with microscope removed. 


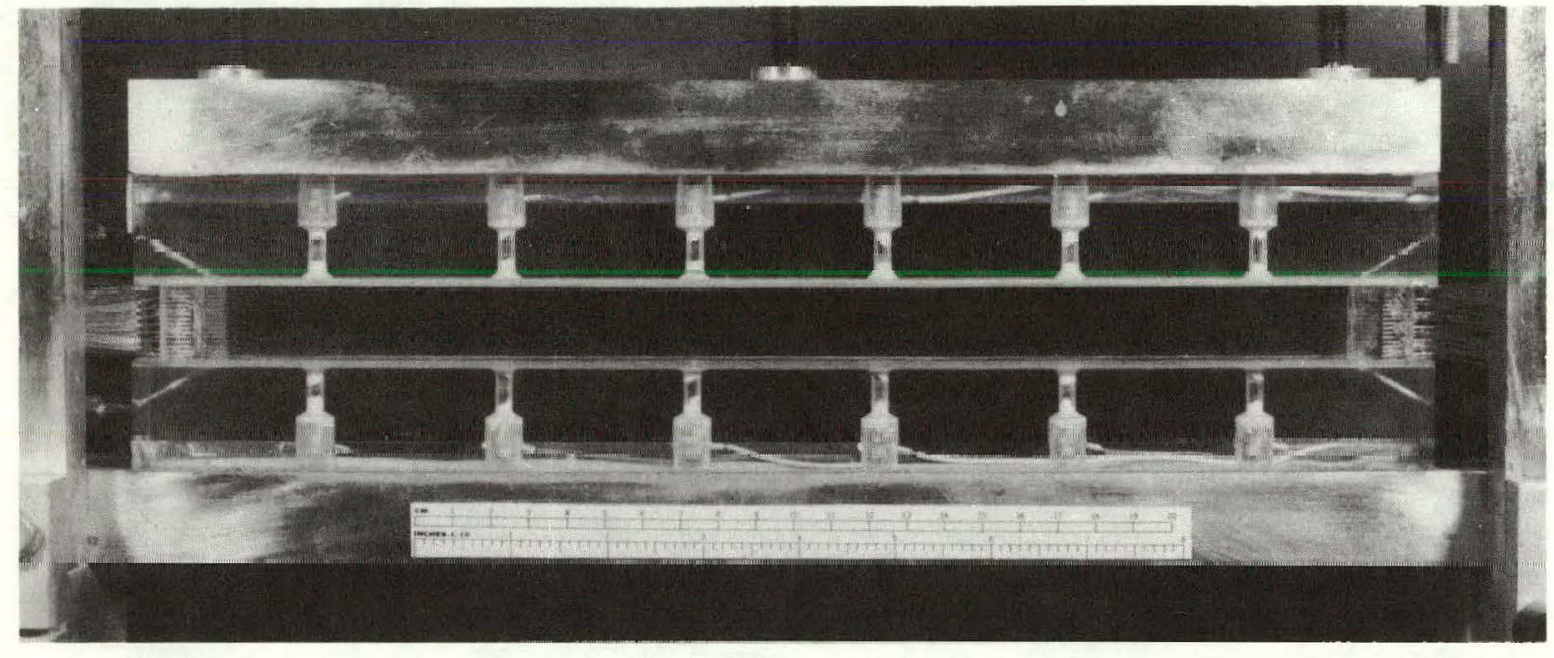

C BB $6812-7726$

Fig. 7. Closeup of flow-cell showing flow-chamber and part of wiring to electrodes. 


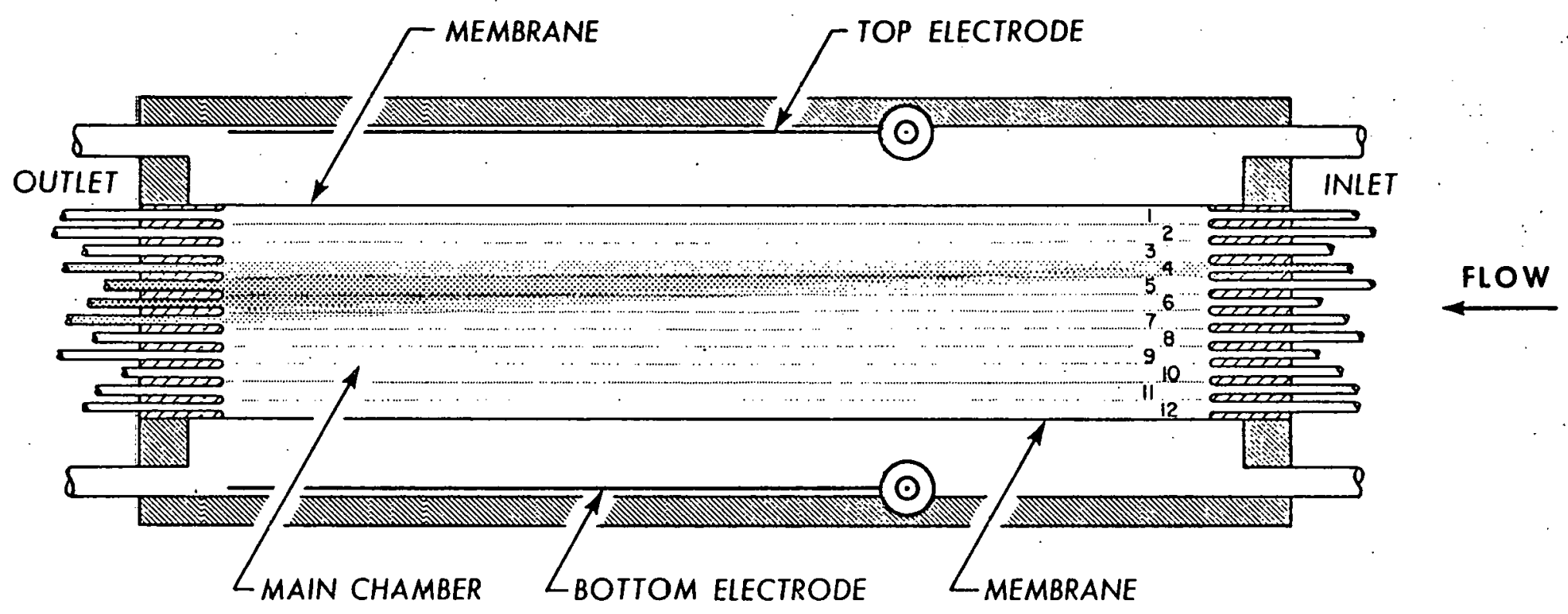

$$
M U-32018-B
$$

Fig. 8. Schematic diagram $0 \equiv$ flow-cell showing spermatozoa migration pattern. 
systems. Each system consists of a rack of plastic syringes containing solutions for the respective inlets on the flow-cel1 (see Figure 3). The rack is driven by a variable speed motor which allows a wide range of accurately controlled solution flow rates. The pumping system used with the $1 \times 12$ flow-cell consisted of a linear rack containing twelve $20 \mathrm{ml}$ plastic syringes (TOMAC Disposable Syringe, American Hospital Supply Corp., Evanston, I11.). It is similar in design to that used with the $3 \times 16$ flow-cell which is described in more detail below. The pumping system used with the $3 \times 12$ flow-cell holds thirty-six $20 \mathrm{ml}$ plastic syringes.

The pump rack for the $3 \times 16$ flow-cell is shown in Figure 3 (A) and has holders for a linear array of gixteen $5 U . m \perp$ plastic syringes (YLASILHAK, Discardit, $50 \mathrm{ml}$, Becton, Dickinson and Co., Rutherford, N.J.). The syringes are connected by three-way valves to the flow-cell inlets and to solution reservolis. The pump rack is driven by a $1 / 70$ horsepower, 0 to 1725-rpm shunt-wound electric motor (Bodine Electric Co., Chicago, I11.), with a varlable-speed power supply (Minarik Electric Co., Los Angeles, Calif., Model Sh-14). The low speed drive rate is controlled by a variable speed Zero-Max Torque Converter (Revco Incorporated, Minneapolis, Minn., Model 14 Standard) giving a solution flow rate range of $0.075-2.62 \mathrm{ml} / \mathrm{minute} j$ inlet with $50 \mathrm{ml}$ syringes. The pump rack can be driven in reverse to fill the syringes from solution reservoirs.

The sample suspension can be introduced into the flow-cell with one of the syringes on the pump rack giving a sample stream $0.125 \mathrm{~cm}$. high. In order to preserve the viability of many biological species, it is desirable to reduce the length of inlet tubing by mounting the sample syringe near the inlet and controlling its flow rate from the pump rack. For greater resolution of the migrating particles, often it is desirable to reduce 
the height of the sample stream by using a syringe size smaller than that used for the other 15 inlets ${ }^{263}$. For instance, 10 and $20 \mathrm{ml}$ plastic syringes give solution flow rates approximately $37 \%$ and $52 \%$ that of the 50 ml plastic syringe, respectively. The corresponding sample streams would be 0.046 and $0.065 \mathrm{~cm}$ high compared to $0.125 \mathrm{~cm}$ in the other layers. This arrangement is shown in Figure 7 where syringe $\$$ is on the rack has been replaced by a $20 \mathrm{ml}$ plastic syringe which drives the $20 \mathrm{ml}$ sample syringe mounted on the right side of the flow-cell near the inlet. Generally, magnetic stirring is used to maintain a uniform suspension in the sample syringe throughout the experiment.

c. Collection Systems. An adequate collection system for the outlet solutions from the flow-chamber is a level platform holding one plastic syringe barrel (with plugged tip) for each outlet tubing. This system has the shortest possible outlet tubing and was used effectively with the $3 \times 12$ and $3 \times 16$ flow-cells with $10 \mathrm{ml}$ and $20 \mathrm{ml}$ plastic syringe barrels, respectively.

If use of longer tubing is compatible with the type of particle being studied, operation of the Staflo apparatus is simplified by a valved collection system such as that shown for the $3 \times 16$ flow-cell in Figure 3 . In this dual. collection system, the outlet flow can be directed to either of the two sets of forty-elght $20 \mathrm{ml}$ collection containers (plastic syringe barrels). One set is used for setting up ("SET UP" side) and the other for collection ("COLLECTION" side) of the fractionated sample. Prior to introducing the sample suspension; density gradient solutions are run to both the SET UP and COLLECTION sides to establish steady state conditions in the flow-chamber. This condition is ascertained by observing the level of entrance and exit of fluld in one layer. This is fac1litated by introduclng a blue dextran solution in the sample layer. Only enough solution 
is run to the COLLECTION side to insure steady state conditions in order to minimize the volume of solution in the collection containers. Flow is switched to the SET UP side for the introduction of the sample suspension. After the sample stream has attained the desired migration pattern across the flow-cell and has been malntained for $1 / 6$ of the average transit time across the flow-cell, the outlet flow is switched to the COLLECTION side. Thus, any initial instability can be corrected without contaminating the final collection fractions. Generally, only the center fraction of each layer is saved for evaluation, e.g., that coming from the middle outlet channel of the three horizontal ones in each layer.

Both oidco of the collection systeï can le emplied ldyldly by a vacuum system to facilitate cleaning in place. The central row of containers on the COLLECTION side can be removed for more thorough cleaning and for working with the fractionated samples.

d. Electrode/Electrical system. The electrical system for electrophoresis experiments consists of electrodes in the electrode chambers, a power supply unit and an electrode wash system. As noted above, the electrode chambers are separated from the flow-chamber by cellophane membranes (dialysis tubing). The electrode chambers are $0.25 \mathrm{~cm}$ deep and have the same length and width as the flow-chamber. On the $3 \times 16$ flow-cell, there are 6 platinum electrodes $0.8 \mathrm{~cm}$ wide and $4.95 \mathrm{~cm}$ long, leaving spaces of approximately 0.05 $\mathrm{cm}$ between adjacent electrodes. The 6 sections of electrode area can be controlled individually, giving the flexibility of a programmed electric fle $1 \mathrm{~d}^{263,229}$. As discussed by Tippetts, a bubble forme in each of the $0.05 \mathrm{~cm}$ gaps due to extreme polarization at those sites and effectively insulates the shorted-out electrodes from the active electrophoresis electrodes $^{263}$. Thus, one can get a time average uniform electric field with 
high currents and without lethal polarization. Power is supplied to the electrodes by a regulated DC power supply (Power Designs, Inc., Model 5015S) monitored by a General Electric voltmeter and ammeter.

The electrode wash flow system consists of two 2,000 ml separatory funnelis, acting as reservoirs, connected to the electrode chambers by plastic tubing. Solutions flowing in the top and bottom electrode chambers generally are the same as the solutions in layers 1 and 16 , respectively, of the flow-chamber. The flow rates can be regulated over the range $0-34$ $\mathrm{ml} / \mathrm{min}$ with floating-ball flow meters (Fischer and Porter Co., Holbord, Pa.). Changes in $\mathrm{pH}$ and temperature in the flow-chamber due to the electric field can be controlled by increasing the electrode wash flow rate (EWFR) without excess hydrodynamic shock.

Temperature measurements were made in the $3 \times 16$ flow-chamber with a Varian Temperature Recorder connected to a probe at the level of layer 5 approximately $3 \mathrm{~cm}$ from the outlet. The results showed that the temperature rise in the flow-chamber depends upon the electric field strength, the solution flow rate and especially the EWFR.

With room and solution temperatures of $4-6^{\circ} \mathrm{C}$, an average transit time of 21 minutes, an electric field over the last $25 \mathrm{~cm}$ of the flow-chamber giving a heat production of 1,200 watt-sec, and an EWFR of $20 \mathrm{ml} / \mathrm{min}$, the maximum and average changes in temperature in the flow-chamber would be $+1.0^{\circ} \mathrm{C}$ and $+0.5^{\circ} \mathrm{C}$, respectively. With rare exceptions, the operating conditions used in this study gave temperature rises of this or lesser magnitude.

e. Optical Equipment. The slit lamp technique was used for observations and measurements of the gross migration pattern and profile $229,263$.

The acquisition of several long working distance objectives and a 
reduction of flow-cell wall thickness made microscopic observations of cells in the flow-chamber possible. Inftially, the microscopic system consisted of a horizontally mounted microscope and an Independent light source. Because difficulties in alignment resulted in poor observation and were time consuming, the microscope shown in Figures 9 and 10 was constructed specifically for use with the Staflo apparatus. An adjustable light source, a condenser and the microscope were aligned on a carriage which can move laterally the length of the flow-chamber. Complete vertical movement and depth focusing makes it possible to observe and locate cells anywhere in the flow-chamber within the accuracy of the millimeter scales on all 3 axes of movement. Although excellent viewing was possible at magnifications up to $640 \mathrm{X}$, a magnification of $320 \mathrm{X}$ with dark field illumination was generally used for observing spermatozoa.

The stand to which the microscope carrlage 1s attached can be fasteried by set screws to the Staflo mount to prevent Inadvertent movement and is interchangeable with all current Staflo apparatuses.

\section{Modes of Operation}

The versatility of use of the Staflo apparatus in electrophoresis, sedimentation and sedimentation-electrophoresis experiments has been described by Mel ${ }^{158}$. With only a change in the medium used in the flow-chamber, a varlety of studies can be conducted for either analytical or preparative purposes. The experimental design considerations presented in Chapter II with regard to studies on spermatozoa are applicable to, and place many restrictions upon, Staflo experiments with those biological cells. This section presents general experimental design considerations pertaining to different modes of operation of the Staflo apparatus without regard to the type of cell being studied. 


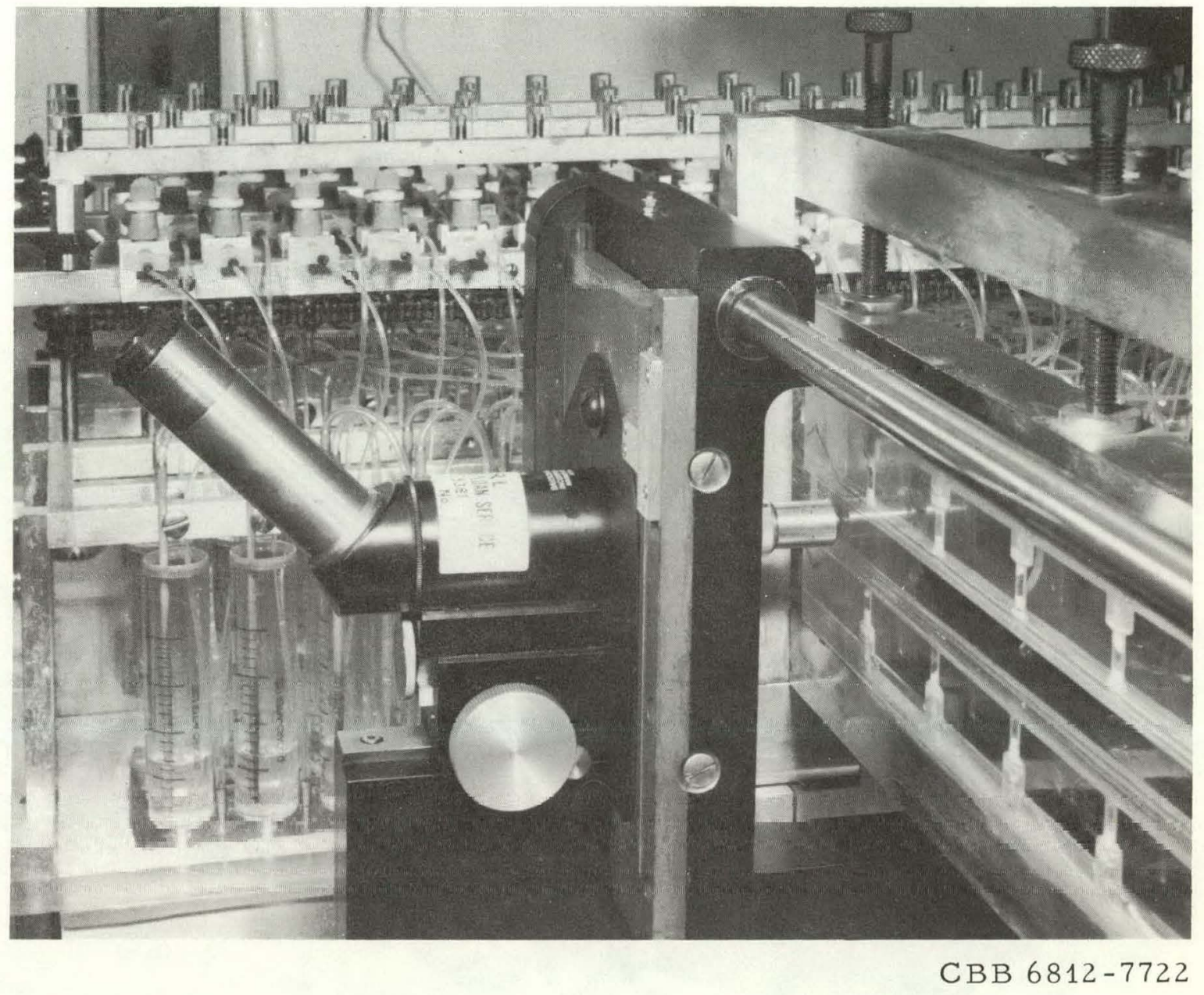

Fig. 9. Closeup of Staflo microscope mounted on Staflo apparatus. 


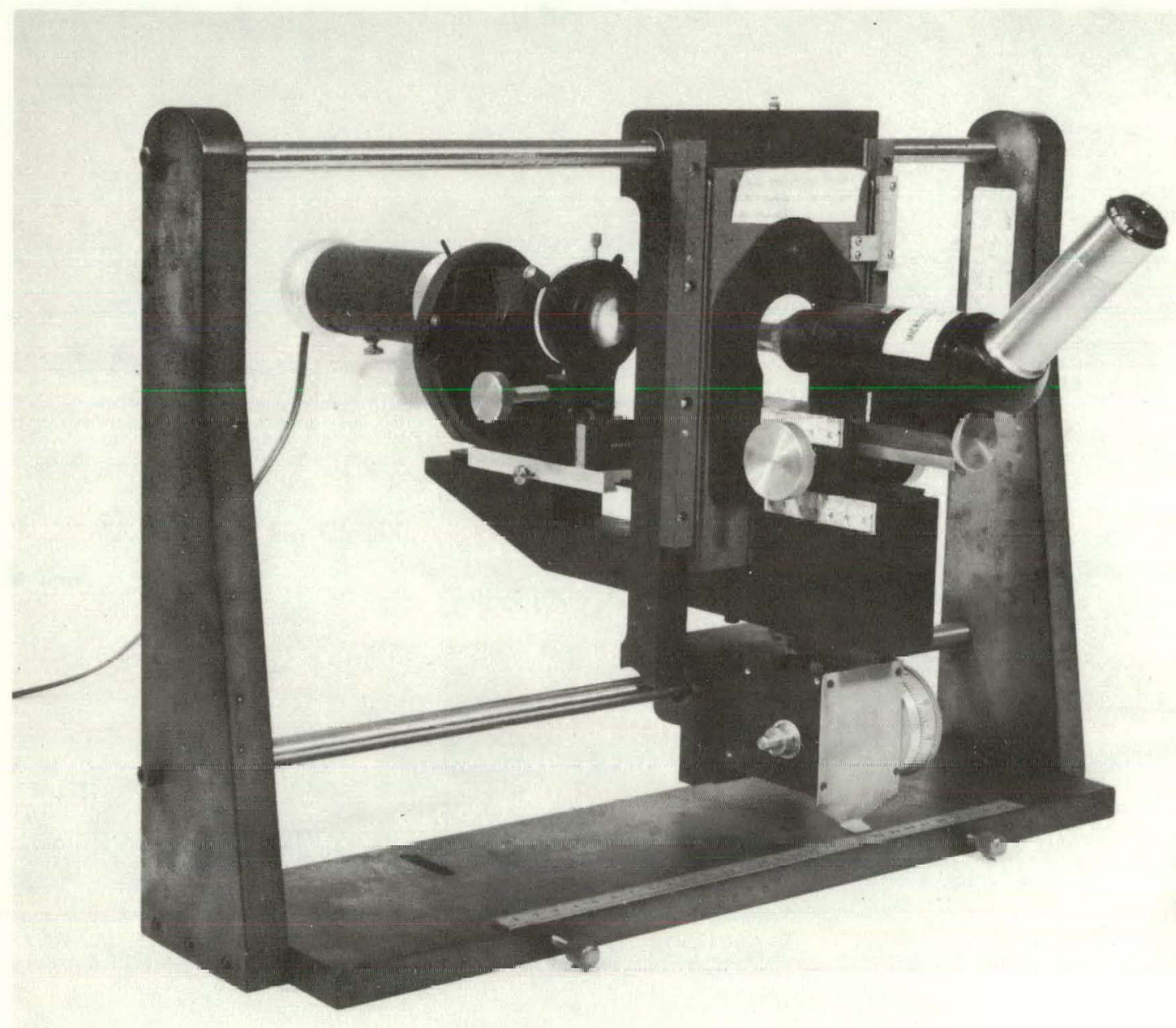

XBB 689-5752

Fig. 10. Photograph of Staflo microscope showing component parts. 
a. Types of Experiments. The types of Staflo experiments which can be conducted with populations of cells or particles include:

(1) 18 sedimentation

(2) Electrophoresis with upward and/or downward migration

(a) Single $\mathrm{pH}$

(b) Two or three $\mathrm{pH}$ regions within the Staf1o chamber

(c) $\mathrm{pH}$ gradient

(d) Conductivity gradient

(3) Sedimentation-upward-electrophoresis (SUE)

(4) Sedimentation-downward-electrophoresis (SDE)

(5) Exploitation of intrinsic motility, alone or in combination with sedimentation, electrophoresis, or both.

b. Experimental Variables. Experimental variables and the ways in which they influence each of the above groups of Staflo experiments are as follows:

(1) Transit time: Under a fixed set of conditions, the migration distance will be proportional to the transit time in all types of experiments. The appropriate transit time for any one experiment will depend upon the specific migration properties and the requirements for the preservation of vital functions of the specles being studied.

(2) Staflo medium: The speciflc gravity of the medium will determine whether one gets sedimentation and/or flotation of particles and the degree thereof depending upon the difference between the specific gravities of the medium and the particles. Because of the limited height of the flow-chamber, large differences between the specific gravities of the particles and the medium would give high migration rates for all particles, and consequently, poor resolution in fractionation. Ideally, an intermediate specific gravity would give flotation of the light particles and sedimentation of the heavy particles in the population. The osmolarity 
of the medium may influence the specific gravity of cells through changes in hydration.

Increases in viscosity of the medium will decrease all migration rates, thereby requiring longer transit times, especially in sedimentation experiments. Results from experiments with media of different viscosities can be normalized for comparison.

In aqueous solutions, the effects of changes in temperature are primarily reflected in changes of viscosity and conductivity; relatively smaller changes occur in speciflc gravity. The changes in conductivity are very important in electrophoresis experiments so the temperature and the fonfc strength of the medium must be known.

For microscopic observations in the flow-chamber, a clear medium must be used.

The $\mathrm{pH}$ of the medium primarily affects the alectrophoretic mobility of the migrating species and the conductivity of the medium and thus, is important in electrophorests and sedimentation-electrophoresis experiments. Its effect on the vital functions of cells should be considered, also.

(3) Density gradient: Materials added to the medium in low concentratIons to give a density gradient usually do not cause significant changes In the specific gravity. Sucrose, a common density gradient constituent, increases osmolarity with little effect on viscosity, while dextron has the opposite effect. These substances and other common density gradient materlals have little effect on conductivity and $\mathrm{pH}$ other than through volume changes. Too large an increment between layers can give a density shelf which acts as a barrier to migration of particles. However, diffusion of the density gradient molecules tends to minimize this problem, especlally if a low molecular weight substance such as sucrose is used for the density 
gradient $^{157}$

In sedimentation-upward-electrophoresis experiments, large density gradient increments may maintain the differential migration achieved against gravity by preventing convection 263 .

(4) Temperature: In addition to its effects on the medium as noted above, the temperature has a strong influence on the intrinsic motility of spermatozoa and other motile organisms. As the temperature approaches $0^{\circ} \mathrm{C}$, spermatozoan motility ceases. Low temperatures also aid in the dissipation of heat generated by the electric field.

(5) Electric field: The strength of the electric field determines the rate of electrophoretic migration and dictates the transit time. It also affects the temperature in the flow-chamber. A programmed electric field can be used for special purposes 229,263 .

c. Interrelationships Among Variables. As noted in Chapter II, the viability, motility and fertilizing capactty of spermatozoa are affected strongly by the characteristics of the medium. If a migrating species has both a significant sedimentation rate and electrophoretic mobility, the relative contributions of each can be controlled most readily by variations in the electric field strength. If 1 t is desired to minimize or eliminate the sedimentation component of migration in electrophoresis experiments, one can increase the specific gravity and viscosity of the medium, increase the density gradient or reduce the transit time. To do the latter, the electric field strength may have to be increased to get sufficient migration of the particles.

In experiments combining electrophoresis and/or sedimentation, and/or intrinsic motility, all of these variables must be considered in terms of their effects on the physical and blological properties of the migrating 
spectes and on the physical stabllity of the experimental system. These interrelationships are 1llustrated in Chapters IV, V, VI and VIII. .

\section{Staflo Migration-Fractionation Principles}

As noted above, the Staflo migration profile is fractionated at the outlet flow divider into $0.125 \mathrm{~cm}$ high layers which are collected separately. In addition to evaluating the particles in each collection fraction for differences in morphology, size and biological activity, it is often desirable to characterize each fraction by an average and range of sedimentation rates or electrophoretic mobilities. The limits of migration can be measured with the slit lamp technique 229 and intermediate measurements are calculated from the known positions of the outlet flow dividers. The figures presented in this section show the range and distribution of migration distances in the flow-chamber and the interplay of various combinations of sedimentation, electrophoresis and intrinsic motility. Txpressions for calculating the minimum and maximum migration distances for palllules in each layer in sedimentation and sedimentation-electrophoresis experiments are developed below. In addition, equations for calculating the average migration rates for particles in each layer are presented for populations having a uniform distribution of migration rates. These equations are applicable, to the fractionation of all types of partirles in the Staflo apparatus within the limitations upon which they are based. Only migration profiles exiting the flow-chamber in 2 or 3 adjactit layers are considered but the equations can be readily extended to 4 or more adjacent layers. The special problem of the effect of the intrinsic motility of spermatozoa on sedimentation and sedimentation-electrophoresis migration patterns is considered separately.

1. Sedimentation Experiments. 
Figures 11 and 12 are schematic diagrams of Staflo-sedimentation migration pattérns spreading over 2 and 3 adjacent layers, respectively. Figures $11 \mathrm{~A}$ and $12 \mathrm{~A}$ depict sedimenting particles exiting from the layer in which they enter and 1 or 2 adjacent layers below, respectively. Figures $11 \mathrm{~B}$ and $12 \mathrm{~B}$ show general conditions in which the particles exit the flow-chamber in any 2 or 3 adjacent layers below the inlet layer, respectively. The following discussion develops equations for calculating (1) the range and distribution of sedimentation migration distances, (2) the average migration distances for the appropriate collection fractions and (3) the weighted average sedimentation distance for the migration pattern as a whole for each of the conditions shown in Figures 11 and 12 .

a. Migration Distances. Because the average sedimentation migration distance for most collection fractions is not a simple average of the minimum and maximum migration distances, expressions for these distances will be developed for migration patterns spreading over 2 or 3 adjacent layers. Then expressions for the average migration distances will be developed for the same migration patterns.

Assumptions applicable in hoth 2- and 3-layer migration patterns are as follows: (1) The bottom of the inlet sample stream is at the bottom of the inlet layer under steady state conditions. (2) The maximum migration distance in layer $I$ is less than or equal to $h$, the height of the sample stream at the inlet. (3) Upon entry into the flow-chamber, the sample suspension is homogeneous. (4) The leading (bottom) edge of the migration pattern is determined by the fastest migrating particles, some of which were at the lower border of the sample stream at the inlet. (5) The top edge of the migration pattern is determined by the slowest migrating particles, some of which were at the upper border of the sample 
STAFLO "MIGRATION PATTERNS"

Sedimentation and sedimentation-downward-electrophoresis
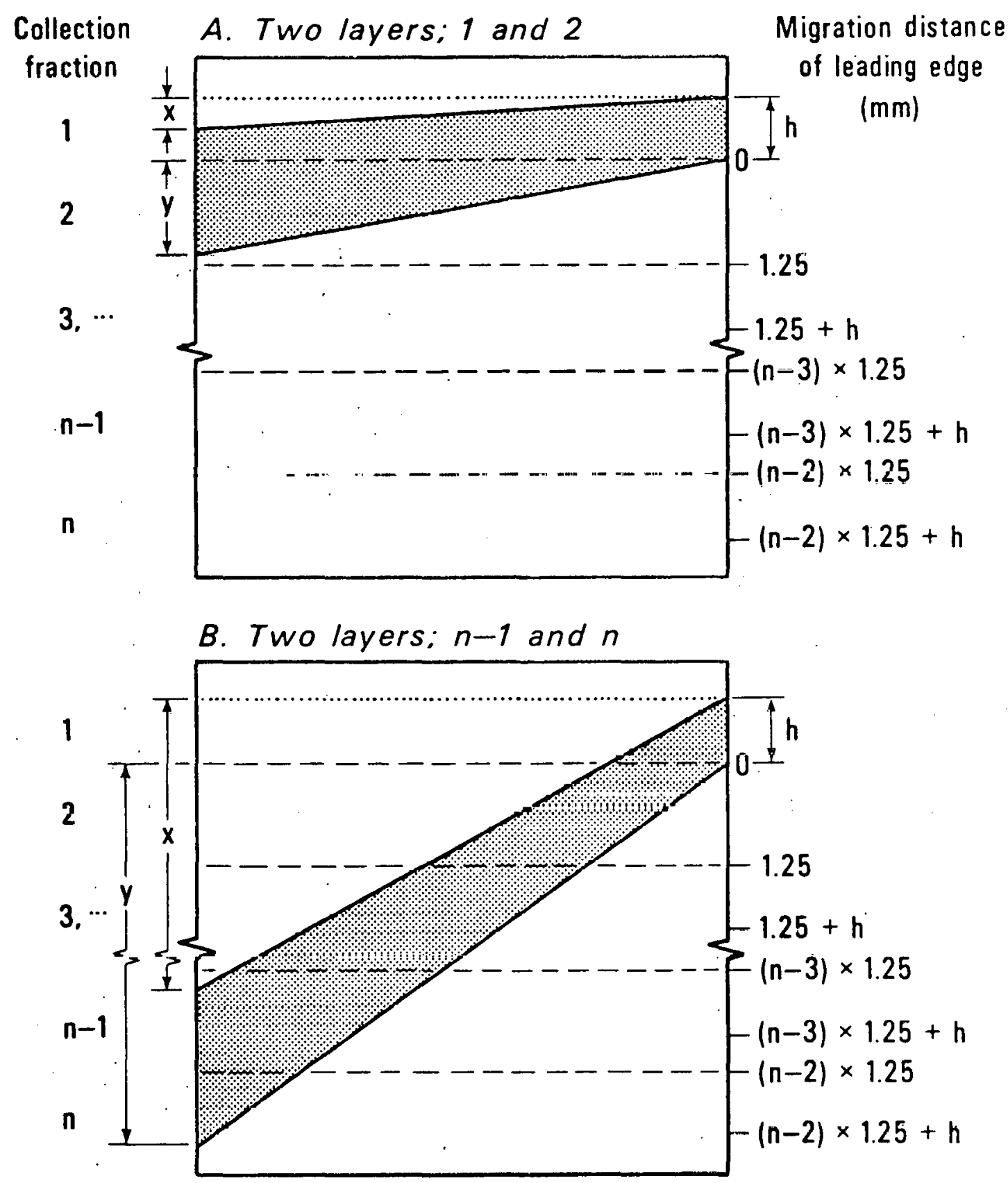

DBL 689-5473

Fig. 11. Schematic diagram of sedimentation or sedimentation-downwardelectrophoresis migration pattern; two-layer spread. A. Exit in layers 1 and 2. B. Exit in layers $n-1$ and $n$. 
STAFLO "MIGRATION PATTERNS"

Sedimentation and sedimentation-downward-electrophoresis
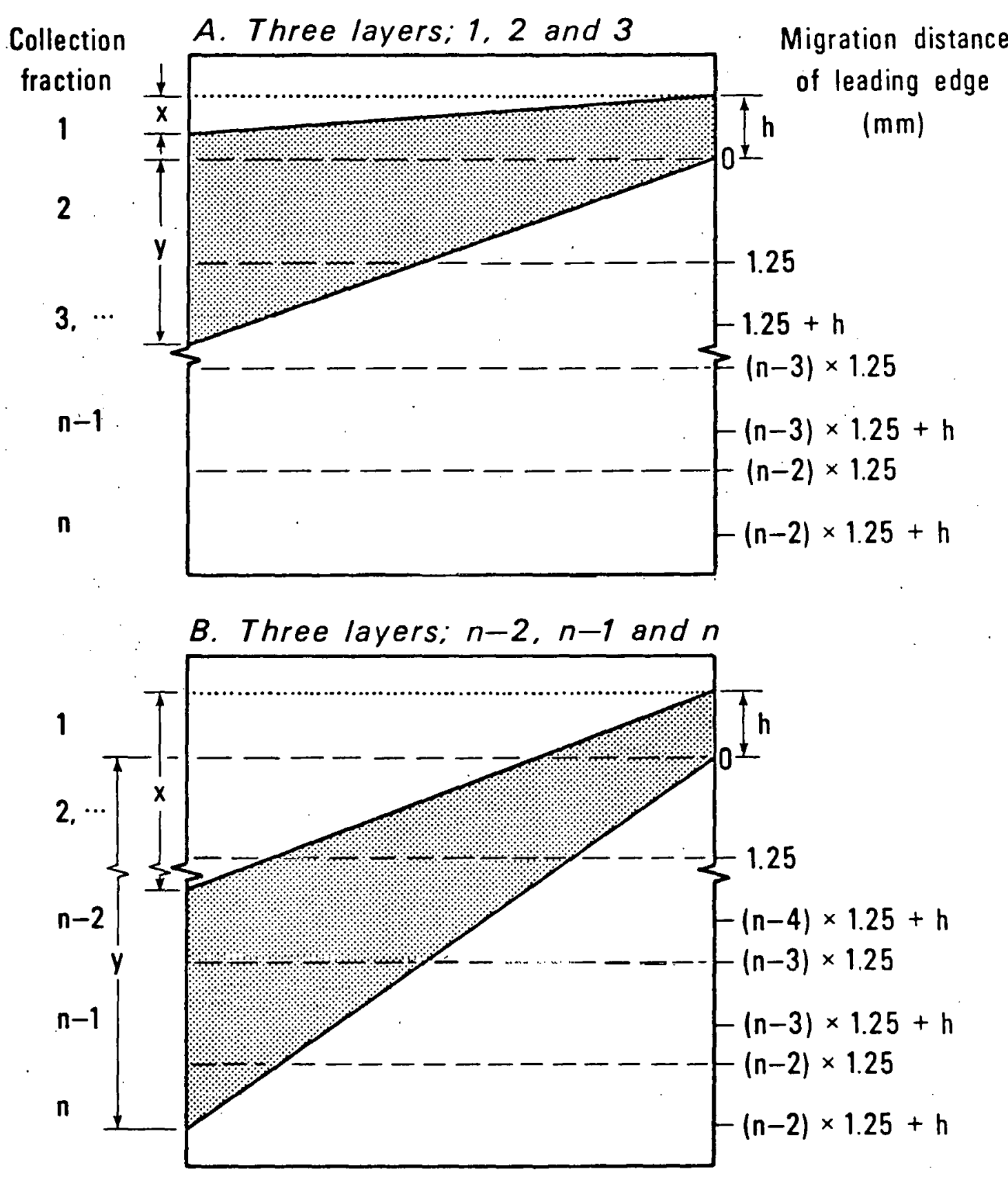

DBL 689-5474

Fig. 12. Schematic diagram of sedimentation or sedimentation-downwardelectrophoresis migration pattern; three-1ayer spread. A. Exit in layers 1, 2 and 3. B. Exit in layers $n-2, n-1$ and $n$. 
stream at the inlet.

(1) Minimum and Maximum Mgration Distances. Referring to Figure 11A, the minimum migration distance for both collection fractions in the 2-1ayer migration pattern is $\mathrm{x}$. The maximum migration distance in both fractions depends upon the distance the particles migrate into layer 2 since, by reason of assumption (4), this is the maximum sedimentation distance for the population of particles. Thus, if $y<h, y$ is the maximum migration distance for both fractions. If $y>h$, the maximum migration distances are $h$ and $y$ for particles in fractions 1 and 2 , respectively. These migration distances are summarlzed in Appendix El.

The minimum and maximum migration distances for the collection fractions of the 3-1ayer migration pattern shown in Figure 12A are determined as follows:

Fraction 1: The minimum and maximum migration distances are $\mathrm{x}$ and $\mathrm{h}$, respectively.

Fraction 2: The minimum migration distance is $x$. As in the case of fraction 1 for a 2-layer migration pattern, the maximum migration distance for particles in fraction 2 depends upon the relationship between the distances $h$ and $y$.

If $(y-1.25)<\mathrm{h}, \boldsymbol{\lambda}_{2}=\mathrm{ym}$. If $(y-1.25)>\mathrm{h}, \boldsymbol{\lambda}_{2}=1.25+\mathrm{hmm}$.

Fraction 3: The minimum and maximum migration distances are $1.25 \mathrm{~mm}$ and $y \mathrm{~m}$, respectively.

These migration distances are summarized in Appendix EL.

The same principles apply to the determination of the minimum and maximum migration distances for particles exiting in layers $n-1$ and $n$ in Figure $11 B$ and $n-2, n-1$ and $n$ in Figure 12B. These expressions are also summarized in Appendices E1 and E2, respectively. 
(2) Range and Distribution of Migration Distances. An understanding of the range and distribution of sedimentation distances characterizing each of the collection fractions is helpful in developing equations for the average migration distances. In Figures 13 and 14, the abscissa represents the total range of migration distances and the ordinate represents the migration distance of the leading edge of the migration pattern starting at the bottom of the sample stream at the inlet (see assumptinn (1) j. 'lhus, assuming a homogeneous distribution of particles in the sample stream at the inlet, particles characterized by a specific migration distance will be found in a column "h" mm high with the bottom of the column at the intersection of the lines representing that migratinn distance drawn from both the ordinate and the abscissa. For example, referring to Figure 13A, particles characterized by a sedimentation velocity that gives a maximum sedimentation distance of $1.25 \mathrm{~mm}$ will be distributed throughout the bottom $\mathrm{h} \mathrm{mm}$ of layer 2. It should be noted that in Figures 13 and 14, the migration distances $x$ and $y$ are both drawn from the origin (o) even through they are measured differently in Figures 11 and 12.

Figure 13A shows that the particles characterized by the slowest sedimentation velocity (mlgration distance $x^{\prime}$ ) are distributed between layers 1 and 2 in inverse proportion to the ratio $\frac{x^{\prime}}{h}$. If $x^{\prime}=0$, all of the particles will be in layer 1 ; whereas, if $x^{\prime}=h$, all of the particles will be in layer 2. For $0<x^{\prime}<h$, the percentage of particles in layer 1 characterized by a sedimentation velocity giving a migration distance $x^{\prime}$ is $\left(1-\frac{x^{\prime}}{h}\right) \cdot 100 \%$ and in layer $2, \frac{x^{\prime}}{h} \cdot 100 \%$. This relationship 15 more obvious in Figure 15 which presents the same information as Figures 13 and 14 in a different manner. The area of the distribution band falling within each collection fraction in Figures 13 and 14 is shown in Figure 


\section{RANGE AND DISTRIBUTION \\ OF STAFLO MIGRATION DISTANCES \\ Sedimentation and}

sedimentation-downward-electrophoresis

A. Two layers; 1 and 2

Migration distance $(\mathrm{mm})$
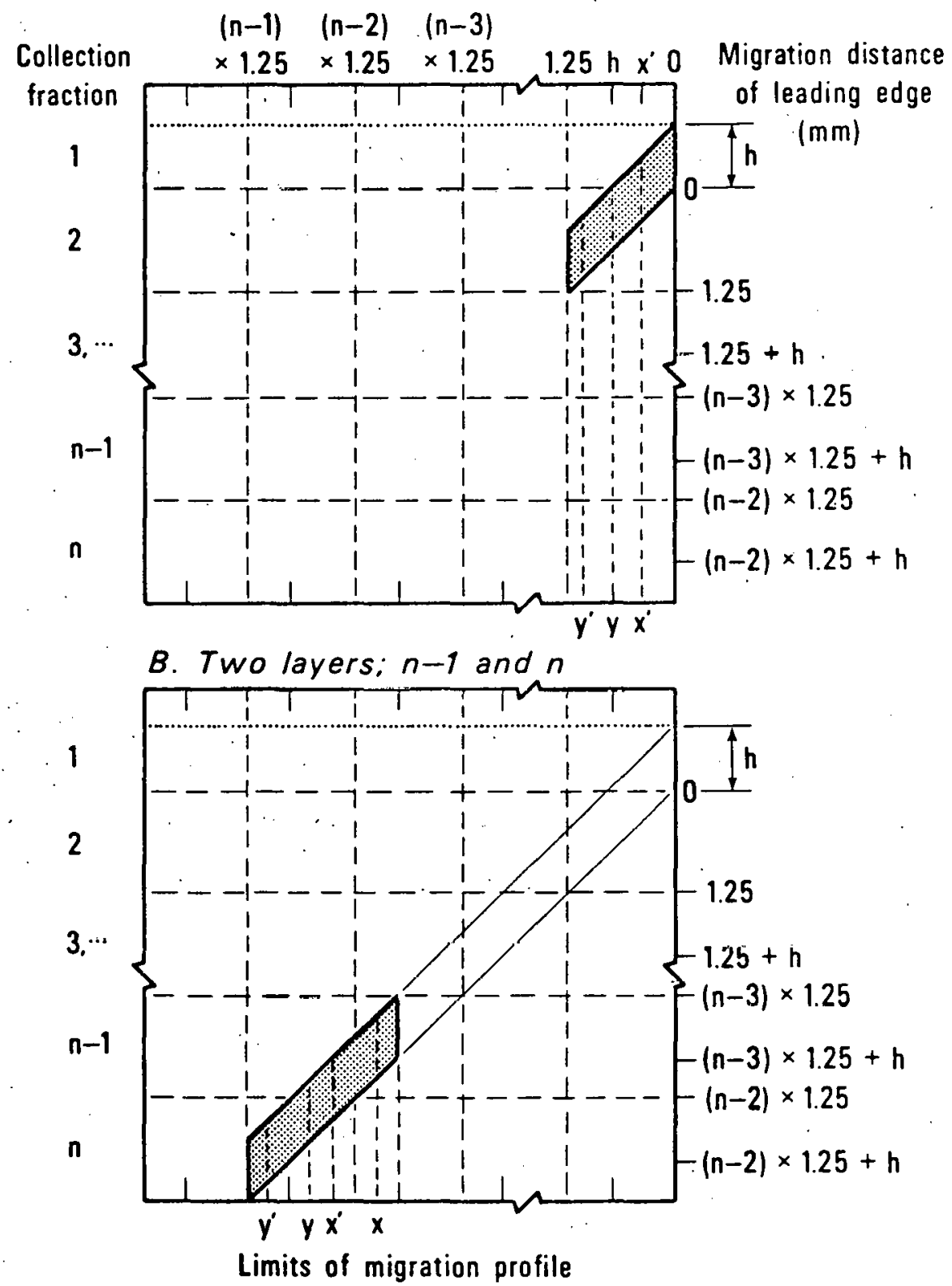

DBL 70115968

Fig. 13. Range and distribution diagram for migration distances in . sedimentation and sedimentation-downward-electrophoresis experiments; two-layer spread. A. ExIt In layers 1 and 2. B. Exit in layers $n-1$ and $n$. 


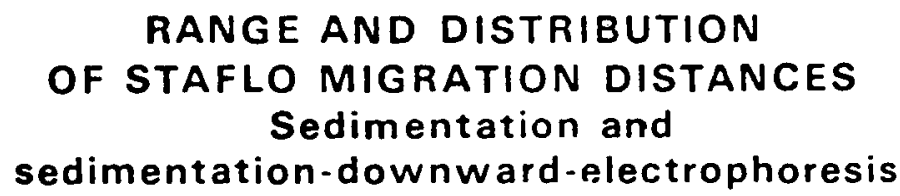

A. Three layers; 1,2 and 3

Migration distance $(\mathrm{mm})$

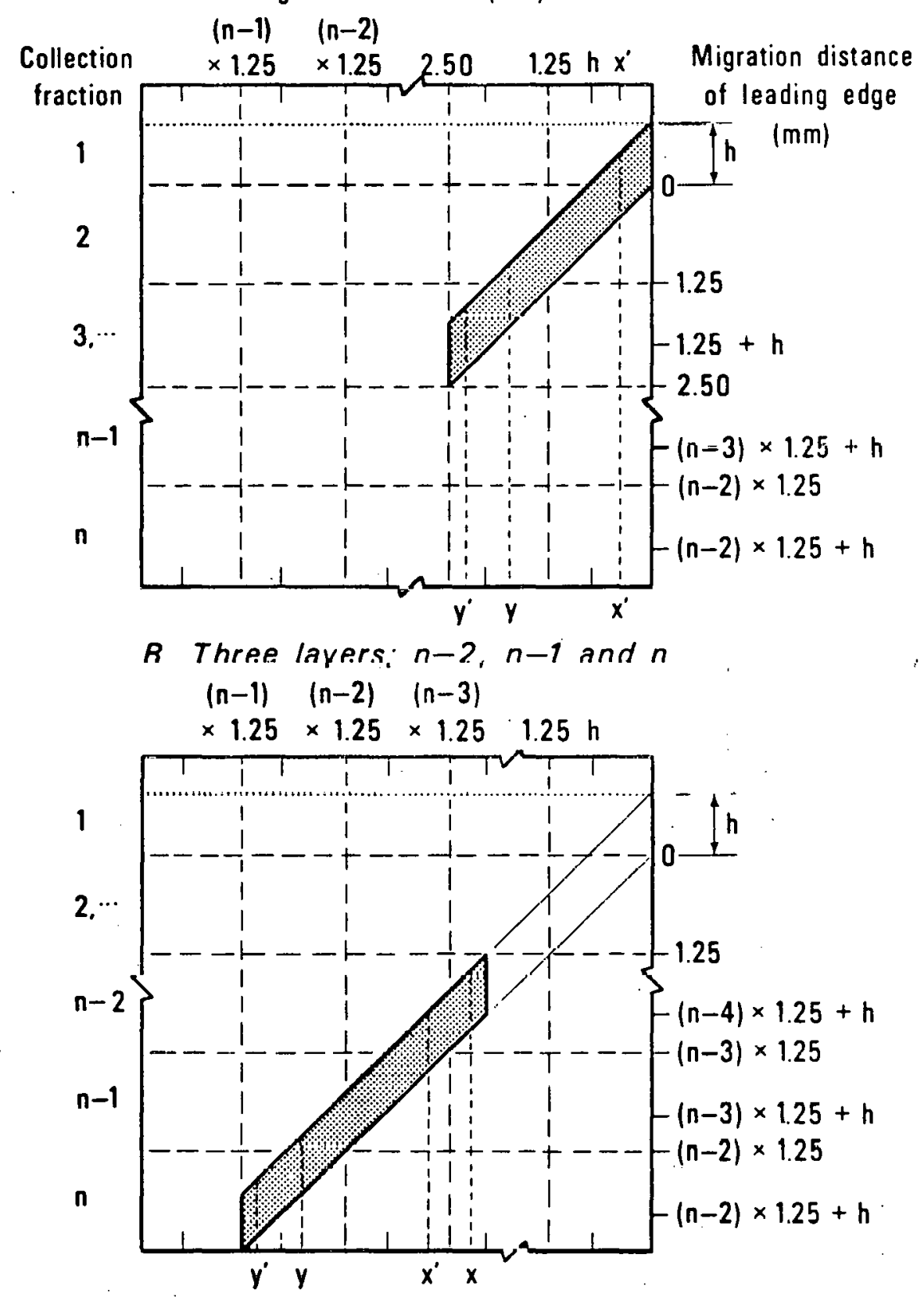

Limits of migration profile

DBL 70115969

F1g. 14. Range and distribution diagram for migration distances in sedimentation and sedimentation-downward-electrophoresis experiments; three-layer spread. A. Exit in layers 1, 2 and 3. B. Exit in layers $n-2, n-1$ and $n$. 


\section{RANGE AND DISTRIBUTION \\ OF STAFLO MIGRATION DISTANCES \\ Sedimentation and}

sedimentation-downward-electrophoresis

Migration distance $(\mathrm{mm})$

(n) $\times \quad(n-1) \quad(n-2) \quad(n-3)$

1.25

$\times 1.25 . \times 1.25 \times 1.25$

$1.25 \mathrm{~h}$

10

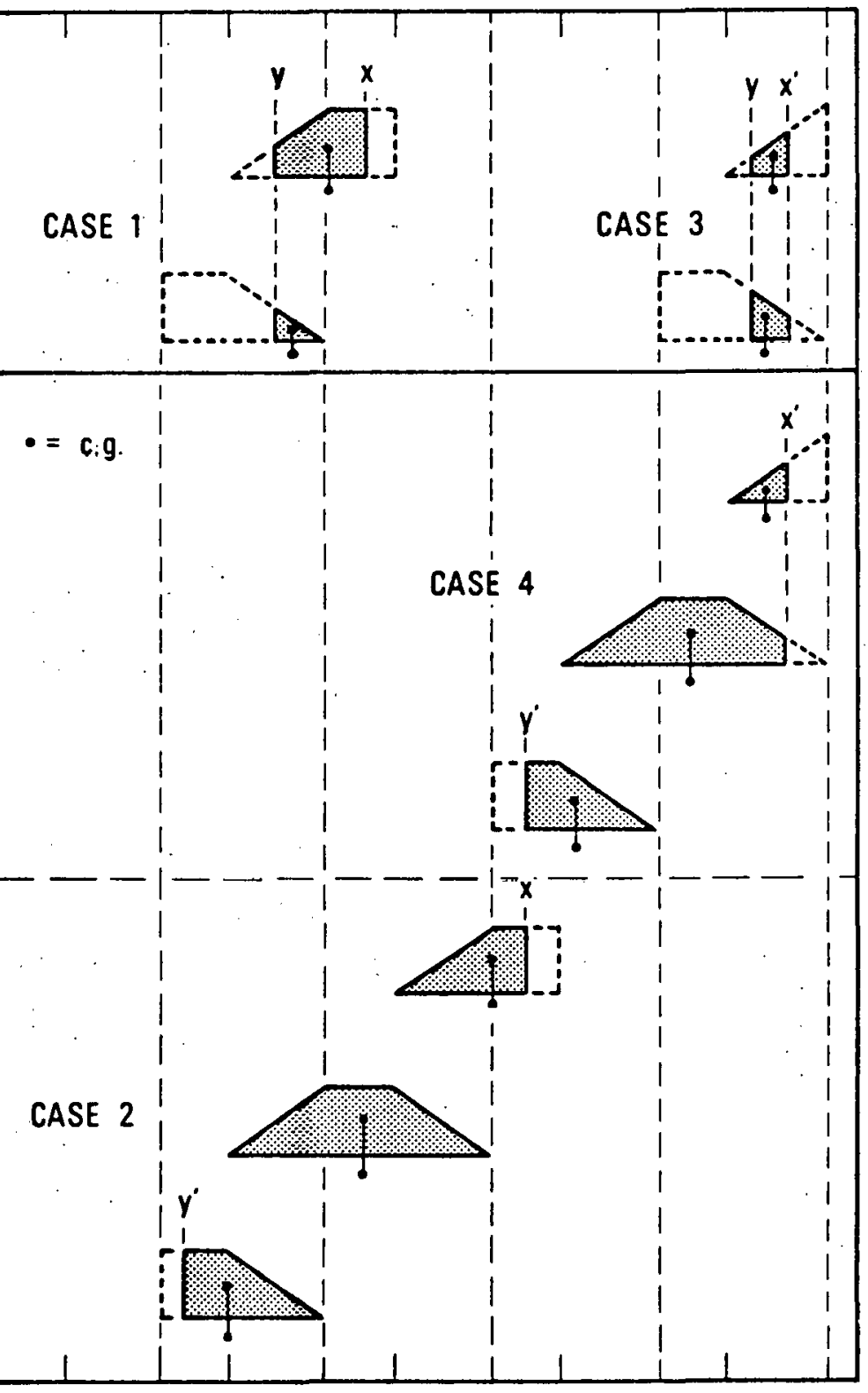

A. Two-layer spread Collection

fraction

$1(n-1)$

$2(n)$

B. Three-layer spread

1

2

3

$n-2$

$n-1$

n

DBL 70115970

F1g. 15. Alternative range and distribution diagram for migration distances in sedimentation and sedimentation-downward-electrophoresis experiments; two- and three-layer spread. Dots show the centers of gravlty of the respective areas. 
15 in such a way that the relative height of the geometrical figure represents the fraction of particles characterized by a specific migration distance found in that collection fraction.

Figure 15 shows that the extent of the overlap in the distribution of migration distances among the collection fractions for 2- and 3-layer migration patterns depends upon the relationships among the distances $h$, $\mathbf{x}$ and $\mathbf{y}$. Figure 15A shows that, for 2-layer migration patterns, Case 1 (layers $n-1$ and $n$ ) and Case 3 (layers 1 and 2) give similar average migration distances for the examples shown. Iff the madirm, density gradient and operating conditions are selected to give the maximum distribution of migration distances (represented by the entire area shown in Figure 15A), the overlap is relatively less and the difference between the average migration distances is much greater.

Figure 15B showo that the extreme collectiun fractions of a 3-1ayer migration pattern have completely different ranges of migration distances while the center fraction overlaps the other 2 . Conditions that give the widest spread of the 3-layer migration pattern will give the greatest number of particles in the top and bottom fractions. This would make available for analysis the largest number of particles with distinctly different migration properties.

(3) Average Migration Dietances. The basic assumption of the center of gravity method of calculating the average migration distance is that the cells are uniformly distributed throughout the migration band. For this to be true, the range of sedimentation velocities characterizing the particles must be distributed unfformly throughout the population of particles. With this assumption, the fractional area of the migration band in each layer is equal to the fraction of the total number of particles 
exiting in that layer. In the absence of specific information about the characteristics of the population of particles, this method is believed to give a good approximation of the average migration distance. This assumption is discussed in Chapter VIII in regard to the results of a specific Staflo sedimentation experiment.

Using Figures 13 and 14 and the center of gravity principle, equations can be derlved for the average sedimentation migration distance for particles in each collection fraction. In fractions with 2 or 3 different geometrical areas representing particles with different ranges of sedimentation velocities, the average migration distance for the fraction as a whole 18 calculated as follows:

$$
\lambda_{\text {ave }}=\frac{\mathrm{A}_{1} \lambda_{1}+\mathrm{A}_{2} \lambda_{2}+\mathrm{A}_{3} \lambda_{3}}{\mathrm{~A}_{1}+\mathrm{A}_{2}+\mathrm{A}_{3}}
$$

where $A_{1}, A_{2}$ and $A_{3}$ are the areas for ranges of sedimentation distances such as $0 \rightarrow \mathrm{h}, \mathrm{h} \rightarrow 1.25$ and $1.25 \rightarrow(1.25+\mathrm{h}) \mathrm{mm}$ for collection fraction 2 in Figure 14A. $\boldsymbol{\lambda}_{1}, \boldsymbol{\lambda}_{2}$ and $\boldsymbol{\lambda}_{3}$ are the migration distances to the centers of gravity of the respective areas.

Equations for 2- and 3-layer migration patterns are given in Appendices EI and E2, respectively.

(4) Weighted Average Migration Distance. In experiments for which migration proflle measurements and the distribution of particles among rnllection fractions are available, the weighted average sedimentation distance $\left(\overline{\boldsymbol{\lambda}}_{\mathrm{S}}\right)$ is determined as follows:

$$
\overline{\boldsymbol{\lambda}}_{\mathrm{S}}=\mathbf{\Sigma} \mathrm{f}_{1} \boldsymbol{\lambda}_{\boldsymbol{8}_{1}}
$$

where $f_{i}$ is the fraction of the total sample in collection fraction " 1 "; and $\boldsymbol{\lambda}_{\mathbf{g}_{1}}$ is the average sedimentation distance for particles in collection 
fraction "I".

(5) Correction of Sedimentation Migration Distances for Temperature and Viscosity. The viscosity of solutions used in the Staflo apparatus changes significantly with temperature, whereas the change in spectfic gravity is relatively small and generally can be ignored. For the purpose of these calculations, it is believed adequate to correct the sedimentation migration distances $\left(\lambda_{S}\right)$ solely for changes in viscosity as follows:

$$
\left(\lambda_{S}\right)_{\mathrm{k}^{\circ} \mathrm{C}}=\left(\lambda_{\mathrm{S}}\right)_{\mathrm{I}^{\circ} \mathrm{C}} \cdot \frac{\eta \mathrm{k}^{\circ} \mathrm{C}}{\eta \mathrm{r}^{\circ} \mathrm{C}}
$$

where $\eta=$ viscosity at temperature $k^{\circ} \mathrm{C}$ or $r^{\circ} \mathrm{C}$, as appropriate.

An additional refinement can be made by correcting for differences In viscosity among the solutions in different layers in the flow-chamber. For Instance, if the sedimentation rate $(\mathrm{dx} / \mathrm{dt})_{\mathrm{k}^{\circ} \mathrm{C}}$ is determined from measurements made in layers 8,9 and 10, a corrertion is necessary with upward electrophoresis where the sedimentation would take place in layers 6, 7 and 8. Depending upon the component of the migration pattern for which the calculation is being made, it may be appropriate to correct $\left(\boldsymbol{\lambda}_{9 S}\right)_{\mathrm{k}^{\circ} \mathrm{C}}$ to $\left(\boldsymbol{\lambda}_{7 S_{\mathrm{r}^{\circ} \mathrm{C}}}\right.$. This is done by using the viscosities of the medium for the appropriate solutions and temperatures in equation (3). b. Calculation of Sedimentation Velocity. The sedimentation velocity $(\mathrm{dx} / \mathrm{dt})$ is calculated by dividing the sedimentation distance $\left(\lambda_{\mathrm{g}}\right)$ hy the minimum transit time $\left(\boldsymbol{\tau}_{\mathrm{min}}\right)$ for the experiment:

$$
(\mathrm{dx} / \mathrm{dt})_{i}=\frac{\boldsymbol{\lambda}_{\mathrm{S}_{1}}}{\boldsymbol{\tau}_{\mathrm{min}}} .
$$

where 1 indicates that the equation is general and can be used to calculate the minimum, maximum or average sedimentation velocities for individual collection fractions or the entire migration pattern as desired. 


\section{Sedimentation-Electrophoresis Expertments:}

The relative contributions of sedimentation and electrophoresis to the net migration of particles in the flow-chamber depend primarily upon the particle sedimentation rate, the average transit time and the strength and length of the electric field. Additional factors were considered in Section 1 above. A general formula for relating the observed sedimentationelectrophoresis migration distance $\left(\boldsymbol{\lambda}_{\mathrm{OBS}}\right)$ to the true electrophoretic $\left(\lambda_{E}\right)$ and sedimentation $\left(\lambda_{S}\right)$ migration distances is:

$$
\boldsymbol{\lambda}_{\text {OBS }}=\boldsymbol{\lambda}_{E} \pm \boldsymbol{\lambda}_{S}
$$

where ( + ) applies to downward electrophoresis $\left(\boldsymbol{\lambda}_{\mathrm{OBS}}=\boldsymbol{\lambda}_{\mathrm{SDE}}\right)$; and (-) applies to upward electrophoresis $\left(\lambda_{\text {OBS }}=\lambda_{\text {SUE }}\right)$.

General statements with regard to equation (5) include the following: 1) It is applicable only if the migrating species has no intrinsic motIlity under the conditions of the experiment. 2) As $\boldsymbol{\lambda}_{S} \rightarrow 0, \boldsymbol{\lambda}_{\text {OBS }} \rightarrow \boldsymbol{\lambda}_{E}$, 1.e., true electrophoresis is achieved only when the term $\lambda_{S}$ approaches zero. Inasmuch as a finite time is required for transit across the flow-chamber, one must reduce $\lambda_{S}$ in order to approach true electrophoresis. This can be done by Increasing the spectfic gravity and/or the viscosicy of the medium. 3) $\boldsymbol{\lambda}_{\mathrm{s}}$ can be calculated from sedimentation experiments. Thus, If one assumes that the electrophoretic mobility and sedimentation rate of Individual particles are independent, $\boldsymbol{\lambda}_{\mathrm{E}}$ can be calculated from equation (5) if $\lambda_{S}$ is known.

a. Sedimentation-Downward-Electrophoresis Migration Distances. The migration pattern in sedimentation-downward-electrophoresis (SDE) experiments is similar to that shown in Figures 11 and 12 for 2 and 3-layer patterns, respectively, except the electric fleld generally starts $1 / 6$ or $1 / 3$ of the way across the flow-chamber. The range and distribution of migration 
distances are as shown in Figures 13, 14 and 15. The equations presented in Appendices E1 and E2 apply to SDE experiments, also. The calculation of electrophoretic mobilities is discussed below.

Figure 16 shows the relationships between the sedimentation and electrophoretic components of the migration pattern in a SDE experiment in which there is a significant sedimentation component. The minimum and maximum sedimentation distances, $\lambda_{\operatorname{Smin}}$ and $\lambda_{\text {Smax }}$, respectively, which would have been observed in the absence of an electric fleld are shown in Figure 16. The minimum and maximum SDE migration distances, $\boldsymbol{\lambda}_{\mathrm{SDE}}$ min $\boldsymbol{\lambda}_{\mathrm{SUE}}$, are given by $x$ and $y$, respectively. The minfmum electrophoretic migration distance $\left(\boldsymbol{\lambda}_{\mathrm{E}_{\min }}\right)$ is equal to the minimum observed migration ( $\boldsymbol{\lambda}_{\mathrm{SDE}}$ min less the expected minimum sedimentation distance $\left(\lambda_{S_{\min }}\right)$. The maximum electrophoretic migration distance $\left(\boldsymbol{\lambda}_{\mathrm{E}_{\max }}\right)$ is equal to the maximum observed migration distance $\left(\lambda_{S_{D F}}\right)$ ) less the expected maximum sedimentation distance $\left(\boldsymbol{\lambda}_{S_{\max }}\right)$. These relationships are summarized in equations (6) and (7) be.low.

The lines in Figure 16 labelled $s$ and $S$ represent the expected distribution of the slowest and fastest sedimenting spermatozoa in the absence of an electric field and are equal. in height to " $h "$. The lines labelled $E \cdot S, e \cdot s, E \cdot s$ and $e \cdot S$ represent the expected distribution of the fastest electrophoresing-fastest sedimenting, slowest electrophoresingslowest sedimenting, fastest electrophoresing-slowest sedimenting, slowest electrophoresing-fastest sedimenting populations of spermatozoa, respectively.

b. Sedimentation-Upward-Electrophoresis Migration Distances. In electrophorests experiments, the electric field generally is applied over the last $20-25 \mathrm{~cm}$ of the flow-chamber to allow density gradient stabilizing 


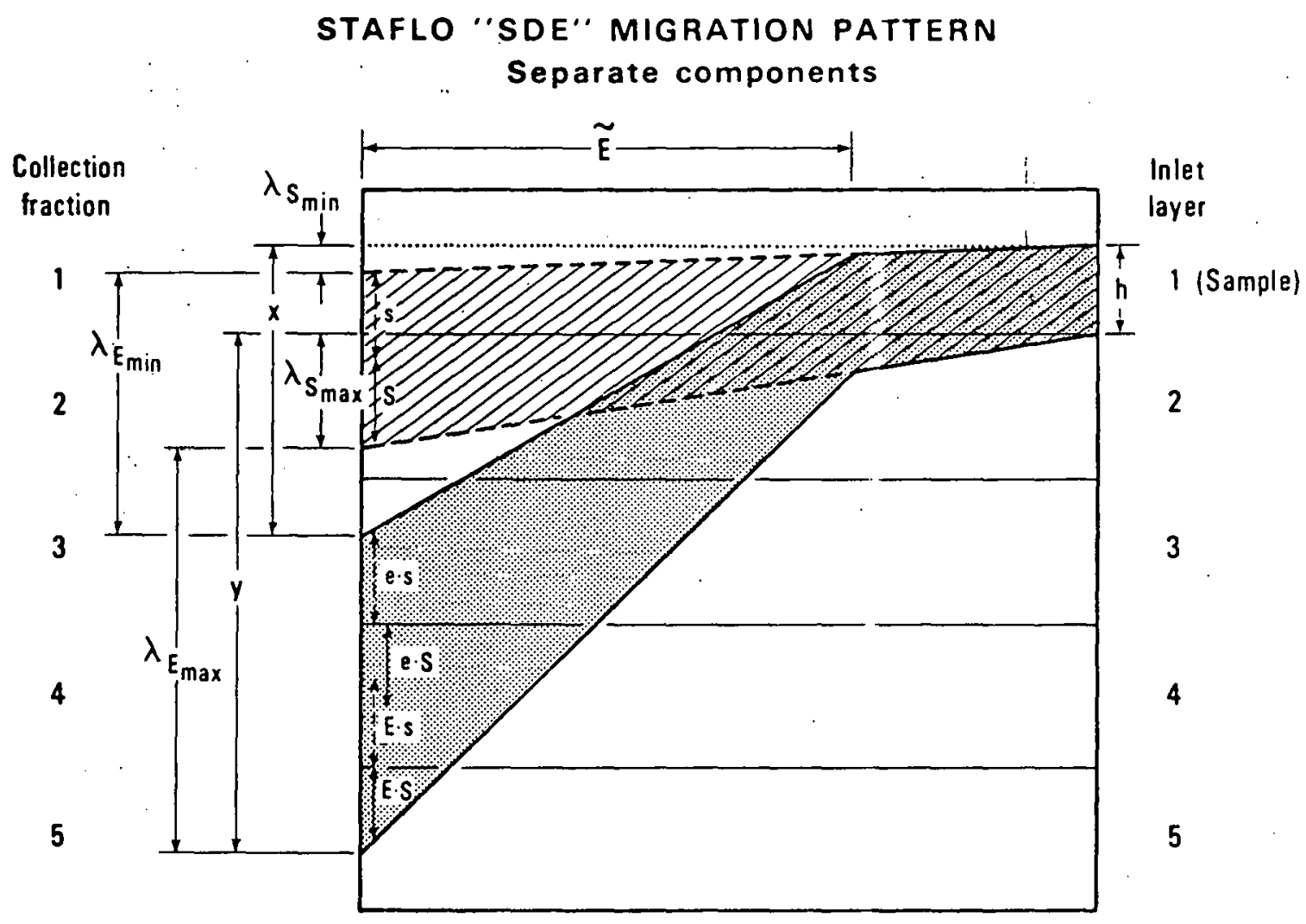

DBL 70115971

F1g. 16. Schematic diagram of sedimentation-downward-electrophoresis migration pattern showing separate components. 
diffusion to take place. ${ }^{157}$ The upward migration is illustrated in Figures 17 and 18 which represent a vertical plane in the centerline through the long axis of the flow-chamber in 2- and 3-layer sedimentation-upwardelectrophoresis (SUE) migration patterns, respectively. In addition to the assumptions given above for sedimentation experiments, it is assumed that the leading and tralling edges of the mlgration pattern determine. the maximum and minimum SUE migration distances, respectively.

\section{(1) Minimum and Maximum Migration Distances. Figure 19 shows the} relationships among the various migration distances in a SUE experiment in which there is a significant sedimentation component. The minimum and maximum sedimentation distances $\left(\boldsymbol{\lambda}_{\mathrm{S}_{\min }}\right.$ and $\left.\boldsymbol{\lambda}_{\mathrm{S}_{\max }}\right)$ which would have been observed in the absence of an electric field are shown in Figure 19. The distances $x$ and $y$ are the minimum and maximum SUE migration distances, $\boldsymbol{\lambda}_{\mathrm{SUE}_{\mathrm{min}}}$ and $\boldsymbol{\lambda}_{\mathrm{SUE}}$ max , respectively.

The minimum electrophoretic migration distance is equal to the sum of the maximum sedimentation distance and the minimum SUE distance. The maximum electrophoretic migration distance is equal to the sum of the minimum sedimentation distance and the maximum SUE migration distance. These relationships are summarized in equations ( 8 ) and ( 9 ) below.

In Figure 19 the lines labelled s, S, E.S, E.s, e.S and e.s have the same meaning as in Figure 16 which was discussed above.

(2) Range and Distribution of Migration Distances. The same general principles, discussed in relation to sedimentation, concerning overlapping ranges of migration distances and the need for special equations depending upon the relationships between the location of the sample stream at the inlet and the limits of the migration pattern at the outlet apply to SUE experiments. Because the sample stream is at the bottom of the inlet 


\section{STAFLO "SUE" MIGRATION PATTERN}
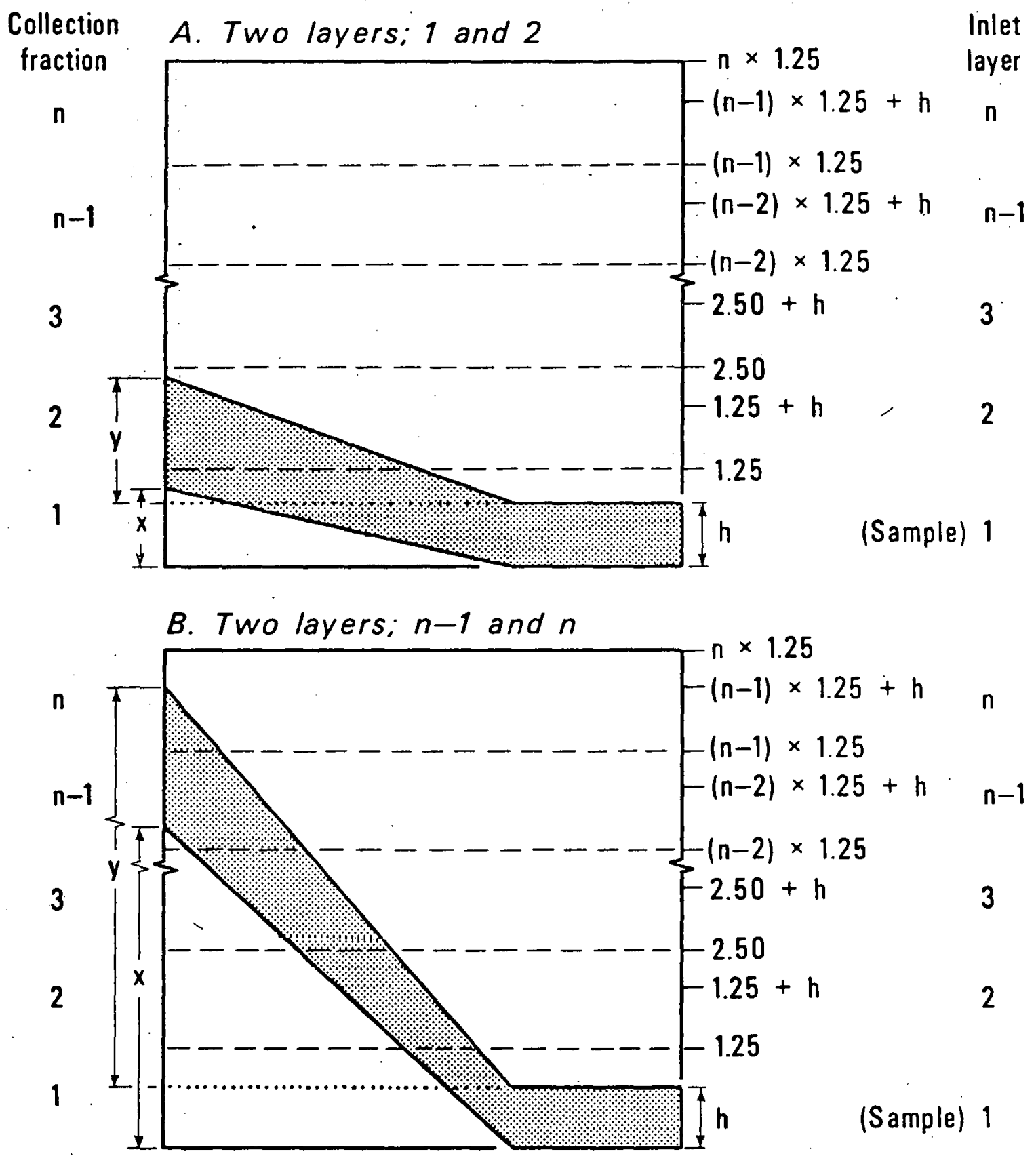

DBL 70115972

Fig. 17. Schematic diagram of sedimentation-upward-electrophoresis migration pattern; two-layer spread. A, Exit in layers 1 and 2. B. Exit in layers $n-1$ and $n$. 


\section{STAFLO "SUE" MIGRATION PATTERN}

Collection
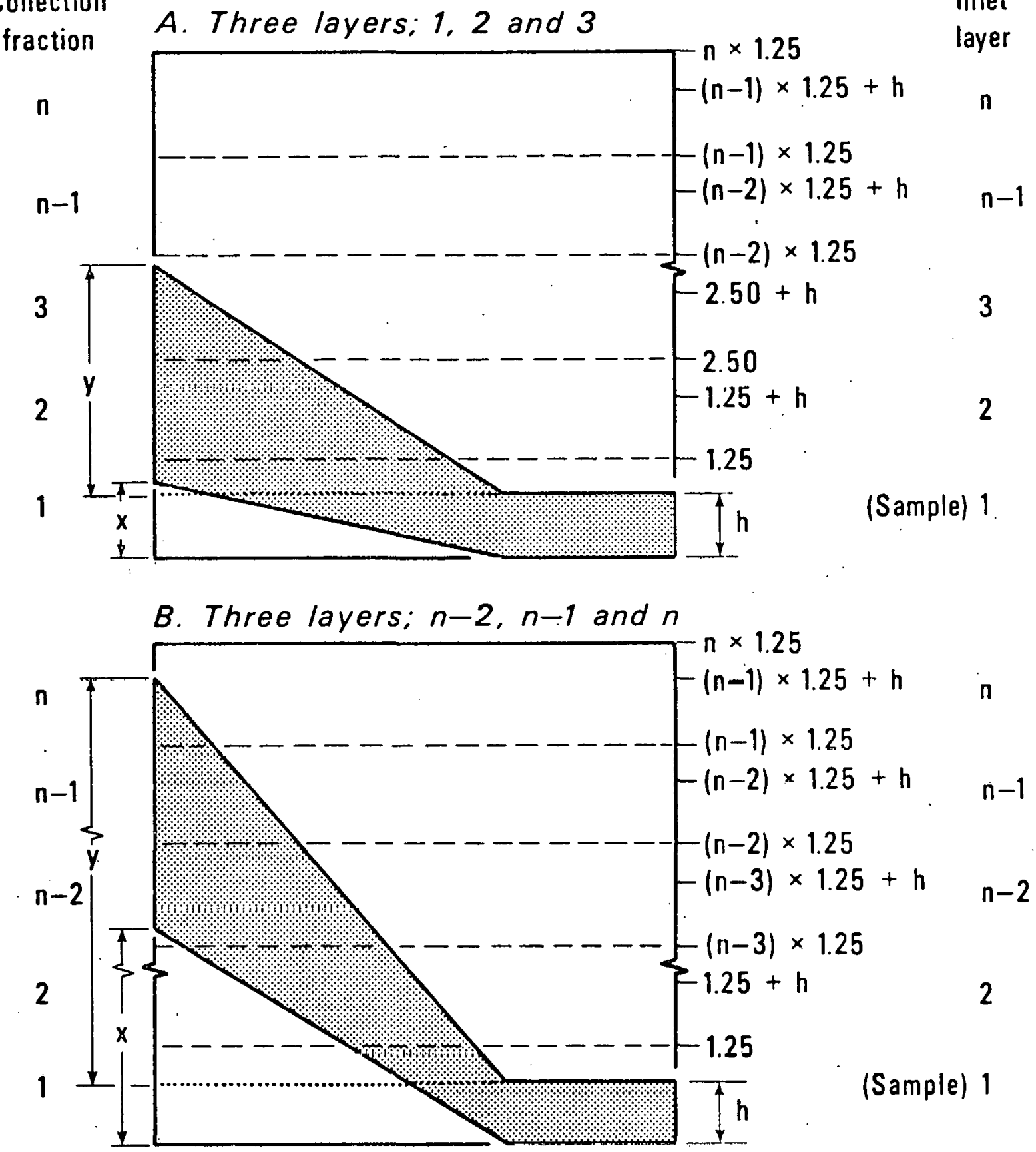

DBL 70115973

Fig. 18. Schematic diagram of sedimentation-upward-electrophoresis migration pattern; three-layer spread. A. Exit in layers 1, 2 and 3. B. Exit in 1ayers n-2, n-1 and n. 


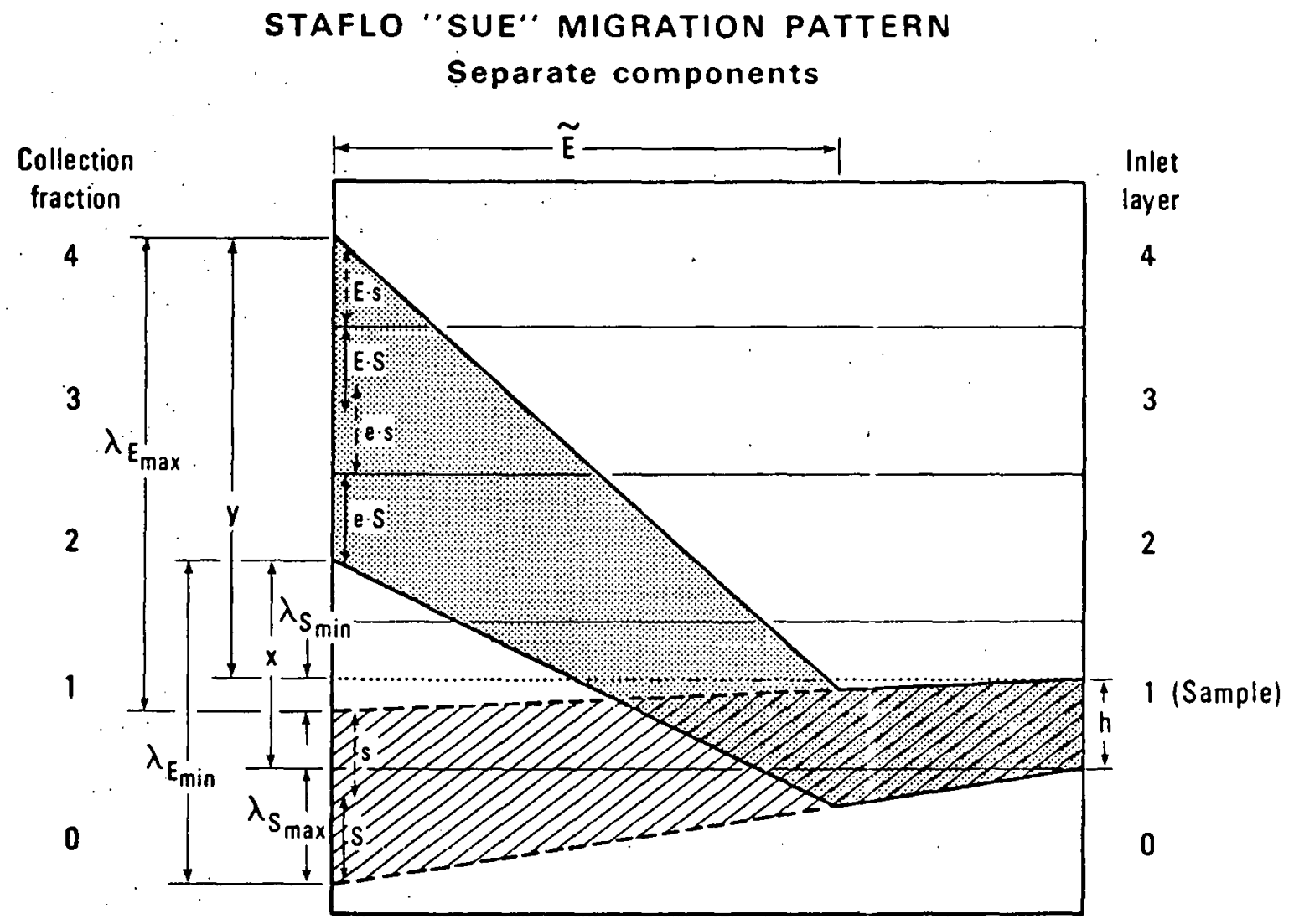

DBL 70115974

F1g. 19. Schematic diagram of sedimentation-upward-electrophoresis migration pattern showing separate components. 
layer under steady state conditions, the distribution of SUE migration distances can have any 1 of 4 different patterns. These are shown in Figures 20 and 21 which are diagrams of the possible ranges of SUE migration distances. The relationships among the possible distributions for 2- and 3-layer migration patterns are summarized mathematically in Appendices E3 and E4, respectively. Note that $x$ and $y$ in Figures 20 and 21 are both drawn from the origin (o) even though they are measured differently in Figures 18 and 19.

In F1gures 20 and 21, the migration distance of the trailing edge of the column of particles is plotted on the ordinate against the same migration distance on the abscissa for migration patterns spreading over the 2 and 3 layers, respectively. For example, when those particles at the bottom of the sample stream at the inlet, which are characterized by an electrophoretic mobility $(\mu)$ giving a migration distance of $1.25 \mathrm{~mm}$, reach the bottom of the next layer, the remainder of the particles characterized by that value of $\mu$ will be found in the lower part of that layer up to height " $h$ " as shown in Figures 20 and 21.

The geometry of the upward migration pattern makes it possible to write general expressions for the minimum, maximum and average SUE migration distances regardless of the outlet layers. These equations are presented in Appendices E3 and E4 for 2- and 3-layer migration patterns, respectively.

Figures 22 and 23 1llustrate al1 4 combinations of minimum and maximum migration distances for 2- and 3-1ayer upward migration patterns, respectively. For each migration distance, the relative heights of the figures represent the fraction of particles in each collection fraction characterized by that migration distance. As shown in Figure 22 for a 2-1ayer migration pattern, Case 2 gives the least overlap and the great- 
RANGE AND DISTRIBUTION OF STAFLO "SUE"' MIGRATION DISTANCES

A. Two layers; 1 and 2

Migration distance $(\mathrm{mm})$

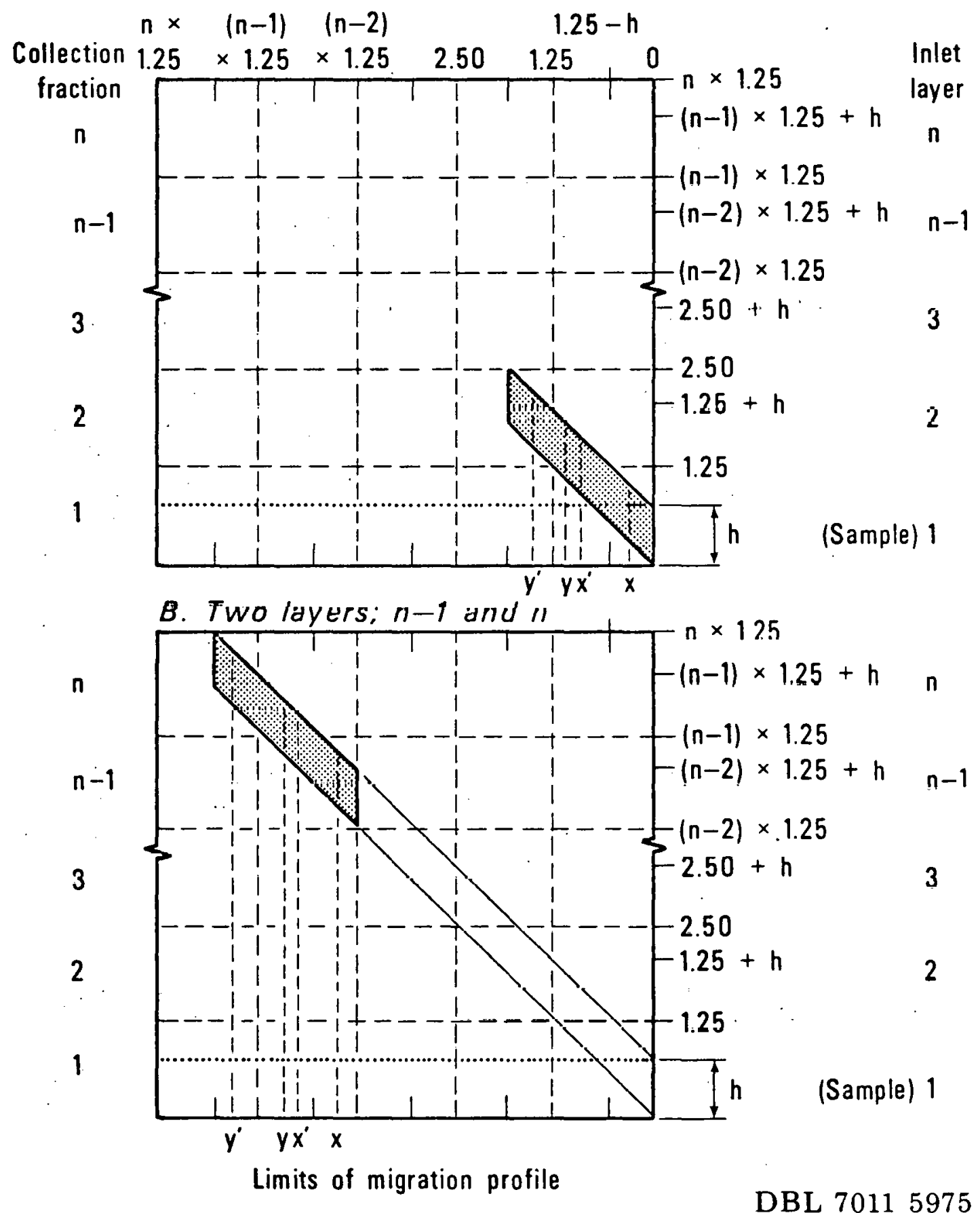

DBL 70115975

FIg. 20. Range and distribution diagram for sedimentation-upward electrophoresis migration distances; two-layer spread. A. Exit in layers 1 and 2. B. Exit in layers $n-1$ and $n$. 


\section{RANGE AND DISTRIBUTION OF STAFLO "SUE" MIGRATION DISTANCES}

A. Three layers; 1,2 and 3

Migration distance $(\mathrm{mm})$

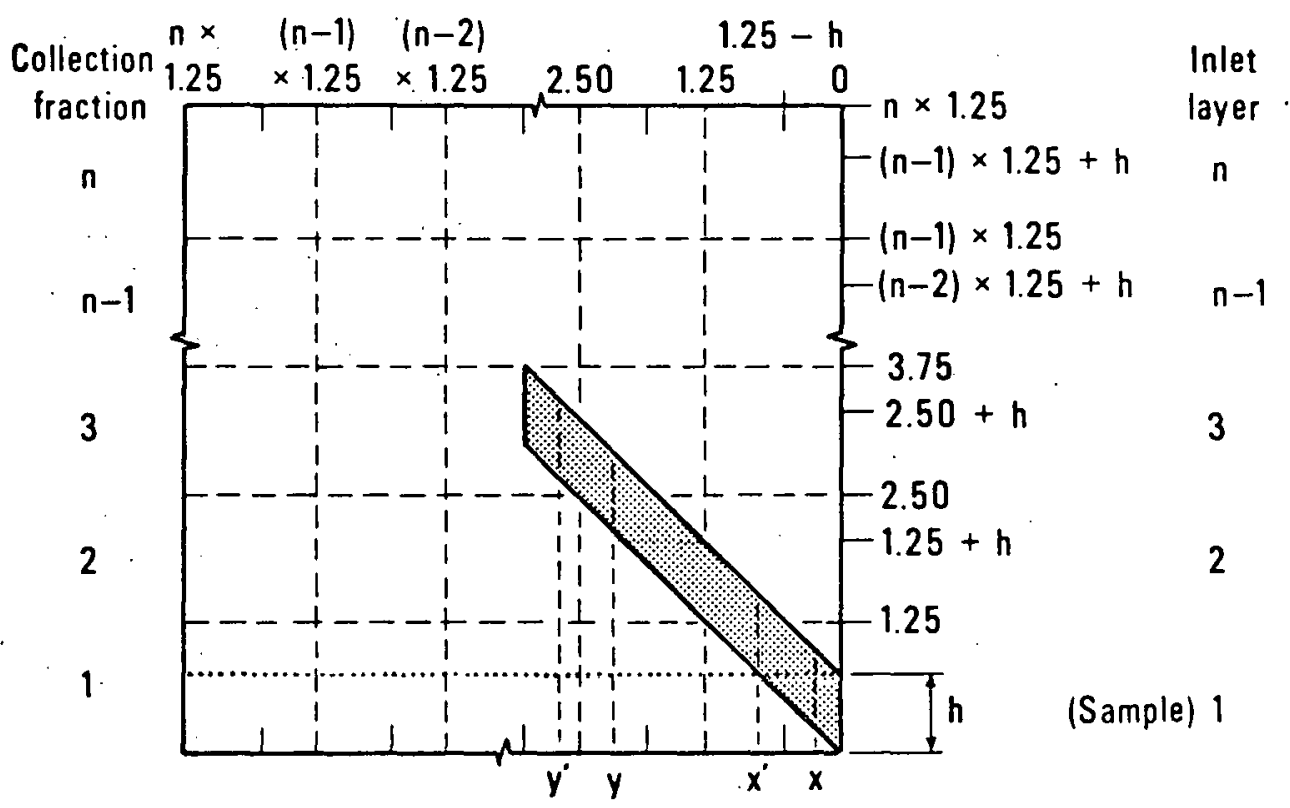

B. Three layers; $n-2, n-1$ and $n$

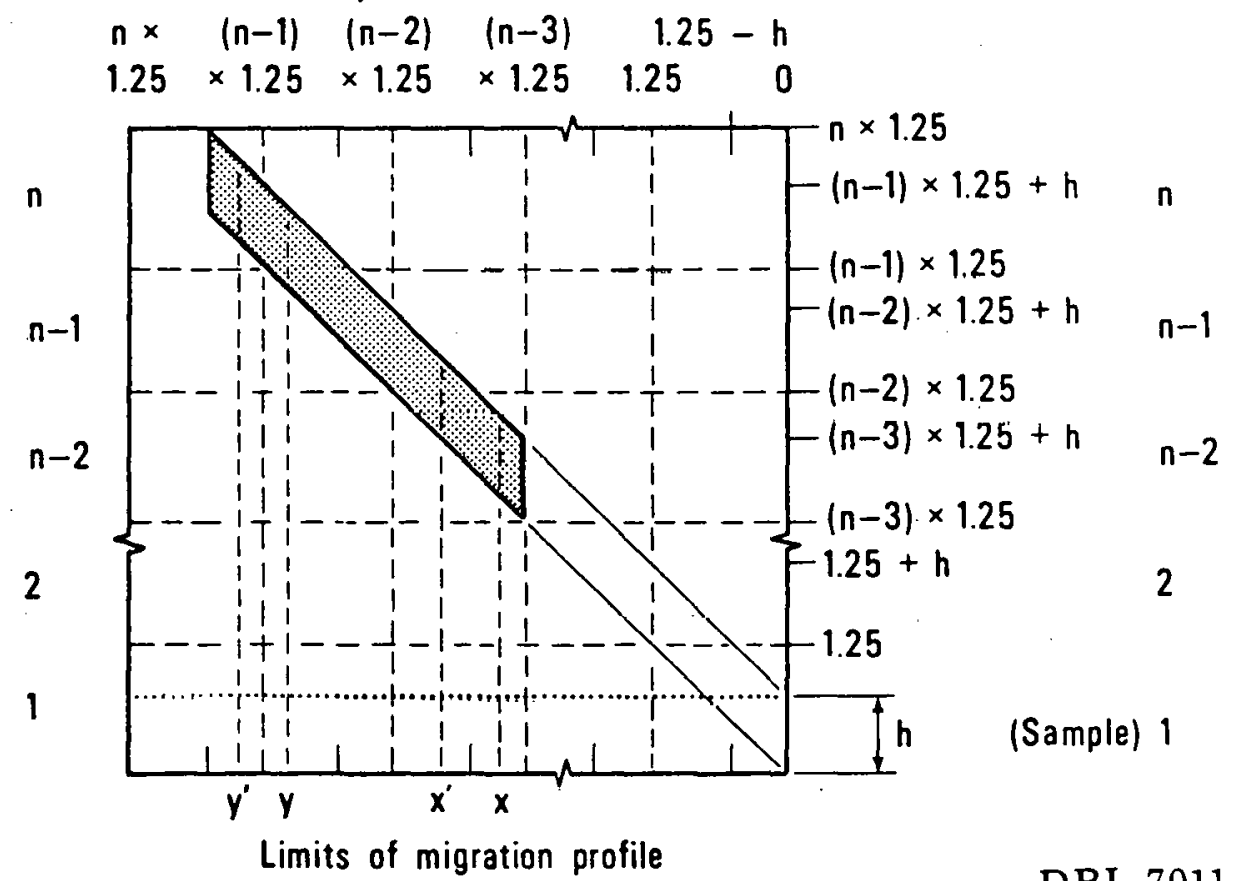

DBL 70115976

Fig. 21. Range and distribution diagram for sedimentation-upwardclcctrophoresis migration, distances; three-layer spread. A, Exit in layers 1, 2 and 3. B. Exit in layers $n-2, n-1$ and $n$. 
RANGE AND DISTRIBUTION OF STAFLO "SUE" MIGRATION DISTANCES

Migration distance $(\mathrm{mm})$

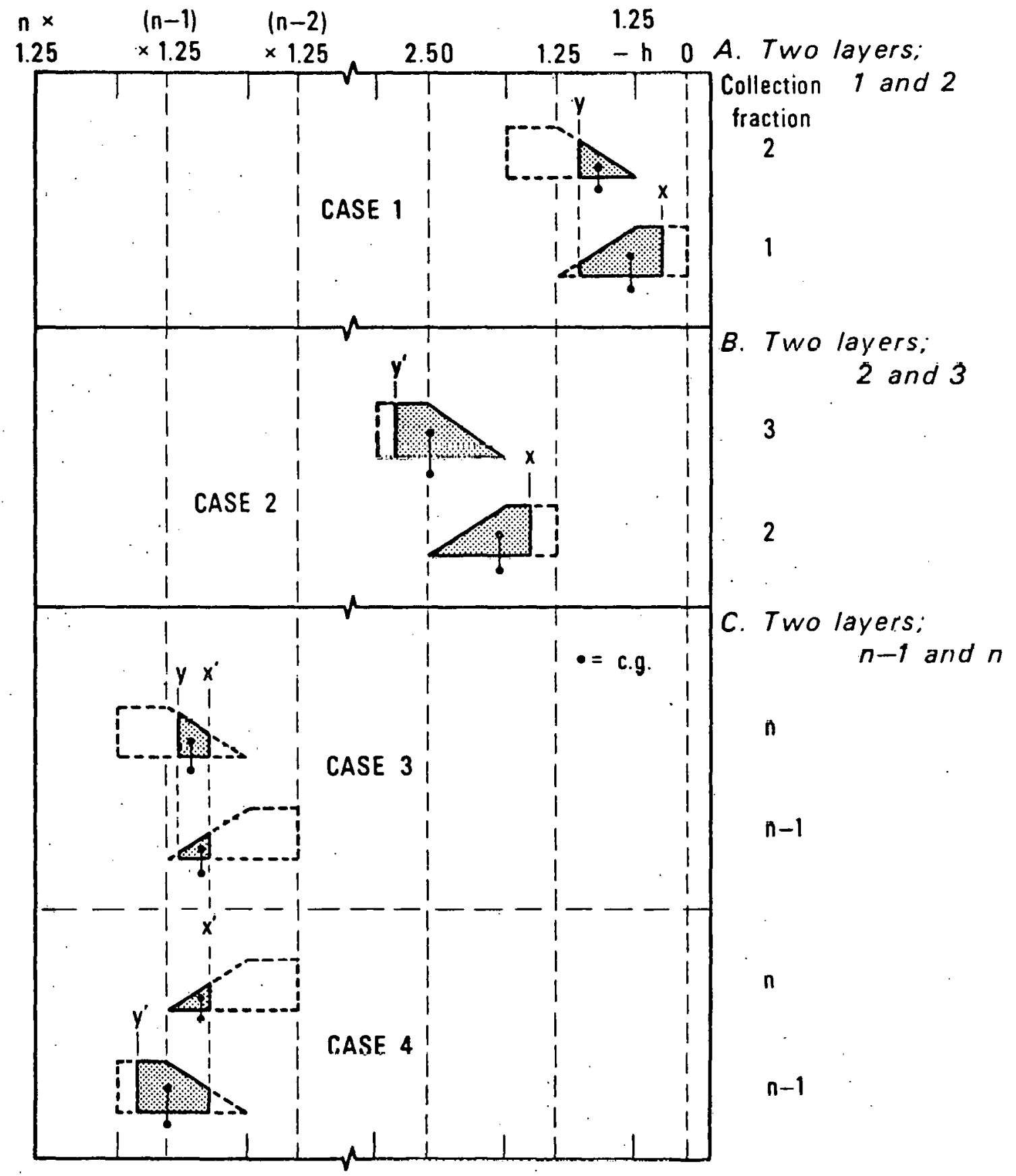

DBL 70115977

Fig. 22. Alternative range and distribution diagram for migration. distances in sedimentation-upward-electrophoresis experiments; two-layer spread. Dots Indicate centers of gravity of the respective areas. 


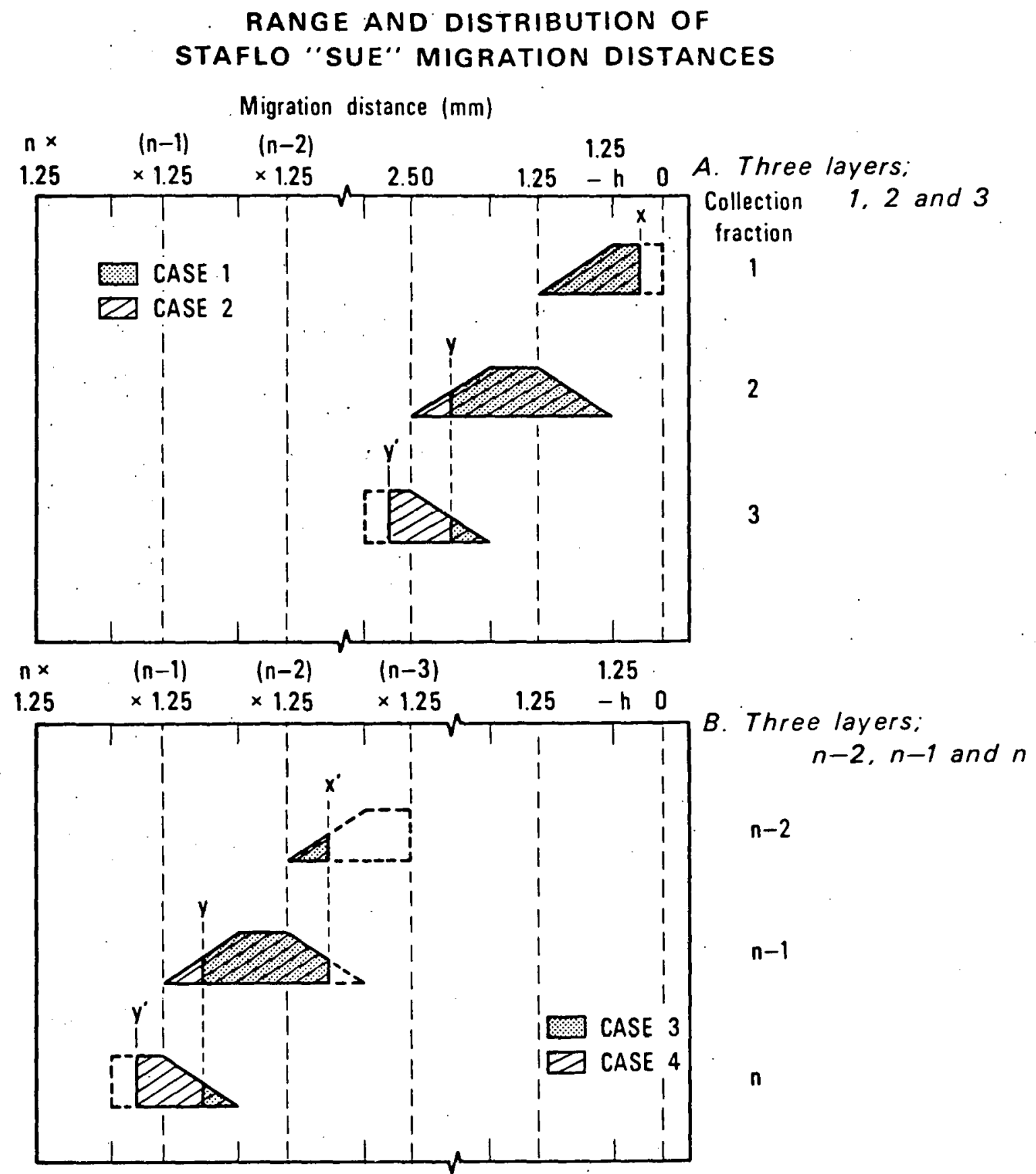

DBL 70115978

Fig. 23. Alternative range and distribution diagram for migration distances in sedimentation-upward-electrophoresis experiments; three-layer spread. Dots show centers of gravity of the réspective areas. 
est difference between the average migration distances of the 2 collection fractions. Case 3 gives the least difference. Figure 23 shows that Cases 2 and 3 give the largest and smallest numbers of particles, respectively, In the extreme fractions of the 3-1ayer migration pattern.

c. Comparison of Sedimentation-Downward and -Upward-Electrophoresis.

The relationships among the sedimentation, electrophoresis and sedimentation-electrophoresis migration distances are as follows:

$$
\begin{aligned}
& \boldsymbol{\lambda}_{\mathrm{SDE}}=\boldsymbol{\lambda}_{E_{\mathrm{min}}}+\boldsymbol{\lambda}_{\mathrm{S}} \\
& \boldsymbol{\lambda}_{\mathrm{SDE}}=\boldsymbol{\lambda}_{\mathrm{E}_{\max }}+\boldsymbol{\lambda}_{\mathrm{S}_{\max }} \\
& \lambda_{\mathrm{SUE}_{\mathrm{min}}}=\boldsymbol{\lambda}_{\mathrm{E}_{\mathrm{min}}}-\boldsymbol{\lambda}_{\mathrm{S}_{\max }} \\
& \boldsymbol{\lambda}_{\mathrm{SUE}_{\max }}=\boldsymbol{\lambda}_{\mathrm{E}_{\max }}-\boldsymbol{\lambda}_{\mathrm{S}_{\mathrm{mIn}}} \\
& \boldsymbol{\lambda}_{\mathrm{SDE}}=\boldsymbol{\lambda}_{\mathrm{E}_{\text {ave }}}+\boldsymbol{\lambda}_{\mathrm{S}} \\
& \boldsymbol{\lambda}_{\mathrm{SUE}}=\boldsymbol{\lambda}_{\mathrm{E}_{\text {ave }}}-\boldsymbol{\lambda}_{\mathrm{S}}
\end{aligned}
$$

These equations apply to individual collection fractions or to the migration pattern as a whole.

For spherical objects and for non-spherical particles if the value of $\boldsymbol{\lambda}_{\mathrm{S}_{\text {ave }}}$ is not changed by a reorientation of particles upon changing the direction of the electric field, the average electrophoretic migration distance can be determined from a combination of upward- and downwardelectrophoresis experiments. Adding equations (10) and (11) gives 


$$
\lambda_{E_{\text {ave }}}=1 / 2\left(\lambda_{\mathrm{SDE}_{\text {ave }}}+\lambda_{\mathrm{SUE}_{\mathrm{ave}}}\right)
$$

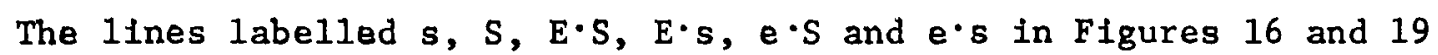
were explained above. With particles having a signiflcant sedimentation rate, a transit time can be selected to give a partial separation of the slowest and fastest sedimenting particles shown by lines $s$ and $S$ in Figures 16 and 19. There will be overlap between the 2 groups as discussed in relation to Figure 15 . If a downward electrophoretic migration is superimposed upon the sedimentation pattern as shown in Figure 16, the extreme fractions will contain predominantly $e^{\cdot s}$ and $E \cdot S$ groups. With upward electrophoretic migration as shown in Figure 19, the extreme fractions will contain primarily $E \cdot s$ and $e \cdot S$ groups. In table form, the distribution would be as follows:

$\begin{array}{ccc}\begin{array}{c}\text { Collection } \\ \text { Fraction }\end{array} & \text { SDE } & \text { SUE } \\ \text { Top } & \text { e.s } & \mathrm{E} \cdot \mathrm{s} \\ \text { Upper MLddle } & \mathrm{e} \cdot \mathrm{S} & \mathrm{E} \cdot \mathrm{S} \\ \text { Lower Middle } & \mathrm{E} \cdot \mathrm{s} & \mathrm{e} \cdot \mathrm{s} \\ \text { Bottom } & \mathrm{E} \cdot \mathrm{S} & \mathrm{e} \cdot \mathrm{S}\end{array}$

Thus, by conducting SDE and SUE experiments one can get $e \cdot s, E \cdot S, E \cdot s$ and e.S groups of particles for evaluation. Because of the overlap of migration distances discussed above in relation to Figures 15,22 and 23 , it may be desirable to shave the top or bottom of the migration pattern at a flow-divider to get a more homogeneous population of particles for study. It should be noted that only if the sedimentation rate and electrophoretic mobility of the particles are Independent, would the segregation of the 4 groups of particles occur as described.

d. Calculation of Electrophoretic Mobility. The minimum, maximum and 
average electrophoretic migration distances $\left(\boldsymbol{\lambda}_{E}\right)$ can be calculated from equations (6)-(11) as appropriate. Using these migration distances, the electrophoretic mobility $(\mu)$ can be calculated as follows:

$$
\mu=\frac{w}{\widetilde{E}}=\frac{\lambda_{E} / \mathcal{Z} E_{\operatorname{mIn}}}{I / A \cdot \sigma} \frac{(\mathrm{cm} / \mathrm{sec})}{(\mathrm{V} / \mathrm{cm})}
$$

where $v$ is the particle migration rate $(\mathrm{cm} / \mathrm{sec})$ and $\tilde{E}$ is the electric field strength $(\mathrm{V} / \mathrm{cm})$. I is the electric current (amps), A is the electrode area $\left(\mathrm{cm}^{2}\right)$ and $\sigma=\frac{1.46}{\mathrm{R}}\left(\mathrm{ohm}^{-1} \mathrm{~cm}^{-1}\right)$. $\mathrm{R}$ is the resistance of the medium (ohms) and $1.46 \mathrm{~cm}^{-1}$ is the cell constant for the conductivity meter in our laboratory.

According to Bier" 38 "because nearly all the effect of temperature on mobility is due to the change in viscosity of $\mathrm{H}_{2} \mathrm{O}$, the following relationship can be used to correct the mobility to any temperature as long as is not too large: $\eta_{1} \mu_{1}=\eta_{2} \mu_{2}$." This relationship can be used to normalize electrophoretic mobilities determined at different temperatures in the same medium or in different media if the viscosities are known.

3. Effects of Motility on Migration and Fractionation.

In early Staflo experiments conducted at temperatures of $6-7^{\circ} \mathrm{C}$, motile spermatozoa were observed in the flow-chamber with a microscope. At these temperatures and with $\mathrm{pH} 6$ or greater, the orfentation of all spermatozoa, including the motile spermatozoa, appeared to be predominantly vertical with tails upward in the sedimentation and SUE experiments regardless of the electric field strength in the latter. In SDE experiments, the orientation was uniformly vertical with tails pointing downward at $\mathrm{pH} 7$ with high electric field strengths only. In SDE experiments with low electric field strengths, the spermatozoa assumed a variety of orientations as discussed in Chaptep VTTT. 
In order to quantitate the effect of intrinsic motility of spermatozoa in Staflo experiments, one must know the true range of sedimentation velocities and/or of electrophoretic mobilities in the medium and density gradient. These can be obtained in experiments conducted at or below $3^{\circ} \mathrm{C}$ depending upon the medium. Then if the temperature is controlled to eliminate random swiming, the orientation of the spermatozoa is determined by gravity and/or the electric field, and the motility vector is parallel to the sedimentation and/or electrophoretic vectors.

a. Sedimentation Experiments. Figure 24 is a schematic diagram of a Staflo sedimentation experiment with an intrinsic motility component showing minimum and maximum spermatozoa migration distances, $x$ and $y$, respectively. The expected maximum sedimentation distance, $\boldsymbol{\lambda}_{\mathrm{S}_{\max }}$, is assumed to have been determined in experiments at very low temperatures. Then, from Figure 24, the maximum contribution of motility to the net migration is given by the expression:

$$
\lambda_{\mathrm{MOT}_{\max }}=\mathrm{y}-\boldsymbol{\lambda}_{\mathrm{S}_{\max }}
$$

In Figure 24, one must be careful to interpret the maximum migration distance attributable to motility as the distance from $\boldsymbol{\lambda}_{\mathrm{S}_{\max }}$ to $\mathrm{y}$ and not from zero to $y$. The sample rarely has $100 \%$ motile spermatozoa, but if it did, the minimum contribution of motility $\left(\lambda_{M_{\text {OT }}}\right)$ would be observed as a displacement of $\mathbf{x}$ from the expected minimum sedimentation distance $\left(\boldsymbol{\lambda}_{\mathrm{S}_{\mathrm{min}}}\right)$ which it represents in Figure 24 . The relationship between these components is:

$$
\boldsymbol{\lambda}_{\text {MOT }}=x-\boldsymbol{\lambda}_{S_{\min }}
$$

b. Sedimentation-Electrophoresis Experiments. Unfortunately, the differential orientation of spermatozoa in an electric field under varying 


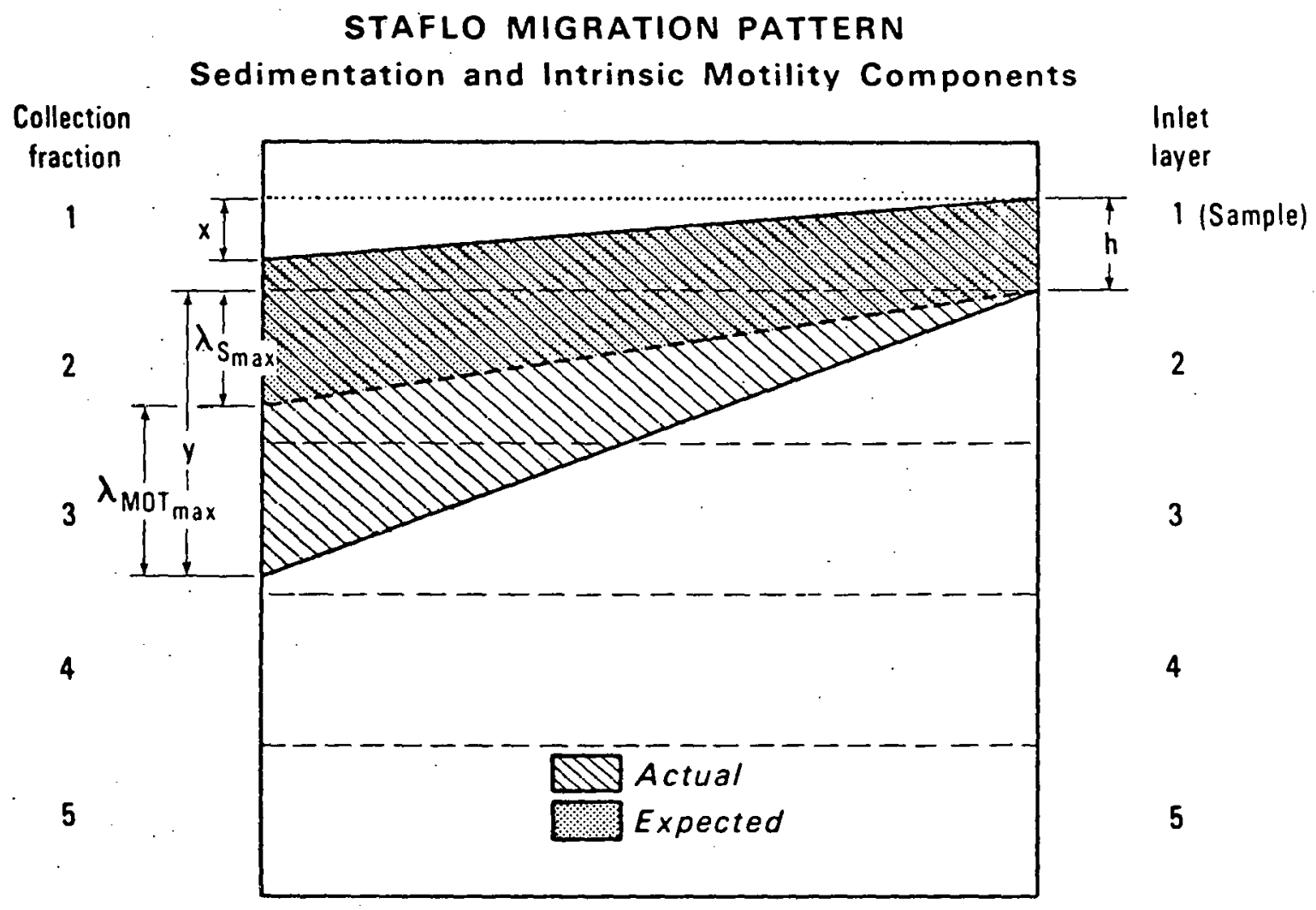

DBL 70115979

Fig. 24. Schematic diagram of a sedimentation migration pattern showing the effect of intrinsic motility on migration. 
conditions of $\mathrm{pH}$ and fonic strength is further complicated by the random orientation of strongly swimming spermatozoa if their intrinsic motility is sufficient to overcome the polarizing effects of the electric field as mentioned by Bangham ${ }^{18}$; and Vessilinovitch ${ }^{233}$. However, one can control the head-tail orientation by proper selection of $\mathrm{pH}$ and ionic strength and can control the random swimming by using a temperature at which the intrinsic motility is insufficlent to change the orientation imposed by gravity and/or the electric field.

Figures 25 and 26 show a motillty component to the net migration in addition to the electrophoretic and sedimentation migration distances for $S \mathrm{SH}$ and SUE experiments, respectively. In both downward- and upwardelectrophoresis under conditions of $\mathrm{pH}$ and ionic strength which cause the sperm to be tall anode, the direction of the motility component is opposite that of the electrophoretic component. Because most spermatozoa samples contaln a significant number of immotile spermatozoa which would experience the maximum electrophoretic migration, the observed migration pattern would extend from $x$ to $y$ in both flgures. The minimum expected electrophoretic migration distance $\left(\lambda_{E_{\min }}\right)$ in both cases is reduced by the intrinsic motilfty component $\boldsymbol{\lambda}_{\text {MOT }_{\max }}$. Thus, if intrinsic motilit.y and elertrophoretic mobility are independent, one would get an enrichment of the lower collection fractions with regard to motility in upward-electrophoresis and of the upper collection fractions in downward-electrophoresis experiments. With a range of Intrinsic velocities one would expect to find a range of degrees of intrinsic motillty among the spermatozoa in the collection fractions with the least motile sperm having migrated the greatest distance in the directions shown in Figures 25 and 26.

Expressions relating intrinsic motility to sedimentation-electrophoresis 


\section{STAFLO "SDE" MIGRATION PATTERN}

Sedimentation, Electrophoresis and Intrinsic Motility Components

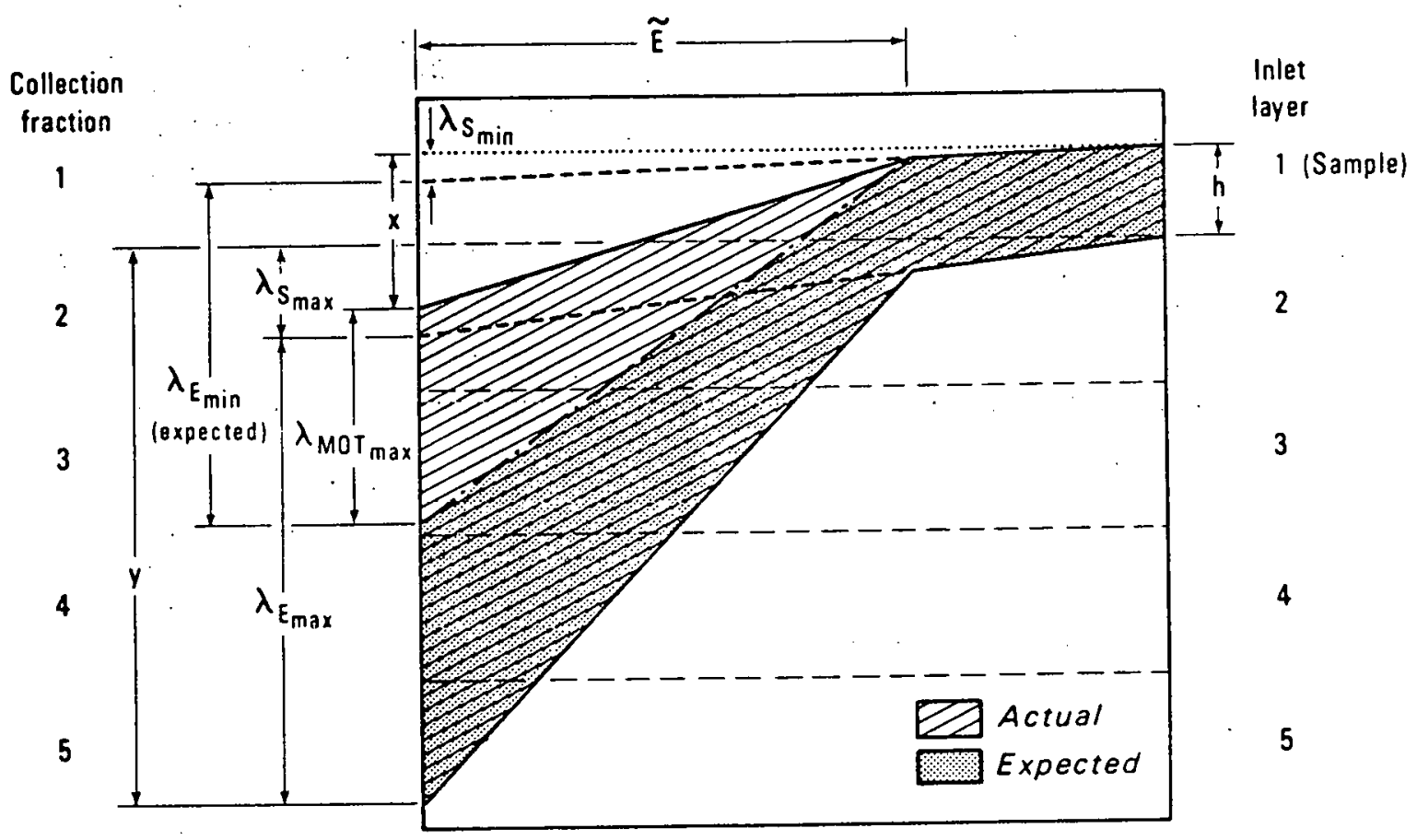

DBL 70115980

Fig. 25. Schematic diagram of a sedimentation-downward-electrophoresis migration pattern showing the interrelationships among the sedimentation, electrophoresis and intrinsic motility components. 


\section{STAFLO "SUE" MIGRATION PATTERN}

Sedimentation. Electrophoresis and Intrinsic Motility Components

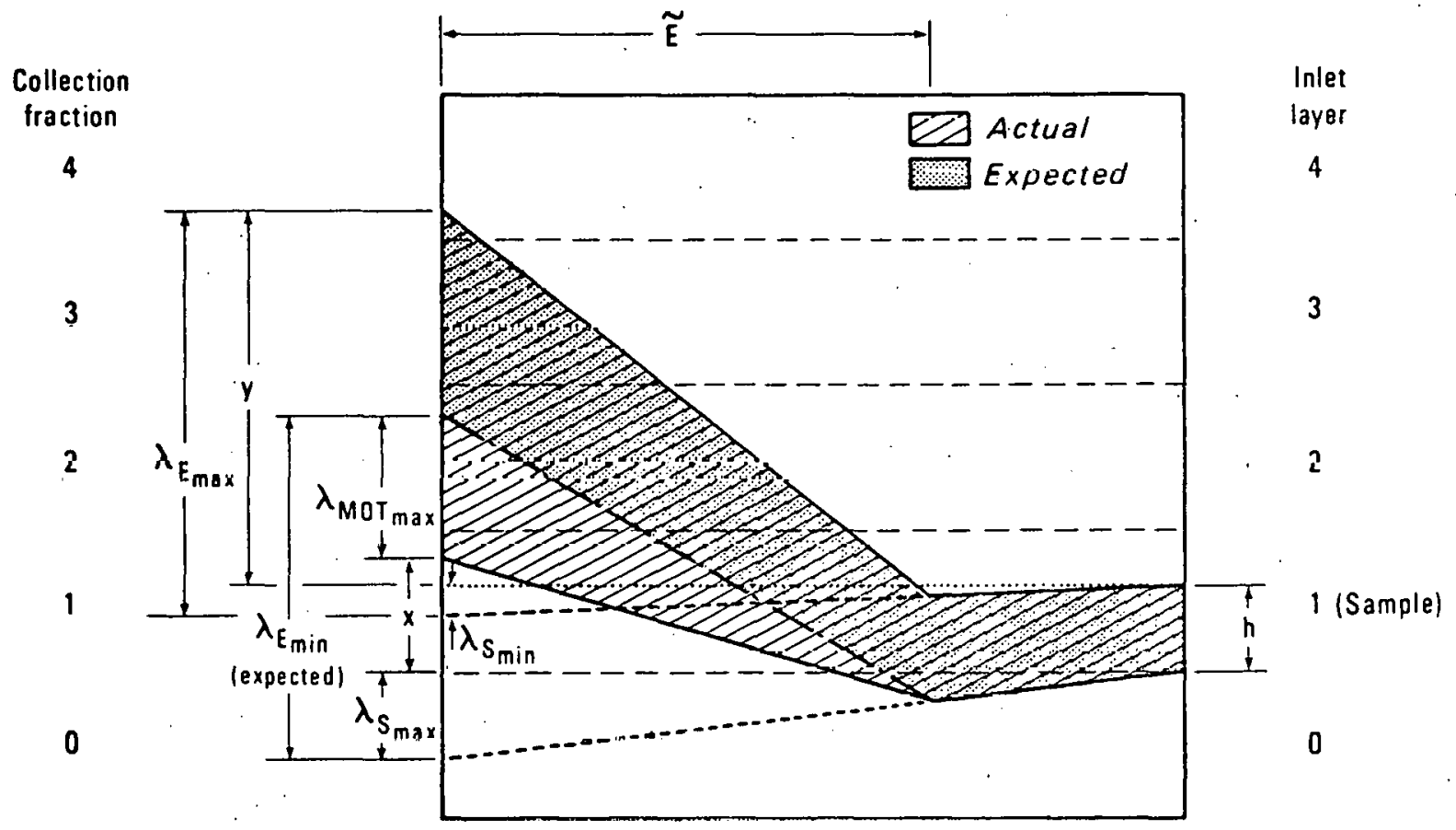

DBL 70115981

Fig. 26. Schematic diagram of a sedimentation-upward-electrophoresis migration pattern showing the interrelationships among the sedimentation, electrophoresis and intrinsic motility components. 
migration distances are as follows:

Sedimentation-downward-electrophoresis (Figure 25):

$$
\lambda_{\mathrm{MOT}_{\max }}=\boldsymbol{\lambda}_{E_{\min }} \text { (expected) }-x+\boldsymbol{\lambda}_{\mathrm{S}_{\min }}
$$

Sedimentation-upward-electrophoresis (F1gure 26):

$$
\lambda_{\text {MOT }_{\max }}=\boldsymbol{\lambda}_{\mathrm{E}_{\min }} \text { (expected) }-\mathrm{x}-\boldsymbol{\lambda}_{\mathrm{S}_{\max }}
$$

4. Significance of This Method of Analysis.

From the several theoretical analyses developed in this chapter, certain conclusions can be drawn.

Figure 15B shows that for most particle populations, a 3-layer spread of the migration pattern is the minimum from which one can obtain collection fractions with completely different populations. Thus, if possible, one should attempt to achieve at least a 3-layer spread of the pattern. However, Figure 15A shows that the difference between the collection fractions in a 2-layer spread can be quite meaningful if the pattern is spread over the entire 2 layers. This is determined largely by the relative height of the sample stream at the inlet (h) compared to the layer thickness. Therefore, if the physical or biological properties of the sample limit the spread to 2 layers, one should use the "thin sample technique" 263 and select operating conditions that will give the widest spread within those 2 layers.

Figures 16 and 19 show that, in sedimentation-electrophoresis experiments, the sedimentation and electrophoretic migration components can be separately identified if one of the 2 properties is known. Similarly, Figures 24-26 demonstrate the interrelationships among the intrinsic motility and sedimentation and/or electrophoretic migration vectors. The 
effects of motility on sedimentation and on sedimentation-electrophoresis experiments are discussed in Chapters IV and VIII, respectively.

Separation of the fastest eloctrophoresing-slowest sedimenting (E.S) particles and of particles possessing the other combinations of sedimentation-electrophoretic properties $(E \cdot S, e \cdot S$ and $e \cdot s)$ from a population are discussed in Section C2c. An initial attempt to use this analysis to separate these sub-populations of semen spermatozoa is reported in Chapter VIII.

The most important assumption in deriving the equations presented in Appendix $E$ is that the distribution of migration properties among the population is uniform. Migration distance range and distribution diagrams of the form shown in Figures 15, 22 and 23 provide a visual method for comparing actual and hypothetical distributions of properties among populations. Such a comparison 18 made in Chapter VIII, where the actual distribution of spermatozoa among the collection fractions in sedimentation Experiment 12-26-67 is compared to the expected distributions of (hypothetical) uniform, bimodal and normal populations of cells with the same range of sedimentation velocities.

Within their stated limitations, the equations in Appendix E provide an accurate method of calculating Staflo migration distances. Representative calculations using these equations and the guideline in sub-sertions 1 and 2 , above, for sedimentation and sedimentation-electrophoresis experiments are given in Appendices $F$ and $G$, respectively.

A computer program, usting the equations in the text and in Appendix E would greatly simplify calculations of the sedimentation velocities or electrophoretic mobilities of individual collection fractions and of the average for the migration pattern as a whole. The equations in Appendix $\mathrm{E}$ 
can be extended to migration patterns encompassing 4 or more adjacent layers. In addition, the equations for patterns extending over 2,3 or more adjacent layers can be generalized so that the inlet position need not be specified as it was in the assumptions in this chapter. Both of these extensions should be made in adapting the equations to a computer program to give even greater flexibility and simplicity in quantitating the migration properties of particles studied in Staflo experiments.

This method of analysis of the migration properties of a heterogeneous mixture of particles should also be applicable to a variety of fractionation techniques; for example, the sedimentation column used by Bhattacharya ${ }^{35}$, by Schilling ${ }^{205}$ and by Bedford and Bibeau ${ }^{29}$. Other applicable techniques include rate and zonal centrifugation, and column, paper and starch-gel electrophoresis.

\section{Summary}

In addition to describing the Staflo apparatus and its many modes of operation, this chapter signiflcantly extends the Staflo method in the direction of quantitation of design and interpretation of experiments. It presents the concept of range and distribution diagrams for particle migration distances (e.g. Figures 13 and 15). These diagrams are used in developing the equations in Appendix $\mathrm{E}$. The alternate form for presenting the range and distribution information (e.g. Figures 15, 22 and 23) clarifies the requirements for optimum fractionation of migration patterne and provides a method of analyzing the distribution of migration properties among particle populations. The remaining contribution is a method of identifying individual components of complex migration patterns involving separate identification of 2 or more of the properties of sedimentation rate, electrophoretic mobility and intrinsic motility, when they occur together in various combinations. 
IV. EARLY STAFLO SEDIMENTATION EXPERIMENTS: RABBIT SPERMATOZOA

\section{Contents}

A. Introduction. . . . . . . . . . . . . . 106

B. Materials and Methods ................. 107

1. Sample Preparation. ................ 107

2. Slaflu Apparatus. . . . . . . . . . . . . . 108

3. Medium and Density Gradient . . . . . . . . . . . 108

4. Temperature Control ................ . 110

5. Observation and Evaluation of Spermatozoa . . . . . . 110

c. Resules...................... . . . . . . 112

1. Fractionation in a Sucrose Denslty Gradient. . . . . . . 112

2. Fractionation in a Dextran Density Gradient . . . . . . 120

3. Artfflcial Insemination . . . . . . . . . . 126

D. Discussion. . . . . . . . . . . . . . . 128

1. Variables Affecting Sedimentation Experiments . . . . . 128

a. Quality of the Spermatozoa Sample.......... 128

b. Spermatozoa Concentration . . . . . . . . 129

c. Temperature and Motility, . . . . . . . . . 131

2. Comparisons with Results of Other Investigators . . . . 135

E. Summiary and Conclusions . . . . . . . . . . . . . 138

\section{A. Introduction}

The first Staflo study of spermatozoa was a series of sedimentation experiments with rabbit spermatozoa using the $1 \times 12$ flow-cell. The interest in the sedimentation properties of rabbit spermatozoa was stimulated by Bhattacharya's report of significant control of the sex ratio of offspring from the insemination of rabbit spermatozoa fractionated in a column 
sedimentation device ${ }^{35}$. In addition, Gordon reported dimorphism of spermatozoa ejaculate populations which was not related to the electrophoretic properties of the spermatozoa ${ }^{92}$. At the time of these studies, Schilling's report of the partial control of the sex ratio of offspring from the insemination of fractionated bull spermatozoa ${ }^{205}$ and the report by Bedford and Btbeau showing no difference in the sex ratio from the insemination of fast and slow sedimenting rabbit spermatozoa ${ }^{29}$ had not been published.

Ancillary purposes of these early experiments were to develop and improve procedures for handling spermatozoa in all phases of Staflo experiments and to explore the effects of the numerous experimental variables (Table I) affecting biophysical studies of spermatozoa. At the same time, an evaluation of media for the preservation of motility and fertilizing capacity of spermatozoa was being conducted (Appendix D).

As will be evident in the results, the maintenance of motility and fertilizing capacity was very poor in most of these experiments. Thus, the most interesting biological assay system, the sex ratio of offspring, could not be correlated with other biological results and the physical properties in these studies. However, these experiments demonstrate a number of important interrelationships among the biological and physical properties of spermatozoa and the numerous experimental variables listed in Table. $I$. Of special interest is the effect of the intrinsic motility of spermatozoa upon "sedimentation -migration"rates.

\section{B. Materials and Methods}

\section{Sample Preparation}

Spermatozoa samples were obtained from mature Dutch Belt rabbits using the artificial vagina described in Appendix C. Each ejaculate was diluted immediately by adding $2.0 \mathrm{ml}$ of medium (see below) at $37^{\circ} \mathrm{C}$ 
to the collection vial. The suspension was cooled to room temperature In an insulating nest of glass wool. A $0.1 \mathrm{ml}$ aliquot of this sample was diluted 1:10 for counting and for evaluation of the percentage and degree of motility. While the sample was being cooled slowly to the temperature of the experiment, it was diluted in several stages to the desired concentration. In order to obtain a minimum of $1.5 \mathrm{~m} 1$ containing $20 \times 10^{6}$ spermatozoa/ml, it was usually necessary to pool ejaculates from 2 or more rabbits. After cooling and dilution, the spermatozoa suspension was subjected to Staflo sedimentation.

\section{Staflo Apparatus}

The $1 \times 12$ flow-cel1 (designated No. VII C), described in Cliapter III, was used in this serles of experiments. The spermatozoa suspension was introduced into inlet 4 , using the same size syringe $(20 \mathrm{~m} 1)$ as used for the other inlet solutions. Thus, the height of the sample stream at the inlet was $1.25 \mathrm{~mm}$. A typical migration pattern for this series of experiments is illustrated in Figure 27, where the spermatozoa suspension enters via inlet 4 and spreads over layers 4-7. In all experiments a set-up period equivalent to 2 times the average steady-state transit time through the flow-cell was allowed before collection of the fractionated spermatozoa was begun.

\section{Medium and Density Gradient}

A sucrose density gradient was used in 4 of the 7 sets of experiments described in this chapter and a dextran density gradient was used in the other 3. These experiments are hereafter referred to as "sucrose" or "dextran" experiments, respectively. In the sucrose experiments, the primary medium (medium S) conststed of 1/3 Hank's' solution and $2 / 3$ isotonic saline supplemented with $0.1 \%$ fructose. The flowing layers, numbered $1-12$ 
STAFLO FLOW-CELL SHOWING SPERMATOZOA MIGRATION PATTERN

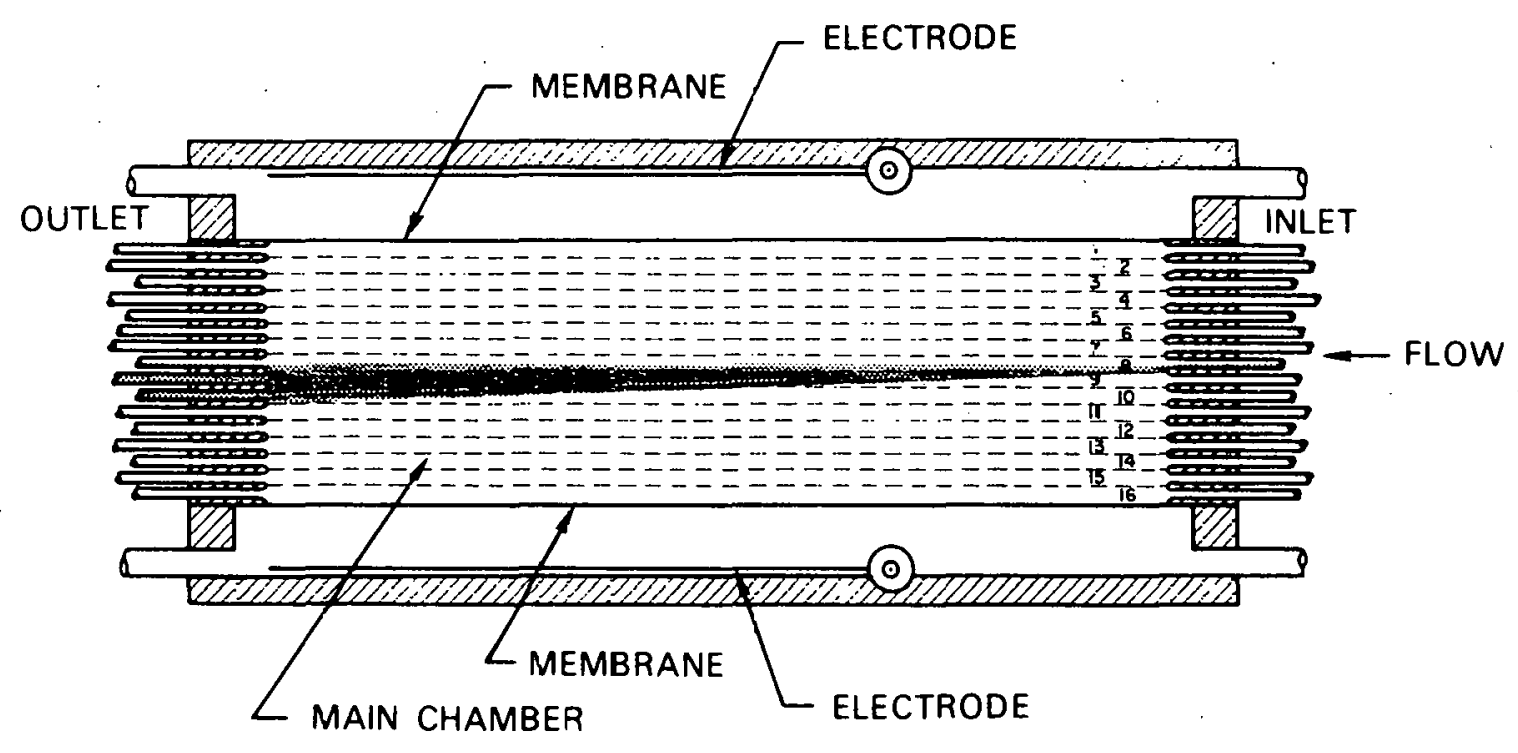

DBL 688-5418

Fig. 27. Schematic diagram of Staflo flow-cell showing typical rabbit spermatozoa sedimentation migration pattern at $7^{\circ} \mathrm{C}$; sucrose density gradient. 
from top to bottom, contained sucrose in weight/volume percent as follows: No. 1, 0\% (distilled water); Nos. 2 and 3,0\% (medium S); No. 4, 0\% (spermatozoa suspended in medium S); No. 5, 0.800\%; No. 6, 0.875\%; No. 7, 0.950\%; No. $8,1.025 \%$; No. $9,1.100 \%$; No8. 10-12, 3.0\%. The viscosity, osmolarity and specific gravity of the solutions in layers 3-9 are shown in Figure 28 for both the sucrose and dextran density gradients. Under the conditions employed in these experiments, few spermatozoa migrated below the seventh (at $7^{\circ} \mathrm{C}$ ) or ninth (at $15^{\circ} \mathrm{C}$ ) outlets. The "density barrier" in layers 10-12 was designed to stop spermatozoa or other particles that migrated that distance in order to minimize contamination of the membrane over the bottom oloctrodo compartmont.

In the dextran experiments, the primary medium (medium D) consisted of a mixture of $10 \% \quad 0.154 \mathrm{M}$ glycine and $90 \%$ medium $\mathrm{S}$. The layers contained dextran (MW 73,000) in weight/volume percent as follows: No. 1, $0 \%$ (dist11led water); Nos. 2 and 3, $0 \%$ (medium D); No. 4, $0 \%$ (spermatozoa suspended In medfum D); No. 5, $0.70 \%$; No. $6,0.75 \%$; No. $7,0.80 \%$; No. $8,0.85 \%$; No. $9,0.90 \%$; Nos. $10-12,3.0 \%$.

\section{Temperature Control}

In the experiments conducted at $4^{\circ} \mathrm{C}$ or above, the temperature of the solutions in the syringe on the pump rack was controlled by enclosing the pump rack in a plastic canopy and cooling with dry ice. The flow-cell was cooled with dry lce in a doorless refrigerator in the sucrose experiments and in a lucite box in the dextran experiments.

The experiments at $2.5^{\circ} \mathrm{C}$ were conducted in a newly constructed refrigerated room which was large enough for the entire apparatus, accessory equipment and several investigators.

5. Observation and Evaluation of Spermatozoa 
CHARACTERISTICS OF DEXTRAN AND SUCROSE GRADIENTS

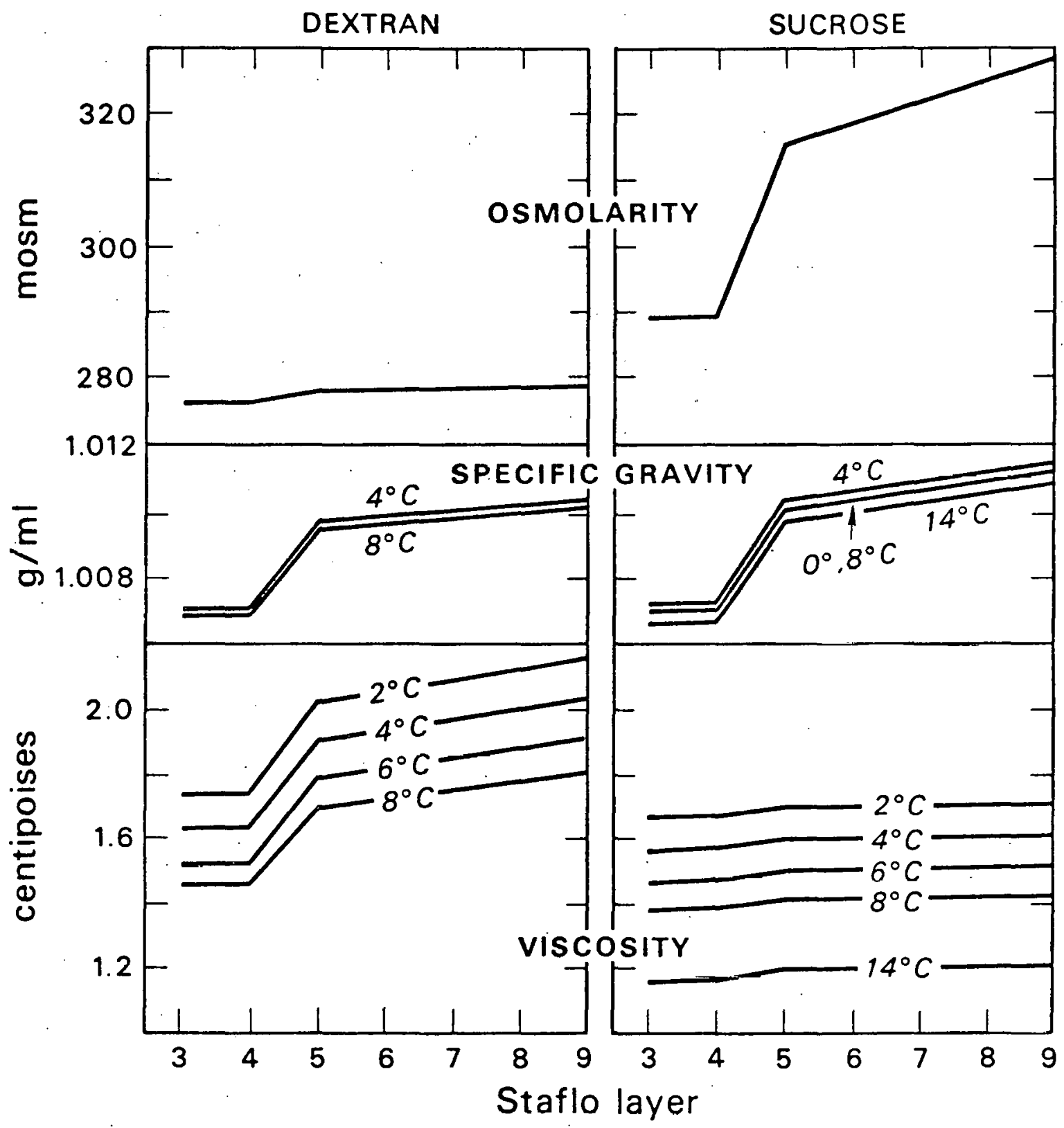

DBL 688-5422

Fig. 28. Characteristics of dextran and of sucrose density gradients for early sedimentation experiments. 
The migration pattern was observed with the "slit lamp technique"263 and observations of individual spermatozoa and the microscopic steady-state pattern were made with a portable microscope at magnifications up to $640 \mathrm{X}$. This portable microscope was a prototype for the Staflo microscope described in Chapter III. After collection, the spermatozoa concentration and motility in each fraction was determined with a hemocytometer and the viability was evaluated by nigrosfn-eosin staining as described in Appendix $B$. The categories of spermatozoa in the nigrosin-eosin stained preparations are as follows: unstained (U) or viable, morphologically normal but stained (MNS) and stained (S) or non-viable.

\section{Resulis}

1. Fractionation in a Sucrose Density Gradient.

Experiments were conducted at 4 temperatures in the following sequence: $7^{\circ} \mathrm{C}, 15^{\circ} \mathrm{C}, 4^{\circ} \mathrm{C}$ and $2.5^{\circ} \mathrm{C}$. The resulls are presented here and are compared in section $D$.

a. $7^{\circ} \mathrm{C}$. The results of a typical experiment $(10-21)$ at $7^{\circ} \mathrm{C}$ with a sucrose density gradient and a spermatozoa concentration of $20 \times 10^{6} / \mathrm{m} 1$ are shown in Figure 29. The most striking feature is the absolute enrichment in fractions 5, 6 and 7 with regard to viable spermatozoa relative to the unfractionated sample both before and after the experiment. The curve for stained spermatozoa is almost a mirror image of the curve for viablc spermatozoa. The determination of motility was made 90 minutes after collection stopped and shows an increase from fraction 4 to fraction 5 . The concentrations shown in Figure 29 for collection fractions $4-7$ represent $35.6 \%, 50.4 \%, 12.7 \%$ and $1.3 \%$ of the collected spermatozoa, respectively. It may be helpful to refer to Figure 27 to visualize the spermatozoa migration pattern. 


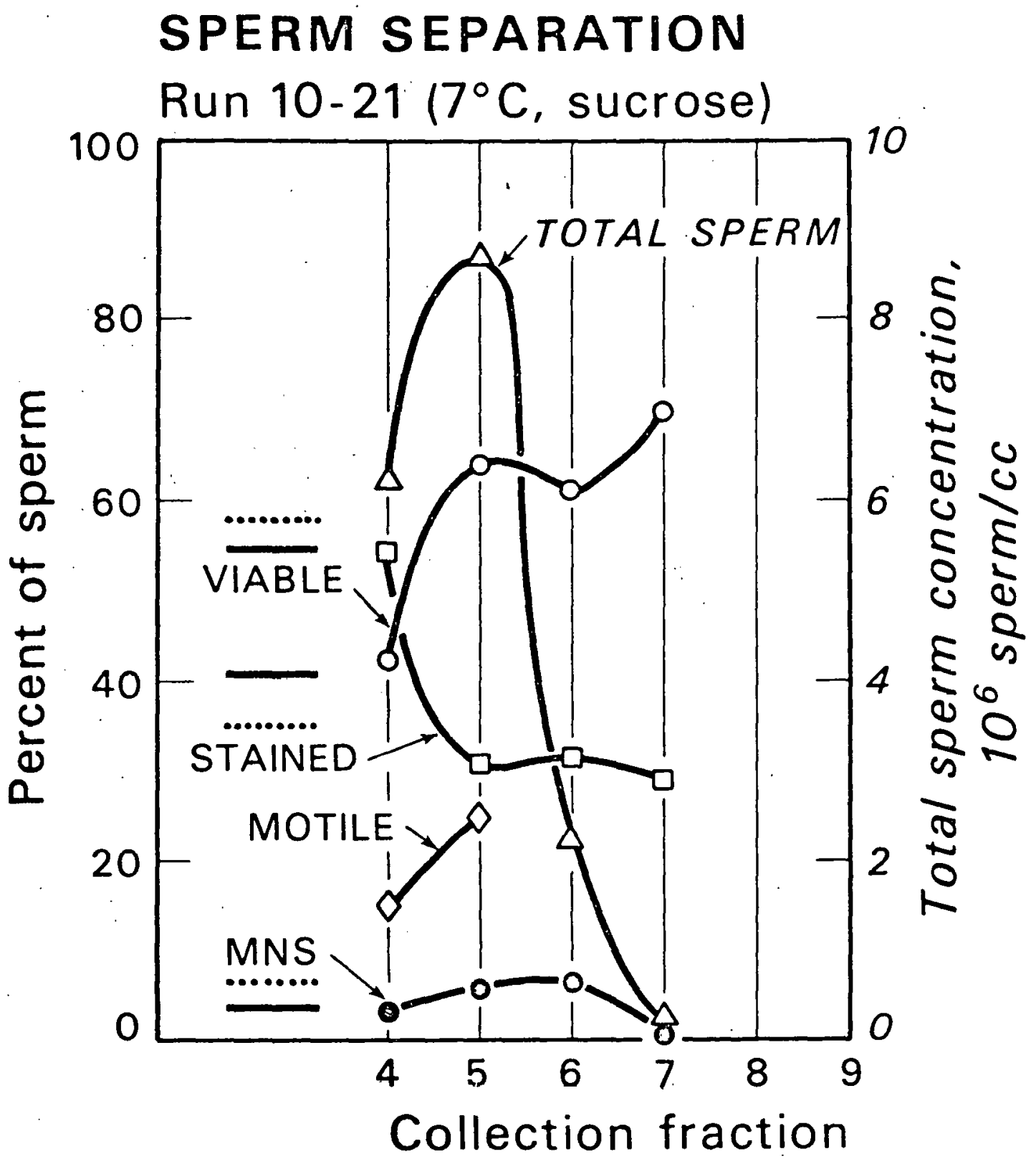

DBL 70115982

Fig. 29. Results of Sedimentation Experiment 10-21 with rabbit spermatozoa at $7^{\circ} \mathrm{C}$; sucrose density gradient. Also shown are the characteristics of the unfractionated sample before $(\ldots \ldots)$ and after (—) the experiment. 
The results of this experiment and 4 others run at $7^{\circ} \mathrm{C}$ with the same sucrose density gradient and spermatozoa concentrations ranging from 19$26 \times 10^{6} / \mathrm{ml}$ are shown in Figure 30 . The spermatozoa samples consisted of an ejaculate from rabbit $H$ in all experiments mixed with ejaculates from rabbit $G$ in 3 experiments and from rabbits 0 and $P$ in 1 experiment each. Samples were of approximately equal initlal viability and motility. Despite great changes in spermatozoa viability during the course of some experiments, the corresponding curves for viable and stained spermatozoa in Figure 30 show the same qualitative features seen in Figure 29. A consistent feature of the motility curves is their qualitative similarity to their respective total sperm distribution curves. The viability consistently. increases from fraction 6 to 7 while both spermatozoa concentration and motility decrease. Included among and indistinguishable from the curves of total sperm distribution is the result of one experiment (shown by the arrow) conducted under the same conditions with a spermatozoa concentration of about $20 \times 10^{6} / \mathrm{ml}$, all of which were immotile upon ejaculation.

The general features of the sets of curves in Figure 30 are clearly seen in Figure 31 which shows the averages for all 5 experiments. The relative composition of stained spermatozoa (U, MNS and $S$ ) in each fraction can be determined from this figure.

Nominal sedimentation rates can be calculated for the spermatozoa in each collection fraction using a layer thickness of $1.25 \mathrm{~mm}$ and an average steady-state transit time of 23.3 minutes. All spermatozoa entered the main chamber via inlet 4. Spermatozoa leaving via outlet 4 must have sedimented between 0 and $1.25 \mathrm{~mm}$. For these cells, the average extreme sedimentation rate is approximately $1.08 \mathrm{~mm} / \mathrm{hr}$. This and sedimentation 


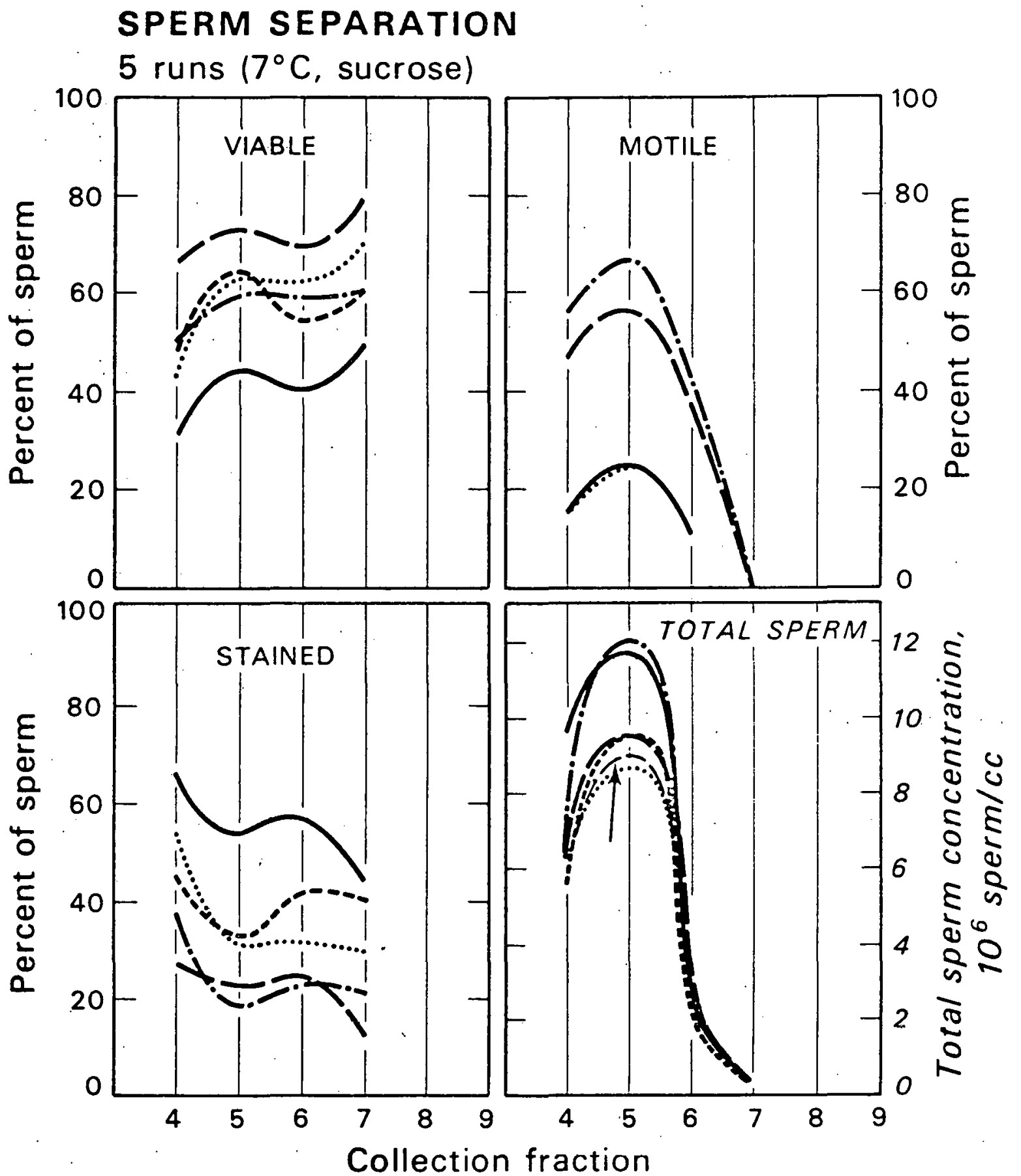

DBL 70115983

Fig. 30. Individual results of five sedimentation experiments with rabbit spermatozoa at $7^{\circ} \mathrm{C}$; sucrose density gradient. Key for experiments; $9-15(-)$; 9-22 (- - ); 10-5 (- $(-)$; $10-12(--)) ; 10-21(\cdots \ldots)$. 
SPERM SEPARATION

Average of 5 runs

$\left(7^{\circ} \mathrm{C}\right.$, sucrose)

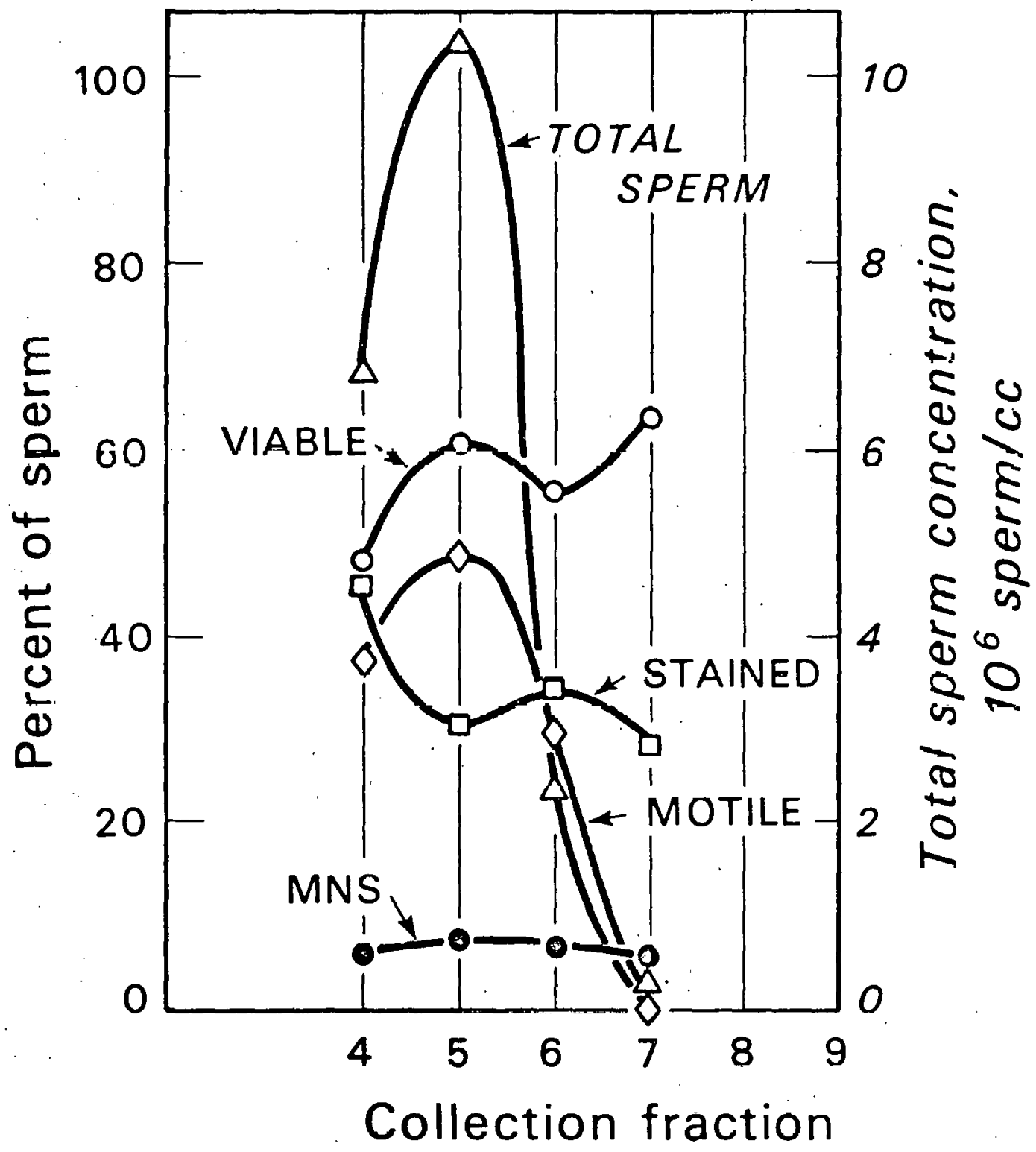

DBL 70115984

Fig. 31. Average results of five sedimentation experiments with rabbit spermatozoa at $7^{\circ} \mathrm{C}$; sucrose density gradient. 
rates for spermatozoa in the other collection tubes were calculated with the equations in Chapter III and Appendix E. They are sumarized in Table III. The percentages of spermatozoa in collection fractions 4-7 ranged from $31.5-38.6 \% ; 47.6-55.9 \%, 11.4-12.7 \%$ and $0.9-1.4 \%$, respectively, in the 5 experiments, demonstrating good reproduçibility.

M1croscoplc observations in the flow-cell showed that about $90 \%$ of the spermatozoa sedimented vertically with heads down and the remainder were obliquely orfented with their heads below the horizontal.

The evaluation of motility generally was made 60-90 minutes after collection stopped. At this time, 5-10\% of the spermatozoa in fraction 4 showed vigorous forward motion, whereas the remainder of the motility was primar1ly tail-wagging. Even though the spermatozoa in fraction 5 generally had a higher percentage of motility, the degree thereof was less than in fraction 4.

b. $15^{\circ} \mathrm{C}$. The average composition of the collection fractions in two experiments and the percentage of motile spermatozoa in each fraction for one experiment are shown in Figure 32A. Again, the curves for viable and stained spermatozoa change in opposite directions. Because of the limited data on motility, there is probably no slgnificance to the second peak in motility in fraction 8 . In Figure $32 \mathrm{~B}$, the average distribution of sperm in these 2 experiments at $15^{\circ} \mathrm{C}$ is shown wh the average for the 5 experiments at $7^{\circ} \mathrm{C}$ (from Figure 31). There is a significantly greater number of sperm In fractions 6,7 and 8 at $15^{\circ} \mathrm{C}$ than at $7^{\circ} \mathrm{C}$. The cell concentrations at $15^{\circ} \mathrm{C}$ were $25 \times 10^{6}$ and $28 \times 10^{6}$ spermatozoa/ml and were mixtures of ejaculates from rabblts $G, 0$ and $P$. Slight "cluster sedimertation" was observed in both experiments. Actively swiming spermatozoa were observed microscopically in layers 4 through 10 during the collection period in both 
Table III. Average Migration Velocities: Intrinsic Motility-Sedimentation, for Rabbit Spermatozoa; Sucrose Density Gradient; $2.5,4,7$ and $15^{\circ} \mathrm{C}$

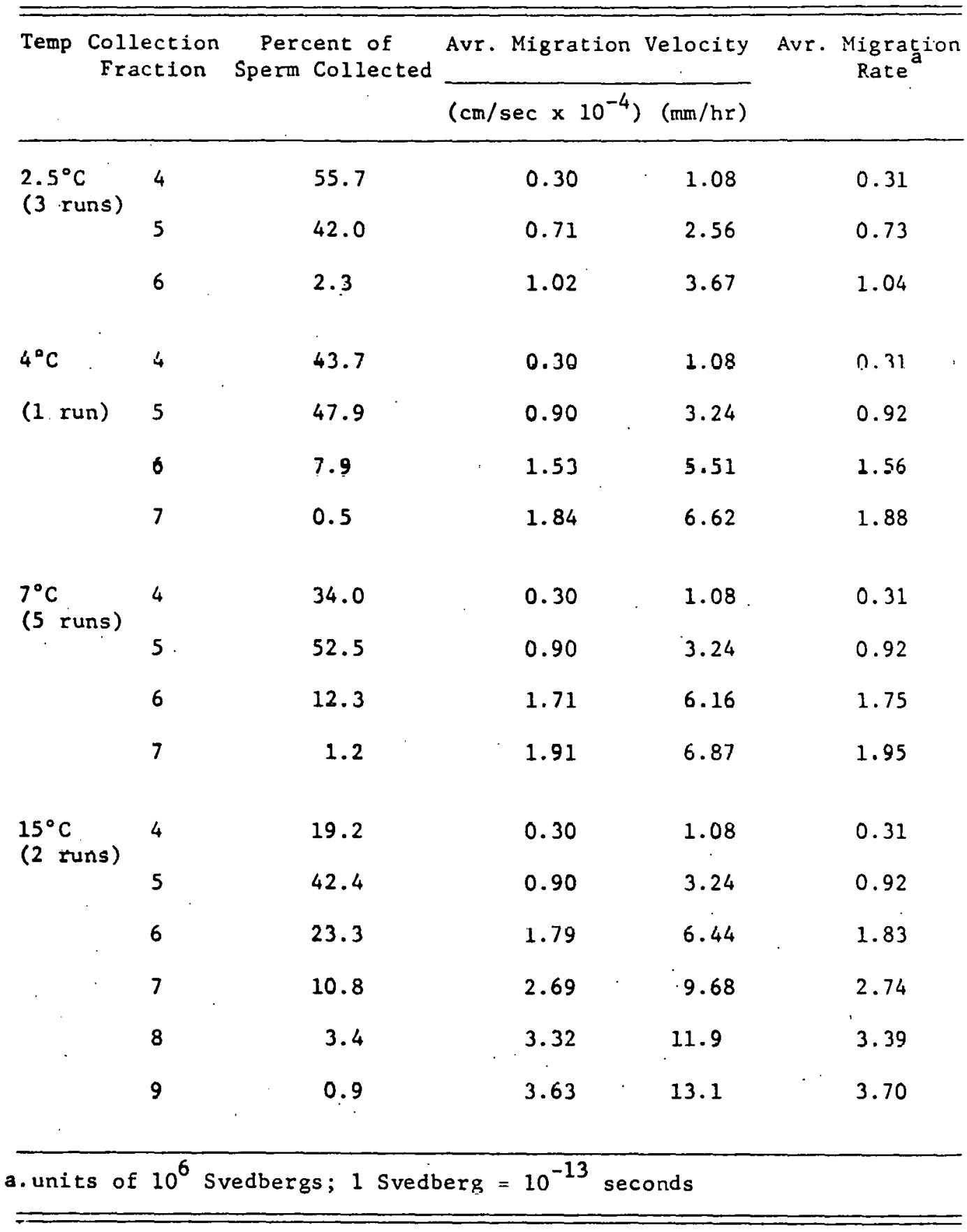




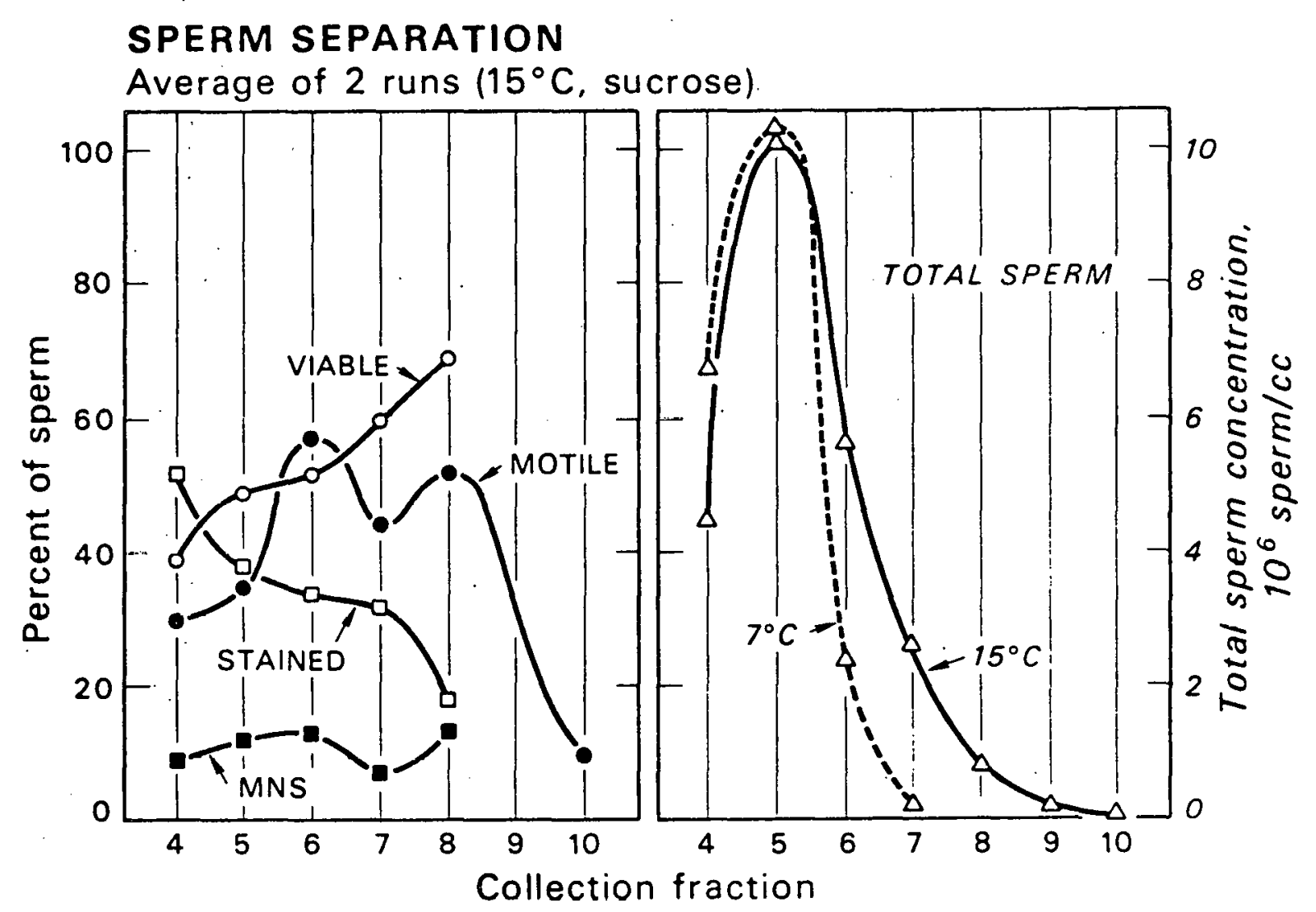

DBL 70115985

Fig. 32. Average results of two sedimentation experiments at $15^{\circ} \mathrm{C}$ and a comparison of the average total rabbit spermatozoa distribution at $15^{\circ} \mathrm{C}$ with that from five experiments at $7^{\circ} \mathrm{C}$; sucrose density gradient. 
experiments.

c. $4^{\circ} \mathrm{C}$. The results of a single experiment at $4^{\circ} \mathrm{C}$ are shown in Figure 33. Comparing Figure 33 with Figure 31 , it is seen that at $4^{\circ} \mathrm{C}$, a greater proportion of spermatozoa remained in layer 4 and fewer reached layer 6 . As at $7^{\circ} \mathrm{C}$, a higher percentage of spermatozoa were motile and viable in fraction 5 compared to fraction 4.

d. $2.5^{\circ} \mathrm{C}$. The individual results of 3 experiments at $2.5^{\circ} \mathrm{C}$ with a sucrose density gradient are shown in Figure 34 and the average values for all 3 experiments are shown in Figure 35. At room temperature, there were no motile spermatozoa in the Initial samples in Experiments 12-17-66 and 12-18-66. In Experiment 12-19-66, only $20 \%$ of the spermatozoa initially showed moderate tall-wagging with no forward progress. As shown in Figure 34 , only $2 \%$ of the total spermatozoa were in fraction 6 and they were of lower viability than those in either fraction 4 or 5 . Even though no motility was observed microscopically in the flow-cell in Experiment 12-19-66, the total spermatozoa distribution for that experiment differs from the 2 others in Figure 34 and is similar to that observed in experlments at $4^{\circ} \mathrm{C}$ and $7^{\circ} \mathrm{C}$. Figure 35 shows a slight rise in viability going from fraction 4 to 5 , as in experiments at higher temperatures, and a marked drop in viability from fraction 5 to 6 .

e. Calculation of Sedimentation Velocities. Using the fractionationmigration principles outlined in Chapter III, the intrinsic motilitysedimentation velocities were calculated for the rabbit spermatozoa in each of the collection fractions in the experiments at $2.5^{\circ} \mathrm{C}, 4^{\circ} \mathrm{C}$, and $15^{\circ} \mathrm{C}$. These results are presented in Table III with the values for the experiments at $7^{\circ} \mathrm{C}$.

2. Fractionation in a Dextran Density Gradient 


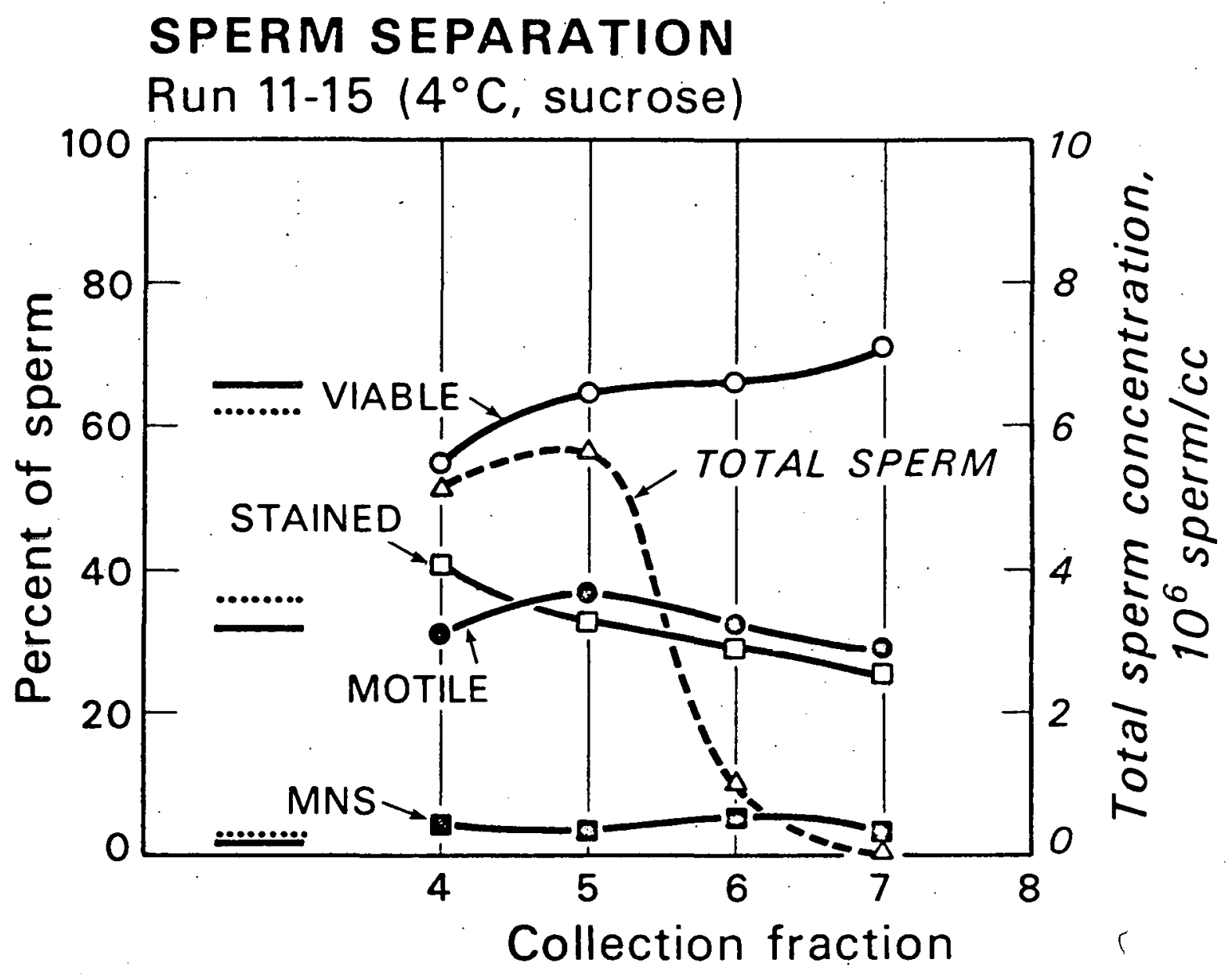

DBL 70115986

Fig. 33. Results of one sedimentation experiment with rabbit spermatozoa at $4^{\circ} \mathrm{C}$; sucrose density gradient. Characteristics of the unfractionated sample: before $(\longrightarrow$ ) and after $(\ldots . .$.$) the$ experiment. 


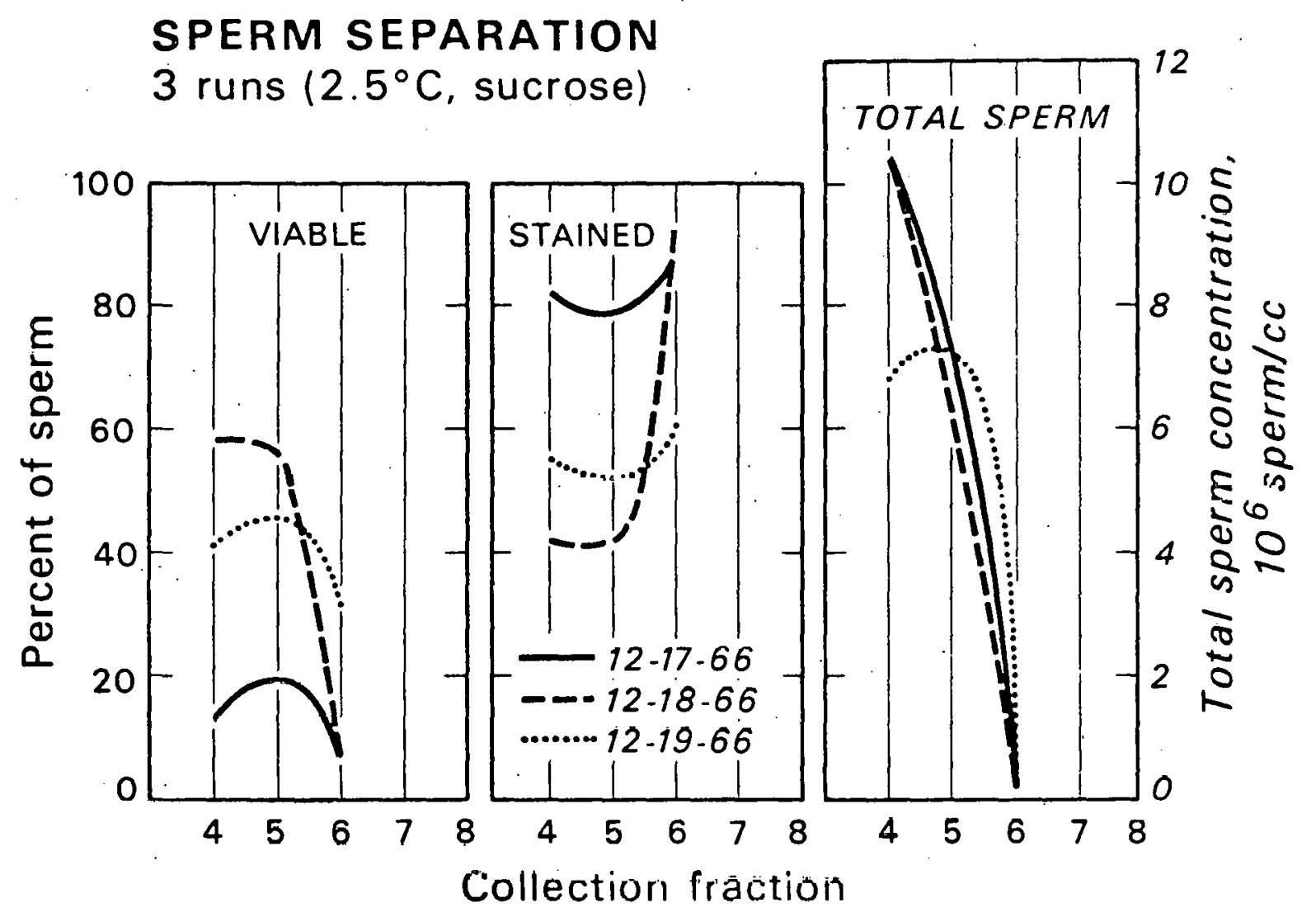

DBL 70115987

Fig. 34. Individual results of three sedimentation experiments with rabbit spermatozoa at $2.5^{\circ} \mathrm{C}$; sucrose density gradient. 


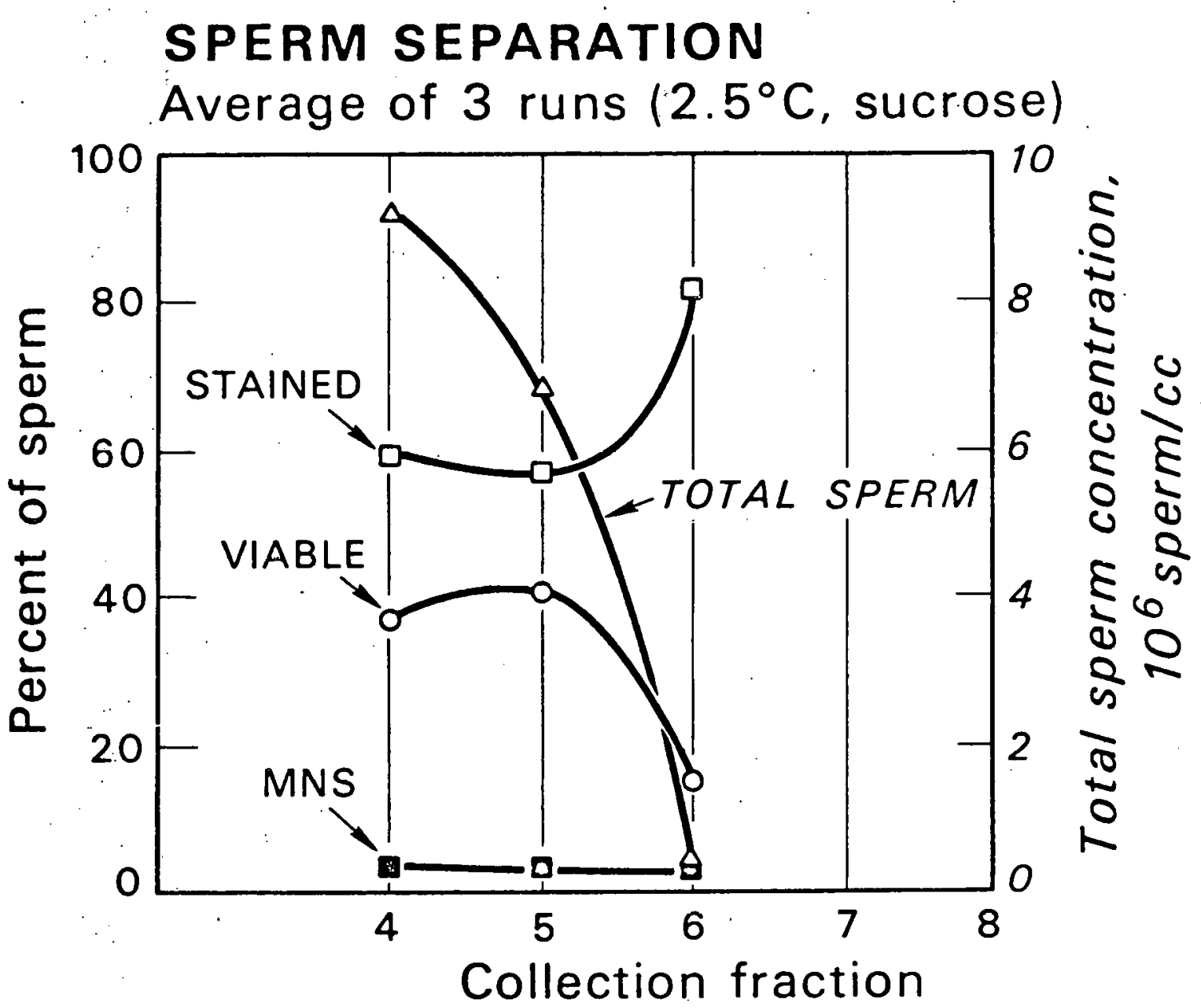

DBL 70115988

Fig. 35. Average results of three sedimentation experiments with rabbit spermatozoa at $2.5^{\circ} \mathrm{C}$; sucrose density gradient. 
Experiments were conducted only at temperatures of $7{ }^{\circ} \mathrm{C}, 4^{\circ} \mathrm{C}$ and $2.5^{\circ} \mathrm{C}$ in this density gradient.

a. $7^{\circ} \mathrm{C}$. The results of 2 experiments at $7^{\circ} \mathrm{C}$ using a glycine supplemented mediun and dextran density gradient with a 27 minute steady-state transit time are shown in Figure 36. Ejaculates from rabbits Chi, $O$ and $P$ were diluted to concentrations of $18 \times 10^{6}$ and $22 \times 10^{6}$ spermatozoa/m1 for Experiments 1-4 and 1-14, respectively. In Figure $36 \mathrm{~A}$, there is an increase in viability from fractions 4-6. In contrast, the percent motility decreases from fraction 5 to 6 , again showing that the loss of motility precedes the loss of viability. The curves for total spermatozoa distribution and motility in $\overline{\mathrm{F}}$ igure 36 are qualitatively similar. A high percentage of the spermatozoa in the flow-cell showed slight tail-wagging. In both experiments, over $25 \%$ of the spermatozoa in fraction 4 and a few percent In fractions 5 and 6 showed vigorous forward movement. The activity of the spermatozoa in the collection fractions in this glycine supplemented medium was much greater than ever observed in the collection fractions in the sucrose experiments.

b. $4^{\circ} \mathrm{C}$. A single experiment was conducted with the glycine-dextran medium at $4^{\circ} \mathrm{C}$ with a spermatozoa sample of fair initial quality. Eighty perrent of the spermatozoa exited the flow-chamber from layer 4 (the inlet layer), $19 \%$ extted from layer 5 and only $1 \%$ of the spermatozoa exited in layer 6.

c. $2.5^{\circ} \mathrm{C}$. After construction of a refrigerated room, 2 experiments were conducted at $2.5^{\circ} \mathrm{C}$ using the same dextran density gradient, a spermatozoa concentration of $20 \times 10^{6} / \mathrm{ml}$ and an average transit time of 66 minutes. A $10 \mathrm{ml}$ syringe was used to Introduce the spermatozoa suspension; thus, the sample stream at the inlet was only $0.75 \mathrm{~mm}$ high. At the outlet, the sample stream was $1.55 \mathrm{~mm}$ high but spermatozoa were observed with a micro- 


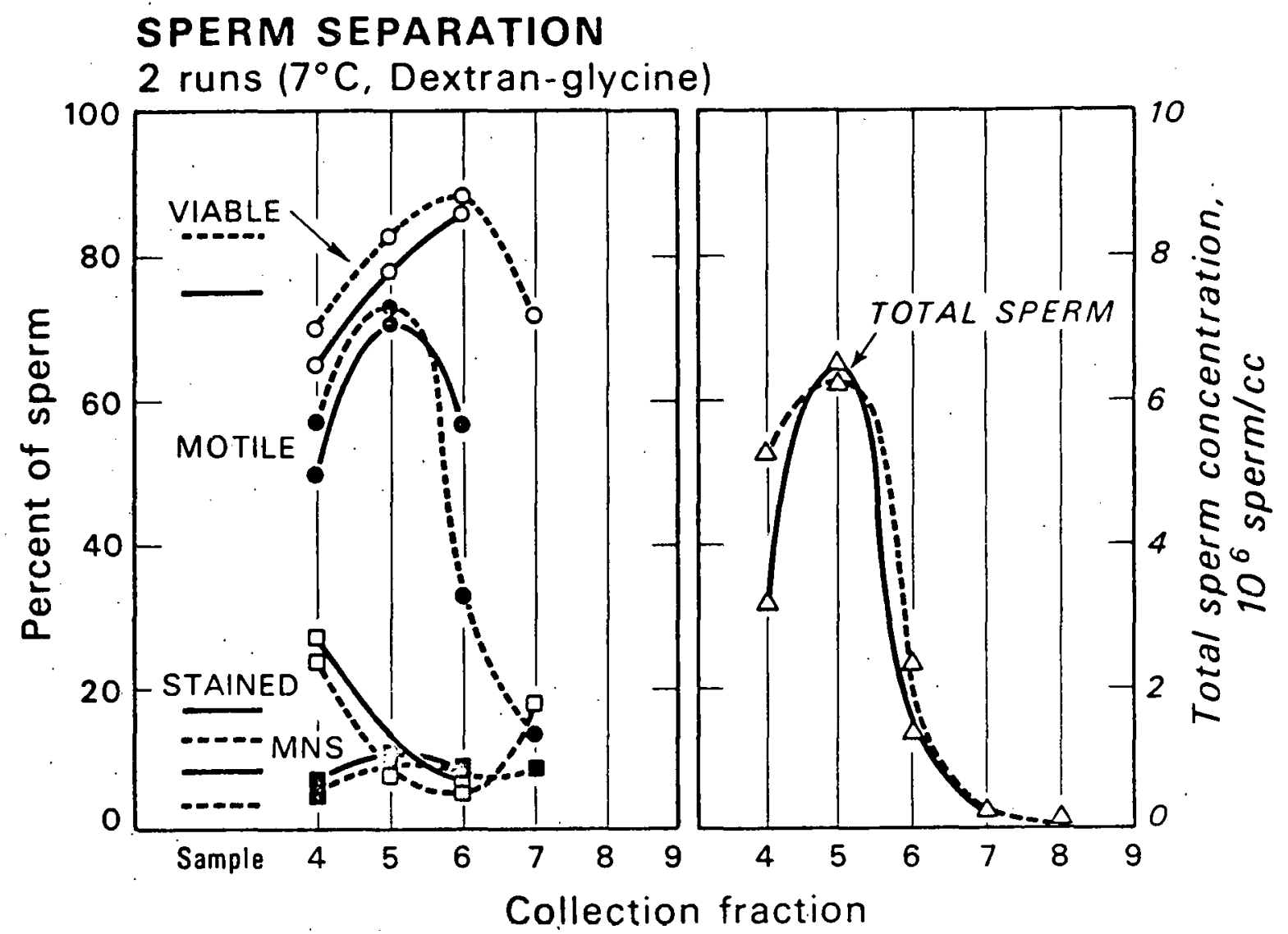

DBL. 70115989

Fig. 36. Individual results of two sedimentation experiments with rabbit spermatozoa at $7^{\circ} \mathrm{C}$; dextran density gradient. Initial sample values are also given. Key: Exp. 1-4 (־) ; Exp. 1-14 $(---)$ 
scope to be in the bottom $0.95 \mathrm{~mm}$ only. Thus, the minimum migration distance of the spermatozoa was $0.60 \mathrm{~mm}$ and the maximum migration distance from the bottom of the sample stream at the inlet to the bottom of the sample stream at the outlet was $0.80 \mathrm{~mm}$. Using these migration distances and the average transit time of 1.1 hours, calculations give minimum, maximum and average sedimentation rates of $0.82,1.09$ and $0.97 \mathrm{~mm} / \mathrm{hr}$, respectively. Because the spread of the spermatozoa migration pattern was less than the thickness of 1 Staflo layer, the fractionation was not meaningful in these 2 experiments.

\section{d. Calculation of Sedimentation Velocities. Using the fractionation-} migration princlples discussed In Chapter III, the average intrinsic mot1lity-sedimentation rates were calculated for the spermatozoa in each of the collection fractions in the experiments at $2.5^{\circ} \mathrm{C}, 4^{\circ} \mathrm{C}$ and $7^{\circ} \mathrm{C}$. These rates are presented in Table IV in units of $\mathrm{cm} / \mathrm{sec} \times 10^{-4}, \mathrm{~mm} / \mathrm{hr}$ and Svedbergs.

\section{J. ALlifleldi Tnseminar1oñ.}

Despite inseminating a large number of rabbits with spermatozoa from the collection fractions in many of the experiments presented above, only 4 1itters were obtained. In the $4^{\circ} \mathrm{C}$ sucrose experiment, both rabbits Inseminated with spermatozoa from fraction 4 gave litters, one of 4 males and 3 females, and the other, 1 male and 4 females. Insemination of spermatozoa from traction 5 in that experiment gave no offspring. In 2 dextran experiments, one each at $4^{\circ} \mathrm{C}$ and $2.5^{\circ} \mathrm{C}$, with $80 \%$ and $95 \%$ of the spermatozoa in fraction 4, respectively, insemination of spermatozoa from those collection fractions gave 2 litters of 3 females and 2 males each. This small number of offspring from several different experiments was insufficient for correlation with the physical results. 
Table IV. Average Migration Velocities: Intrinsic Motility-Sedimentation, for Rabbit Spermatozoa in a Dextran Density Gradient at $2.5,4$ and $7^{\circ} \mathrm{C}$

\begin{tabular}{|c|c|c|c|c|c|c|}
\hline \multirow[t]{2}{*}{ Temp $\mathrm{Cc}$} & \multirow[t]{2}{*}{$\begin{array}{l}\text { Collection } \\
\text { Fraction }\end{array}$} & \multirow{2}{*}{$\begin{array}{c}\text { Percent of } \\
\text { Sperm Collected }\end{array}$} & \multicolumn{2}{|c|}{ Avr. Migration Velocity } & \multirow[t]{2}{*}{ Avr. } & \multirow[t]{2}{*}{$\begin{array}{c}\text { Migration } \\
\text { Rate }^{a}\end{array}$} \\
\hline & & & $\left(\mathrm{cm} / \mathrm{sec} \times 10^{-4}\right)$ & $(\mathrm{mm} / \mathrm{hr})$ & & \\
\hline $\begin{array}{l}2.5^{\circ} \mathrm{C} \\
(2 \text { runs) }\end{array}$ & - & 100 & 0.27 & 0.97 & & 0.27 \\
\hline \multirow{3}{*}{$\begin{array}{l}4^{\circ} \mathrm{C} \\
(1 \text { run })\end{array}$} & 4 & 80 & 0.22 & 0.78 & & 0.22 \\
\hline & 5 & 19 & 0.48 & 1.73 & & 0.49 \\
\hline & 6 & 1 & 0.71 & 2.56 & & 0.73 \\
\hline \multirow{4}{*}{$\begin{array}{l}7^{\circ} \mathrm{C} \\
(2 \text { runs })\end{array}$} & 4 & 32.7 & 0.26 & 0.94 & & 0.27 \\
\hline & 5 & 51.3 & 0.77 & 2.77 & & 0.79 \\
\hline & 6 & 14.1 & 1.34 & 4.82 & & 1.37 \\
\hline & 7 & 1.3 & 1.60 & 5.76 & & 1.63 \\
\hline \multicolumn{7}{|c|}{ a. units of $10^{6}$ Svedbergs; I Svedberg $=10^{-13}$ seconds } \\
\hline
\end{tabular}




\section{Discussion}

The similarity of the shapes of the curves in Figure 30 , and the small range of values for the average distrịbution of spermatozoa among the collection fractions are demonstrations of experimental reproducibility under specified conditions. However, this series of experiments has shown that in the multi-vartable Staflo system, a large number of parameters Influence the sedimentation-migration pattern of spermatozoa. These, In turn, affect the biological results and must be considered in planning experiments. Variables of importance include the initial sample quality (the ratio of viable to stained spermatozoa and the degree of motility) and durability (a measure of the loss of viability and motility during the experiment), the spermatozoa concentration, and the temperature. Related factors include the average steady-state transit time, and the medium and density gradient. After considering the influence of these variables on the experiments, a comparison w111 be made with the results of other inyestigators on the sedimentation of rabbit spermatozoa.

\section{Variables Affecting Sedimentation Experiments.}

a. Quality of the Spermatozoa Sample. In addition to great variations in the initial quality of ejaculates from the same rabbit on different days, or different rabbits on the same day, ejaculates of the same apparent inftial quality varied in their ability to withstand the stresses in Staflo experiments. This unpredictability of spermatozoa quality is discussed in more detall in Chapter IX.

In this series of experiments, the varlable durability of spermatozoa samples is illustrated in the sucrose experiments using the percentage of viable (unstained) spermatozoa for comparison. In Experiments 9-15 and 9-22 (Figure 30) and in the 2 experiments at $15^{\circ} \mathrm{C}$ (Figure 32), the percentages 
of viable spermatozoa in the collection fractions were approximately $20 \%$ less than in the corresponding intial samples. In the other sucrose experiments, there was essentially no degradation of spermatozoa quality during the experiment as Judged by nigrosin-eosin staining.

Using the degree of motility after collection relative to the initial sample as a criterion, the durability of the spermatozoa sample in all of the sucrose experlments was poor. The relatively higher degree of motility of the spermatozoa in the collection fractions in the dextran experiments may have been a result of (1) a beneficlal effect of the glycine or dextran, or (2) more durable spermatozoa samples obtained at a different time of year.

The poor results from the insemination of spermatozoa from the collection fractions in this series of experiments is believed to be due primarily to dilution in media not suitable for the preservation of motility and fertilizing capacity. In retrospect, in most experiments, the spermatozoa in the collection fractions had such a poor degree of motility that Insemination was not warranted.

\section{b. Spermatozoa Concentration. The spermatozoa concentration affects}

both the migration pattern and the quality of spermatozoa in the collection fractions at the termination of the experiment. Based upon the results of the $7^{\circ} \mathrm{C}$-sucrose experiments, the migration pattern appears independent of concentration over the range $19-26 \times 10^{6}$ spermatozoa/ml. In the $15^{\circ} \mathrm{C}-$ sucrose experiments, slight "cluster sedimentation" was observed with spermatozoa concentrations of $25 \times 10^{6}$ and $28 \times 10^{6} / \mathrm{ml}$. The critical concentration of yeast cells referred to in Mel's discussion of "cluster" sedimentation ${ }^{158}$ is $26-27 \times 10^{6} / \mathrm{ml}$, suggesting a similar threshold for the 2 types of cells in simflar low density-low viscosity media. 
Other experiments in which there is a suggestion that "cluster sedimentation"occurred are the experiment at $7^{\circ} \mathrm{C}$ with immotile sperm (arrow in Figure 30) and Experiment $12-19-66$, at $2.5^{\circ} \mathrm{C}$, where the total sperm distribution resembles that at higher temperatures. In the latter experiment, it is unlikely that there was sufficient motility to explain the increased migration:

The effect of low spermatozoa concentrations (dilution effect) on the quality of the spermatozoa at the termination of the experiment is also illustrated in this series of experiments. In all sucrose experiments at $7^{\circ} \mathrm{C}$, the percentage of motile spermatozoa decreased from fractions 5-7 with changes in concentration from over $8 \times 10^{6} / \mathrm{ml}$ in fraction 5 to about $2 \times 10^{6}$ and $0.5 \times 10^{6} / \mathrm{ml}$ in fractions 6 and 7 , respectively. A similar loss of motility was also observed in the $7^{\circ} \mathrm{C}$ dextran experiments (Figure 36) in suspensions of less than about $4 \times 10^{6}$ spermatozoa/ml and is attributed primarily to dllution. This dilution effect has been reported by others to occur at concentrations of less than $4 \times 10^{6}$ spermatozoa/ml and to be prevented by certain macromolecules such as gum-arabic ${ }^{125}$. The recent studies by Quinn, White and Wirrick showed that dilution of ram and bull spermatozoa caused an influx of sodium and an efflux of potassium, calcium and magnesium from the cells, and was associated with loss of moti11ty ${ }^{193 .}$ The macromolecule dextran may have had a beneficial effect in the dextran density gradient experiments as noted in "a", above.

Because the spermatozoa concentrations in fractions 4 and 5 were greater than $4 \times 10^{6} / \mathrm{ml}$ in all experiments except in the Dextran Experiment 1-4, there was no dilution effect in those fractions. The percentage of motile spermatozoa in fraction 5 was greater than in fraction 4 in all experiments; however, the spermatozoa in fraction 4 had a higher degree of motility in 
all cases. The presence of seminal plasma in fraction 4 is the most likely explanation for the higher degree of motility in that fraction.

In all of the sucrose experiments at temperatures of $4^{\circ} \mathrm{C}$ or higher, and in all the dextran experiments at $7^{\circ} \mathrm{C}$ (except for fraction 7 in Experiment 1-14), the percentage of viable spermatozoa increased with increasing migration distance below layer 5 . This is in contrast to the loss of motility observed with decreasing spermatozoa concentrations in those same fractions. This shows that at least during a minimum period averaging 36 minutes at low concentrations, the loss of motility preceded the loss of viability, judged by nigrosin-eosin staining. It is postulated that at these temperatures, the best spermatozoa judged by degree of intrinsic motility swam downward, thereby giving an increase in the percentage of unstained (viable) spermatozoa in the lower fractions.

c. Temperature and Motillty. The temperature affects the sedimentation migration pattern primarily through its influence on motility. Figure 37 is a comparison of the total spermatozoa distribution for sucrose experiments at $2.5^{\circ} \mathrm{C}, 4^{\circ} \mathrm{C}, 7^{\circ} \mathrm{C}$ and $15^{\circ} \mathrm{C}$. It shows a significant increase in the percentage of the total number of spermatozoa appearing in the lower collection fractions at the higher temperatures. Because.the spermatozoa used in the 3 experiments at $2.5^{\circ} \mathrm{C}$ were of poor initial quality and no motflity was observed in the flow-chamber, the migration patterns observed In those experiments are belleved to be free of a motility component. In the experiment at $4^{\circ} \mathrm{C}$, spermatozoa were observed to have a rolling motion in the flow-cell and had sufficient motility to move forward slightly in a hemocytometer at that temperature. No motility was observed microscopically in the flow-cell during the $7^{\circ} \mathrm{C}$ sucrose experiments, but the optical system was in a very preliminary stage of development at the time of those 


\section{SPERM SEPARATION}

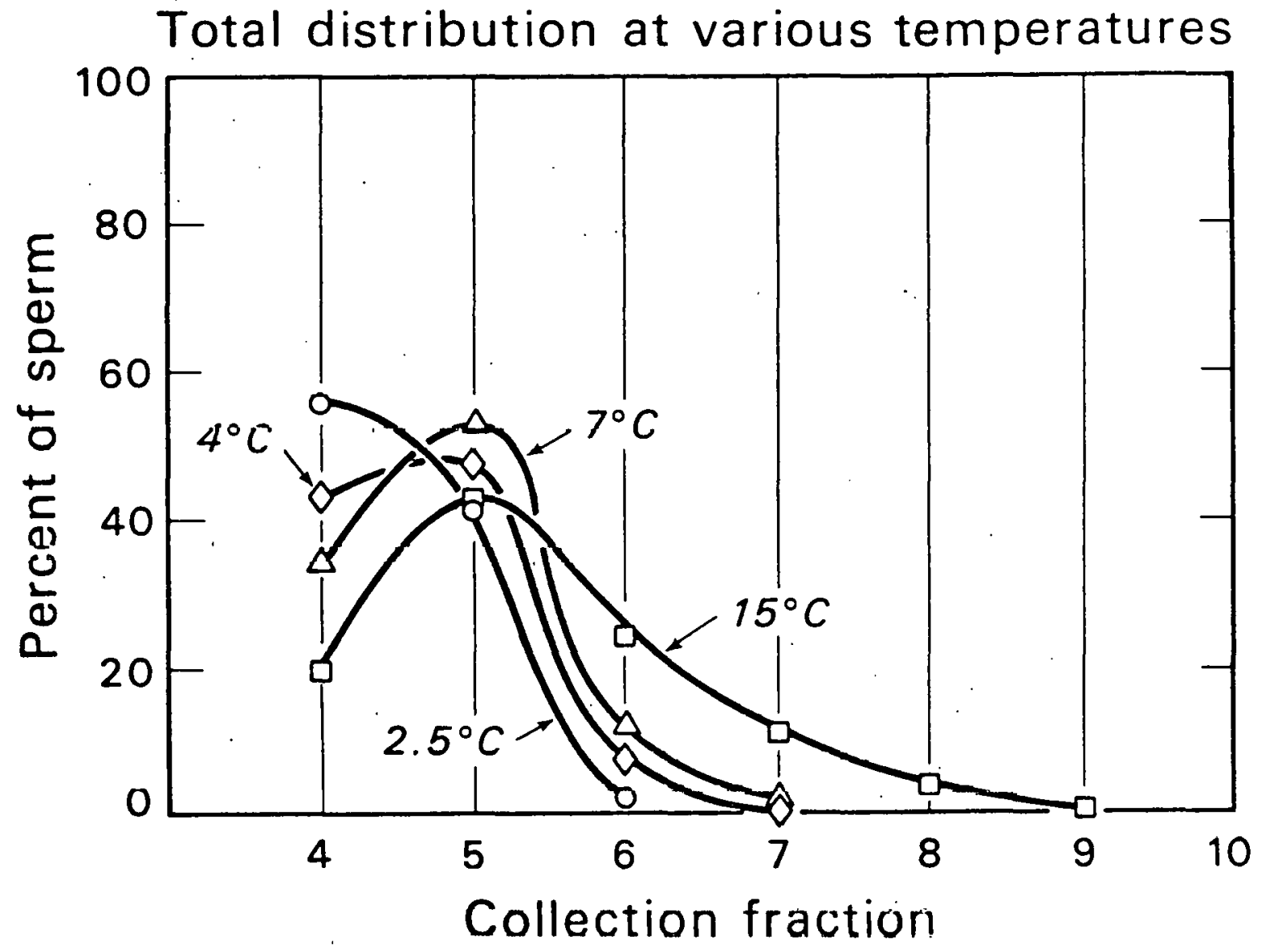

DBL 70115990

Fig. 37. Average distribution of rabbit spermatozoa among the collection fractions in sedimentation experiments at $2.5^{\circ} \mathrm{C}, 4^{\circ} \mathrm{C}$, $7^{\circ} \mathrm{C}$ and $15^{\circ} \mathrm{C}$; sucrose density gradient. 
experiments. Thus, motility could have contributed to the migration patterns in the $7^{\circ} \mathrm{C}$ experiments. A high percentage of actively swimming spermatozoa was observed microscoplcally in the flow-cell in both experiments at $15^{\circ} \mathrm{C}$. Thus, even though there was slight "cluster sedimentation" in both experiments, the greater migration at $15^{\circ} \mathrm{C}$ is attributed primarily to the intrinsic motility of the spermatozoa at that temperature.

Figure 38 is a comparison of the different-temperature sucrose experIments using the migration distance diagrams discussed in Chapter III. I belleve that the experiments at $2.5^{\circ} \mathrm{C}$ represent true sedimentation. Thus, the observed and expected distribution of spermatozoa at that temperature would be the same. As shown In Figure 28, the viscosity of the sucrose density gradient changes from 1.675 centipoises at $2.5^{\circ} \mathrm{C}$ to 1.175 centipoises at $15^{\circ} \mathrm{C}$. Using these differences in viscosity and the distribution at $2.5^{\circ} \mathrm{C}$, the expected distributions at $4^{\circ} \mathrm{C}, 7{ }^{\circ} \mathrm{C}$ and $15^{\circ} \mathrm{C}$ were calculated. They are shown in Figure 38 along with the actual distributions of spermatozoa in the 4 sets of experiments. Even though the decrease in viscosity contributes more to the distribution of the spermatozoa than one might expect, the migration is much greater than can be explained by viscosity alone. Therefore, the greater migration is attributed to the intrinsic motility of the spermatozoa at the respective temperatures.

The effect of motility upon the distribution of spermatozoa in the dextran experiments is similar to that observed in the sucrose experiments. In both dextran experiments at $2.5^{\circ} \mathrm{C}$, the spermatozoa were of poor initial quality and the migration pattern was not influenced by motility. Thus, the observed and expected migration distances are consilered to be the same at $2.5^{\circ} \mathrm{C}$. At $4^{\circ} \mathrm{C}$, a small percentage of the spermatozoa showed a slight degree of tail-wagging in the flow-cell. At $7^{\circ} \mathrm{C}$, a high percentage of 


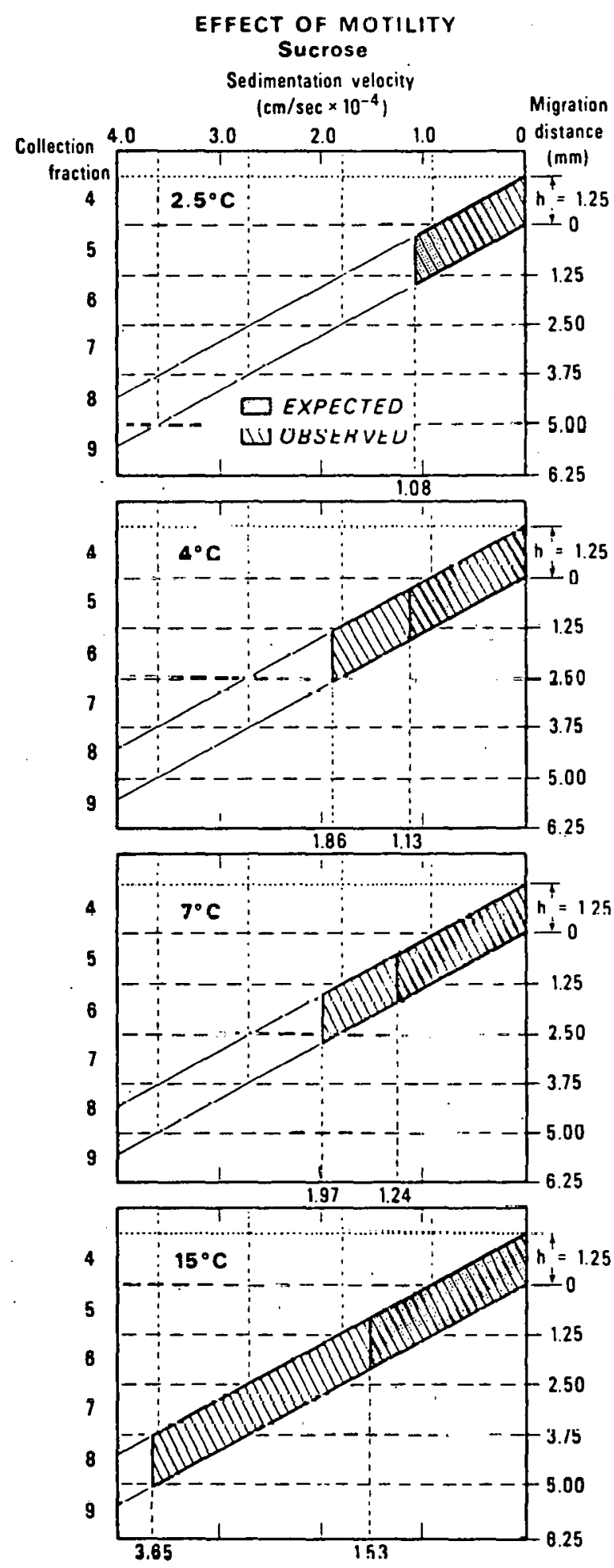

DBL 70115991

Fig. 38. Comparison of expected and actual distributions of rabbit spermatozoa in sucrose density gradient sedimentation experiments at various temperatures. 
spermatozoa in the flow-cell showed gentle rolling or tail-wagging. The effects of the motility on the migration distances are shown in Figure 39. Using the known differences in viscosity at the 2 temperatures, the expected distributions of spermatozoa at $4^{\circ} \mathrm{C}$ and $7^{\circ} \mathrm{C}$ were calculated and are shown in Figure 39 with the respective observed migration distances. The contribution of motility at the higher temperatures in the dextran experiments is even more pronounced than in the sucrose experiments.

The observations of motility of varying degrees in the flow-cell at $4^{\circ} \mathrm{C}$ and at the higher temperatures supports the hypothesis that the difference between the expected and observed migration distances in Figures 38 and 39 are due to motility. However, as noted earlier, one experiment conducted at $7^{\circ} \mathrm{C}$ with immotile spermatozoa gave a distribution similar to the average of the 5 experiments conducted with spermatozod samples of fair initial quality. This suggests that with migration distances as small as those achieved in most of these experiments, "cluster sedimentation" can have a significant relative influence on the migration pattern.

\section{Comparisons with Results of Other Investigators.}

In the sucrose density gradient at $2.5^{\circ} \mathrm{C}$, the sedimentation velocity ranged from $0-3.86 \mathrm{~mm} / \mathrm{hr}$ assuming that the $2.3 \%$ of spermatozoa in fraction 6 had sedimented a maximum distance of $1.50 \mathrm{~mm}$ (this is probably a generous estimate). At this temperature, the viscosity and specific gravity of the medium in layer 5 are $1.70 \mathrm{cp}$ and 1.010, respectively.

Calculations based upon the data reported by Bhattacharya ${ }^{35}$ give a "sedimentation velocity" range of $0-5 \mathrm{~mm} / \mathrm{hr}$ for rabbit spermatozoa in his apparatus at $0^{\circ} \mathrm{C}$. The medium had a viscosity of 75 centipoises and a spectfic gravity of 1.0304 . This estimate of the sedimentation velocity of rabbit spermatozoa in his medium is greater than that in my sucrose 


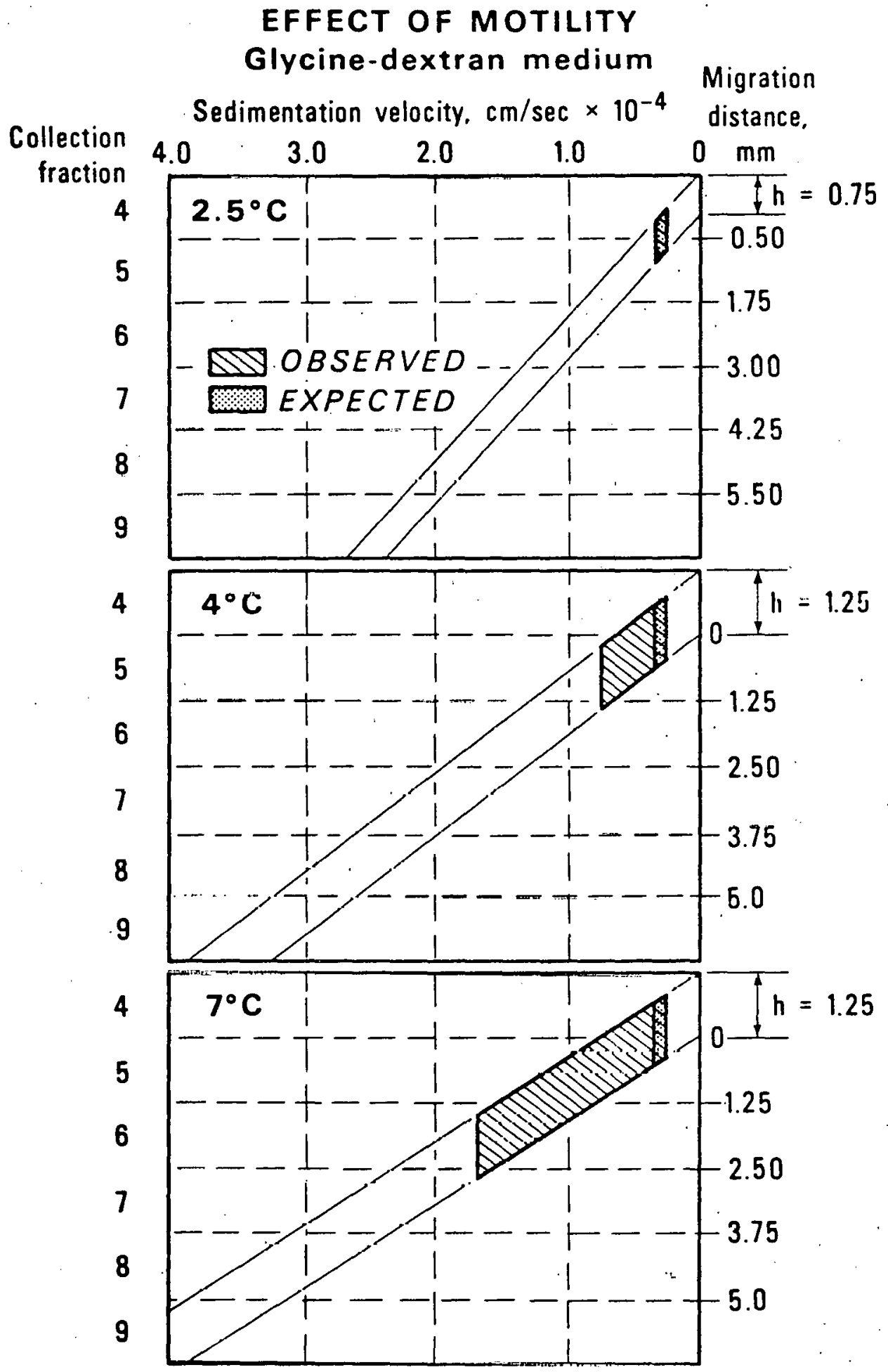

DBL 689-5467

Fig. 39. Comparison of expected and actual distributions of rabbit spermatozoa in dextran density gradient sedimentation experiments at various temperatures. 
density gradient even though the specific gravity and viscosity, in particular, were much higher in his apparatus.

Schilling reported large sedimentation distances for bull spermatozoa in a simflar device in 60 minutes at $0^{\circ} \mathrm{C}$, in a medium with a viscosity of 7-10 centipoises and a specific gravity of $1.037-1.044^{205}$. If interpretation of his report is correct, the maximum "sedimentation velocities" in his apparatus would have been $50-60 \mathrm{~mm} / \mathrm{hr}$.

Bedford and Bibeau reported rabbit spermatozoa sedimentation experiments at $2^{\circ} \mathrm{C}$ in a medium with a viscosity of $6.7 \mathrm{cp}$ and a specific gravity of $1.048^{29}$. The sedimentation distances are not reported but the apparent "sedimentation velocities" appear to be comparable to those in Schilling's experiments even though rabbit and bull spermatozoa were used in the respective studies.

Compared to Bhattacharya's results, the proportionate increase in sedimentation velocity in the experiments by Schilling and by Bedford and Bibeau is consistent with the lower viscosity of their media. Thus, the relative differences between the sedimentation velocities observed in my experiments and those of the three other groups of experiments are comparable. All three are incompatible with my results and suggest that more than true sedimentation occurred in their experiments.

The other investigators layered spermatozoa at concentrations of $200-300 \times 10^{6} / \mathrm{ml}$ on top of their media in a burette. Even though the greater viscosities and specific gravities of their media would raise the threshold for "cluster sedimentation" above the $25-30 \times 10^{6}$ spermatozoa/m1 threshold of my sucrose density gradient, that phenomenon is considered the most likely explanation of the higher "sedimentation velocities" in 
their experiments. Because of the low temperatures used by those investigators, there should have been no contribution of motility to the migration.

As noted in Chapter II, Bhattacharya obtained a significant differ-. ence in the sex of offopring from the extreme fractions of his column sedimentation device ${ }^{35}$. Schilling had less success ${ }^{205}$, and Bedford and Bibeau reported none 29 . It is interesting to speculate that "cluster sedimentation' accentuated the sedimentation of the faster migrating species ${ }^{158}$, while at the same time giving a false high sedimentation velocity for the spermatozoa. Even though this phenomenon may have given a differentlal distribution of slow and fast sedimenting spermatozoa the separation of the $\mathrm{X}$ - and $\mathrm{Y}$-chromosome bearing spermatozoa by Bhattacharya and by Schflling is more likely related to the aqueous polymer two-phase system described by Albertsson 270 or to some undefined cell-cell interactions related to certain constituents of the medium.

\section{E. Sümary and Conclusions}

The $7^{\circ} \mathrm{C}$ sucrose experiments demoristrate good reproducibility under the same operating conditions (e.g., medfum, $\mathrm{pH}$, density gradient and average transit time) with spermatozoa concentrations ranging from 19$26 \times 10^{6} / \mathrm{ml}$. The differences among the different-temperature experiments with both sucrose and dextran density gradients are attributed primarily to the intrinsic motillty of the spermatozoa. In addition to this variable, the spermatozoa concentration is an important factor, especially at temperatures below $2.5^{\circ} \mathrm{C}$ where motility is negligible in the sucrose and dextran media.

In these low density-low viscosity media, the upper limit of the cell concentration is approximately $25 \times 10^{6} / \mathrm{ml}$, above which "cluster sedimen- 
tation"was observed with the slit lamp technique. Examples were given where it is believed that "cluster sedimentation", in certain experiments at $2.5^{\circ} \mathrm{C}$ and $7^{\circ} \mathrm{C}$, gave distributions simflar to those expected with motile spermatozoa. Thus, depending upon the medium and density gradient, the threshold for "cluster sedimentation"will determine the maximum desirable spermatozoa concentration. At the other extreme, the motility and fertilizing capacity were not preserved adequately at concentrations below $4 \times 10^{6}$ spermatozoa/ml in these media. In certain fractions the presence of diluted seminal plasma enhanced the preservation of motility, but not of fertilizing capacity. In view of the dilution inherent in multi-layer fractionation of any kind, the "dilution effect" dictates using an initial sample of the highest posstble concentration. Thus, one is faced with the contradictory objectives of maintaining the vital functions of the spermatozoa without compromising the ideal migration pattern. The resolution of these problems is described in later chapters.

At the higher temperatures, "cluster sedimentation'still should generally be avolded but the key factors are the direction and magnitude of the intrinsic motility vector, which depend upon the temperature, medium and quality of spermatozoa. Since the microscopic optical system available for the $7^{\circ} \mathrm{C}$ and $15^{\circ} \mathrm{C}$ sucrose experiments was very primitive and unreliable, the degree of motility in the flow-chamber in those experiments was not we11 defined. The orientation of spermatozoa at both temperatures appeared to be primarily downward. In the later dextran experiments at temperatures of $7^{\circ} \mathrm{C}$ and lower, the spermatozoa were seen to be oriented primarily downward which suggests that the same was the case for the $7^{\circ} \mathrm{C}$ sucrose experiments.

The relative enrichment of the lower collection fractions in many 
experiments, with respect to motility and viability, is attributed to intrinsic motility. This interpretation of the differential distribution was made difficult by the fact that motility was lost prior to viability. However, in the $2.5^{\circ} \mathrm{C}$ sucrose experiments, where there was no motility in the flow-chamber, there was no difference between the two "high concentration" fractions with respect to viable spermatozoa. This suggests that the differential fractionation of viable spermatozoa at higher temperatures was due to motility. Th1s is consistent with Bhattacharya's report that there was no difference in the relative distribution of live and dead spermatozoa in his "sedimentation" device.

Figures 37 and 38 are 2 ways of displaying temperature-dependent migration information. The range and distribution diagram (Figure 38) indicates a clear distinction between the expected and actual spermatozoa distributions at these temperatures and demonstrates the feasibility of quantitatively defining the intrinsic motility of spermatozoa as a function of temperature, for the population as a whole. This would be of particular Interest in fractionations with media capable of preserving both motility and fertilizing capacity. Under these conditions, the temperature dependence of the degree of motility could be correlated with the migration distance. In addition, it would also be possible to determine the fertilizing capacity of the spermatozoa, and the sex ratio of offspring derived therefrom, e.g., as a function of the relative degrees of intrinsic motility.

The difficulty of deallng quantitatively with "cluster sedimentation" argues for modifying the cell concentration, medium and/or density gradient to avold the problem 158,263 . The much greater "sedimentation velocities" observed by other investigators are no doubt due to "cluster sedimentation" or sedimentation processes other than sedimentation of Individual cells. 
It does not appear that the separation of $X$ - and $Y$-chromosome bearing rabbit (Bhattacharya ${ }^{35}$ ) and bull (Schilling ${ }^{205}$ ) spermatozoa is a simple function of differential sedimentation of the two types of spermatozoa. 


\section{EARLY STAFLO-ELECTROPHORESIS EXPERIMENTS:}

RABBIT AND FOWL SPERMATOZOA

\section{Contents}

A. Introduction. . . . . . . . . . . . . . . . 143

B. Materials and Methods . . . . . . . . . . . . . . . 144

1. Sample Preparation. . . . . . . . . . . . . . . 144

2. Staflo Apparatus. . . . . . . . . . . . . . 144

3. Media and Density Gradients . . . . . . . . . . . . 145

4. Temperature Control .............. 146

5. Observation and Evaluation of Spermatozoa . . . . . 146

c. Results . . . . . . . . . . . . . . . . . . 147

1. 1 x 12 Flow-Cell Experiments. . . . . . . . . . . 147

a. Experiments at 5,6.25 and $7.5 \mathrm{~V} / \mathrm{cm}$. . . . . . 147

b. Comparison of Vitterent fieid strèngth Experiments. . 150

2. $3 \times 16$ Flow-Cel1 Experiments. . . . . . . . . . 158

a. Preliminary Flow-Cell Evaluation. . ......... 158

b. Downward-Electrophoresis: Rabbit and Fowl Spermatozoa............................. 163

c. Upward-Electrophoresis: Rabbit and Fow1 Spermatozoa . . . . . . . . . . . . . . 166

D. Discussion. . . . . . . . . . . . . . . . . 169

1. Factors Influencing the Distribution of Spermatozoa in . the Flow-Chamber. . . . . . . . . . . . . 169

2. Electrophoretic Mobility Comparisons. . . . . . . . 174

E. Summary and Conclusions . . . . . . . . . . . . . 176 


\section{A. Introduction}

Studies by others of the electrophoretic properties of spermatozoa were summarized in Chapter II. This included the work by Sçhröder 206,207 and by Gordon 91,92 showing a variation from the normal sex ratio upon the insemination of anodic and cathodic fractions of spermatozoa separated in an electrophoretic apparatus. However, their work was not confirmed in studies by many other Investigators $18,174,233$. Bedford demonstrated changes in the electrophoretic properties of spermatozoa durfing maturation ${ }^{24}$, while Bangham showed changes in the relative surface charge distribution between heads and tails of spermatozoa in media of different lonic strength and at different $\mathrm{pH}^{\prime} \mathrm{s}^{18}$. He also found differences in the electrophoretic mobllity under varying conditions of $\mathrm{pH}$ and lonic strength ${ }^{18}$.

This chapter is devoted to a series of experiments conducted during a transition phase in the development of Improved staflo equipment and techniques. While experiments with the $1 \times 12$ flow-cell were providing knowledge on the Staflo electrophoresis properties of spermatozoa and the requirements for handling spermatozoa IN VITRO, work was in progress concurrently, on the following: (1) the construction of a $3 \times 16$ staflo apparatus to provide greater resolution in fractionation; (2) the development of new buffers to preserve the viability, motility and fertilizing capacity of the spermatozoa, as well as giving greater $\mathrm{pH}$ stabllity in the Staflo apparatus; (3) the design and construction of a microscope to be used with the Staflo apparatus for better observation of spermatozoa within the flow-chamber and (4) the construction of a refrigerated room to provide accurate temperature control during experiments. 
Because many of the spermatozoa samples used in the series of $1 \times 12$ flow-cell experiments had high initial quality, these experiments demonstrate many of the interrelationships among the biophysical properties of spermatozoa and the experimental variables, especially the effects of motility and temperature on the distribution of spermatozoa in the flowchamber. Subsequent experiments provide information on the characteristics of the $3 \times 16$ Staflo apparatus, the orientation of spermatozoa in the flow-chamber, and the electrophoretic mobility of both rabbit and fowl spermatozoa during electrophoresis in upward and downward directions.

\section{B. Materials and Methods}

\section{Sample Preparation}

Spermatozoa samples were obtained from mature Dutch Belt rabbits using the artificial vagina described in Appendix $C$. Each ejaculate was diluted immedlately by adding $2.0 \mathrm{ml}$ of medium (see bêlow') at $37^{\circ} \mathrm{C}$ to the collection vial and then was allowed to cool to room temperature in an insulating nest of glass wool. A $0.1 \mathrm{ml}$ aliquot of this sample was diluted to $1.0 \mathrm{ml}$ and evaluated for percent and degree of motility as described in Appendix B. After the sample had been cooled slowly in a water bath to the experiment temperature, it was diluted to approximately $20 \times 10^{6}$ spermatozoa/ml. In order to obtain a sample suspension of at least $15 \mathrm{ml}$ at that concentration, it was usually necessary to pool ejaculates from 2 or more rabbits. The cooled, diluted spermatozoa suspension then was subjected to Staflo electrophoresis. All work with the spermatozoa was accomplished using minimal lighting in order to reduce the deleterious effects of visible light ${ }^{176}$.

\section{Staf10 Apparatus}

The $1 \times 12$ and $3 \times 16$ flow-cell experiments were conducted with flow-cell 
number VII-C and XI, respectively. The characteristics of these flow-cells and the Staflo microscope are described in Chapter III.

\section{Media and Density Gradients}

a. $1 \times 12$ Flow-Cell Experiments. The $1 \times 12$ flow-cell experiments were conducted with a Hanks-glycine primary medium using a dextran density gradient. The Hanks-glycine electrophoresis medium consisted of $100 \mathrm{ml}$ of Hanks solution, $10 \mathrm{ml}$ of $0.35 \% \mathrm{NaHCO}_{3}, 9.25 \mathrm{~g}$ glycine and $25.0 \mathrm{~g}$ glucose diluted to $1,000 \mathrm{ml}$ with distilled water, including adjustment of $\mathrm{pH}$ with $1.0 \mathrm{~N} \mathrm{NaOH}$. The density gradient consisted of dextran (MW, 73,000, Sigma Chemical Company) in weight/volume percent in the staflo layers as follows: No. $1,0 \%$; No. 2 , $0.1 \%$; No. $3,0.3 \%$; No. $4,0.6 \%$; No. $5,0.7 \%$; No. $6,0.8 \%$; No. $7,0.9 \%$; No. 8 , $1.0 \%$; No. $9,1.0 \%$; Nos. $10-12,2 \%$. The top electrode compartment contained Hanks-glycine medium without dextran, while the bottom electrode compartment contained the same medium with $2 \%$ dextran. Even though this density gradient supported a suspension of up to $30 \times 10^{6}$ spermatozoa/ml in layer 9 , lower cell concentrations were used to avoid "cluster sedimentation'described in Chapter IV.

b. $3 \times 16$ Staflo Experiments. Low ionic strength glycine-phosphate and glycine-citrate buffers were used in this series of experiments.

(1) Glycine-phosphate buffer. The glycine phosphate buffer consisted of $2.20 \mathrm{~g} \mathrm{Na}_{2} \mathrm{HPO}_{4} .12 \mathrm{H}_{2} \mathrm{O}, 0.20 \mathrm{~g} \mathrm{NaCl}, 0.20 \mathrm{~g} \mathrm{NaHCO}, 0.016 \mathrm{~g} \mathrm{KH}_{2} \mathrm{PO}_{4}$, $9.25 \mathrm{~g}$ glycine, $23.0 \mathrm{~g}$ fructose, $10^{6}$ units of penicillin $\mathrm{G}$, all diluted to $1,000 \mathrm{ml}$ with distilled water. $\mathrm{pH}$ adjustments were made with concentrated $\mathrm{HCl}$.

(2) Glycine-citrate buffer. The glycine-citrate buffer consisted of $2.0 \mathrm{~g}$ sodium citrate dihydrate, $9.25 \mathrm{~g}$ glycine, $3.0 \mathrm{~g}$ fructose, $0.25 \mathrm{ml}$ $10 \%$ citric acid solution, $48.6 \mathrm{~g}$ sucrose, $10^{6}$ units penicillin G diluted 
to $1,000 \mathrm{ml}$. with distilled water. $\mathrm{pH}$ was approximately 6.94 .

Different density gradients consisting of dextran (MW, 73,000, Sigma Chemical Company) or sucrose were used in the $3 \times 16$ flow-cell experiments, and, therefore, are described in the appropriate sections below.

\section{Temperature Control}

In the $1 \times 12$ flow-cell experiments conducted at $4^{\circ} \mathrm{C}$ or above, the temperature of the solutions in the feed syringes was controlled by enclosing the pump rack in a plastic canopy and cooling with dry ice. The flow-cell was housed in a lucite box and also cooled with dry 1ce. The experiments at $2.5^{\circ} \mathrm{C}$ were conducted in a newly constructed refrigerated room which was large enough for the entire apparatus, accessury tyuipilent and several investigators.

All experiments with the $3 \times 16$ Staflo apparatus were conducted in the refrigerated room. Measurements with a temperature recorder (Varian Associates) showed that under the maximum electric field conditions used In this series of experiments, the average and maximum temperature rises near the outlet of the flow-cell were $0.5^{\circ} \mathrm{C}$ and $1.0^{\circ} \mathrm{C}$, respectively.

\section{Observation and Evaluation of Spermatozoa}

The gross electrophoresis pattern was observed by eye using the slit lamp technique 229,263 . Observations of individual spermatozoa and the microscopic steady-state pattern were made with the optical equipment described in Chapter III. After collection, the concentration and degree of motility of the spermatozoa in each collection fraction were determined by counting with a hemocytometer. Vital staining of spermatozoa in each fraction was accomplished with the nigrosin-eosin staining technique descrlbed in Appendix B. 


\section{Results}

\section{1. $1 \times 12$ Flow-Cell Experiments.}

The characteristics of the spermatozoa sample and Staflo operating parameters for the $1 \times 12$ flow-cell upward-electrophoresis experiments are presented in Table V. Except for Experiments 12-21-65-1 \& 2, which were conducted at $2.5^{\circ} \mathrm{C}$ in a refrigerated room, all experiments were conducted with marginal temperature control. In all experiments, spermatozoa were introduced into layer 9 with electrophoresis in an upward direction. Because geometrical considerations prevented the use of the slit lamp, quantitative measurements of the migration pattern were not made.

\section{a. Experiments at $5,6.25$ and $7.5 \mathrm{~V} / \mathrm{cm}$.}

(1) $5 \mathrm{~V} / \mathrm{cm}$. Figure 40 presents the individual results of the first 4 experiments in Table V. As shown in this figure, the total sperm distribution was upward out of layer 9 except in Experiment 4-29-65. In that experiment, the higher percentage of spermatozoa remaining in layer 9 might be attributed efther to "cluster" sedimentation, because the spermatozoa concentration was $30 \times 10^{6} / \mathrm{ml}$, and/or to a high degree of intrinsic motility at $7^{\circ} \mathrm{C}$. The upward distribution was most pronounced in Experiment 5-6-65 which was conducted at $5^{\circ} \mathrm{C}$ with the poorest quality spermatozoa sample. The distribution of spermatozoa in Experiment $5-13-65$ at $5^{\circ} \mathrm{C}$ was similar to that in Experiment 5-6-65. The upward distribution in Experiment 5-20-65 at $7^{\circ} \mathrm{C}$ was less than in the 2 experiments at $5^{\circ} \mathrm{C}$, also suggesting that there is less upward migration at the higher temperature.

W1th regard to the distribution of viable spermatozoa, the least difference among the collection fractions was observed in Experiment 5-6-65 at $5^{\circ} \mathrm{C}$. In Experiment $4-29-65$ at $7^{\circ} \mathrm{C}$, using high quality spermatozoa, there was a higher percentage of viable spermatozoa in fractions 8 and 9 
Table V. Spermatozoa Sample and Staflo Operating Parameters for ix 12 Flow-Cell Upward-Electrophoresis Experiments

\begin{tabular}{|c|c|c|c|c|c|c|c|c|c|}
\hline \multirow[t]{2}{*}{ Experiment No. } & \multicolumn{3}{|c|}{ Spermatozoa Sample } & \multicolumn{6}{|c|}{ Staflo Operating Parameters } \\
\hline & Rabbit (s) & Initlal Quality & $\begin{array}{c}\text { Initial Sperm } \\
\text { Cancentration } \\
\text { (Units of } 10^{6} / \mathrm{ml} \text { ) }\end{array}$ & $\mathrm{pH}$ & $\begin{array}{l}\text { Temp } \\
\left({ }^{\circ} \mathrm{C}\right)\end{array}$ & $\begin{array}{c}\tau \text { ave } \\
(\min )\end{array}$ & $\begin{array}{l}\tau_{\mathrm{E}} \\
(\min )\end{array}$ & $\begin{array}{c}\mathrm{L} \\
(\mathrm{cm})\end{array}$ & $\begin{array}{c}\tilde{E} \\
(\mathrm{~V} / \mathrm{cm})\end{array}$ \\
\hline $4-29-65$ & $0 \&$ Psi & $70 \%$ motile & 30 & 6.5 & 7 & 20 & 13.3 & 20 & 5 \\
\hline $5-6-65$ & $P \&$ Psi & $40 \%$ motile & 23 & 6.5 & 5 & 20.5 & 13.7 & 20 & 5 \\
\hline $5-13-65$ & $P$ & Excellent & 22 & $6 . .5$ & 5 & 20 & 13.3 & 20 & 5 \\
\hline $5-20-65$ & $0 \& \operatorname{Ch} 1$ & Excellent & 22 & 6.5 & 7 & 20 & 13.3 & 20 & 5 \\
\hline $7-28-65$ & $0 \&$ Psi & $80 \%$ motile & 16 & 7.0 & 5 & 20.5 & 13.7 & 20 & 6.25 \\
\hline $8-4-65$ & $0 \& \operatorname{Ch} 1$ & Excellent & 15 & 7.0 & 3.5 & 20.5 & 13.7 & 20 & 6.25 \\
\hline $6-23-65$ & Chi & $80 \%$ motile & 14 & 6.5 & 6 & 17 & 11.3 & 20 & 7.5 \\
\hline $7-14-65$ & $P$ & $60 \%$ motilie & 13 & 6.5 & 4 & 17 & 11.3 & 20 & 7.5 \\
\hline $7-21-65$ & Psi & Very poor & 13 & 6.5 & 4 & 17. & 11.3 & 20 & 7.5 \\
\hline $7-23-65$ & $\mathbf{P}$ & $85 \%$ motile & 13 & 6.5 & 5 & 17 & 11.3 & 20 & 7.5 \\
\hline $12-21-65-1$ & Ch1 & Very poor & 11 & 7.0 & 2.5 & 20 & 13.3 & 20 & 6.25 \\
\hline $\begin{array}{c}12-2.1-65-2 \\
:\end{array}$ & $\therefore \operatorname{Ch} 1$ & Very poor & 9 & 7.0 & 2.5 & 18 & 12.0 & 20 & 7.5 \\
\hline
\end{tabular}




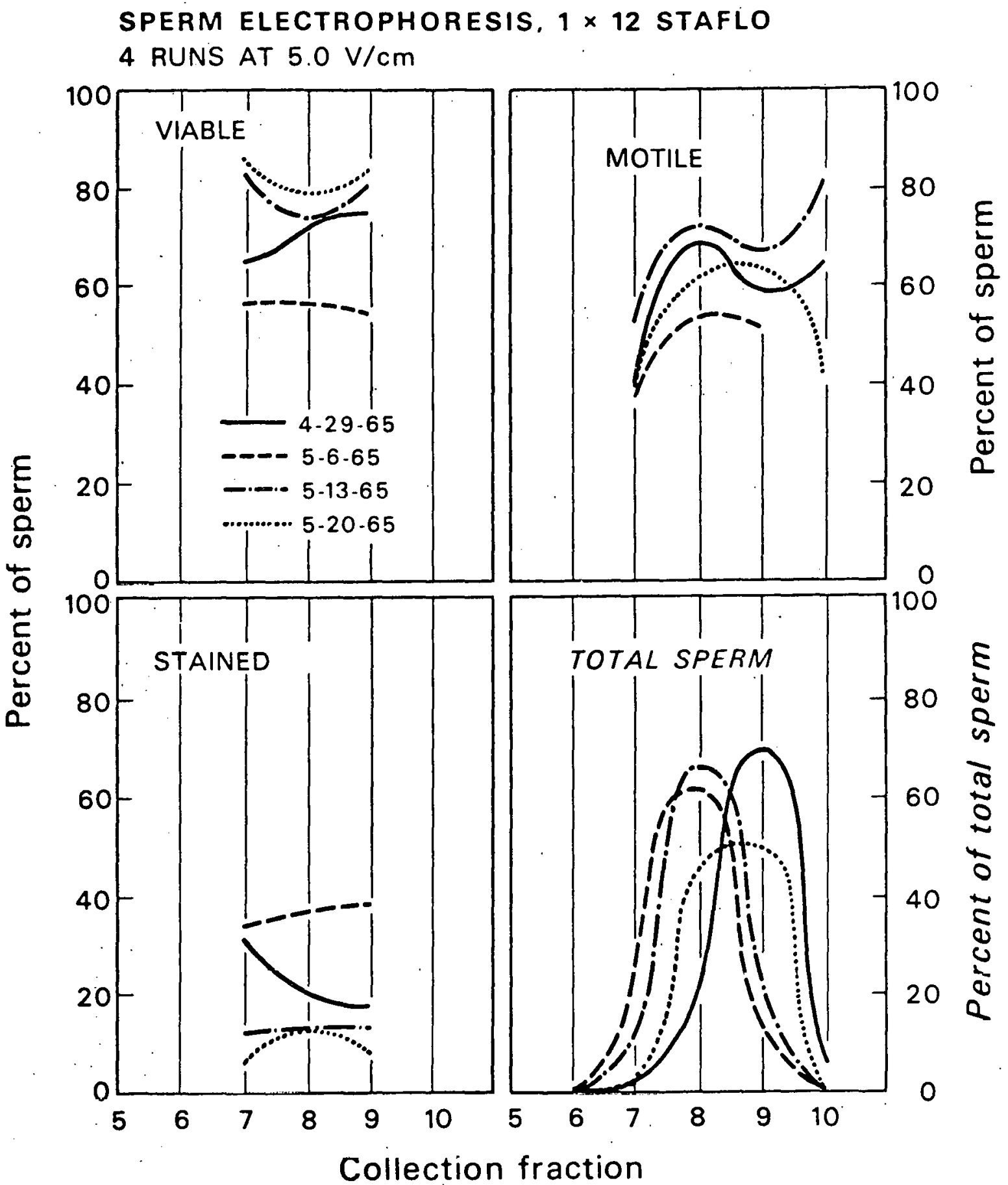

DBL 688-5379

F1g. 40. Individual results of four upward-electrophoresis experiments with rabbit spermatozoa at $5 \mathrm{~V} / \mathrm{cm} ; 1 \times 12$ staflo apparatus. 
compared to fraction 7 . In the other 2 experiments with samples of comparable initial quality, the distribution of viable spermatozoa was similar despite the difference in temperature $\left(5\right.$ vs. $7^{\circ} \mathrm{C}$ ). The distribution of stained spermatozoa was essentially a mirror image of that of the viable spermatozoa.

Except for a lower percentage of motile spermatozoa in fraction 7 compared to fractions 8 and 9 , Figure 40 shows no definite pattern in the distribution of motile spermatozoa among the collection fractions. In 2 experiments, the percentages of motile spermatozoa in fractions 8 and 9 are approximately the same, while in the other 2 experiments, there is a higher percentage of motile spermatozoa in fraction 8 . In Experiment 4-29-65, approximately $5 \%$ of the total number of spermatozoa migrated downward into layer 10 against the electrophoretic force, suggesting a strong intrinsic motility component. This variability in the distribution of motile spermatozoa and the influence of intrinsic motility on the distribution of other categories of spermatozoa are considered in detäil in the discussion.

In Experiment 5-6-65, a few spermatozoa in the flow-cell were oriented horfizontally, while over $95 \%$ were oriented with head down and tail upward in the direction of the positive electrude. A high percentage of the spermatozoa had vigorous tall-wagging, but the significance of this degree of motilfty in terms of migration against the electric field could not be assessed with the microscope. At the conclusion of the experiment, the degree of motility of the spermatozoa in the collection fractions was relatively poor. Thus, the degree of motility observed in the collection fractions at the end of the experiment does not correlate well with the apparent interaction between the intrinsic motility and electrophoretic 
force in the flow-cell. This lllustrates the need for microscopic observations in the flow-chamber during experiments.

(2) $6.25 \mathrm{~V} / \mathrm{cm}$. Figure 41 presents the results of 2 experiments at 6.25 $\mathrm{V} / \mathrm{cm}$. The distribution of the various categories of spermatozoa is similar in the 2 experiments despite the difference in temperatures $\left(3.5^{\circ} \mathrm{C}\right.$ and $\left.5.0^{\circ} \mathrm{C}\right)$. Despite the lower percentage of viable spermatozoa in fraction 9 compared to 8 , in both experiments, there is an unequivocal increase in the percentage of motile spermatozoa going from fraction 7 through 10 .

In Experiment 7-28-65, observations with the microscope showed that in layer 7, all spermatozoa were oriented vertically with the tail toward the top (positive) electrode and that some spermatozoa had tail-wagging. In layer 9, most spermatozoa were oriented vertically, while a few were in a horizontal position. Nearly all spermatozoa in layer 9 showed some degree of motility at $5^{\circ} \mathrm{C}$. In both experiments, seminal plasma was distributed primarily in layers 7 and 8 with only large "blobs of junk" in 1ayer 9 .

(3) $7.5 \mathrm{~V} / \mathrm{cm}$. Figure 42 presents the individual results of 4 experiments at $7.5 \mathrm{~V} / \mathrm{cm}$. There is a slightly greater upward total sperm distribution In Experiments 7-14-65 and 7-21-65, both of which were conducted at $4^{\circ} \mathrm{C}$ with poorer quality spermatozoa samples than in the other 2 experiments. The least migration was observed in Experiment $6-23-65$ conducted at $6^{\circ} \mathrm{C}$ with the spermatozoa sample having the highest percentages of viable and motile spermatozoa at the conclusion of the experiment.

There is little reproducibility in the distribution of viable and motile spermatozoa among the collection fractions. Desplte the low cell concentrations, viable spermatozoa were observed in fractions 6 and motile spermatozoa were observed in fractions 6 and 5 . There is some similarity 


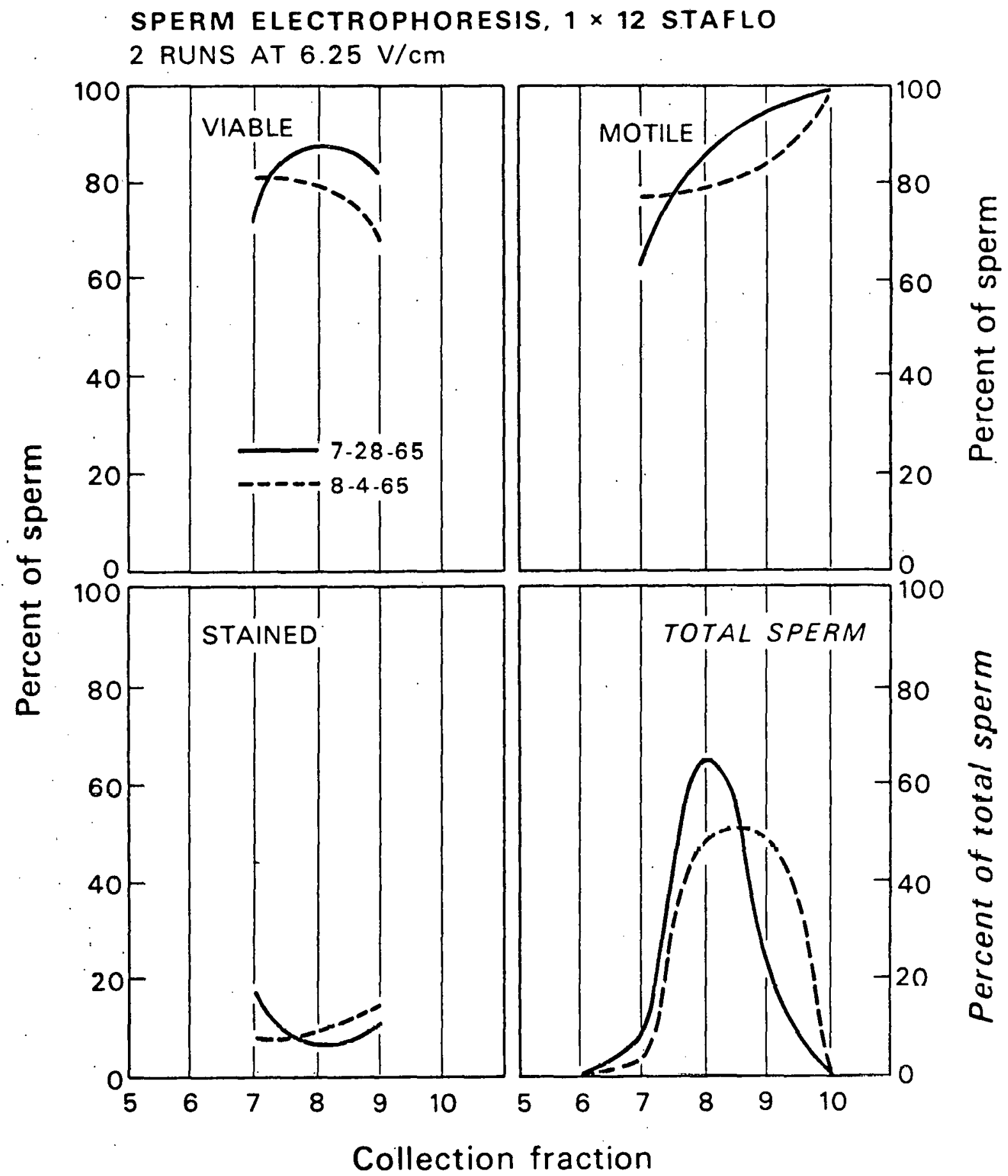

DBL 688-5378

Fig. 41. Individual results of two upward-electrophoresis experiments with rabbit spermatozoa at $6.25 \mathrm{~V} / \mathrm{cm} ; 1 \times 12$ Staflo apparatus. 


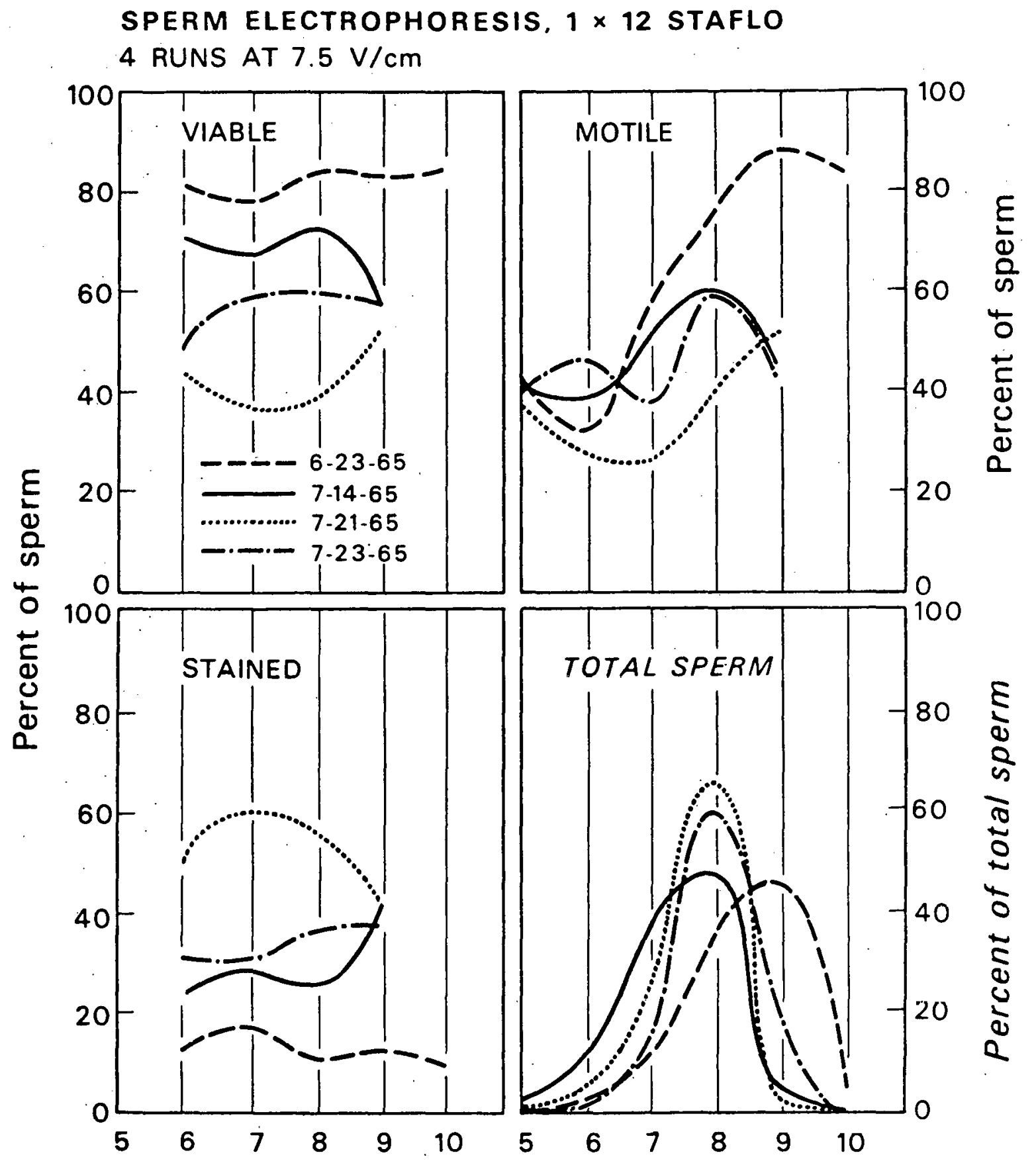

Collection fraction

DBL 688-5380

Fig. 42. Individual results of four upward-electrophoresis experiments with rabbit spermatozoa at $7.5 \mathrm{~V} / \mathrm{cm} ; 1 \times 12$ Staflo apparatus. 
between the distribution of viable and motile spermatozoa in the respective experiments. The distribution of stained spermatozoa mirrors that of viable spermatozoa.

(4) Experiments at 6.25 and $7.5 \mathrm{~V} / \mathrm{cm}$ and a Temperature of $2.5^{\circ} \mathrm{C}$. After construction of a refrigerated room, upward electrophoresis experiments were conducted at 6.25 and $7.5 \mathrm{~V} / \mathrm{cm}$ at a temperature of $2.5^{\circ} \mathrm{C}$. In both experiments, the initial quality of the spermatozoa samples was poorer than in previous experiments and the degree of motility observed in the collection fractions at the termination of the experiments was extremely poor. The results are presented in Figure 43 and show a slightly greater upward total migration of spermatozoa at an electric field strength of $7.5 \mathrm{~V} / \mathrm{cm}$.

As shown in Figure 43, there is a slight increase in the percentage of viable spermatozoa going from collection fraction 7 to 9 , while the distribution of stained spermatozoa is a mirror image thereof. In both experiments, there is a higher percentage of motile spermatozoa in fraction $/$ than in either fraction 8 or 9 . This distribution of motile spermatozoa is the opposite of that observed in the series of experiments at higher temperatures at both electric fleld strengths as shown in Figures 41 and 42 .

In the experiment at $6.25 \mathrm{~V} / \mathrm{cm}$, essent1ally $100 \%$ of the spermatozoa near the wall in the single cell suspension were oriented vertically with their tails towards the top (positive) electrode. The spermatozoa showed a slight degree of twlsting at the lowest levels of the migration pattern. Spermatozoa in the collection fractions inftially had a fair degree of motility which was observed to decrease rapidly over a period of 30 minutes. In the experiment at $7.5 \mathrm{~V} / \mathrm{cm}$, swaying spermatozoa were observed in the lower part of the migration pattern. Before the experiment started, only 12 of 900 spermatozoa showed feeble tail-wagging in a hemocytometer at $2{ }^{\circ} \mathrm{C}$. 


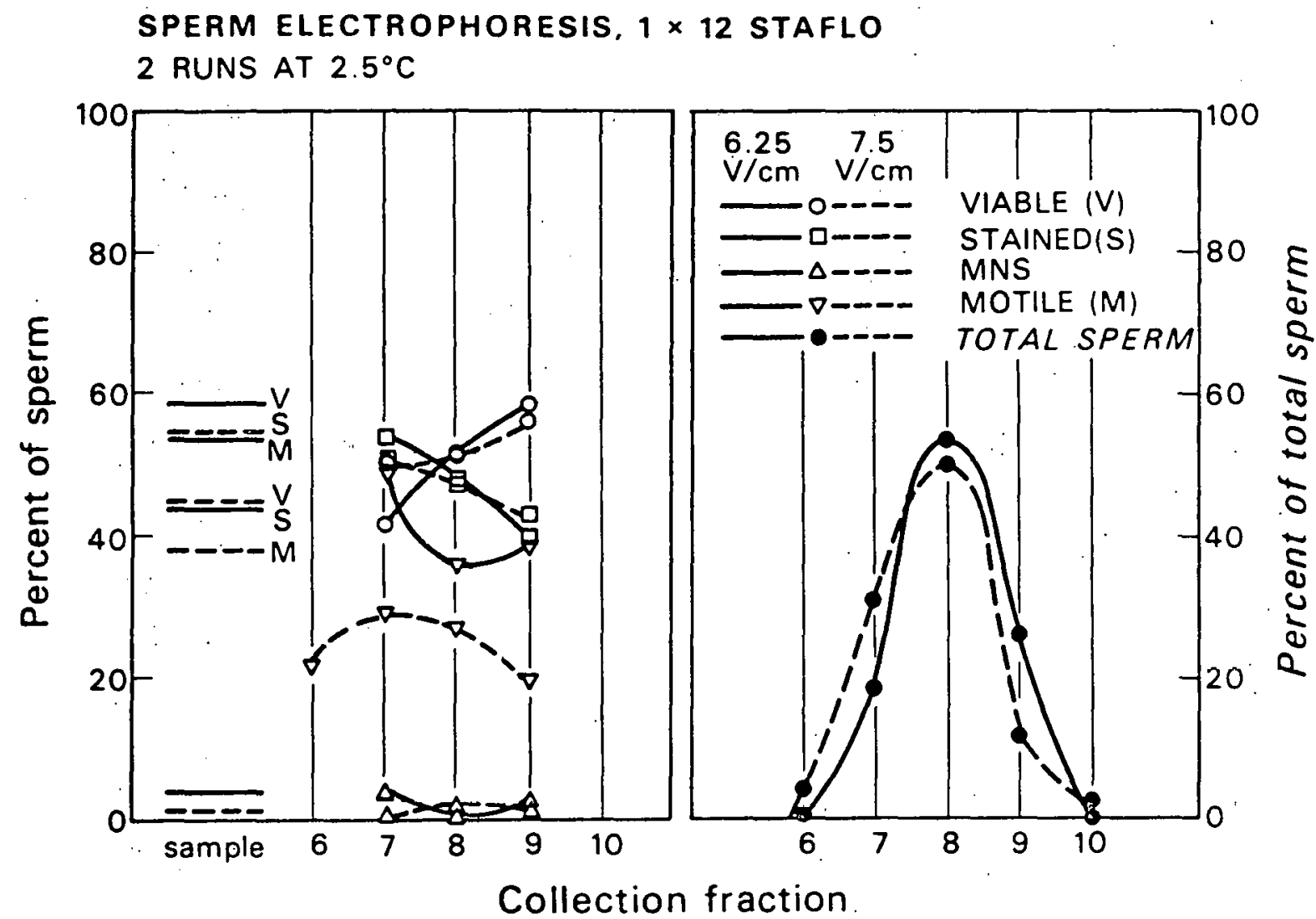

DBL 688-5384

Fig. 43. Comparison of results of upward-electrophoresis experiments with rabbit spermatozoa at 6.25 and $7.5 \mathrm{~V} / \mathrm{cm}$, at $2.5^{\circ} \mathrm{C} ; 1 \times 12$ Staflo apparatus. 
The swaying motion is attributed to the slightly higher temperature in the flow-chamber due to the electric field.

b. Comparison of Different-Fleld Strength Experiments. Figures 44,45 and 46 present average values for the total distribution, and the distribution of several categorles of spermatozoa among the collection fractions for the upward-electrophoresis experiments at $5,6.25$ and $7.5 \mathrm{~V} / \mathrm{cm}$ shown In Figures 40, 41 and 42, respectively. Compared to the experiments at $5 \mathrm{~V} / \mathrm{cm}$, there is a slight increase in the upward distribution of spermatozoa among the collection fractions at $6.25 \mathrm{~V} / \mathrm{cm}$ and a definite upward shift at $7.5 \mathrm{~V} / \mathrm{cm}$. There is little difference among the collection fractions with regard to the distribution of viable spermatozoa at 5 and $7.5 \mathrm{~V} / \mathrm{cm}$, while at $6.25 \mathrm{~V} / \mathrm{cm}$, there is a higher percentage of viable spermatozoa in fraction 8 than in 7 or 9 . In Figures 44-46, the percentage of motility in fraction 7 is lower than in fractions 8 and 9 . The differential distribution of motile spermatozoa is most pronounced in the experiments at $6.25 \mathrm{~V} / \mathrm{cm}$ where a much higher percentage of motility was found in fraction 9 compared to 8 . In all 3 figures, there are marked differences in the distribution of viable and motile spermatozoa. The distribution of morphologically normal but stained (MNS) spermatozoa is essentially the same In all collection fractions at 5 and $7.5 \mathrm{~V} / \mathrm{cm}$ but is slightly higher in fractions 7 and 9 at $6.25 \mathrm{~V} / \mathrm{cm}$, thereby accentuating the differential distribution of viable spermatozoa in that group of experiments.

In many of the early electrophorests experiments, the quality of spermatozoa at the end of the experiment appeared to be adequate for artiflclal insemination. However, only one insemination of dozens resulted In offspring. In Experiment 7-28-65, the migration pattern for which is shown in Figure 41, insemination of spermatozoa from fraction 8 gave 4 male offopring from the same female. Insemination of spermatozoa from 
SPERM ELECTROPHORESIS, $1 \times 12$ STAFLO AVERAGE OF 4 RUNS AT $5.0 \mathrm{~V} / \mathrm{cm}$ $20.5 \mathrm{~min}$ TRANSIT

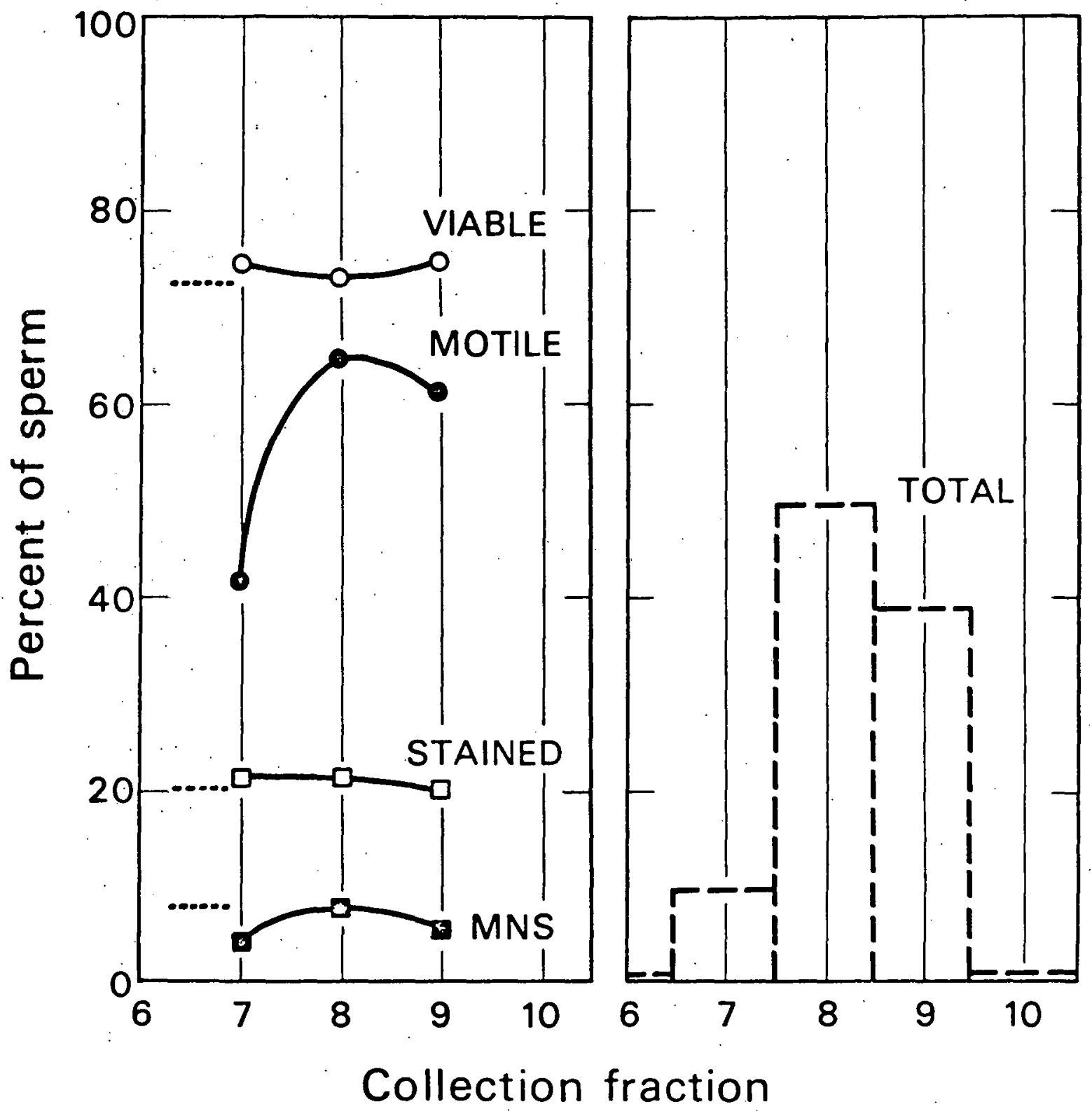

DBL 688-5410

Fig. 44. Average results for four upward-electrophoresis experiments with rabbit spermatozoa at $5 \mathrm{~V} / \mathrm{cm} ; 1 \times 12$ Staflo appratus. 


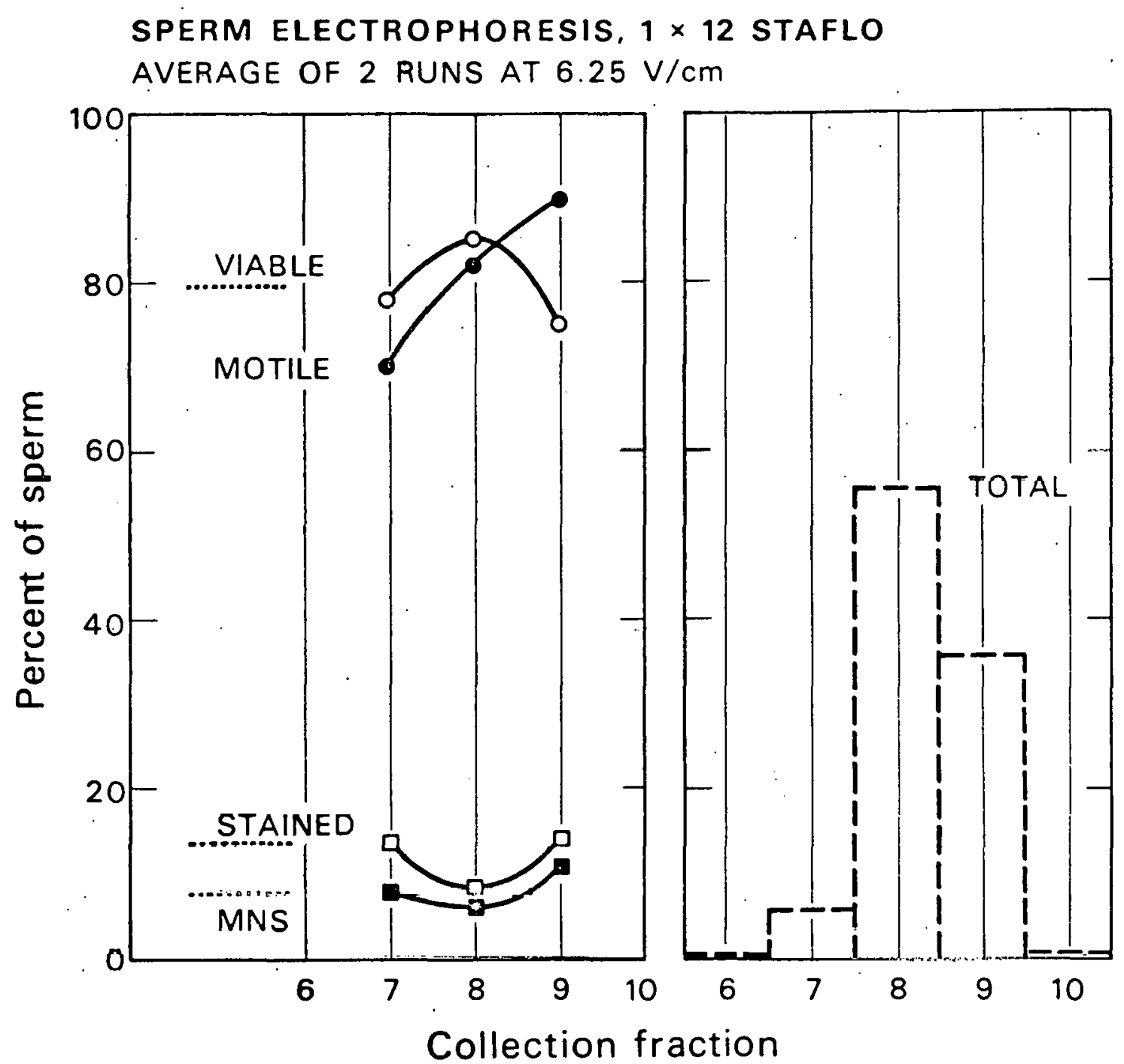

DBL 688-5409

Fig. 45. Average results for two upward-electrophoresis experiments with rabbit opcrmatozoa at $6.25 \mathrm{~V} / \mathrm{cm} ; 1$ x 12 Staflo apparatus. 


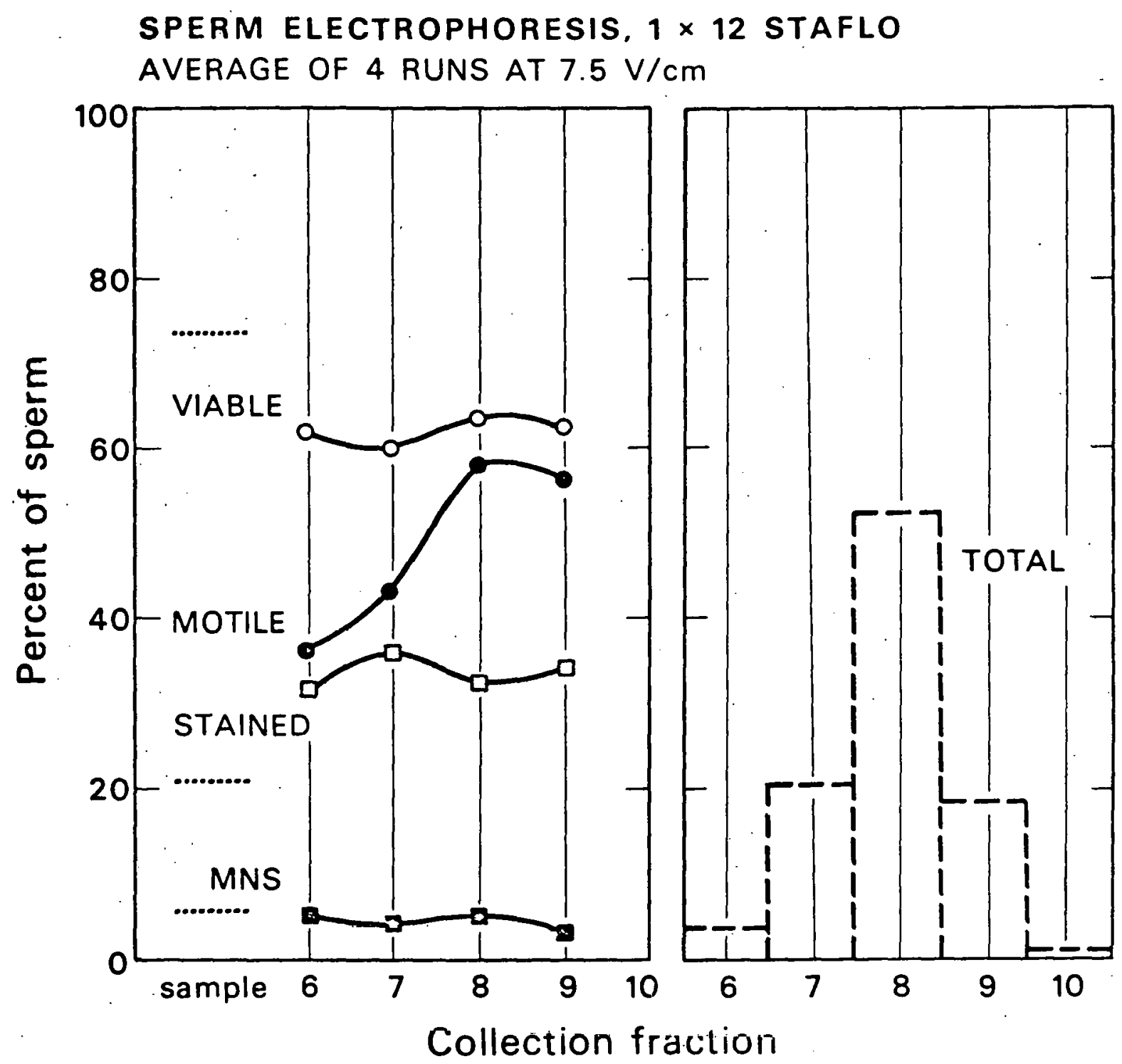

DBL 688-5408

Fig. 46. Average results for four upward-electrophoresis experiments with rabbit spermatozoa at $7.5 \nabla / \mathrm{cm} ; 1 \times 12$ Staflo apparatus. 
fraction 9 gaye no offspring.

2. $3 \times 16$ Flow-Ce11 Experiments.

As mentioned in Chapter III, greater resolution in the fractionation of migration patterns is achieved with the $3 \times 16$ flow-cell. This section reports the results of several preliminary experiments using the $3 \times 16$ flow-cell and new electrophoresis media providing greater pH stability and better preservation of the vital functions of spermatozoa. The availability of a refrigerated room facilitated the use of the slit lamp technique for macroscopic observations and the Staflo microscope for the observation of individual spermatozoa in the flow-chamber.

In addition to the preliminary evaluation of the $3 \times 16$ flow-cell, results are presented below for: (1) upward electrophoresis of rabbit spermatozoa at $8 \mathrm{~V} / \mathrm{cm}$ with a dextran density gradient in a glycine-phosphate buffer, (2) downward electrophoresis of rabbit and fow1 spermatozoa with a dextran density gradient in the glycine-phosphate buffer, and (3) upward electrophoresis of rabbit and fowl spermatozoa with a sucrose density gradient in the glycine-citrate buffer.

a. Preliminary Flow-Cell Evaluation. Figure 47 shows the average distribution of the total number and different categories of spermatozoa among the centerline collection fractions in 2 experiments at $8 \mathrm{~V} / \mathrm{cm}, 3.5^{\circ} \mathrm{C}, \mathrm{pH} 7$, an average transit time of 21.5 minutes, and an average concentration of $25 \times 10^{6} / \mathrm{ml}$. The density gradient, prepared in glycine-phosphate-buffer, consisted of dextran (MW, 73,000) in weight/volume percent in the staflo layers as follows: No. $1,0 \%$; No. $2,0.1 \%$; No. $3,0.2 \%$; No. $4,0.3 \%$; No. 5 , $0.4 \%$; No. $6,0.5 \%$; No. $7,0.6 \%$; No. $8,0.7 \%$; No. $9,0.8 \%$; No. $10,0.9 \%$; No. $11,1.25 \%$; No. $12,1.5 \%$; Nos. 13 and $14,1.75 \%$ and Nos. 15 and 16, $2.0 \%$. The top and bottom electrode compartments contained the same medium and percent dextran as layers 1 and 16 , respectively. 


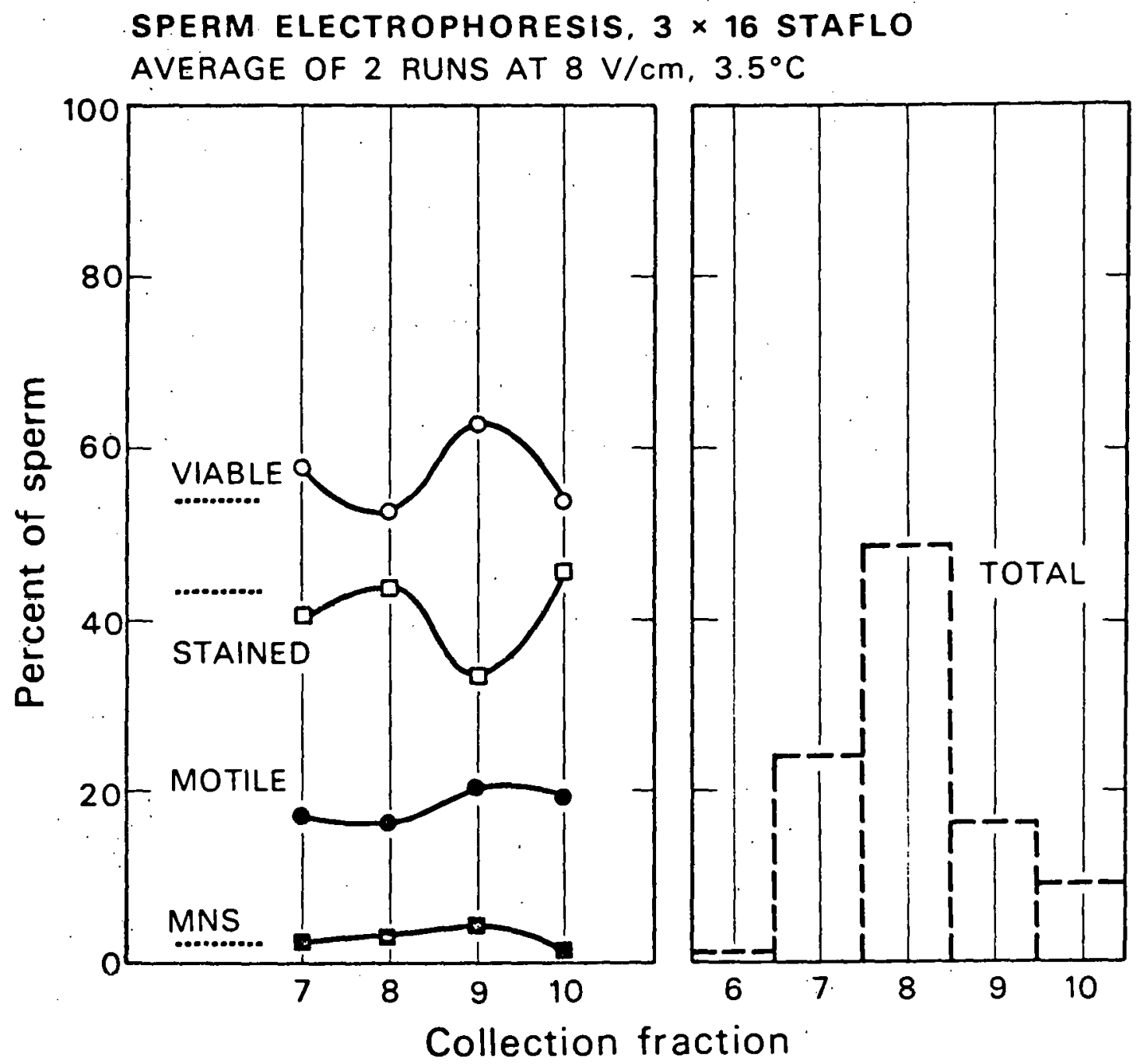

DBL 688-5407

Fig. 47. Average results for two upward-electrophoresis experiments with rabbit spermatozoa at $8 \mathrm{~V} / \mathrm{cm} ; 3 \times 16$ Staflo apparatus. 
In both of these experiments, the spermatozoa were introduced into layer 10 with electrophoresis in an upward direction causing approximately $75 \%$ of the spermatozoa to migrate from layer 10 through layer 9 into layers 8,7 and 6 . The highest percentage of viable spermatozoa was found in fraction 9, one layer below that having the highest spermatozoa concentration. This is the direction in which motile spermatozoa would have been expected to swim against the electric field. However, the initial degree of motility of the spermatozoa was relatively poor, and the difference in motility among the spermatozoa in the collection fractions was not significant. The distribution of stained spermatozoa is a mirror image of the distribution of viable spermatozoa, while that of MNS spermatozoa is essentially constant from fractions $7-10$.

In both experiments, all spermatozoa within the region of the electric field were orlented vertically with the tall towards the top (positive) electrode. Table.VI shows the differential distribution of mature spermatozoa, spermatozoa with cytoplasmic droplets, heads, and tails among the centerline collection fractions in one of the two experiments. The average migration appears to be greatest for tails and least for heads, and to be slightly greater for mature spermatozoa than for those with cytoplasmic droplets. The distribution of the spermatozoa with the cytoplasmic droplets appears to be slightly more uniform than that of the mature spermatozoa.

The vertical and horizontal distributions of spermatozoa in the $3 \times 16$ flow-chamber are given in Table VII. Table VII A gives the spermatozoa concentrations in all 3 horlzontal channels of layers 7-10. As shown in Table VII B, the horizontal distribution of spermatozoa in the layers is somewhat symmetrical. $48.4 \%$ of the spermatozoa exited the flow-chamber in the centerline. This is slightly lower than the prediction by Tippetts 
Table VI. Differential Distribution of Mature Sperm, Sperm with Cytoplasmic Droplets (CPD), and Sperm Heads and Tails in Upward-Electrophoresis Experiment $2-28-66,3.5^{\circ} \mathrm{C}$.

\begin{tabular}{|c|c|c|c|c|c|c|c|c|}
\hline \multirow[t]{2}{*}{$\begin{array}{l}\text { Collection } \\
\text { Fraction. }\end{array}$} & \multicolumn{2}{|c|}{$\begin{array}{c}\text { Mature } \\
\text { Spermatozoa }\end{array}$} & \multirow{2}{*}{$\begin{array}{l}\text { Sperm } \\
\text { No. }\end{array}$} & \multirow{2}{*}{ with } & \multirow{2}{*}{$\begin{array}{l}\text { Sperm } \\
\text { No. }{ }^{a}\end{array}$} & \multirow{2}{*}{$\begin{array}{c}\text { Heads } \\
\%\end{array}$} & \multirow{2}{*}{$\begin{array}{l}\text { Sperm } \\
\text { No. }^{a}\end{array}$} & \multirow{2}{*}{$\begin{array}{c}\text { Tails } \\
\%\end{array}$} \\
\hline & No. ${ }^{a}$ & $\%$ & & & & & & \\
\hline 6 & 0.7 & 0.03 & 0 & 0 & 0 & 0 & 5.0 & 5.7 \\
\hline 7 & 396 & 17.0 & 26.7 & 18.9 & 7.0 & 13.5 & 31.5 & 35.9 \\
\hline 8 & 1160 & 49.9 & 58.7 & 41.5 & 18.3 & 35.3 & 33.4 & 38.0 \\
\hline 9 & 475 & 20.4 & 32.1 & 22.7 & 16.5 & 31.9 & 12.5 & 14.2 \\
\hline $10^{\mathrm{b}}$ & 294 & 12.6 & 23.8 & 16.9 & 10.0 & 19.3 & 5.5 & 6.2 \\
\hline 11 & 0 & & 0 & & 0 & & 0 & \\
\hline
\end{tabular}

a. in units of $10^{4}$ sperm $/ \mathrm{ml}$; b. sample inlet layer 
Table VII. Two-Dimensional Distribution of Spermatozoa in

Upward-Electrophoresis Experiment 2-28-66 in the $3 \times 16$

Staflo Flow-Ce11, $3.5^{\circ} \mathrm{C}$.

Staflo Layer

A. Sperm Concentration

(units of $10^{6} / \mathrm{m} 1$ )

6
7
8
9
10

0
1.24
1.77
1.24
0.60
0

0
1.78
Collection Fraction

Lenterline Kighe

lotal

B. Percent of Sperm

in Rows (Horizontal)

7
8
9
10

Average

C. Percent of Sperm

in Columns (Vertical)

\begin{tabular}{l}
7 \\
8 \\
9 \\
10 \\
\hline
\end{tabular}

Total
27.5

20.3

25.8

24.0

24.4

25.6

36.5

25.6

$\underline{12.3}$

100
5.21

7.07

1.32

0

39.4

59.4

42.1

52.8

48.4

27.2

33.1

20.3

32.1

23.2

1.50

1.77

1.34

0.58

0
0.003

4.52

8.75

4.80

2.50

0 
that $62 \%$ of particles should exit from the central channel of each layer ${ }^{263}$. The vertical distribution given in Table VII $C$ shows less variation on the 2 sides of the flow-chamber than in the centerline. A slightly higher percentage of spermatozoa is found on the sides of layer 7 than in the centerline of that layer. This would be expected from the parabolic migration pattern giving a slower longttudinal flow of the medium along the sides of the flow-chamber. 229,263

The $\mathrm{pH}$ stability of the glycine-phosphate buffer under the conditions of these $3 \times 16$ flow-cell upward-electrophoresis experiments was excellent. With an electric fleld strength of $8 \mathrm{~V} / \mathrm{cm}$, an average transit time of 21 minutes, and at $3.5^{\circ} \mathrm{C}$, the maximum $\mathrm{pH}$ change in the layers of interest (4-11) was $0.1 \mathrm{pH}$ units or less.

\section{b. Downward-Electrophoresis: Rabbit and Fow1 Spermatozoa}

(1) Rabbit Spermatozoa: Two downward-electrophorests experiments using the glycine-phosphate buffer were conducted with rabbit spermatozoa introduced Into layer 6 over 1\% dextran, with increments of $0.2 \%$ dextran in successively lower layers constituting the density gradient. The operating conditions were an electric field strength of $10 \mathrm{~V} / \mathrm{cm}$ using the last $25 \mathrm{~cm}$ of the flow-chamber, a spermatozoa concentration of $15 \times 10^{6} / \mathrm{ml}$, a chamber temperature of $6^{\circ} \mathrm{C}$ and average transit times of 22 and 34 minutes. As shown In Table VIII, the longer transit time resulted in both a greater spread of the migration pattern and a greater downward distribution of the total number of spermatozoa. In both experiments, there is a suggestion of a normal distribution of electrophoretic mobilities, the averages of which are calculated to be $0.62 \times 10^{-4}$ and $0.60 \times 10^{-4}(\mathrm{~cm} / \mathrm{sec}) /(\mathrm{V} / \mathrm{cm})$ for the 22 and 34 minute transit times, respectively. When the electrophoretic mobilities are corrected, as suggested by Bier ${ }^{38}$, to that expected at $3^{\circ} \mathrm{C}$ 
Table VIII. Distribution of Rabbit Spermatozoa in Centerline Collection Fractions with Downward-Electrophoresis In a Dextran Density Gradient, Average Transit Times of 22 and 34 Minutes, $10 \mathrm{~V} / \mathrm{cm} ; 6^{\circ} \mathrm{C}$.

\begin{tabular}{ccc}
\hline $\begin{array}{c}\text { Collection } \\
\text { Fraction }\end{array}$ & \multicolumn{2}{c}{$\begin{array}{c}\text { Percent of Sperm Collected } \\
\text { T ave }=22 \text { minutes }\end{array}$ ave $=34$ minutes } \\
\hline 5 & 0 & 0 \\
6 & 3.2 & 0.3 \\
7 & 3.1 & 0.3 \\
8 & 6.4 & 1.6 \\
9 & 59.8 & 19.5 \\
10 & 27.4 & 32.2 \\
11 & 0.1 & 33.3 \\
12 & 0 & 12.8 \\
13 & 0 & 0.03 \\
\hline
\end{tabular}


in this glycine-phosphate buffer, with a sucrose concentration of $1 \%$, one gets mobility values of $0.82 \times 10^{-4}$ and $0.79 \times 10^{-4}(\mathrm{~cm} / \mathrm{sec}) /(\mathrm{V} / \mathrm{cm})$, respectively. Because the sedimentation rate of rabbit spermatozoa in a medium containing $1 \%$ or greater dextran is very low, the contribution of sedimentation to these values is believed to be very slight.

Observations with the Staflo microscope showed almost all spermatozoa to be orfented vertfcally with the tail pointing downward in the direction of the bottom (positive) electrode. Because the initial quality of the spermatozoa sample was very poor, no motility was observed in the flowchamber. After warming to room temperature, the spermatozoa in the collection fractions showed less than $10 \%$ tail-wagging. The migration profile in both experiments was parabolic and the mild degree of "cluster sedimention"observed at both transit times is not believed to have affected the distribution of spermatozoa significantly.

In another experiment using the same density gradient, a severe degree of convection was observed ${ }^{263}$. At $15 \mathrm{~cm}$ from the inlet, the migration profile was a neat parabola. At $20 \mathrm{~cm}$, migration of the spermatozoa downward along both sides of the flow-chamber and then into the centerline formed a tube-like profile. At $25 \mathrm{~cm}$, the profile again was essentially parabolic w1th marked central sharpening and it remained that way over the last $5 \mathrm{~cm}$ of the flow-chamber. These changes in the migration pattern illustrate the Importance of examining the migration profile at frequent intervals throughout the chamber length by the slit lamp technique 22,263 .

(2) Fowl Spermatozoa. A downward-electrophoresis experiment using the glycine-phosphate buffer was conducted with fowl spermatozoa introduced into layer 5 over $2 \%$ dextran with Increments of $0.2 \%$ dextran in successively lower layers constituting the density gradient. W1th an electric field 
strength of $12 \mathrm{~V} / \mathrm{cm}$, an average transit time of 21 minutes and a flowchamber temperature of $6.8^{\circ} \mathrm{C}$, the spermatozoa were distributed over layers 9-12 as follows: No. $9,4.3 \%$; No. $10,24.6 \%$; No. $11,62.1 \%$ and No. 12 , 8.7\%. Approximately $50 \%$ of the spermatozoa in each collection fraction were mot1le with Grade II motility in fraction 11 and Grade $I$ in the other 3 fractions. At this time, the Inftial sample had Grade III motility.

Under the conditions given above, the average electrophoretic mobility of fowl spermatozoa was calculated to be $1.14 \times 10^{-4}(\mathrm{~cm} / \mathrm{sec}) /(\mathrm{V} / \mathrm{cm})$ at $6.8^{\circ} \mathrm{C}$. When a correction is made for viscosity, the average electrophoretic mobility is estimated to be $1.68 \times 10^{-4}(\mathrm{~cm} / \mathrm{sec}) /(\mathrm{V} / \mathrm{cm})$ in this glycinephosphate buffer with a sucrose concentration of $1 \%$ at $3^{\circ} \mathrm{C}$.

\section{c. Upward-Electrophoresis: Rabbit and Fowl Spermatozoa. In one pair}

of separate experiments using the glycine-citrate buffer, rabbit and fowl spermatozoa were introduced into layer 12 in $3.3 \%$ sucrose and were electrophoresed upward through a density gradient formed by decrements of $0.3 \%$ eucroes por layer. Oporating conditions included an clectric ficld gtrcngth of $12 \mathrm{~V} / \mathrm{cm}$ over the last $20 \mathrm{~cm}$ of the flow-chamber, an average transit time of approximately 21 minutes, a temperature of $6^{\circ} \mathrm{C}$, and spermatozoa concentrations of $20 \times 10^{6} / \mathrm{m} 1$ and $30 \times 10^{6} / \mathrm{ml}$ for rabbit and fowl spermatozoa, respectively. Under these conditions, the electrophoretic mobilities for rabbit and fowl spermatozoa were $0.99 \times 10^{-4}$ and $1.75 \times 10^{-4}(\mathrm{~cm} / \mathrm{sec}) /(\mathrm{V} / \mathrm{cm})$, respectively. When corrected for viscosity, the electrophoretic mobilities at $3^{\circ} \mathrm{C}$ in the same primary medium with only $1 \%$ sucrose were $0.95 \times 10^{-4}$ and $1.68 \times 10^{-4}(\mathrm{~cm} / \mathrm{sec}) /(\mathrm{V} / \mathrm{cm})$ for rabblt and fowl spermatozoa, respectively.

With the microscope, it appeared that all spermatozoa of both species were oriented vertically with the tails pointing toward the top (positive) electrode. Most fowl spermatozoa had a significant degree of motility in 
the flow-chamber and, therefore, the motility may have given a decrease in the net migration distance observed for those spermatozoa. The initial quality of the rabbit spermatozoa was very poor and the effect of intrinsic motility on the migration of that species is believed to be negligible in this experiment.

In another pafr of separate experfments, using the glycine-citrate buffer, rabbit and fowl spermatozoa were introduced into layer 12 in $3.8 \%$ sucrose and electrophoresed upward through a density gradient with decrements of $0.5 \%$ sucrose per layer. Operating conditions included an electric field strength of $12 \mathrm{~V} / \mathrm{cm}$ over the last $20 \mathrm{~cm}$ of the flow-chamber, an average transit time of 19.3 minutes, and $\mathrm{pH}$ 6.94. Under these conditions, the average electrophoretic mobility of the rabbit spermatozoa was 0.78 $x 10^{-4}(\mathrm{~cm} / \mathrm{sec}) /(\mathrm{V} / \mathrm{cm})$ at $5.4^{\circ} \mathrm{C}$ and that of the fowl spermatozoa was 1.58 $x 10^{-4}(\mathrm{~cm} / \mathrm{sec}) /(\mathrm{V} / \mathrm{cm})$ at $4.3^{\circ} \mathrm{C}$. When corrections for viscosity were made, the estimated electrophoretic mobilities at $3^{\circ} \mathrm{C}$ in the same medium with $1 \%$ sucrose were $0.76 \times 10^{-4}$ and $1.58 \times 10^{-4}(\mathrm{~cm} / \mathrm{sec}) /(\mathrm{V} / \mathrm{cm})$ for the rabbit and fowl spermatozoa, respectively.

Table IX shows the distribution of tail-wagging and capped rabbit spermatozoa among the collection fractions in this experiment. Although there were no significant differences among the percentages of capped spermatozoa in the fractions, a much higher percentage of tail-wagging was observed among the spermatozoa in fraction 9 than in 10 or 11 . The infitial sample had been stored in egg yolk and had a high percentage of tall-wagging, but extremely poor forward motion, even at room temperature. These results suggest a greater electrophoretic mobility for tail-wagging rabblt spermatozoa than for viable spermatozoa without that property. Approximately $90 \%$ of the fowl spermatozoa in this experiment exited 
Table IX. Distribution of Tail-Wagging and Capped Rabbit Spermatozoa in Upward-Electrophoresis Experiment $5-14-67 ; 5.4^{\circ} \mathrm{C}$.

\begin{tabular}{|c|c|c|c|}
\hline $\begin{array}{l}\text { Collection } \\
\text { Eractinn }\end{array}$ & $\begin{array}{c}\text { Total } \\
\text { Sperm } \\
\text { Collected } \\
(\%)\end{array}$ & $\begin{array}{c}\text { Tail-Wagging } \\
(\%)\end{array}$ & $\begin{array}{c}\text { Capped } \\
(\%)\end{array}$ \\
\hline 9 & 24.5 & 50 & 90 \\
\hline 10 & 59.0 & 40 & y5 \\
\hline 11 & 16.5 & 32 & 94 \\
\hline $12^{\mathrm{a}}$ & 0 & - & - \\
\hline
\end{tabular}

a. Sample in 1et. layer 
the flow-chamber in layer 7. The spermatozoa in this layer had a very high percentage and high degree of motility, whereas approximately $99 \%$ of the spermatozoa in fraction 6 (concentration $10 \times 10^{6} / \mathrm{ml}$ ) were immotile. At $4.3^{\circ} \mathrm{C}$, a "sea of motion" was observed in the flow-chamber with the microscope. Thus, the separation of motile and immotile fowl spermatozoa is belleved to be due primarily to intrinsic motility. This may explain the slight difference in the average electrophoretic mobility observed in the 2 upward-electrophoresis experiments with fowl spermatozoa. The average electrophoretic mobility of the 1mmotile fowl spermatozoa which exited the flow-chamber in layer 6 is estimated to be $1.67 \times 10^{-4}(\mathrm{~cm} / \mathrm{sec}) /$ $(\mathrm{V} / \mathrm{cm})$ compared to an average of $1.58 \times 10^{-4}(\mathrm{~cm} / \mathrm{sec}) /(\mathrm{V} / \mathrm{cm})$ for the sample as a whole at $3^{\circ} \mathrm{C}$ in a $1 \%$ sucrose solution.

\section{Discussion}

1. Factors Influencing the Distribution of Spermatozoa in the F1ow-Chamber

The most important lesson to be gained from the experiments reported in this chapter is an understanding of the factors influencing the distribution of spermatozoa in the flow-chamber. The lack of reproducibility among. the $1 \times 12$ flow-cell experiments introduces many of the problems related to that apparatus and to work with spermatozoa. For convenience, this section of the discussion is divided into sections concerned with the Staflo apparatus and the intrinsic motility of spermatozoa.

a. Staflo Apparatus. The characteristics of the $3 \times 16$ flow-cell, which underwent its initial evaluation in this series of experiments, were discussed in the Results section. The following remarks pertaining to density gradient stability, $\mathrm{pH}$ stability and the magnitude of the electric field pertain equally well to both the $1 \times 12$ and $3 \times 16$ flow-cells.

Because of the iimfted means for observation in the flow-chamber during 
the $1 \times 12$ flow-cell experiments, the role of "cluster sedimentation"in those experiments could not be assessed, but is believed to be slight. In several of the later experiments, a few small clumps of spermatozoa were observed with the microscope in the lower part but not the upper part of the migration pattern. Similar observations were made in the sedimentation experiments reported in Chapters IV and VIII. A specific example of convection, in which spermatozoa migrated from the sides of the migration profile into the center of the flow-chamber, was described in the results. Because this increases the apparent net migration in the centerline of the flow-chamber, this phenomenon, as well as that of "cluster sedimentation", distorts the migration profile. By influencing the net migration of the spermatozoa, both of these processes interfere with the interpretation of the fractionation. Thus, heterogeneity of the population could be masked by these irregularities in migration.

The excellent pH stability of the glycine-phosphate buffer was demonstrated in the $3 \times 16$ flow-cell experiments. Because of the effects of $\mathrm{pH}$ on electrophoretic mobilfty and on the viability and intrinsic motility of spermatozoa, it is essential to have good pH stability in the staflo apparatus. Within limits, changes in the flow-cell temperature and in the $\mathrm{pH}$ of the medium due to increased electric field strength can be compensated for by increasing the electrode wash flow rate. $\mathrm{pH}$ changes were greater wh the medium used in the $1 \times 12$ flow-cell experiments than with the glycine-phosphate buffer and also may have influenced the distribution of spermatozoa slightly in those experiments. No temperature measurements were made in the $1 \times 12$ flow-cell and 1 it is probable that the poor reproductbility in that series of experiments, espectally at $7.5 \mathrm{~V} / \mathrm{cm}$, is also due in part to temperature differences among experiments. 
b. Intrinsic Motility. With the same medium, density gradient and $\mathrm{pH}$, the effect of intrinsic motility upon the distribution of spermatozoa in the Staflo apparatus is Influenced by temperature and by the orlentation and quality of the spermatozoa. Many examples of these interrelationships are presented in Section C.1.a above.

(1) Temperature. The magnitude of the intrinsic motility of individual spermatozoa can be controlled by temperature regulation. The degree of motility at any specific temperature depends strongly upon the suspension medium as reported by Bhattacharya ${ }^{35}$. At temperatures of $2^{\circ} \mathrm{C}$ or lower, there was no motility in any of the low lonic strength buffers used in this series of experiments. In the experiments at 6.25 and $7.5 \mathrm{~V} / \mathrm{cm}$, at $2.5^{\circ} \mathrm{C}$, the inftial quality of the spermatozoa was quite poor, and motility should have played no part in the distribution of the spermatozoa. Thus, in those 2 experiments, there was not a higher percentage of motile spermatozoa in the lower collection tubes as observed in the experiments at higher temperatures (e.g., Figure 41) under the same conditions of electric field strength. On the contrary, at $2.5^{\circ} \mathrm{C}$, spermatozoa in the upper collection fractions had a higher percentage of motility (Figure 43).

(2) Orientation. In all of the electrophoretic media used in the experiments reported in this chapter at $\mathrm{pH} \mathrm{6-7,} \mathrm{and} \mathrm{at} \mathrm{an} \mathrm{electric} \mathrm{field}$ strength of $5 \mathrm{~V} / \mathrm{cm}$ or greater, all spermatozoa were orfented vertically in the electric field with the talls pointing toward the positive electrode. The orfentation was not affected by the direction of migration. The differential distribution of the component parts of spermatozoa in one of the early $3 \times 16$ flow-cell experiments confirms the presence of a relatively greater net negative charge on the tall of the spermatozoa. The suggestion in Table VI of a lesser migration for the spermatozoa with cytoplasmic 
droplets, which are consldered a sign of the final stage of maturation, is consistent with Bedford's report of a lower electrophoretic mobility for epididymal than for mature spermatozoa ${ }^{24}$.

Under the proper conditions of temperature and electric field strength, one can use the tail anode property to orient the intrinsic motility vector. As shown in Experiment 4-29-65 at $5 \mathrm{~V} / \mathrm{cm}$ (Figure 40), the conditions of temperature and electric fleld were such that $5 \%$ of the spermatozoa migrated Into layer 10 , the layer below the inlet stream.

As reported in Chapter IV, the spermatozoa generally assume a headdown orientation in a $\mathrm{Ig}$ field. Because of the similar conditions of transit time and temperature in the $1 \times 12$ flow-cell experiments, the contribution of sedimentation to the migration should have been approximately the same in all experiments. The dextran density gradients used in some of the $3 \times 16$ flow-cell experiments greatly retarded the sedimentation of spermatozoa and, therefore, the sedimentation component can probably be ignored in those experiments.

(3) Quality. 'The abllity to control the magnitude and orientation of the intrinsic motility vector is hypothetical unless the initial spermatozoa quality is excellent. The same protocol was followed in ejaculate collection and handling in all of the experiments in this series, and, therefore, should not have caused unnatural differences in the Initial quality of spermatozoa samples. The characteristics by which quality is defined include the percentage of motility, the degree of motility of individual spermatozoa and the durability of the spermatozoa. The latter implies the survival of the vital functions (motility, viability and fertilizing capacity) during the experiments.

It is rare to obtain an ejaculate in which all of the spermatozoa have 
forward motion. Therefore, in electrophoresis experiments, a certain percentage of the sample w111 undergo the maximum electrophoretic or sedimentation-electrophoretic migration. In order to observe significant effects on the net migration of the sample, a substantial fraction of the population must have a high degree of forward motion. Some of the differences among the $1 \times 12$ flow-cell experiments can be attributed to the variable percentages of motile spermatozoa in the initial samples. The degree of motility 18 important for an initial sample with only tail-wagging at room temperature is going to have no motility at lower temperatures. In Experiment 4-29-66 in the $1 \times 12$ flow-cell series, the spermatozoa had a high degree of intrinsic motility and a significant downward migration of motile spermatozoa was evident from the distribution thereof.

The importance of the durability of spermatozoa, or the preservation of intrinsic motility, viability and fertilizing capacity, was well illustrated in these experiments. As observed in the early sedimentation expertments, the spermatozoa lost their intrinsic motility prior to losing viability, fudged by a nigrosin-eosin staining technique. In most cases, there was a great difference between the Initial quality of the spermatozoa sample and the qualfty of spermatozoa in the collection fractions immediately after the experiment. When the spermatozoa in the collection fractions initially did have some degree of motility, it was often lost within 30 minutes after termination of the experiment. Unfortunately, this loss of motility prevented analysis of the interrelationships among the motility, temperature and electric field strengths in the flow-chamber. In addition, the rapid loss of motility was associated with an frreversible loss of fertilizing capacity, demonstrated by the poor fertilization rate achieved In the $1 \times 12$ flow-cell experiments. Because the spermatozoa were insem-: Inated 1-2 hours after the female was mated with a vasectomized buck, the 
spermatozoa would have had to remain motile IN VIVo for $8-9$ hours in order to fertllize ova. However, the loss of fertilizing capacity was observed with spermatozoa samples in concentrations greater than $4 \times 10^{6} / \mathrm{ml}$ in the $1 \times 12$ flow-cell experiments, suggesting a deficlency in the medium rather than a dilution effect. Thus, the loss of fertilizing capacity may not be just a simple reflection of the loss of motility.

The preservation of the motllity and viability of opermatozoa was greatly enhanced by the glycine-phosphate buffer. Observations on the evaluation of this buffer are presented in Appendix D and in Chapter VI. In the early.sedimentation experiments, there appeared to be an enhancement of motility and viability by the seminal plasma Lu cullection fraction 4. There is a suggestion of a simflar effect in many of the $1 \times 12$ flowcell experiments in which the seminal plasma migrated into layers 8 and 7 . Although seminal plasma may be beneflclal from the point of view of preserving vital functions of the spermatozod, in high concentrations it would, unfortunately, change the ionic strength of the medium.

\section{Electrophoretic Mobility Comparisons}

The results of the upward and downward-electrophoresis experiments with rabbit and tow spermatozoa using the $3 \times 16$ [ low-cell are summarizcd in Table $X$. In all of the experiments with rabbit spermatozoa, the intrinslc motility was very poor and should not have attected the electrophurelic moblifty measurements. Fowl spermatozoa, however, had a higl percentage and degree of motility at room temperature in all exporiments, and the calculated average electrophoretic mob1lity, therefore, may be slightly 1ow.

In Table $X$, it is seen that under similar experimental conditions, the electrophoretic mobllity of fowl spermatozoa is approximately 2 times 
Table X. Average Electrophoretic Mobilities of Rabbit and Fow1 Spermatozoa Normalized to a Density Gradient of $1 \%$ Sucrose at $3^{\circ} \mathrm{C}$.

\begin{tabular}{|c|c|c|c|c|}
\hline \multirow[t]{2}{*}{$\begin{array}{l}\text { Medium and } \\
\text { Density Gradient }\end{array}$} & \multirow[t]{2}{*}{$\begin{array}{l}\text { Electrophoresis } \\
\text { Direction }\end{array}$} & \multirow[t]{2}{*}{ Temp } & \multicolumn{2}{|c|}{$\begin{array}{l}\text { Electrophoretic Mobility } \\
\text { [ Units of } 10^{-4}(\mathrm{~cm} / \mathrm{sec}) /(\mathrm{V} / \mathrm{cm}\end{array}$} \\
\hline & & & Rabbit & Fow1 \\
\hline Glycine-phosphate buffer; & Downward & $6^{\circ} \mathrm{C}$ & 0.82 & - \\
\hline \multirow[t]{2}{*}{ dextran density gradient } & Downward & $6^{\circ} \mathrm{C}$ & 0.79 & - \\
\hline & Downward & $6.8^{\circ} \mathrm{C}$ & - & 1.68 \\
\hline Average & & & 0.81 & 1.68 \\
\hline Glycine-citrate buffer; & Upward & $6^{\circ} \mathrm{C}$ & 0.95 & 1.68 \\
\hline \multirow[t]{2}{*}{ sucrose density gradient } & Upward & $5.4^{\circ} \mathrm{C}$ & 0.76 & - \\
\hline & Upward & $4.3^{\circ} \mathrm{C}$ & - & 1.58 \\
\hline Average & & & 0.86 & 1.63 \\
\hline
\end{tabular}


that of rabbit spermatozoa. Additional measurements of electrophoretic mobility of both rabbit and fowl spermatozoa are reported in subsequent chapters and confirm this impression.

Considering the use of different buffers and different density gradlent materials, the average values of the electrophoretic mobility for rabbit spermatozoa in upward and downward directions agree within the limits of the measurement in the Staflo apparatus. Although only one downward electrophoresis experiment was conducted with fowl spermatozoa, the agreement between that value and the average of the 2 upward-electrophoresis experiments, as shown in Table $\mathrm{X}$, is also very good. These values are normalized to that expected in a density gradient containing $1 \%$ sucrose at $3^{\circ} \mathrm{C}$ for comparison with other electrophoresis and sedimentation results reported in Chapter VIII.

\section{E. Sumiary and Conclusions}

The different-electric fleld groups of $1 \times 12$ flow-cell upward electrophoresis experiments were characterized by a lack of reproducibility which is explained, in part at least, by varlations both in temperature and in the quality of spermatozoa in individual experiments. Because the initlal spermatozoa concentrations were $20.5 \times 10^{6} / \mathrm{ml}$ or less in all experiments except one (Experiment 4-29-65), there should have been no "cluster sedimentation"with this one possible exception.

The lack of reproducibility within, and differences among, groups of experiments prevented drawing firm conclusions about the distribution of viable spermatozoa among the collection fractions as judged by nigrosineosin staining. As was also observed in the early sedimentation experiments (Chapter IV), the rapid loss of motility before loss of viability further complicated interpretation of the distribution of viable spermatozoa. 
St1ll prior to development of a good Staflo microscopic system, this loss of motility also prevented understanding the possible interplay between intrinsic motility and electric field, in the flow-chamber. As before, the very poor results of artificial insemination suggest that the loss of motility was irreversible. Thus, the Hanks-glycine medium used in these experiments was highly unsatisfactory for biological work. As also noted in Chapter. IV, the presence of diluted seminal plasma appeared to enhance the relative preservation of mot1lity in the respective fractions.

The differences among the $5,6.25$ and $7.5 \mathrm{~V} / \mathrm{cm}$ groups of experiments are consistent with the known relationships between the intrinsic motility and electrophoretic mobility of spermatozoa. Even at temperatures of $5-6^{\circ} \mathrm{C}$, there was a significant decrease in the net electrophoretic migration because of the oppositely directed intrinsic motility vector. The most highly motile spermatozoa showed very little net migration in the electric field, and, either through "exhaustion" or because of subsequent dilution, lost their motility before or shortly after the runs were completed. Relative enrichment of the lower collection fractions with regard to motile and viable spermatozoa was demonstrated in the $6.25 \mathrm{~V} / \mathrm{cm}$ experiments at $3.5^{\circ} \mathrm{C}$ and $5^{\circ} \mathrm{C}$.

The 1 × 12 flow-cell experiments further demonstrated the need for accurate temperature control which later became possible upon construction of a cold room. With temperature control, the glycine-phosphate and several greater-increment density gradients, good reproducibility was achieved in predominantly electrophoresis experiments.

The $3 \times 16$ flow-cell and ancillary equipment were tested in a series of upward- and downward-electrophores1s experiments with both rabbit and fowl spermatozoa. The horizontal and vertical distributions of spermatozoa 
In the flow-chamber approximated those predicted for "ideal" migration patterns by Tippetts 263 . The glycine-phosphate buffer used in the $3 \times 16$ flow-cell experiments, in addition to being far superior to the Hanksglycine medium for the preservation of spermatozoa, also demonstrated excellent $\mathrm{pH}$ stability (less than $0.1 \mathrm{pH}$ unit change during an experiment). This was without the "high conductivity" barrier used in subsequent experiments (Chapters VI and VIII).

The Staflo microscope and slit lamp technique were both used to measure migration distances in the flow-chamber. The slit lamp was more practical for observing aberrations of the gross migration profile, whereas the microscopic system gave the additlonal capability of observing the orlentation and degree of motillty, and cell-cell interactions such as microscopic "cluster sedimentation". Several experiments in this series 111ustrated the need for such observations if proper interpretation of the net migration was to be made.

Al1 rabbit and fowl spermatozoa had a net negative charge from $\mathrm{pH}$ 6-7 in all buffers used and, at temperatures of $2-7^{\circ} \mathrm{C}$, had a tailanode orientation in the electric field regardless of the direction of electrophoretic migration. With both upward and downward migration, the total spermatozoa distribution suggested a unimodal distribution of electrophoretic mobilities, the averages of which are summarized in Table $X$. The values have been normalized as described in Chapter III to those expected in the glycine-phosphate buffer with $1 \%$ sucrose at $3^{\circ} \mathrm{C}$ in order to make some subsequent comparisons.

The electrophoretic mobility of fowl spermatozoa in these experiments was seen to be approximately twice that of rabbit spermatozoa. Additional electrophoretic determinations are given in Chapter VI and VIII for fowl and rabbit spermatozoa, respectively. 
VI. STAFLO ELECTROPHORESIS: FOWL SPERMATOZOA

\section{Contents}

A. Preservation of Motility and Fertilizing Capacity under

Conditions of Low Temperature and Low Spermatozoa Concentrations

1. Introduction. . . . . . . . . . . . . . . . 180

2. Materials and Methods ................. 182

a. Characterlstics of Ejaculates.............. 182

b. Evaluation of Spermatozoa . . . . . . . . . . 182

c. Cooling of Spermatozoa. . . . . . . . . . . . 183

d. Media ................... 183

3. Results . . . . . . . . . . . . . . . . 184

a. Comparison of the Two "High" Ionic Strength Media . . . . 184

b. Comparison of the Two "Low" Ionfc Strength Media. . . . 185

c. Comparison of the Best "High" and "Low" Ionic Strength 189 Med1a at 1:30.D1lution .............. 189

4. Discussion. . . . . . . . . . . . . . . . . 189

a. Effectiveness of Various Media. . . . . . . . . . 189

b. Factors Affecting Fertilization Rates.......... 191

1) Artificial Insemination . . . . . . . . . 191

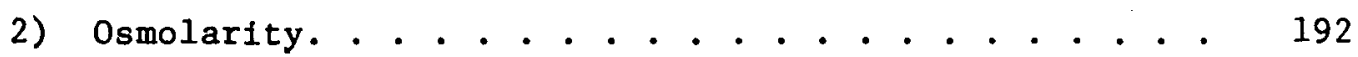

3) Dilution and Reconcentration. ........... 193

c. Additional studies. . . . . . . . . . . . . . 194

B. Electrophoresis Experiments . . . . . . . . . . . . . 195

1. Introduction. . . . . . . . . . . . . . 195

2. Materials and Methods .. . . . . . . . . ... 196

a. Sample Preparation. . . . . . ......... 196

b. Staflo Apparatus. ................ 196 
c. Medium and Density Gradient. . . . . . . . . .

d. Temperature Control. . . . . . . . . . 198

e. Observation and Evaluation of Spermatozoa. . . . 198

3. Results. . . . . . . . . . . . . . . . 199

a. Electrophoresis Experiments. . . . . . . . . 199

b. Electrophoresis-Enrichment Experiments ....... 200

4. Discussion ................... . . . . . 201

a. Technical Considerations ............ 201

b. Electrophoretic Mobility Measurements. . . . . . 202

C. Summary and Conclusions. . . . . . . . . . . . . . 206

A. Preservation of Motility and Fertilizing Capacity Under Conditions of Low Temperature and Low Spermatozoa Concentrations

\section{Introduction}

It is well recognized that knowledge of the biophysical properties of many types of cells has contributed greatly to an understanding of their role in blological systems. In this regard, the properties of spermatozoa should be of great interest in the blology of reproduction. In the study and practice of mammalian reproduction, considerable flexibility is provided in work with a number of specles by the ability to store spermatozoa for weeks or longer at low temperatures, with maintenance of their vital functions, prior to artificial insemination. In contrast, for fowl spermatozoa, such success has not been achieved and the comon practice is to Inseminate concentrated semen spermatozoa immediately after collection. The Inability of fowl spermatozoa to survive low temperature storage conditions also severely limits the fearibility of conducting many blophysical studies 
on these cells. This section is concerned with the preservation of the motility and fertilizing capacity of fowl spermatozoa not only at low temperatures, but in low concentrations and in low fonic strength media.

A convenient means of reversibly controlling motility is through the control of temperature. For complete immobilization of spermatozoa, depending upon the suspension medium, temperatures near $0^{\circ} \mathrm{C}$ are required 35 . Varying specified degrees of motility, can be achieved by selection of sultable temperatures above $0^{\circ} \mathrm{C}$.

Even though the blological qualities of spermatozoa are most easily preserved at high sperm concentrations, for certain purposes, it is desirable or essential to work at low concentrations and still maintain these properties. For example, In both analytical and preparative electrophoresis, the requirements for observation of Individual spermatozoa and for hydrodynamic stability demand spermatozoa concentrations ranging from about $1 / 300-1 / 30$ of those in undiluted ejaculates ${ }^{156-158}$. Many electrophoresis experiments further require media with ionic strengths much less than those of physiological solutions. The inability to preserve motility and fertilizing capacity under such extreme conditions severely limits the possible kinds of experimental manipulations of spermatozoa.

Many investigators interested primarlly in artificlal insemination have studied the effects of cooling or dilution of fowl spermatozoa but not combinations of the two factors as noted above. With regard to temperature, Van Wambeke observed almost no loss of fertilizing capacity of fow1 spermatozoa diluted with equal volumes of either of two diluents containing skimmed milk and stored at $2-5^{\circ} \mathrm{C}$ for 24 hours $^{23 I}$. Even though the semen was diluted $1: 2$, the cell concentration is very high in the sense used in this paper. Lorenz, in his review, mentions work by others at room 
temperature which showed the following: (1) $1: 3$ dilution with seminal plasma gave a slight decrease in fertility but no additional loss with dilutions of up to $1: 63$, provided that the same number of spermatozoa was inseminated; (2) dilutions of up to $1: 50$ in an artificlal medium resulted in only a slight loss of fertility for inseminations of at least $10^{8}$ spermatozoa; poor results were obtained with greater dilutions or with insemination of fewer spermatozoa and (3) 1:1250 dilution in saline or in Tyrode's or Baker's solutions produced immediate irreversible immobilization ${ }^{140}$.

Thus, from the above, it is seen that the fertilizing capacity of fowl spermatozoa has been maintained for brief periods in media of high Ionic strength during storage under conditions of (1) low temperature-high concentration, or (2) room temperature-low concentration. In this section, we report a signfficant extension of the limiting experimental conditions of this kind, namely, preservation of motility and fertilizing capacity under the combined conditions of low temperature and low sperm concentrat1on, in 2 low tonic strength media sultable for electrophoresis experiments, In addition, 2 high lonic strength media were evaluated for storage of spermatozoa at low temperature and high concentrations.

\section{Materials and Methods.}

a. Characteristics of Ejaculates. The characteristics of the ejaculates from the White Leghorn roosters used in these experiments were as follows: volume, $0.4-1.0 \mathrm{ml}$; spermatozoa concentration, $2.5-3.0 \times 10^{9} / \mathrm{ml}$; motility, $80 \%-90 \%$; fertilizing capacity, $85 \%-95 \%$ with insemination of $0.1 \mathrm{ml}$ of normal, undiluted semen at 4-5 day intervals.

b. Evaluation of Spermatozoa. The customary evaluation scale was used for hfgh concentration populations of spermatozoa. It ranges from Grade I (extremely poor mot1lity) to Grade VI (a "vigorous swarming pattern"). In 
addition, with low concentrations it was possible to estimate the percentage of individually motile spermatozoa and to utilize a scale of degrees of motility as follows: (1) lmmotile, (2) feeble tail-wagging, (3) vigorous tall-wagging, (4) feeble forward motion and (5) vigorous forward motion.

c. Cooling Spermatozoa. Initial cooling of spermatozoa in all experiments was accomplished by adding an equal volume of dilution medium to pooled ejaculates in a $15 \mathrm{ml}$ glass centrifuge tube which then was capped and placed In the center of a 2 lb coffee can full of sawdust. The coffee can was covered and immersed to the brim in ice water. Cooling curves determined with a temperature recorder (Varian Assoclates) showed a temperature decrease from $29^{\circ} \mathrm{C}$ to $2.5^{\circ} \mathrm{C}$ in 120 minutes, with a maximum cooling rate of $0.30^{\circ} \mathrm{C}$ per minute.

\section{d. Media.}

(1) Egg yolk medium. The egg yolk-glycine-citrate medium is a modification of that used by Bhattacharya ${ }^{35}$ : $40 \%$ by volume whole egg yolk and $60 \%$ glycine-citrate solution consisting of $20 \mathrm{~g}$ sodium citrate dihydrate, $20 \mathrm{~g}$ glycine, $5 \mathrm{~g}$ Fructose, $0.25 \mathrm{~g}$ citric actd, $1.5 \times 10^{6}$ units penicillin $\mathrm{G}$, diluted to $1000 \mathrm{ml}$ with distilied water; a "high" ionic strength medium.

(2) Vitalizing medium. The glucose vitalizing medium is a modification of the Krebs-Henseleit-Ringer solution used by Bhattacharya ${ }^{35}: 0.9 \mathrm{~g} \mathrm{NaCl}$, $0.05 \mathrm{~g} \mathrm{KC} 1,0.05 \mathrm{~g} \mathrm{Na}_{2} \mathrm{HPO}_{4} \cdot 12 \mathrm{H}_{2} 0,0.04 \mathrm{~g} \mathrm{MgSO}_{4}, 0.03 \mathrm{~g} \mathrm{NaHCO}, 0.5 \mathrm{~g}$ glucose, $10^{5}$ untts penicillin $G$, diluted to $100 \mathrm{ml}$ with distilled water. $\mathrm{pH}$ adjusted to 7.0 with $\mathrm{HCl}$; a "high" ionic strength medium.

(3) Glycine-citrate buffer. A "low" Ionic strength solution consisting of $2.0 \mathrm{~g}$ sodium citrate dihydrate, $9.25 \mathrm{~g}$ glycine, $3.0 \mathrm{~g}$ fructose, $0.25 \mathrm{ml}$ of $10 \%$ citric acid solution, $48.6 \mathrm{~g}$ sucrose, $10^{6}$ units of penicillin $\mathrm{G}$, diluted to $1,000 \mathrm{ml}$ with distilled water; $\mathrm{pH} 6.94$.

(4) Glycine-phosphate buffer. A "low" Ionic strength solution consisting 
of $2.2 \mathrm{~g} \mathrm{Na}_{2} \mathrm{HPO}_{4} \cdot 12 \mathrm{H}_{2} \mathrm{O}, 0.20 \mathrm{~g} \mathrm{NaCl}, 0.20 \mathrm{~g} \mathrm{NaHCO}, 0.016 \mathrm{~g} \mathrm{KH}_{2} \mathrm{PO}_{4}$,

$9.25 \mathrm{~g}$ glycine, $23 \mathrm{~g}$ fructose, $10^{6}$ units penicillin $\mathrm{G}$, diluted to 1,000 $m l$ with distilled water. $\mathrm{pH}$ adjusted to 7.1 with $\mathrm{HCl}$.

As mentioned in the text, dextran (MW 73,000; Sigma Chemical Company) or sucrose was added to the glycine-citrate and glycine-phosphate buffers In some instances to simulate density gradient conditions for electrophorests experiments.

\section{Results.}

a. Comparison of the two "high" Ionic strength media. Egg yolk medium and vitallzing medium, both of which have been found useful in work with rabbit spermatozoa ${ }^{35}$ were evaluated for use in the initial dilution and cooling of fowl spermatozoa and for the temporary storage of these spermatozoa reconcentrated after dilution in low lonic strength media. Semen was obtained by abdominal massage ${ }^{266}$. It was pooled and then diluted with equal volumes of either egg yolk medium or vitalfzing medium and placed in separate $15 \mathrm{ml}$ glass centrifuge tubes. Both samples were cooled continuously from room temperature to $4^{\circ} \mathrm{C}$ in 2 hours and were held at that temperature for 2 additional hours. The tubes then were removed from the cooling container and allowed to warm naturally to room temperature. After warming there were no apparent differences in morphology or in the high degree of motility of the spermatozoa in the 2 samples. Two groups of 2 chickens each were insemlnated with $0.1 \mathrm{ml}$ of the respective samples. The procedure was repeated twice with fresh spermatozoa at 4-day intervals and eggs were collected from the day after the first insemination unt11 4 days after the third insemination. The results are presented in Table XI. Only $9.1 \%$ of the eggs were fertilized by spermatozoa stored in the egg yolk medium compared to $80 \%$ fertility in the vitalizing medium. 
Table XI. Preservation of Fertilizing Capacity of Fowl Spermatozoa Diluted $1: 2$ and Stored in "High" Ionic Strength Media at $4^{\circ} \mathrm{C}$.

\begin{tabular}{lcc}
\hline & Egg Yolk Medium & Vitalizing Medium \\
\hline Total number of eggs & 33 & 40 \\
Number of fertile eggs & 3 & 32 \\
Percentage of fertile eggs & 9.1 & 80 \\
\hline
\end{tabular}

b. Comparison of the two "low" ionic strength media.

(1) Motility. The preservation of motility at $3^{\circ} \mathrm{C}$ in "1ow" lonic strength glycine-citrate and glycine-phosphate buffers was evaluated in solutions of each adjusted with sucrose to 310,368 and 427 mosm. Pooled semen was diluted $1: 2$ with vitalizing medium and cooled from room temperature to $3^{\circ} \mathrm{C}$ In 2 hours. Aliquots were then diluted in each of the 6 solutions, to concentrations of $80 \times 10^{6}$ spermatozoa/ml. Additional dilutions were made on each of the suspensions resulting in concentrations of $40,27,20,13$ and $8 \times 10^{6}$ spermatozua/ml, giving a total of 36 samples.

After 1 hour at $3^{\circ} \mathrm{C}$, aliquots of selected 310,368 and 427 mosm solutions of both buffers were allowed to warm to room temperature. Spermatozoa In the 310 and 368 mosm solutions showed no change from their initial motility ( $80 \%-90 \%$ vigorous forward motion). On the other hand, those in all of the 427 mosm solutions exhibited a marked decrease in motility with approximately $10 \%$ having feeble forward motion and $15 \%-20 \%$ feeble tail-wagging. Intermittent observations made over the next 5 hours established a continuous trend of loss of sperm quality in some solutions, and a relative constancy in others. These were culminated by the final observations at room temperature 
following storage for 6 hours at $3^{\circ} \mathrm{C}$. In the 310 mosm solutions of both buffers, and at a11 6 concentrations, approximately $70 \%$ of the spermatozoa had vigorous forward motion. In all 368 mosm solutions of both buffers, approximately $50 \%$ of the spermatozoa had vigorous forward motion and $20 \%$ exhibited vigorous tail-wagging. Moderately severe tail-tail agglutination occurred in all 368 mosm solutions whereas agglutination was rare in the 310 mosm solutions. For the 427 mosm solutions of both buffers, less than $5 \%$ of the sperm had either tail-wagging or forward motion. In summary, no qualitative differences were found between any of the corresponding 310 , 368 or 427 mosm solutions of "low" ionic strength glycine-citrate and glycine-phosphate buffers. Furthermore, both 310 mosm solutions gave excellent preservation of motility; i.e. less than a $15 \%$ decrement from the initial values was observed, in concentrations ranging from $8-80 \times 10^{6} / \mathrm{ml}$ after 6 hours storage at $3^{\circ} \mathrm{C}$.

(2) Fertilizing capacity. The glycine-citrate and the glycine-phosphate buffers were also compared to each other and to vitalizing medium with regard to preservation of fertilizing capacity after dilution and brief storage. Pooled semen was diluted 1:2 with vitalizing medium and cooled. to $3^{\circ} \mathrm{C}$ in 2 hours. One-third of the sample was diluted in vitalizing medium to approximately $250 \times 10^{6}$ spermatozoa/ml and stored at $3^{\circ} \mathrm{C}$ for $21 / 2$ hours. The remainder of the sample was divided between the two $40 \mathrm{ml}$ glass centrifuge tubes. One suspension was diluted with glycine-citrate and the other with glyclne-phosphate buffer to spermatozoa concentrations of approximately $350 \times 10^{6} / \mathrm{ml}$. After 1 hour, these samples were further diluted to $80-90$ $x 10^{6} / \mathrm{ml}$ and held at $3^{\circ} \mathrm{C}$ for an additional $11 / 2$ hours. This dilutionstorage protocol was repeated in 4 and 8 days. For each of the 3 trials, the same 3 groups of 6 hens each were inseminated. Eggs were collected from the day after the first insemination until 4 days after the third 
insemination. In the first trial, reconcentration of the diluted spermatozoa was not attempted because the $2 \%$ dextran, which was used to simulate density gradient conditions, Interfered with the centrifugation process. Each hen was inseminated with approximately $1.0 \mathrm{ml}$ of suspension containing a total of $86-100 \times 10^{6}$ spermatozoa as indicated in Table XII A. In trials 2 and 3 , where sucrose was used to simulate the density gradient conditions, reconcentration of the spermatozoa in all 3 suspensions was accomplished by centrifugation ( 50 minutes at $400 \mathrm{~g}$ in trial 2, 30 minutes at $1,500 \mathrm{~g}$ in trial 3 ). The volumes and total number of spermatozoa insemInated in trials 2 and 3 are shown in Table XII B.

The results of trial 1 are shown in Table XII $A$. It is seen that a much higher percentage of eggs was fertilized by spermatozoa diluted and stored in the glycine-citrate buffer than in elther of the 2 other media. The differences between the fertilization rate of $56.3 \%$ wth glycine-citrate buffer and the rates of $15.8 \%$ (glycine-phosphate) and $27.2 \%$ (vitalizing medium) are significant at the $P<0.01$ and $P=0.07$ levels, respectively, In a one-tail test ${ }^{90}$.

The pooled results of trials 2 and 3 are shown in Table XII B. The largest difference among the fertilization rates, i.e. that between the $41.9 \%$ in vitalizing medium and $26.5 \%$ in glycine-citrate buffer, is significant only at the $P=0.10$ level. The spermatozoa used in trials 2 and 3 did exhibit increased evidence of certain morphological changes following. centrifugation, the most common of which was severe bending of the spermatozoa to give apposition of head and proximal tail segments.

The average results of all 3 trials are given in Table XII $\mathrm{C}$. There is no difference between the fertilization rates with glycine-citrate buffer and vitalizing medium $(P \gg 0.10)$. The fertilization rates with 
Table XII. Preservation of Fertilizing Capacity of Fowl Spernatozoa Diluted 1:30 and Stored in "High" and "Low" Ionic Strength Media at $3^{\circ} \mathrm{C}$.

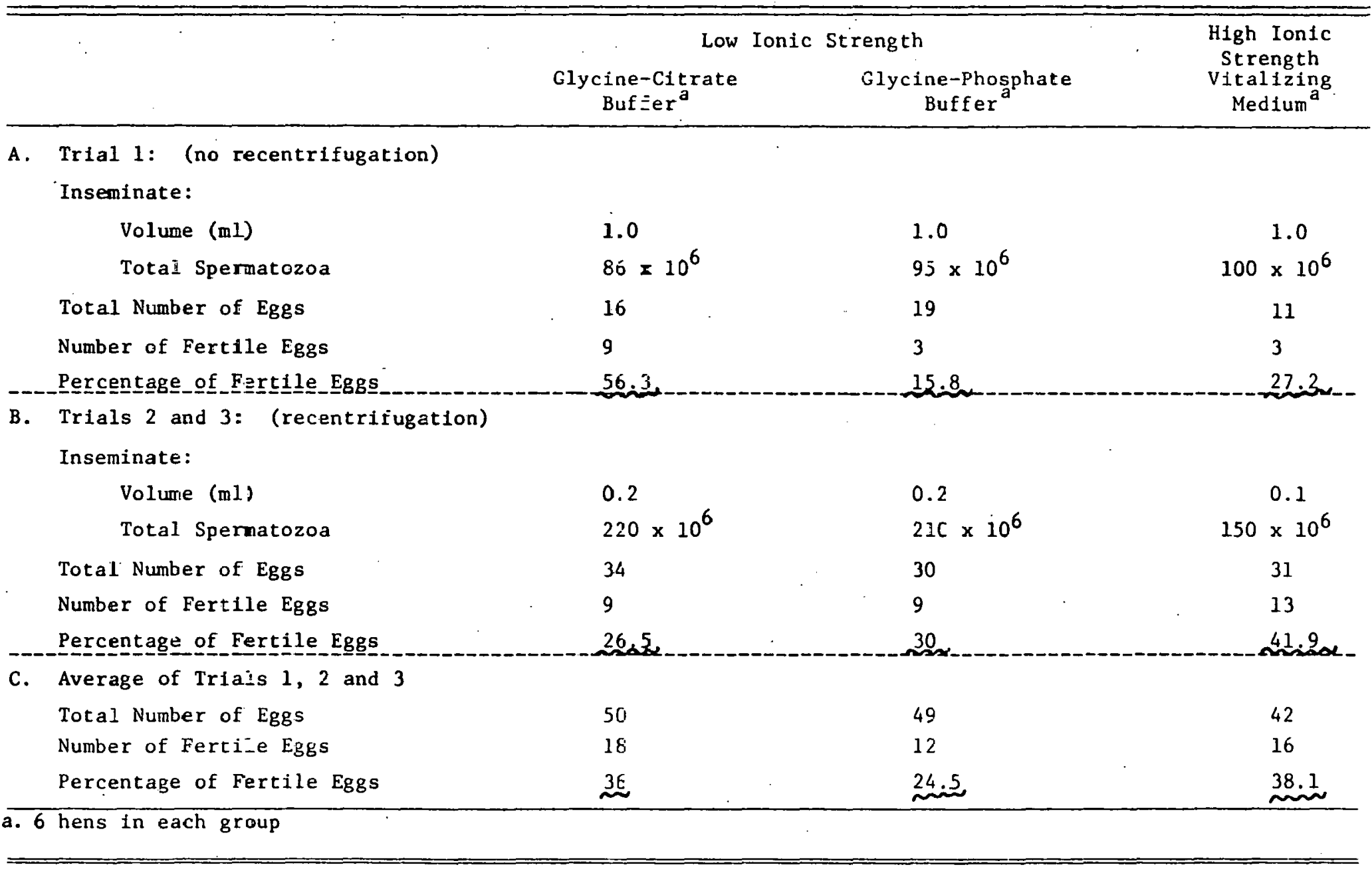


the glycine-citrate and glycine-phosphate buffers differ only at the $P=0.12$ level while the latter and the vitalizing medium differ at the $P=0.08$ level. Thus, any advantage of using the glycine-citrate buffer must be considered slight.

\section{c. Comparison of best "high" and "low" lonic strength media at 1:30} dilutions. The effects of moderate dilution in vitalizing medium and in glycine-citrate buffer on the fertilizing capacity of fowl spermatozoa were compared, using a 1:2. dilution control. Pooled semen was diluted $1: 2$ in vitalizing medium and cooled from room temperature to $3^{\circ} \mathrm{C}$ in 2 hours: One-third of the sample (the control) was stored at this temperature without further dilution. The remainder was divided equally between two $40 \mathrm{ml}$ glass centrifuge tubes and diluted 1:30. with either vitalizing medium or with glycine-citrate buffer. After 1 hour, the diluted spermatozoa were reconcentrated by centrifugation for 50 minutes at 250 8. Recovery was approximately $85 \%-90 \%$. After removal of the supernatant, both samples of spermatozoa were resuspended in vitalizing medium. This dilution-storage protocol was repeated 3 days later. On each occasion, the same 3 groups of 6 chickens each were inseminated with $0.20 \mathrm{ml}$ of suspension containing approximately $200 \times 10^{6}$ spermatozoa. Eggs were collected from the day after the first insemination until 7 days after the second insemination. The results are shown in Table XIII. There are no significant differences among the 3 fertilization rates.

\section{Discussion.}

a. Effectiveness of varlous media. The poor preservation of fertilizing capacity by media containing egg yolk, despite maintenance of motility, has been reported by others ${ }^{140}$. In addition to its superiority in preservIng fertllizing capacity, vitalizing medium is a clear, colorless solution of low viscosity. In contrast, egg yolk medium exhibits decreased light 
Table XIII. Effects of Dilution and Media on the Preservation. of the Fertilizing Capacity of Fowl Spermatozoa Stored at $3^{\circ} \mathrm{C}$.

Ionic Strength

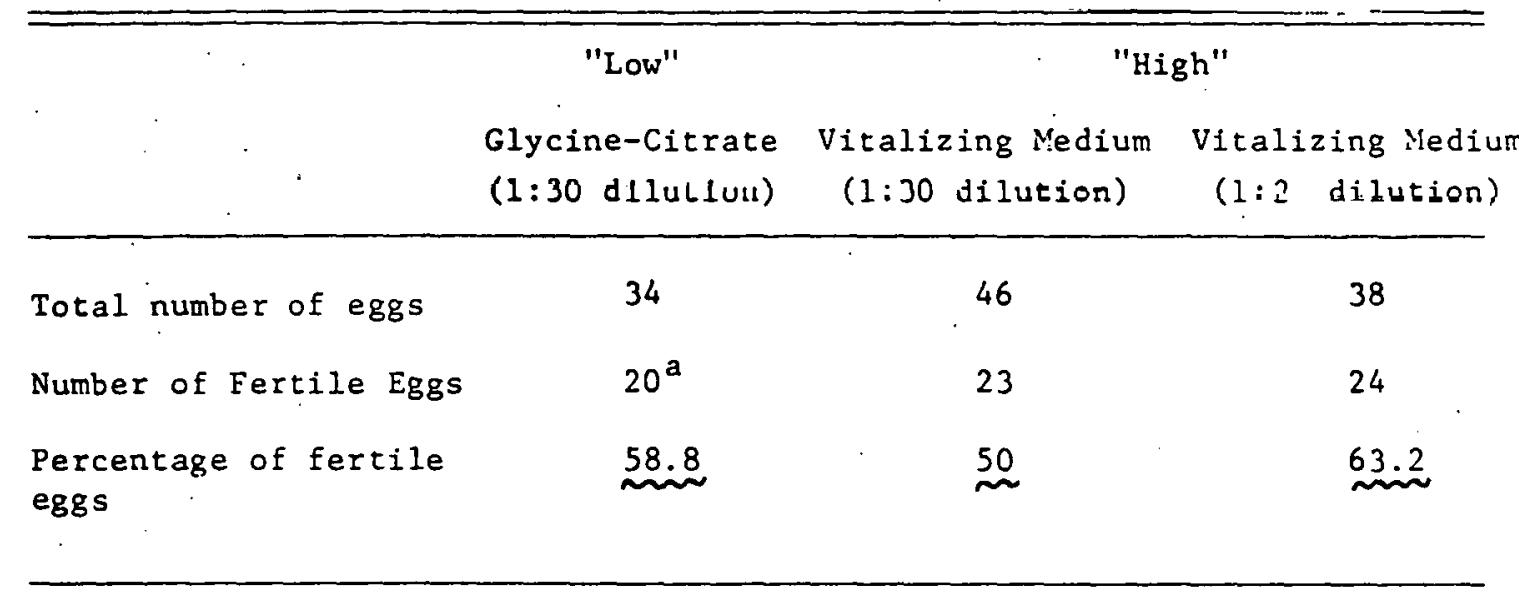

a. 3 of 20 eggs contained dead embryos whereas all other fettile eggs containcd viable embryos on the 17 th day of Incubaliun. 
transmission, a high viscosity and a potential for coating equipment with a protein film - all undesirable properties in certain experimental systems.

For the preservation of motility, no difference was found between the "low" ionfc strength glycine-citrate and glycine-phosphate buffers. For the preservation of fertilizing capacity, the data in Table XII C suggest a possible slight advantage to using the glycine-citrate buffer. Consequently, that medium was selected for subsequent dilution studies and for electrophoresis experiments to be reported in Section $B$.

In the study summarized in Table XIII, the fertilizing capacity of spermatozoa diluted 1:30 in "low" Ionic strength glycine-citrate buffer, stored for $11 / 2$ hours at $3^{\circ} \mathrm{C}$ and reconcentrated by centrifugation is comparable to that obtained for spermatozoa diluted $1: 2$ in vitalizing medium and stored under the same time-temperature conditions without centrifugation: This suggests that fowl spermatozoa can withstand the stresses expected in Staflo electrophoresis experiments: low ionic strength, low temperature, moderate dilution and reconcentration by centrifugation.

Comparison of the data in Table XIII for the $1: 30$ dilution (with centrifugation) and the $1: 2$ dilution (no centrifugation) in vitalizing medium reveals no difference $(P>0.10)$. Thus, if use of a "high" ionic strength medium is indlcated, vitalizing medium could be used for studies with fowl spermatozoa under conditions of moderate dilution.

\section{b. Factors Affecting Fertilization Rates.}

(1) Artificial insemination. The fertilization rate of $80 \%$ achieved with spermatozoa diluted $1: 2$ in vitalizing medium (Table XI) hardly differs from the optimum range of $85 \%-95 \%$ expected with our flock. If there is a slight difference, it might be due entirely to the cooling to $4^{\circ} \mathrm{C}$ (2 hours) and storage at $4^{\circ} \mathrm{C}(2$ hours $)$. 
The difference between the fertilization rates of $80 \%$ (Table XI) and 63.2\% (Table XIII), with 1:2 dilutions in vitalizing medium and storage under the same conditions, is attributed primarlly to using young hens of unknown fertility in the latter study. Thus, it is expected that a fertilization rate approaching $80 \%$ would have been obtained if hens of proven fertility had been avallable. Likewise, a comparable improvement could be anticipated in the $58.8 \%$ fertilization rate for spermatozoa diluted 1:30 In the "low". Ionlc strength glycine-citrate buffer (Table XIII).

(2) Osmolarity. For the preservation of mottlity, the 310 mosm solutions of both the glycine-citrate and glycine-phosphate buffers were far superior to the 368 and 427 mosm solutions, with storage at $3^{\circ} \mathrm{C}$ for 6 hours in concentrations from $8 \times 10^{6}-80 \times 10^{6}$ spermatozoa/ml: In other studies, using 310 mosm solutions of both buffers, the fertilizing capacity was preserved for 2 hours at $3^{\circ} \mathrm{C}$ at $1: 30$ dilutions. Even though the preservation of this property was not evaluated in the 368 mosm solutions, the more rapid loss of motility therein suggests that a corresponding loss of fertility would also occur. Other investigators, using different media, have reported different optimum osmolarities for the preservation of motility and fertilizing capacity -300 and 350 mosm, respectively ${ }^{140}$. Our observations favor use of the lower (310) mosm solutions for sustaining both biological properties.

The marked decrease in motility, after 1 hour at $3^{\circ} \mathrm{C}$, for all spermatozoa concentrations in the 427 mosm glycine-citrate and glycine-phosphate buffers is in contrast with the excellent preservation of motility for 4 hours in the 415 mosm egg yolk medium. Thus, egg yolk has an important protective effect on motility in overcoming otherwise adverse effects of high osmolarity. Other observations in this study indicated excellent preservation of fowl sperm motility in egg yolk medium for more than 24 hours. In 
spite of this, the observed loss of fertilizing capacity (Table XI) suggests that irreversible damage was done to the spermatozoa during the 4-hour exposure to egg yolk medium. (The probable dilution and/or absorption of the medium in the female genital tract should have largely eliminated the influence of the medium shortly after artificial insemination). It is recognized that these IN VITRO observation times are short considering that good fertility is maintained in chickens for 5-6 days after mating and that some fertile eggs may be obtained as late as 35 days thereafter 220 . Never-. theless, these results point to a probable differential vulnerability of fowl spermatozoa with respect to the 2 key blological characteristics: motility and fertilizing capacity.

(3) Dilution and Reconcentration. Because the usual practice with fowl is to Inseminate $0.05-0.10 \mathrm{ml}$ of highly concentrated spermatozoa (undiluted or 1:2 dilution), immediately after collection, dilute suspensions of spermatozoa in this study were generally reconcentrated by centrifugation prior to insemination. An important question is whether or not the morphological changes seen following the centrifugation process negate the advantages of insemfnating small volumes. Comparison of the fertilization rates in Table XII for dilute (56.3\%) and reconcentrated (26.5\%) spermatozoa suggests that, at least for the glycine-citrate buffer, centrifugation is detrimental (significant at the $P=0.02$ level). Although the reverse appears to be true for the spermatozoa in vitalizing medium and in glycinepliosphate buffer, the diffcrenceg aro not oignificant $(P>0.10)$.

The results of this part of the study indicate that successful insemination of low concentrations of spermatozoa is possible in glycine-citrate buffer. Furthermore, if centrifugation is required to achieve a high concentration sample, insemination with a moderately dilute sample actually 
appears to be preferred provided that at least $80 \times 10^{6}$ spermatozoa can be Inseminated in a volume of $1 \mathrm{ml}$ or less.

\section{c. Additional Studies. Because the 415 mosm "high" Ionic strength egg} yolk medium preserved the motility of the fowl spermatozoa much better than did the 427 mosm "low" ionfc strength glycine-citrate and glycinephosphate buffers, the preservation of both motility and fertilizing capactty in an eg8 yolk medium adjusted to 310 mosm should also be evaluated. This would shed light on the question of whether osmolarity or some other property was responsible for the loss of fertilizing capacity in media containing egg yolk.

The excelient preservation of motility in the 310 mosm "low" ionic strength glycine-citrate and glycine-phosphate buffers at concentrations as low as $8 \times 10^{6}$ spermatozoa/m1 shows that meaningful analytical electrophoresis studies can be conducted. The analytical information is of interest on 2 accounts: (1) In providing physical characterizations of spermatozoa populations and (2) in revealing optimum conditions for preparative electrophoresis separations. It is expected that information obtained in analytical studies can be exploited in preparative experiments.

It was seen that the "low" ionic strength glycine-citrate buffer excellently preserved both motility and fertilizing capacity. In view of the fertilization results achieved with different media by others at room temperature with dilutions of $1: 50$ and $1: 63^{140}$; successful insemination may be possible after even greater dilutions at low temperature in glycinec1trate buffer. Furthermore, one can speculate that this buffer might be an even better diluent than vitalizing medium for storage of spermatozoa at high concentrations. For this reason, it would be useful to extend the present study to a comparison of the 2 media at $1: 2$ dilutions with longer 
storage times at $3^{\circ} \mathrm{C}$. Effective extension of successful spermatozoa storage, as well as insemination at lower concentrations, could be of considerable practical benefit for work in poultry husbandry and genetics.

\section{B. Electrophoresis Experiments}

\section{Introduction}

In contrast to the abundance of Information concerning the electrophoretic properties of mammalian spermatozoa, there have been few such reports on fowl spermatozoa. Undoubtedly, major reasons for the greater interest in mammalian spermatozoa are the economic and sociological consequences of finding a way to separate genetically differing, e.g. X- and Y-chromosome bearing, spermatozoa. Despite the work of Schröder ${ }^{206}$ and of Gordon ${ }^{91}$, it is uncertain that apparent differences in the electrophoretic properties of rabbit spermatozoa can be attributed to different chromosomal composition ${ }^{18,174,233}$. Because a11 fowl spermatozoa contain $\mathrm{X}$-chromosomes, the sex control Incentive is lacking in the study of this species. However, in addition to basic knowledge about the properties of fowl spermatozoa, electrophoretic studies are motivated by the availability of cocks with a translocation heterozygote producing spermatozoa with differing chromosomal composition and fertilizing capacity ${ }^{120}$. It would also be of interest to compare the electrophoretic properties of spermatozoa from cocks of lines of poor fertility with those from normal cocks.

The stable-flow free-boundary (Staflo) method has been used to characterize cell populations such as rat bone marrow 159 and rabbit spermatozoa (Chapters IV, V and VIII). The flexibility of this method provides an opportunity for either analytical or preparative studies using sedimentation or electrophoresis or a combination of the two. In addition, one can achieve a fractionation of spermatozoa populations using differing degrees of 
intrinsic motility at specified controlled temperatures.

In Section A, it was shown that fowl spermatozoa, which were subjected to cooling, dilution and storage at $3^{\circ} \mathrm{C}$ for $11 / 2$ hours in low ionic strength glycine-citrate medium at concentrations expected in Staflo electrophoresis experiments and then reconcentrated by centrifugation, gave a fertilization rate of $58.8 \%$, as compared to a control value of $63.2 \%$. This section reports a series of experiments which 1) confirm the conclusion from Section A that the fertilizing capacity of spermatozoa could be maintained during Staflo electrophoresis experiments; 2) characterize the spermatozoa populations with regard to average and extreme values of electrophoretic mobility; and 3) partially fractionate and relatively enrich the spermatozoa populations by utilizing a combination of electrophoresis and intrinsic motility.

\section{Materials and Methods}

a. Sample Preparation. Ejaculates were collected from mature White Leghorn cocks by abdominal massage 266 and pooled until $2.0 \mathrm{ml}$ or more of undiluted semen was obtained. A $0.5 \mathrm{ml}$ aliquot of pooled semen was diluted 1:2 with modified Krebs solution for a control specimen and the remainder was diluted 1:2 with glycine-citrate buffer. A $0.10 \mathrm{ml}$ aliquot was diluted $1: 100$ in a solution of $1 \%$ formalin in normal saline for counting in a hemocytometer. Both samples were cooled from room temperature to $2^{\circ} \mathrm{C}$ in 45 minutes. The glycine-citrate sample then was diluted to a spermatozoa concentration of approximately $250-300 \times 10^{6} / \mathrm{ml}$ and subjected to Staflo electrophoresis.

b. Staflo Apparatus. The essential features of the Staflo apparatus employed in these experiments are a $3 \times 16$ channel flow-cell (designated No. XI), duplicate 48-channel collection systems and a 16-channel, multisyringe pumping system. Sixteen $50 \mathrm{ml}$ plastic syringes on the pump rack 
contaln the density gradient solutions which are delivered by polyethylene tubing to the respective inlets on the flow-cell. A variable speed drive allows a wlde range of accurately controlled solution flow rates. A $20 \mathrm{ml}$ syringe is often used for the spermatozoa sample suspension in order to achleve an inftial sample stream thickness approximately $1 / 2$ that of other fluld layers with resulting greater resolution of the migrating species ("thin sample technique" ${ }^{229}$ ). The spermatozoa suspension is stirred magnetically to maintain a uniform concentration throughout the experiment.

The inlets to the flow-chamber are arranged vertically as shown in Figure 48, thereby forming flowing layers, each $0.125 \mathrm{~cm} \mathrm{high,} 0.80 \mathrm{~cm}$ wide and $30 \mathrm{~cm}$ long. The multiple, horizontally flowing layers are stabilized by hydrodynamic feedback principles, rather than by use of any solfd supporting medium ${ }^{156,159}$. The fluid in each of the 16 layers exits the flow-cell through 3 horizontal channels, thereby allowing separation of the faster moving fluid in the central region from the slower moving fluld near each sidewall. In this study, only the collection fractions from the central channel of the flow-cell were evaluated.

In these experiments, only 4 of the 6 electrode segments were activated giving an electric fleld across the downstream $2 / 3^{\prime} \mathrm{s}(20 \mathrm{~cm})$ of the flowcell as shown schematically in Figures 48 and 49.

c. Medium and Density Gradient. Using the low lonic strength glycinecitrate buffer described in Section A, a sucrose density gradient was established by weight/volume percent in the flowing layers as follows: No. 4, $1.0 \%$; No. $5,1.5 \%$; No. $6,2.0 \%$; No. $7,2.5 \%$; No. $8,3.0 \%$; No. $9,4.5 \%$; No. $10,4.8 \%$; No. $11,5.1 \%$; No. $12,5.4 \%$; and No. $13,5.7 \%$. Layers $1-3$ contained a higher conductivity medium resulting from a glycine-citrate content approximately 3 times that of layers 4-12. Layers 14-16 contained the 
STAFLO FLOW-CELL; UP'NARD ELECTROPHORESIS OF FOWL SPERM

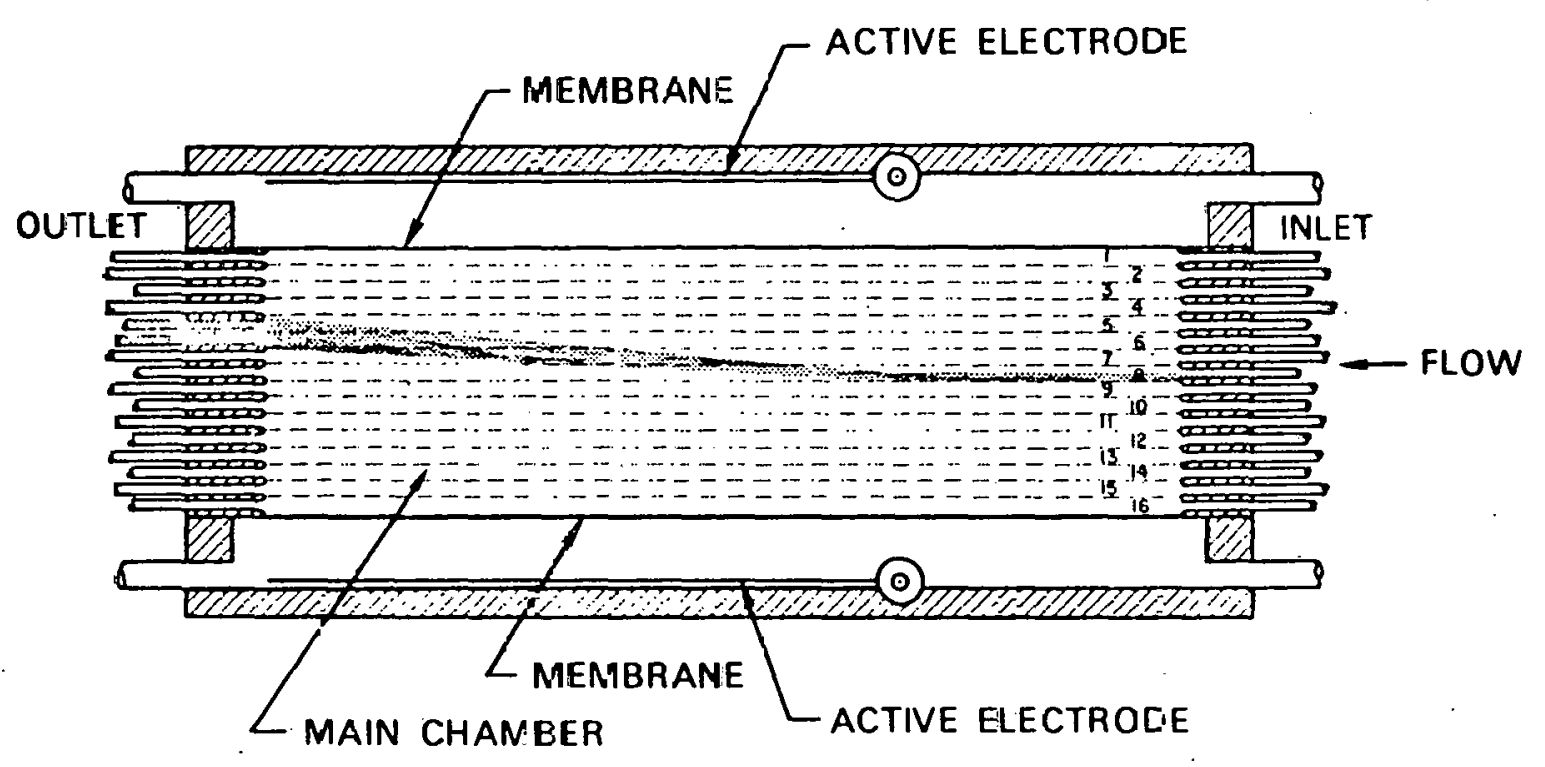

DBL 688-5417

Fig. 48. Schematíc diagram of Staflo Elow-cell illustrating Eow1 spermatozoa upward-electrophoresis migration pattern. 


\section{THREE-DIMENSIONAL STAFLO MIGRATION PATTERN}

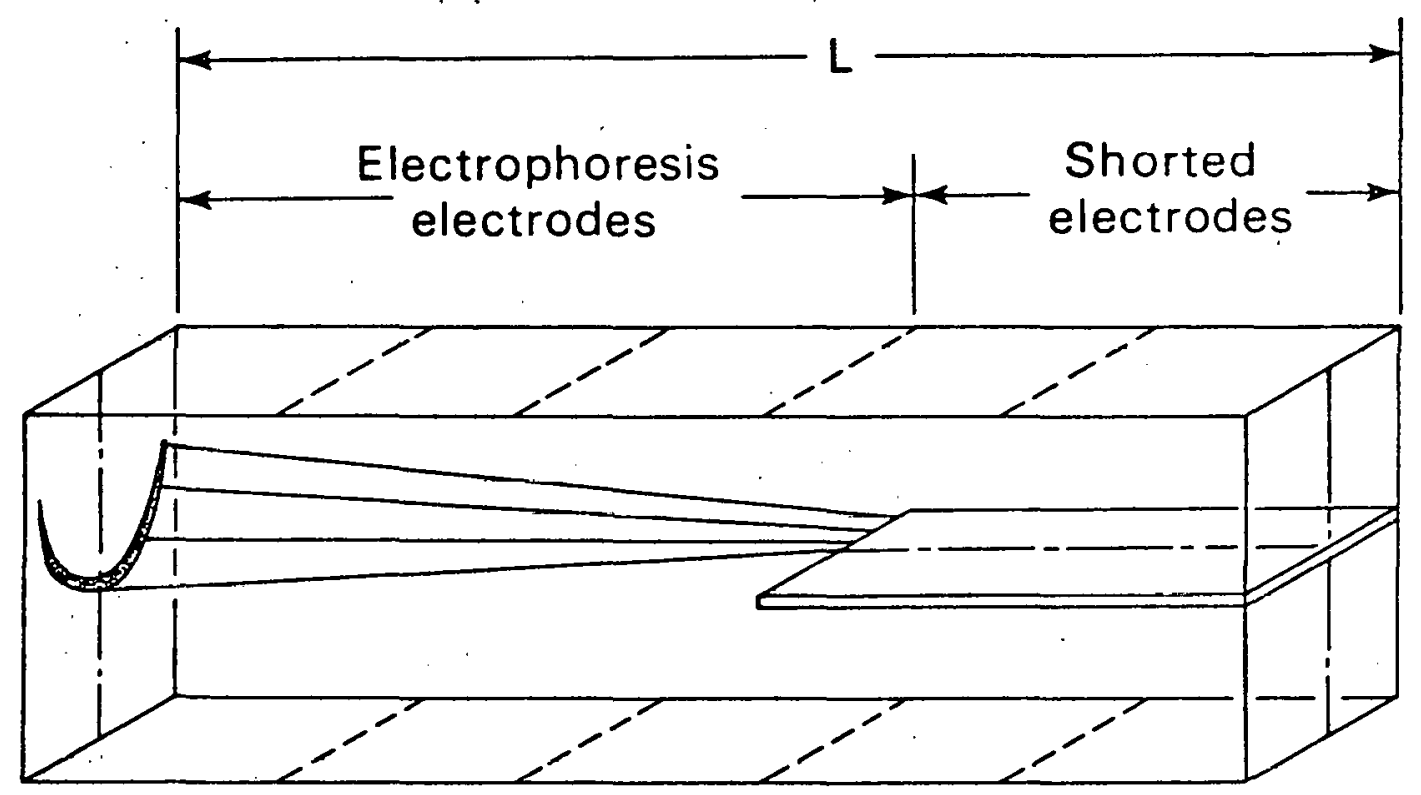

DBL $688-5381$

Fig. 49. Schematic diagram showing ideal three-dimensional Staflo upward-electrophoresis migration pattern of fowl spermatozoa. 
same medium. with $6 \%$ sucrose. The resulting top and bottom "conductivity barriers" buffer the intermediate layers against changes in $\mathrm{pH}$ during the experiment and confine the migrating species to the intermediate layers of the flow-cel1 ${ }^{159}$. Initial discontinuities between layers are smoothed by gradient-stabilizing diffusion of sucrose during the first $1 / 3(10 \mathrm{~cm})$ of the flow-chamber prior to reaching the region of the "programmed" electric field 229 . The spermatozoa suspension was introduced into layer 8 and was Initially supported by the "density shelf" provided by the sucrose increment from layer 8 to 9 .

d. Temperature Contro1. A11 experiments were conducted in a refrigerated room with good temperature regulation. Temperature measurements inside the flow-chamber during electrophoresis have shown the average temperature rise to be less than $0.5^{\circ} \mathrm{C}$ under the operating conditions used in these experiments.

e. Observation and Evaluation of Spermatozoa. The gross electrophoresis pattern was observed by eye, using the "slit lamp technique"229. Individual spermatozoa and the microscopic steady-state pattern were observed with a spectal mfcroscopic optical system developed for use with the Staflo apparatus (Chapter III). Immedlately after each run, the spermatozoa concentration in each collection container was determined with a hemocytometer. The spermatozoa in each fraction were reconcentrated by centrifugation in the cold room and subsequently evaluated at room temperature for motility and viability (nigrosin-eosin staining ${ }^{109}$ ). The quality of motility was judged on a scale of I-VI ranging from essentlally no motility (I) to a vigorously swarming pattern (VI). The nigrosin-eosin stained spermatozoa were categorlzed as unstained (viable) or stained (non-viable). The spermatozoa from each fraction and from the control sample were inseminated into separate groups of White Leghorn hens between 1300 and 1500 hours on the 
day of the experiment.

3. Results

Two groups of electrophoresis experiments are reported. The first group was conducted at $4^{\circ} \mathrm{C}$ or lower to minimize the effect of intrinsic motility on migration. The second group of experiments was conducted at $5.5^{\circ} \mathrm{C}$ in a deliberate attempt to achieve enrichment of one collection fraction with regard to motile spermatozoa. Figure 48 illustrates the migration pattern (overall side-view appearance of a continuously flowing sample stream in the flow-chamber) observed in both sets of experiments. The parabolold migration profile (cross-section of the migration pattern) observed with the "slit lamp technique" is illustrated in Figure 49 . Vertical migration distances measured in the longitudinal centerline by this technique were used to calculate the electrophoretic mobility values.

a. Electrophoresis Experiments. General observations during the series of experiments at temperatures of $4^{\circ} \mathrm{C}$ or lower indicated that 1 ) the migration patterns determined by the "slit lamp technique" were essentially the same at spermatozoa concentrations of $25 \times 10^{6}$ and $250 \times 10^{6} / \mathrm{ml}$; 2) in all experiments, the helght of the migration profile at the outlet in the centerline of the flow-chamber was less than $2.0 \mathrm{~mm}$, thereby giving a 2-layer fractionation of the sample; 3) at $\mathrm{pH} 7.0$ and with the top electrode positive, all spermatozoa were oriented vertically with heads down and talls upward; and 4) at a flow-chamber temperature of $4^{\circ} \mathrm{C}$, a high percentage of Individual spermatozoa showed a moderate degree of tail-wagging.

Despite an attempt to effect an even division of the sample between the 2 layers at the outlet, the upper collection fraction generally had a lower spermatozod concentration than did thebottom nne. In all experiments, the percentage and degree of motility in thebotton fraction were consistently 
greater than in the upper one (though less than in the control sample). In one experiment, the upper fraction, containing only $10 \%$ of the spermatozoa, at a concentration of $10 \times 10^{6} / \mathrm{ml}$ had only $1-2 \%$ motile spermatozoa as compared to about $70 \%$ motility in the bottomfraction. When comparable numbers of spermatozoa were collected in the 2 fractions, for experiments at $4^{\circ} \mathrm{C}$ or lower, there were no significant differences in viability (staining) among the 2 fractions and the control spermatozoa. Even though the spermatozoa fractions in several experiments had Grade II or III. motility, insemination of $150-200 \times 10^{6}$ spermatozoa $1 \mathrm{n} 0.15 \mathrm{ml}$ failed to give any fertile eggs. Control inseminations with Grade III or IV spermatoroa did give fertile eggs. Possible reasons for the loss of fertilizing capacity of the spermatozoa in the collection fractions in this first group of experiments are considered in the Discussion.

The average and ranges of apparent electrophoretic mobilities for one palr of electrophoresis runs at $4^{\circ} \mathrm{C}$ with the same Staflo operating conditions but differing spermatozoa concentrations are shown in Table XIV.

Table XIV. Apparent Electrophoretic Mobilities of Fow1 Spermatozoa at $4^{\circ} \mathrm{C}$.

\begin{tabular}{llr}
\hline $\begin{array}{c}\text { Spermatozioa } \\
\text { Concentration }\end{array}$ & $\begin{array}{c}\text { Apparent Electrophoret1c Mobility } \\
\left.\text { [Unfts of } 10^{-4}(\mathrm{~cm} / \mathrm{sec}) /(\mathrm{V} / \mathrm{cm})\right] \\
\text { Range }\end{array}$ \\
\hline $25 \times 10^{6} / \mathrm{ml}$ & 1.56 & $1.50-1.61$ \\
$250 \times 10^{6} / \mathrm{ml}$ & 1.69 & $1.50-1.79$ \\
\hline
\end{tabular}

b. Electrophoresis-Enrichment Experiments. Two enrichment experiments were conducted using upward electrophoresis at $5.5^{\circ} \mathrm{C}$. Figure 50 is a photograph of the steady-state migration pattern during one of these experiments. 


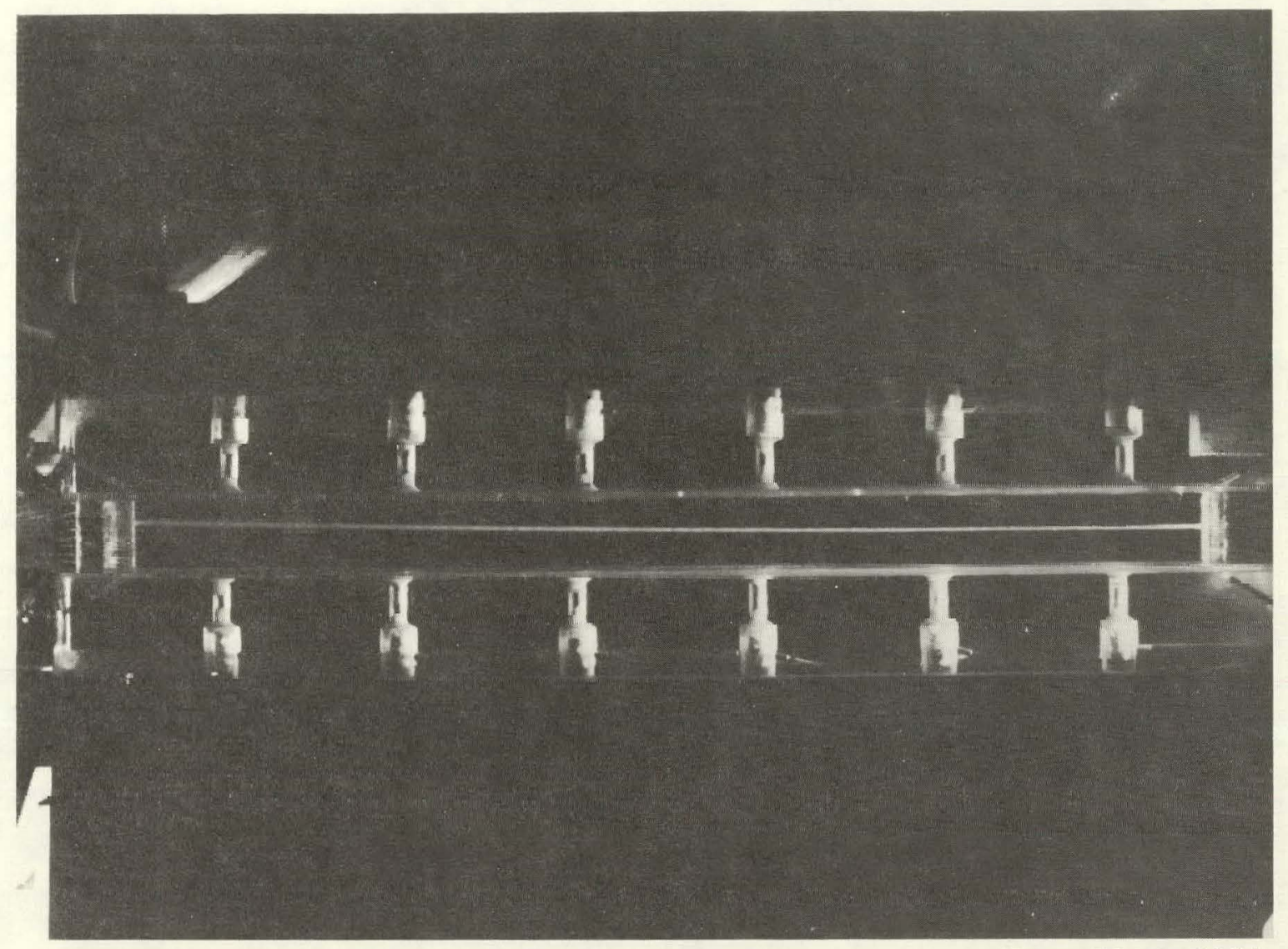

XBB 7011-5006

Fig. 50. Photograph of upward-electrophoresis migration pattern with fow1 spermatozoa in a $3 \times 16$ Staflo apparatus enrichment experiment at $5.5^{\circ} \mathrm{C}$. 
Agaln, because the pattern spread was small, only 2 fractions were obtained (containers 5 and 6 ). The spermatozoa in the fractions were reconcentrated by centrifugation for 5 minutes at $1,400 \mathrm{~g}$ at $5^{\circ} \mathrm{C}$ prior to evaluation at room temperature, and artificial insemination. The same 3 groups of 6 hens each were insemlnated on each occasion with the respective collection fractions or control spermatozoa. Eggs were collected from the day after the first insemination unt1l 4 days after the second insemination (a total of 8 days). The fractionation results with respect to spermatozoa distribution, motility, viability, fertility and hatchability are summarized in Table XV.

In Table XV, it is seen that fraction 6 has both a higher concentration and a better quality of spermatozoa than fraction 5, judged by mot1lity and viability. In addition, the percentages of fertility and hatchability for fraction 6 are significantly higher than those for fraction $5(P<0.01$ in a one-tall test ${ }^{90}$ ). There was no difference between fraction 6 and the control with regard to motflity, viabilfty, fertility or hatchability. Following one of these experiments, which used a concentration of $250 \times 10^{6}$ spermatozoa/m1, a comparison run was made using $1 / 10$ the initial concentration. Although the pattern spread was slightly greater for the higher concentration, the average migration distances were the same. The mobility, calculated from the average migration distance was slightly greater $(\approx 2 \times$ $10^{-4} \mathrm{~cm} / \mathrm{sec} / \mathrm{V} / \mathrm{cm}$ at $5.5^{\circ} \mathrm{C}$ ) than in Table XIV. However, the measurements in Table XIV are considered more rellable because the migration distances Involved were greater.

\section{Discussion}

a. Technical Considerations. In view of the report by Proudfoot and Stewart ${ }^{189}$ that the fertilizing capacity of fowl spermatozoa did not appear 
Table XV. Average Enrichment-Fractionation and Artificial Insemination Results of Two Electrophoresis Experiments with Fowl Spermatozoa at $5.5^{\circ} \mathrm{C}$.

Fraction $5 \quad$ Fraction $6 \quad$ Control

Sperm Conc. (units of $10^{6} / \mathrm{m} 1$ )

87

140

1500

Percent of Sperm Collected:

Average

Range

Mulplily (ytade)

Viability (unstained) $(\%)$

Artificial Insemination:

Volume (m1)

No. of Sperm (units of $10^{6}$ )
38.3

$36.2-39.8$

II

77
61.7

$60.2-63.8$

IV

90.5

Evaluation of Eggs:

Total Number

39

0.15

0.15

0.10

Number Fertile

5

170

150

Percent Fertile

Number Hatchable

12.8

3

7.7

46

46

26.

25

Percent Hatchable

56.6

19

$41 \cdot 3$
IV

91.8 
to be affected by a rapid temperature fall during collection, cooling from room temperature to $3^{\circ} \mathrm{C}$ was accomplished in 45 minutes in these experiments as compared to 2 hours in Section A. The fertilization rates in Table XV are comparable to those in the dilution experiments in Section $A$ and confirm the report that rapid cooling is not detrimental.

Another consideration to bear in mind in comparing the results of Sections $A$ and $B$ is the $25^{\circ}-45$ minute shorter centrifugation time used In the present experiments. This reduction was possible in part because the collection fractions contained only 8 - $10 \mathrm{ml}$ compared to the $15-20 \mathrm{ml}$ volumes used in Section A.

It was noted that for several of the earlier "electrophoresis experiments" no fertile eggs were obtained following insemination of adequate numbers of reconcentrated spermatozoa of Grade II or III motility. Control inseminations, however, gave fertile eggs. Furthermore, in the subsequent "electrophoresis enrichment experiments"; a fertilization rate of $56.6 \%$ was achieved from Staflo collection fraction 6 as compared with the $54.4 \%$ control value. It is possible that the loss of fertilizing capacity (but not motility) in the early experiments was due to trace contamination of the apparatus either by Roccal (bengalkonium chloride) used in cleaning or by a toxic substance assoclated with new polyethylene tubing.

\section{b. Electrophoretic Mobility Measurements. Even though there was good} agreement between the average values of electrophoretic mobility for the simflar pairs of high and low concentration runs, the range of mobilities was somewhat greater for both high concentration experiments. The greater spread In the steady-state migration pattern of the high concentration runs may reflect a small degree of density gradient instability. (Alternatively, this may be a consequence of the relative difficulty in defining 
the limits of the low concentration migration pattern). Only having a slight instability at a concentration as high as $250 \times 10^{6}$ spermatozoa/m1 is remarkable in view of the threshold for instability of $25 \times 10^{6} / \mathrm{ml}$ for rabbit spermatozoa in other density gradients (Chapters IV, V and VIII). The increased stability in the fowl spermatozoa experiments may be attributed to 1) a larger increment in the density gradient between layers (0.5\% sucrose/layer vs. $0.10-0.15 \%$ sucrose/layer for rabbit spermatozoa experiments) and 2) a lower sedimentation rate for fowl spermatozoa ${ }^{35}$.

In addition to the apparent electrophoretic mobility of $1.69 \times 10^{-4}$ $\mathrm{cm} / \mathrm{sec} / \mathrm{V} / \mathrm{cm}$ for the $250 \times 10^{6} / \mathrm{ml}$ spermatozoa sample shown in Table XIV, an average value of $1.52 \times 10^{-4} \mathrm{~cm} / \mathrm{sec} / \mathrm{V} / \mathrm{cm}$ was obtained in another experiment under simflar conditions, at $4^{\circ} \mathrm{C}$. When these values are normalized to conditions of a $1 \%$ sucrose density gradient at $3^{\circ} \mathrm{C}$, they become $1.75 \times 10^{-4}$ and $1.57 \times 10^{-4} \mathrm{~cm} / \mathrm{sec} / \mathrm{V} / \mathrm{cm}$, respectively. These latter values compare favorably with the $1.68 \times 10^{-4} \mathrm{~cm} / \mathrm{sec} / \mathrm{V} / \mathrm{cm}$ observed in a downward electrophorests experiment using the glycine-phosphate buffer described in Section A, corrected to $1 \%$ sucrose at $3^{\circ} \mathrm{C}$ (Chapter V). They also agree well with the value of $1.55 \times 10^{-4} \mathrm{~cm} / \mathrm{sec} / \mathrm{V} / \mathrm{cm}$ reported by $B$ ey, also in a low ionic strength medium 251 .

It is difficult to evaluate precisely the relative electrophoretic mobilities of live and dead spermatozoa because even at $4^{\circ} \mathrm{C}$, a mild degree of tall-wagging was observed in the flow-chamber. It was noted that in such low temperature experiments, where substantial numbers of spermatozoa were found in both collection fractions, there was no difference between them in staining properties. In contrast, in the enrichment experiments at $5.5^{\circ} \mathrm{C}$, a significant difference in viabllity was observed between fractions 5 and 6 as shown in Table XV. This difference probably results from the 
greater degree of intrinsic motility of (viable) spermatozoa at $5.5^{\circ} \mathrm{C}$.

The electrophoretic mobility of immotile (non-viable) spermatozoa at $4^{\circ} \mathrm{C}$ can be estimated from the experiment in which the upper collection fraction contained $99 \%$ immotile spermatozoa, at a concentration of $10 \mathrm{x}$ $10 \%$ m 1 . Because 1 was shown in section $A$ that concentrations as low as $8 \times 10^{6} / \mathrm{ml}$ could be tolerated for up to 6 hours at $3^{\circ} \mathrm{C}$, it is probable that the spermatozoa were immotile at the beginning of the experiment. The average electrophoretic mobility of these immotile spermatozoa was $1.67 \times 10^{-4} \mathrm{~cm} / \mathrm{sec} / \mathrm{V} / \mathrm{cm}$ compared to average and minimum apparent mobility values of $1.52 \times 10^{-4}$ and $1.36 \times 10^{-4} \mathrm{~cm} / \mathrm{sec} / \mathrm{V} / \mathrm{cm}$ for the whole sample. Theoretically, at least, the minimum value represents the slowest electrophoresing-fastest swimming spermatozoa. In order to define the true contribution of motility to the net migration, experiments should be conducted at temperatures ranging from less than $1^{\circ} \mathrm{C}$ to about $15^{\circ} \mathrm{C}$, and with lower spermatozoa concentrations to insure that the migration is not influenced. by any kind of density gradient instability.

c. Relative Enrichment. Because of the superior quality of the initial spermatozoa suspensions (Table XV), absolute enrichment of a collection Eraction, compared to the control sample, was not achieved. However, with an Intial sample of about $50 \%$ motile spermatozoa rather than $90 \%$, especially if a slightly higher temperature and/or greater migration distance is used, absolute enrichment would be expected. Such enrichment has been obtained in experiments with rabblt spermatozoa (Chapter IV). It is significant that despite the additional stresses of dilution, electrophoresis and centrifugation, the spermatozoa in collection fraction 6 were as good as the control sample by all of the criteria in Table XV.

Because the minimum. spermatozoa concentration encountered in the en- 
richment experiments was comparable to those at which motility and fertilizing capacity were shown to be maintained in Section $A$, all of the differences between fractions 5 and 6 in Table XV are most likely a consequence of intrinsic motility. Thus, at $5.5^{\circ} \mathrm{C}$ in the glycine-citrate medium, the downward oriented intrinsic motility vector is believed to have been of suffictent magnitude to give the depletion in layer 5 and the relative enrichment in layer 6 with regard to all of the criteria enumerated in Table XV.

The fertilization rate of $56.6 \%$ obtained with spermatozoa from collection fraction 6 in the enrichment experiments is essentially the same as the $58.8 \%$ from spermatozoa diluted 1:30 in glycine-citrate buffer, stored at $3^{\circ} \mathrm{C}$ for $11 / 2$ hours and reconcentrated by centrifugation prior to insemination (Section $A$, Table XIII). The difference between the respective control fertilization rates of $54.4 \%$ and the $63.2 \%$ in the 2 studies was also not significant $(P \gg 0.10)$. Therefore, it can be said that staflo electrophoresis has not adversely affected the motility and fertilizing capacity of the spermatozoa.

The combined results of Sections $A$ and $B$ have shown that analytical experiments can be conducted at $3^{\circ} \mathrm{C}$ using spermatozoa concentrations as low as $8 \times 10^{6} / \mathrm{ml}$, and that the results can be exploited in Staflo electrophoresis or electrophoresis-enrichment experiments. A natural extension of the present fractionation studies is the evaluation of the fertilizing ability of sub-populations of spermatozoa characterized by differing degrees of intrinsic motility, obtained by differential fractionation in Staf1oelectrophoresis-motility or sedimentation-motility experiments. With regard to fowl spermatozoa, this extension would be of particular interest in studying the "translocation heterozygote" spermatozoa. For mammalian 
spermatozoa, there is the additional possibility of obtaining information on the relationship of motility to the sex ratio of offspring.

\section{Summary and Conclusions}

Although both egg yolk medium and vitalizing medium preserved the mot1lity of fowl spermatozoa during storage for 2 hours at $4^{\circ} \mathrm{C}$, vitalizing medium was far superior in preserving the fertilizing capacity ( $80 \%$ vs. $9.1 \%$ for egg yolk medium).

310 mosm solutions of low lonic strength glycine-citrate and glycinephosphate buffers were far superior to 368 and 427 mosm solutions in the preservation of motility during storage at $3^{\circ} \mathrm{C}$ for spermatozoa concentrations of $8-80 \times 10^{6} / \mathrm{m} 1$. After storage for 6 hours in the 310 mosm solutions, there was only a $15 \%$ decrement from initial motility values at all concentrations. The percentages of motility were much lower in the 368 and 427 mosm solutions.

In regard to the preservation of fertilizing capacity there was no difterence between the glycine-citrate and glycine-phosphate buffers after storage at $1: 30$ dilutions for $11 / 2$ hours at $3^{\circ} \mathrm{C}$. In a second study, the preservation of fertilizing capacity of spermatozoa after storage at 1:30 dilutions in glycine-citrate medium for $11 / 2$ hours at $3^{\circ} \mathrm{C}$, followed by reconcentration by centrifugation, was the same as that of spermatozoa diluted 1:2 in vitalizing medium and stored under the same time-temperature conditions without further handling. This suggested that fow1 spermatozoa could be subjected to the stresses of low temperature, low concentration and centrifugation expected in Staflo electrophoresis experiments, with low ionic strength media, and still malntain their vital functions. This was confirmed in the enrichment-electrophoresis experiments where a fertilization rate of $56.6 \%$ was obtained with spermatozoa in the lower collection 
fraction, compared to $54.4 \%$ for the control.

The electrophoresis experiments showed the same average electrophoretic mobllities for fowl spermatozoa at concentrations of $25 \times 10^{6}$ and $250 \times 10^{6} / \mathrm{ml}$ and showed comparable mobllities for upward and downward electrophoresis. The higher concentration gave an apparently slightly greater spread of the migration pattern with associated greater range of electrophoretic mobilities. The average apparent electrophoretic mobility for fowl spermatozoa in these experiments is about $1.6 \times 10^{-4}(\mathrm{~cm} / \mathrm{sec}) /(\mathrm{V} / \mathrm{cm})$. Th1s value compares favorably with those obtained in Chapter $V$ (Table X).

At a flow-chamber temperature of $5.5^{\circ} \mathrm{C}$, relative enrichment in the lower of 2 collection fractions, with an upward-electrophoresis migration pattern, was obtalned with regard to motility, viability, fertilizing capacity and hatchability. The enrichment is attributed to the intrinsic motility of the spermatozoa at that temperature: Because of the high quality of the inftial spermatozoa sample, the lower fraction did not differ from the control, according to these criteria.

These studies showed that the motility of fowl spermatozoa could be well preserved after storage for 6 hours at $3^{\circ} \mathrm{C}$ in concentrations as low as $8 \times 10^{6} / \mathrm{ml}$ in low lonic strength media - conditions desirable for analytical electrophoresis studies. Furthermore, the excellent fertilization results with "Staflo spermatozoa" In the enrichment experiments show that information learned in analytical studies can be exploited in preparative electrophoresis experiments using the Staflo apparatus. 
VII. EQUILIBRIUM DENSITY GRADIENT CENTRIFUGATION:

RABBIT AND FOWL SPERMATOZOA

Contents

A. Introduction. . . . . . . . . . . . . 208

B. Materials and Methods ............... 209

1. Media and Density Gradients ........... 209

2. Centrifugation. ............... 209

3. Evaluation of Spermatozoa ............ 210

C. Results ................. . . 210

1. Dextran Gradient Experiments. . . . . . . . . 210

2. Sodium Iothalamate Gradient Experiments . . ...... 211

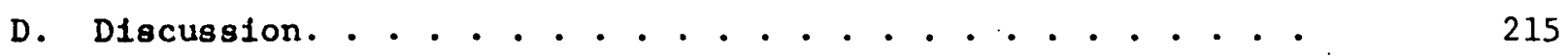

1. Technical Considerations. . . . . . . . . 219

2. Comparison of Fowl and Rabbit Experiments....... 219

3. Correlation with Staflo-Sedimentation Studies .... 222

4. Comparison with Work by Other Investigators . . . . . 223

E. Sumary and Conclusions . . . . . . . . . . . . 225

\section{A. Introduction}

The review of the 1iterature on the centrifugation of spermatozoa in Chapter II, A, 4, summarizes the work by many investigators to determine the apparent buoyent density of bull and rabbit spermatozoa. There were marked differences in the reported speciflc gravities determined in different density gradients. In order to better understand the behavior of rabbit and fowl spermatozoa in Staflo sedimentation and sedimentationelectrophoresis experiments, the work in this chapter was done to determine the magnitude and range of specific gravities of both rabbit and 
fowl spermatozoa. At the time this work was done (summer, 1966), the reports by Benedict, Schumaker and Davies ${ }^{32}$, and by Lavon, Volcani, Amir and Danon 132,133 had not been published.

Th1s chapter presents results of equilibrium density gradient centrifugation experiments with rabbit and fowl spermatozoa using both dextran and sodium lothalamate density gradients. The studies showed both similarities in the specific gravity of fowl and viable rabbit spermatozoa and marked differences in the properties of the spermatozoa of the 2 species when under the influence of osmotic stress.

\section{B. Materials and Methods}

\section{Media and Dens1ty Gradients}

Bovine serum albumin (BSA), dextran (Sigma Chemical Company, MW $15-20,000 ; 73,000$ and 215,000$),$ Ficoll and several radiographic contrast media were evaluated for use in equilibrium density gradient centrifugation. In general, sufficiently dense solutions of BSA, Ficoll and dextran could not be prepared without encountering limiting degrees of viscosity. The work reported below was accomplished with density gradients prepared with dextran or sodium lothalamate (Ang1o-Conray or Conray-400) in $0.3 \%$ or $0.9 \% \mathrm{NaCl}$. Because the motility of both fowl and rabbit spermatozoa was not maintained well in $0.3 \%$ saline-sodium iothalamate solutions, $0.9 \%$ saline solutions were used for most experiments. The effects of radiographic contrast media on the motility of fowl spermatozoa and on the motility and fertlizing capactty of rabbit spermatozoa are reported in Appendix D.

\section{Centrifugation}

Linear density gradients were preformed at room temperature with a gradient machine using dextran or sodium lothalamate in normal saline. 
The centrifuge tubes then were cooled to approximately $3^{\circ} \mathrm{C}$ in a refrigerated room. Spermatozoa, which had been collected and cooled slowly to $3^{\circ} \mathrm{C}$ as described in Chapter III, were suspended in a gradient material solution slightly less dense than the top of the linear density gradient. The suspension was layered on the density gradient and the boundary was stirred carefully to eliminate the interface. Centrifugation was accomplished at $4^{\circ} \mathrm{C}$ with the Beckman Model L U1tra-centrifuge using the SW39 swinging-bucket rotor. After centrifugation, the tubes were examined in the cold room where the migration bands were measured, photographed and fractionated by pipetting. After removal from the centrifuge tubes, spermatozoa in the separate bands were resuspended in approximate1y $25 \mathrm{ml}$ of a $0.5 \%$ glucose-normal saline solution and were centrifuged for 15 minutes at 3,300 rpm in a clinical centrifuge. The pellet of spermatozoa was resuspended in egg yolk medium (see Chapter VI) and evaluated for motility and vital stalning characteristics. The total time of exposure of the spermatozoa to the sodium lothalamate, when it was used, ranged from 2b-3b minutes, approximately 20 minutes longer than the centrifugation time.

\section{Evaluation of Spermatozoa}

Spermatozoa were evaluated by phase contrast microscopy and by nigrosin-eosin stainfing as described in Appendix B. No artificial insemInation studies were done in the experiments described in this chapter.

\section{Results}

1. Dextran Gradient Experiments

a. Fow1 Spermatozoa. In preliminary experiments using a linear density gradient of $15-20,000 \mathrm{MW}$ dextran and a force of $1,500 \mathrm{~g}$ in an International Centrifuge, a centrifugation time of nearly 60 minutes was required for 
fowl spermatozoa to reach equilibrium. Fowl red blood cells, on the other hand, reached equilibrium in 15 minutes or less. The apparent specific gravitles for spermatozoa and red blood cells were $1.15-1.17$ and 1.14 , respectively.

b. Rabbit Spermatozoa. Centrifugation of 2 samples of rabbit spermatozoa In Inear density gradients of $15-20,000 \mathrm{MW}$ dextran in a phosphate buffered medium gave low-density bands corresponding to a speciflc gravity range of 1.16-1.21. Spermatozoa also were found at the bottom of the centrifuge tube which corresponded to a specific gravity of 1.23. Because some spermatozoa appeared to have a specific gravity greater than the maximum specific gravity of the solution (1.23) and the viscosity of this dextran solution was very high, sodium iothalamate was used in subsequent studies.

\section{Sodium Iothalamate Gradient Experiments}

a. Fowl Spermatozoa. The results of the centrifugation of fractions of Individual ejaculates from White Leghorn cocks are presented in Figure 51 and Table XVI. Figure 51A shows that, with centrifugation times of 5 , 9 , and 15 minutes at $5,800,6,600$ and $6,700 \mathrm{~g}$, respectively, the apparent specific gravity of spermatozoa from the same efaculate increased progressively to a maximum range of $1.19-1.21$ at the 15 minute centrifugation time. The last 4 ranges of apparent speciflc gravities shown in Figure 51 A agree within the accuracy of measurements in these experiments. Figure 51 B shows the results of 3 centrifugation runs with the same fow 1 spermatozoa sample using a density gradient prepared with sodium iothalamate in $0.3 \%$ saline. The $0.3 \%$ saline was used to reduce the osmolarity of the linear density gradient. There appears to be a slight increase in the apparent specific gravity with an increase in centrifugation time from $61 / 4$ to $103 / 4$ minutes but no further increase with time. The maximum range of speciflc gravities is $1.19-1.20$, slightly less than in the gradient 
RANGES OF APPARENT SPECIFIC GRAVITIES OF FOWL SPERMATOZOA

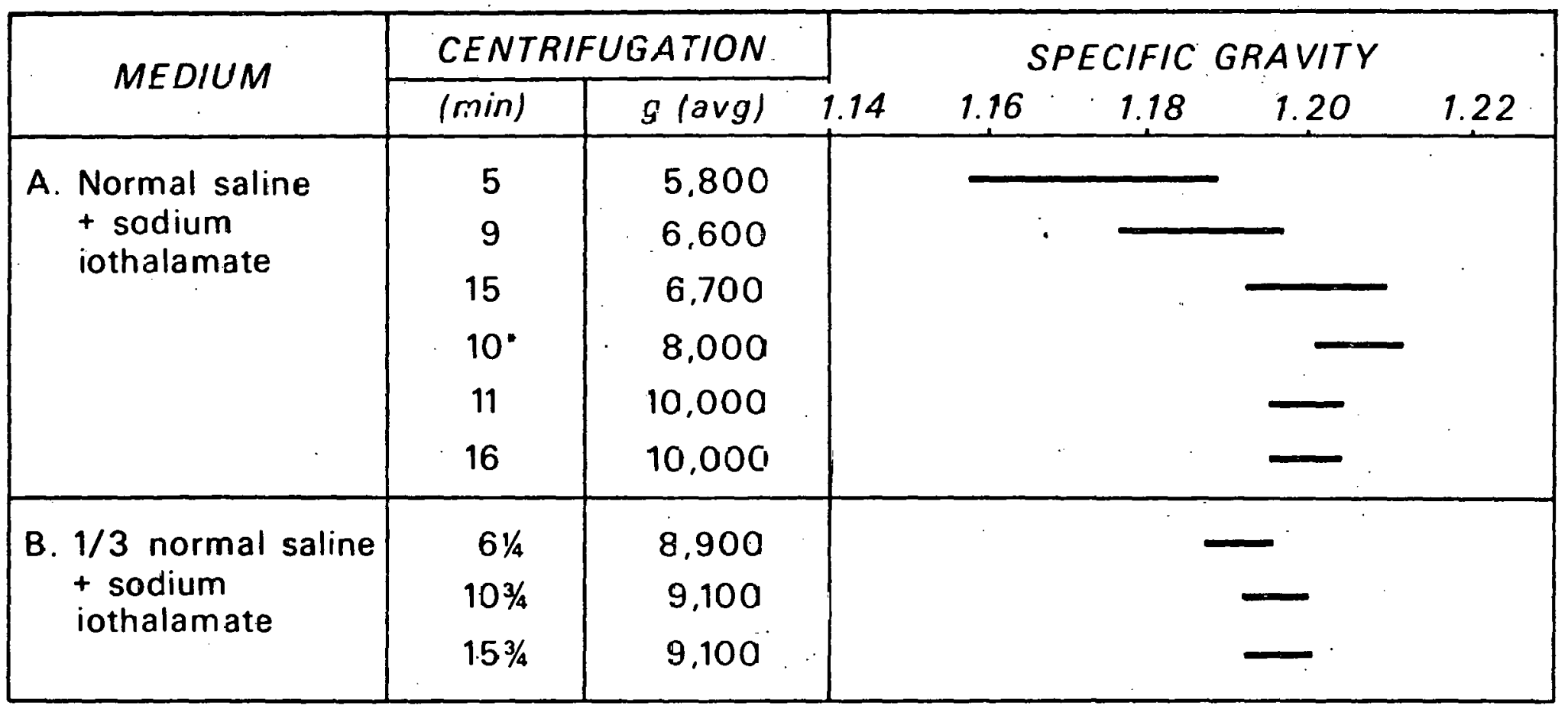

* 2 tubes

Fig. 51. Ranges of apparent specific gravities of fowl spermatozoa in sodium iothalamate density gradients in Ai normal saline and B) one--hird normal seline background solutions.

DBL 689-5469 
Table XVI. Ranges of Apparent Specific Gravities of Fowl Spermatozoa in Sodium Iothalamate Density Gradients.

\begin{tabular}{|c|c|c|c|c|}
\hline \multicolumn{3}{|c|}{ Centrifugation } & \multicolumn{2}{|c|}{ Specific Gravity } \\
\hline Time & $(\min )$ & $g\left(a v_{.}\right)$ & Range & Average \\
\hline A. Norma & 1 Salin & & & \\
\hline & 5 & 5,800 & $1.158-1.189$ & 1.174 \\
\hline & 9 & 6,600 & $1.176-1.197$ & 1.187 \\
\hline & 15 & 6,700 & $1.192-1.210$ & 1.201 \\
\hline & $10(2)$ & 8,000 & $1.201-1.212$ & 1.207 \\
\hline . & 11 & 10,000 & $1.195-1.205$ & 1.200 \\
\hline & 16 & 10,000 & $1.195-1.205$ & 1.200 \\
\hline
\end{tabular}

B. $1 / 3$ Normal Saline

$\begin{array}{cccc}6,1 / 4 & 8,900 & 1.187-1.196 & 1.192 \\ 103 / 4 & 9,100 & 1.192-1.200 & 1.196 \\ 153 / 4 & 9,100 & 1.192-1.201 & 1.197\end{array}$


using $0.9 \%$ saline.

Fowl spermatozoa gave a continuous band in the density gradient, generally 2-4 mim wide. The narrowest bands were obtalned with the highest quality ejaculates and, after centrifugation and washing, most spermatozoa still were intact and had a high degree of motility. With poor quality ejaculates, a differential distribution of spermatozoa and component parts thereof was observed. Above the top of the discrete band, there were sperm tails. In the top of the band, spermatozoa were intact and had excellent mot1lity. In the bottom of the band, spermatozoa showed signs of deterforating and had efther a poor degree of motility or were 1mmotile. At the bottom margin nf the band and olightly below, all syytuatozoa were deteriorating or were represented by talls with remnants of heads. Deterforation was manifested first by swelling of the sperm head to 2-3 times its normal width, later by the formation of bead-like masses of nuclear material and finally by scattering of the bead-like material, leaving the tail with a knob projecting toward the former head region. Rreause of this fruglifey of tilu luwl spermacozoa heads, no intact heads were ever observed separated from talls. Swelling and disintegration of fowl spermatozoa heads was also observed after sturage for 24 hours or more in egg yolk and other media, suggesting that this deterioration is a general phenomenon. Distortion of this differential distribution was observed In the presence of clumping for it appeared thal vlable spermatozoa were carried to the lower margin of the band by clumps. No clumps were ever observed in the upper part of the band.

In fresh ejaculates of poor quality, several percent of the spermatozoa were bent in the vicinity of the neck plece, giving a hairpin shape. In centrifuged samples, the frequency of this abnormality was 
greatly increased. Despite this damage, many such individual spermatozoa often had a high degree of motility.

b. Rabbit Spermatozoa. The results of rabbit spermatozoa experiments are summarlzed in Figure 52 and Table XVII. Three or more discrete bands were observed in each experiment. Spermatozoa from the same ejaculate were used in the first 3 experiments in Figure 52 (and Table XVII). It Is seen that the apparent specific gravity of the upper band increases with Increasing centrifugation time unt1l a range of $1.195-1.210$ is reached. This range was observed even with centrifugation for 90 minutes at $27,000 \mathrm{~g}$. The middle band appears to plateau at a specific gravity range of 1.26-1.29. There is a suggestion that the apparent specific gravity of the lower band continues to increase over the range 1.31-1.37, but the formation of sub-bands in that range complicates interpretation thereof.

In addition to the differences in specific gravity, there was a marked difference in the quality of the spermatozoa in the 3 bands. Table XVIII A summarizes the results of one experiment in which the upper and lower bands were at the top and bottom of the density gradient. There is a dramatic decrease in the quality of the spermatozoa, judged by vital staining, morphology and motility, from top to bottom. By all criteria, the upper band was enriched with regard to the initial sample. This differential distributinn tis confirmed by the phase contrast observations of morphology and motility for a second experiment, as shown in Table XVIII B. Again, there is a marked difference between the upper and lower bands and absolute enrichment of the upper band.

\section{Discussion}

The results of the experiments reported above are considered in terms 


\section{RANGES OF APPARENT SPECIFIC GRAVITIES OF RABBIT SPERMATOZOA}

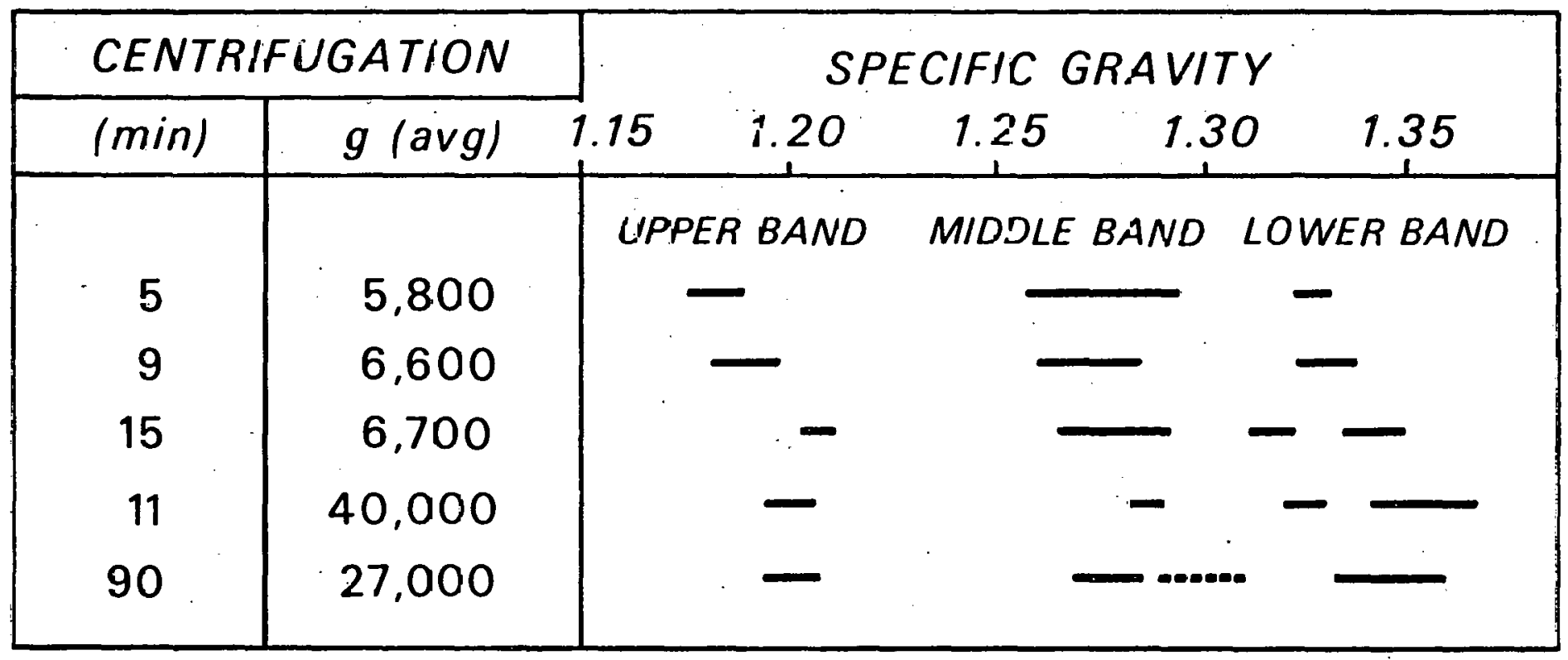

DBL 689-5468

Fig. 52. Ranges of apparent specific gravities of rabbit spermatozoa in a sodium iothalamate density gradient. 
Table XVII. Ranges of Apparent Specific Gravities of Rabbit Spermatozoa in a Sodium Iothalamate Density Gradient in $0.9 \%$ Saline.

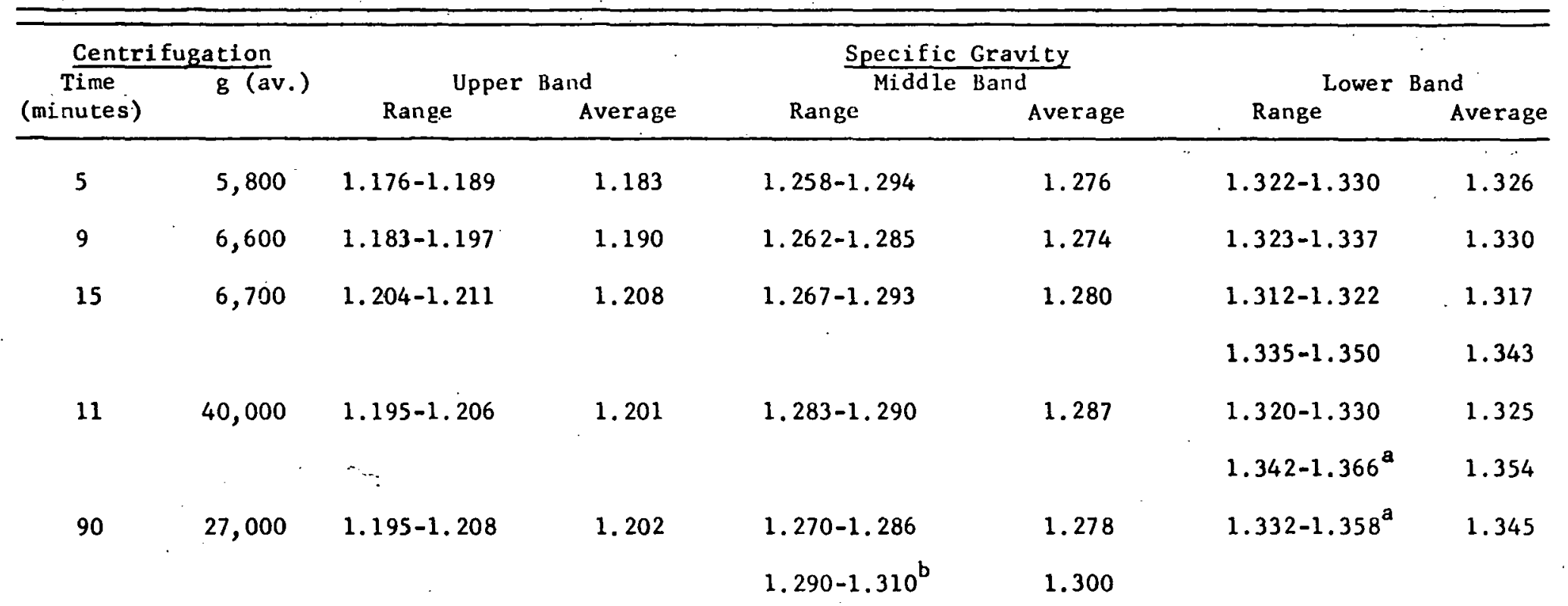

a. A very small number of spermatozoa were slightly lower in the gradient.

b. Second part of band was thinly scattered. 
Table XVIII. Viability and Morphology of Rabbit Spermatozoa in a Linear Density Gradient. ${ }^{a}$

\begin{tabular}{cc|ccc|cccc}
\hline \hline Sample & Percent of & \multicolumn{2}{|c|}{ Nigrosin-Eosin } & Staining & \multicolumn{2}{|c|}{ Phase contrast } & \\
& Sperm & $\mathrm{U}$ & MNS & $\mathrm{S}$ & $\mathrm{C}$ & $\mathrm{CL}$ & $\mathrm{FM}$ & $\mathrm{TW}$ \\
& & $(\%)$ & $(\%)$ & $(\%)$ & $(\%)$ & $(\%)$ & $(\%)$ & $(\%)$ \\
\hline
\end{tabular}

A. Specimen $\| 1$

$\begin{array}{lcccccccc}\text { Upper tand } & 44.0 & 91.5 & 4.5 & 1.0 & 94 & 6 & 11.6 & 29.5 \\ \text { Int-band 1) } & 3.4 & 65.5 & 18.2 & 16.3 & 68.2 & 31.8 & 0 & 1 \\ \text { Int-band 2) } & 2.5 & 22 & 16 & 62 & 53.2 & 46.8 & 0 & 0 \\ \text { Lower band } & 50.1 & 6.0 & 13.5 & 81.5 & 15.5 & 84.5 & 0 & 0 \\ \begin{array}{l}\text { Initial } \\ \text { Sample }\end{array} & 100 & 37 & 12 & 51 & 65.2 & 34.8 & 7.5 & 17.5 \\ & & . & & & & & & \end{array}$

B. Specimen 非2

\begin{tabular}{|c|c|c|c|c|c|}
\hline Upper band & 51.2 & 99 & 1 & 0 & 90 \\
\hline Int-band & 18.2 & 31 & 69 & 0 & 8 \\
\hline Lower band & 30.6 & 7 & 93 & 0 & 3 \\
\hline $\begin{array}{l}\text { Initial } \\
\text { Sample }\end{array}$ & 100 & 62 & 38 & $5-10$ & $50-60$ \\
\hline
\end{tabular}

a. See Glossary for explanation of abbreviations 
of (1) technical aspects of equilibrium density gradient centrifugation, (2) a comparison of fowl and rabbit spermatozoa centrifugation results, (3) a comparison with Staflo sedimentation results and (4) a comparison with the work of other investigators.

\section{Technicai Considerations}

As shown in Figures 51 and 52 , when sufficient time and centrifugal force are used to assure that equilibrium is reached, reproducible results are obtained with both rabblt and fowl spermatozoa. There were no signiflcant variations in results using spermatozoa from different male rabbits ur frum differenl cucks.

The mechanical preparation of the linear density gradient was uncomplicated with the low viscosity contrast media, but difficult with high concentrations of dextran because of the increased resistance to flow through the small bore tubing of the gradient former. The high viscosity of dextran density gradients also significantly increased the time required for fowl spermatozoa to reach equilibrium. This is due primarlly to the extreme axial ratio $\left(f / f_{0}^{204}\right.$ ) from the length of approximately $150 \mathrm{microns}$ and thlckness of 0.5 microns. The high viscosity also made it difficult to remove spermatozoa from the centrifuge tubes. In addition to these problems due to viscosity, the dextran density gradient did not give a sufficiently high specific gravity to study the most dense rabbit spermatozoa. Therefore, sodium lothalamate was used for the equilibrium centrifugation of both rabbit and fowl spermatozoa because solutions of high spectflc gravity and low viscosity could be prepared readily:

2. Comparison of Fowl and Rabbit Experiments

The most striking difference between the results of equilibrium centrifugation of fowl and rabbit spermatozoa in the sodium iothalamate density gradient is the formation of a single band with the former compared 
to 3 or more bands with the latter. As shown in Tables XVI and XVII, the apparent specific gravity of fowl spermatozoa ranges from $1.192-1.212$ which is approximately the same as the range of 1.195-1.211 for the lowdensity band of rabbit spermatozoa. Thus, the simflarity applies only to the highest quality rabbit spermatozoa.

Even though there was only 1 band of fowl spermatozoa in the centrifuge tube, the difference in the quality between the top and the bottom of that band suggested a differential distribution of viable and non-viable cells. The observation of deteriorating fowl spermatozoa at the high density border of the band suggests that either (1) disintegration occurs before further migration takes place or (2) swelling decreases the specific gravity and arrests migration. The finding of tails slightly below the band, as well as above it, suggests that non-viable spermatozoa incorporated some sodium lothalamate, migrated to the lower margin of the band, remained intact (although swollen) during centrifugation and then disintegrated during the pipetting and washing of the fractions of the migration band. These observations show that fowl spermatozoa are quite fragile compared to rabbit spermatozoa, which maintain their physical integrity, except for loss of the acrosomal cap, even after loss of viability.

In all experiments using the sodium lothalamate density gradients, rabbit spermatozoa segregated into at least 3 major bands characterized by different ranges of specific gravities. The marked differences in the quality of the spermatozoa in the 3 bands, as shown in Table XVIII, suggests that their formation is dependent primarily upon the relative viablifty of the spermatozoa. Inasmuch as the spermatozoa were immobilIzed both by the. low temperature and by the high concentration of sodium lothalamate, there was no influence of motility on the distribution. It Is easy to postulate a low-density group consisting of viable spermatozoa 
whlch are affected uniformly to a slight degree, or not at all, by the sodium fothalamate, and a high-density group consisting of non-viable spermatozoa which experience a marked increase in apparent specific gravity due to the hyperosmolar environment.

Mechanisms by which hyperosmolar solutions of low molecular weight substances used In density gradients might modify the apparent specific gravity of spermatozoa include the following: (1) simple dehydration, (2) attachment of the molecules to the membranes of both viable and nonviable cells and (3) selective penetration of the cells depending upon their relative viability. In regard to dehydration, the estimated difference of about 100 mosm between the $0.3 \%$ and $0.9 \%$ saline-iothalamate solutions gives a difference in the average specific gravity of only 0.004 in Table XVI. This is much less proportionately (2\%) than the 15-20\% change in the osmolarity and suggests a non-11near relationship of the 2 factors. There is a suggestion of dehydration also because of the difference in the apparent spectflc gravity range of $1.190-1.210$ in the sodium fothalamate gradient compared to the range of $1.15-1.17$ observed in the dextran density gradient.

The formation of a discrete intermediate'band of rabbll syermatozoa suggests the loss of 1 or more selective mechanisms functioning in the viable cells to exclude fothalamate, but not a state of complete penetrance as In the spermatnzoa in the lower band. Th1s phenomenon may be related to the observation in nigrosin-eosin stained preparations of morphologically normal but stained spermatozoa in which the post-nuclear cap region Is stained, while the remainder of the head is unstained. It is possible that attachment of or penetration by the iothalamate molecules in that region of the spermatozoon could give a population of cells with a relatively 
discrete intermediate range of specific gravities. The analogy with vital staining is not complete for viable rabbit spermatozoa take up eosin at $4^{\circ} \mathrm{C}$, whereas, if they do take up some iothalamate, they are more selectlve than non-viable cells. Benedict and co-workers report no difference in the apparent specific gravity at $5^{\circ} \mathrm{C}$ and $37^{\circ} \mathrm{C}$, suggesting that the influence of certain low molecular welght substances on the specific gravity of rabbit spermatozoa is not temperature dependent ${ }^{32}$.

\section{Correlation with Staflo-Sedimentation Studies}

In Staflo-sedimentation experiments reported in Chapter VTTT, a range of sedimentation velocities from $0-1 \times 10^{-4} \mathrm{~cm} / \mathrm{sec}$ was observed for rabbit spermatozoa in a sucrose density gradient. The distribution of spermatozoa observed in those experiments suggested a uniform distribution of sedimentation velocities among the spermatozoa population. This is in contrast to the suggestion of a trimodal distribution of sedimentation velocities in these equilibrium centrifugation experiments. Because of the short migration distances in the Staflo-sedimentation cxperiments, uverlap among several dfferent-density groups of spermatozoa could have occurred, giving the appearance of a uniform distribution. Nevertheless, the formation of 3 discrete bands in the linear density gradient is attributed to a differential vulnerability of viable and nnn-viablo celio to penetration by sodium iothalamate rather than to distinct grnups of spermatozoa with different specific gravities.

Bhattacharya reported partial bimodal distributions of rabbit and bull spermatozoa and a unimodal distribution of fowl spermatozoa in sedimentation experiments ${ }^{35}$. Bedford and Bibeau observed a suggestion of a bimodal distribution of rabblt spermatozoa using a simflar apparatus, but most distributions could be interpreted as unimodal or uniform ${ }^{29}$. Schilling 
observed a unimodal distribution of bull spermatozoa using a similar device but different medium from that used by Bhattacharya ${ }^{205}$. The disagreement among many investigators using simflar equipment shows that there is still some question as to the sedimentation properties of both rabbit and bull spermatozoa. The results of these centrifugation experiments illustrate a need for considering the effects of the medium on the sedimentation as well as centrifugation properties of rabbit and other spermatozoa.

\section{Comparison with Work by Other Invest1gators}

Table XIX summarlzes the wide range of specific gravities of rabbit and bull spermatozos reported by other investigators and also includes my results. As shown In Table XIX, Benedict, Schumaker and Davies obtained 3 bands of rabbit spermatozoa in a linear density gradient prepared with umbradilic acid ${ }^{32}$. They observed a wider low-density band which overlapped the one I obtained. The intermediate bands were very similar and I observed a greater specific gravity for the higher density spermatozoa. I did not observe a splitting of the low-density band of rabbit spermatozoa, as reported by Benedict and co-workers, in any of my experiments.

The range of 1.09-1.18 for the specific gravity of rabbit spermatozoa reported by Beatty using high molecular weight dialyzed colloidal silica or dextran solutions ${ }^{20}$, is significantly less than the range observed by Benedict, et $\mathrm{al}^{32}$ and me with low molecular weight substances. As noted earlier; using a density gradient prepared with 15-20,000. MW dextran, I observed a specific gravity range of 1.16-1.21 for the low-density band of rabbit spermatozoa. Inasmuch as many spermatozoa formed a pellet at the bottom of the centrifuge tube, it appears that some spermatozoa had a specific gravity greater than 1.23. Unfortunately, the viability of the spermatozoa in the bottom of the centrifuge tube was not evaluated to see 
Table XIX. Comparison of Specific Gravity Measurements on Rabbit and Bul1

Spermatozoa in Different Density Gradients.

\begin{tabular}{|c|c|c|c|c|c|}
\hline \multirow{2}{*}{$\begin{array}{l}\text { Sperm } \\
\text { Source }\end{array}$} & \multicolumn{2}{|c|}{ Specific Gravity } & \multirow[t]{2}{*}{ Sub-Gromps } & \multirow[t]{2}{*}{ Main Constituents of Density Gradient } & \multirow[t]{2}{*}{ Reference } \\
\hline & Range & Mean & & & \\
\hline Bul1 & $1.24-1.35$ & $\begin{array}{l}1.2867 \\
1.2897 \\
1.2668\end{array}$ & $\begin{array}{l}\text { First eja:ulate } \\
\text { Second ejacjlate } \\
\text { Third ejazulate }\end{array}$ & $\begin{array}{l}\text { Methyl glucamine salt of umbradil } \\
(2,5 \text {-dilodine-4-pyridone-N-acetic acid) }\end{array}$ & $\begin{array}{l}\text { Lindahl and } \\
\text { Kinls trom }\end{array}$ \\
\hline Bul1 & & & $\begin{array}{l}\text { Heads } 1.276 ; \\
\text { tails \& widpieces, } \\
1.035-1.0 .56\end{array}$ & $"$ & Kihlstrom 124 \\
\hline Bull & $1.10-1.125$ & - & Epididymal sperm & Ficoll (synthet1c polysacchardae) & $\begin{array}{l}\text { Lindah1 and } \\
\text { Thunqvist }\end{array}$ \\
\hline \multirow[t]{2}{*}{ Bull } & $\begin{array}{l}1.150-1.226 \\
1.255-1.318\end{array}$ & $\begin{array}{l}1.18 ? \\
1.283\end{array}$ & $\begin{array}{l}\text { Low density band } \\
\text { High density sub- } \\
\text { band }\end{array}$ & $\begin{array}{l}\text { Hethy } 1 \text { glucamine salt of } 3,5-d 1-10 d o-4- \\
\text { pyridone-N-acet1c acid (umbradilic acid) }\end{array}$ & $\begin{array}{l}\text { Benedict, Schumaker } \\
\text { and Davies } 32\end{array}$ \\
\hline & $1.286-1.324$ & 1.303 & $\begin{array}{l}\text { High density sub- } \\
\text { band }\end{array}$ & " & $"$ \\
\hline Rabbit & $\begin{array}{l}1.150-1.210 \\
1.273-1.306 \\
1.280-1.313\end{array}$ & $\begin{array}{l}1.185 \\
1.283 \\
1.29\end{array}$ & $\begin{array}{l}\text { Low density band } \\
\text { High density band } \\
\text { High density band }\end{array}$ & " & " \\
\hline Bull & $1.037 \epsilon-1.0927$ & - & & $\begin{array}{l}\text { D1-N-butyl phthalate, di-ethyl-phthalate, } \\
\text { di-1so-butyl-phthalate }\end{array}$ & $\begin{array}{l}\text { Lavon, Volcan1, Am1 r } \\
\text { and Danon } 132\end{array}$ \\
\hline Rabb1t & $1.09-1.18$ & 1.132 & & Dialysed collolda- s1lica, dextran & Beatty 20 \\
\hline Rabbit & $\begin{array}{l}1.195-1.211 \\
1.267-1.310\end{array}$ & $\begin{array}{l}1.203 \\
1.288\end{array}$ & $\begin{array}{l}\text { Low density band } \\
\text { Intermediate } \\
\text { density band }\end{array}$ & $\begin{array}{c}\text { Sodion Iothalamate } \\
" 1 \\
"\end{array}$ & $\begin{array}{c}\text { Pistenma - this report } \\
" 1\end{array}$ \\
\hline & $\begin{array}{l}1.312-1.330 \\
1.332-1.366\end{array}$ & $\begin{array}{l}1.321 \\
1.35: 1\end{array}$ & $\begin{array}{l}\text { Bottom band } t i 1 \\
\text { Botton band } t i 2\end{array}$ & " & $"$ \\
\hline Rabbit & $\begin{array}{c}1.155-1.215 \\
>1.23\end{array}$ & $\begin{array}{l}- \\
-\end{array}$ & $\begin{array}{l}\text { Upper baad } \\
\text { Bottom band (s) }\end{array}$ & Dextran $(20,000 \mathrm{H} / \mathrm{W}$, S1 gma $)$ & $"$ " \\
\hline
\end{tabular}


If they were non-viable as expected from the sodium iothalamate density gradient experiments. The dextran gradent values of the apparent specific gravity of rabbit spermatozoa are higher than those reported by Beatty yet lower than those obtained with the sodium lothalamate density gradients. These differences 1llustrate the need for further study of the effect of osmotic pressure on the apparent specific gravity of spermatozoa and other cells.

Similar differences in Table XIX are found in the reported apparent spectfic gravities of bull spermatozoa which vary from a range of $1.0376-$ 1.0927 , reported by Lavon and co-workers using a density gradient of din-butyl phthalate and di-ethyl-phthalate ${ }^{133}$ to a range of $1.24-1.35$ reported by Lindahl and KIhlström using MGU ${ }^{138}$.

The differences in quality of the spermatozoa in the several bands observed in my experiments, examples of which are given in Table XVIII, appear to be greater than that observed by Benedict and co-workers ${ }^{32}$. Spermatozoa from the high-density band in my experiments had almost no motility and very low viabllity, suggesting that the fertilizing capacity was negligible. My Impression is that any viable and motile spermatozoa In the high-density band reached that level in the centrifuge tube by droplet sedimentation or by being in a clump with "dead" spermatozoa. Thus, I. am surprised that Benedict and co-workers were able to get offspring from the Insemination of spermatozoa from the high-density bands even though the fertility rate was only $4-6 \%$.

\section{E. Summary and Conclusions}

For physiological reasons, it is desirable to use dextran density gradients rather than hyperosmolar solutions of radiographic contrast media or similar substances. However, In addition to severe technical problems 
with high concentrations of dextran, a $65.8 \%$ (weight/volume) solution of 15-20,000 MW dextran (specific gravity 1.23 at $4^{\circ} \mathrm{C}$ ) was not sufficient $1 \mathrm{y}$ dense for isopycnic centrifugation of all of the rabbit spermatozoa. In this linear density gradient, the lightest band of rabbit spermatozoa had a specific gravity range of 1.16-1.21 while that for fow 1 spermatozoa ranged from 1.15-1.17.

In a linear density gradient of sodium iothalamate in saline, centrifugation for approximately 10 minutes at $9,000 \mathrm{~g}$ was required to reach equilibrium with both fowl and viable rabbit spermatozoa. Fowl spermatozoa gave a single band in the density gradient with an apparent specific gravity range of 1.19-1.21. Under the same conditions, rabbit spermatozoa always gave 3 or more bands, the lightest of which had this same apparent specific gravity range of $1.19-1.21$. The two other bands formed by rabbit spermatozoa generally were in the ranges of $1.26-1.29$ and $1.32-1.36$. There was a suggestion that the apparent specific gravity of non-viable rabbit spermatozoa increased with increasing centrifugal force and centrifugation time in this density gradient.

The discrepancy between results using dextran and sodium iothalamate density gradients are similar to those sumarlzed in Table XIX. Pussible mechanisms were examined for the apparent high specific gravity in the sodium iothalamate density gradient.

There was a marked differential distribution of viable and non-viable rabbit spermatozoa anong the 3 bands with unequivocal absolute enrichment of the upper band in regard to viable and motile spermatozoa. All sperm- 
atozoa in the lowest band were essentially non-viable. Thus, with equilibrium density gradient centrifugation in a sodium iothalamate gradient one can obtain a population of high quality rabbit spermatozoa from a lesser quality starting sample. The homogeneity of the most dense (nonviable) band of rabbit spermatozoa can be modified by clumps which tend to carry viable cells into the higher density regions of the gradient, along with the non-viable ones.

A differential distribution of fowl spermatozoa within the single, continuous band was also found. The highest quality spermatozoa were on top and deteriorating cells were on the bottom. The disintegration of nonviable fowl spermatozoa is in marked contrast to the morphological integrity of non-viable rabbit spermatozoa except for the latter's loss of the acrosomal cap. Thus, the discreteness of the band of fowl spermatozoa in the density gradient is an Indication of the quality of the sample.

It was shown (Appendix D) that for rabblt spermatozoa, neither the motillty nor fertilizing capacity was affected by exposure to low concentrations of sodium lothalamate at $2-4^{\circ} \mathrm{C}$ for many hours. Fowl spermatozoa experfenced only a mild loss of motility after exposure for about 30 minutes to high concentrations of several contrast media. The preservation of vital functions in these media suggests that they might be useful for the separation of viable and non-viable cells with other cell systems, as well. 
VIII. MULTI-CHARACTERIZATION STAFLO STUDIES: RABBIT SPERMATOZOA Contents

A. Introduction. . . . . . . . . . . . . . . . 229

B. Materials and Methods . . . . . . . . . . . . 230

1. Sample Preparation. . . . . . . . . . . . 230

2. Staflo Apparatus. ................. 231

3. Media and Density Gradient. . . . . . . . . . . . 232

4. Temperature Conlrul . . . . . . . . . . . . 234

5. Evaluation of Spermatozoa ............. 234

C. Results..................... . . . . . 235

1. Sedimentation .................. . . . . . 237

a. Three Individual Experiments. . . . . . . . 237

b. Calculation of Sedimentation Velocities . . ... . 241

2. Sedimentation-Upward-Electrophoresis: High Electric

Field Strengths. . . . . . . . . . , , , . . . . . . 244

a. Ten Individual Experiments. . . . . . . . . . 244

b. Calculation of Electrophoretic Mobilfties . . . . 253

3. Sedimentation-Electrophoresis: Low Electric Field 255

Strengths . . . . . . . . . . . . . . . 255

a. Upward-Electrophoresis. . . . . . . . . . 255

b. Downward-Electrophoresis. . . . . . . . . 258

D. Discussion. . . . . . . . . . . . . . . 259

1. Sedimentation . . . . . . . . . . . . 259

a. Comparison of Three Experiments . . . . . . . 259

b. Migration Comparison: Actual vs Hypothetical

Spermatozoa Populations . . . . . . . . . . . 
2. Sedimentation-Upward-Electrophoresis: High Electric

Field Strengths. . . . . . . . . . . . . . 263

a. Comparison of Experiments . . . . . . . . . 263

b. Interpretation of Electrophoretic Mobilities. . . 265

c. Concentration of Spermatozoa: Effect on

Artificial Insemination. . . . . . . . . . .

3. Sedimentation-Electrophoresis: Low Electric Field

Strengths . . . . . . . . . . . . . . . .

a. Upward Electrophores1s. . . . . . . . . . 272

b. Downward Electrophores1s. . . . . . . . . 274

4. Orientation of Spermatozoa. . . . . . . . . . . 274

5. Quality of Spermatozoa. . . . . . . . . . . . 277

E. Summary and Conclusions . . . . . . . . . . . . 279

\section{A. Introduction}

A review of work by others on the sedimentation and electrophoretic properties of spermatozoa 18 presented in Chapter II. The results of early Staflo-sedimentation and -electrophoresis studies on rabbit spermatozoa are reported in Chapters IV and V, respectively. These early studies suggested a poorly defined heterogeneity in the sedimentation and electrophoret1c properties approximately resembling a normal distribution. Interesting observations included a near-vertical orientation of the spermatozoa with the head down and tall up in sedimentation experiments, and a vertical orientation in sedimentation-electrophoresis experiments with the tall pointing toward the positive electrode, irrespective of the direction of migration. In addition, a significant effect of motility on the fractionatton of the spermatozoa in the Staflo apparatus was observed at temperatures 
of $4^{\circ} \mathrm{C}$ and above where some spermatozoa had sufficient intrinsic motility to overcome the force of the electric fleld. Some of the influences of the $\mathrm{pH}$ and fonic strength on the electrophoretlc properties of spermatozoa, as reported by Bangham ${ }^{18}$, were also observed In these early studies.

The purpose of the experiments reported in this chapter was to explore the relationships among the sedimentation rate, electrophoretic mobility and Intrinsic motility of rabbit spermatozna utilizing the flexibility of the Staflo method. A serles of sedimentation and sedimentation-electrophoresis experiments, the possible types of which were discusocd in Chapter III, was conducted, using the same medium and density gradient. The "low" ionic strength glycine-phosphate buffer evaluated in Chapters $V$ and VI was selected because of its good $\mathrm{pH}$ stability in electrophoresis experiments and its excellent preservation of motility. A sucrose density gradient was selected to minimize discontinuitles between layers and to enhance the sedimentation of spermatozoa.

\section{B. Malerlals and Methods}

\section{Sample Preparation}

Spermatozoa samples were obtained from mature Dutch Belt, California or New Zealand White rabbits using the artificial vagina described in Appendix C. Each efaculate was diluted immediately by adding $1.0 \mathrm{ml}$ of the glyclne-phosphate buffer at $37^{\circ} \mathrm{C}$ tn the collection vinl. The samplo then was allowed to $c 001$ to room temperature in an insulating nest of glass wool. A $0.05 \mathrm{ml}$ aliquot of this sample was then diluted to $1.0 \mathrm{ml}$ in egg yolk medium and was used for the initial evaluation of forward motility, for the inftial estimate of the spermatozoa concentration in the sample, and for control inseminations. Further cooling to the experimental temperature was accomplished as described in Chapter III. 
After the spermatozoa had been cooled to the temperature of the experiment, the sample was diluted to a concentration of approximately $20 \times 10^{6} / \mathrm{ml}$. Even though the density gradient used in these experiments supported a suspension of up to $30 \times 10^{6} / \mathrm{ml}$, lower concentrations were used to avoid "cluster sedimentation'discussed in Chapter IV. Generally, a single ejaculate provided suffictent spermatozoa for an experiment. If 1 t was necessary to pool efaculates from 2 or more rabbits, only spermatozoa from rabbits of the same breed were used.

A $1.0 \mathrm{ml}$ allquot of the $20 \times 10^{6} / \mathrm{m} 1$ suspension, called the initial sample (IS), was set aside for Immediate evaluation and for later comparison with the fractionated spermatozoa. The remainder of the initial sample was Introduced into layer 8 in all experiments. over the small density shelf between layers 8 and 9 , and was subjected to Staflo sedimentation or sedimentation-electrophoresis, as desired. The aliquot of the initial sample which had been set aside then was evaluated in the hemocytometer to determine the motility or lack thereof at the temperature of the experiment, and was characterized with regard to the percentage of capped and capless spermatozoa by phase contrast microscopy.

\section{Staflo Apparatus}

The Staflo apparatus, characteristics of operation and accessory equipment are described in Chapter III. Therefore, this section makes note only of the equipment used in the multi-characterization studies.

a. Flow-Cells. The $3 \times 16$ flow-cell (No. XI) was used in 1 sedimentation experiment and in all sedimentation-electrophoresis experiments discussed below. The $3 \times 12$ flow-cell (No. XIII) was used only for 2 sedimentation experiments. The principles of operation of the 2 flow-cells are the same in a11 respects. 
b. Optical Accessorles. The gross migration patterns were observed by eye, using the slit lamp technique 229,263 . Observations of individual spermatozoa and the microscopic steady-state migration pattern were made with the microscopic syotem described in Chapter III.

Ueing dark field 1llumfnation, observation of individual spermatozoa was possible up to magnifications of $640 \mathrm{X}$. In addition to observing the orlentation and movement, if any, of spermatozoa, the microscope was used to measure the vertical migration distances in the centerline of the flowchamber.

\section{Media and Density Gradient}

a. Glyclne-Phosphate Buffer (GPB). This buffer is the primary medium for the density gradient solutions used in this series of experiments. The composition and initial evaluation, and additional studies on the glycine-phosphate buffer are reported in Chapters V and VI, respectively. b. Sucrose Density Gradient. The density gradient contains sucrose by weight/volume percent in the flowing layers numbered 1 through 16 from top to bottom, as follows: Nos. 1-3 contalned no sucrose. A higher conductivity solution with 3 times as much modifled Baker's medium was used In these layers and also in the top electrode wash compartment as a "conductivity barrier" ${ }^{229,263}$; No. 4, 0.05\%; No. 5, 0.20\%; No. 6, 0.35\%; No. 7, $0.5 \%$; No: $8,0.65 \%$; No. $9,1.0 \%$; No. $10,1.15 \%$; No. $11,1.3 \%$; No. $12,1.45 \%$; No. 13, 1.60\%; Nos. 14-16, and the bottom electrode compartment contained the same high conductivity medium used in the top electrode wash compartment plus $2.0 \%$ sucrose.

F1gure 53 shows the following characteristics of the glycine-phosphate buffer and this density gradient at $3^{\circ} \mathrm{C}$ : apecific gravity, viscosity and osmolarity. 


\section{CHARACTERISTICS OF DENSITY GRADIENT}

Multicharacterization studies

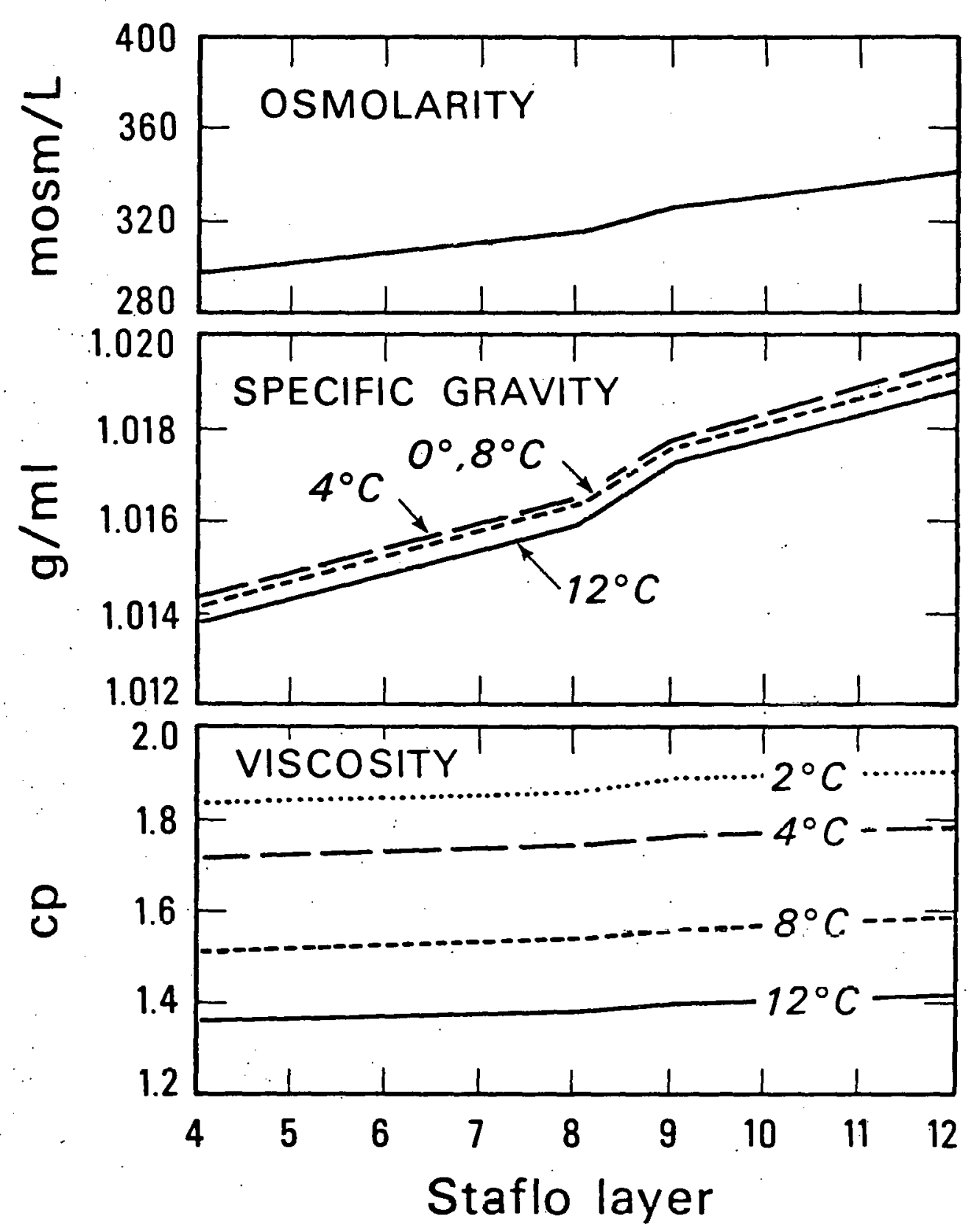

DBL 70115992

Fig. 53. Characteristics of the glycine-phosphate buffer and sucrose denșlty gradient för multi-characterization studies of rabbit spermatozoa. 
c. Egg Yolk Medium (EYM). The egg yolk medium used in these experiments was the same as described in Chapter VI. Because of the ability of the egg yolk medium to maintain the viabllity, motility and fertilizing capacity of rabbit spermatozoa, the spermatozoa in the collection fractions were reconcentrated by centrifugation and resuspended in egg yolk medium. This eliminated the necessity for immediate artificial insemination (AI) and provided more time for evaluation of the spermatozoa prior to AI.

\section{Temperature Contro1.}

All experiments were conducted in a refrlgerated room which was large enough for the entire Staflo apparatus, accessory equipment and several Investigators. In the sedimentation-electrophoresis experiments with high electric fleld strengths, the average and maximum temperature rises in the flow-chamber were $0.5^{\circ} \mathrm{C}$ and $1.0^{\circ} \mathrm{C}$, respectively. For sedimentation-electrophoresis experiments with low electric field strengths there was essentially no change in temperature. Flow-chamber temperatures reported below are corrected for the estimated temperature rise depending upon the electric field strengths and other operating conditions.

\section{Evaluation of Spermatozoa}

a. Morphology, Mot1lity and Viability. Immediately after completion of the Staflo fractionation phase of the experiment, the suspensions in the central row of collection containers were evaluated in a hemocytometer for spermatozoa concentration and for percentage of capped and capless. spermatozoa by phase contrast microscopy. Each collection fraction, the $1.0 \mathrm{ml}$ initial sample and any remaining stirred suspension (SS) then was concentrated by centrifugation for 5 minutes at $1,400 \mathrm{~g}$ in a clinical centrifuge in the refrigerated room. The supernatant was removed and the spermatozoa were re-suspended in egg yolk medium to a concentration of 
approximately $20 \times 10^{6} / \mathrm{ml}$. The re-suspended spermatozoa then were evaluated at room temperature for motility, percent capped and capless by phase contrast microscopy and for vital staining characteristics by a nigrosin-eosin staining technique. The stained spermatozoa were categorized as unstained (U), morphologically normal but stained (MNS) and stained (S). All work with spermatozoa was accomplished using minimal lighting in order to reduce the deleterious effects of visible light ${ }^{176}$. b. Fertilizing Capacity. If $10 \%$ or more of the spermatozoa in each collection fraction had a high degree of motility at room temperature, artificial insemination was attempted. Ovulation was induced by an intravenous injection of $0.5 \mathrm{mg} / \mathrm{kg}$ of luteinizing hormone (ovine or bovine), generally less than 1 hour prior to insemination. A $0.5-1.0 \mathrm{ml}$ aliquot of each suspension, containing $10-20 \times 10^{6}$ spermatozoa, was inseminated in as many rabbits as there was suspension, using a plastic pipette (Falcon Plastics, Division of B-D Laboratories, Inc.), on which the tip had been rounded. A control insemination was made with $0.5 \mathrm{ml}$ of the spermatozoa suspension initially diluted and stored in egg yolk medium.

\section{Results}

The order of presentation of the results is as follows: (1) sedimentation experiments, (2) sedimentation-upward-electrophoresis (SUE) experiments with high electric field strengths, and (3) sedimentation-upwardelectrophoresis (SUE) and sedimentation-downward-electrophoresis (SDE) experiments with low electric field strengths.

Because the migration distances were small in all experiments, the migration pattern shown in Figure 54 (SUE with low electric field strength) is representative of that encountered in all 3 series of experiments. The 


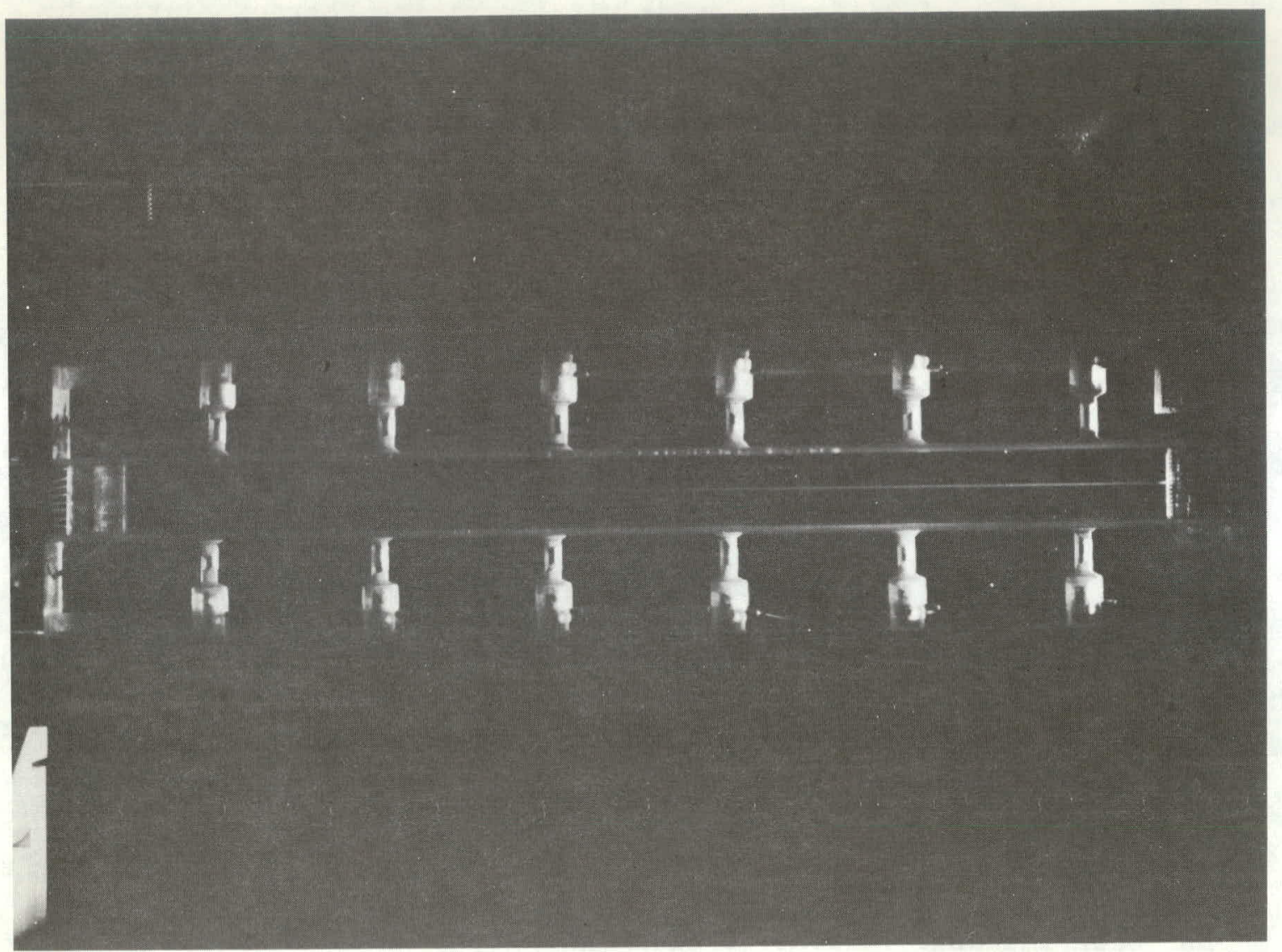

XBB $7011-5007$

Fig. 54. Photograph of the migration pattern in a sedimentationupward-electrophoresis experiment with rabbit spermatozoa using a low electric field strength in the $3 \times 16$ Staflo apparatus. 
orfentation of the parabolold migration profile (e.g. Figure 49) depended upon the direction of net migration.

\section{Sedimentation}

Table $\mathrm{XX}$ is a summary of the characteristics of the spermatozoa sample and Staflo operating parameters for 3 sedimentation experiments. The quality of the spermatozoa samples was high initially only in Experiment 8-10-67 and that sample deteriorated rapldly during the course of the experiment. Consequently, motility is considered to have contributed little, if anything, to the migration patterns in this series of experiments. There was no opportunity for artificlal insemination at the conclusion of any of the 3 experiments because of the poor quality of the spermatozoa.

Experiment 12-26-67 was conducted with the $3 \times 16$ flow-cell. The sample stream Inftially was $0.7 \mathrm{~mm}$ high at the bottom of layer 8 , which is the desired location for downward migration, as discussed in Chapter III. The $3 \times 12$ flow-cell, in which the inlet solutions are provided by thirtysix $20 \mathrm{ml}$ syringes, was used for the other two experiments. The sample was introduced into layer 6 with a single $20 \mathrm{ml}$ syringe, giving a $0.4 \mathrm{~mm}$ high sample stream which was in the middle of layer 6 at steady-state conditions. Thus, even though technically acceptable migration patterns were observed in both experiments with the $3 \times 12$ flow-cell, three-layer fractionation was not achieved because the spermatozoa had to sediment through part of layer 6 and all of layer 7 before reaching layer 8 . If the sample migration had started at the bottom of layer 6 , a significant number of spermatozoa would have been recovered in collection fraction 8 .

\section{a. Three Individual Experiments.}

(1) Experiment 12-26-67. Immediately after collection, the ejaculate had 1 ess than $50 \%$ forward motion and tail-wagging combined at room temperature 
Table XX. Spermatozoa Sample and Staf lo Operating Parameters for Multi-Characterization Sedimentation Experiments

\begin{tabular}{|c|c|c|c|c|c|c|c|c|c|c|c|c|}
\hline \multirow[b]{2}{*}{$\begin{array}{l}\text { Exper imen } \\
\text { Number }\end{array}$} & \multicolumn{5}{|c|}{ Characteristics of Spermatozoa Sample } & \multicolumn{7}{|c|}{ General Staflo Operating Parameters } \\
\hline & Rabbi= & Breed & $\begin{array}{l}\text { Medium } \\
\text { for } \\
\text { Cooling }\end{array}$ & $\begin{array}{l}\text { Qualit } \\
\text { Initial }\end{array}$ & Final & \multicolumn{2}{|c|}{$\begin{array}{l}\text { Indtial Sperm } \\
\text { Conc. (unlts } \\
\left.\text { of } 10^{6} / \mathrm{ml}\right) \mathrm{pH}\end{array}$} & \multirow{2}{*}{$\frac{\begin{array}{l}\text { Tempe } \\
\text { Roon } \\
\left({ }^{\circ} \mathrm{C}\right)\end{array}}{3}$} & \multirow{2}{*}{$\begin{array}{c}\begin{array}{c}\text { rature } \\
\text { Staflo } \\
\left({ }^{\circ} \mathrm{C}\right)\end{array} \\
3\end{array}$} & \multirow{2}{*}{$\frac{\begin{array}{c}\tau_{\text {ave }} \\
\text { (min) }\end{array}}{43}$} & \multirow{2}{*}{$\frac{\begin{array}{c}\tau_{\min } \\
(\min )\end{array}}{28.7}$} & Remarks \\
\hline $12-26-67$ & Kar1 & $\begin{array}{l}\text { Dutch } \\
\text { Belt }\end{array}$ & GPB & $\begin{array}{l}\text { Less } \\
\text { than } 50 \% \\
\text { tail-wag- } \\
\text { ging and } \\
\text { forward } \\
\text { motion. }\end{array}$ & $\begin{array}{l}\text { Very } \\
\text { Poor }\end{array}$ & 20 & 7.1 & & & & & $\begin{array}{l}\text { Sample } 0.7 \mathrm{~mm} \\
\text { high at inlet } \\
\text { in bottom of } \\
\text { layer } 8 \text {. } \\
(3 \times 16 \text { flow-ce } 11)\end{array}$ \\
\hline $7-29-67$ & otte. & $\begin{array}{l}\text { Dutch } \\
\text { Belt }\end{array}$ & GPB & $\begin{array}{l}\text { Less tr.an } \\
40 \% \text { tad1- } \\
\text { wagging } \\
\text { and } \\
\text { forward } \\
\text { motion }\end{array}$ & Poor & 17 & 7.1 & $\leq$ & 5 & 34 & 22.7 & $\begin{array}{l}\text { Sample } 0.4 \mathrm{~mm} \\
\text { high at inlet } \\
\text { in middle of } \\
\text { layer } 6 \text {. } \\
(3 \times 12 \text { flow-cel1) }\end{array}$ \\
\hline $8-10-67$ & Ps 1 & $\begin{array}{l}\text { Dutch } \\
\text { Belt }\end{array}$ & EYM & $\begin{array}{l}60 \% \text { fo:- } \\
\text { ward } \\
\text { motion }\end{array}$ & $\begin{array}{l}\text { Poor } \\
\text { (ques- } \\
\text { tion of } \\
\text { toxin } 1 \\
\text { apparat } \\
\text { us). }\end{array}$ & in & 7.1 & $\dot{j}$ & 6 & 34 & 22.7 & $\begin{array}{l}\text { Sample } 0.4 \mathrm{~mm} \\
\text { h1gh at inlet } \\
\text { in middle of } \\
\text { layer } 6 . \\
(3 \times 12 \text { flow-ce } 11)\end{array}$ \\
\hline
\end{tabular}

a. See Glossary for explanation of abbreviations. 
in GPB. No motility was observed at $3^{\circ} \mathrm{C}$ in a hemocytometer. After steadystate conditions had been reached, there was no change in the migration pattern. The migration profile was parabolold throughout the experiment. Observations in the flow-chamber with the Staflo microscope showed the following: (1) spermatozoa were at the top of the migration pattern but at a lower concentration than in the middle; (2) there was no rolling, tall-wagging or other Indication of Intrinsic motility; (3) there were a small number of microscopic streamers, in which spermatozoa were spread well apart, at the bottom of the migration pattern only; (4) spermatozoa were oriented randumly from a horizontal to a vertical position with the head down and the tail up. (Most tails were oriented within $45^{\circ}$ of the vertical position and no spermatozoa were seen with the tail down and the head up.) and (5) there was no effect of location within the flow-chamber upon the orientation of the spermatozioa.

Table XXI summarizes the distribution of spermatozoa among the collection fractions and the phase contrast observations and results of nigrosineosin staining thereof. Despite the range of concentrations from $0.72-$ $2.91 \times 10^{6} / \mathrm{ml}$, there were no significant differences among the 3 fractions with regard to the distribution of capped spermatozoa immediately after collection. Deterforation of the spermatozoa sample at a concentration of $20 \times 10^{6} / \mathrm{ml}$ is shown by the change from $79.2 \%$ capped in the initial sample (IS) to $52.4 \%$ capped in the stirred sample (SS) at the conclusion of the experiment. After reconcentration and suspension in egg yolk medium, no motile spermatozoa were observed at room temperature. There were no significant differences in the nigrosin-eosin staining results.

(2) Experiment 7-29-67. As shown in Table XXI, there was no significant difference between the 2 collection fractions with regard to the per- 
Table XXI. Phase Contrest Observations and N1grosin-Eosin Staining of Spersacozoa in Sedimentation Exper iments.

\begin{tabular}{|c|c|c|c|c|c|c|c|c|c|c|c|}
\hline \multirow[t]{2}{*}{$\begin{array}{l}\text { Experiment } \\
\text { Number }\end{array}$} & \multirow[t]{2}{*}{$\begin{array}{c}\text { Spermatozoa } \\
\text { Sample }\end{array}$} & \multirow{2}{*}{$\begin{array}{l}\text { Sperm so:lected } \\
\text { Soncentration } \\
\text { (untts of } 10^{5} / \mathrm{nl} \text { ) }\end{array}$} & \multirow[t]{2}{*}{$(7)$} & \multirow[t]{2}{*}{$\begin{array}{l}\text { Temp. } \\
\left({ }^{\circ} \mathrm{C}\right)\end{array}$} & \multicolumn{4}{|c|}{$\begin{array}{l}\text { Phase Zontrast } \\
\text { Observations }\end{array}$} & \multicolumn{3}{|c|}{ Nigrosin-Eosin Staining } \\
\hline & & & & & $\begin{array}{c}c \\
(x) \\
\end{array}$ & $\begin{array}{l}\mathrm{CL} \\
(\%) \\
\end{array}$ & $\begin{array}{c}\text { TW } \\
(\%)\end{array}$ & $\begin{array}{l}F M \\
(\%)\end{array}$ & $\begin{array}{c}U \\
(\%)\end{array}$ & $\begin{array}{l}\text { MNS } \\
(\%) \\
\end{array}$ & $\begin{array}{c}S \\
(\%) \\
\end{array}$ \\
\hline $12-26-67$ & $\begin{array}{ll}\text { IS } & \\
\text { CF } & 8 \\
\text { CF } & 9 \\
\text { CF } & 10 \\
\text { SS } & \\
\end{array}$ & $\begin{array}{r}- \\
1.09 \\
2.91 \\
0.72 \\
- \\
\end{array}$ & $\begin{array}{c}- \\
23.1 \\
61.6 \\
15.3 \\
- \\
\end{array}$ & $\begin{array}{l}3 \\
3 \\
3 \\
3 \\
3\end{array}$ & $\begin{array}{l}79.2 \\
4 L .5 \\
4 i .2 \\
4 E .7 \\
52.4\end{array}$ & $\begin{array}{l}20.8 \\
55.5 \\
52.8 \\
51.3 \\
47.6 \\
\end{array}$ & $\begin{array}{l}0 \\
0 \\
0 \\
0 \\
0 \\
\end{array}$ & $\begin{array}{l}0 \\
0 \\
0 \\
0 \\
0\end{array}$ & $\begin{array}{l}29.4 \\
31 \\
26 \\
25\end{array}$ & $\begin{array}{l}1.6 \\
9 \\
5 \\
1\end{array}$ & $\begin{array}{l}68.7 \\
60 \\
69 \\
74\end{array}$ \\
\hline $7-27-67$ & $\begin{array}{l}\text { IS } \\
\text { CF } 6 \\
\text { CF } 7 \\
\text { Control }\end{array}$ & $\begin{array}{l}1 . \overline{90} \\
1.23\end{array}$ & $\begin{array}{l}- \\
60.7 \\
39.3\end{array}$ & $\begin{array}{l}5 \\
5 \\
23 \\
5 \\
23 \\
23\end{array}$ & $\begin{array}{c}85.6 \\
90 \\
- \\
92.1 \\
- \\
-\end{array}$ & $\begin{array}{c}14.4 \\
10 \\
- \\
7.9 \\
- \\
-\end{array}$ & $\begin{array}{c}0 \\
0 \\
5.2 \\
0 \\
10.5 \\
-\end{array}$ & $\begin{array}{c}0 \\
0 \\
2.0 \\
0 \\
2.3 \\
-\end{array}$ & $\begin{array}{l}\overline{2} \\
\overline{-} .5 \\
29 \\
45\end{array}$ & $7^{-}$ & $\begin{array}{l}\overline{-} \\
71.5 \\
- \\
64 \\
53\end{array}$ \\
\hline $8-10-67$ & $\begin{array}{l}\text { IS } \\
\text { CF } 6 \\
\text { CF } 7\end{array}$ & $\begin{array}{c}- \\
5.62 \\
0.71\end{array}$ & $\begin{array}{l}88.7 \\
11.3\end{array}$ & $\begin{array}{l}6 \\
23 \\
6 \\
23 \\
6 \\
23\end{array}$ & $\begin{array}{l}43.0 \\
11.5 \\
73.3 \\
13.1 \\
70.5 \\
11.5\end{array}$ & $\begin{array}{l}14.7 \\
19.1 \\
25.2 \\
29.9 \\
27.5 \\
57.6\end{array}$ & $\begin{array}{l}38.9 \\
48.2 \\
1.5 \\
56.5 \\
2.5 \\
28.6\end{array}$ & $\begin{array}{l}0 \\
21.2 \\
0 \\
0.5 \\
0 \\
2.0 \\
\end{array}$ & $\begin{array}{r}- \\
90 \\
- \\
93 \\
- \\
92 \\
\end{array}$ & $2^{-}$ & $5^{9}-$ \\
\hline
\end{tabular}

a. CF: Collection Fraction; IS: Inftlal Sample; SS: Stirred Suspension;

b. See Glossary for explanation of abbreviaclons. 
centages of capped spermatozoa at $5^{\circ} \mathrm{C}$ or of forward motility at room temperature. There were slightly higher percentages of both tail-wagging and unstained spermatozoa in fraction 7 relative to 6 . The large difference between the percentage of capped spermatozoa at $5^{\circ} \mathrm{C}$ and the percentage of unstained spermatozoa at $23^{\circ} \mathrm{C}$ is attributed primarily to deterioration during centrifugation. In the control sample, $53 \%$ of the spermatozoa stained by the nigrosin-eosin technique, indicating that the inftial quality of the sample was poor.

(3) Experiment 8-10-67. Desplte the 8-fold difference in spermatozoa concentration, there was no significant difference between the 2 fractions with regard to the percentages of capped spermatozoa at $6^{\circ} \mathrm{C}$ or of unstained spermatozoa at room temperature. Although the preservation of motility and viability was better in this experiment than in either of the other 2 sedimentation experiments, the motility was almost all tail-wagging. The lower percent tall-wagging in fraction 7 is probably due to the lower concentration in that fraction.

b. Calculation of Sedimentation Velocities... A sample calculation of sedimentation velocities for Experiment $12-26-67$ is presented in Appendix F. Th1s experiment was selected as a sample for the calculations because the spermatozoa suspension entered the flow-cell at the bottom of the inlet layer, satisfying the requirements for the formulae discussed in Chapter III. Table XXII presents the minimum, maximum and average sedimentation velocities for the spermatozoa in each collection fraction. The weighted average sedimentation velocity at $3^{\circ} \mathrm{C}$ was calculated to be 0.53 $\times 10^{-4} \mathrm{~cm} / \mathrm{sec}$.

Table XXIII presents the minimum, maximum and weighted average sedimentation velocities, normallzed to $3^{\circ} \mathrm{C}$, for all 3 experiments. The maximum sedimentation velocity ranges from $0.87-1.24 \times 10^{-4} \mathrm{~cm} / \mathrm{sec}$ and the 
Tabl $=$ XXII. Sedimentation Velocities for Spermatozoa in Collection Fractions;

Sedimentation Experiment $12-26-67,3^{\circ} \mathrm{C}$.

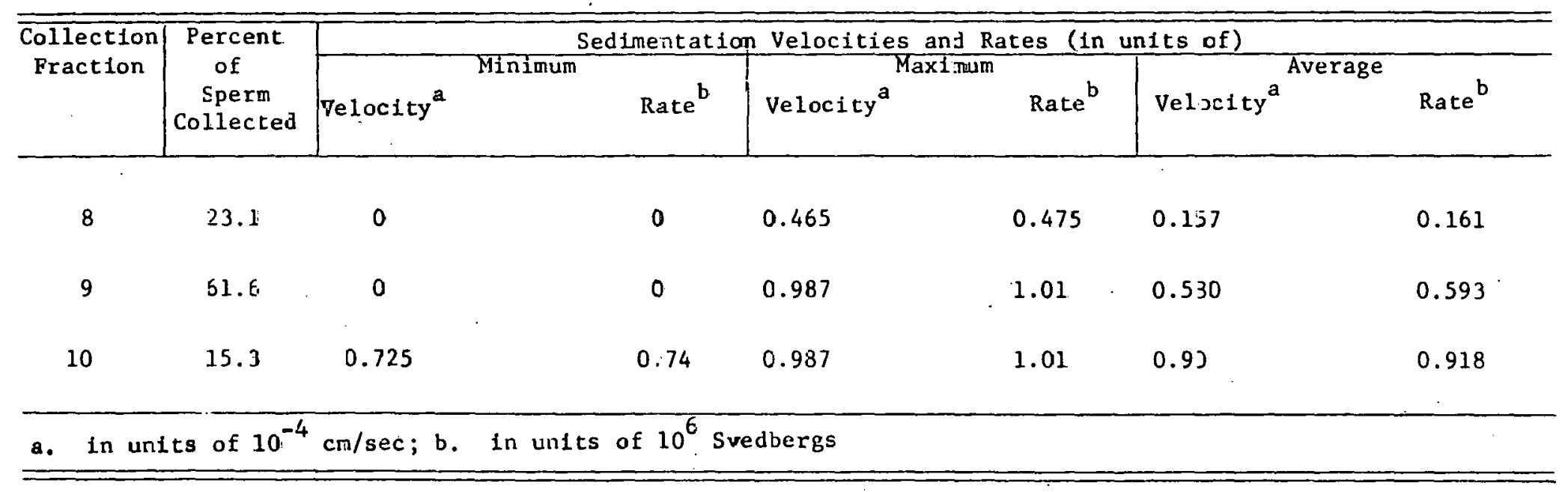


Table XXIII. Summary of Calculated Sedimentation Velocities

Normallzed to Values Expected at $3^{\circ} \mathrm{C}$.

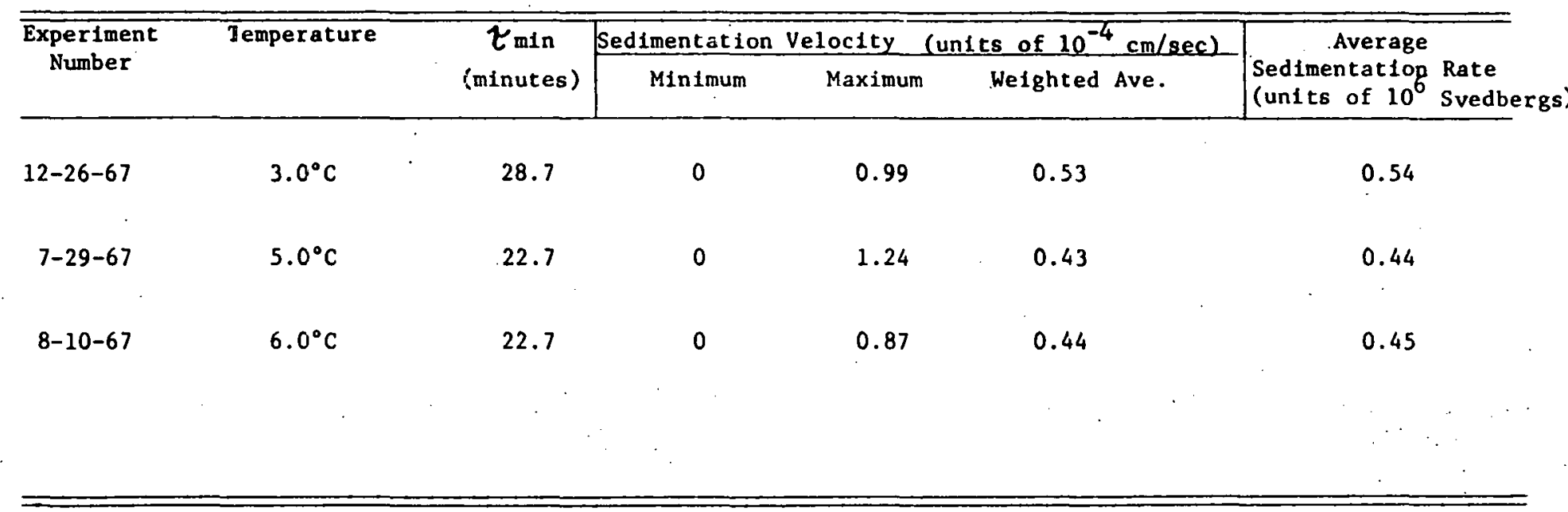


weighted average sedimentation velocity from $0.43-0.53 \times 10^{-4} \mathrm{~cm} / \mathrm{sec}$.

\section{Sedimentation-Upward-Electrophores18: High Electric Field Strengths}

The characteristics of the spermatozoa sample and Staflo operating parameters for this series of experiments are given in Table XXIV. In all experiments in this serles, the spermatozoa sample stream initially was $0.7 \mathrm{~mm}$ high and, during steady-state conditions, usually was at the bottom of layer 8 at the 1nlet. Most experiments were conducted near

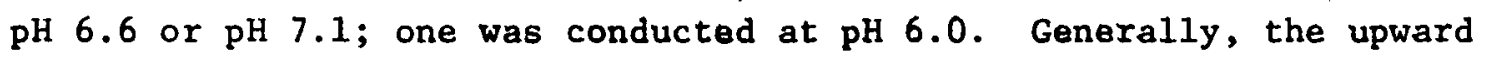
migration pattern spread over 2 layers and exited the flow-chamber $1 n$ layers 6 and 7 .

Unless it was desired to use the intrinsic motility of spermatozoa as a downward migration vector against the upward electrophoretic force, the experiment was conducted as close to $1^{\circ} \mathrm{C}$ as possible. On many occasions, the lowest temperature maintalned by the cold room was from $4-6^{\circ} \mathrm{C}$, which was higher than desired. Table XXV summarizes the distribution of spermatozoa among the collection fractions and the phase contrast observations and results of nigrosin-eosin staining thereof, in all experiments of this series. Offopring were obtained after artificial insemination of spermatozoa from the collection fractions in the 4 experiments listed last in Tables XXIV and XXV. Individual experiments, including the results of artificial insemination, will be considered in the order listed in those tables. The calculation of electrophoretic mobilities w111 be constdered separately.

\section{a. Ten Individual Experiments}

(1) Experiment 10-2-67. This experiment was conducted at $\mathrm{pH} 6.0$ with a spermatozoa sample having a high inftial quality. Observations with the Staflo microscope showed no motility in: the flow-chamber and that, after 
Table XXIV. Spernatozoa Sample and Staflo Operating Parameters for SedimentationUpward-Electrophoresis Experiments Using High Electric Field Strengths.

\begin{tabular}{|c|c|c|c|c|c|c|c|c|c|c|c|c|c|c|}
\hline \multirow{3}{*}{$\begin{array}{c}\text { Experiment } \\
\text { Number }\end{array}$} & \multicolumn{5}{|c|}{ Characteristics of Spermatozoa Sample } & \multicolumn{9}{|c|}{ General Staf 10 Operating Parameters } \\
\hline & \multicolumn{2}{|c|}{ Rabbit } & \multirow[t]{2}{*}{$\begin{array}{l}\text { Cooling } \\
\text { Mediuma }\end{array}$} & \multirow{2}{*}{\multicolumn{2}{|c|}{ Quality }} & \multirow{2}{*}{\multicolumn{2}{|c|}{$\begin{array}{l}\text { Initial } \mathrm{pH} \\
\text { Sample Conc. } \\
\text { (units } \\
\text { of } 10^{6} / \mathrm{ml} \text { ) }\end{array}$}} & \multirow{2}{*}{\multicolumn{2}{|c|}{$\begin{array}{l}\text { Temp. } \\
\text { RT Staflo } \\
\left({ }^{\circ} \mathrm{C}\right)\left({ }^{\circ} \mathrm{C}\right)\end{array}$}} & \multirow{2}{*}{$\begin{array}{c}\tau \text { ave } \\
\text { (min) }\end{array}$} & \multirow[t]{2}{*}{$\begin{array}{c}\overline{\min } \\
(\min )\end{array}$} & \multirow[t]{2}{*}{$\begin{array}{c}\tau E \\
(m i n)\end{array}$} & \multirow[t]{2}{*}{$\begin{array}{l}\mathrm{L} \\
(\mathrm{cm})\end{array}$} & \multirow[t]{2}{*}{$(\mathrm{E} / \mathrm{cm})$} \\
\hline & Name & Breed & & & & & & & & & & & & \\
\hline $10-2-67$ & Psi & Dutch & GPB & Excellent & Poor & 24 & 6.0 & 2.4 & 3.3 & 20 & 13.3 & 8.84 & 20 & 7.6 \\
\hline $8-18-67$ & otto & Dutch & EYN: & Falr & Poor & 20 & 6.60 & 3.5 & 4.1 & 20.5 & 13.7 & 9.10 & 20 & 6.2 \\
\hline $7-26-67$ & otto & Dutch & GPB & Fair & Poor. & 18.5 & 7.05 & 4.5 & 4.9 & 21.7 & 14.5 & 9.64 & 20 & 5.4 \\
\hline $7-29-67$ & Larry & Cal1f. & $\mathrm{GPB}$ & Excellent & Fair & 22.8 & 7.05 & 5.0 & 5.4 & 21.3 & 14.2 & 9.48 & 20 & 5.4 \\
\hline $8-10-67$ & Psi & Dutch & EYM & Excellent & Poor & 26 & 7.10 & 6.0 & 6.4 & 20.5 & 13.7 & 9.10 & 20 & 5.3 \\
\hline $9-22-67$ & Sam & $\mathrm{NZW}$ & GPB & Fair & Poor & 23.0 & 7.10 & 7.0 & 7.2 & 20 & 13.3 & 8.87 & 20 & 4.0 \\
\hline $8-29-67$ & otto & Dutch & $\mathrm{GPB}$ & Excellent & Good & 22.8 & 6.63 & 3.5 & 4.2 & 20.7 & 13.8 & 9.10 & 20 & 6.3 \\
\hline $9-13-67 \mathrm{~s}$ & Snoopy & Calif. & GPB & Superior & Excellent & 21.1 & 6.55 & 2.1 & 2.8 & 20.5 & 13.7 & 9.10 & 20 & 8.7 \\
\hline $9-13-67 P$ & Psi & Dutch & GPB & Excellent & Fair & 20.8 & 6.55 & 2.7 & 3.1 & 20.5 & 13.7 & 9.10 & 20 & 5.1 \\
\hline $9-18-67$ & Psi & Dutch & $\mathrm{GPB}$ & Excellent. & Fair & 25.6 & 7.15 & 2.8 & 3.2 & 20.5 & 13.7 & 9.07 & 20 & 5.5 \\
\hline
\end{tabular}


Table XXV. Summary of D1stribution, Phase Contrast Observations and Nigrosin-Eosin Staining of Spermatozoa in Sedimentation-Upward-Ileccrophoresis Experiments Using High Electric Field Strengths.

\begin{tabular}{|c|c|c|c|c|c|c|c|c|c|c|c|c|}
\hline \multirow{2}{*}{$\begin{array}{l}\text { Experiment } \\
\text { Number }\end{array}$} & \multirow[t]{2}{*}{$\mathrm{pH}$} & \multirow{2}{*}{$\begin{array}{c}\text { Spermatozoa } \\
\text { Sample }\end{array}$} & \multirow{2}{*}{\multicolumn{2}{|c|}{$\begin{array}{c}\text { Spera } \\
\text { Collected } \\
\text { Conc. } \quad(\%)\end{array}$}} & \multirow{2}{*}{$\begin{array}{l}\text { Temp. } \\
\left({ }^{\circ} \mathrm{C}\right)\end{array}$} & \multicolumn{4}{|c|}{ Phase Contrast Observations } & \multicolumn{3}{|c|}{ Nigrosin-Eosin Staining } \\
\hline & & & & & & $\begin{array}{c}C \\
(\%)\end{array}$ & $\begin{array}{l}\mathrm{CL} \\
(\%)\end{array}$ & $\begin{array}{l}\text { TW } \\
(\%)\end{array}$ & $\begin{array}{c}\mathrm{FM} \\
(\%)\end{array}$ & $\begin{array}{c}\mathrm{U} \\
(\%)\end{array}$ & $\begin{array}{l}\text { MNS } \\
(\%)\end{array}$ & $\begin{array}{c}S \\
(\%)\end{array}$ \\
\hline $10-2-67$ & $6 . C$ & $\begin{array}{l}\text { CF } 6 \\
\text { CF } 7 \\
\text { IS }\end{array}$ & $\begin{array}{l}0.5 \\
6.0\end{array}$ & $\begin{array}{r}3.3 \\
91.7\end{array}$ & $\begin{array}{l}3.3 \\
23 \\
3.3 \\
23 \\
3.3 \\
23\end{array}$ & $\begin{array}{l}50 \\
13.5 \\
79.7 \\
38.1 \\
91.5 \\
41.1\end{array}$ & $\begin{array}{r}50 \\
83.5 \\
20.5 \\
40 . \tilde{z} \\
8.5 \\
36.5\end{array}$ & $\begin{array}{c}0 \\
3.5 \\
0 \\
21.7 \\
0 \\
22.1\end{array}$ & $\begin{array}{c}0 \\
0 \\
0 \\
0 \\
0 \\
\ll 1.0\end{array}$ & $\begin{array}{c}- \\
30.5 \\
- \\
66.5 \\
- \\
68\end{array}$ & $\begin{array}{c}- \\
0.6 \\
- \\
0 \\
- \\
0.5\end{array}$ & $\begin{array}{c}- \\
68.9 \\
- \\
33.5 \\
\overline{1} .5\end{array}$ \\
\hline $8-18-6 ?$ & 6.60 & $\begin{array}{l}\text { CF } 5 \\
\text { CF } 6 \\
\text { IS }\end{array}$ & $\begin{array}{l}1.36 \\
3.10\end{array}$ & $\begin{array}{l}30.5 \\
59.5\end{array}$ & $\begin{array}{l}3.8 \\
22 \\
3.8 \\
22 \\
3.8 \\
22\end{array}$ & $\begin{array}{l}67.5 \\
62.2 \\
67.7\end{array}$ & $\begin{array}{l}32.5 \\
37.5 \\
32.5\end{array}$ & $\begin{array}{c}0 \\
\text { Poor } \\
0 \\
\text { Poor } \\
0 \\
\text { Poor }\end{array}$ & $\begin{array}{c}0 \\
\text { Poor } \\
0 \\
\text { Poor } \\
0 \\
\text { Poor }\end{array}$ & $\begin{array}{l}29 \\
45.5 \\
44.5\end{array}$ & $\begin{array}{l}2 \\
0 \\
11.5\end{array}$ & $\begin{array}{l}69 \\
54.5 \\
44.0\end{array}$ \\
\hline $7-26-6 ?$ & 7.015 & $\begin{array}{l}\text { CF } 5 \\
\text { CF } 6 \\
\text { CF } 7 \\
\text { IS }\end{array}$ & $\begin{array}{l}0.70 \\
4.0 \\
1.34\end{array}$ & $\begin{array}{c}11.6 \\
56.2 \\
22.2 \\
.\end{array}$ & $\begin{array}{l}4.5 \\
22 \\
4.5 \\
22 \\
4.5\end{array}$ & $\begin{array}{l}86.5 \\
23.9 \\
84.5 \\
21.0 \\
92.5\end{array}$ & $\begin{array}{r}13.5 \\
51.8 \\
15.5 \\
48.9 \\
7.5\end{array}$ & $\begin{array}{c}0 \\
10.8 \\
0 \\
11.3 \\
0\end{array}$ & $\begin{array}{c}0 \\
13.5 \\
0 \\
18.8 \\
0\end{array}$ & & & \\
\hline $7-29-6 ?$ & 7.05 & $\begin{array}{l}\text { CF } 6 \\
\text { CF } 7 \\
\text { IS }\end{array}$ & $\begin{array}{l}5.04 \\
3.53\end{array}$ & $\begin{array}{l}58.6 \\
41.4\end{array}$ & $\begin{array}{l}5.4 \\
23 \\
5.4 \\
23 \\
5.4 \\
23\end{array}$ & $\begin{array}{l}65.4 \\
26.9 \\
57.0 \\
32.2 \\
64.8\end{array}$ & $\begin{array}{r}10.7 \\
16.5 \\
6.6 \\
26.2 \\
4.4\end{array}$ & $\begin{array}{l}23.9 \\
48.7 \\
36.4 \\
24.3 \\
30.7\end{array}$ & $\begin{array}{c}0 \\
8.3 \\
0 \\
17.3 \\
0\end{array}$ & $\begin{array}{l}47 \\
45 \\
46\end{array}$ & $\begin{array}{l}1 \\
6\end{array}$ & $\begin{array}{l}50 \\
54 \\
48\end{array}$ \\
\hline
\end{tabular}


Table Xxv. (Continued)

\begin{tabular}{|c|c|c|c|c|c|c|c|c|c|c|c|c|}
\hline \multirow{2}{*}{$\begin{array}{l}\text { Experiment } \\
\text { Number }\end{array}$} & \multirow[t]{2}{*}{$\mathrm{pH}$} & \multirow{2}{*}{$\begin{array}{c}\text { Spermatozoa } \\
\text { Sample }\end{array}$} & \multirow{2}{*}{\multicolumn{2}{|c|}{$\begin{array}{l}\text { Sperm } \\
\text { Collected } \\
\text { Conc. }^{\mathrm{b}}(\%)\end{array}$}} & \multirow{2}{*}{$\begin{array}{l}\text { Temp. } \\
\left({ }^{\circ} \mathrm{C}\right)\end{array}$} & \multicolumn{4}{|c|}{ Phase Contrast Observations } & \multicolumn{3}{|c|}{ Nigrosin-Eosin Staining } \\
\hline & & & & & & $\begin{array}{c}\mathrm{C} \\
(\%)\end{array}$ & $(\%)$ & $\begin{array}{l}\text { TW } \\
(\%)\end{array}$ & $\begin{array}{c}F M \\
(\%)\end{array}$ & $\begin{array}{c}U \\
(\%)\end{array}$ & $\begin{array}{l}\text { MNS } \\
(\%)\end{array}$ & $\begin{array}{c}S \\
(\%)\end{array}$ \\
\hline $8-10-67$ & 7.10 & $\begin{array}{l}\text { CF } 6 \\
\text { IS }\end{array}$ & 5.0 & 99 & $\begin{array}{l}6 \\
23 \\
6 \\
23 \\
\end{array}$ & $\begin{array}{l}71.8 \\
11.7 \\
43.0 \\
11.5\end{array}$ & $\begin{array}{l}28.2 \\
34.1 \\
14.7 \\
19.1\end{array}$ & $\begin{array}{c}0 \\
50.0 \\
38.9 \\
48.2 \\
\end{array}$ & $\begin{array}{c}0 \\
4.2 \\
0 \\
21.2 \\
\end{array}$ & $\begin{array}{l}91 \\
90 \\
\end{array}$ & $\begin{array}{l}1 \\
1\end{array}$ & $\begin{array}{l}8 \\
9\end{array}$ \\
\hline $9-22-67$ & 7.10 & $\begin{array}{l}\text { CF } 6 \\
\text { CF } 7 \\
\text { IS }\end{array}$ & $\begin{array}{l}3.42 \\
3.72\end{array}$ & $\begin{array}{l}47.9 \\
52.1\end{array}$ & $\begin{array}{l}7 \\
23 \\
7 \\
23 \\
7 \\
23 \\
\end{array}$ & $\begin{array}{l}71 \\
32.2 \\
74 \\
34.8 \\
68 \\
25.3 \\
\end{array}$ & $\begin{array}{l}29 \\
63.5 \\
26 \\
58.7 \\
28.2 \\
71.5 \\
\end{array}$ & $\begin{array}{l}0 \\
4.3 \\
0 \\
6.5 \\
3.8 \\
3.2 \\
\end{array}$ & $\begin{array}{l}0 \\
0 \\
0 \\
0 \\
0 \\
0 \\
\end{array}$ & $\begin{array}{c}- \\
34 \\
- \\
35 \\
- \\
32.5 \\
\end{array}$ & $\begin{array}{l}- \\
1.5 \\
- \\
0.5 \\
- \\
4\end{array}$ & $\begin{array}{c}-\overline{64.5} \\
- \\
64.5 \\
\overline{64.5} \\
\end{array}$ \\
\hline $8-29-67$ & 6.63 & $\begin{array}{l}\text { CF } 6 \\
\text { CF } 7 \\
\text { IS }\end{array}$ & $\begin{array}{l}1.71 \\
5.60\end{array}$ & $\begin{array}{l}23.4 \\
76.6\end{array}$ & $\begin{array}{l}4.2 \\
23 \\
4.2 \\
23 \\
4.2 \\
23 \\
\end{array}$ & $\begin{array}{c}96.3 \\
- \\
97.5 \\
- \\
93.8 \\
- \\
\end{array}$ & $\begin{array}{l}3.7 \\
- \\
2.5 \\
- \\
6.2 \\
- \\
\end{array}$ & $\begin{array}{l}- \\
- \\
- \\
- \\
-\end{array}$ & $\begin{array}{r}40 \\
60 \\
> \\
-\end{array}$ & $\begin{array}{r}5 \overline{-} \\
- \\
75 \\
- \\
64 \\
\end{array}$ & $\begin{array}{l}- \\
3 \\
3.5 \\
- \\
1\end{array}$ & $\begin{array}{c}- \\
38 \\
- \\
21.5 \\
- \\
35 \\
\end{array}$ \\
\hline $9-13-675$ & 6.55 & $\begin{array}{l}\text { CF } 6 \\
\text { CF } 7 \\
\text { IS }\end{array}$ & $\begin{array}{l}1.9 \\
8.0\end{array}$ & $\begin{array}{l}19.2 \\
80.8\end{array}$ & $\begin{array}{l}2.8 \\
22 \\
2.8 \\
22 \\
2.8 \\
22 \\
\end{array}$ & $\begin{array}{c}92.8 \\
- \\
97.0 \\
- \\
96.3 \\
- \\
\end{array}$ & $\begin{array}{l}7.2 \\
- \\
3.0 \\
- \\
3.7 \\
- \\
\end{array}$ & $\begin{array}{l}- \\
- \\
- \\
- \\
-\end{array}$ & $\begin{array}{r}>50 \\
>50 \\
>50\end{array}$ & $\begin{array}{c}- \\
87 \\
\overline{7} \\
72.5 \\
\overline{6} \\
\end{array}$ & $\begin{array}{l} \\
1.0 \\
- \\
6 \\
- \\
4\end{array}$ & $\begin{array}{c}\overline{1} \\
- \\
21.5 \\
\overline{2} \\
29 \\
\end{array}$ \\
\hline $9-13-67 \mathrm{P}$ & 6.55 & $\begin{array}{l}\text { CF } 7 \\
\text { CF } 8 \\
\text { IS }\end{array}$ & $\begin{array}{l}4.25 \\
5.20\end{array}$ & $\begin{array}{l}45.0 \\
55.0\end{array}$ & $\begin{array}{l}3.1 \\
22 \\
3.1 \\
22 \\
3.1 \\
22 \\
\end{array}$ & $\begin{array}{c}88.3 \\
- \\
92.5 \\
- \\
- \\
-\end{array}$ & $\begin{array}{l}11.7 \\
- \\
7.5 \\
- \\
- \\
\end{array}$ & $\begin{array}{l}- \\
- \\
- \\
- \\
-\end{array}$ & $\begin{array}{c}\sim \\
\sim \\
"- \\
-\end{array}$ & $\begin{array}{c}- \\
52.5 \\
- \\
59.5 \\
- \\
51\end{array}$ & $\begin{array}{l}\overline{-} \\
1.5 \\
\overline{1} \\
1.5 \\
\overline{0}\end{array}$ & $\begin{array}{r}- \\
46 \\
- \\
39 \\
- \\
49\end{array}$ \\
\hline $9-18-67$ & 7.15 & $\begin{array}{ll}\text { CF } & 6 \\
\text { CF } & 7 \\
\text { IS }\end{array}$ & $\begin{array}{l}2.5 \\
4.6\end{array}$ & $\begin{array}{l}35.2 \\
64.8\end{array}$ & $\begin{array}{l}3.0 \\
23 \\
3.0 \\
23 \\
3.0 \\
23 \\
\end{array}$ & $\begin{array}{l}80 \\
91.5\end{array}$ & $\begin{array}{c}20 \\
8.5 \\
12.6 \\
- \\
\end{array}$ & $\begin{array}{l}- \\
- \\
- \\
- \\
-\end{array}$ & $\begin{array}{r}2 \overline{-} \\
- \\
40 \\
- \\
50\end{array}$ & $\begin{array}{c}\overline{61.5} \\
- \\
75.5 \\
- \\
66.5\end{array}$ & $\begin{array}{l}\overline{0} \\
\overline{0} \\
0.5 \\
\overline{2} \\
\end{array}$ & $\begin{array}{c}3 \overline{8} .5 \\
\overline{24} \\
\overline{31.5} \\
\end{array}$ \\
\hline See Gl & Iry & explan & 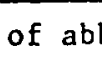 & $a$ & s; & in ur & ts of & spe & 1 . & & & \\
\hline
\end{tabular}


passing into the region of the electric field, essentialiy all spermatozoa were orlented vertically with their talls polnting toward the top (positive) electrode. The migration pattern was difficult to control and only $8 \%$ of the spermatozoa $\left(0.5 \times 10^{6} / \mathrm{ml}\right)$ exited in layer 6 . In Table XXV, it is seen that the percentage of capped spermatozoa at $3.3^{\circ} \mathrm{C}$ and the percentages of tall-wagging and unstained spermatozoa at $23^{\circ} \mathrm{C}$ were significantly less In CF 6 than in 7. This is attributed primarily to the low spermatozoa concentration In CF 6. Although the percentage of capped spermatozoa at $3.3^{\circ} \mathrm{C}$ was 81 ightly 1 ess in CF 7 than in the Inftial sample, the percentages of tall-wagging and unstained spermatozoa after centrifugation were essentially the same in those 2 samples. There were no motile spermatozoa at room temperature in CF 6 or 7 and less than $1 \%$ in the initial sample. Because there was little change in the control sample which had been stored In egg yolk medium, the deterioration of the spermatozoa in the Staflo apparatus and in the initial sample is believed to be due to an irreversible loss of motility at $\mathrm{pH} 6.0$.

(2) Experiment 8-18-67. Th1s experiment was conducted with a spermatozoa sample of poor Initial quality. The migration pattern was highly acceptable throughout the experiment with $30.5 \%$ and $69.5 \%$ of the spermatozoa recovered in fractions 5 and 6 , respectively. As shown in Table $X X V$, there were no significant differences between the percentages of capped spermatozoa in fractions 5 and 6 and among those fractions and the Inttial sample at $3.8^{\circ} \mathrm{C}$. After centrifugation, too little supernatant was removed and the addition of the egg yolk medium resulted in a precipitate which hindered the evaluation of the low percentage and poor degree of motility present. CF 6 had a much higher percentage of unstained. spermatozos than CF 5 and was comparable to the initial sample. 
(3) Experiment 7-26-67. Because Inlet syringe No. 6 was left off inadvertently, the inlet sample stream reached a steady-state position at the boundary between layers 8 and 9. Otherwise, the migration pattern was technically acceptable. No observations were made with the Staflo microscope. The spermatozoa were distributed among fractions 5, 6 and 7, but fraction 5 was not evaluated. There was no significant difference between fractions 6 and 7 with regard to the percentage of capped spermatozoa at $4.5^{\circ} \mathrm{C}$ or the percentages of capped, tall-wagging and motile spermatozoa at $22^{\circ} \mathrm{C}$. The large increases in the percentages of capless spermatozoa between the observations at $4.5^{\circ} \mathrm{C}$ and $22^{\circ} \mathrm{C}$ in fractions 6 and 7 are attributed to damage during centrifugation.

(4) Experiment 7-29-67. Immediately after collection of the ejaculate, the spermatiozoa swam in very small circles, but eventually strafghtened out. Because a $3 \times 12$ sedimentation experiment was being conducted simultaneously, no microscoplc observations were made in the flow-chamber. The migration pattern was stable throughout the experiment and the concenLrations of sperinatozoa recovered in fractions 6 and 7 were $5.04 \times 10^{6} / \mathrm{ml}$ and $3.53 \times 10^{6} / \mathrm{ml}$, respectively. At $5.4^{\circ} \mathrm{C}, 23.9 \%$ and $34.6 \%$ of the sperm-. atozoa In fractions 6 and 7 , respectively, had tall-wagging. The 5\% and $20 \%$ Increases in capless spermatozoa in fractions 6 and 7, respectively, because of centrifugation were less than usually encountered in this series of experiments. At room temperature, after centrifugation, the spermatozoa In CF 6 had a higher percentage of tall-wagging, but a lower percentage of forward motion than CF 7. With regard to nigrosin-eosin staining results, there was no signiflcant difference between the 2 fractions or between those fractions and the initial sample. However, the percentages of stained spermatozoa were much greater than the percentages of capless spermatozoa for both collection fractions, suggesting deterloration during the staining 
procedure.

(5) Experiment 8-10-67. Inlet syringe No. 6 was turned off because of mechanical difflculty and, therefore, the sample stream at the inlet was high in layer 8. After steady-state conditions had been reached, the migration pattern remained stable. Essentlally 100\% of the spermatozoa were oriented vertically with the talls toward the top (positive) electrode. A low percentage of the spermatozoa showed slight rolling and tail-wagging at a temperature of $6.4^{\circ} \mathrm{C}$. Approximately $99 \%$ of the 1ntact spermatozoa exited in layer 6 while fractions 5 and 7 contained many tails and heads, respectively. At $6^{\circ} \mathrm{C}, 38.9 \%$ of the spermatozoa in the initial sample showed tall-wagging but no motility was observed at that temperature in CF 6. After centrifugation and re-suspension in egg yolk medium, the initial sample had about the same percentage of tall-wagging as CF 6 , but had a higher percentage of motile spermatozoa. However, the percentages of unstained spermatozoa were essentially the same in the 2 samples.

(6) Experiment 9-22-67. Th1s experiment was conducted at $7.5^{\circ} \mathrm{C}$ with the intention of separating the highly motile spermatozoa from the remainder of the sample by allowing downward swlming of the spermatozoa against the electric fleld. The inftial percentage and degree of motility of the spermatozoa was excellent, but the sample deteriorated markedly during the course of the experiment, both in the GPB and in the egg yolk medium. No motility was observed in the flow-chamber at $7.4^{\circ} \mathrm{C}$ and essent1ally all spermatozon were orfented vertically with the tall pointing toward the top (positive) electrode. Only $3.8 \%$ of the spermatozoa in the inftial sample had tail-wagging at $7^{\circ} \mathrm{C}$ and all samples had less than $7 \%$ tail-wagging at room temperature. After collection, there were no differences among the 8amples. The centrifugation resulted in a $33 \%-43 \%$ increase in capless spermatozoa. 
(7) Experiment 8-29-67. Inlet 6 did not flow because of mechanical difficulty. However, after reaching equilibrium conditions, the migration pattern appeared stable and resulted in concentrations of $1.71 \times 10^{6}$ and $5.60 \times 10^{6}$ spermatozoa/ml in fractions 6 and 7 , respectively. No observations were made in the flow-chamber. Evaluation in a hemocytometer at $3.5^{\circ} \mathrm{C}$ showed no motile spermatozoa in the initial sample. As shown in Table XXV, there were no significant differences among the samples at $4.2^{\circ} \mathrm{C}$. After centrifugation and resuspension in egg yolk medium, the spermatozoa in fractions 6 and 7 had $40 \%$ and $60 \%$ forward motion, respectIvely, as compared to slightly greater than $50 \%$ in the initial sample. Thus, CF 7 was slightly enriched with regard to motile sperm. The perrentage of unstained spermatozoa was greater in CF 7 than in either CF 6 or the Infial sample, agaln, ouggesting absolute enrichment. The insemination of spermatozoa from CF 6 and the control sample resulted in no offojrisy. Huwever, as showin in lable XXVI, the insemination of spermatozoa from CF 7 resulted in 2 litters with a total of 5 offspring.

(8) Experfment 9-13-67s. The inftial quality of the spermatozod sample used in this experiment was superior. No observations were made in the flow-chamber with the microscope; however, at $2^{\circ} \mathrm{C}$, no motile spermatozoa were observed in a hemocytometer in GPB. Even though the spermatozoa concentrations were $1.9 \times 10^{6}$ and $8.0 \times 10^{6} / \mathrm{ml}$ in fractions 6 and 7 , respect= ively, thero were no signilicant dfferences among the fractions and the inftial sample with regard to capped spermatozoa at $2.8^{\circ} \mathrm{C}$ and motile spermatozoa at room temperature. The percentage of unstained spermatomoa was greater in CF 6 than in CF 7 and both were greater than the initial sample. Having both fractions better than the initial sample suggests an artifact of staining. 
Table XXVI. Summary of Artificial Insemination (AI) Results.

\begin{tabular}{|c|c|c|c|c|c|c|c|c|c|}
\hline \multirow{2}{*}{$\begin{array}{l}\text { Exper1ment } \\
\text { Number }\end{array}$} & \multirow{2}{*}{$\begin{array}{c}\text { Spermatozoa } \\
\text { Sample }^{\mathrm{a}}\end{array}$} & \multicolumn{3}{|c|}{ Artificial Insemination } & \multicolumn{5}{|c|}{ Insemination Results } \\
\hline & & $\begin{array}{l}\text { No. of } \\
\text { Females }\end{array}$ & $\begin{array}{l}\text { Sperm } \\
\text { per Female }\end{array}$ & Comments & $\begin{array}{l}\text { Litters } \\
\text { (\#) }\end{array}$ & Total & Males & Females & Comments \\
\hline \multirow[t]{3}{*}{$8-29-67$} & CF 6 & 1 & $8 \times 10^{6}$ & Good AI & 0 & 0 & & & One eaten. \\
\hline & CF 7 & 2 & $8 \times 10^{6}$ & Good AI & 2 & 5 & 4 & 0 & \\
\hline & Control & 1 & $16 \times 10^{6}$ & Good AI & 0 & 0 & & & \\
\hline \multirow[t]{4}{*}{$\begin{array}{c}9-13-67 \\
S\end{array}$} & & 1 & $5 \times 10^{6}$ & $\begin{array}{l}\text { Urinated } \\
\text { during AI }\end{array}$ & 0 & 0 & & & \\
\hline & CF 7 & 3 & $8 \times 10^{6}$ & $\begin{array}{l}1 \text { urinated } \\
\text { during AI } \\
2 \text { partial }\end{array}$ & 0 & 0 & & & \\
\hline & & & & $\begin{array}{l}\text { loss of in- } \\
\text { seminate }\end{array}$ & 2 & 8 & 4 & 4 & \\
\hline & Control & 1 & $15 \times 10^{6}$ & Good AI & 1 & 1 & 1 & 0 & \\
\hline \multirow[t]{2}{*}{$\begin{array}{c}9-13-67 \\
P\end{array}$} & $\therefore C F 7$ & 1 & $14 \times 10^{6}$ & $\begin{array}{l}\text { Re-insemin- } \\
\text { ation after } \\
\text { sample was } \\
\text { squirt out }\end{array}$ & 1 & 3 & 1 & 1 & One eaten. \\
\hline & Control & 1 & $25 \times 10^{6}(?)$ & $\begin{array}{l}\text { Entire sam- } \\
\text { ple squirt } \\
\text { out by fe- } \\
\text { male. } \\
\text { Good AI } \\
\end{array}$ & 0 & 0 & & & Pseudopregnant \\
\hline \multirow[t]{3}{*}{$9-18-67$} & CF 6 & 2 & $7 \times 10^{6}$ & $\begin{array}{l}\text { Lost } 1 / 2 \text { of } \\
1 \text { inseminate }\end{array}$ & 0 & 0 & & & \\
\hline & CF 7 & 2 & $13 \times 10^{6}$ & Good AI & 1 & 2 & 1 & 1 & \\
\hline & Control & 1 & $14 \times 10^{6}$ & Good AI & 0 & 0 & & & \\
\hline
\end{tabular}

a. CF: Collection fraction 
Flve rabbits were inseminated with spermatozoa of the highest quality seen in any collection fractions in any. Staflo experiment. As shown in Table XXVI, the oingle Insemination with spermatozoa from CF 6 gave no offspring. However, two of the three inseminations with spermatozoa from CF 7 gave 2 litters with a total of 8 offspring; 4 males and 4 females. The single control insemination gave 1 male offepring.

(9) Experiment 9-13-67P. The Inttial quality of the spermatozoa sample used in this experiment was excellent, but not quite so good as that used in Experiment 9-13-67S. Because the electric field strength was lower than that used in Experiment 9-13-67S, the spermatozoa were distributed between fractions 7 and 8 , in concentrations of $4.25 \times 10^{6}$ and $5.20 \times 10^{6} / \mathrm{m} 1$, respectively. No observations were made in the flow-chamber. There were no motile spermatozoa in either of the 2 fractions at $2.6^{\circ} \mathrm{C}$ at the termination of the experiment. However, it cannot be stated that there was no motility in the flow-chamber where the average temperature was $3.1^{\circ} \mathrm{C}$. There was no significant difference between fractions 7 and 8 with regard to capped spermatozoa at $3.1^{\circ} \overline{\mathrm{C}}$, or unstalned spermatozoa at room temperature.

Because of slight precipitation of the egg yolk medium in the phosphate buffer, it was difficult to assess the motility of the spermatozoa at room temperature. Because only about $30 \%$ of the spermatozoa in each fraction and in the intial sample had an excellent degree of motility, only 1 rabbit was inseminated with spermatozoa from each sample. Despite difficulty. In. insemination, 1 litter of 3 offspring was obtained from CF 7. No offspring were obtained from the control sample or CF 8; however, both samples were refected by the females during insemination.

(10) Experiment 9-18-67. The spermatozoa sample used in this experiment initially had approximate1y $60 \%$ forward motion at room temperature, but had 
no motility at $2.8^{\circ} \mathrm{C}$ in $\mathrm{GPB}$. No observations were made in the flowchamber with the microscope. The percentage of capped spermatozoa in CF 7 was slightly higher than in elther CF 6 or the initial sample. At room temperature, the spermatozoa in CF 7 had approximately $40 \%$ motility compared to $24 \%$ in CF 6 and 50\% in the initial sample. CF 7 had a higher percentage of unstained spermatozoa than CF 6 or the initial sample. Thus, CF 7 contained higher quality spermatozoa than CF 6 by all criteria, and was almost as good as the inftial sample.

Two rabbite were Inseminated with spermatozoa from each fraction and one from the control sample. The only litter was obtained from the insemInation of spermatozoa from CF 7 and consisted of 2 offspring, 1 male and 1 female.

b. Calculation of Electrophoretic Mobilities. Table XXVII presents the minimum, maximum and average observed and theoretical electrophoretic mobillties for the SUE experiments with high electric fleld strengths. Appendix $G$ is a sample calculation of the electrophoretic mobilities for Experiment 7-29-67, using the formulae presented in Chapter III. All values In Table XXVII have been normalized to $3^{\circ} \mathrm{C}$ to facilitate comparison within this series and with other experiments. The observed minimum, maximum and average electrophoretic mobilities are calculated from the minimum and maximum SUE migration distances. Thus, they are not corrected for the contribution of sedimentation to the net migration.

The theoretical electrophoretic mobllities are corrected for the expected sedimentation component to the migration pattern. The corrections were made using the sedimentation velocity range of $0-0.99 \times 10^{-4} \mathrm{~cm} / \mathrm{sec}$ and the weighted average sedimentation velocity of $0.53 \times 10^{-4} \mathrm{~cm} / \mathrm{sec}$ from Experiment 12-26-67. As shown in Figure 19, (Chapter III), the minimum electrophoretic migration distance is equal to the sum of the minimum SUE 
Table XXVII. Observed (Uncorrected) and Theoretical Electrophoretic Mobilities of Rabbit Spermatozoa; Sedimentation-Upward-Electrophoresis Experiments Using High Electric Field Strengths.

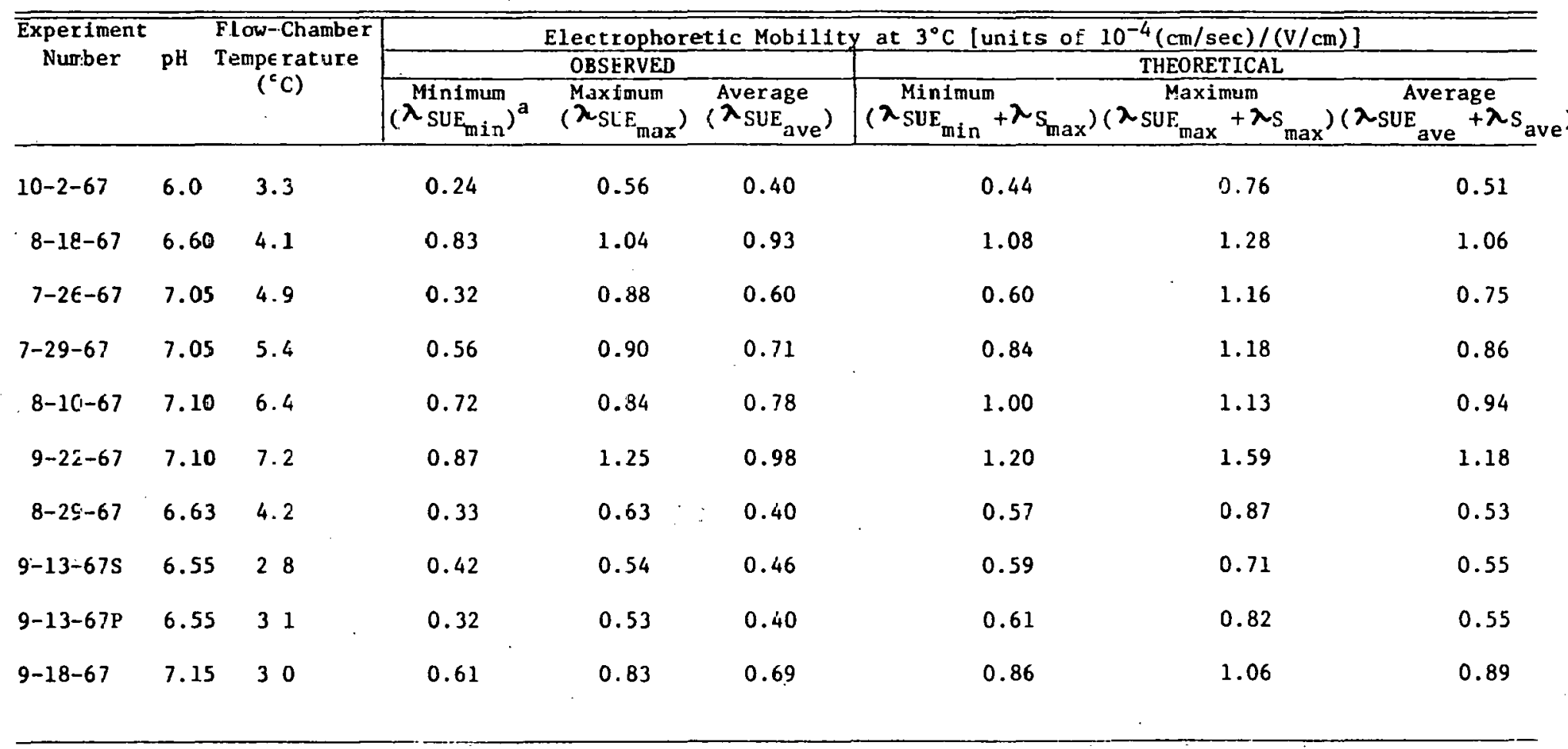

a.Migration distances used in calculations are shown in parentheses. 
and maximum sedimentation migration distances. The maximum theoretical electrophoretic migration distance is equal to the sum of the maximum SUE and maximum sedimentation migration distances, assuming independence of the 2 properties. The welghted average electrophoretic migration distance is equal to the sum of the weighted average SUE and welghted average sedimentation distances.

The effect of motility is not considered in the calculations of either the observed or the theoretical electrophoretic mobility. A comparison of the electrophoretic moblifies and an interpretation of the results are presented in the Discussion.

\section{Sedimentation-Electrophores1s: Low Electric Field Strengths.}

Table XXVIII is a summary of the characteristics of the spermatozoa sample and the Staflo operating parameters for sedimentation-upward- and -downward-electrophoresis experiments with low electric field strengths. Table XXIX summarlzes the distribution of spermatozoa among the collection fractions and the phase contrast observations and results of $\mathrm{n}$ igrosin-eosin staining thereof, in those experiments.

\section{a. Upward Electrophoresis}

(1) Experiment 7-9-67. The spermatozoa sample used in this experiment inftially had a fair degree of forward motion at room temperature and a slight degree of tall-wagging in a hemocytometer at $3.5^{\circ} \mathrm{C}$. Observations with the Staflo microscope showed that after the spermatozoa entered the electric field, all had a vertical orientation with head down and tall up. Mild twisting and rolling of spermatozoa was observed in the lower part of the migration pattern only. The migration pattern at $\mathrm{pH} 7.1,3.9^{\circ} \mathrm{C}$ and an electric field strength of $1.2 \mathrm{~V} / \mathrm{cm}$ was stable throughout the experiment and gave spermatozoa concentrations of $4.32 \times 10^{6}$ and $2.65 \times 10^{6} / \mathrm{m} 1$ in 
Table XXVIII. Spermatozoa Sample and Staflo Operating Parameters for SedimentationElectrophoresis Expe=iments Using Low Electric Field Strengths. ${ }^{a}$

\begin{tabular}{|c|c|c|c|c|c|c|c|c|c|c|c|}
\hline \multirow{3}{*}{$\begin{array}{l}\text { Experiment } \\
\text { Number }\end{array}$} & \multicolumn{5}{|c|}{ Characteristics of Spermatozoa Sample } & \multicolumn{6}{|c|}{ General Staf lo Operating Parameters } \\
\hline & \multicolumn{2}{|c|}{ Rabbit } & \multirow{2}{*}{$\begin{array}{l}\text { Cooling } \\
\text { Medium }\end{array}$} & \multicolumn{2}{|c|}{ Quality } & \multirow{2}{*}{$\begin{array}{l}\text { Initial pH } \\
\text { Sample Conc. } \\
\text { (units } \\
\text { of } 10^{6} / \mathrm{ml} \text { ) }\end{array}$} & \multirow{2}{*}{$\begin{array}{l}\text { Temp. } \tau \text { ave } \\
\text { RT Staflo (min) } \\
\left({ }^{\circ} \mathrm{C}\right)\left({ }^{\circ} \mathrm{C}\right)\end{array}$} & \multirow{2}{*}{$\tau_{\min }$} & \multirow{2}{*}{$\tau_{\mathrm{E}}$} & \multirow{2}{*}{$\begin{array}{l}\mathrm{L} \\
(\mathrm{cm})\end{array}$} & \multirow{2}{*}{$\begin{array}{l}\tilde{E} \\
(\mathrm{~V} / \mathrm{cm}\end{array}$} \\
\hline & Nane & Breed & & Inttial & Ir.al & & & & & & \\
\hline
\end{tabular}

A. SUE

\begin{tabular}{|c|c|c|c|c|c|c|c|c|c|c|c|c|c|c|}
\hline $7-9-67$ & Larry & N2W & EYM & Fair & Pocr & 20.5 & 7.10 & 3.8 & 3.9 & 30.7 & 20.5 & 17.1 & 25 & 1.2 \\
\hline $10-3-67$ & Snoopy & Callf. & GPB & Poor & Pocir & 21.7 & 6.60 & 2.2 & 2.3 & 37 & 24.7 & 20.6 & 25 & $1: 7$ \\
\hline
\end{tabular}

B. SDE

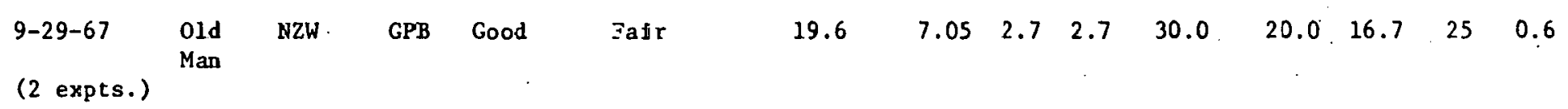

a. See clossary for explanation of definitions. 
Table XXIX. Summary of Distribution, Phase Contrast Observations and Nigrosin-Eosin Staining of Spermatozoa; Sedimentation-Electrophoresis Experiments Using Low Electric Field Strengths.

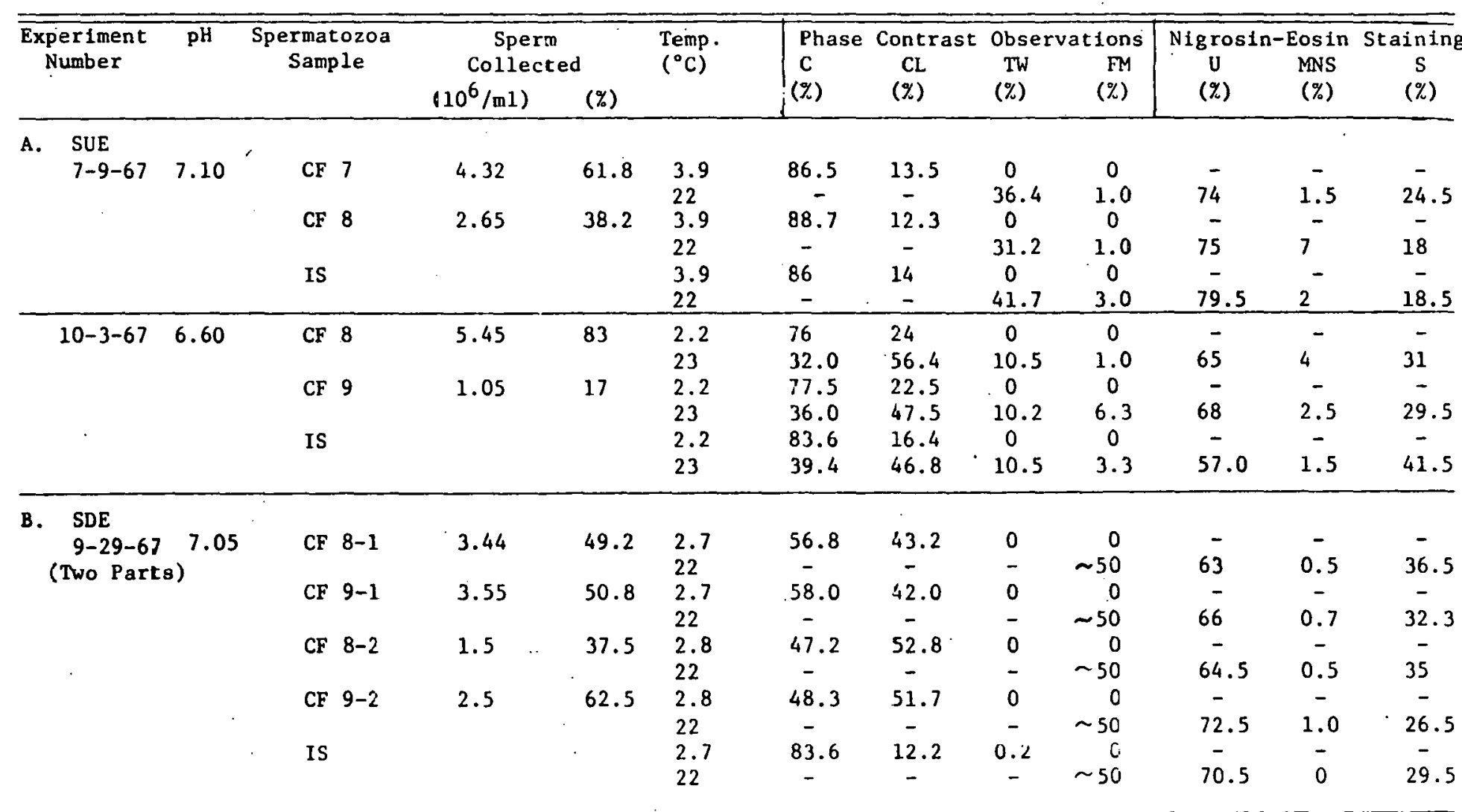

a. See Glossary for explanation of abbreviations. 
fractions 7 and 8 , respectively. At room temperature, the spermatozoa In CF 7 had a slightly higher percentage of tal1-wagging than CF 8 , and a slightly lower percentage than in the initial sample. There were no other differences among the spermatozoa samples at room temperature or at $3.9^{\circ} \mathrm{C}$, as shown in Table XXIX A.

(2) Experiment 10-3-67. This experiment was conducted at $\mathrm{pH} 6.6,2.3^{\circ} \mathrm{C}$ and an electric field strength of $1.7 \mathrm{~V} / \mathrm{cm}$. Even though the sample stream at the inlet was in the same location as in Experiment 7-9-67, the migration pattern was lower and gave spermatozoa concentrations of $5.45 \times 10^{6}$ and $1.05 \times 10^{6} / \mathrm{ml}$ in fractions 8 and 9 , respectively. In the region of the electric fleld, spermatozoa were orlented vertically with the tail up and head down. No motflity was observed in the flow-chamber or in a hemocytometer at $2.2^{\circ} \mathrm{C}$.

There were no signiflcant differences among the collection fractions and the initial sample with regard to the percentages of capped spermatozoa at $2.2^{\circ} \mathrm{C}$ or capped and tall-wagging spermatozoa at $23^{\circ} \mathrm{C}$. Because of the extremely poor motility, the differences among the samples is not belleved to be slgniflcant. The slightly higher percentages of unstained spermatozoa In the fractions compared to the initial sample are probably due to the staining procedure.

The differences between Experiment 7-29-67 and 10-3-67 are considered In the Discussion.

b. Downward-Electrophores1s. Experiment 9-29-67 consisted of 2 separate Staflo fractionations using the same spermatozoa sample and the same operating conditions. Even though there was no change in the location of the sample stream at the inlet during the second experiment, there is a slight possibility of a change in the migration pattern during that experiment because of the very slow rate at which equilibrium is re-established in 
in the flow-cell after emptying the collection contalners. This may explain the slight difference in distribution of spermatozoa between the 2 collection fractions in the 2 experiments as shown in Table XXIX B. Approximately $50 \%$ of the spermatozoa had a high degree of forward motion at room temperature before the inftial cooling, but no motility was observed in a hemocytometer at $2.7^{\circ} \mathrm{C}$. Except for a slightly higher percentage of unstained spermatozoa in CF 9-2 compared to CF 8-2, there were no differences between the respective fractions in the 2 parts of the experiment at $2.8^{\circ} \mathrm{C}$ or at room temperature.

In the region of the electric field, with the bottom electrode positive, there was a slight differentlal orientation of the spermatozoa in the top and bottom of the migration profile. Near the top, the orientation ranged from nearly horizontal to a $45^{\circ}$ angle from the horizontal with the tall angled downward. Near the bottom, the orlentation ranged from the last mentloned $45^{\circ}$ angle to just beyond the horizontal so that the head was in a slightly downward position. A higher percentage of spermatozoa was obliquely or horizontally orfented than in any other type of Staflo experiment reported in this thesis.

The expected average sedimentation migration distance is greater than the observed average SDE migration distance. This unexpected result and the quantitative aspects of the experiment are considered in the Discussion.

\section{Discussion}

\section{Sedimentation}

a. Comparison of Three Experiments. As shown in Table XXI, there were no significant differences among the collection fractions in individual experiments with regard to the distribution of capped spermatozoa at low temperatures or of unstained spermatozoa at room temperature. Insufficient 
observations were made to evaluate fractionation with regard to motility. Thus, within the limitations of the two- and three-layer fractionations of spermatozoa samples in this series of experiments, there does not appear to be any influence of morphology or of viability on the sedimentation velocity. Because the quality of the spermatozoa was inadequate for artificial insemination, the relationship between sedimentation velocity and chromosomal content of the spermatozoa could not be evaluated.

In Experiment 12-26-67, the minimum sedimentation velocity was estimated to be $0 \mathrm{~cm} / \mathrm{sec}$ inasmuch as some spermatozoa were observed in the top part of the migration pattern. The maximum sedimentation velocity for the three experiments ranges from $0.87-1.24 \times 10^{-4} \mathrm{~cm} / \mathrm{sec}$. This wide range may be a partial reflection of the difficulty in accurately measuring small migration distances in the flow-chamber. The minimum and maximum sedimentatIon velocities calculated for the $2.5^{\circ} \mathrm{C}$ sucrose sertes of early sedimentation experiments (Chapter IV), In a simflar medium, are 0 and $1.07 \mathrm{x}$ $10^{-4} \mathrm{~cm} / \mathrm{sec}$, respectively, indicating good agreement between the two series of experiments. As expected, the weighted average sedimentation velocity range of $0.43-0.53 \times 10^{-4} \mathrm{~cm} / \mathrm{sec}$ for the three experiments at $3^{\circ} \mathrm{C}$, encompasses the average of $0.49 \times 10^{-4} \mathrm{~cm} / \mathrm{sec}$ for the $2.5^{\circ} \mathrm{C}$ sucrose experiments (Chapter IV). The agreement between the two sets of sedimentation experiments in the low density-low viscostty media strengthens the impression stated In Chapter IV that the apparent sedimentation velocities in the experiments by Bhattacharya ${ }^{35}$ and by others 29,205 are high.

In Experiment 12-26-67, the average sedimentation velocity calculated from the minimum and maximum sedimentation distances $180.523 \times 10^{-4} \mathrm{~cm} / \mathrm{sec}$ compared to the welghted average sedimentation velocity of $0.53 \times 10^{-4} \mathrm{~cm} / \mathrm{sec}$. The excellent agreement between these 2 methods of calculating the average 
sedimentation velocity suggests a unfform distribution in the migration pattern. However, the small migration distance is a limiting factor in this interpretation of the results.

b. Mygration Comparison: Actual vs. Hypothetical Spermatozoa Populations Figure 55 is a comparison of the actual distribution of spermatozoa in Experiment 12-26-67 with the expected distribution of hypothetical spermatozoa populations characterlzed by uniform, blmodal and normal (Gaussian) distributions of sedimentation velocities over the range $0-0.99 \times 10^{-4} \mathrm{~cm} /$ sec. As shown in Figure 55, the observed distribution of spermatozoa among collection fractions 8,9 and 10 was $23.1 \%, 61.6 \%$ and $15.3 \%$, respectively. With a uniform population, one would expect $23.6 \%, 68.9 \%$ and $7.5 \%$ of the spermatozoa in fractions 8,9 and 10 , respectively, under the same Staflo operating conditions. The 2 distributions compare favorably except for the higher percentage of spermatozoa in fraction 10 in Experiment 12-26-67. This might be due either to the observed mild degree of microscopic streaming, or to a skewed distribution of the actual sedimentation velocities toward the maximum.

Comparison of the actual and the hypothetical bimodal distributions in Figure 55 shows good agreement in fraction 10 but not in fraction 8 where there is a higher percentage of spermatozoa in the bimodal distribution. A difference of the same magnitude, but in the opposite direction, is seen in fraction 9 . In Figure 55, spermatozoa are shown distributed evenly throughout layer 9 because a sperm count in that collection fraction would not give information on the differential distribution of spermatozoa In the $0.125 \mathrm{~cm}$ high layer represented by that fraction.

As shown in Figure 55, the expected diatribution of spermatozoa among the collection fractions in the case of population with a normal 
COMPARISON OF ACTUAL SPERM DISTRIBUTION AND HYPOTHETICAL POPULATIONS

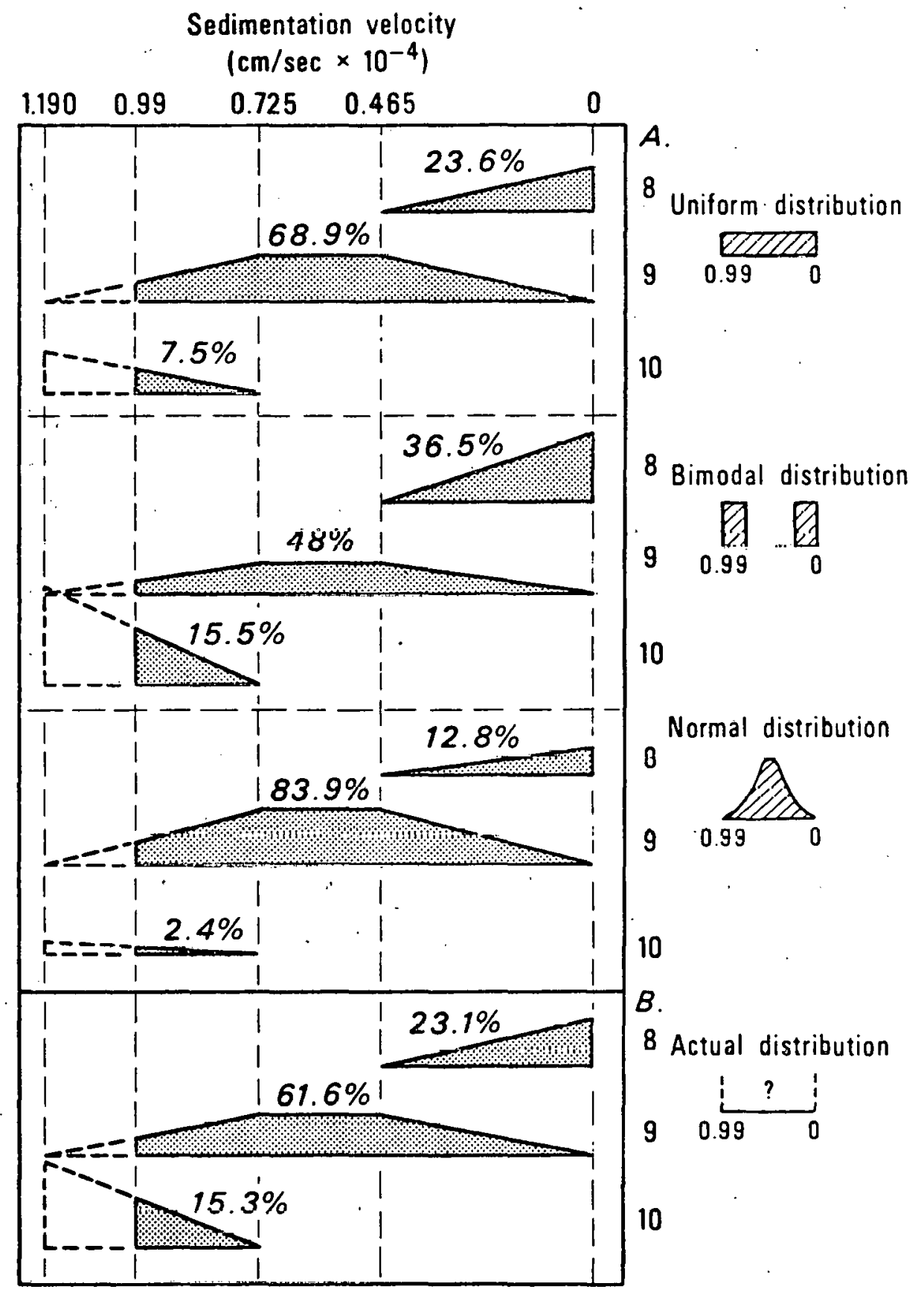

DBL 70115993

F1g. 55. Distributions of rabbit spermatozoa for actual, and three hypothetical distributions of sedimentation velocities. 
distribution of sedimentation velocities differs widely from the actual distribution. This is the least likely possibility of the three hypothetical distributions considered. Even recognizing the limitations of a 3-layer fractionation of the spermatozoa population in the actual experiment, the comparison of the normal distribution with the actual distribution 18 very unfavorable.

Of the three hypothetical distributions in Figure 55, the uniform distribution compares most favorably with the actual experiment. A better evaluation of the type of distribution of sedimentation velocities In a population of spermatozoa would be possible if the sedimentation migration pattern spread over 5 or 6 layers, thereby allowing a more quantitative comparioon of actual and hypothetical distributions.

\section{Sedimentation-Upward-Electrophores1s (SUE): High Electric Field} Strengths.

As shown In Table XXIV, the spermatozoa samples and Staflo operating conditions differed considerably among the experiments in this series. The migration profile generally spread from an inlet sample thickness of $0.7 \mathrm{~mm}$ to a final height of $2.0 \mathrm{~mm}$ or less in the centerline of the flow-chamber. Except for one experiment, in which essentially all of the spermatozoa exited the flow-chamber in one layer, the fractionation was between two layers. The ten experiments will be evaluated in terms of (1) the distribution of categories of spermatozoa between the collection fractions; (2) the effects of $\mathrm{pH}$, egg yolk medium, breed of rabbit and temperature on the electrophoretic mobilities; and (3) the relationships between the concentration of spermatozoa and biological results.

a. Comparison of Experiments. The distribution of capless, tail-wagging, highly motile and viable (unstained) spermatozoa in 9 experiments was 
evaluated using a difference of $10 \%$ between the 2 collection fractions as an arbitrary level of signiffcance. In 7 of the 9 experiments, there was no difference with regard to the distribution of capless spermatozoa Immediately after collection (at the low temperature). In the 2 other experlments, there was a lower percentage of capless spermatozoa in the lower collection fraction. Thus, the presence of the acrosomal cap appears to have Ifttle influence on the distribution of spermatozoa between the 2 collection fractions.

In 8 of the 10 experiments, there are nigrosin-eosin staining results on 2 fractions. In 4 experiments, there was a higher percentage of unstained spermatozoa in the lower of the 2 collection fractions. In 3 experiments; there was 1 ess than a $10 \%$ difference between the 2 fractions, and, in 1 experiment, the bottom fraction contained a lower percentage of unstained spermatozoa. Although not conclusive, these results suggest that the lower of the 2 collection fractions contained spermatozoa of slightly better quality.

In 8 of the 10 experiments, there was sufflclent tall-wagging and/or forward motion to assess the relative distribution of these properties. In Experiment 10-2-67, one of 2 experiments in which there was no forward motion, the percentage of tall-wagging spermatozoa at $23^{\circ} \mathrm{C}$ was $18.2 \%$ higher in the lower collection fraction. In Experiment 9-22-67, the percent tall-wagging was less than $7 \%$ at room temperature, and there was no signiflcant difference between fractions 6 and 7 . Both tail-wagging and forward motion were evaluated in Experiments 7-26-67 and 7-29-67. As shown in Table XXV, there was a slightly higher percentage of spermatozoa with forward motion in the lower fraction (CF 7) in both experiments. In contrast, CF 6 in Experiment 7-29-67 had 24\% moro tal1-wagging spermatozoa than did CF 7. There was no difference In Experiment 7-26-67 in regard to 
the distribution of tall-wagging spermatozoa.

In 4 experiments with high quality spermatozoa, only forward motion was evaluated. In Experiments 8-29-67 and 9-18-67, there was a significantly higher percentage of motile spermatozoa in the lower of the 2 collection fractions whereas there was no difference in Experiments 9-1.3-67S and 9-13-67P. The temperature in the flow-chamber was approximately $4.2^{\circ} \mathrm{C}$ in Experiment 8-29-67 compared to 2.8 and $3.1^{\circ} \mathrm{C}$ in Experiments 9-13-67S and 9-13-67P, respectively. Th1s could explain the differences among those experiments; however, there was relative enrichment of the lower fraction in Experiment 9-18-67 even though the flow-chamber temperature was only $3.2^{\circ} \mathrm{C}$.

Therefore, the strongest statement that one can make with regard to the distribution of motile opermatozoa is that there is a tendency for the lower of the 2 collection fractions to have a higher percentage of spermatozoa with forward motion. This is consistent with the idea that in these experiments the intrinsic motility vector is directed downward agalnst the upward force of the electric fleld. With spermatozoa samples of the same Initial quality, the important variable then becomes the flowchamber temperature which determines the magnitude of the intrinsic moti11ty vector.

b. Interpretation of Electrophoretic Mobilities. It is seen in Table XXVII that the theoretical average electrophoretic mobilities (Colum 9) are significantly greater than the uncorrected (observed) average SUE electrophoretic mobilities in column (6). As shown in Appendix $G$, the correction for sedimentation in the calculation of the weighted average electrophoretic migration distance in Experiment 7-29-67 1s $20.8 \%$ of the observed average migration distance. In other experiments, this correction ranges from 12-28\%. As expected, the average, uncorrected electrophoretic 
mobility (6) calculated from the observed migration distance lies between the minimum (4) and maximum (5) observed electrophorettc mobilities.

W1th regard to the theoretical electrophoretic mobility calculations, the minfmum (7) is greater than the average (9) In 7 of the 10 experiments. The theoretical minimum electrophoretic migration distance was assumed to be equal to the sum of the minimum observed SUE migration distance and the expected maximum sedimentation distance. The discrepancy between columns 7 and 9 suggests that the maximum sedimentation velocity from Experiment 12-26-67, which was used in the calculations, is too great. The small degree of microscoplc streaming observed at the bottom of the sedimentation migration pattern in that experiment may have given a false maximum sedimentation velocity. This supports the suggestion that the difference between the actual and unfform distributions of spermatozoa in Figure 55 is due to a small degree of microscoplc streaming.

The theoretical electrophoretic mobility ranges from $0.51 \times 10^{-4}(\mathrm{~cm} /$ $\mathrm{sec}) /(\mathrm{V} / \mathrm{cm})$ at $\mathrm{pH} 6$ to $1.18 \times 10^{-4}$ (cm/sec)/(V/cm) at pH 7.10 . Three or 4 electrophoretic moblities determined at $\mathrm{pH} 6.6$ range from $0.53-0.55 \mathrm{x}$ $10^{-4}(\mathrm{~cm} / \mathrm{sec}) /(\mathrm{V} / \mathrm{cm})$, while one measurement at that $\mathrm{pH}$ gave a value of $1.06 \times 10^{-4}(\mathrm{~cm} / \mathrm{sec}) /(\mathrm{V} / \mathrm{cm})$. Simllarly, 4 of the 5 values of electrophoretic mobility determined at $\mathrm{pH} 7.05-7.15$ range from $0.75-0.94 \times 10^{4}$ (cm/sec)/. $(\mathrm{V} / \mathrm{cm})$, while the fifth value is $1.18 \times 10^{-4}(\mathrm{~cm} / \mathrm{sec}) /(\mathrm{V} / \mathrm{cm})$. Inaccuracy In measuring the migration distance may explain some, but not all, of the discrepancy in the 2 values of electrophoretic mobility which appear to be somewhat higher than other values in the series of measurements. Other variables to consider are $\mathrm{pH}$, the effect of egg yolk medium, the breed of the rabbit and temperature.

(1) Effect of $\mathrm{pH}$. The two apparently high values of electrophoretic 
mobility in Experiments 8-18-67 and 9-22-67 were obtained at $\mathrm{pH}$ 6.6 and $\mathrm{pH} 7.1$, respectively. The agreement among the other experiments at each of those $\mathrm{pH}^{\mathrm{s}} \mathrm{s}$ was quite good. There is no question about the $\mathrm{pH}$ of the medium in those 2 experiments.

Experiments 10-2-67, 9-13-67P and 9-18-67 were conducted with spermatozoa from rabbit $\mathrm{Psi}$ at $\mathrm{pH} 6, \mathrm{pH} 6.55$ and $\mathrm{pH} 7.15$, respectively. The calculated theoretical average electrophoretic mobllities for these 3 experiments are $0.51,0.55$ and $0.89 \times 10^{-4}(\mathrm{~cm} / \mathrm{sec}) /(\mathrm{V} / \mathrm{cm})$, respectively. The 3 experiments were conducted at temperatures ranging from $3.1^{\circ} \mathrm{C}-3.3^{\circ} \mathrm{C}$, and, therefore, the normalization to $3^{\circ} \mathrm{C}$ was a minor correction. A simflar increase in the electrophoretic mobility with increase in $\mathrm{pH}$ was observed in the other experiments in this serles. Because only one experiment was conducted at $\mathrm{pH} 6$, the small increment in electrophoretic mobility from $\mathrm{pH} 6$ to $\mathrm{pH} 6.55$ compared to the increment between $\mathrm{pH} 6.55$ and $\mathrm{pH} 7.15$ is of questionable significance.

(2) Effect of Egg Yolk Medium. Spermatozoa from rabbit 0 were used in Experiments 8-18-67, In which egg yolk medium was used for the Initial dilution and cooling, and in 8-29-67, In which the initial dilution and cooling was with the glycine-phosphate buffer. Both experiments were conducted at approximately $\mathrm{pH} 6.6$ and gave electrophoretic mobilities of 1.06 and $0.53 \times 10^{-4}(\mathrm{~cm} / \mathrm{sec}) /(\mathrm{V} / \mathrm{cm})$, respectively. Th1s suggests an increased electrophoretic mobllity for spermatozoa cooled and diluted in egg yolk medium. However, comparison of the electrophoretic mobility of the spermatozoa from rabbit Psi in Experiment 8-10-67, using egg yolk medium, and In Experiment 9-18-67, using glycine-phosphate buffer for cooling shows only a difference between 0.94 and $0.89 \times 10^{-4}(\mathrm{~cm} / \mathrm{sec}) /(\mathrm{V} / \mathrm{cm})$, respectively. In Experiments 7-26-67 and 9-22-67, the spermatozoa were cooled in glycinephosphnte buffer and electrophorettc mobilities of 0.75 and $1.18 \times 10^{-4}$ 
$(\mathrm{cm} / \mathrm{sec}) /(\mathrm{V} / \mathrm{cm})$, respectively, were obtained at $\mathrm{pH} 7.10$. Therefore, factors other than egg yolk.medium are responsible for the two high values of electrophoretic mobility observed in this series of experiments. As noted in Chapter II, Nevo did not observe any influence of egg yolk medium on the electrophoretic mobilities of rabbit and bull spermatozoa ${ }^{174}$.

(3) Effect of Breed of Rabbit. In Experiments 9-13-67S and 9-13-67P, Identical Staflo operating conditions except for the strength of the electric field were used with spermatozoa from $S$, a Calffornia rabbit, and PsI, a Dutch Belt rabbit. The calculated average theoretical electrophoretic mobilities were $0.55 \times 10^{-4}(\mathrm{~cm} / \mathrm{sec}) /(\mathrm{V} / \mathrm{cm})$ at $\mathrm{pH} 6.55$ for the spermatozoa from both rabbits. Experfment 7-29-67, at pH 7.05 with spermatozoa from L, a Calffornia rabbit, and Experiment 9-18-67, at pH 7.15, with spermatozoa from Psi gave electrophoretic mobilities of 0.86 and 0.89 $x 10^{-4}(\mathrm{~cm} / \mathrm{sec}) /(\mathrm{V} / \mathrm{cm})$, respectively. The agreement between these two gets of experiments at different pH's with spermatozoa from California and Dutch Belt rabbits suggests that there is no differential electrophoretic mobility because, of breed, at least within the range of accuraoy of these measurements.

(4) Effect of Temperature. In approximately une-half of these experiments, there was a higher percentage of motile and/or tail-wagging spermatozos and of unstained spermatozoa in the lower of the two collection fractions. Experiment 9-22-67 was conducted at $7^{\circ} \mathrm{C}$ in a deliberate attempt to enrich the lower collection fraction with regard to motile spermatozoa. However, because the spermatozoa sample as a whole lost motility rapidly during the experiment, no enrichment was obtained. Inadvertent enrichment with regard to both forward motion and unstained spermatozoa was obtained In Experiment 8-29-67. At $4.2^{\circ} \mathrm{C}, 60 \%$ of the spermatozoa in $\mathrm{CF} 7$ had for- 
ward motion compared to only $40 \%$ in CF 6 . A similar differential distribution of motile spermatozoa was observed in Experiment 9-18-67 at $3.2^{\circ} \mathrm{C}$. The role of intrinsic motility in the latter experiment is questionable because observations in a hemocytometer inftially at $2.8^{\circ} \mathrm{C}$ and later at $3^{\circ} \mathrm{C}$ showed no motility. Also, there was no enrichment of the lower collection fraction in Experiments $9-13-67 \mathrm{~S}$ and $9-13-67 \mathrm{P}$, at $2.8^{\circ} \mathrm{C}$ and $3.1^{\circ} \mathrm{C}$, respectively, in which the spermatozoa were of extremely high initial and final quality. These observations suggest that with electric fleld strengths of about $6 \mathrm{~V} / \mathrm{cm}$, the intrinsic motility of spermatozoa becomes a significant factor at temperatures of approximately $4^{\circ} \mathrm{C}$ and above. In addition to temperature control, it is necessary to have high quality spermatozoa in order to achieve enrichment of collection fractions.

c. Concentration of Spermatozoa: Effect on Artificial Insemination. The higher quality' of spermatozoa in the lower of the two collection fractions is also demonstrated by the relatively greater number of successful inseminations with spermatiozoa from that fraction. As shown in Table XXX, 4 of 5 inseminations with spermatozoa from the upper collection fraction were adequate technically, but only 1 resulted in a litter ( 3 offspring). Six of 8 inseminations with spermatozoa from the luwer fraction were adequate and 5 resulted in litters with a total of 1.5 offspring. All 4 control inseminations were good but only I resulted in a litter.

$$
\text { . }
$$

Un further analyois, all of the inseminations with. "Staflo spermatozod' which resulted in offspring were from collection fractions in which the spermatozoa concentration was always greater than $4 \times 10^{6} / \mathrm{m} 1$, even in the Staflo apparatus. In Experiment 9-13-67, in which the only 1itter resulted from the insemination of apermatozoa from the upper collection fraction, the concentration in the collection container was $4.25 \times 10^{6} / \mathrm{ml}$ 
Table XXX. Analysis of Artificial Insemination Results by Spermatozoa Sample Source.

\begin{tabular}{|c|c|c|c|c|c|c|c|}
\hline $\begin{array}{l}\text { Sperm } \\
\text { Sample }\end{array}$ & $\begin{array}{c}\text { Number of } \\
\text { Total }\end{array}$ & $\begin{array}{c}\text { Inseminations } \\
\text { Good }\end{array}$ & $\begin{array}{c}\text { Litters } \\
\text { (No.) }\end{array}$ & $\sigma^{\circ}$ & $\begin{array}{c}\text { f fs } \\
\text { o }\end{array}$ & $\begin{array}{l}\text { ring } \\
\text { Unk }\end{array}$ & $\begin{array}{l}\text { (No.) } \\
\text { Total }\end{array}$ \\
\hline Upper Fraction & 5 & 4 & 1 & 1 & 1 & 1 & 3 \\
\hline Lower Fraction & 8 & 6 & 5 & 9 & 5 & 1 & 15 \\
\hline Control & 4 & 4 & 1 & 1 & 0 & 0 & 1 \\
\hline Totals & 17 & 14 & 7 & 11 & 6 & 2 & 19 \\
\hline
\end{tabular}


before centrifugation.." In the other 3 experiments, no offspring were obtained from the insemination of spermatozoa from the upper collection fractions which had pre-centrifugation concentrations ranging from $1.71-$ $2.5 \times 10^{6} / \mathrm{ml}$. In the 3 experiments in which insemination of spermatozoa from the lower collection fraction resulted in offspring, the concentrations in the fractions prior to centrifugation were $4.6,5.6$ and $8.0 \times 10^{6} / \mathrm{ml}$. In the fourth experiment, the spermatozoa concentration in the lower fraction was $5.2 \times 10^{6} / \mathrm{m} 1$. Unfortunately, the inseminate in the latter experiment was rejected by the female and no litter was obtained: In sumary, 6 of the 7 inseminations with spermatozoa from collection fractions having Inftlal concentrations of greater than $4 \times 10^{6} / \mathrm{ml}$ gave Iitters and the one Insemination that did not was refected by the female rabbit. Inasmuch as only 1 of 4 control inseminations, In which the spermatozoa had been stored In egg yolk medium, gave litters, the success rate of inseminations with the "Staflo spermatozod" is consldered excellent. Thus, it appears that the minfmum concentration of spermatozoa in a Staflo collection fraction at which fertilizing capacity 18 preserved is approximately $4 \times 10^{6} / \mathrm{ml}$.

The loss of fertillzing capacity at concentrations of less than $4 x$ $10^{6}$ spermatozoa/ml in the collection fractions is in marked contrast to the preservation of motility at concentrations as low as $1.71 \times 10^{6} / \mathrm{ml}$ (Experiment 8-29-67). In addition, In Experiment 9-13-675, a superior degree of motility was preserved in fraction 6 at a spermatozoa concentration of $1.9 \times 10^{6} / \mathrm{ml}$ for over 2 hours.

As noted in Table XXX, 14 of the 17 inseminations in the 4 experiments with high quality spermatozoa were considered to be technically adequate. Litters were obtained in 7 of the 14 good inseminations (50\%) but the number of offspring was disappointingly low. This is believed to be a 
reflection of the anestrous condition of many female rabbits which had not littered in the preceding year.

\section{Sedimentation-Electrophoresis: Low Electric Field Strengths}

a. Upward Electrophoresis (SUE). The operating parameters and distribution of spermatozoa among the collection fractions in Experiments 7-9-67 and 10-3-67 are presented in Table XXXI. Despite the higher electric field strength and longer electrophoresis time in Experiment 10-3-6\%, the migration pattern was. signdficantly lower in the flow-chamber than in Experiment 7-9-67. The difference lo nut completcly croplainod by the lower electrophoretic mobility of spermatozoa at $\mathrm{pH} 6.6$. Even though no abnormality in the migration pattern was observed, Lt is probable that a olight shift in the pattern accounts for the discrepancy in these results. Thus, these experiments 1llustrate the necessity for unequivocal steady-state conditions in the Staflo apparatus, particularly when the migration distances are very small. In addition, In experiments such as these, 1t would be desirable to fractionate the samples with greater resolucion than lit $1.25 \mathrm{~mm}$ layer height in current flow-cell models.

In Experiment 7-9-67, the estimated average sodimentation distance was approximately $42 \%$ of the total estimated electrophoret1c migrallui distance. In Experiment 10-3-67, the estimated average sedimentation distance was significantly greater than the weighted average SUE migration distance. This confirms the impression that steady-state conditions did not prevall In the flow-chamber during the experiment.

The strength of the electric field required to balance. the sedimentation of spermatozoa In the Staflo apparatus has been calculated under conditions of a temperature of $3^{\circ} \mathrm{C}$ using an average transit time of 28 minutes, an electrode length of $25 \mathrm{~cm}$, and a weighted average sedimentation velocity 
Table XXXI. Comparison of Staflo Operating Parameters and Results of Sedimentation-Upward-Electrophoresis Experiments Using Low Electric Field Strengths.

\begin{tabular}{lcc}
\hline \multicolumn{2}{c}{ Parameter } & \multicolumn{2}{c}{ Experiment Number } \\
\hline$\tilde{E}(\mathrm{~V} / \mathrm{cm})$ & $7-9-67$ & $10-3-67$ \\
$\mathrm{pH}$ & 1.2 & 1.7 \\
Temperature $\left({ }^{\circ} \mathrm{C}\right)$ & 7.1 & 6.6 \\
Electrophoresis Time (min) & 17.1 & 2.3 \\
Motility in Staflo & 3.9 & 20.6 \\
Distribution \\
Collection fraction 7 \\
Collection fraction 8
\end{tabular}


of $0.53 \times 10^{-4} \mathrm{~cm} / \mathrm{sec}$. The welghted average electrophoretic mobilities used in the calculations are 0.55 and $0.90 \times 10^{-4}(\mathrm{~cm} / \mathrm{sec}) /(\mathrm{V} / \mathrm{cm})$, at $\mathrm{pH} 6.6$ and $\mathrm{pH} 7.10$, respectively. The electric fleld strengths required under these conditions are 0.96 and $0.59 \mathrm{~V} / \mathrm{cm}$ at $\mathrm{pH} 6.6$ and 7.10 , respectively.

\section{b. Downward Electrophoresis (SDE). The differences in the migration} pattern and in the distribution of the various categories of spermatozoa among the respective collection fractions in the 2 parts of Experiment 9-29-67 are not remarkable. They may be due to a delayed return to steady-state conditions in the second part of the experiments after emptying the collection containers between runs, as mentioned earlier.

The expected average sedimentation distance of $0.064 \mathrm{~cm}$ is greater than the observed average SDE migration distance of $0.046 \mathrm{~cm}$ in part 1 of Experiment 9-29-67. Inasmuch as the electrophoretic migration was in the same direction as sedimentation, one would have expected a greater combined (SDE) migration distance than with elther the sedimentation or electrophoretic component alone. It is belleved that the change from the primarily vertical orfentation oeen in the sedimentation experiments to the more horizontal orlentation observed in this experiment significantly changed the hydrodynamic properties of the migrating spermatoma. Thus, the increased frictional force due to the "broadside" orientation of the spermatozoa to the direction of migration may have reduced the net migration rate to less than that expected with elther sedimentation or electrophoresis in a more vertical orlentation.

\section{Oriertation of Spermatozoa}

The differential orientation of spermatozoa in the flow-chamber under conditions of different electric field strength and direction of migration is shown in Figure 56. In SUE experiments with both high and 


\section{ORIENTATION OF SPERMATOZOA IN STAFLO EXPERIMENTS} $\left(3-5^{\circ} \mathrm{C}\right)$

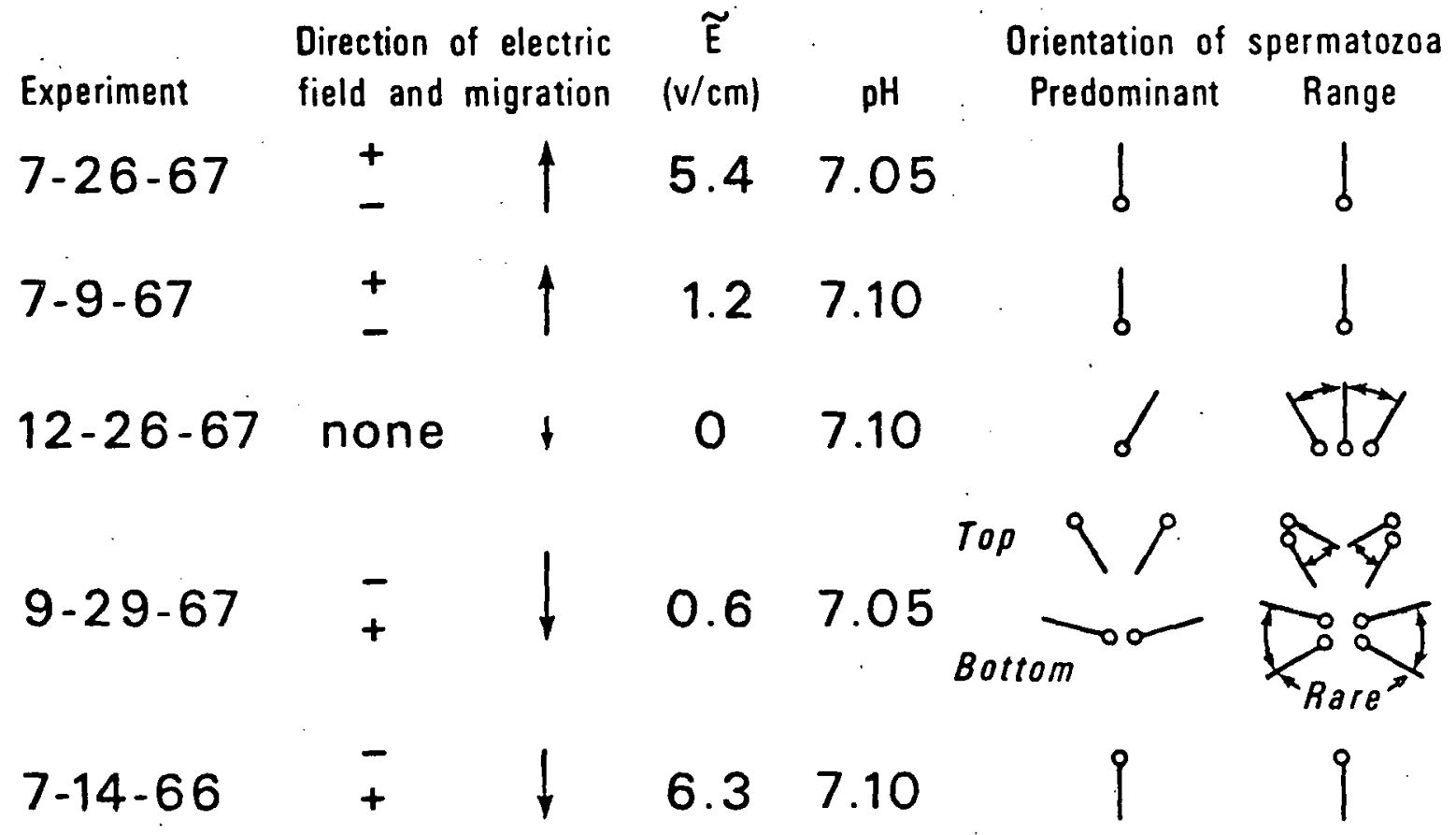

DBL 70115994

F1g. 56. Orientation of rabbit spermatozoa in Staflo sedimentation and sedimentation-electrophoresis experiments at $3-5^{\circ} \mathrm{C}$. 
low electric fleld strength, essentially all of the spermatozoa were oriented in a vertical direction with the tall pointing toward the top (positive) electrode. An orientation different from this was extremely rare at low temperatures, even when there was a slight degree of twisting or other expression of intrinsic mot111ty of spermatozoa in the flowchamber. No difference in orientation was observed at different locations within the migration pattern in the region of the electric fleld.

In sedimentation experiments, the spermatozoa were oriented with the heads down and talls upward, ranging up to $45^{\circ}$ on either side of the vertical position. Usually the head pointed in the direction of the flow with the tall trafling slightly.

In SDE experiments with high electric fleld strengths, the spermatozoa were oriented vertically with the tall pointing toward the bottom (positive) electrode. In SDE experiments with low electric fleld strengths, however, the orfentation of the spermatozoa differed slightly at the top and bottom of the migration profile. At the top, it ranged from a horizontal position to primarily a $45^{\circ}$ angle with the head up and the tall pointing obliquely toward the bottom (positive) electrode. Very few spermatozoa were observed In positions outside of this range. At the bottom of the profile, some spermatozoa heads were pointing slightly downward as shown in Figure 56. Because SDE experiments were not conducted with electric fleld strengths between $0.6-6.0 \mathrm{~V} / \mathrm{cm}$, the minimum electric field strength required to give a vertical orientation with the tail pointing downward toward the bottom (positive) electrode was not determined.

The partially horizontal position assumed by spermatozoa in the SDE experiments with low electric field strengths, shown in Figure 56, is believed to result from the competition between the force of gravity acting 
on the very dense head and the electrophoretic force on the tail which has relatively greater negative charge than the head at $\mathrm{pH} 7$. This orfentation is belleved to explain the discrepancy between the expected SDE migration distance and the observed migration distance in that series of experiments. Thus, it appears that in order to test the hypothesis presented in Chapter III with regard to the separation of spermatozoa characterized by the fastest sedimenting-fastest electrophoresing and alternate combinations of these properties, it will be necessary to determine the minimum electric field strengths at which the spermatozoa assume a vertical orlentation in SDE experiments.

\section{Quality of Spermatozoa}

On many occasions, spermatozoa initially had a high percentage and degree of forward motion which was lost shortly after starting the experiment. The effect of the environment and other factors on the inftial quality of spermatozoa and factors influencing the preservation of viability, motility and fertilizing capacity are consldered in Chapter IX.

The effects of spermatozoa concentration on the preservation of viability, motility and fertilizing capacity were discussed in $2, \mathrm{c}$ above. In experiments of this series, with concentrations as different as 1.05 $\times 10^{6}$ and $5.45 \times 10^{6}$ spermatozoa/ml, there was no difference between the 2 collection fractions with regard to the percentage of capless and unstained spermatozoa. In Experiment 9-13-675, spermatozoa in CF 6 with a concentration of $1.9 \times 10^{6} / \mathrm{ml}$ had essentlally the same percentage and degree of motility as the spermatozoa in CF 7 with a concentration of $8 \times 10^{6} / \mathrm{ml}$. In Experiment $8-29-67, \mathrm{CF} 6$ with a concentration of $1.71 \times$ $10^{6} / \mathrm{ml}$ had the same percentage of capped spermatozoa as CF 7 with 5.60 $\times 10^{6} / \mathrm{ml}$. However, after reconcentration and suspension in egg yolk medium, 
CF 6 had a lower percentage of motile and unstained spermatozoa, suggesting that the stress of centrifugation was more detrimental to the spermatozoa which had been exposed to the lower concentration. Thus, morphology, viability and motility appear to be preserved in the Staflo apparatus at spermatozoa concentrations above $1 \times 10^{6} / \mathrm{ml}$. This is considerably lower than the concentration of $4 \times 10^{6} / \mathrm{ml}$ apparently required for the preservation of fertilizing capacity.

In several SUE experiments with short average transit times, the percentage and degree of motility of the spermatozoa in the collection fractions was essentially the same as in the initial sample which had been stored at a concentration of approximately $20 \times 10^{6} / \mathrm{ml}$. The longest time during which spermatozoa were exposed to low concentrations in the Staflo apparatus and maintained their motility was approximately 6 hours. This was observed in Experiment 9-13-67S in which the spermatozoa quality was supertor. In 4 experiments with high quality spermatozoa, artificial insemination of "Staflospermatozos" gave litters with viable offspring. Thus, this series of experiments shows that glycine-phosphate buffer preserves the motility and fertilizing capacity of rabbit spermatozoa for several hours during Staflo sedimentation-electrophoresis experiments if the Initial quality of the spermatozoa is excellent.

Reconcentration of the spermatozoa in the collection fractions by centrifugation prior to insemination resulted in $5-40 \%$ increases in the percentages of capless spermatozoa with the greatest increases occurring In the samples of poorest quality. This confirms the general observation that a spermatozoa sample of high inftial quality remains good throughout the procedure involved In Staflo experiments, whereas spermatozoa of poor inftal quality deterforate rapldiy. Th1s observation is discussed 
in more detail in Chapter IX.

Clumping rarely was a problem in sedimentation or sedimentationelectrophoresis experiments. No clumping was observed in the glycinephosphate buffer at any temperature, or in the egg yolk medium at cold room temperatures. In Experiment 9-29-67, after resuspension in egg yolk medium, the spermatozoa were not clumped in the cold room but clumped immediately upon warming to room temperature. The spermatozoa used in that experiment were from an old New Zealand White rabbit whose sample happened to be the highest quality on that particular day. Such clumping was not observed in any other experiment.

\section{E. Summary and Conclusions}

The glycine-phosphate buffer evaluated in Chapters $V$ and VI was used In all of these experiments. In a 3-layer sedimentation-fractionation of rabbit spermatozoa at $3^{\circ} \mathrm{C}$ with concentrations in the collection containers ranging from $0.72-2.91 \times 10^{6} / \mathrm{ml}$, there were no differences among the fractions with regard to morphology (evaluated by phase contrast microscopy) or viability (Judged by nigrosin-eosin staining). The distribution of the spermatozoa among the collection fractions compared favorably to that expected with a hypothetical population of cells having a uniform distribution of sedimentation velocities. The slight difference between the actual and hypothetical distributions may be due to a smali degree of microscoplc "cluster sedimentatiod" observed in the flow-chamber. Comparison of the actual with hypothetical blmodally or normally distributed populations showed poor agrement.

For 3 experiments, the minimum eedimentation velocity was $0 \mathrm{~cm} / \mathrm{sec}$, the average ranged from $0.43-0.53 \times 10^{-4} \mathrm{~cm} / \mathrm{sec}$, and the maximum from $0.87-1.24 \times 10^{-4} \mathrm{~cm} / \mathrm{sec}$ at. $3^{\circ} \mathrm{C}$. Average values for all 3 experiments were 
$0,0.48 \times 10^{-4}$ and $1.06 \times 10^{-4} \mathrm{~cm} / \mathrm{sec}$, respectively. Within the limits of measurement in the Staflo apparatus, these averages are the same as those obtained in the $2.5^{\circ} \mathrm{C}$ sucrose experiments in a similar low densityLow viscosity medium (Chapter IV): $0,0.49 \times 10^{-4}$ and $1.07 \times 10^{-4} \mathrm{~cm} / \mathrm{sec}$, respectively.

In these particular experiments, the calculation of electrophoretic mobilities included a correction for sedimentation which was estimated to be $12-28 \%$ of the average "true" electrophoretic migration distance. After making these corrections, the average electrophoretic mobilities of rabbit spermatozoa at $\mathrm{pH} 6.0,6.6$ and 7.10 are $0.51,0.54$ and $0.86 \times 10^{-4}(\mathrm{~cm} / \mathrm{sec}) /$ $(\mathrm{V} / \mathrm{cm})$, respectively, corrected to $3^{\circ} \mathrm{C}$. The latter value is essentially the same as that obtained in Chapter $V$ for both upward- and downwardelectrophoresis. The numerical values of electrophoretic mobility were not influenced by the breed of the rabbit or by prior exposure of the spermatozoa to egg yolk medium.

In 7 of 9 sedimentation-upward-electrophoresis (SUE) experiments wh high electric field strengths, no differences were found in percentages of capped spermatozoa between the different fractions from a given experiment, evaluated 1mmediately after collection. The evaluation at ronm temperature suggested that the lower collection fraction was better with respect to morphology, viability, motility and fertilizing capacity. As was previously suggested in Chapter VI, this may be a reflection of motility in the flow-chamber and/or generally higher spermatozoa concentrations therein. No differential distribution of motile spermatozoa between fractions was found where the average flow-chamber temperature was $2.8^{\circ} \mathrm{C}$, even with superior quality opormatozoa. However, at an average temperature of $4.2^{\circ} \mathrm{C}$, absolute enrichment of the lower fraction was achieved. Thus, in the glycine-phosphate buffer with electric field strengths of about $6 \mathrm{~V} / \mathrm{cm}$, 
intrinsic motility appears to become a significant factor at about $4^{\circ} \mathrm{C}$ and becomes increasingly important at higher temperatures.

Although motility was well preserved in this glycine-phosphate buffer at concentrations of $1 \times 10^{6}$ spermatozoa/ml for 2-3 hours during staflo runs, fertilizing capacity was lost at concentrations of less than $4 \times 10^{6}$, nl. Because of this 1085 , the number of offspring was too small to attach any significance to the slightly greater number of males resulting from the insemination of lower-fraction spermatozoa.

In the low electric field strength sedimentation-upward-electrophoresis (SUE) and sedimentation-downward-electrophoresis (SDE) experiments, no differences were found between the respective collection fractions with regard to capped, viable, tall-wagging or highly motile spermatozoa. In the SUE experiments, all spermatozoa were oriented vertically. The calculated electric field strengths needed to exactly balance the average contribution of sedimentation to the observed migration at $3^{\circ} \mathrm{C}$ were 0.96 and $0.59 \mathrm{~V} / \mathrm{cm}$ at $\mathrm{pH} 6.6$ and 7.1 , respectively.

In the low electric field strength SDE experiments, the observed average migration distances were less than either of the expected average sedimentation or electrophoretic migration distances. This discrepancy is attributed to the more horizontal orfentation of the spermatozoa in these experiments (Figure 56) as compared to the orientation observed in sedimentation experiments or SDE experiments with high electric field strengths.

Because of the small spread of the migration pattern in both the SUE and SDE experiments, it was not possible to obtain sub-populations having the combinations of sedimentation and electrophoretic properties $(E \cdot S, E \cdot S$, $\mathrm{e} \cdot \mathrm{S}$ and $\mathrm{e} \cdot \mathrm{s}$ ) discussed in Chapter III. Furthermore, the apparent influence of spermatozoa orientation on SDE experiments indicates that in such experiments, a suffictently high electric field must be used to orient all spermatozoa "tall down" to eliminate the differential migration effect of orlentation. 
IX. THE QUALITY OF RABBIT SPERMATOZOA POPULATIONS:

A CRITICAL DISCUSSION

\section{Contents}

A. Introduction. . . . . . . . . . . . . . . . .

B. Comparison of Optical-Cytological Methods Used to Evaluate Spermatozoa. . . . . . . . . . . . . . . . .

1. Comparison of Scoring Nigrosin-Eosin Preparations with Ordinary Light and Phase Contrast Mirrnscopy : .

2. Comparison of Phase Contrast Observations of Unstained and Nfgisulu-Eugin stained Spermatozoa. . . . . .

C. Environmental and Procedural Factors Affecting Rabbit

Spermatozoa Quality...................

1. Miscellaneous Facturs. . . . . . . . . . . .

2. Frequency of Collection. . . . . . . . . . . .

3. Centrifugation .................

4. Environmental Temperature. . . . . . . . . .

D. Summary and Conclusions . . . . . . . . . . . . .

\section{A. Introduction}

One of the most perplexing and frustrating problems encountered In this study of spermaluzod has been the unpredictability of the quality of efaculates from different rabbits on the same day, of ejaculates from the same rabbit on different days and of consecutive ejaculates from the same rabbit on the same day. Observations over a year suggested that the quality of ejaculates was consistently good during the winter and early spring, and was extremely variable in the summer and autumn months. In addition, spermatozoa samples which inftially appeared to be of high quality 
with regard to percentage and degree of motility, demonstrated variable resistance to the stresses encountered in Staflo experiments. This variable durability of epermatozoa eamples contributed to many unhappy days because spermatozoa which Initially appeared to be adequate for an experiment lost their motility before the completion of the experiment. Thus, they were inadequate for artificial insemination and the blological assay system of most interest was lost. The purpose of this section is to review the many factors which influence the quality of spermatozoa, and, Insofar as possible, to draw conclusions from the many observations of spermatozoa quality made in this study, most of which were opportunistic in confunction with staflo experiments.' In the review of the biologIcal properties of opermatozoa presented in Chapter II, much information pertinent to the subject of this chapter was presented. It is appropriate at this time to refer to or present additional information from those Btud1es.

Doggett found a cyclic vartation of and correlation between the efaculate volume and the concentration and motility of spermatozoa there1n ${ }^{68}$. The total eperm count varled approximately $\pm 50 \%$ from the mean with an apparent correlation of good and poor motility with high and low sperm counts, respectively. With maturation of the rabbit, the periods generally shortened and showed a predominance of 3 and 4-day intervals. The timing of the peaks appeared to be an inherent characteristic of the rabbit and not related to the enviromment. 68 .

Degerman and K1hlström found cycllc variations in the body temperatures of male rabbits. The cycles varied in length from 3-9 days with a mean of 7 days, and were correlated with vartations in the dally semen volume ${ }^{63}$. The mot1lity of the opermatoroa was not reported. 
Asdell and Sallsbury studied the duration of spermatogenesis in the rabbit by observing the regeneration of the spermatogenetic process in testes which had been damaged by exposure to the relatively higher temperature of the abdominal cavity, by anchoring testes therein, for 1-7 days 12 . Although their technique was not so preclse as current methods using tritiated thymidine, their studies are enlightening. The effect of exposure of testes to the intra-abdominal temperature for periods less than 1 day was not reported. After exposure for 1 day to the elevated intra-abdominal temperature, testes showed clumping and detachment of spermatids, some derangement in lacunae formation in spermatocytes and cessation of spermatogenesis even in the spermatogonial layer. In testes exposed to the elovated temperature for 7 days, there were no spermatozoa or spermatids, spermatocytes were clumped and the layers of spermatogonia showed some signs of damage. The rate of recovery of the spermatogenic process after return of the testes to the scrotum was roughly proportional to the length of stay in the abdominal cavity. They estimated that 35 daye wcre necessary to observe new spermatozoa in the testes ${ }^{12}$. Remembering that the male rablil can, and often does, pull his testes into his abdomen when handled or frightened, the significance of exposure to intra-abdominal temperatures as noted above is of great concern in regard to animal care.

The influence of the environment on the reprndurtive porformance of rabbits has been repurted by sittman and co-workers ${ }^{216}$ and is disrissed in Chapter II. It is interesting to note that Rulfer has observed a marked decrease in the percentage of litters obtained from rabbits mated from early morning to late afternoon on very hot days; however, he experienced fair success with matings in the evenings of those same days ${ }^{267}$. A major section which follows presents observations during this study on the effects 
of the environment on sperm quality. However, the first section is concerned.w1th differences among several methods of evaluating the quality of spermatozoa.

B. Comparison of Optical-Cytological Methods Used to Evaluate Spermatozoa

In the experiments reported in Chapter VIII, spermatozoa in the collection fractions and the Initial sample were evaluated in the cold room immediately after collection, and again at room temperature after re-concentration by centrifugation. Evaluation in the cold room and at room temperature, by phase contrast microscopy, determined the percentages of capped (C), capless (CL), tail-wagging (TW) and highly motile (FM) spermatozoa. At room temperature, the reconcentrated spermatozoa were also evaluated by the nfgrosin-eosin vital staining technique. By light microscopy, stalned spermatozoa preparations were categorized as unstained (U), morphologically normal but stained (MNS), and stained (S). It was assumed that capless spermatozoa would take up the vital stain, and, therefore, that the percentage of capless (CI) spermatozoa by phase contrast microscopy would be approximately equal to the percentage of stained (S) spermatozoa (e.g., CL = S). However, differences ranged from S being $33.9 \%$ greater than CL (CF 6, Experiment 7-29-67, Table XXV) to S $52.6 \%$ less than CL (CF 7, Experiment 8-10-67, Table XXI). In the former, the spermatozoa concentration was $5.04 \times 10^{6} / \mathrm{ml}$ and in the 1 atter, $0.71 \times 10^{6} / \mathrm{ml}$, suggesting a differential effect of concentration. However, in another experiment (CF 6, Experiment 8-10-67, Table XXV), S was 22.1\% less than $\mathrm{CL}$ even though the spermatozoa concentration was $5 \times 10^{6} / \mathrm{ml}$. Thus, there was a marked lack of correlation between the 2 methods of evaluating spermatozoa and it was not explained, solely at least, by differences in cell concentration. Further evaluation of these discrepancies includes(1) a comparison of scoring nigrosin-eosin stained preparations by both ordinary 
light and phase contrast microscopy, and (2) a comparison of phase contrast observations of unstained spermatozoa and nigrosin-eosin stained preparations.

\section{Comparison of Scoring Nigrosin-Eosin Preparations with Ordinary Light and Phase Contrast Microscopy.}

As noted above, scoring of nigrosin-eosin stained preparations by light microscopy led to 3 categories of spermatozoa (U, MaNS and S), whereas scoring by phase contrast microscopy gave 4 , categories of spermatozoa as follows: (1) unstained and capped (UC), (2) unstained and capless (UCL), (3) morphologically normal but stained (MNS) and (4) stained and capless (SCL). One would expect agreement between the unstained categories on slides assayed by both techniques, (e.g., $U=U C+U C L)$. This hypothesis was tested by comparing light microscope and phase contrast scoring of 23 palrs of slides with 100 spermatozoa belng scored on each slide by each method, or 200 spermatozoa per sample. The results are shown in Table XXXII. Columns 10 and 11 compare the percentages of unstained spermatozoa representing the "U" category (3) from light microscope scoring and the "UC+ UCL" categorias ( $6+8)$, trom the phase contrast scoring, respectively. Using a one-tail test ${ }^{90}$, the differences were signiflcant at the $P \leqslant 0.01$ level In only $3(13.6 \%)$ of the 22 samples, whereas thero was no difference $(P>0.10)$ in $16(72.7 \%)$ of the samples. In the 3 other samples, the differences were significant at the $P=0.03-0.05$ level.

Generally, there was a higher percentage of MNS spermatozoa by phase contrast scoring. This is consistent with the better observation of the acrosomal cap region with this method. Despite these differences, there Is falr agreement between the 2 methods of scoring in regard to determining the percentage of unstalned spermatozoa in the sample.

2. Comparison of Phase Contrast Observations of Unstained and NigrosinEogin Stalned Spermatozoa. 
Table XXXII. Comparison Light and Phase Contrast Microscopy

Scoring of Nigrosin-Eosin Stained Rabbit Spermatozoa."

\begin{tabular}{|c|c|c|c|c|c|c|c|c|c|c|c|}
\hline$(1)$ & \multicolumn{2}{|r|}{ (2) } & (3) & $(4)$ & (5) & $(6)$ & $(7)$ & (8) & (9) & $(10)$ & $(11)$ \\
\hline \multirow{2}{*}{$\begin{array}{l}\text { Experiment } \\
\text { Number }\end{array}$} & \multirow{2}{*}{\multicolumn{2}{|c|}{ Sample }} & \multicolumn{3}{|c|}{ Ordinary Light } & \multicolumn{4}{|c|}{ Phase Contrast } & \multicolumn{2}{|c|}{ Unstained } \\
\hline & & & $\begin{array}{l}U \\
(\%)\end{array}$ & $\begin{array}{l}\text { MNS } \\
(\%)\end{array}$ & $\begin{array}{c}S \\
(\%)\end{array}$ & $\begin{array}{l}\text { UC } \\
(\%)\end{array}$ & $\begin{array}{l}\text { MNS } \\
(\%)\end{array}$ & $\begin{array}{l}\text { UCL } \\
(\%)\end{array}$ & $\begin{array}{l}\mathrm{SCL} \\
(\%)\end{array}$ & $\begin{array}{l}(3) \\
(\%)\end{array}$ & $\begin{array}{c}(6+8) \\
(\%)\end{array}$ \\
\hline \multirow[t]{3}{*}{$9-13-67 \mathrm{~s}$} & $\mathrm{CF}$ & 6 & 87 & $1: 0$ & 12 & 24.3 & 2.3 & 63.4 & 10 & 87 & 87.7 \\
\hline & CF & 7 & 72.5 & 6 & 21.5 & 21 & 3 & 44 & 32 & 72.5 & 65 \\
\hline & IS & & 67 & 4 & 29 & 30.5 & 4 & 30 & 35.5 & 67 & 60.5 \\
\hline \multirow[t]{3}{*}{$9-13-67 P$} & C.F & 7 & 52.5 & 1.5 & 46 & 18 & 11 & 30 & 41 & 52.5 & 48 \\
\hline & CF & 8 & 59.5 & 1.5 & 39 & 31.5 & 3 & 33 & 32.5 & 59.5 & 64.5 \\
\hline & IS & & 51 & 0 & 49 & 28 & 9 & 21 & 42 & 51 & 49 \\
\hline \multirow[t]{3}{*}{$9-18-67$} & $\mathrm{CF}$ & 6 & 61.5 & 0 & 38.5 & 38 & 15.5 & 23 & 23.5 & 61.5 & 61 \\
\hline & $\mathrm{CF}$ & 7 & 75.5 & 0.5 & 24 & 63 & 12 & 12.5 & 12.5 & $75: 5$ & 75.5 \\
\hline & IS & & 66.5 & 2 & 31.5 & 67 & 3 & 16 & 14 & 66.5 & 83 \\
\hline \multirow[t]{3}{*}{$9-22-67$} & CF & 6 & 34 & 1.5 & 64.5 & 8.5 & 10.5 & 22 & 59 & 34 & 30.5 \\
\hline & $\mathrm{CF}$ & 7 & 35 & 0.5 & 64.5 & 14.5 & 9 & 26.5 & 50 & 35 & 41 \\
\hline & IS & & 32.5 & 4 & 64.5 & 17.5 & 2.5 & 25 & 55 & 32.5 & 42.5 \\
\hline \multirow[t]{5}{*}{$9-29-67$} & CF & $8-1$ & 63 & 0.5 & 36.5 & 24.5 & 6.5 & 44.8 & 24.2 & 63 & 69.3 \\
\hline & CFE & $8-2$ & 66 & 0.7 & 32.3 & 32.6 & 4 & 36.4 & 27 & 66 & 69.0 \\
\hline & CF & $9-1$ & 64.5 & 0.5 & 35 & 25.4 & 8.2 & 40.7 & 25.7 & 64.5 & 66.1 \\
\hline & CF & $9-2$ & 72.5 & 1.0 & 26.5 & 35 & 7.7 & 37.4 & 20 & 72.5 & 72.4 \\
\hline & IS & & 70.5 & 0 & 29.5 & 34.5 & 11 & 30.8 & 23.7 & 70.5 & 65.3 \\
\hline \multirow[t]{3}{*}{$10-2-67$} & CF & 6 & 30.5 & 0.6 & 68.9 & 7.3 & 22.6 & 37 & 33.1 & 30.5 & 44.3 \\
\hline & CF & 7 & 66.5 & 0 & 33.5 & 44.5 & 16 & 24.5 & 15 & 66.5 & 69 \\
\hline & IS & & 68 & 0.5 & 31.5 & 39 & 8 & 30 & 23 & 68 & 69 \\
\hline \multirow[t]{3}{*}{$10-3-67$} & CF & 8 & 65 & 4 & 31 & 13.5 & 13.5 & 43.5 & 29.5 & 65 & 57 \\
\hline & $C F$ & 9 & 68 & 2.5 & 29.5 & 15 & 10.5 & 53 & 21 & 68 & 68 \\
\hline & IS & & 57.5 & 1.5 & 42 & 14 & 9.5 & 42 & 34.5 & 57.5 & 56 \\
\hline
\end{tabular}


Twelve ejaculates were diluted 1:2 with modffled Baker's medium. In less than 1 hour, both unstained and stained preparations were evaluated by phase contrast microscopy. As noted above, with phase contrast scoring one gets 4 categories of spermatozoa in fresh specimens (C,CL,TW and FM) and 4 categories in nigrosin-eosin preparations (UC, UCL, MNS and SCL). The results of scoring the 12 specimens are given in Table XXXIII. In this table, capped spermatozoa are represented by columns 3,5 and 6 in phase contrast observations and columns 7 and 8 in the nigrosin-eosin stained preparations. Capless spermatozoa by phase contrast are listed in column 4 and by the nigrosin-eosin staining technique in columns $y$ and 10. For convenience, a comparison of the percentages of capless spermatozoa 1s given in colums 11 and 12 . The same difference would be observed between the total percentages of capped spermatozoa.

The percentages of capless spermatozoa determined by the 2 methods agree within $4 \%$ in 9 of the first 10 efaculates listed in Table XXXIII. In the first 10 ejaculates, the percentages of tall-wagging and forward motfon were very low. In the last 2 ejaculates listed in Table XXXIII, the percentages of capless spermatozoa by. phase contrast microscopy (11) are much less than those of the capless spermatozod by nigrosin-eosin staining (12). The percentage and degree of motility in the last 2 efaculates was considerably better than all but 1 of the 10 previous efaculates. Thus, agreement between the 2 methods of evaluation appears to be good only for poor spermatozoa samples.

The question of correlation between the quality of spermatozoa samples, judged by motility alone, and the discrepancy between the percentages of capless spermatozoa determined by the 2 methods of evaluation was explored by analyzing 61 ejaculates. A difference of $\pm 5 \%$ between the percentages of capless spermatozoa determined by the 2 techniques 
Table XXXIII. Comparison of Phase Contrast Observations of Unstained Spermatozoa and Nigrosin-Eosin Stained Spermatozoa.

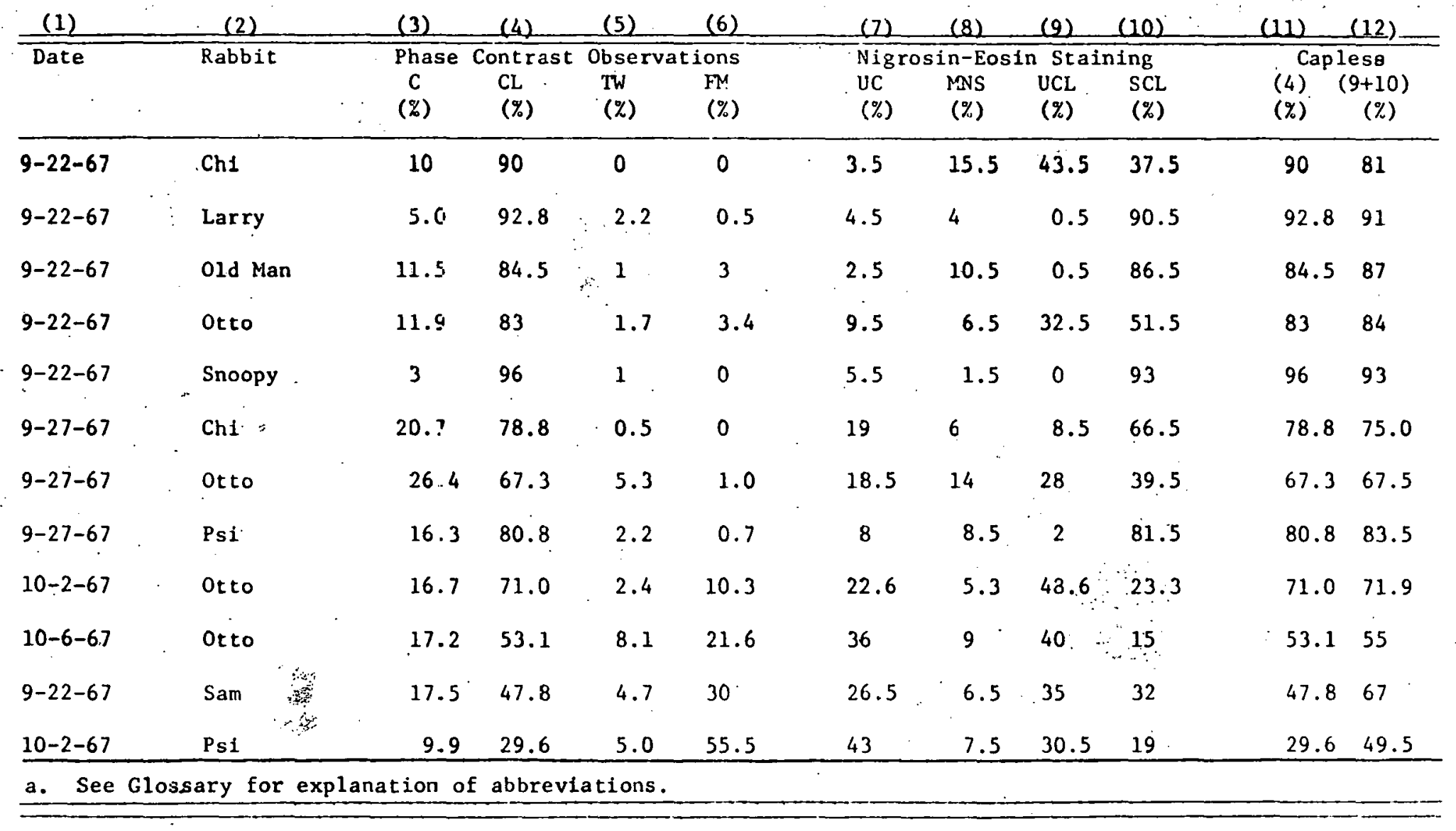


was considered to be within the accuracy of counting. Three relationships between the 2 evaluation techniques are presented in Table XXXIV. In the first, the percentage of capless spermatozoa by phase contrast observation is less than the percentage of capless spermatozoa by the nigrosin-eosin staining technique. Twelve of 61 efaculates or $19.7 \%$ fell within this category, $8(66.7 \%)$ of which had $20 \%$ or more motile spermatozoa. Twentytwo of the 61 ejaculates fell into the classification of approximately equal percentages of capless spermatozoa by both methods. Of the 22,6 (27.3\%) had $20 \%$ or more mot1le spermatozoa. Of the 27 ejaculates falling into the third category (CL $>$ UCL + SCL), only 1 (3.7\%) had more than $20 \%$ motile spermatozoa.

The conclusion from this comparison is that, if the spermatozoa sample is of good quality judged by forward motion, the percentage of capless spermatozoa by. phase contrast observation of unstained spermatozoa Is lese than by phase contrast scoring of nigrosin-eosin stained spermatozoa. This 1s illustrated by the last 2 ejaculates listed in Table XXXIII, both of which had a fair percentage of spermatozoa with forward motion. This suggests that staining has a detrimental effect, possibly mechanical, which causes some unstained spermatozoa to lose their acrosomal caps, thereby Increasing the UCL category. When the spermatozoa are of very poor quality, a higher percentage appears to be capless by phase contrast observation of unstained spermatozoa.

\section{Environmental and Procedural Factors Affecting Spermatozoa Quality}

\section{Miscellaneous Factors}

a. Animal Care. The environment, illness, feeding and type of pellet, unnecessary moving while cleaning and posstbly other peculiartites of animal care can influence the Inftial quality of ejaculates. Even when all of these 
Table XXXIV. Correlation of Scoring of Caplessness by Phase Contrast Observations of Unstained (CL) and N1grosin-Eosin Stained (UCL+SCL) Spermatozoa.

\begin{tabular}{|c|c|c|c|c|}
\hline $\begin{array}{c}\text { Relationship of Categories } \\
\text { of Spermatozoa }\end{array}$ & $\begin{array}{l}\text { Sam } \\
\text { (No.) }\end{array}$ & es $(\%)$ & $\begin{array}{l}\text { Samples with } \\
\text { More Motile } \\
\text { (No.) }\end{array}$ & $\begin{array}{c}20 \% \text { or } \\
\text { Sperm } \\
(\%)\end{array}$ \\
\hline $\mathrm{CL}<\mathrm{UCL}+\mathrm{SCL}$ & 12 & 19.7 & 8 & 67 \\
\hline $\mathrm{CL} \approx \mathrm{UCL}+\mathrm{SCL}$ & 22 & 36 & 6 & 27.3 \\
\hline $\mathrm{CL}>\mathrm{UCL}+\mathrm{SCL}$ & 27 & 44.3 & 1 & 3.7 \\
\hline Totals & 61 & 100 & 15 & 100 \\
\hline
\end{tabular}

a. See Glossary for explanation of abbreviations: 
1tems were under close supervision, variations in the quality of spermatozoa in ejaculates were observed.

b. Sperm Collection. Polge reported that rabbit spermatozoa will not regain motility if more than approximately $2.5 \%$ glycerol is introduced rapidly into the semen ${ }^{188}$. Inasmuch as I used glycerol as a lubricant In the artificial vagina in most experiments, it is conceivable that excessive lubrication contributed to the deterioration of spermatozoa samples early in the study. However, later on, a standard number of drops of glycerol was used to lubricate each lumen, thereby eliminating the possiblitty of excess glycerol.

A constant danger in the collection of spermatozoa is too high a temperature in the artifictal vagina. Brackett, Weinman and Williams observed a decrease in motility after exposure of spermatozoa to $48^{\circ} \mathrm{C}$ temperature for only 40 seconds 265 . The artiflclal vagina shown in Appendix $\mathrm{C}$ was generally. used w 1 th a temperature of $40-42^{\circ} \mathrm{C}$. Temperatures below $38^{\circ} \mathrm{C}$ discouraged the bucks, but did not appear to affect the spermatozoa. Occastonaliy, temperatures as high as $45^{\circ} \mathrm{C}$ were used, but, unfortunately, no correlation of that temperature and the quality of the sample was made. However, Walton recommended using a temperature of $45^{\circ} \mathrm{C}^{239}$.

Because of the well known phenomenon of "cold shock", which occurs with rapid cooling of some mammalian spermatozoa, great care was taken to avold this problem. Stnce the same procedure was used for all samples (see Appendix B), the great varlability in sample quality noted above does not appear to be attributable to the cooling procedure.

c. Staflo Handling Procedures. An attempt was made to avoid low spermatozoa concentrations, because of the deleterious effects thereof on motility, viability and fertilizing capacity. Dilution was accomplished slowly 
and in several stages. The medium generally was prepared by the same Individual and; whenever there was a question of contamination of glassware, 1t was washed by the author.

In numerous Staflo experiments, spermatozoa which initially had a high degree of motility lost essentialiy all motility before the end of the experiment. At the conclusion of other Staflo experiments, both fowl and rabbit spermatozoa appeared to have mot1lity suggestive of good fert1lizing capacity but insemination produced no offspring. During the period in which these experiments were conducted, other Staflo experiments In this laboratory, using bone marrow cells, showed a marked loss of colony formation using procedures which previously had led to a high percentage of colony formation. Studies conducted with the bone marrow cells showed that exposure to a very short segment of new polyethylene tubing resulted in a marked decrease in colony formation. Other observations by me showed that spermatozoa suspended in egg yolk medium, when stored for several hours in new polyethylene syringes, lost essentially all motility. In contrast, spermatozoa from the same efaculate stored in the same medium in a clean glass vial maintained a high degree of motility for over 48 hours. These problems with polyethylene tubing and syringes were avoided by using old tubing and old syringes which apparently had lost the toxic substance.

Another toxin, Roccal (benzalkonlum chloride) could have caused deterioration of spermatozoa samples on occasion. It was used to prevent bacterial growth in the Staflo apparatus between experiments. Evaluation of 1ts effects in saline solutions showed that a $1: 1,000$ concentration of Roccal caused Immobilization of all spermatozoa.within 45 seconds. In a 1:2,500 concentration, after 10 minutes, spermatozoa showed reduced degree of motility. After 80 minutes, a high percentage of spermatozoa 
had only a poor degree of tall-wagging. After 90 minutes, on $1 y 27 \%$ of the spermatozoa were unstained (n1grosin-eosin stain) compared to $64 \%$ of the control sperm. At a Roccal concentration of 1:5,000, after 80 minutes, the percentage and degree of motility was less than in the saline control suspension. After 90 minutes, $50 \%$ of the spermatozoa were unstained (viable) compared to $64 \%$ of the control sperm. At concentrations of $1: 10,000$ and less, there appeared to be no effect of Roccal within 90 minutes. The fertilizing capacity of spermatozoa exposed to Roccal was not studied.

\section{Frequency of Collection.}

A desire to have the best possible spermatozoa for an experiment led to the collection of a second ejaculate within an hour, on some occasions, or the next morning from rabbits which had given the best spermatozoa sample on a particular day. Hopes of obtaining an abundance of excellent spermatozoa for experiments were shattered by the results shown in Table XXXV. It is seen that there were marked variations in the quality of consecutive ejaculates from the same rabbit collected at Intervals of 24 hours or of 15-60 minutes during the sumer and early aurumn .

Table XXXV A shows a striking difference in the quality of consecutive efaculates with an interval of 15-60 minutes between collections. All rabblts were housed in outdoor hutches with a sprinkler system for limited temperature control. Even though the percentage of motile spermatozoa in the flret efaculate ranged frum $13.6 \%-85 \%$, there were no mot1le spermatozoa in any of the second ejaculates. The absolute increases In the percentages of capless spermatozoa in 4 of the pairs of ejaculates ranged from $26.4 \%-82.5 \%$. 
Table XXXV. Varlations in the Quality of Consecutive

Ejaculates from the Same Rabbit.

\begin{tabular}{|c|c|c|c|c|c|}
\hline \multirow{3}{*}{$\begin{array}{l}\text { Interval } \\
\text { Between } \\
\text { Collections } \\
\text { and Date }\end{array}$} & \multirow{3}{*}{ Rabbit } & \multicolumn{2}{|c|}{ Percent Forward Motion } & \multicolumn{2}{|c|}{ Percent Capless } \\
\hline & & Ejaculate & Difference $(2-1)$ & Ejaculate & Difference $(2-1)$ \\
\hline & & 1st 2nd & & ist 2nd & \\
\hline
\end{tabular}

A. $15-60 \mathrm{Min}$.

$\begin{array}{llllllll}6-24-67 & \text { Whitey } & 65 & 0 & -65 & & & \\ 7-7-67 & \text { Bill } & 41 & 0 & -41 & & & \\ 7-7-67 & \text { Otto } & 13.6 & 0 & -13.6 & 24.4 & 50.8 & +26.4 \\ 7-8-67 & \text { Larry } & 85 & 0 & -85 & 7.5 & 90 & +82.5 \\ 7-8-67 & \text { Robert } & 73.4 & 0 & -73.4 & 15.7 & 91.5 & +75.8 \\ 7-15-67 & \text { Red } & 24(\mathrm{TW})^{\mathrm{a}} & 0 & -24(\mathrm{TW}) & 48.2 & 86 & +37.8\end{array}$

B. 15-60 Min.

$\begin{array}{cccccccc}10-13-67 & \text { 0tto } & 50 & 50 & 0 & 25 & 22.5 & -2.5 \\ 10-26-67 & \text { Psi } & 85 & 17.5 & -67.5 & 10.5 & 37.5 & +27\end{array}$

C. 24 Hours

\begin{tabular}{llllllll}
$8-30-67$ & Otto & 60.0 & 16.0 & -44.0 & 18.4 & 45.0 & +26.6 \\
$9-18-67$ & Larry & 16.7 & 1.0 & -15.7 & 50.0 & 77.0 & +27.0 \\
$9-18-67$ & Otto & 67.2 & 16.5 & -50.7 & 11.6 & 39.1 & +27.5 \\
$9-18-67$ & Psi & 59.4 & 18.8 & -40.6 & 27.1 & 47.1 & +20.0 \\
\hline
\end{tabular}

a. TW: Tail wagging. 
The pairs of ejaculates listed in Table XXXV B were collected approximately 2 months after the rabbits had been placed in an air-conditioned room. The second ejaculate collected from rabbit ot to less than 1 hour after the first was essentially unchanged, whereas the second ejaculate collected from Psi showed a $67 \%$ decrease in the percentage of motile spermatozoa and a $27 \%$ increase in the percentage of capless spermatozoa. In the 2-week interval between the collection of the ejaculates from Otto and Psi, there was a transient temperature rise of about $13^{\circ} \mathrm{F}$ in the airconditioned room when the alr-conditioner was inadvertently turned off. The spermatozoa samples from other rabbits in the air-conditioned room on 10-26-67 showed a decrease in quality which suggests that relative, as well as absolute, temperature changes are important. Thus, it is belleved that the relative temperature rise on $10-17-67$ was the primary cause of the difference In the quality of the 2 ejaculates from Psi on $10-26-67$.

Table XXXV C shows that the absolute loss of forward motion ranged from $15.7 \%-50.7 \%$ and the relative loss trom $68.4 \%-94 \%$ in 4 palrs of ejaculates collected from rabbits on consecutive days. The absolute increase In the percentage of capless spermatozoa ranged from 20-27.5\%. At the time of collection, rabbits Otto and $\mathrm{P} g i$ had been in an air-conditioned room for about 5 weeks, whereas Larry was in an outdoor hutch cooled by a sprinkler system. Thuo, although being in the c001 room did appear to improve the quality of the first ejaculates from Otto and Psi, even 5 weeks in a controlled environment did not prevent a significant decrease in the quality of the second ejaculates.

These observations showed that in hot weather there was a marked decrease in the quality of the second ejaculate collected from a rabbit within 
one hour after the first, and that there was a significant decrease in the quality of ejaculates collected approximately 24 hours later. This suggests that there is a selective process within the genital duct system of the male rabbit enhancing the migration of the highest quality spermatozoa toward the distal portion of the duct. This may be related to the observation by Orgebin-Crist that, during epididymal passage, young and old sperm cell populations are mixed ${ }^{181}$. Consequently, the first efaculate is of the best possible quality. In view of the frequent development of pseudo-pregnancy in female rabbits following a sterile mating, one mlght speculate that "getting off a good first shot" so to speak, is very important for the survival of the species, especially in hot weather. However, one could offer a counter-argument to the effect that such a phenomenon 18 designed to minimize the reproductive rate in the rabbit population, at least in the summer. Under these circumstances, an overly energetic young.buck, despite his ambitions, could fertilize only 1 female a day, If that often.

\section{Centrifugation.}

As noted in Chapter VIII, the percentages of capped, capless and motile opcrmatozoa in each collection fraction were determined by phase contrast microscopy, immediately after Staflo operation ceased. In order to have a sample with a high concentration of spermatozoa for artificial insemination, each collection fraction was centrifuged for 5 minutes at $1,400 \mathrm{~g}$ In a clinical centrifuge in the refrigerated room. After the supernatant was removed and the spermatozoa resuspended in egg yolk medium, they were warmed to room temperature for evaluation which included a second determination of the percentages of capped, capless and motile spermatozoa, and nigrosin-eosin vital staining.

In most experiments, it was observed that the percentage of capless 
spermatozoa increased significantly between the evaluations prior to and after centrifugation, a period of approximately 30 minutes. Because the spermatozoa were In egg yolk medium for about half of that interval, the deterioration is attributed solely to centrifugation. Even though the centrifugation time and speed were the same in all experiments, the changes in the percentages of capless spermatozoa were not constant from sample to sample. As shown in Table XXXVI, the increases in the percentages of capless spermatozoa in selected experiments ranged from $4.4 \%-43.3 \%$. In assessing these changes in the initial samples (IS), it should be noted that the first determination of the percentages of capless spermatozoa was made shortly after the inftlal dilution which was generally 2-3 hours prior to centrifugation. Therefore, some deterioration could have occurred. in the Initial sample prior to centrifugation, whereas the collection fractions were assayed immediately prior to and after centrifugation.

With regard to Table XXXVI, the following observations on individual experiments are pertinent:

a. Experiment $7-26-6 \%$ Despite the much lower spermatozoa concentration, CF 7 had a slightly smaller increase In capless spermatozoa. This suggests that with a spermatozoa sample that undergoes significant deterioration during centrifugation, there is no differential effect of concentrations 6 as low as $1.34 \times 10 / \mathrm{ml}$. Even though the cold room temperature was $4.5^{\circ} \mathrm{C}$. no motile spermatozoa were seen in the collection fractions. Thus, it is unlikely that CF 7 was enriched by motile spermatozoa during electrophoresis. b. Experiment 7-29-67. In this experiment, both collection fractions had relatively high spermatozoa concentrations. Thus, the greater increase in capless spermatozoa in CF 7 is not belfeved to be related to the slightly lower sperm concentration in that fraction.

c. Experiment 8-10-67. The increase in capless spermatozoa was less 
Table XXXVI. Effect of Low Temperature Centrifugation for 5 Minutes at $1400 \mathrm{~g}$ on the Morphology of Rabbit Spermatozoa. ${ }^{a}$

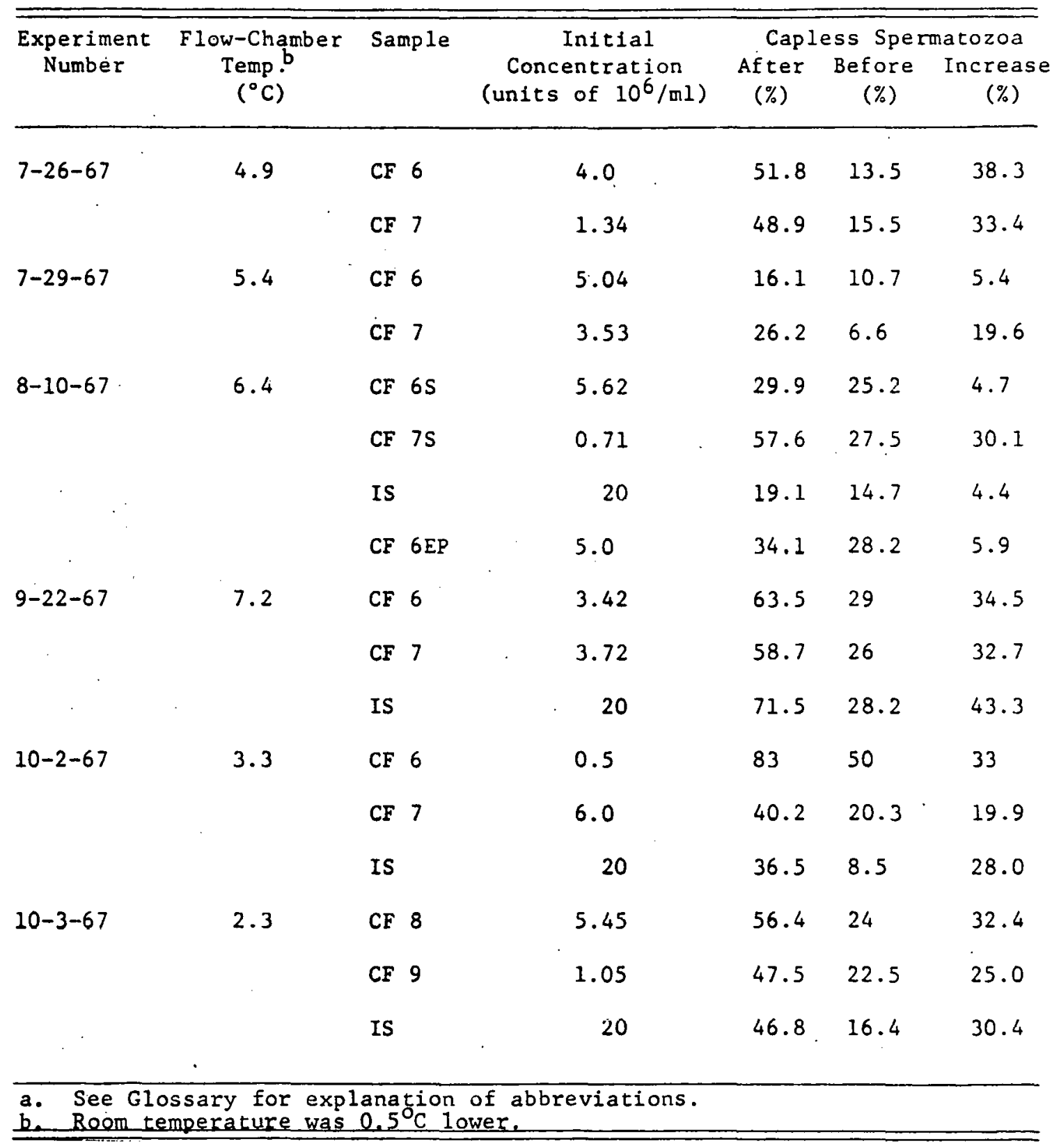


than $5.9 \%$ for 3 samples with spermatozoa concentrations equal to or greater than $5 \times 10^{6} / \mathrm{ml}$ prior to centrifugation. There was a $30.1 \%$ increase in CF $7 \mathrm{~S}$ which had an inttial concentration of on $1 \mathrm{y} 0.71 \times 10^{6} / \mathrm{ml}$, suggesting that, In this experiment with relatively high quality spermatozoa, the low concentration was detrimental.

d. Experiment 9-22-67. Even though the concentrations of spermatozoa in the collection fractions prior to centrifugation were 3.42 and 3.72 $x 10^{6} / \mathrm{ml}$, the absolute increases in the percentage of capless spermatozoa were very large, $34.5 \%$ and $32.7 \%$, respectively. A slightly greater change was observed in the initial fraction, part of which may be explained by the time difference between evaluations.

e. Experiment 10-2-67. In addition to a greater increase during centrifugation, CF 6 also had a higher pre-centrifugation percentage of capless spermatozoa than did CF 7. This suggests a detrimental effect of a concentration of $0.5 \times 10^{6} / \mathrm{ml}$ even with the highest quality spermatozoa of any experiment listed in Table XXXVI.

f. Experiment 10-3-67. As observed in Experiment 7-26-67, the 1ower collection fraction had a smaller increase in capless spermatozoa, despite a lower concentration. The sample as a whole experienced a significant Increase In capless spermatozoa, suggesting poor spermatozoa quality. Thus, with poor quality sperm, a concentration as low as $1.05 \times 10^{6} / \mathrm{ml}$ is not detrimental. The flow-chamber temperature of $2.3^{\circ} \mathrm{C}$ was too low for the spermatozoa to have had any intrinsic motility, thereby ruling out enrichment in CF 9 with regard to motility.

Conclusions from these results, pertaining to the centrifugation of spermatozoa under the conditions specifled above, are as follows: (1) With spermatozoa concentrations of less than $1 \times 10^{6} / \mathrm{ml}$, there 1 is a dispropor- 
tionate Increase in the percentage of capless spermatozoa compared to samples of higher concentrations if the initial quality of the sample as a whole is good. (2) With spermatozoa concentrations of $1.05 \times 10^{6}$ and $1.34 \times 10^{6} / \mathrm{ml}$, there is no differential increase in capless spermatozoa as compared to higher concentrations if the quality of the sample as a whole is poor. This observation appears unrelated to possible effects of Intrinsic motility on spermatozoa distribution. (3) With spermatozoa concentrations ranging from $3-6 \times 10^{6} / \mathrm{ml}$, the increases in the percentage of capless spermatozoa were generally comparable in the 2 collection fractions and ranged from $4.4 \%-38.3 \%$. The magnitude of the increase is belleved to reflect the quality of the spermatozoa. (4) The changes in the percentages of capless spermatozoa do not appear to be related to the initial percentages thereof or to the temperature over the range $2.3^{\circ} \mathrm{C}-7.2^{\circ} \mathrm{C}$.

\section{Environmental Temperature}

Observations over several summers showed a marked variation of efaculate quality from extremely good to very poor in an irregular manner unt1l well after cool weather had returned. This variation in quality persloted even when a roof eprinkler was nsed to cool the rabbit hutches. In an attempt to improve the quality of spermatozoa available for Staflo experiments and to gain information concerning the effect of environmental temperature on sperm quality, a study was designed with 6 rabbits divided between 2 subgroups matching breed and history. Three rabbits remalned out-of-doors in the hutches cooled by the sprinkler, and hereafter are referred to as the "outside" group. The other 3 rabbits, the "Inside". group, were moved into an afr-conditioned room with controlled artfficial lighting and 1 small window without direct sunlight. The temperature was recorded continuously in both locations. Insofar as 
possible, ejaculates were collected from matched pairs on the same day, usually within minutes of each other.

Prior to August 15, all 6 rabbits were housed cut-of-doors and experienced the same temperatures to that date. From the period July 1August 15 , significantly elevated temperatures were as follows: $87^{\circ} \mathrm{F}$ on July $3 ; 91^{\circ} \mathrm{F}$ on July $11 ; 83^{\circ} \mathrm{F}$ on July 20,26 and 28 , and $90^{\circ} \mathrm{F}$ on August 14, the day before the "inside" group was moved into the airconditioned room. Ejaculates were collected from each of the rabbits on August 1.5 prior to moving the "Indoor" group into the air-conditioned room. For administrative reasons, the "outdoor" group was moved into the air-conditioned room on October 14.

All ejaculates were evaluated by phase contrast microscopy for percentages of capped, capless, tall-wagging and highly motile spermatozoa and by the nigrosin-eosin vital staining technique described in Appendix B. Figures 57 and 58 show the porcentages of mottle and capless spermatozoa; respectively, for individual rabbits of the "Indoor" and "outdoor" groups. The curves for Otto in Figures 57 and 58 will be discussed in detall and then generalizations will be made with reference to the information on the other rabbits in both groups.

Before discussing the figures, it is necessary to know the relative Ilfespan of each of the stages of spermatogenesis. Swierstra and Foote arbitrarily divided the cycle of seminiferous eptthelium of the rabbit Into 8 stages and determined the relative duration of each stage ${ }^{222}$. They observed that the Type A spermatogonia divided to produce 2 Type A spermatogonia, 1 of which remalned the starting cell for the next generation, while the other underwent 4 spermatogonial divisions to give (theoretically) 16 primary spermatocytes and, in turn, 64 spermatids. However, they estimated that, because of degenerative processes, only 15.6 primary spermatocytes 

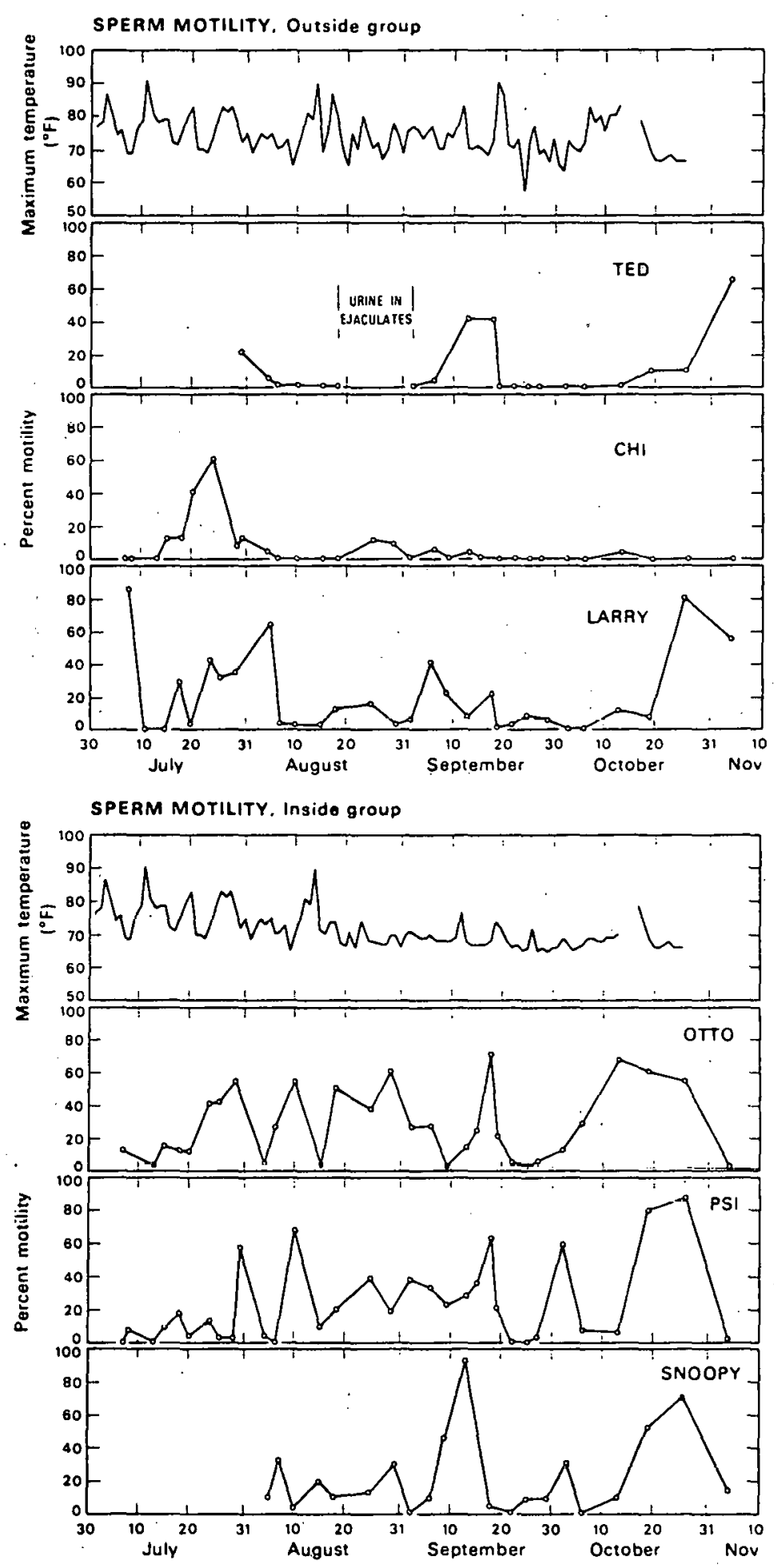

Fig. 57. Effects of environmental temperature on the motility of cabbil sperillatuzud. A. "Outside" group. B. "Inside" group. Lifespan scale (Figure 59 reduced) in rear-cover envelope may be used with this figure. 

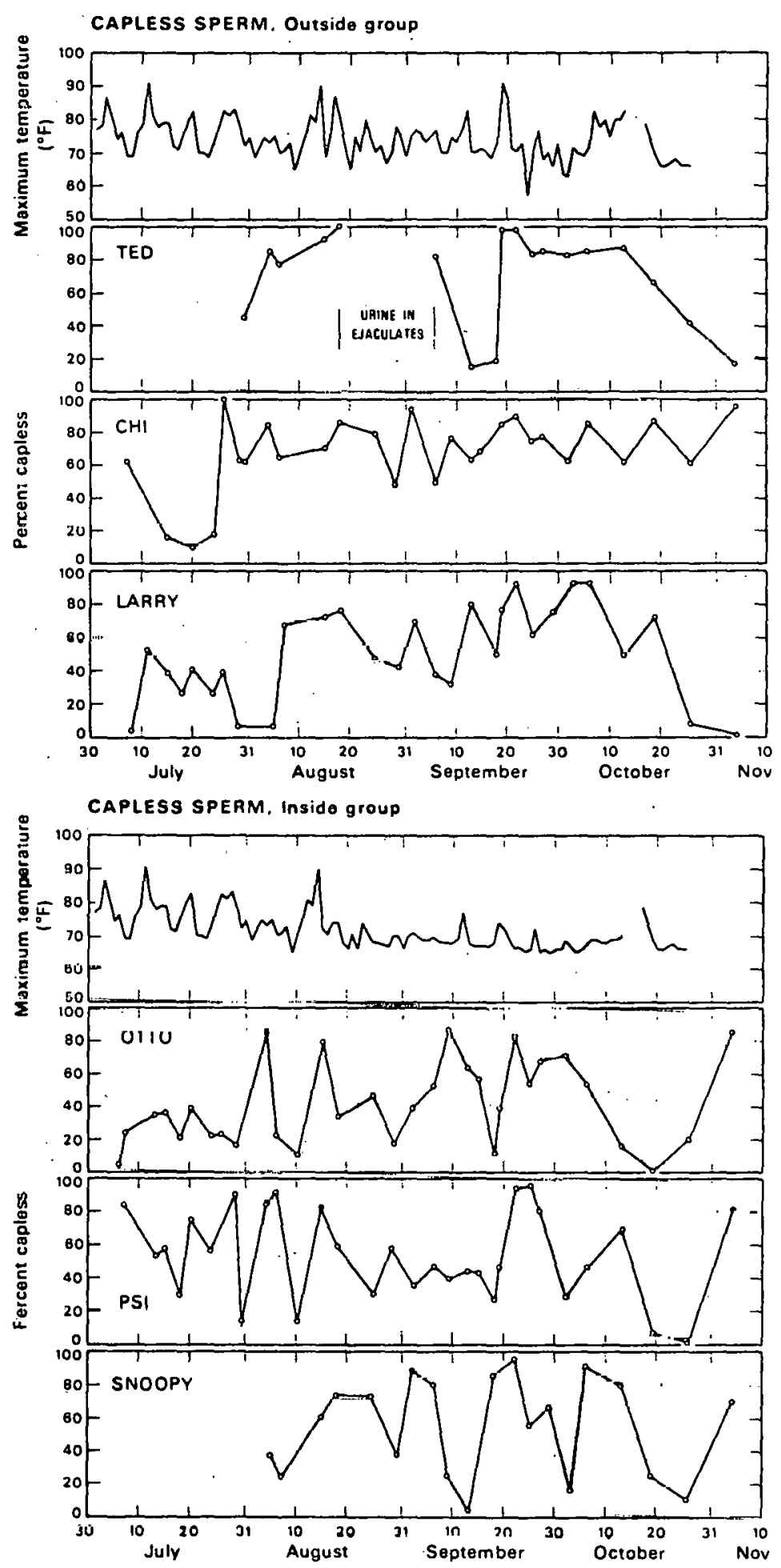

Fig. 58. Effects of DBL 00116010 capless spermatozoa in rabbit ejaculates. A. "Jutside" group. B. "Inside" group. Lifespan scale (Figure 59 reduced) in rearcover envelope may be used with this figure. 
and 47.5 spermat1ds were produced 222 . Subsequently, they studied the duration of spermatogenesis and of spermatozoa transport in the male rabbit using tritiated thymidine ${ }^{223}$. The estimated life span of the different cells in the spermatogenetic process are given in Table XXXVII. After formation from spermatids, spermatozoa spend approximately 5.6 days in the lumen of the seminiferous tubule and then 8-10 days in transport through the ducts. As noted by the authors, the estimate of the total duration of spermatogenesis depends upon the polnt chosen as the onset of the process. If it is considered to begin with the first of the series of spermatogonial divisions leading to the production of primary spermatocytes, the duration is approximately 43.6 days. If it is assumed that spermatogenesis starts with the formation of spermatogonlal stem cells, and that the life span of these cells is 1 cycle of the seminiferous epithelium, then spermatogenesis would extend over approximately 51.8 days. Thus, adding 10 days for transport in the duct system would make the total time from the onset of spermatogenesis to the appearance of spermatozos in the ejaculate exther 54 or 62 days. Figure $59 \mathrm{R}$ is a linear representation of the time scale for spermatogenesis and operm transport on the same scale as Figures 57 and 58 . When the right border of the time scale is placed at the date on which an ejaculate was collected, one can visualize the relationship between individual environmental temperature peaks and the corresponding stage of development of the spermatozoa in that particular ejaculate.

Swlerstra and Foote found that the first labeled spermatozoa appeared In the efaculate from 39-42 days after infection of tritiated thymidine ${ }^{223}$. The earilest observation of labeled spermatozoa in the testis by OrgebinCrist was 30.6 days after thymidine injection ${ }^{181}$. Adding time for spermatozoa release and transport in the duct system to the 30.6 days would 
Table XXXVII. Estimated Lifespan of Rabbit Spermatogenetic Cells ${ }^{223}$

\begin{tabular}{cc}
\hline Type & $\frac{\text { Estimated Lifespan }}{(\text { Days })}$ \\
Spermatogonia & 3.4 \\
A & 0.6 \\
Intermediate-1 & 2.8 \\
Intermediate-2 & 1.1 \\
B & 16.5 \\
Primary Spermatocytes & 0.8 \\
Secondary Spermatocytes & 10.0 \\
Spermatids & \\
Spermatozoa & 5.6 \\
In Testes & 10.0 \\
In Transport & 50.8 \\
T. & \\
\hline
\end{tabular}




\section{LIFESPAN OF SPERMATOGENETIC CELLS}

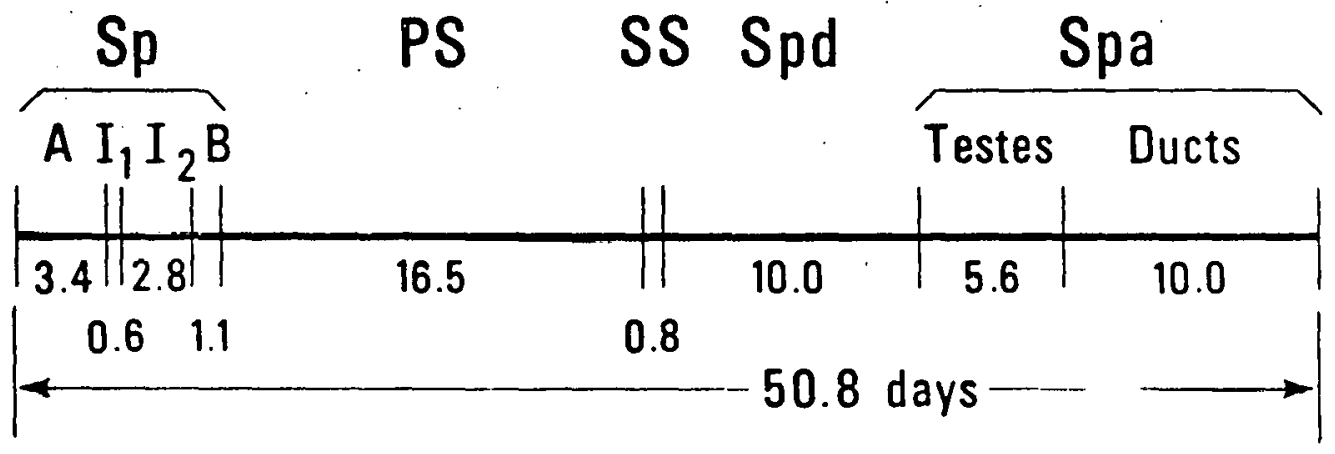

DBL 70115995

Fig. 59. Lifespan of rabbit spermatogenetic cells. See also Figure 59 (reduced) in rear-cover envelope, for use with Figures 57 and 58. 
give a time of appearance in the ejaculate similar to that observed by Swierstra and Foote. Takeda, Lutwak-Mann and Mann labelled mammalian spermatozoa with $32 \mathrm{p}$ in order to study the so-called leakage of intracellular sperm compounds which is known to accompany sperm aging or senescence ${ }^{225}$. The level of radioactivity in rabblt spermatozoa separated from seminal plasma by centrifugation and washed 3 times with Ringer's solution remained low until 40-42 days after the injection of $\mathrm{p}^{32}$, at which time the radioactivity rose steeply ${ }^{225}$. This time span corresponds favorably to the results reported by other investigators.

Referring to Figures 57 and 58, the ejaculate from Otto on August 15, the day after the temperature was $90^{\circ} \mathrm{F}$, contained less than $4 \%$ motile spermatozoa and $80 \%$ capless spermatozoa. Efaculates collected from then unt11 September 6 contained from 25-60\% motile spermatozoa and from 18-52\% capless spermatozoa. Spermatozoa appearing in the ejaculates from August 15-29 should have been in the spermatogonial stage at the time of the $91^{\circ} \mathrm{F}$ temperature on July 11 and in varying primary spermatocyte stages durlng the $83^{\circ} \mathrm{F}$ days on July 20,26 and 28 . The spermatozoa appearing in ejaculates from August 29-September 6 would have been in spermatogonial stages during the $83^{\circ} \mathrm{F}$ days in the latter part of July; therefore, the $91^{\circ} \mathrm{F}$ temperature on July 11 would have had no effect.

On September 9, the quallty of Otto's ejaculate was very poor, with less than $4 \%$ motile spermatozoa and $87 \%$ capless spermatozoa. Most of the spermatozoa in that ejaculate would have been undergoing the second maturational (meiotic) division on August 14 when it was $90^{\circ} \mathrm{F}$ and in the spermatogonial diyision stages on July 26 and 28 when it was $83^{\circ} \mathrm{F}$. The effects of increased environmental temperature on efther of these stages could have contributed to the decreased motflity and high percentage of capless spermatozoa In that efaculate. This suggests that spermatozoa 
and spermatids are relatively resistant to increased environmental temperatures. It appears that spermatozoa in the intra-abdominal part of the duct system are damaged, thereby demonstrating the importance of the lower scrotal temperatures in sperm survival.

After slight improvement in the next 2 ejaculates, the ejaculate on September 18 contained $72 \%$ motile spermatozoa and only $10 \%$ capless spermatozoa. The decrease in quality on the following day to only $22 \%$ motile and $40 \%$ capless spermatozor is probably an example of the second ejaculate phenomenon discussed in subsection 2, above. The spermatozoa in this transient peak of high motility would have been in the middle stages of primary spermatocyte development on August $14\left(90^{\circ} \mathrm{F}\right)$ and may well have gtarted their development after the elevated temperatures on July 26 and 28 . This suggeste that elevated environmental temperatures during the intermediate stages of primary spermatocyte development have little effect on the subsequent motility of the spermatozoa.

The downward trend observed on September 19 continued such that 3 ejaculates between September 22-27 had less than $6 \%$ motile spermatozoa and from 55-84\% capless spermatozoa. Spermatozoa appearing in those ejaculates would have been in the early spermatocyte and late spermatogonial division stages of development on August 14 when 1 was $90^{\circ} \mathrm{F}$. This suggests that the spermatogonial and early spermatocyte stages of development are very sensitive to increased environmental temperature.

The improvement in the quality of the ejaculates after October 5 reflects the appearance of spermatozoa which started their spermatogonial development after August 14. One can speculate that the failure of the quality of the spermatozoa to reach a superior level, e.g., approaching 90\% motile, reflects a prolonged influence of that particular high environmental temperature on the stem cell layer. 
Although the curves for Psi and Snoopy in Figures 57 , and 58 are not identical to those for Otto, the simllarity is evident and analyses of those curves lead to stmilar conclusions.

Spermatiozoa samples collected from the "indoor" group on November 4 showed a significant decrease in quality with an average of $8 \%$ motile spermatozoa and an Increase in percentage of capless spermatozoa to $80 \%$. This is attributed to a transient relative temperature rise to $79^{\circ} \mathrm{F}$ in the air-conditioned room, which was generally kept at $66^{\circ} \mathrm{F}$, due to an error on the part of the caretaker. On the $79^{\circ} \mathrm{F}$ day (October 17), the spermatozoa would have been in the final stages of morphogenesis from spermatids.

In general, the quality of the efaculates from the rabbits in the "outdoor" group was extremely poor until after they had been moved into the air-conditioned room on October 14. Shortly thereafter, there was a marked increase in the quality of the ejaculates from Ted and Larry, whereas those from Chi remained poor. However, the average quality of the ejaculates Increased considerably and did not show a drop on November 4, as did the ejaculates from the "Indoor" group. It is probable that the relative temperature rise of $13^{\circ} \mathrm{F}$ did not seem significant to the rabbits in the "outdoor" group which had been exposed to much higher temperature ranges prior to coming into the alr-conditioned room 3 days previously. This introduces the concept of relative, as compared to absolute, temperature changes. Thus, for rabbits acclimatized to consistently low temperatures, a relative temperature rise can be significant. In contrast, rabbits accustomed to wide daily temperature variations are not affected by moderate eleyations of temperature.

In retrospect, this ancillary study on the influence of the environmental temperature on the quality of spermatozoa is one of the most interest- 
Ing and most important parts of this thesis. Putting rabbits in an air-conditfoned room greatly improved the quality of spermatozoa available for Staflo experiments. In addition, the observations strongly suggest that a very high environmental temperature for part of one day can cause marked variations in the quality of spermatozoa of rabbits, kept thereafter in a controlled environment, for 7-8 weeks and probably to some degree for several weeks longer. There were no changes in animal care or sperm collection to explain the varlations in quality. The probable differentlal effects of the environmental temperature on the stages of spermatogenesis explain the random variation in spermatozoa quality prevlosly observed in warm weather.

Within the obvious limitations of this study, it appears that several stages of spermatogenesis are sensitive to increased testicular and abdominal temperatures secondary to elevated environmental temperatures. It is probable that the spermatogonial divisions are critical periods considering the improvement in sperm quality of the "Indoor" group after October 5. However, it 18 difficult to separate that phase of development from the early primary spermatocyte stage in this study because of the several hot days to constder. The maturational division stages and the very early (and possibly very late) phase of spermatid development appear to be sensitive to elevated temperatures. Whereas late spermatids and Immature spermatozoa appear to be protected to some degree in the scrotum, mature opermatozoa in the intra-abdominal duct system are very sensitive to temperature changes. For Instance, nearly complete disintegration of spermatozoa was observed in many ejaculates collected the morning after a day with a $90^{\circ} \mathrm{F}$ or higher temperature.

The difficulty in dissecting the effects of various temperature peaks during the period shortly after the rabbits were moved into the air-conditioned 
room. emphasizes the need for a controlled study. In general, it should include the following: (1) establishing a steady-state baseline with regard to sperm quality and collection frequency in a controlled low temperature $\left(60-65^{\circ} \mathrm{F}\right)$ environment, (2) exposure of the experimental group to a temperature of $85-90^{\circ} \mathrm{F}$ for 6-10 hours on one day only, and (3) dally collection of efaculates from both control and experimental groups until full reproductive performance returns or at least for 60 days.' Dally spermatozoa collection is necessary to avoid the second ejaculate phenomenon which would mask less dramatic actual changes in sperm quality. Such a study would give a comparioon of the differential sensitivity of the stages of spermatogenesis to temperature, determined in this manner, and also to external radiation of the testes.

\section{Summary and Conclusions}

Even after eliminating obvious possible adverse factors in animal care and sperm handing, the quality of ejaculates showed marked and unpredictable variability in warm weather. In addition to differences in the quality of ejaculates from different rabbits, a dramatic decrease in the quality of consecutive ejaculates from the same rabbit was observed if the Interval between collections was 60 minutes or less. Only partial recovery was observed after an interval of 24 hours, suggesting a selective process in the genital duct system favoring ejaculation of the highest qualitiy spermatozoa with the first emission.

Substantial differences in measured sperm quality were found, according to the methods used for evaluation. For example, there was a lack of correlation between the percentages of capless (CL) spermatozoa determined by phase contrast microscopy and of stained. (S) spermatozoa from light microscopy scorling of nigrosin-eosin stained preparations. Capless 
percentages ranged from $33.9 \%$ less than to $22.1 \%$ more than "stained" percentages, with the differences apparently unrelated to spermatozoa concentration.

Subsequent studies showed falr agreement between phase contrast and light microscope scorlng of nigrosin-eosin stained spermatozoa. Comparison of phase contrast scoring of unstained spermatozoa with nigrosin-eosin stained preparations of the same sample showed differences related to spermatozoa quality. W1th poor quality ejaculates, the unstained samples showed a higher percentage of capless spermatozoa than did the nigrosineosin preparations $(e .8 ., \mathrm{CL}>\mathrm{UCL}+\mathrm{SCL}$ in Table XXXIV). The reverse was true for high quality samples. The deterioration of spermatozoa samples during centrifugation is a function of the inherent quality of the sample even at concentrations above $4 \times 10^{6} / \mathrm{ml}$. It does not appear to be related to the Initial percent of capless sperm in the sample or to temperature, over the small range of $2.3-7.2^{\circ} \mathrm{C}$.

It was shown in this chapter that elevated environmental temperatures caused marked varlations in sperm quality for 7-8 weeks (and possibly longer) after exposure, even if the rabbits were placed in an.air-conditloned room at $66^{\circ} \mathrm{F}$ right after the high temperature exposure. Therefore, the relatively sensitive stages of spermatogenesis would appear to be the spermatogonial divisions, the maturational divisions and the early spermatid stage (Flgure 57 and 59). The late spermatid and spermatozoa stages appear relatively resistent to elevated body temperatures except for the mature spermatozoa in the Intra-abdominal part of the genital duct system. The latter spermatozoa undergo marked deterioration as evidenced by 1088 of motility and Increased percentages of caplessness In the efaculate. The delayed and intermittent expression of the effects of envirommental temperature on spermatozoa quality explains, at least 
partially, the observations mentloned in previous chapters and in the first paragraph of this section. 


\section{SUMMARY}

Since a number of detailed conclusions and recommendations were presented in the individual chapters, only the most important are recapitulated here, along with some summary findings.

The motility and fertilizing capacity of rabbit spermatozoa were preserved for several hours during separation experiments at $3^{\circ} \mathrm{C}$ at concentrations of approximately $1 \times 10^{6}$ and $4 \times 10^{6} / \mathrm{ml}$, respectively. These experiments were carried out in low ionic strength glycinephosphate buffer. Fowl spermatozoa motility was preserved at concentrations as low as $8 \times 10^{6} / \mathrm{ml}$ for 6 hours at $3^{\circ} \mathrm{C}$ in 310 mosm solutions of both low ionic strength glycine-citrate and glycine-phosphate buffers. Under the same media and temperature conditions, fertilizing capacity was preserved for about 2 hours at concentrations down to $90 \times 10^{6}$ sperm/m1. The establishment of these conditions rendered it possible to conduct analytical biophysical experiments on low concentration preparations of rabbit and fowl spermatozoa, and to exploit the results of such studies at higher concentrations in preparative Staflo experiments with maintenance of vital cellular functions, especially the fertilizing capacity.

In Staflo experiments at $3^{\circ} \mathrm{C}$ or lower, migration was not significantly influenced by intrinsic motility and there was no differential distribution of live and dead rabbit spermatozoa among collection fractions in three-layer sedimentation and two-layer electrophoresis migration experiments. Under these temperature conditions a most important variable in experiments using low density-low viscosity media is the cell concentration. For instance, concentrations above $25 \times 10^{6}$ rabbit spermatozoa/ml may result in "cluster sedimentation", 
i.e., cooperative rather than individual cell sedimentation.

At $4^{\circ} \mathrm{C}$ and higher, the intrinsic motility vector may have a significant contribution to the migration pattern. Sperm orientation was influenced by gravity in all $\mathrm{Staflo}$ experiments and by the strength and direction of the electric field in electrophoresis experiments. By proper selection of the medium, $\mathrm{pH}$, density gradient, temperature and electric field strength (if used), the intrinsic motility vector of both rabbit and fowl spermatozoa was controlled so as to give enrichment of certain collection fractions with regard to motility, viability and fertilizing capacity.

The best values obtained for selected physical propertico of rabbit and fowl spermatozoa are given in Table XXXVIII. The sedimentation and electrophoretic values were determined in Staflo experiments, the specific gravities in sodium iothalamate gradient. Within the single (specific gravity) band of fowl sperm, a higher percentage and degree of motllity were found in the top of the band. Among the different bands of rabbit spermatozoa, there was a marked differential viability distribution with the low density band containing nearly all of the viable sperm. Thus centrifugation in a sodium iothalamate density gradient provides a ready means for separating live and dcad rabbit spermatozoa. Although there was no evident bimodal distribution of viable sperm in the low density band, (as suggested by others) it is possible that, with an expanded density gradient of physiological osmolarity, different groups of spermatozoa might be found in thi.s band. The high density band, containing essentially only non-viable sperm, appeared to migrate farther with increasing centrifugation time suggesting a non-constant effective density. 
Table XXXVIII. Best Values of Physical Properties of Rabbit and Fowl Spermatozoa."

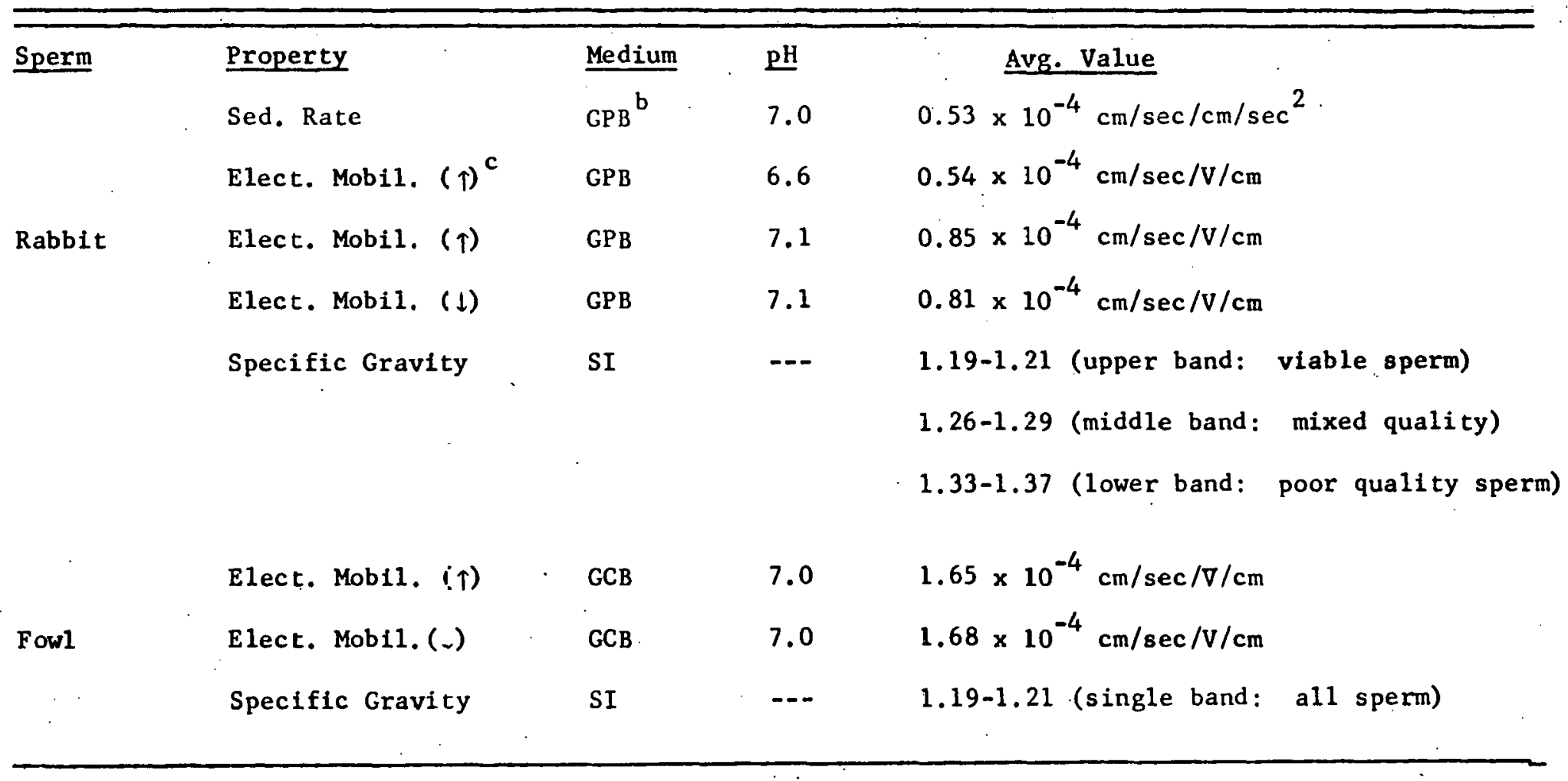

a) normalized to conditions of $3^{\circ} \mathrm{C}$ and $1 \%$ sucrose.

b) $\mathrm{GPB}=$ glycine-phosphate buffer; $\mathrm{GCB}=$ glycine-citrate buffer; both of resistivity $960 \mathrm{ohm} \mathrm{cm}$. SI = sodium iothalamate density gradient.

c) $\uparrow=$ upward migration. $\quad \downarrow=$ downward migration. 
The number of offspring obtained from the artificial insemination of separated rabbit spermatozoa was insufficient for the correlation of physical properties with the X- and Y-chromosome content of the spermatozoa. Observations in these experiments strongly suggest that the partial separation of the two types of spermatozoa reported by other investigators is not a simple function either of differential sedimentation rates or of differing electrophoretic mobilities. Thus the partial separation of $\mathrm{X}$ - and $\mathrm{Y}$-chromosome bearing spermalozoa in their sedimentation experiments must have been influenced by "cluster sedimentation" or some process other than individual cell sedimentation, such as an aqueous polymer two phase separation as described by Albertson 270 . My observation of a significant effect of motility on electrophoretic migration, at temperatures as 1 ow as $4^{\circ} \mathrm{C}$ depending upon the medium, suggests that the $X-Y$ separations reported by Schröder 206 and by Gordon ${ }^{91}$ using temperatures generally $10-15^{\circ} \mathrm{C}$, were most likely a consequence of a differential swimming rate of, rather than an opposite charge on, the two types of spermatozoa.

A frequent obstacle to obtaining offspring from separated spermatozoa was the marked variability and unpredictabllity in the qualicy of rabbit ejaculates, especially during warm weather. The effects of elevated environmental temperatures appeared to be intermittent over a 7-8 week period. Relative as well as absolute elevations of temperature may be important in this regard. The most sensitive phases of spermatogenesis appeared to be the spermatogonial and maturational divisions, and the early spermatid stage. In addition, mature spermatozoa in the intra-abdominal part of the genital duct system appeared to undergo marked deterioration because of elevated 
environmental temperatures, as evidenced by the immediate loss of motility and increased caplessness of ejaculate sperm.

Several theoretical analyses applicable to free solution zonal migration and fractionation processes were made. Range and distribution diagrams for particle migration distances were developed which make possible visualization of the requirements for optimum fractionation, and subsequent quantitation thereof. The diagrams also provide a means for analyzing the distribution of migration properties among particle populations. They emphasize the fact that migration patterns spreading over only two adjacent layers can be meaningful but that a three-layer pattern is the minimum that will give distinct sub-populations in the extreme fractions.

As in all investigative fields, each project generates innumerable ideas for additional work. Many suggestions for expanding the different methods of studying spermatozoa used in this thesis are made in individual chapters. It is hoped that some of the suggestions will be implemented in the near future. 


\section{ACKNOWLEDGMENTS}

Because I lived and studied at Stanford throughout most of the time during which this work was done, my dependence upon and appreciation of the assistance provided by the persons in Berkeley, mentioned below, is more than ordinary.

I wish to express my deepest appreciation to my advisor; Dr. Howard C. Mel, for his continued encouragement, guidance and assistance, and for making available the invaluable and frequent help from his research assistants, Mrs. Gretchen Hylton, Mrs. Anne Poley and Mr. Ralph Reynolds.

Other persons to whom I am greatly indebted are: Miss Sylvia $J$. Spengler for working with me during the academic years 1964-65 and 196566; the late Roger D. Tippetts for frequent consultation on Staflo operation and design; Mr. Johnny Maxion and his staff at Building 74 for the excellent care given the rabbits; Mr. Pete Dowling and others in the Donnor Laboratory Mach1ne Shop; Mr. John Flambard for drawing all of the figures; and Mr. Roger Sherman and Mrr. Will1am Love for dsslslanle in developing the Staflo microscopic system.

I am very grateful to Dr. Nachum Snapir, Faculty of Agriculture, Hebrew University of Jerusalem, who collaborated with me on the work with fowl spermatozoa. We were gractously assisted by the personnel at the Department of Youltry Husbandry, Berkeley, especlally Dr. Samuel Lepkoviky, Mrs. Dorothy Lowry, Mr. Ralph Len and Mr. Fred Furuta.

The PLH used in these studies was provided by the Endocrinology Study Section of the National Institutes of Health (NIAMD).

The support of the U.S. Atomic Energy Commission, Donner Laboratory and Lawrence Radiation Laboratory (W 7405-eng-48) is gratefully acknowledged. 


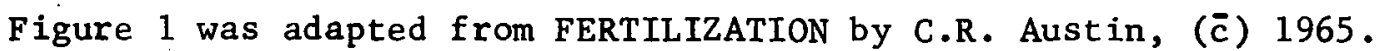
Reprinted by permission of Prentice-Ha11, Inc., Englewood,Cliffs, N.J. Finally, to Miss Sherrie Casaurang, who typed many preliminary drafts as well as this final one, I am especially grateful both for the superior quality of typing and for sacrificing many evenings and weekends to accomplish the job expeditiously. 
GLOSSARY

Symbo1

or Term

AI

C

CF

CL

Collection container

rp

CPD

$\frac{d x}{d t}$

$\dddot{\mathrm{E}}$

e.s

e.s

$\mathrm{E} \cdot \mathbf{8}$

$E \cdot S$

EWFR

EYM

F1ow-ce11

Flowchamber
Meaning

Artificial insemination

Capped (spermatozoa)

Collection fraction. The cells or particles in an individual collection container

Capless (spermatozos)

Receptacle in collection system for outflow from one outlet channel

Contipoioc (viscosity)

Cytoplasmic droplet (sperm)

$\left(\frac{\mathrm{b}}{\mathrm{cm} \cdot \sec }\right) \times 10^{-2}$

p 21

Sedimentation velocity

Electric field strength

$\mathrm{V} / \mathrm{cm}$

Slowest electrophoresing-

sluwest sedimenting (particle)

Slowest electrophoresing-

fastest sedimenting (particle)

Fastest electrophoresingslowest sedimenting (particle)

Fastest electrophoresingfastest sedimenting (particle)

Elcctrode wash solution flow rate

Egg yolk medium

Consists of flow-chamber and upper and lower electrode chambers

Chamber in which electro phoretic and/or sedimentation migration occurs
Where First Used

p 234

Table XVIII, p 218

p 65

Table XVIII, p 218

p 65
$\mathrm{Eq}(4), \mathrm{p} 84$

Fig 16, P 87

p 86

p 86

p 86

p 86

p 67

$\mathrm{mI} / \mathrm{mIn}$

p 234

p 55

p 55 
Symbo1

or Term

FM

Fraction

$\mathrm{g}$

$\mathrm{GCB}$

GPB

h

IS

L

Layer

MBM

Migration pattern

Migration profile

MNS

$\mathrm{NH}-1,2$

NZW

s
Meaning

Forward motion (spermatozoa motility)

Units

Short for "collection fraction"

Acceleration of gravity $980 \mathrm{~cm} / \mathrm{sec}^{2}$

Gilycine-citrate buffer

Glycine-phosphate, buffer

Height of sample stream at inlet

Initial sample. Sample suspension that is introduced into Staflo inlet

layer

Length of flow-chamber

$\mathrm{cm}$

Horizontally flowing fluid

layer in Staflo flow-chamber

Modified Baker's Medium

$\mathrm{cm}$

Where First

Used

Table XVIII, P 218

p 66

App F, p 381

App D, p 358

p 232

p 75

p 231

Table V, p 148

P 58

App D, p 358

The overall side view appearance of a continuously flowing sample migrating in the flow-chamber

The appearance or shape of the cross section of a migrating sample

p 58

Morphologically normal but stained (spermatozoa)

p 112

Norman-Johns on Solutions

1 or 2

p 37

New Zealand White (rabbit)

Table XXIV, p 245

Slowest sedimenting (particle)

Fig 16, p 87

Fastest sedimenting (particle) 
Symbo1

or Term

S

SCL

SDE

SI

SS

Staf1o

SUE

'I'W

$\mathrm{U}$

UC

UCL

$\mathrm{v}$

V

$\eta$

$\lambda$ $\frac{\text { Meaning }}{\text { Sedimentation coefficient }}$

Units.

Svedbergs

$\left(=10^{-1} 3 \mathrm{sec}\right)$

Stained (spermatozoa)

Stained, capless

(spermatozoa)

Sedimentation-downward-

electrophoresis

Sodium iothalamate (medium)

Stirred sample. Suspension

that remains in staflo

sample (feed) syringe at conclusion of experiment (usually stirred magnetically throughout experiment)

Short for "Stable-flow free-boundary"

Sedimentation-upward

clcctrophoresis

Te11-waggling (uplitumaluad motility)

Uns tained (spermatozoa)

Unstaincd, capped

(spermatozoa)

Unstained, capless

(sperma luzua)

Farticle migration ratc

Vo1ts

Absolute viscosity

Migration distance
Where First

Used

Table III, p 117

P 112

P 286

p 85

Tab1e XXXVIII, P 317

P 234 p 2

p 85

Tnhln virI, p 218

p 112

ก 2 ถูก

p 286

$\mathrm{cm} / \mathrm{sec}$

Eq (13),

p 97

Vo1ts

Eq (13),

p 97

poise $\left(\frac{\mathrm{g}}{\mathrm{cm} \cdot \mathrm{sec}}\right)$

Eq (3),

p 84

$\mathrm{cm}$

p 77 
Symbo1

or Term

$\lambda_{E}, \lambda_{S}$,

etc.

、

$\lambda_{\text {9SUE-min }}$

$\mu$

$\mu$

$\tau_{\min }$

$\tau_{\text {av }}$

${ }^{\tau} E_{\text {min }}$

\section{Meaning}

Units

Migration distances:

E (electrophoresis),

S. (sedimentation),

SDE (sedimentation-downward-

electrophoresis), SUE

(sedimentation-upward-

elec trophoresis)

Minimum $\lambda$ for spermatozoa

in collection fraction 9

in sedimentation-upward-

electrophoresis

Electrophoretic mobility

Micron

Minimum residence time; the time any given volume element resides in the flow-chamber

Average residence time; time required to pump a volume of fluid equal to the volume of the flowchamber

Minimum residence time for sec electrophoresis
Where First Used

Eq (2), p 83 p 86

$\frac{\mathrm{cm}^{2}}{\mathrm{sec} \cdot \mathrm{volt}}$

$10^{-4} \mathrm{~cm}$

p 42

sec

Eq (4), p 84

sec

Table V, p 148

Eq (13), p 97 
APPENDIX A

Considerations in Selecting the Rabbit

as a Sperm Donor

1. Rabbit spermatozoa have been studied extensively with regard to both physiology and attempted separation of X- and Y-chromosome bearing sperm.

2. The rabbit is large enough to work with conveniently. Sperm can be collected with an artificlal vagina, allowing reuse of the animal. Rats and mice generally are sacrificed to get sperm.

3. Rabbit semen does not coagulate ${ }^{92}$ as does that of the rat ${ }^{168}$. Guinea pig spermatozoa agglutinate more rapidly than sperm from other apecies in all media, including saline ${ }^{52}$.

4. Rat spermatozoa survive only 2-3 hours in saline, whereas spermatozoa from other species generally survive for longer time periods ${ }^{52}$.

5. The rabbit is usually in estrus if there is sufficient light. Ovulation can be Induced in the rabbit, whilc thc mouse and hamster ovulate sponn ţano ous ly.

6. Normal ovulation in the rabb1t yields an average of 10.2 ova. More can be obtained with superovulation.

7. Fertilized rabbit ova spend about 3 days in the oviduct and implant in the uterus in the morula ( 3 day) stage or blastula ( 4 day) stage. This is also true for rat and mouse ova.

8. The gestation period of the rabbit is 30-32 days; slightly greater than those of the rat, mouse and hamster which are 22,19 and $16-19$ days, respectively.

9. The litter slze of the rabbit averages from 4.0-8.1 offspring, compared to $6.1-11.1$ for the rat and $4.5-7.4$ for the mouse. 
APPENDIX B

Supplemental Information on Work with Germ Cells

Contents

1. Spermatozoa. . . . . . . . . . . . . 328

a. Collection ................ . . 328

b. Cooling. . . . . . . . . . . . . . 329

c. Counting ................. 330

d. Evaluation ................. 331

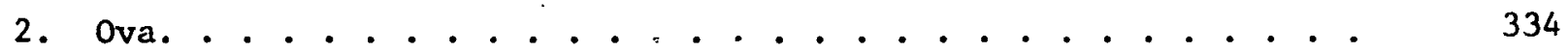

a. Normal Fertility of the Female Rabbit. . . . . . . 334

b. Inducing ovulation ............... . . 337

c. Obtaining ova................. 340

d. Cultivating Ova IN vITRO .............. 340

e. Storage of ova................. 341

f. Transplanting Ova............... 342

3. Fertilization. ................ 343

a. Fertilization IN VIVo. ............. 343

1). Capacitation of Spermatozoa. .......... 344

2) Spermatozoa Transport in the Female. . . . . . 346

3) Penetration of Ova by Spermatozoa. . . . . . . 348

4) Effects of Female Hormones . . . . . . . . . 349

b. Fertilization IN VITRO ................. 349

1) Parthenogenests. . . . . . . . . . . . . 349

2) Example of Procedure Used for IN VITRO Fertilization of Rabbit Ova .............. . 350

4. Artifictal Insemination. . . . . . . . . . . 351

a. Time of Insemination . . . . . . . . . . . 351

b. Place and Number of Spermatozoa. ........... 351 
5. Sexing offspring. . . . . . . . . . . . . . . . . .

a. Gonads. . . . . . . . . . . . . . . . 353

b. Chromosomes .................... 353

c. Sex Chromatin .. . . . . . . . . . . . 354

\section{Spermatozoa}

a. Collection. Designs of artificial vaginas which have proved successful are discussed by Gordon ${ }^{92}$; Walton ${ }^{239}$; Bredderman, et a1 ${ }^{46}$; and Pistenma (Appendix C).

Foote used glycerol as a lubricant in an artificial vagina, and found a large percentage of bull spermatozoa adherent to the rubber surface ${ }^{81}$. Using silicon as a lubricant did not decrease the tendency for adhesion of spermatozoa to the lumen ${ }^{81}$. Polge observed that rabbit spermatozoa did not regain motility if more than approximately $2.5 \%$ glycerol is rapidiy introduced into the semen. ${ }^{188}$. Thus, it is conceivable that liberal lubrication of the lumen of the artificial vagina with glycerol could be detrimental to the sperm. KY jelly and similar sterile lubricants have been used by me with no observable effect on the spermatozoa.

Fox collected rabbit semen between $b: 30$ and $\%: 30$ A.M. when distracting noises and influences were minimal ${ }^{85}$. He evaluated the semen for motility, concentration (rejected if less than $100 \times 10^{6} / \mathrm{ml}$ ) and live sperm, using a differential (live-dead) fast-green FCF and Eosin B stain and rejected the sample if less than $50 \%$ were viable. 85

W1Iliame and Hamner used a blackened Warburg flask for collection, centrifugation and washing spermatozoa ${ }^{243}$. They observed a transient increase in respiration of rabbit spermatozoa with a 5-30 second exposure to white light and observed almost a 4-fold increase in respiratory rate 
after a 3 minute exposure to white 1 ight $(100 \text { watt bulb at } 20 \mathrm{~cm})^{243}$. Norman and Goldberg showed that a wave length of $4400 \mathrm{~A}^{\circ}$ produced the greatest effect which is belleved to be a photosensitized oxidation ${ }^{176}$. Impairment of motility is exponential with exposure as if the light Initiated an autocatalytic reaction. Brief exposure to light initiates a reaction which continues at a slower rate in the dark. The red end of the spectrum had little effect on the spermatozoa ${ }^{176}$.

Brackett and WIlliams observed that the temperature of the artificial vagina used in semen collection changed the effect of light on respiration. Exposure to $48^{\circ} \mathrm{C}$ for 40 seconds reduced motility. Heat stimulated respiration In the absence of light and depressed respiration upon exposure to light later 265 . Walton 239 suggested a temperature of $45^{\circ} \mathrm{C}$ for the artificial vagina. In view of Brackett and W1lliams' observation, I would recommend staying well below $45^{\circ} \mathrm{C}$ in order to avold the possibility of heat damage to spermatozoa. Male rabbits are not very happy if the temperature of the artificial vagina is below $38^{\circ} \mathrm{C}$, so a temperature of $40-42^{\circ} \mathrm{C}$ appears to be a good compromise.

b. Cooling. The loss of viability and motility of spermatozoa after sudden cooling has been deacribed for many mamalian species, including the rabbit ${ }^{92}$ and the bul1 101,109 .

Tanaka and Okamoto have shown that rapid cooling of fowl spermatozoa from $25^{\circ} \mathrm{C}$ to $5^{\circ} \mathrm{C}$ has no effect on fertillty of undiluted and diluted spermatozoa ${ }^{269}$. Rapid cooling of spermatozoa to 0 and $-2^{\circ} \mathrm{C}$ resulted in lowered fertility with undiluted semen, but no change in motility with 4-fold dilution of the semen with Wilcox's buffer. Cooling to $-2^{\circ} \mathrm{C}$ at a rate of $0.5^{\circ} \mathrm{C} /$ minute resulted in no significant decrease in fertility of diluted or undiluted semen 269 . 
Cooling curves determined for $2 \mathrm{ml}$ of buffer in a $15 \mathrm{ml}$ centrifuge tube in the center of 1 and 2 pound coffee cans filled with sawdust and immersed in ice water have shown maximum cooling rates of less than $0.4^{\circ} \mathrm{C} /$ min. Cooling from room temperature $\left(23^{\circ} \mathrm{C}\right)$ to $3^{\circ} \mathrm{C}$ takes approximately 90 and 110 minutes in the 1 and 2 pound coffee cans, respectively. This is a simple and convenient way to cool spermatozoa gradually.

c. Counting. Rothschild 197 and Maude ${ }^{150}$ observed nonrandom distributions of spermatozoa in a Hawksley hemocytometer. Rothschild observed that gravity did not affect the distribution of the spermatozoa and that bull spermatozoa appeared to be attracted to the large glass surfaces of the cover slip and microscope slide ${ }^{197}$. Freund and Carol reported that in a study of the variance in counts by different technicians using a hemocytometer, pipetting accounted for $22 \%$ of the varfance, and handling of the chamber $26 \% 87$. $57.4 \%$ of the variance in hemocytometer technique was due to variance among techniclans, and $42.6 \%$ was due to variance among duplicate hemocytometer determinations by the same technician ${ }^{86}$. The $95 \%$ contidence interval for hemocytometer counts among technicians on a single specimen was $\pm 52 \%^{86,87}$.

Segal and Laurence analyzed human spermatozoa using a Coulter Coint$\mathrm{er}^{209}$. The addition of $1 \mathrm{ml}$ of $1 \%$ saponin/100 $\mathrm{ml}$ of diluted semen to prevent clumping caused Immediate loss of motility of the spermatozoa wh thout an observable change in morphology. Estimates of the spermatozoa concentration, manually, with a hemocytometer, and by counting in the Coulter Counter gave agreement within $25 \%$ in approximately $1 / 2$ of the samples counted. The variance in counts ranged from $25-50 \%$ in approximately $28 \%$ of the samples and was greater than $50 \%$ in approximately $22 \%$ of the samples 209 .

I have found a $1 \%$ solution of formalin in saline to be excellent 
for counting spermatozoa. The solution stops all mot1lity 1mmediately and does not change the morphology of the spermatozoa, so that differential counts of capped and capless spermatozor can be made conveniently.

\section{d. Evaluation.}

(1) Motility. The motility of spermatozoa can be evaluated quantitatively in low concentrations or qualitatively in high concentrations. The quantitative evaluation can be done with elther a light microscope or phase contrast microscope. The degrees of motility of individual spermatozoa are categorized as follows: a) imotile, b) feeble tall-wagging, c) vigorous tal1-wagging, d) slowly progresslve forward motion, and e) vigorous forward motion.

It is customary to evaluate fowl spermatozoa in high concentrations on a scale of I to VI corresponding to essentially no motilfty (Grade I) and a vigorous swarming motion (Grade VI), respectively, in the specimen under low or intermediate power in the microscope.

(2) Phase Contrast Observations. Using phase contrast microscopy, individual spermatozoa in a sample can be categorized as follows: a) immotile spermatozoa: capped (C) and capless (CL); b) tall-wagging (TW); and, c) forward motility (FM). The tail-wagging and forward motion are assessed degrees as noted in sub-paragraph (1) above. All spermatozoa with tall-wagging or forward mot1lity observed by the author were capped. However, the possibility of motile capless spermatozoa is not doubted for motile tails with midpleces but nn heads have been observed.

\section{(3) Nigrosin-Eosin Staining.}

a) Procedure. I used the stain recommended by Hancock which is prepared by dissolving 5 grams of water soluble. Eosin Y (Gurr) in 300 $\mathrm{ml}$ of $10 \%$ aqueous Nigrosin (Gurr) ${ }^{109}$. One drop of spermatozoa suspension is placed in 8 drops of the stain for 5 minutes. It is then smeared on 
a glass slide and allowed to dry in air. Other methods of differential staining of spermatozoa are presented in references $21,58,131$ and 152 . b) Scoring Stained Spermatozoa. Stained slides can be scored by either light microscopy or phase contrast microscopy, but there is a difference in the 2 methods with nigrosin-eosin stained samples. The correlation of the scoring by the 2 methods is discussed in Chapter IX.

With the light microscope; 3 categories of stained spermatozoa are observed as follows: unstalned (U), morphologically normal but stained (MNS) and "stained" (S) spermatozoa. These are 11lustrated in Figure 60 which shows the acrosomal cap present on the unstained and on the MNS spermatozoa. The MNS spermatozoa arc stained only in the post-nuclear cap region, whereas the "stained" spermatozoa have no acrosomal cap and the entire head is stained homogeneously.

Four categories of spermatozoa are observed with phase contrast microscopy as follows: unstained and capped (UC), unstained and capless (UCL), morphologically normal but stained (MNS) and stained and capless (SCL). This categorization differs from the light microscope scoring of the nigrosin-eosin stained spermatozoa by the division of the unstained spermatozoa into capped and capless groups.

(4) Signs of Deterioration or Damage of Spermatozoa. Signs of deterioration of mammalian spermatozoa in order of increasing severity are loss of motflity; loss of the acrosomal cap; bending of the tall, usually at the neck; and complete severance of the tall from the head. The heads of mammallan spermatozoa remained intact despite considerable stress.

The deterioration of fowl spermatozoa is somewhat similar to that of mammalian spermatozoa. However, in addition to the loss of motility and bending of the tall (resembling a halmpin In many instances), the heads 


\section{SPERM STAINING WITH NIGROSIN-EOSIN}

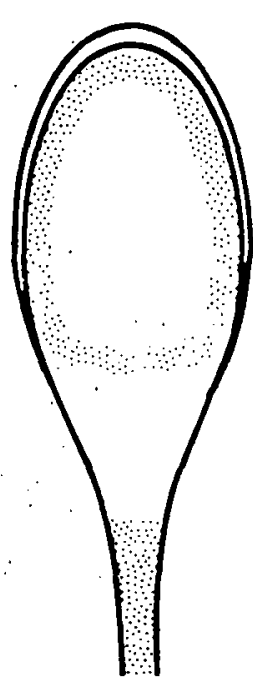

a.

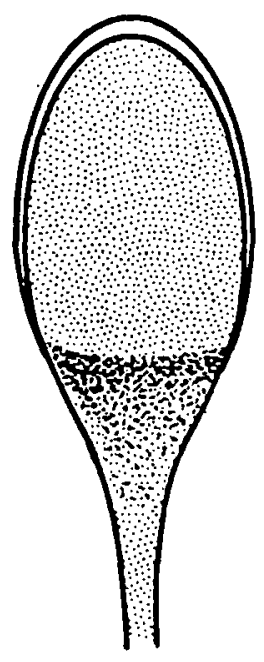

b.

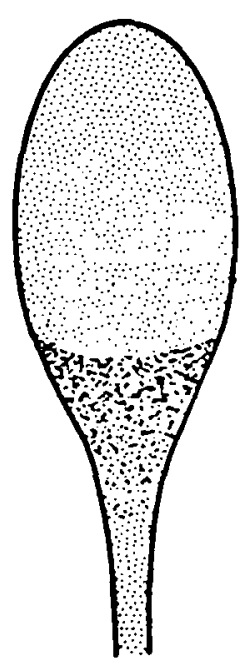

c.

\section{DBL 70115996}

F1g. 60. Schematic diagram of categories of rabbit spermatozoa observed with light microscopy scoring of nigrosin-eosin preparatIons: unstained (U), morphologically normal but stained (MNS) and stained (S). 
of fowl spermatozoa undergo complete disintegration. This change in morphology is characterized Initially by marked swelling of the head to 2-3 times the normal diameter. This is followed by a beady appearance and finally complete disintegration of the head with small beads of chromatin-appearing material scattered in the medium.

(5) Fertilization Capacity. The ultimate test of the spermatozoa of any species is their ablifty to fertilize ova. This is discussed in other sections below.

2. Ova

a. Normal Fertility of the Female Rabbit. Rabbits are practically $100 \%$ fertile when mated $1-4$ days after parturition if the offspring have been removed from the nest. They remain in heat up to 36 days after parturition but the fertility is less than days $1-4$ thereafter ${ }^{105}$. If the young are to be removed from the nest, they should be removed immediately after birth. The slightest delay may allow letting down of milk and lead to caked breasts in the mother.

Rabbits mated at the end of pseudopregnancy (approximately day 20) have a very low percentage of infertile matings, whereas one gets an average of $31 \%$ infertile matings in rabbits which have not been pregnant recently ${ }^{105}$ Foote and coworkers observed that a high proportion of does, even though not pseudopregnant, may refuse to copulate 82 .

The age of the female is important for the following reasons: (1) Females less than 100 days give few offspring ${ }^{170}$. (2) The age of the female at the flrst fertile coltus varies according to season of birth:

Month of Birth

March-April

Oct. -Dec.

\section{Age at First Coitus}

8.5 months

5.2 months

Natural copulation is more likely from April through July, whereas anoestrum 
normally prevalls in late fall and winter ${ }^{105}$. (3) Young does give an average of $55 \%$ normal offspring, whereas 19 month old does give only $36 \%$ normal of spring ${ }^{105}$.

Staples and Holtkamp found a positive correlation between the number of Dutch Belt does with corpora lutea and increasing welght of the females $^{218}$. It is interesting that 1 doe weighing only $1.05 \mathrm{~kg}$ maintained 2 fetuses. They suggested that if the age of Dutch Belt does is unknown, 1t is important to use does that weigh $1.75 \mathrm{~kg}$ or more for reproduction studies 218

There is a positive correlation between the number of eggs released from the left and the right ovaries ${ }^{170}$. The fertilizability of the ovum is greatest up to 4 hours after ovulation and after approximately 8 hours is not fertilizable ${ }^{4}$. This may be related in part to the furmation of a mucin coat around the ovum from 5-14 hours after ovulation if the rabbit is mated normally. Formation of this mucin coat is delayed until about the 17 th hour if ovulation is artificially induced and no mating occurs ${ }^{15}$. Fertilization of aged ova gives a higher mortality rate of the embryos ${ }^{4}$.

Shaver and Carr found a marked decrease in the number of blastocysts recovered when mating of the rabbits took place more than 10 hours after injection of gonadotropin ${ }^{212}$. They found 7 blastocysts with chromosome abnormaifties from mating 6 or more hours after gonadotropin injection and 6 blastocysts with chromosome abnormalities with mating 2 hours or less after injection. A total of 135 blastocysts were examined ${ }^{212}$. Although not specifled, it is believed that the 2 groups had approximately the same number of blastocysts.

Chang and Bedford showed that the cumulus oophorus does not play a part in fertilization but the corona radiata is important in protecting the fertilizability of ova ${ }^{55}$. Dickmann discusses the morphology of the 
zona pellucida of the rabbit ovum ${ }^{66}$. Szollosi and Ris discuss the disappearance of most of the cortical granules found within the plasma membrane after sperm penetration ${ }^{224}$. Austin presents a thorough review of all aspects of the mammalian egg $^{15}$.

Thirty hours after insemination of the doe, eggs should be approachIng the 4-cell stage 170 . Additional information on cleavage rates may be found in reference 15 . The rate of development of fertilized ova is related to the number of eggs fertilized, the number shed and to the age and weight of the female, in that order of importance ${ }^{170}$.

Hafez and Rajakoski have studied the effects of many maternal factors on the growth and survival of blastocysts in the domestic rabbit ${ }^{103}$. The ovulation rate tended to increase with increase in parity. The number of degenerating blastocysts per doe increased in all superovulated groups and the percentage of viable blastocysts among the total number recovered did not vary in normally ovulated groups and in one superovulated group ${ }^{103}$. Hafez showed that salpingectomy, unilateral ovariectomy, subtotal ovariectomy or administration of progesterone in intact rabbits had no significant effect on the viability and size of blastocysts or on the diameter of the embryonic disk 100 .

Maurer, Hunt, Van Vleck and Foote studied the developmental potential of superovulated rabbit ova after transfer to rectpient females. They found that fertilized ova from the donors exhibited a normal appearance at the time of transfer, revealed no subsequent defects or deficiences and were as capable of developing into viable young as ova obtained by routine ovulation 264 .

McGaughey and Daniel have shown that a concentration of $25 \mu \mathrm{g} / \mathrm{ml}$ of estradiol inhibits cleavage of single-cell rabbit eggs IN VITRO while most 
eggs are apparently unaffected by an estradiol concentration of $5 \mu \mathrm{g} / \mathrm{m}{ }^{153}$. Equimolar concentrations of progesterone appear to be antagonistic to the effects of estradiol 153 .

Dukelow showed that the lambing percentages of mated ewes increased by $12.9 \%$ and the number of ewes lambing by $7.6 \%$ after oral administration of extracted wheatgerm oil over 2 breeding seasons ${ }^{69}$. He observed no effect in Guernsey cows given the wheatgerm oil ${ }^{69}$. He did not evaluate its effect on rabbits; however, this is an interesting possibility to improve results of reproductive studies.

b. Inducing Ovulation. Two apparently satisfactory means of inducing ovulation are the use of a vasectomized buck and the use of hormones.

1) Using a Vasectomized Buck. The advantages of using a vasectomized buck are that it simulates natural mating and no injection is needed. In addition, the doe can be mated immediately after parturition without concern about the development of immunity to induction of ovulation by hormones, which is noted below.

The disadvantages of using a vasectomized buck are: (a) The timing and control of the experiment are subject to an expression of love by the rabbits and sometimes as many as $50 \%$ of the does refuse to mate. (b) One must check the vasectomized buck of ten in order to insure that there are no sperm in the ejaculate. Gordon recommends careful checking for the f1rst 3 weeks and periodic checks thereafter (Telecon with Mel, March 11, 1964).

2) Use of Hormones. Because a high percentage of does may refuse to copulate with a vasectomized buck, Foote recommends the use of luteinizing hormone as a means for carrying out an experiment with sound statistical design ${ }^{82}$. However, the use of luteinizing hormone to irduce ovulation is 
reliable only if the female rabbits are maintained in estrus by natural or artificial mating when not being used in experiments. If there is a question as to whether or not a doe is in estrus, Foote has found that a doe that accepts a vasectomized buck will ovulate. Most does that refuse to mate with the vasectomized buck will not ovulate ${ }^{i, 2}$. Ihe use of LH avoids the possibility of inadvertent fertilization by an incompletely vasectomized buck.

Foote, et al., used supplemental lighting to $i$ isure 12 hours of simulated daylight to minimize seasonal effects of undogenous hormone production $^{82}$. $0.5 \mathrm{mg} / \mathrm{kg}$ of pituitary luteinizing hormone (PLH), (Armour), in $0.9 \% \mathrm{NaCl}$, was injected in a marginal ear vein. This was follnwed immediately by artificial insemination of greater than $3 \times 10^{6}$ sperm in a 0.1-0.2 $\mathrm{ml}$ vol. Normal ovulation rates and numbers of offspring were obtained. With injection of a total of 2.5 ing PLH, $91 \%$ of 57 does kindled ${ }^{82}$. Following a second injection, 16 does snowed normal fertility and gave a normal litter size. Up to 4 injections over a 2-year period did not affect fertility ${ }^{82}$.

Even though Foote observed no decrease in fertility with successive injections of luteinizing hormone, there is evidence that repeated injections of LH leads to immunity in the rabbit. Adams observed a failure of does to produce litters with continued use of $\mathrm{LH}$, as shown below:

Fallure of Does to Produce Litters With Continued Use of $\mathrm{LH}^{1}$

\begin{tabular}{lcccccc}
\hline $\begin{array}{l}\text { Attempted number: } \\
\begin{array}{l}\text { Days between } \\
\text { injections: }\end{array}\end{array}$ & 1st & 2nd & 3 rd & 4th & 5 th & 6 th \\
$\begin{array}{l}\text { Females failing to } \\
\text { produce litters }(\%)\end{array}$ & 8 & 87 & 91 & 40 & 37 & 102 \\
\hline
\end{tabular}


Some does were mated naturally at the time of the 5 th injection of luteinizing hormone and all conceived despite immunity to the hormone ${ }^{1}$.

Adams and Chang injected 25 iu of luteinizing hormone (LH) (Lutormone or Follutein), mated does naturally from 5 hours before to 2 hours after ovulation and evaluated results by removing Fallopian tubes and examining ova $^{4}$. They observed from 7 to 15 ovulations (mean of 10.3) per doe and estimated that ovulation occurred 9.5 to 13 hours after LH injection. With mating at 8 hours after LH injection, $85 \%$ of the does conceived and $74 \%$ of all eggs (including the eggs in does having no fertilized eggs) were fertilized ${ }^{4}$.

Adams injected 20 iu of Pregny1 (Organon Laboratories) intravenously and Inseminated $20 \times 10^{6}$ fresh spermatozoa in $0.5 \mathrm{ml}$ from 3 hours before to 6 hours after injection of the hormone ${ }^{1}$. $83.6 \%$ of the does kindled with an average litter size of 7.3 , which is normal ${ }^{1}$.

In order to Induce superovulation, Foote, et al, injected $0.2 \mathrm{mg}$ FSH (Armour) subcutaneously twice daily for 5 days prior to injecting PLH the morning of the 6 th day ${ }^{82}$. Napier gave $2 \mathrm{mg}$ horse anterior pituitary extract for 5 days subcutaneously and, on the 6 th morning, he inseminated spermatozoa and injected 25 iu of chorionic gonadotropin 170 . The results at 30 hours showed the mean number of eggs shed and fertilized to be 32.6 and 21.8 per doe, respectively. 15 of 72 does gave no fertilized cggs. He obtalned $75 \%$ and $88 \%$ conception rates with homospermic and heterospermir. Inseminations, respectively ${ }^{170}$.

Mauer, Hunt and Foote studied the development of refractoriness to Induction of ovulation by exogenous hormones in Dutch-Belted rabbits using brief administration of FSH-LH or PMSG-HCG at three 16-week intervals followed by one 8-week interval ${ }^{151}$. LH infections were used as a control. The number of ovulation points per doe decreased with both regimens but 
the refractoriness was most pronounced in the PMSG-HCG group $^{151}$.

c. Obtaining Ova. Most workers usually remove the oviducts and uteri, and flush with Krebs-Ringer bicarbonate solution 2-3 hours after ovulation 15 Chang has a good discussion of this procedure ${ }^{53}$. Moricard discusses removal of ova but does not say that the animal is alive ${ }^{163}$. Avis and Sawin used a surgical technique in order to use the does over again. $81 \%$ of ova they transplanted gave normal offspring ${ }^{16}$. This is a feasible technique for getting blastocysts.

In larger anfmals, such as the cow, a multiple lumina tube can be used to flush the eggs from the uterus.

d. Culturing Ova IN VITTÓ. Gwatkin and Biggers used mouse Fallopian tubes to culture mouse eggs through the morula stage ( 3 rd day) and blastocyst stage (4th day) 97 . Biggers, Gwatkin and Brinster showed that development of blastocysts IN VITRO occurs in the normal time span. 39

Chang used diluted blood serum to study the maturation of rabbit ova IN VITRO ${ }^{53}$. Biggers, Gwatkin and Brinster found that mouse embryos developed for 4 days IN VITRO in a chemically defined medium containing Fallopian tubes, but did not develop in the chemically defined medium alone ${ }^{39}$. The presence of the ovary had no influence on the development of the embryos $^{39}$. Daniel and olson found that the first cleavage of the fertilized rabbit ovum will occur in an amino acid-free medium, but that the second and subsequent cleavages require the addition of certain amino acids 59 . Ahlgren transferred fertilized tubal rabbit eggs to millipore filter chambers and compared the effect of emplacement for 48 hours in the abdominal cavity or in the uterus ${ }^{5}$. Development was significantly retarded in the abdominal cavity. He suggests that a tubal factor is necessary. for the eggs to develop 5 . 
e. Storage of Ova. Several different media are discussed by Hafez in conjunction with work primarily related to storage of 2-16 blastomere stage ova at $10^{\circ} \mathrm{C}^{99,101,102}$. Some observations were that: (1) Serum was indispensible for survival of ova and whether the serum was autologous or heterologous did not change the implantation or survival rates 101,102 . (2) $\mathrm{CO}_{2}$ did not improve the survival rate of ova ${ }^{102}$. (3) The survival of ova was not affected when stored in media having antibiotics in the following concentrations or lower: streptomycin, $7.5 \mathrm{mg} / \mathrm{ml}$; chloromycetin, $4 \mathrm{mg} / \mathrm{ml}$; paramomycin, $6.5 \mathrm{mg} / \mathrm{ml}$ or penicillin G, $23.9 \mathrm{mg} / \mathrm{ml}$. However, at higher concentrations, the survival was partially or completely inhibited 99 .

With a $1: 1$ serum-sallne basic solution, Hafez found that $1 \%$ and $7 \%$ gelatine were better than $5 \%$ gelatine 102 and that $2 \%$ glycerol proved more helpful than $7 \%$ glycerol $^{101}$. The gelatine medium was better for storage than plain liquid and he recomends addition of $1 \%$ gelatine even though embryo survival is about the same for gelled or liquid media ${ }^{102}$. The implantation rate is higher for gelled than for liquid media ${ }^{102}$.

The importance of temperature control in the survival of ova is 1llustrated by the following: (1) Unfertilized ova are more resistant to freezing and thawing than are fertilized ova ${ }^{53}$. (2) slow cooling of ova in a yolk-buffered medium is the best method of preventing temperature shock $^{101}$. (3) Slow warming of stored ova is harmful as shown by an $8 \%$ implantation rate for ova warmed slowly after storage for 144 hours at $10^{\circ} \mathrm{C}$. In contrast, a rate of $31 \%$ was achieved with fast warming (insertion directly into recipient female) ${ }^{102}$.

Hafez discusses changes observed in fertilized ova during storage in references 101 and 102. Some of the observed abnormalities are belleved 
secondary to faulty fertilization or aging of gametes ${ }^{102}$. The mucin coat on fertilized rabbit eggs may enhance their survival IN VITRO. Most other mammalian ova of interest do not have this coat ${ }^{102}$. The implantation rate of abnormal eggs determined at 8 days is simflar to that of normal ova; however, the survival rate at 15 days is lower ${ }^{101}$. Cleavage is arrested In rabbit ova stored at $25^{\circ} \mathrm{C}$ but at $37-39^{\circ} \mathrm{C}$ one sees $2-16$ cell ova develop to the blastocyst stage ${ }^{101}$. Other observations on abnormal development of ova are found in reference 99.

It appears that the ability of stored ova to implant IN VIVO cannot be measured accurately by their ability to cleave IN VITRO. This may be due to the requirement for correlation between endometrial growth and ovum development at the time of transplantation ${ }^{102}$.

f. Transplanting Ova. Chang showed that there is little or no chance for a transplanted ovum or blastocyst to develop into young when transferred to the genital tract of the recipient female 1 or 2 . days before or after the corresponding corpus luteal stage 50 . Staples has shown that the viäbilitey of 5-day rảbile blastocyses was thaintained during culture IN VITRO for at least 16 hours, as judged by development of viable fetuses after subsequent transfer to recipient females ${ }^{218}$. There was no significant difference in the percentage of viable fetuses developing from 5-day blastocysts cultured for at least 8 hours at $37^{\circ} \mathrm{C}$ compared to the development of noncultured blastocysts 218 .

Pavlok has reported some improvements in the fertilization IN VITRO, cultivation and transplantation of mouse ova ${ }^{184^{\prime}}$. In some cases, normal embryos were obtained after transplantation ${ }^{184}$. Hunter, Polge and Rowson achieved moderate success in transplanting 7 and 8 -day-old pig blastocysts to unmated recipients 118 . 


\section{Fertilization}

a. Fertilization IN V.IVO. In natural mating, ovulation is induced in the rabbit by the act of copulation and occurs from 9.5 to 13 hours thereafter. The time of ovulation after the injection of luteinizing hormone is approximately the same. Fertile mating requires coordination of the capacitation of the spermatozoa, the maintaining of fertilizability of the ovum and spermatozoa, and the transport of an adequate number of spermatozoa to the site of fertilization ${ }^{15}$. Spermatozoa capacitation, transport of spermatozoa in the female rabbit and penetration of ova by spermatozoa are discussed below. Reference 15 contains an excellent discussion of the mammalian egg and the details of oogenests.

Roche, Dziuk and Lodge evaluated the effectiveness of fertilization of rabbit eggs by fresh and aged spermatozoa ${ }^{195}$. Spermatozoa aged by storage for 24 hours at $5^{\circ} \mathrm{C}$ in a diluent composed of $0.5 \%$ fructose and $2.9 \%$ sodium citrate in distilled water fertilized a significantly smaller proportion of eggs than fresh spermatozoa. However, the decreased effectiveness of aged spermatozoa, in competition with fresh spermatozoa, does not appear to be due to a lack of capacitation, transport or fertilizing capacity, or to embryonic losses ${ }^{195}$.

Dzuik, by double mating does to 2 different bucks which were distinguishable from each other, showed that when the interval between serving the does to the bucks was 2 hours, the first buck sired $86 \%$ of the offspring 72 When the interval between males was shortened, the first male sired the majority of the offspring, but the proportion was less than after a 2-hour interval. Thus, it appears that spermatozoa which have resided in the genital tract of the doe for up to 10 hours have a distinct competitive advantage over newly inseminated spermatozoa ${ }^{72}$. 
1) Capacitation of Spermatozoa. Spermatozoa must be in the genital tract of the female rabbit for approximately 6 hours before they are capable of fertilizing eggs ${ }^{4}$. Estimates range from 3-6 hours for the time it takes rabbit sperm to reach the site of fertilization ${ }^{4,247}$. This slowness of transport compared to other species may be related to capacitation ${ }^{4}$.

Capacitation in the uterus is more efficient than in the Fallopian tube. This was shown by a higher fertilization rate from deposition of semen into the uterus 10 hours before ovulation than from deposition in the Fallopian tube at the same time ${ }^{3}$. Noyes, Walton and Adams have shown that spermatozoa can be capacitated in the spayed female, in the intact male and in organs unrelated to the genital system ${ }^{177}$. A $41 \%$ fertilization rate was achieved with spermatozoa capacitated in an excised rabbit uterus maintained in a saline medium. Small numbers of sperm $(2,000-10,000)$ were Inseminated surgically and the evaluation of capacitation was judged by fertilization of ova IN VIVO ${ }^{177}$.

Chang showed that seminal plasma is beneficial to both motility and fertility as a medium for artificial insemination ${ }^{49}$. However, after capacitation of spermatozoa in the female genital tract, seminal plasma is detrimental both to motility and fertilizing capacity ${ }^{49}$. Dukelow, Chernoff and Williams showed that seminal plasma from the bull, boar, stallion, rabbit and monkey all contained a decapacitation factor, but human, rooster and dog seminal plasma did not contain this factor ${ }^{70}$. The authors suggested that the presence, or absence, of decapacitation factor in the seminal plasma is indicative of the need for or lack of capacitation in that species. They also discussed the properties of the decapacitation factor ${ }^{70}$.

Hamner and Williams showed that rabbit spermatozoa respire at a greater rate in oviduct fluid than in calcium-free Krebs-Ringer phosphate solution ${ }^{108}$. 
They suggested that the stimulating factor was bicarbonate and that $1 t$ stimulated maximally at a concentration of $2.36 \times 10^{-3} \mathrm{M}$. It appears to stimulate respiration by increasing the concentration of TCA cycle intermediates $^{108}$. $0_{2}$ uptake by sperm increased 4 times after incubation in a rabbit uterus and gave a fertilization rate of $18 \%$ compared to $0 \%$ for freshly ejaculated sperm ${ }^{107}$. Murdoch and White showed that the metabolism of labelled glucose by rabbit spermatozoa after incubation in the uterus was significantly higher than the rate of metabolism by fresh spermatozoa 167 . The time required for the development of those metabolic changes was similar to that required for the capacitation of rabbit spermatozoa ${ }^{167}$.

Howarth, Alliston and Ulberg found no significant difference in fertilization capacity of spermatozoa recovered from females maintained at $21^{\circ}$ and $32^{\circ} \mathrm{C}$ during $6-8$ hour capacitation period in the uterus ${ }^{116}$. There was a significant reduction, however, in pre-implantation embryo survival as a result of uterine environment on sperm ${ }^{116}$.

Dukelow and Williams showed that spermatozoa incubated for 12 hours in the uterus and inseminated into the rabbit oviduct 4 hours before ovulation gave a fertilization rate of $4.5 \%$ compared to a rate of $77 \%$ when the spermatozoa had been incubated in the uterus for 16 hours and inseminated at the time of ovulation 71 . This observation and others, using different time factors, suggest that the oviduct has a detrimental effect upon capacitated spermatozoa.

Bedford and Shalkovsky evaluated the species-specificity of rabbit sperm capacitation using the cat, ferret, guinea pig and rat ${ }^{31}$. Spermatozoa did not survive for more than 12 hours in the cat, ferret and guinea pig, and spermatozoa removed from these species prior to that time did not fertilize any rabbit eggs when deposited in the oviducts soon after ovila-, tion. Rabbit spermatozoa survived up to 48 hours in the uterus of estrous 
rats, and spermatozoa which had been in the uterus of the rat for more than 14 hours fertilized a significant proportion of tubal rabbit ova. Although some phases of capacitation appear to occur in the uterus of the rat, it appears that functional capacitation involves another phase which Is species specific and possibly may be restricted to the female tract of the rabbit ${ }^{31}$.

It appears that capacitation has a two-fold effect: (1) alteration of the acrosome, releasing hyaluronidase and thus enabling the spermatozoon to pass through the cumulus and (2) the removal of the acrosome, thus exposing, the perforatorlum, which is apparently the organelle directly concerned in sperm penetration of the zona pellucida ${ }^{14,15}$. The rate of capacitation may vary under different environmental conditions, indicating the probability of a chemlcal or physicochemical process which need not be acrosome removal 3,107 . The correlation between the acrosome removal and capacitation is questioned because the decapacitation by seminal plasma does not replace the acrosome ${ }^{3}$.

2) Epermatozon Transport in the Female. Spernatozoa seldom reach the oviduct of the rabb1t in less than 1 hour after mating and 5-6 hours may be required before sufficient spermatozoa enter the oviducts of all rabbits to fertilize all of the eggs shed ${ }^{247}$. The mechanism of transport of spermatozoa in the female is attributed to muscular contractions of the vagina and uterus and/or to the intrinsic motility of the spermatozoa.

Krehbiel and Carstens reported the transfer of methylene. blue, janus green $B$ and iodochlorol through the reproductive systems of estrous rabbits after artificial stimulation of the vulva to simulate the copulatory reflex (erection of vulvar tissue) $^{128}$. The dyes reached the tubal end of the uterine horns 2-5 minutes after the initiation of stimulation of the vulva which resulted in combined muscular contractions of the vagina and uterus. 
It was necessary to place fluids beyond the vaginal sphincter. In the absence of a response to vulvar stimulation, no oil passed into the uterine horns 128 .

Noyes, Adams and Walton placed radiopaque oil in the vaginas of rabbits and, even though they observed vaginal contraction upon vulvar stimulation, they did not observe filling of the uterine horns with ofl at any time ${ }^{178}$. They even experienced difficulty filling the uterine horns by means of a catheter placed within the uterine lumina. After vaginal insemination of a mixture of $90 \%$ dead and $10 \%$ motile spermatozoa, an increasing number of spermatozoa in the uterine horns over a period of 4 hours was noted when insemination preceded mating with a vasectomized buck. The percentage of motile spermatozoa decreased from $90-100 \%$ in the first hour to $75-85 \%$ at the end of 4 hours. When mating preceded artificial insemination, the percentage of motile spermatozoa in the uterine horns ranged from $67-91 \%$. They concluded that living spermatozoa passed through the cervical barrier as a result of intrinsic motility and that the point at which the rate of progress is first influenced by muscular contractions of the female genital tract remains to be identified ${ }^{178}$.

Moghissi and coworkers studied the relationship between the motility of spermatozoa and migration through the mucus of the human cervix ${ }^{161}$. In summary, their observations were as follows: (1) Spermatozoa penetrate clear, clean, watery, mid-cycle mucus readily and rapidly. (2) With increasing viscosity of the cervical mucus, the degree of sperm penetration was found to decrease. (3) Acid mucus immobilizes spermatozoa and alkaline mucus enhances migration. (4) Incompatible proteins in mucus may cause death or agglutination of sperm. (5) Proteolysis of cervical mucus by seminal plasma enzymes may play a role in sperm migration. They suggest that an IN VITRO test of the compatibility of mucus with sperm and seminal 
plasma may provide clues in infertility problems ${ }^{161}$.

3) Penetration of Ova by Spermatozoa. Observations on sperm penetration are provided by Szollosi and Ris for the rat 224 , by Yanagimachi for the hamster ${ }^{245}$, and by Hunter and Dzuik for the pig ${ }^{119}$. Sperm penetration, the immediate reactions of the mamalian egg upon penetration and the cytology of fertilization are dicussed in reference 14 .

Austin reports that the corona radiata cells need not be off to get sperm penetration ${ }^{14}$. Kabbit eggs IN VITRO generally cannot be denuded by treatment with sperm suspension or hyaluronidase solution alone, suggestIng that a uterine secretion complements the hyaluronidase released by the : sperm ${ }^{14}$. The importance of the corona radiata in egg fextilizability is shown by the fertilization of about $85 \%$ of ova with an intact corona radiata but only about $50 \%$ without the corona radiata from 2-4 hours after ovulation 55 . At 6 hours after ovulation, the percentages were $60 \%$ and $12 \%$, respectively ${ }^{55}$.

Iickmann describes 2 distinct concentric layers in the zona pellucida of the rabbit ovrum ${ }^{6 h}$. The "outer zona" appears granular and the "inner zona" appears fairly homogeneous. The width of the "outer zona" varies relative to the thickness of the "inner zona". Sometimes one can distingulsh 2 or more concentric layers in the "inner zona". Radial striations are also seen in this layer on occasion ${ }^{66}$. It has been postulated that the zona pellucida of the mammalian egg secretes a substance ("fertilizin") which has the primary purpose of enhancing attachment of the spermatozoon to the zona pellucida, thereby aiding penetration" 14 . This "fertilizin" also causes agglutination of sperm IN VITRO but not IN VIVO where it may be neutralized by a secretion of the female genital tract ("antagglutin") 14 For IN VITRO fertilization, it is necessary to wash freshly ovulated eggs 
before introducing the sperm ${ }^{14}$. Soupart has shown that the acquisition of the ability to penetrate the zona pellucida is associated with a shortening of the 11 fe span and fertilizing ability of rabbit spermatozoa 217 .

It is estimated that the fertilizing spermatozoon takes $1 / 2$ hour to cross the peri-vitelline space ${ }^{14}$. With fertilization IN VIVO, the rabbit egg usually shows several spermatozoa in the peri-vitelline space ${ }^{14}$. However, Adams placed as many as $17.6 \times 10^{6}$ sperm at the site of fertilization and got normal fertilization, thereby leading to the belief that a normal egg can prevent polyspermy ${ }^{3}$. The percentage of eggs fertilized is not proportional to the sperm concentration ${ }^{14}$.

4) Effects of Female Hormones. McGaughey and Danlel have observed that estrogens cause tubal locking IN VIVO and also cause fragmentation of fertilized ova IN VITRO ${ }^{153}$. One-stage cells are most susceptible to fragmentation by this treatment. Subsequent stages of development are less susceptible as the number of cells increases ${ }^{153}$. Psychoyos has shown that estrogen is necessary for the dissolution of the zona pellucida in the rat $^{190}$. His results suggest also that delayed implantation is not related to the persistence of the zona pellucida ${ }^{190}$.

b. Fertilization IN VITRO. The main difficulty, in effecting fertilization IN VITRO is the inftiation (penetration) which appears to involve the capacitation of the spermatozoa and the removal or neutralization of egg fertilizin $^{14}$. Difficulties in confirming fertlization IN VITRO arise from technical errors, misinterpretation of artifacts and accidental induction of parthenogenesis.

1) Parthenogenesis. Reportedly, during culture IN VITRO, rabbit eggs can be activated by heat shock and by treatment with hypo- and hypertonic solutions and solutions of butyric actd ${ }^{14}$. Upon sperm penetration, one sees 
the contraction of the vitellus, the expulston of the second polar body, the formation of 2 pronuclei and the Initiation of cleavage. With a few exceptions, the expulsion of the second polar body occurs only with activation by sperm entry. When activation occurs by other means, the second polar body is not expelled and the cells resulting from division are diploid ${ }^{14}$. Incubation of ova in the presence of spermatozoa increases the chance of activation without sperm penetration, whereas incubation of unfertilized ova with Falloplan tube mucosa reduces the incidence of parthenogenesis ${ }^{4}$. Austin suggests that incomplete vasectomy may account for some reports of parthenogenesis ${ }^{4}$.

2) Example of Procedure used for IN VITRO Fertilization of Rabbit Ova. A procedure used with some success is as follows: ${ }^{31}$

(a) Eggs were obtained from the Fallopian tube and placed in a capacitated sperm suspension in a flask and incubated for 3-4 hours at $38^{\circ} \mathrm{C}$ on a rocking device.

(b) Eggs were removed after 3-4 hours and transferred to an $8 \mathrm{~m} 1$ Gatel flusk cuilululng frugh lumologous gerum which earlier had been heated to $55^{\circ} \mathrm{C}$ for 20 minutes.

(c) Following an 18 -hour incubation period at $38^{\circ} \mathrm{C}$ in the Carrel. flasks, the eggs were removed and examined.

(d) Of 266 eggs recovered, 55 appeared to have cleaved to the 4-cell stage, 36 of the 55 fertilized eggs were transferred tn 6 females immediately after examination. Four females gave 15 living young;

2 females did not become pregnant.

(e) Capacitation of sperm was the most important factor; $0_{2}$ partial pressure; the suspending medium and the redox potential appeared to be less important ${ }^{31}$.

In the last few years, investigators have been capacitating spermatozoa 
in the female rabbit for fertilization IN VITRO 30,221 . One method for recovering spermatozoa is to flush the uterine horns with Krebs-Ringer bicarbonate solution giving a recovery rate of about $10 \%{ }^{15}$. Using spermatozoa capacitated in this manner, Bedford and Chang achieved an IN VITRO fertilization rate of $54 \%$ compared to a normal rate of $94 \%$.

\section{Artifictal Insemination}

The technique of artificlal insemination in the rabbit is discussed by Adams ${ }^{1,2}$. The advantages and disadvantages of using a vasectomized buck and hormones to induce ovulation were discussed in Section 2.b. above.

a. Time of Insemination. Ovulation occurs 9.5-13 hours after LH injection and 10-12 hours after mating with a vasertomized buck, The fertilizability of the ovum is greatest the first 4 hours after ovilation. It takes 6 hours in the female genital tract for the sperm to be capacitated and sperm penetration stops about 6-9 hours after ovulation when the ovum becomes coated with a mucin layer ${ }^{65}$. Fox inseminated 15-30 minutes after using a vasectomized buck to stimulate ovulation ${ }^{84}$. Using $L H$ and natural mating as a function of time after infection, Adams observed that $90-100 \%$ of the does yielded fertilized eggs when mated 8-12 hours after LH injection and that no fertilization of eggs occurred when mating took place 14 hours after LH injection ${ }^{4}$. Thus, in the rabbit, successful inseminations can be made from several hours before inducing ovulation to 8-12 hours thereafter.

b. P1ace and Number of Spermatozoa. Most workers deposit spermatozoa at the anterior end of the vagina. Because the rabbit has a primltive reproductive tract (a separate cervix to each horn of the uterus) and it Is easy to inject sperm into the abdominal cavity rather than the uterine horn when trying to inseminate directly into the latter, 1t appears best to use the conventional procedure ${ }^{105}$. With regard to sperm concentrations 
required in artificial insemination, Foote, et al, got good results by depositing $3 \times 10^{6}$ motile sperm (in 0.1 or $0.2 \mathrm{ml}$, either undiluted or diluted with a few drops of $0.9 \%$ saline) in the anterior part of the vagina ${ }^{82}$. Napier inseminated $10 \times 10^{6}$ sperm in $0.5 \mathrm{~m} 1$ of $0.85 \%$ saline solution $^{170}$. Adams obtained good results with 0.5 n:1 inseminate containing $20 \times 10^{6}$ sperm ${ }^{1}$. Both Napier and Adams placed the sperm in the anterior part of the vagina.

Adams and Chang compared the effects of depositing spermatozoa either in the uterus or in the Falloplan tube ${ }^{3}$. They assessed fertilization by examining ova recovered from excised Fallopian tubes. They inseminated at 3 different times after inducing ovulation with $30 \mathrm{mg}$ of $\mathrm{LH}$ (Follutein, Squib). The results were as follows:

Effect of Site of Deposition of Spermatozoa ${ }^{3}$

\begin{tabular}{|c|c|c|c|}
\hline $\begin{array}{l}\text { Time after } \\
\text { LH Injection } \\
\text { (hours) }\end{array}$ & $\begin{array}{c}\text { Sperm } \\
\text { Inseminaţed } \\
\times 10^{6}\end{array}$ & $\begin{array}{c}\text { Ova } \\
\text { Uterine } \\
\text { Insemination } \\
(\%)\end{array}$ & $\begin{array}{c}\text { Fallopian Tube } \\
\text { Insemination } \\
(\%)\end{array}$ \\
\hline $3 / 4$ & $0.1 .2-.8$ & 88.5 & 27.3 \\
\hline 6 & 0.8 & 100 & 16.7 \\
\hline$\therefore 8$ & $0.33-0.65$ & 71 & 10.0 \\
\hline
\end{tabular}

The same low percentage fertilization with Insemination Into the Fallopian tube was observed with higher sperm concentrations, thereby demonstrating that capacitation takes longer in the tubes ${ }^{3}$.

\section{Sexing Offspring}

Methods of determining the sex of offepring in mamals include examination of the external genitalia, gonads, chromosomes or sex chromatin at appropriate stages of development. Bhattacharya describes a method of 
determining the sex of rabbits from the external genitalia at birth without sacrificing them ${ }^{35}$. In order to get feedback on the success of experiments with rabbits, it is desirable to determine the sex of offspring prior to the end of the 31-day gestation period. Thus, determining the sex by studying elther the sex chromatin or chromosomes as soon as possible after conception with accuracy and without loss of ova or offspring is a method of reducing this watting period.

a. Gonads. Bhattacharya found the gonads of fetal rabbits after 18 days could be sexed by staining techniques ${ }^{35}$. Gonads can be fixed, sectioned and stained, either by a perlodic acid-Schiff-hematoxylin technique 222 , or by Delafield's hematoxylin and counterstained with eosin 92 .

Lush reported that the sex of rabbits at birth could be determined by visual examination of the gonads ${ }^{143}$. Neonatal testes could be distinguished by a very conspicuous epididymis wrapped around one end of the testis and the testis was usually attached to a very thick gubernaculum. The neonatal ovary was generally attached to a less conspicuous ligament and had no structure resembling the epididymis. The location of the gonad within the abdominal cavity did not correlate very well with the sex of the offspring ${ }^{143}$.

Comparison of the 2 methods by me showed $100 \%$ agreement in the sex of 51 offspring determined by evaluation of gross morphology and by microscopic examination of stained sections of the gonads.

b. Chromosomes. Vickers describes a method for the direct measurement of the sex ratio in mouse blastocysts by looking at chromosomes of the cells of the blastocysts which have been arrested in the metaphase stage of mitosis with colchicine 234 .

Two methods which might be of assistance in gaining access to the cello of rabbit blastncysts have been reported. Edwards describes a method 
for removing the zona pellucida of 1 - and 2 -celled rabbit eggs IN VITRO 74 Mastrolanni and Ehteshamzadeh discuss the role of fluid from rabbit oviducts in loosening the cells of the corona radiata of recently ovulated ova, pretreated with hyaluronidase ${ }^{148}$.

c. Sex Chromatin. Vickers has found sex chromatin in $67-90 \%$ of ectodermal cell nuclei in the female mouse amnion and in 2-15\% of such cells in the male 235 . She considers that criterion a reliable indicator of the sex of the fetus from the eleventh day of gestation onward ${ }^{235}$.

Gardner and Edwards have been able to sex intact, living rabbit blastocysts by identifying sex chromatin in trophoblast cells approximately 5.75 days after mating 89 .

The methods described by Vickers and by Gardner and Edwards would make avallable information on the sex ratio of offspring 3 weeks earlier than waiting for the full gestation period to elapse. 
APPENDIX C

\section{A New Artificial Vagina for Collecting}

\section{Rabbit Spermatozoa}

The versatility of Teflon has been extended from facilitating the frying of eggs in the kitchen to simplifying the collection of spermatozoa with a Teflon artificial vagina. After the rabbit was selected as the animal to be used in this study of the biophysical properties of spermatozoa, the designs of artificial vaginas used by Gordon ${ }^{92}$ and by Walton $^{239}$ were reviewed. With the assistance of Mr. John Begovitch, the artificial vagina shown in Figure 61 was constructed. A recent communication by Bredderman, Foote and Yassen ${ }^{46}$ presents a number of considerations in the design and use of an artificial vagina for collecting rabbit semen.

The salient features of this artificial vagina are the Teflon shell connected to a removable base by a watertight junction. The base has inlet and outlet water condults and an 0-ring lined hole in its center, which holds a one dram shell vial (15 × $45 \mathrm{~mm}$, No. 60930-L, Owens, Illinois, U.S.A.). The closed end of a tissue finger cot (Latex, 610 Large, Bitner Brand, U.S.A.) is inclsed and secured around the mouth of the one dram vial with masking tape. The open end of the finger cot is secured to the opening of the artificial vagina with a rubber band as shown in the photograph. The space around the rubber lumen and upper part of the shell vial is filled with water at a temperature of $40-42^{\circ} \mathrm{C}$. Filling via one of the conduits connected by rubber tubing to a faucet facilitates control of both temperature and pressure in the shell. If desired, the rubber lumen and vial can be washed and autoclaved prior to insertion into the artificial vagina. The rubber lumen and vial are rinsed with sterile saline after assembly. Glycerol, KY Jelly, or another acceptable lubricant is spread over the mouth and into the artificial vagina with a small thermometer 


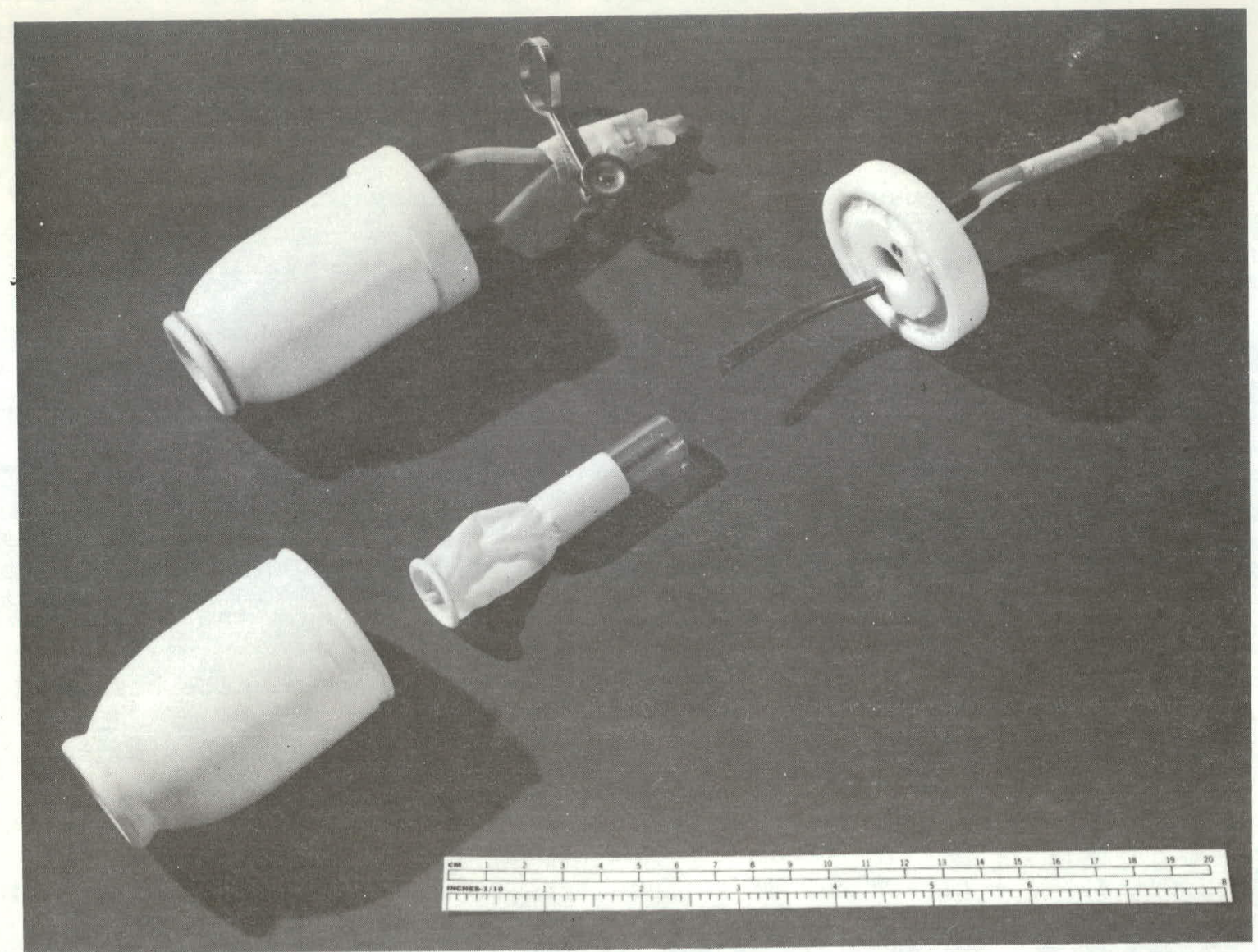

JHL -4935

Fig. 61. Photograph of two Teflon artificial vaginas showing one assembled apparatus and the component parts of another. 
which is also used to measure the temperature in the lumen.

Because Teflon is a good insulator, the temperature change in the artificial vagina at room temperature is $0.5^{\circ} \mathrm{C} / \mathrm{min}$ for the first 8 minutes. This rate of cooling is compatible with collecting spermatozoa some distance from the source of warm water if necessary.

Among the advantages of this artificial vagina are the following: (1) Ejaculation is close to the mouth of the shell vial reducing the loss of spermatozoa by adhesion to the lumen. In addition, the lumen can be washed with a small volume of diluent to enhance the recovery of spermatozoa. (2) Because the vial is kept at approximately the same temperature as the water in the chamber, the spermatozoa are not subjected to cold shock during collection. (3) Between collections, the Teflon shell can be washed with alcohol or hot water to minlmize the chance of passing infection from one rabbit to another. (4) The shell vials and tissue finger cots are inexpensive and can be discarded after using once. (5) Many lumens (vial + finger cot) can be prepared in advance, thereby facilitating the collectIon of large numbers of ejaculates consecutively. Using this artificial vagina, one individual can collect ejaculates from rabbits at a rate of one every 5 minutes or less without assistance. 
APPEND IX D

Evaluation of Media

Contents

1. Introduction. ...................

2. Results and Discussion. . . . . . . . . . . . . .

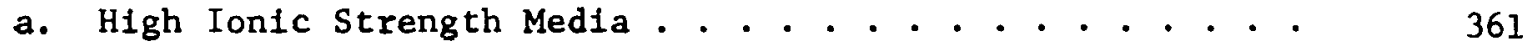

(1). Egg Yolk Medium (EYM). . . . . . . . . . . . 361

(2) Modified Baker's Medium (MBM). . . . . . . . . 363

(3) Norman-Johnson Solutions (NJ-1, NJ-2). . . . . . 364

(4) Vitalizing Medium. . . . . . . . . . . . 364

b. "Low" Lonic Strength Media. . . . . . . . . . . . . 364

(1) Glycine-Ring $\mathrm{z}^{\mathrm{d}}$ Solution . . . . . . . . . . 364

(2) 5\% Dextrose in Distilled Water . . . . . . . 365

(3) Glycine-Citrate Buffer (GCB) .......... 365

(4) Glyclne-Phosphate Buffer (GPB) . . . . . . . . 365

(5) Lower Ionic Strength Glycine-Phosphate Butters . . . 365

c. Density Gradient Materials. .. . . . . . . . . . 366

(1) Staflo Density Gradient Materials. . . . . . . 366

(2) Equilibrium Centrifugation Density Gradient Materials : 367

\section{Introduction}

The ability to preserve the fertllizing capacily of spermaluzud for long pertods of time has been of great benefit in the field of mammallan reproduction and genetics. In poultry husbandry, it is a much desired, but at present relatively unavallable, tool. Consequently, the voluminous literature on the subject attests to the tremendous effort that has been devoted to the evaluation of media for the long-term storage of both mammalian and fowl spermatozoa. 
In regard to mammalian spermatozoa, both natural and artificial media have been evaluated. Napier showed that adding seminal fluid to the suspending medium enhanced the preservation of rabbit spermatozoa. He obtained better fertilization results with a medium consisting of $50 \%$ saline and $50 \%$ plasma than with saline alone ${ }^{169}$. Even though blood serum usually is an excellent medium for the IN VITRO culture of many animal cells, Including rabbit ova, Chang found a spermocidal factor in fresh human, bovine, rabbit, guinea pig and rat sera ${ }^{52}$. In addition, Bedford observed a great tendency for agglutination of rabbit spermatozoa in media containing serum 27 .

Gulyas reported excellent maintenance of motility of rabbit spermatozoa at room temperature for up to 6 days using Norman-Johnson Solution No. 2, a completely artificial medium. During this period, the percentage of live spermatozoa decreased from $89 \%$ to $69 \%$, the percentage of motility from $91 \%$ to $72 \%$ and the quality of motility from 4.9 to 1.8 on a scale of $5^{95}$.

Foote and coworkers found that sperm survival in all buffers was Improved by the addition of egg yolk ${ }^{83}$. Bhattacharya describes an egg yulk-ylycine-citrate medium that matntained the fertilizing capacity of rabbit spermatozoa at temperatures near $0^{\circ} \mathrm{C}$ for over 24 hours. He put the spermatozoa in a glucose vitalizing medium at room temperature for approximately 2 hours prior to insemination ${ }^{35}$. Other reports on the use of additives include those by Davis, Bratton and Foote concerning the successful use of tris-buffered and citrate-buffered yolk-glycerol media for the preservation of bovine spermatozoa 60,61 , by Sawada and Chang on the use of dimethyl sulfoxide in the storage of rabbit spermatozoa at temperatures below $0^{\circ} \mathrm{C}^{203}$, and by Roussel and Austin on the preservation of primate spermatozoa during storage in an egg yolk-sodium glutamate- 
glycero]. medium at or near $-79^{\circ} \mathrm{C}^{199}$.

Another common class of additives is antibiotics even though Sharma found only a slight benefit with them ${ }^{211}$. Adams reports that adding 1,000 untes/ml of penicillin to a diluent consisting of physiologic saline or Ringer's solution is not detrimental with sperm dilutions up to $1: 10^{1}$. However, a high concentration of antibiotics is harmful to the survival of spermatozoa ${ }^{233}$. Bhannasiri, Bogart and Krueger studied the effects of antiblotics on semen production in rabbits and concluded that any effects noted during the treatment of the disease were probably due to the disease and not to the antibiotics. They evaluated oxytetracycline (Terramycin), Chlortetracycline, Aureomycin and streptomycin ${ }^{34}$.

In regard to the storage of high concentrations of fowl spermatozoa, Rowell and Cooper observed reductions in fertilizing capacity from $61.3 \%$ to $32.1 \%$ and from $31.1 \%$ to $12.5 \%$ with the insemination of equal volumes of undiluted and 1:3 dilutions of fowl spermatozoa in seminal plasma and in a solution of glycine and distilled water, respectively ${ }^{200}$. Van Wambeke observed almost no loss of fertilizing capacity of fowl spermatozoa diluted with equal volumes of either of 2 diluents containing skimmed milk and stored at $2-5^{\circ} \mathrm{C}$ for 24 hours. Fertilization results were based upon eggs collected from days $2-8$ after insemlnation ${ }^{231}$. Polge diluted cock semen 1:3 with either of 2 diluents contalning egg white or milk and observed average fertility rates of $89 \%$ and $53 \%$ after storage at $5^{\circ} \mathrm{C}$ for 1 and 24 hours, respectively, based upon eggs lald from days 2-7 following insemina$\operatorname{tion}^{140}$.

Lorenz, in his review, mentions work by others at room temperature showing that (1) 1:3 dilution with seminal plasma gave a slight decrease in fertility but no additional loss occurred with dilution of up to $1: 63$ 
provided that the same number of spermatozoa was inseminated; (2) dilution of up to $1: 50$ in an artifictal medium resulted in only a slight loss of fertility. with insemination of at least $10^{8}$ spermatozoa; poor results were obtained with greater dilutions or with insemination of fewer spermatozoa and (3) 1:1,250 dilution in saline or In Tyrode's or Baker's solutions produced immediate irreversible immobilization ${ }^{140}$.

Recent studies have analyzed the fluids secreted in the female genital tract in fowl and epididymal fluid in mammals ${ }^{238}$, organ systems in which the motility and fertilizing capacity of spermatozoa are preserved for long periods of time naturally.

The purpose of this appendix is to consolidate all of my observations on the evaluation of media where the information is of a supplemental nature. In addition, this appendix is a directory to the presentation of additional information on, or the evaluation of, media when it is an integral part of the experiments presented in the maln body. Requirements peculiar to special experimental systems are discussed in the appropriate parts of the text.

\section{Results and Discussion}

a. "High" Ionic Strength Media.

(1) Egg Yolk Medium (EYM). This medium is described in Chapter VI A in confunction with an evaluation of the preservation of the fertilizing capacity of fowl spermatozoa by "high" fonic strength media.

In regard to the preservation of rabbit spermatozoa in EYM, there was essentially no $10 s 8$ of motillty after storage for 48 hours at $2^{\circ} \mathrm{C}$ in concentrations ranging from $1.5 \times 10^{6}-88 \times 10^{6} / \mathrm{ml}$ in one study. The results of another study using an egg yolk and glycine-citrate medium in $25 \%-75 \%$ proportions, respectively, are shown in Table XXXIX. It is seen (Column 6) that there was no change in the quality of the spermatozoa after storage 
Table XXXIX. Preservazion of Morility of Rabbit Spermatozoa in Mcdified Baker's Medium (MBM), Egg Yolk Medium (EYM) and Varying Proportions of the Two Media a $=2^{\circ} \mathrm{C}$.

\begin{tabular}{|c|c|c|c|c|c|}
\hline$(1 ;$ & (2) & (3) & (4) & (5) & $(6)$ \\
\hline $\begin{array}{c}\text { Storage } \\
\text { Time } \\
\text { (hours) }\end{array}$ & $.100 \% \mathrm{MBM}$ & $90 \%$ MBM - 10\% EYM & $75 \% \mathrm{MBM}-25 \% \mathrm{EYM}$ & $50 \%$ MBM $-52 \%$ EYM & $100 \%$ EYM \\
\hline 2 & $\begin{array}{l}\text { 15\% FM, 70\% } \mathrm{xW} \\
15 \% \text { 1mmotile }\end{array}$ & $>75 \%$ FM & $>75 \% \mathrm{FM}$ & $>75 \% \mathrm{FM}$ & $>75 \%$ FM \\
\hline 17 & $0 \% \mathrm{FM}, 2-3 \%=\mathrm{W}$ & $\begin{array}{l}14 \% \text { FM, B0\% TN } \\
26 \% \text { immotile }\end{array}$ & $\begin{array}{l}25 \% \mathrm{FM}, 35 \% \mathrm{Tw} . \\
40 \% \text { immotile }\end{array}$ & $\begin{array}{l}40 \% \text { FM, } 30 \% \mathrm{TW} \\
\text { Bad Clumping }\end{array}$ & $>75 \%$ FM \\
\hline 40 & $0 \% \mathrm{FM}, 0 \% \mathrm{TW}$ & $2-3 \% \mathrm{Tw}$ & $2-3 \% \mathrm{FM}, 5-6 \% \mathrm{TW}$ & $2-3 \%$ FM, $5-6 \%$ TW & $\begin{array}{l}\sim 50 \% \text { FM of } \\
\text { high degree }\end{array}$ \\
\hline
\end{tabular}

a.25\% Egg Yolk - 75\% Glycine-Citrate Medium by Vol-ume. 
for 17 hours and only a slight change after 40 hours. The difference between these results and those of the previous study suggests that $40 \%$ egg yolk is better than $25 \% \mathrm{egg}$ yolk in the egg yolk-glycine-citrate medium.

Despite this excellent preservation of motility, the EYM has many undesirable features which militate against its use in the Staflo apparatus. They are: (1) It is an opaque medium and observations are impossible. (2) It coats the entire apparatus with an opaque film and makes cleaning difficult. (3) It precipitates with almost every medium mentioned in this report except the glycine-citrate buffer. (4) The method of making an optically clear egg yolk medium described by Rikmenspoel 194 involves a tremendous amount of work and is not justified for routine Staflo experiments. (5) The use of EYM for the initial cooling requires additional centrifugation (washing) to get the spermatozua out of EYM before starting the experiment.

The EYM has a very important use after Staflo or centrifugation experiments. If the spermatozoa are reconcentrated and suspended in EYM, they can be left for many hours before insemination, thereby allowing greater flexibility in the conduct of the experiment and artificial insemination thereafter.

(2) Modifled Baker's Medium (MBM). This modification of Baker's medium consists of $22.0 \mathrm{~g} \mathrm{Na}_{2} \mathrm{HPO}_{4} \cdot 12 \mathrm{H}_{2} 0,2.0 \mathrm{~g} \mathrm{NaCl}, 2.0 \mathrm{~g} \mathrm{NaHCO}$, and $0.155 \mathrm{~g}$ $\mathrm{KH}_{2} \mathrm{PO}_{4}$ diluted to $1,000 \mathrm{ml}$ with distilled water. $\mathrm{pH}$ adjustments are made with concentrated HCl.

Table XXXIX compares the effectiveness of modified Baker's medium, $25 \%$ egg yolk-glycine-citrate medium and mixtures of these 2 media in maintaining the motility of spermatozoa over extended time periods at $2^{\circ} \mathrm{C}$ and. at a concentration of $6 \times 10^{6} / \mathrm{ml}$. After 2 hours at $2^{\circ} \mathrm{C}$ in $\mathrm{MBM}$, an aliquot was warmed to room temperature and evaluated. The marked decrease in motility 
was much greater than observed in the "low" Ionic strength glycine-citrate and glycine-phosphate buffers as reported in Chapter VI. As shown in Table XXXIX, the addition of a small amount of EYM to the MBM greatly enhanced the preservation of motility. No difference was observed among the media containing egg yolk at 2 hours; however, at 17 hours, the spermatozoa stored in the $100 \%$ egg yolk-glycine-citrate medium had a much higher percentage and degree of mot1lity than those in any of the mixtures of MBM and EYM.

(3) Norman-Johnson Solutions (NJ-1, NJ-2). Evaluation of NormanJohnson Solutions 1 and $2(N \mathrm{~J}-1,2)^{95}$ showed that they were not so good for preserving motility at room temperature as the egg yolk-glycinecitrate medium. Both NJ-1 and NJ-2 solutions were inferior to the egg yolk-glycine-citrate and to the "low" Ionic strength glycine-phosphate buffer for preservation of spermatozoan motility at temperatures of $2-5^{\circ} \mathrm{C}$.

(4) Vitalizing Medium. This medium is described and evaluated in Chapter VI in regard to use with fowl spermatozoa.

\section{b. "Low" Ionic Strength Media.}

(1) Glycine-Ring $\mathrm{z}^{\mathrm{d}}$ Solution. The glycine-Ring $\mathrm{z}^{\mathrm{d}}$ solution used by Schröder 206 and Gordon 92 was evaluated for use in Staflo-electrophoresis experiments. Equivalent portions of the same ejaculates were diluted simultaneously in normal saline and in glycine-Ring $\mathrm{z}^{\mathrm{d}}$ solution at room temperature. Aflei 30 minutes, spermatozoa in the saline solution had an average of $45 \%$ motility and in the glycine-Ring $\mathrm{z}^{\mathrm{d}}$ solution, on $1 \mathrm{y} 1.5 \%$ motility. Using a nigrosin-eosin vital staining technique, the percentage of viable spermatozoa agreed with the observed motility in the saline solution. The percentage of viable spermatozoa in the glycine-Ring $z^{d}$ solution was approximately $1 / 3$ that in the saline solution, but was still higher than the percentage of motility noted above. It should be noted 
that because the spermatozoa sample had a fairly low percentage of motility initially, it could be expected to deteriorate more rapidly than a good specimen.

(2) $5 \%$ Dextrose in Dist1lled Water. $5 \%$ dextrose and glycine-Ring $\mathrm{z}^{\mathrm{d}}$ solutions were compared at room temperature in regard to the preservation of motility using a normal saline control. Whereas there was essentially no change after 50 minutes in the control sample from the initial values of $50 \%$ forward motion and $65 \%$ viability (nigrosin-eosin stain), all spermatozoa were immotile in less than 15 minutes in the glycine-Ring $\mathrm{z}^{\mathrm{d}}$ solution. After 20 minutes in $5 \%$ dextrose, there was only $30 \%$ forward motion. After 22 minutes, the percentages of viable (unstained) spermatozoa in the glycine-Ring $\mathrm{z}^{\mathrm{d}}$ and $5 \%$ dextrose solutions were $21 \%$ and $29 \%$, respectively. Thus, neither of these 2 "low" lonic strength media preserved motility as well as normal saline at room temperature.

(3) Glycine-Citrate Buffer (GCB). This "low" lonic strength buffer is evaluated in Chapter VI A.

(4) Glycine-Phosphate Buffer (GPB). The properties of this "low" ionic strength buffer are evaluated in Chapter $V$ and its ability to preserve the motility and fertilizing capacity of fowl and rabbit spermatozoa are discussed in Chapters VI and VIII, respectively.

The addition of 1 part of egg yolk medium to 9 parts by volume of the "low" Ionlc strength glycine-phosphate buffer (GPB) preserved a high degree of motillty for 24 hours at $2^{\circ} \mathrm{C}$ at spermatozoa concentrations ranging from $10^{5}-10^{6} / \mathrm{ml}$. After storage at such low concentrations in GPB alone for several hours, the spermatozoa would have lost all motility.

(5) Lower Ionle Strength Glycine-Phosphate Buffers. Fowl spermatozoa of high Initial motility were used to evaluate the effect of varying 
the amount of modified Baker's medium (MBM) mixed with an isotonic glycinedextrose buffer. The spermatozoa were diluted 1:10 in duplicate vials containing the following percentages of $\mathrm{MBM}: 80 \%, 10 \%, 6 \%, 3 \%$ and $0 \%$. After storage for 6 and $1 / 2$ hours at $2{ }^{\circ} \mathrm{C}$ in these media, the spermatozoa were warmed to room temperatures for evaluation. The spermatozoa in the medlum containing $80 \%$ MBM had very poor motility, while those in the soluttons containing $10 \%$ and $6 \%$ MBM had both a high percentage and degree of motility, comparable to their initial values. The spermatozoa in the $3 \%$ and $0 \%$ MBM solutions may have had a slightly lower percentage of motile spermatozoa but the degree of mot1lity was as great as in the $6 \%$ and $10 \%$ solutions. In conclusion, the preservation of the motility of fowl spermatozoa was excellent in solutions containing from $0-10 \% \mathrm{MBM}$ atter $61 / 2$ hours at $2^{\circ} \mathrm{C}$. In contrast, the preservation of motility in the $80 \%$ MBM solutions was poor.

\section{c. Density Gradient Matertals.}

(1) Staflo Density Gradient Materials. Dextrose, fructose and sucrose were evaluated for use as staflo density gradient constituents by comparing the preservation of motflity in $1 \%$ solutions of each sugar in normal saline at concentrations of $8 \times 10^{6}$ and $80 \times 10^{6}$ spermatozoa/ml. After 4 hours at room temperature, there was no difference in motility among the spermatozoa at high concentration in all 3 solutions. At the lower concentration, the degree of motility was greater in the fructose solution than in either of the other solutions.

Dextran (MW 73,000) was also evaluated for use in Staflo density gradients. In one study, with an initial sample having greater than $50 \%$ forward motion and $65 \%$ viabllity by vital staining, there. was no difference In the motility of spermatozoa stored in saline or saline $+6 \%$ dextran after 50 minutes. In Chapter IV, 1t was shown that a high percentage and 
degree of motility is preserved in Staflo sedinentation experiments using a glycine-dextran medium. As shown in Chapter.VI, $2 \%$ dextran did not affect the fertilizing capac1ty of fowl spermatozoa.

\section{(2) Equilibrium Centrifugation Dens1ty Gradient Materials. Among the} materials evaluated for use in equilibrium density gradient centrifugation of spermatozoa were dextran (Sigma Chemical Company), Ficoll, bovine serum albumin (BSA), and the radiographic contrast materials listed in Table XL. The high viscosity of large welght \% solutions of dextran, Ficoll and bovine serum albumin is an important disadvantage in the use of those materials. Radiographic contrast materials can be used to prepare solutions of high specific gravity and low viscosity. Another advantage of the contrast media is that when used in a clear solution such as saline or phosphate buffer, they add no color, thereby enhancing observation and photography. The disadvantage of these compounds is the hyperosmolarity of high concentrations due to their small size compared to dextran, Ficoll and BSA. The knowledge that the contrast media could be used in humans without deleterlous effects gave encouragement for their use with spermatozoa.

The Initial evaluation of the effect of contrast media on spermatozoa consisted of exposure of rabbit spermatozoa to $4.5 \%$ Diodrast in egg yolk medium for approximately 4 hours, 2 hours of which were at $4^{\circ} \mathrm{C}$. After this period, 5 female rabbits were inseminated with approximately $15 \times 10^{6}$ sperm each. These inseminations resulted in 4 litters with 22 offspring. There was no change in the motility or staining characteristics of the spermatozoa after 6 hours in the egg yolk-Diodrast medium.

A longer term study of the effect of Conray 400 was conducted by exposing spermatozoa to $7.5 \%$ and $3.5 \%$ Conray 400 in a $25 \%$ egg yolk-glycine- 
Table XI. Radiographic Contrast Solutions Evaluated for Use as Density Gradient Constituents in Equilibrium Centrifugation Studies of Spermatozoa.

\begin{tabular}{|c|c|c|c|c|}
\hline Brand Name & Chemical Name & 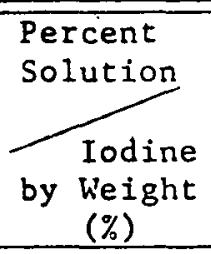 & $\begin{array}{l}\text { Specific } \\
\text { Gravity }\end{array}$ & $\begin{array}{l}\text { Molecular } \\
\text { Weight }\end{array}$ \\
\hline Angio-Conray & $\begin{array}{l}\text { Na 5-acetamido- } 2,4,6- \\
\text { triiodo-N-methyl1so- } \\
\text { phthalam1c ac1d }\end{array}$ & 59.07 & 1.508 & 614 \\
\hline Conray -400 & $"$ & 66. & 1.422 & 614 \\
\hline $\begin{array}{l}\text { Cholografin } \\
\text { meglumine }\end{array}$ & $\begin{array}{l}\text { bis } N \text {-meglumine salt } \\
\text { of } N, N \text { adipyl-bis } \\
\text { (3-amino-2,4,6-trii- } \\
\text { odobenzoic acid) }\end{array}$ & 52 & & 1552 \\
\hline Diodrast & $\begin{array}{l}\text { 3,5-dilodo 4-pyridone } \\
\text { N-acetic acid } \\
\text { dicthanolamino }\end{array}$ & 49.8 & & 305 \\
\hline Hypaque & $\begin{array}{l}\mathrm{Na} 3,5 \text {-diacetamido- } \\
2,4,6 \text {-trilodobenzoate }\end{array}$ & 50 & & 636 \\
\hline $\begin{array}{l}\text { Renografin } \\
60 \text { or } 76\end{array}$ & $\begin{array}{l}\text { Methylglucamine 3,5- } \\
\text { diacetamido-2,4,5- } \\
\text { triiodobenzoic acid }\end{array}$ & $\underbrace{60}_{47}$ or 76 & & 797 \\
\hline Cardiografin & $"$ & 85 & & 797 \\
\hline
\end{tabular}


citrate medium at $2^{\circ} \mathrm{C}$. Table XII summarizes these results. It is seen that $3.5 \%$ Conray 400 in the egg yolk medium had only a slight effect on the survival of spermatozoa over a period of 48 hours. The inftial quality of the spermatozoa used in this evaluation was relatively poor. However, because poor spermatozoa generally tolerate stress less well than high quality spermatozoa, the observation that poor spermatiozoa tolerated exposure to low concentrations of Conray 400 for extended periods of time was encouraging.

The effect of high concentrations of contrast media on fowl spermatozoa was evaluated as follows: $0.1 \mathrm{ml}$ of pooled cock semen, which had been diluted and cooled in egg yolk medium to a temperature of $3^{\circ} \mathrm{C}$, was placed in each of four $1 \mathrm{ml}$ vials containing 30\% Angio-Conray, 45\% Cholografin, $30 \%$ Diodrast or $40 \%$ Renografin, all in normal saline. At $3^{\circ} \mathrm{C}$, the spermatozoa had a moderate degree of motillty in the egg yolk medium but lost this motility in less than 2 minutes 1 all of the media except Cholografin, in which the motility was lost within 5 minutes. After 10 minutes, at $3^{\circ} \mathrm{C}$, the $1.1 \mathrm{ml}$ suspension was placed in $5 \mathrm{ml}$ of a saline-glucose medium and centrifuged to reconcentrate the spermatozoa. The supernatant was removed and the spermatozoa were resuspended in egg yolk medium at $3^{\circ} \mathrm{C}$. All of the spermatozoa which had been exposed to the contrast media showed a percentage and degree of motility that was comparable to that in an egg yolk medium control sample. This suggested that there was no detrimental effect on the motility of fowl spermatozoa for exposures of up to 32 minutes in any of the contrast media. The fertilizing capacity of the spermatozoa was not evaluated in this study.

These studles showed that the motility of fowl spermatozoa is maintained during exposure to high concentrations of radiographic contrast materials for 30 minutes or less and that the fertilizing capacity of rabbit sperma- 
Table XLI. Effect of Sodium Iothalamate (SI) in Egg Yolk Medium (EYM) on the Motility of Rabbit Spermatozoa.

\begin{tabular}{|c|c|c|c|c|c|}
\hline $\begin{array}{c}\text { Storage } \\
\text { Time (Hours) }\end{array}$ & $\begin{array}{l}\text { Egg Yolk } \\
\text { Mediuma }\end{array}$ & $\begin{array}{l}3.5 \% \text { in } \\
\text { in }\end{array}$ & $\begin{array}{l}\text { SI } \\
\text { EYM }\end{array}$ & $\begin{array}{l}7.0 \% \\
1 \mathrm{n}\end{array}$ & $\begin{array}{l}\text { SI } \\
\text { EYM }\end{array}$ \\
\hline
\end{tabular}

Sample $\# 1$

$\begin{array}{llll}48 & \text { Fair FM, High } \% \text { TW } & \text { Same as } 25 \% \text { Egg Yolk } & \text { Few } \% \text { FM and TW } \\ 68 . & 30-40 \% \text { FM, 30\% TW } & 5 \% \text { FM, } 20 \% \text { TW } & 1 \% \text { TW } \\ 144 & 3 \% \text { FM, } \sim 50 \% \text { TW } & 2 \% \text { FM, } 20 \% \text { TW } & 1 \text { motile sperm }\end{array}$

Sample \# 2

$\begin{array}{llcc}28 & 20 \% \mathrm{FM}, 50 \% \text { TW } & 5 \% \mathrm{FM}, 10 \% \text { TW } & 5 \% \mathrm{FM}, 5 \% \mathrm{TW} \\ 96 & 2-3 \% \mathrm{FM}, 50 \% \text { TW } & 2 \% \mathrm{FM}, 15 \% \text { TW } & <1 \% \text { TW }\end{array}$

a.25\% Egg Yolk, 75\% glycine-citrate medium by volume. 
tozoa is preserved after exposure to low concentrations of Diodrast

for at least 4 hours. Thus, these materials can be used in either Staflo or equilibrium centrifugation density gradients. 


\section{APPENDIX E}

Expressions for Minimum, Maximum and Average Migration Distances for Particles in Staflo Collection Fractions

The expressions presented below were derived by using Figures 13 and 14 for Staflo sedimentation and sedimentation-downward-electrophoresis (SDE) experiments and Flgures 20 and 21 for Staflo sedimentation-upward electrophoresis (SUE) experiments. The expressions could be shortened. However, this would ellminate the ability to compare parts of the expressions with the respective parts of the range and distribution diagrams from which they were derived. As the equations are written, the migration distances are given in millimeters.

The restriction that the bottom of the sample stream at the inlet colncide with the bottom of that layer, as discussed in the text, results in restrictions in the use of the equations for sedimentation and SDE experiments when the top of the migration pattern remains in the inlet layer. From Figures 13 and 14 , it can be seen that 1 n Lhis case, there is only one range of values for the minimum migration distance, usiug $x$ ! In Cases 3 and 4.

In the expressions, $n$ is the number of layers from and including the inlet layer to the layer of exit for the particles that have migrated the greatest distance.

1. Two-Layer Migration Pattern in Sedimentation and SedimentationDownward-Electrophorests Experiments.

a. Possible Distributions:

$$
\begin{aligned}
\text { Case 1: } & ([n-3] \times 1.25+h)<x<(n-2) \times 1.25 ; \\
& (n-2) \times 1.25<y<([n-2] \times 1.25+h) \\
\text { Case 2: } & ([n-3] \times 1.25+h)<x<(n-2) \times 1.25 ; \\
& ([n-2] \times 1.25+h)<y^{\prime}<(n-1) \times 1.25
\end{aligned}
$$




$$
\begin{aligned}
& \text { Case 3: }(n-2) \times 1.25<x^{\prime}<y \\
& x^{\prime}<y<([n-2] \times 1.25+h) \\
& \text { Case 4: } \quad(n-2) \times 1.25<x^{\prime}<([n-2] \times 1.25+h) ; \\
& \quad([n-2] \times 1.25+h)<y^{\prime}<(n-1) \times 1.25
\end{aligned}
$$

b. Restrictions: For $\mathrm{n}=2$ or $\mathrm{n}=3$ use Cases 3 and 4 only.

c. Case 1:

$$
\begin{aligned}
& \boldsymbol{\lambda}_{(\mathrm{n}-1)_{\min }}=\mathrm{x} \operatorname{mm}(\mathrm{n}-1)_{\max }=\mathrm{y} \mathrm{mm} \\
& \lambda_{n_{m 1 n}}=(n-2) \times 1.25 \mathrm{~mm} \quad \lambda_{h} \text { max }=y \mathrm{~mm} \\
& \lambda_{(n-1)} \text { ave }=\frac{h([n-2] \times 1.25-x)([n-2] \times 1.25+x)}{2 h([n-2] \times 1.25-x)} x \\
& \frac{+(y-[n-2] \times 1.25)([n-2] \times 1.25+2 h-y)}{+(y-[n-2] \times 1.25)} \times \\
& \frac{\frac{1}{3}(y+[n-1] \times 1.25)}{\left([n-2] \times 1.25+2 \frac{h-y}{2}\right) \mathrm{mm}} \\
& \boldsymbol{\lambda}_{\mathrm{N}_{\text {ave }}}=(\mathrm{n}-2) \times 1.25+\frac{2}{3}(\mathrm{y}-[\mathrm{n}-2] \times 1.25) \mathrm{mm}
\end{aligned}
$$

d. Case 2:

$$
\begin{aligned}
& \boldsymbol{\lambda}_{(\mathrm{n}-1)_{\min }}=\mathrm{x} \mathrm{mm} . \quad \boldsymbol{\lambda}_{(\mathrm{n}-1)_{\max }}=(\mathrm{n}-2) \times 1.25+\mathrm{hmm} \\
& \boldsymbol{\lambda}_{\mathrm{n}_{\mathrm{min}}}=(\mathrm{n}-2) \times 1.25 \mathrm{~mm} \quad \boldsymbol{\lambda}_{\mathrm{n}_{\mathrm{max}}}=\mathrm{y}^{\prime} \mathrm{mm} \\
& \lambda_{(n-1)} \text { ave }=\frac{([n-2] \times 1.25-x)([n-2] \times 1.25+x)}{2([n-2] \times 1.25-x)} \times \\
& \frac{+h\left([n-2] \times 1.25+\frac{h}{3}\right)}{+h} \mathrm{~mm} \\
& \lambda_{n_{\text {ave }}}=\frac{h\left([n-2] \times 1.25+\frac{2 h}{3}\right.}{h} \times \\
& \frac{+\left(y^{\prime}-[n-2] \times 1.25-h\right)\left(y^{\prime}+[n-2] 1.25+h\right)}{+2\left(y^{\prime}-[n-2] \times 1.25-h\right)} \mathrm{mm}
\end{aligned}
$$

e. Case 3:

$$
\begin{array}{ll}
\lambda_{(n-1)_{\min }=x^{\prime} \operatorname{mm}} & \lambda_{(n-1)_{\max }}=y \mathrm{~mm} \\
\lambda_{n_{\text {min }}=x^{\prime \text { mm }}} & \lambda_{n_{\max }}=y \text { mm } \\
\lambda_{(n-1)_{\text {ave }}=x^{\prime}+\frac{1}{3}\left(y-x^{\prime}\right) \mathrm{mm}} &
\end{array}
$$




$$
\lambda_{n_{\text {ave }}}=x^{\prime}+\frac{2}{3}\left(y-x^{\prime}\right) \text { mm }
$$

f. Case 4:

$$
\begin{aligned}
& \lambda_{(n-1)_{\min }}=x^{\prime} \mathrm{mm} \quad \lambda_{(n-1)_{\max }}=(n-2) \times 1.25+h \mathrm{~mm} \\
& \boldsymbol{\lambda}_{n_{\min }}=x^{\prime} \text { mm } \quad \lambda_{n_{\max }}=y^{\prime} \text { mm } \\
& \lambda_{(n-1)} \text { ave }=x^{\prime}+\frac{1}{3}\left([n-2] \times 1.25+h-x^{\prime}\right) \mathrm{mm} \\
& \lambda_{n_{\text {ave }}}=\frac{\left(h+[n-2] \times 1.25-x^{\prime}\right)\left(h-[n-2] \times 1.25+x^{\prime}\right)}{\left(h+[n-2] \times 1.25-x^{\prime}\right)} \times \\
& \frac{\left(x^{\prime}+\frac{2}{3}\left[h+(n-2) \times 1.25-x^{\prime}\right]\right)}{\left(h-[n-2] \times 1.25+x^{\prime}\right)} x \\
& \frac{+h\left(y^{\prime}-[n-2] \times 1.25-h\right)\left(y^{\prime}+[n-2] \times 1.25+h\right)}{+2 h\left(y^{\prime}-[n-2] \times 1.25-h\right)} \mathrm{mm}
\end{aligned}
$$

2. Three-Layer Migration Pattern in Sedimentation and Sedimentation-

Downward-Electrophoresis Experiments.

a. Possible Distributions:

$$
\begin{aligned}
\text { Case 1: } & ([n-4] \times 1.25+h)<x<(n-3) \times 1.25 \\
& (n-2) \times 1.25<y<([n-2] \times 1.25+h) \\
\text { Case 2: } & ([n-4] \times 1.25+h)<x<(n-3) \times 1.25 \\
& (n-2) \times 1.25+h)<y^{\prime}<(n-1) \times 1.25 \\
\text { Case 3: } & \left([n-3] \times 1.25<x^{\prime}<([n-3] \times 1.25+h)\right. \\
& (n-2) \times 1.25<y<([n-2] \times 1.25+h) \\
\text { Case 4: } & \left([n-3] \times 1.25<x^{\prime}<([n-3] \times 1.25+h)\right. \\
& ([n-2] \times 1.25+h)<y^{\prime}<(n-1) \times 1.25
\end{aligned}
$$

b. Restrictions: For $n=3$, use Cases 3 and 4 only.

c. Case 1:

$$
\begin{aligned}
& \lambda_{(n-2)_{\min }}=x_{-}{ }_{\text {um }} \\
& \lambda_{(n-1)_{\min }}=(n-3) \times 1.25 \mathrm{~mm} \\
& \lambda_{(\mathrm{n}-2)_{\max }}=(\mathrm{n}-3) \times 1.25+\mathrm{h} \text {.mm } \\
& \lambda_{n_{\min }}=(1-2) \times 1.25 \mathrm{~mm} \\
& \lambda_{(n-1)_{\max }}=y \cdot \operatorname{mm} \\
& \lambda_{(n-2)}=\frac{([n-3] \times 1.25-x)([n-3] \times 1.25+x)}{2([n-3] \times 1.25-x)([n-3] \times 1.25+x)} \times \\
& \frac{+h\left([n-3] \times 1.25+\frac{h}{3}\right)}{+h} \mathrm{~mm}
\end{aligned}
$$




$$
\begin{aligned}
& \lambda_{(n-1)}=\frac{h^{2}\left([n-3] \times 1.25+\frac{2 h}{3}\right)}{h^{2}} \times \\
& \frac{+h(1.25-h)([2 n-5] \times 1.25+h)}{+2 h(1.25-h)} \times x \\
& \frac{t(y-[n-2] \times 1.25) \times([n-2] \times 1.25+2 h-y)}{+(y-[n-2] \times 1.25)} \times \\
& \frac{\left([n-2] \times 1.25+\frac{1}{3}(y-[n-2] \times 1.25)\right.}{([n-2] \times 1.25+2 h-y)} \mathrm{mm} \\
& \lambda_{n_{\text {ave }}}=(n-2) \times 1.25+\frac{2}{3}(y-[n-2] \times 1.25) \mathrm{mm}
\end{aligned}
$$

d. Case 2:

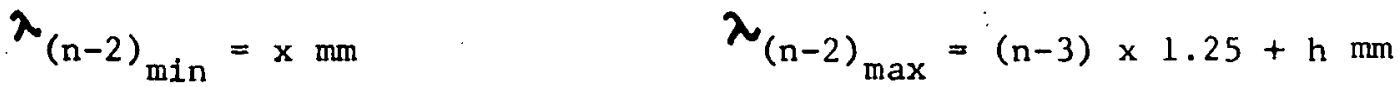

$$
\begin{aligned}
& \boldsymbol{\lambda}_{(\mathrm{n}-1)_{\min }}=(\mathrm{n}-3) \times 1.25 \mathrm{~mm} \quad \boldsymbol{\lambda}_{(\mathrm{n}-1)_{\max }}=(\mathrm{n}-2) \times 1.25+\mathrm{hm} \\
& \lambda_{\mathrm{n}_{\min }}=(\mathrm{n}-2) \times 1.25 \mathrm{~mm} \quad \boldsymbol{\lambda}_{\mathrm{n}}=y_{\max }^{\prime} \\
& \lambda_{(n-2)} \text { ave }=\frac{([n-3] \times 1.25-x)([n-3] \times 1.25+x)}{2([n-3] \times 1.25-x)([n-3] \times 1.25+x)} \times \\
& \frac{+h\left([n-3] \times 1.25+\frac{h}{3}\right.}{t h} \mathrm{~mm} \\
& \lambda_{(n-1)}=\frac{1}{2}([2 n-5] \times 1.25+h) \mathrm{mm} \\
& \lambda_{\text {ave }}=\frac{h\left([n-2] \times 1.25+\frac{2 h}{3}\right)}{2 y^{\prime}} x \\
& \frac{+\left(y^{\prime}-[n-2] \times 1.25-h\right)\left(y^{\prime}+[n-2] \times 1.25-h\right)}{-2(n-2) \times 1.25-h} \mathrm{~mm}
\end{aligned}
$$

e. Case 3:

$$
\begin{aligned}
& \lambda_{(n-2)_{\min }}=x^{\prime} \text { mm } \\
& \lambda_{(\mathrm{n}-2)_{\max }}=(\mathrm{n}-3) \times 1.25+\mathrm{h} \operatorname{mm} \\
& \lambda_{(\mathrm{n}-1)_{\min }}=x^{\prime} \operatorname{mg} \\
& \lambda_{(n-1)_{\max }}=y \mathrm{~mm} \\
& \lambda_{\mathrm{in}}-(\mathrm{n}-2) \times 1.25 \min _{\mathrm{m}} \\
& \lambda_{n_{\max }}-y \text { um } \\
& \lambda_{(n-2)} \text { ave }=x^{\prime} \times \frac{1}{3}\left([n-3] \times 1.25+h-x^{\prime}\right) \mathrm{mm} \\
& \lambda_{(n-1)}=\frac{\left(h+[n-3] \times 1.25-x^{\prime}\right)\left(h-[n-3] \times 1.25+x^{\prime}\right)}{\left(h+[n-3] \times 1.25-x^{\prime}\right)} \times \\
& \frac{\left(x^{\prime}+\frac{2}{3}\left([n-3] \times 1.25+h-x^{\prime}\right)\right)}{\left(h-[n-3] \times 1.25+x^{\prime}\right.} \times \text { (continued) }
\end{aligned}
$$




$$
\begin{aligned}
& \frac{+h(1.25-h) \times([2 n-5] \times 1.25+h)}{2 h(1.25-h)} \times \\
& \frac{+(y-[n-2] \times 1.25)(2 h-y+[n-2] \times 1.25)}{+(y-[n-2] \times 1.25)} \times \\
& \frac{\left([n-2] \times 1.25+\frac{1}{3}(y-[n-2] \times 1.25)\right)}{(2 h-y+[n-2] \times 1.25)} \mathrm{nm}
\end{aligned}
$$

$$
\lambda_{n_{\text {ave }}}=(n-2) \times 1.25+\frac{2}{3}(y-[n-2] \times 1.25) \mathrm{mm}
$$

f. Case 4:

$$
\begin{aligned}
& \lambda_{(n-2)_{\min }}=x^{\prime} \mathrm{mm} \quad \lambda_{(n-2)_{\max }}=(n-3) \times 1.25+h \mathrm{~mm} \\
& \lambda_{(n-1)_{\min }}=x^{\prime} \operatorname{mm} \quad \lambda_{(n-1)_{\max }}=(n-2) \times 1.25+h \text { mm } \\
& \lambda_{n_{\text {min }}}=(n-2) \times 1.25 \mathrm{~mm} \quad \lambda_{n_{\max }}=y^{\prime} \operatorname{mm} \\
& \lambda_{(n-2)}=x^{\prime}+\frac{1}{3}\left([n-3] \times 1.25+h-x^{\prime}\right) \mathrm{mm} \\
& \lambda_{(n-1)} \text { ave }=\frac{\left(h+[n-3] \times 1.25-x^{\prime}\right)\left(h-[n-3] \times 1.25+x^{\prime}\right)}{\left(h+[n-3] \times 1.25-x^{\prime}\right)} \times \\
& \frac{\left(x^{\prime}+\frac{2}{3}\left([n-3] \times 1.25+h-x^{\prime}\right)\right)}{\left(h-[n-3] \times 1.25+x^{\prime}\right)} \times x \\
& \frac{+h(1.25-h)([2 n-5] \times 1.25+h)}{t 2 h(1.25-h)} \text { w } \\
& \frac{+h^{2}\left([n-2] \times 1.25+\frac{h}{3}\right)}{+h^{2}} \\
& \lambda_{\text {ave }}=\frac{h\left([n-2] \times 1.25+\frac{2 h}{3}\right)}{2 y^{\prime}-2(n-2) \times 1.25} \times \\
& \frac{t\left(y^{\prime}-[n-2] \times 1.25-h\right)\left(y^{\prime}+[n-2] \times 1.25-h\right)}{+h} \mathrm{~mm}
\end{aligned}
$$

3. Two-Layer Migration Pattern in Sedimentation-Upward-Electrophoresis Experiments.

a. Possible Distributions:

Case 1: $(n-2) \times 1.25<x<([n-1] \times 1.25-h)$;

$$
([n-1] \times 1.25-h)<y<([n-1] \times 1.25)
$$

Case 2: $(n-2) \times 1.25<x<([n-1] \times 1.25-h)$;

$$
(n-1) \times 1.25<y^{\prime}<(n \times 1.25-h)
$$




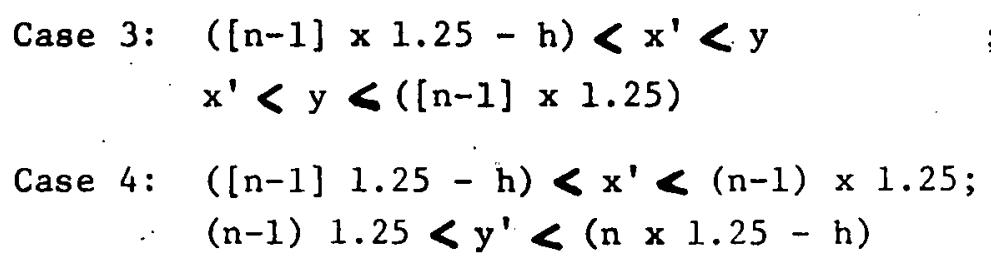

b. Restrictions: None.

c. Case 1:

$$
\begin{aligned}
& \lambda_{(n-1)_{\text {min }}}=x \operatorname{mm} \quad \lambda_{(n-1)_{\max }}=y \mathrm{~mm} \\
& \boldsymbol{\lambda}_{\mathrm{n}_{\mathrm{min}}}=(\mathrm{n}-1) \times 1.25-\mathrm{hmm} \quad \boldsymbol{\lambda}_{\mathrm{n}_{\max }}=\mathrm{ymm} \\
& \lambda_{(n-1)} \text { ave }=\frac{h([n-1] \times 1.25-h-x)([n-1] \times 1.25-h+x)}{2 h([n-1] \times 1.25-h-x)} \times \\
& \frac{t(y-[n-1] \times 1.25+h)([n-1] \times 1.25+h-y)}{+(y-[n-1] \times 1.25+h)} \times \\
& \frac{\left(\frac{2}{3}([n-1] \times 1.25-h)+\frac{y}{3}\right)}{([n-1] \times 1.25+h-y)} \mathrm{mm} \\
& \boldsymbol{\lambda}_{\mathrm{n}_{\text {ave }}}=\frac{1}{3}([\mathrm{n}-1] \times 1.25-\mathrm{h}+2 \mathrm{y}) \mathrm{mm}
\end{aligned}
$$

d. Case 2:

$$
\begin{aligned}
& \lambda_{(n-1)_{\min }}=x \operatorname{man} \lambda_{(n-1)_{\max }}=(n-1) \times 1.25 \mathrm{~mm} \\
& \boldsymbol{\lambda}_{n_{\min }}=(n-1) \times 1.25-h_{m m} \quad \lambda_{n_{\max }}=y^{\prime} \mathrm{mm} \\
& \lambda_{(n-1)}=\frac{([n-1] \times 1.25-h-x)([n-1] \times 1.25-h+x)}{n \times 1.25} \times \\
& \frac{+h\left([n-1] \times 1.25-\frac{2 h}{3}\right)}{-h-2 \times} \mathrm{mm} \\
& \lambda_{\mathrm{n}}=\frac{\mathrm{h}\left([\mathrm{n}-1] \times 1.25-\frac{\mathrm{h}}{3}\right)}{\mathrm{h}} \times
\end{aligned}
$$$$
\frac{+\left(y^{\prime}-[n-1] \times 1.25\right)\left(y^{\prime}+[n-1] \times 1.25\right)}{+2 y^{\prime}-n \times 1.25} \mathrm{~mm}
$$

e. Case 3:

$$
\begin{array}{ll}
\lambda_{(n-1)}=x^{\prime} \operatorname{mm} & \lambda_{(n-1)} \\
\lambda_{n_{\min }}=x^{\prime} \operatorname{mm} & \lambda_{n_{\max }}=y \mathrm{~mm}
\end{array}
$$




$$
\begin{aligned}
& \lambda_{(n-1)} \text { ave }=x^{\prime}+\frac{1}{3}\left(y-x^{\prime}\right) \mathrm{mm} \\
& \lambda_{n_{\text {ave }}}=x^{\prime}+\frac{2}{3}\left(y-x^{\prime}\right) \mathrm{mm}
\end{aligned}
$$

f. Case 4:

$$
\begin{aligned}
& \boldsymbol{\lambda}_{(n-1)_{\min }}=x^{\prime} \mathrm{mm} \quad \boldsymbol{\lambda}_{(n-1)_{\max }}=(n-1) \times 1.25 \mathrm{~mm} \\
& \boldsymbol{\lambda}_{n_{\min }}=x^{\prime} \operatorname{mm} \quad, \lambda_{n_{\max }}=y^{\prime} \text { mm } \\
& \lambda_{(n-1)}=x_{\text {ave }}^{\prime}+\frac{1}{3}\left([n-1] \times 1.25-x^{\prime}\right) \mathrm{mm} \\
& \lambda_{n}=\frac{\left([n-1] \times 1.25-x^{\prime}\right)\left(2 h+x^{\prime}-[n-1] \times 1.25\right)}{\left([n-1] \times 1.25-x^{\prime}\right)} \times \\
& \frac{\left(x^{\prime}+\frac{2}{3}\left[(n-1) \times 1.25-x^{\prime}\right]\right)}{\left(2 h+x^{\prime}-[n-1] \times 1.25\right)} x \\
& \frac{+h\left(y^{\prime}-[n-1] \times 1.25\right)\left(y^{\prime}+[n-1] \times 1.25\right)}{+2 h\left(y^{\prime}-[n-1] \times 1.25\right)} \mathrm{mm}
\end{aligned}
$$

4. Three-Layer Migration Pattern in Sedimentation-Upward-Electrophoresis Experiments.

a. Possible Distributions:

$$
\begin{aligned}
& \text { Case 1: }(n-3) \times 1.25<\times<([n-2] \times 1.25-h) \\
& ([n-1] \times 1.25-h)<y<(n-1) \times 1.25 \\
& \text { Case 2: }(n-3) \times 1.25<x<([n-2] \times 1.25-h) \\
& (n-1) \times 1.25<y^{\prime}<(n \times 1.25-h) \\
& \text { Case 3: }([n-2] \times 1.25-h)<x^{\prime}<(n-2) \times 1.25 \\
& ([n-1] \times 1.25-h)<y<(n-1) \times 1.25 \\
& \text { Case 4: }([n-2] \times 1.25-h)<x^{\prime}<(n-2) \times 1.25 \\
& (n-1) \times 1.25<y^{\prime}<(n \times 1.25-h)
\end{aligned}
$$

b. Restrictions: None.

c. Case 1:

$$
\begin{aligned}
& \lambda_{(n-2)_{\min }}=x_{\operatorname{mm}}^{\lambda} \lambda_{(n-2)}=(n-2) \times 1.25 \mathrm{~mm} \\
& \lambda_{(n-1)_{\min }}=(n-2) \times 1.25-h \operatorname{man} \lambda_{(n-1)}^{\max }=y \operatorname{mm} \\
& \boldsymbol{\lambda}_{\mathrm{n}_{\min }}=(\mathrm{n}-1) \times 1.25-\mathrm{hmm} \quad \boldsymbol{\lambda}_{\mathrm{n}_{\max }}=\mathrm{ymm} \\
& \lambda_{(n-2)} \text { ave }=\frac{([n-2] \times 1.25-h-x)([n-2] \times 1.25-h+x)}{n \times 1.25} \times \text { (continued) }
\end{aligned}
$$




$$
\begin{aligned}
& \frac{+h\left([n-2] \times 1.25-\frac{2 h}{3}\right)}{-h-2 \times} \mathrm{mm} \\
& \lambda_{(n-1)}=\frac{h^{2}\left([n-2] \times 1.25-\frac{h}{3}\right)+h(1.25-h)([2 n-3] \times 1.25-h)}{2.50 h-h^{2}} \times \\
& \frac{t(y-[n-1] \times 1.25+h)(h+[n-1] \times 1.25-y)}{+(y-[n-1] \times 1.25+h)} \times \\
& \frac{\left(\frac{2}{3}[(n-1) \times 1.25-h]+\frac{y}{3}\right)}{(h+[n-1] \times 1.25-y)} \mathrm{mm}
\end{aligned}
$$

$\lambda_{\mathrm{n}_{\text {ave }}}=\frac{1}{3}([\mathrm{n}-1] \times 1.25-\mathrm{h}+2 \mathrm{y}) . \mathrm{mm}$

d. Case 2:

$$
\begin{aligned}
& \boldsymbol{\lambda}_{(\mathrm{n}-2)_{\min }}=x_{\mathrm{mm}}^{\boldsymbol{\lambda}_{(\mathrm{m}-2)_{\max }}}=(\mathrm{n}-2) \times 1.25 \mathrm{~mm}
\end{aligned}
$$

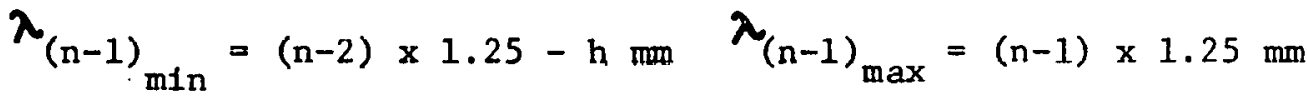

$$
\begin{aligned}
& \boldsymbol{\lambda}_{\mathrm{n}_{\mathrm{min}}}=(\mathrm{n}-1) \times 1.25-\mathrm{hmm} \quad \boldsymbol{\lambda}_{\mathrm{n}_{\max }}=\mathrm{y}^{\prime} \mathrm{mm} \\
& \boldsymbol{\lambda}_{(\mathrm{n}-2)}{ }_{\text {ave }}=\frac{([\mathrm{n}-2] \times 1.25-\mathrm{h}-\mathrm{x})([\mathrm{n}-2] \times 1.25-\mathrm{h}+\mathrm{x})}{\mathrm{n} \times 1.25} \times \\
& \frac{+h\left([n-2] \times 1.25-\frac{2 h}{3}\right)}{-h-2 \times} \mathrm{mm}
\end{aligned}
$$

$$
\begin{aligned}
& \lambda_{(n-1)}=\frac{1}{2}([2 n-3] \times 1.25-h) \mathrm{mm} \\
& \lambda_{n_{\text {ave }}}=\frac{h\left([n-1] \times 1.25-\frac{h}{3}\right)}{h} \times
\end{aligned}
$$

$$
\frac{+\left(y^{\prime}-[n-1] \times 1.25\right)\left(y^{\prime}+[n-1] \times 1.25\right)}{+2 y^{\prime}-n \times 1.25} \mathrm{~mm}
$$

e. Case 3:

$$
\begin{aligned}
& \lambda_{(\mathrm{n}-2)_{\mathrm{min}}}=\mathrm{x}^{\prime} \mathrm{mm} \\
& \boldsymbol{\lambda}_{(\mathrm{n}-2)_{\max }}=(\mathrm{n}-2) \times 1.25 \mathrm{~mm} \\
& \lambda_{(n-1)_{\text {min }}}=x^{\prime} \text { mm } \\
& \lambda_{(n-1)_{\max }}=y \operatorname{mon} \\
& \lambda_{n_{\text {min }}}=(n-1) \times 1.25-h \mathrm{~mm} \\
& \lambda_{\mathrm{n}_{\max }}=\mathrm{ymm} \\
& \boldsymbol{\lambda}_{(\mathrm{n}-2)} \text { ave }=x^{\prime}+\frac{1}{3}\left([\mathrm{~N}-2] \times 1.25-\mathrm{x}^{\prime}\right) \mathrm{mm}
\end{aligned}
$$


$\lambda_{(n-1)}=\frac{\left([n-2] \times 1.25-x^{\prime}\right)\left(x^{\prime}-[n-2] \times 1.25+2 h\right)}{\left([n-2] \times 1.25-x^{\prime}\right)} \times$

$$
\begin{aligned}
& \frac{\left(\frac{x^{\prime}}{3}+\frac{2}{3}[n-2] \times 1.25\right)+h(1.25-h)([2 h-3] \times 1.25-h)}{\left(x^{\prime}-[n-2] \times 1.25+2 h\right)+(1.25-h) \cdot 2 h} \times \\
& \frac{+(y-[n-1] \times 1.25+h)(h+[n-1] \times 1.25-y)}{+(y-[n-1] \times 1.25+h)} \times \\
& \frac{\left(\frac{2}{3}[(n-1) \times 1.25-h]+\frac{y}{3}\right)}{(h+[n-1] \times 1.25-y)} \mathrm{mm}
\end{aligned}
$$

$\lambda_{\text {ave }}=\frac{1}{3}([n-1] \times 1.25-h+2 y) \mathrm{mm}$

f. Case 4:

$\lambda_{(n-2)_{\text {min }}}=x^{\prime} \mathrm{mm}$

${ }^{\lambda}(n-1)_{m 1 n}=x^{\prime} \mathrm{mm}$

$\lambda_{n_{m 1 n}}=(n-1) \times 1.25-h \mathrm{~mm}$.

$\lambda_{(n-2)}=x^{\prime}+\frac{1}{3}\left([n-2] \times 1.25-x^{\prime}\right) \mathrm{mm}$

$\lambda_{(n-1)}$ ave $\frac{\left([n-2] \times 1.25-x^{\prime}\right)\left(x^{\prime}-[n-2] \times 1.25+2 h\right)}{\left([n-2] \times 1.25-x^{\prime}\right)} \times$

$$
\frac{\left(\frac{x^{\prime}}{3}+\frac{2}{3}[n-2] \times 1.25\right)+h(1.25-h)([2 n-3] \times 1.25-h)}{\left(h+[1-2] \times 1.25 x^{\prime}\right)+3.50 h} \times
$$$$
\frac{+h^{2}\left([n-1] \times 1.25-\frac{2 h}{3}\right)}{-h^{2}} \mathrm{~mm} \text {. }
$$

$\boldsymbol{\lambda}_{\mathrm{n}_{\text {ave }}}=\frac{\mathrm{h}\left([\mathrm{n}-1] \times 1.25-\frac{\mathrm{h}}{3}\right)}{\mathrm{h}} \times$

$$
+\frac{\left(y^{\prime}-[n-1] \times 1.25\right)\left(y^{\prime}+[n-1] \times 1.25\right)}{2 y^{\prime}-n \times 1.25} \mathrm{~mm}
$$




\section{APPENDIX F \\ Sample Calculation of Sedimentation Velocitles and Rates for \\ Staflo Sedimentation Experiment 12-26-67}

\section{Summary of Measurements}

$$
\mathrm{h}=0.08 \mathrm{~cm} ; \quad \mathrm{x}=0 \mathrm{~cm} ; \quad \mathrm{y}=0.17 \mathrm{~cm}
$$

\section{Sedimentation Migration Distances}

The sedimentation migration distances $\left(\boldsymbol{\lambda}_{S}\right)$ are calculated in accordance with the discussion in Chapter III and the equations in Appendix $E 2$. They are as follows:

\begin{tabular}{ccccc}
\hline $\begin{array}{c}\text { Collection } \\
\text { Tube }\end{array}$ & $\begin{array}{c}\text { Sperm } \\
\text { collected } \\
(\%)\end{array}$ & $\begin{array}{c}\text { Minimum } \\
(\mathrm{cm})\end{array}$ & $\begin{array}{c}\text { Sedimentation Distance } \\
(\mathrm{cm})\end{array}$ & $\begin{array}{c}\left(\boldsymbol{\lambda}_{\mathrm{S}}\right) \\
\text { Average } \\
(\mathrm{cm})\end{array}$ \\
\hline 8 & 23.1 & 0 & 0.080 & 0.027 \\
9 & 61.1 & 0 & 0.170 & 0.10 \\
10 & 15.3 & 0.125 & 0.170 & 0.155 \\
\hline
\end{tabular}

\section{Sedimentation Veloctities and Rates}

Using a minimum residence time $\left(\boldsymbol{\tau}_{\mathrm{mIn}}\right)$ of $28.7 \mathrm{~min} .(1,722$ seconds $)$ and the expression

$$
\frac{d x}{d t}=\frac{\lambda_{S}}{\tau_{\min }} \frac{(\mathrm{cm})}{(\mathrm{sec})}
$$

one gets minimum, maximum and average sedimentation velocities of $0,0.465 \mathrm{x}$ $10^{-4}$ and $0.157 \times 1.0^{-4} \mathrm{~cm} / \mathrm{sec}$ for collection fraction 8.

The sedimentation velocities can be converted to sedimentation coefficients by the expression

$$
S=\frac{1}{g} \frac{d x}{d t} \quad(\sec )
$$

where $g=980 \mathrm{~cm} / \mathrm{sec}^{2}$ 
Using the average sedimentation velocity of $0.157 \times 10^{-4} \mathrm{~cm} / \mathrm{sec}$ for collection tube 8 , one gets

$$
\mathrm{s}_{8_{\text {ave }}}=0.161 \times 10^{-7} \mathrm{sec}
$$

Converting to Svedberg units ( 1 Svedberg $=10^{-13} \mathrm{sec}$ ) gives

$$
\mathrm{s}_{8_{\text {ave }}}=0.161 \times 10^{6} \text { Svedbergs }
$$

The results of similar calculations for other sedimentation distances and collection fractions are summarized in Table XXII.

4. Weighted Average Sedimentation Velocity

The weighted average sedimentation veloctty $\left(\frac{\overline{d x}}{d t}\right.$ ave $)$ for spermatozoa in Experiment 12-26-67 is determined as follows:

\begin{tabular}{cccc}
\hline $\begin{array}{c}(1) \\
\begin{array}{r}\text { Collection } \\
\text { Fraction }\end{array}\end{array}$ & $\begin{array}{c}\text { Fraction of } \\
\text { Spermatozoa }\end{array}$ & $\begin{array}{c}\left(\frac{\mathrm{dx}}{\mathrm{dt}} \mathrm{ave}\right) \\
(\mathrm{cm} / \mathrm{sec})\end{array}$ & $\begin{array}{c}(4) \\
\mathrm{sec}(3)\end{array}$ \\
\hline 6 & 0.231 & $0.157 \times 10^{-4}$ & $0.0362 \times 10^{-4}$ \\
9 & 0.616 & $0.580 \times 10^{-4}$ & $0.3570 \times 10^{-4}$ \\
1.0 & 0.153 & $0.90 \times 10^{-4}$ & $0.1380 \times 10^{-4}$ \\
\hline
\end{tabular}

The weighted average sedimentation velocity for rabbit spermatozoa is $0.53 \times 10^{-4} \mathrm{~cm} / \mathrm{sec}$ at $3^{\circ} \mathrm{C}$ in a $1 \%$ sucrose solution in glycine-phosphate buffer. 
APPENDIX G

Sample Calculation of Electrophoretic Mobilities for Staflo Sedimentation-Upward-Electrophoresis (SUE) Experiment 7-29-67

\section{Summary of Measurements}

$$
\mathrm{h}=0.07 \mathrm{~cm} ; \quad \mathrm{x}=0.190 \mathrm{~cm} ; \quad \mathrm{y}=0.305 \mathrm{~cm}
$$

2. SUE Migration Distances

The SUE migration distances $\left(\boldsymbol{\lambda}_{\mathrm{SUE}}\right)$ are calculated in accordance with the discussion in Chapter III and the equations. in Appendix E 3 . They are as follows:

\begin{tabular}{ccccc}
\hline $\begin{array}{c}(1) \\
\begin{array}{c}\text { Collection } \\
\text { Tube }\end{array}\end{array}$ & $\begin{array}{c}(2) \\
\text { Sperm } \\
\text { Collected } \\
(\%)\end{array}$ & $\begin{array}{c}\text { Minimum } \\
(\mathrm{cm})\end{array}$ & $\begin{array}{c}(3) \\
\text { SUE }\end{array}$ & $\begin{array}{c}(4) \\
\text { Migration }\end{array}$ \\
\hline 6 & 58.6 & 0.190 & 0.305 & $\begin{array}{c}(5) \\
\text { Distances } \\
(\mathrm{cm})\end{array}$ \\
7 & 41.0 & 0.190 & 0.250 & 0.210 \\
\hline
\end{tabular}

3. Welghted Average SUE MAgration Distance

The weighted average SUE migration distance $\left(\lambda_{\text {SUE }_{a v e}}\right)$ is the sum of the products of columns (2) and (5) above divided by 100 as follows:

$$
\overline{\lambda_{\text {SUE }_{\text {ave }}}}=\frac{58.6 \times 0.259 \times 41 \times 0.210}{100}=0.238 \mathrm{~cm}
$$

4. Weighted Average Electrophoretic Migration Distance

The weighted average electrophoretic migration distance $\left(\overline{\lambda_{\text {Eave }}}\right)$ is given by equation (11) from Chapter III:

$$
\overline{\boldsymbol{\lambda}_{\mathrm{E}}}=\overline{\boldsymbol{\lambda}_{\mathrm{SUE}}}+\overline{\boldsymbol{\lambda}_{\mathrm{Sve}}}
$$

The value of $\overline{\lambda_{\text {SUE }} \text { ave }}$ was determined in paragraph 3 . 
$\overline{\boldsymbol{\lambda}_{\mathrm{S}}}$ is determined by correcting the value of the weighted average sedimentation velocity of $0.53 \times 10^{-4} \mathrm{~cm} / \mathrm{sec}$ calculated in Appendix $\mathrm{F}$ for $3^{\circ} \mathrm{C}$ and $1 \%$ sucrose to that expected at $5.4^{\circ} \mathrm{C}$ and $0.5 \%$ sucrose using the formula of Bier ${ }^{38}$ :

$$
\eta_{1}\left(\frac{d x}{d t}\right)_{1}=\eta_{2}\left(\frac{d x}{d t}\right)_{2}
$$

This gives $\left(\frac{\mathrm{dx}}{\mathrm{dt}}\right.$ ave $) 5.4^{\circ} \mathrm{C}=0.58 \times 10^{-4} \mathrm{~cm} / \mathrm{sec}$. Then, using a minimum residence time of $14.2 \mathrm{~min}$. (852 sec.), one gets a weighted average sedimentation distance at $5.4^{\circ} \mathrm{C}$ of $0.050 \mathrm{~cm}$.

Thus, $\quad \overline{\boldsymbol{\lambda}_{E_{\text {ave }}}}=0.238+0.050=0.288 \mathrm{~cm}$

\section{Weighted Average Electrophoretic Mobility}

In this experiment, the weighted average electrophoretic mobility $\left(\overline{\mu_{a v e}}\right)$ is calculated using equation 13 from Chapter III with the following values: $\lambda_{\mathrm{E}}=0.288 \mathrm{~cm}, \boldsymbol{Z}_{\mathrm{E}}=569 \mathrm{sec}, \mathrm{I}=0.088$ amps, and $\mathrm{R}=$ 1,430 ohms. Ihis gives $\bar{\mu}=0.94 \times 10^{-4} \mathrm{~cm} / \mathrm{sec} / \mathrm{V} / \mathrm{cm}$ al $5.4^{\circ} \mathrm{C}$.

6. Normalization of $\overline{\mu \text { ave }}$ to $3^{\circ} \mathrm{C}$

The formula of Bler ${ }^{38}$ is used to normalize the value of $\overline{\mu \text { ave }}$ at $5.4^{\circ} \mathrm{C}$ to that expected in the same density gradient at $3^{\circ} \mathrm{C}$. The corrected value is $0.852 \times 10^{-4} \mathrm{~cm} / \mathrm{sec} / \mathrm{V} / \mathrm{cm}$.

Simflar calculations have been done for the other SUE experiments in this series and are summarized in Table XXVII. 
REFERENCES

1. Adams, C.E., Artificial Insemination in the Rabbit, J. Reprod. Fert. 2, 521 (1961).

2: Adams, C.E., Artificial Insemination in the Rabbit, Technical Bulletin No. 1, Report of the Commercial Rabbit Association, England, (1961).

3. Adams, C.E. and M.C. Chang, Capacitation of Rabbit Spermatozoa in the Fallopian Tube and in the Uterus, J. Exp. Zool. 151(2), 159(1962).

4. Adams, C.E. and M.C. Chang, The Effect of Delayed Mating on Fert1lization in the Rabbit, J. Exp. 2001. 151(2), 155(1962).

5. Ahlgren, M., Development of Fertilized Tubal Rabbit Eggs in Diffusion Chambers IN VIVO, J. Reprod. Fert. 12, 145 (1966).

6. Ahluwalia, B.S. and E.F. Graham, Free Amino Acids in the Semen of the Fowl and the Turkey, J. Reprod. Fert. 12, 365 (1966).

7. Ahluwalia, B.S. and E.F. Graham, Free Carbohydrates in Seminal Plasma and Sperm Cells of Semen of Fowl, J. Reprod. Fert. 12, 359 (1966).

8. Amann, R.P., Effect of Ejaculation Frequency and Breed on Semen Characteristics and Sperm Output of Rabbits, J. Reprod. Fert. 11, 291 (1966).

9. Amann, R.P. and J.T. Lambiase, Jr., The Male Rabbit. I. Changes in Semen Characteristics and Sperm Output Between Puberty and One Year of Age, J. Reprod. Fert. 14, 329 (1967).

10. Ambrose, E.J., A.M. James and J.H.B. Lowick, Differences Between the Electrical Charge Carried by Normal and Homologous Tumour Cells, Nature 177,576 (1956).

11. Amir, D. and H. Schindler, The Effect of High Sperm Concentrations on the Rates of Respiration and Fructolysis by Ram Spermatozoa, J. Reprod. Fert. 13, 93 (1967).

12. Asde11, S.A. and G.W. Salisbury, The Rate at Which Spermatogenesis Occurs in the Rabbit, Anat. Record 80(2), 145 (1941).

13. Austin, C.R., Acrosome Loss From the Rabbit Spermatozoon in Relation to Entry into the Egg, J. Reprod. Fert. 6, 313 (1963).

14. Austin, C.R., Fertilization of Mammalian Eggs IN VITRO, Int. Rev. of Cytology 10, 337, New York Academic Press (1961).

15. Austin, C.R., The Mammal1an Egg, Charles C. Thomas, Springfield, I11. (1961). 
16. Avis, F.R. and P.B. Sawin, A Surgical Technique for the Reciprocal Transplantation of Fertilized Eggs, The Journal of Heredity 62 (5), 259 (1951).

17. Bahr, G.F. and E. Zeltler, Study of Bull Spermatozoa, Quantitative Electron Microscopy, J. Cell. B1ol. 21, 175 (1964).

18. Bangham, A.D., Electrophoretic Characteristics of Ram and Rabbit Spermatozoa, Proc. Roy. Soc., Ser. B. (Biol. Sci.) 155(959), 292 (1962).

19. Barack, B.M., Transport of Spermatozoa from Seminiferous Tubules to Epididymis in the Mouse: a Histological and Quantitative Study, J. Reprod. Fert. 16(1), 35 (1968).

20. Beatty, R.A., Density Gradient Media for Mammalian Spermatozoa, $V^{\circ}$ Congresso Internazionale per la Riproduzione Animale e la Fecondzione Artificlale, Trento, p 276.(1964).

21. Beatty, R.A., Nigrosin-Eosin Staining of Rabbit Spermatozoa and the Fertility of Semen, Proc. Roy. Soc. Edinburgh, Sec. B Vul, LX VII, 1 (1957).

22. Beatty, R.A. and R.A.N. Napier, I. Genetics of Gametes. I. A Quantitative Analysis of Five Characteristics of Rabbit Spermatozoa, Proc. Roy. Soc. Edinburgh B68 (I-1), 1 (1960).

23. Beatty, K.A. and R.A.N. Napier, II. Geneties of Gametca. II. Strain Differences in Characteristics of Rabbit Spermatozoa, Proc. Roy. Soc. Edinburgh B68 (I-2), 17 (1960).

24. Redford,..T.M., Changes in the Electrophoretic Properties of Rabbit Spermatuzua During Passage Through the Epididymis, Nature 200 (4912), 1178 . (1963).

25. Bedford, J.M., Fine Structure of the Sperm Head in Ejaculate and Uterine Spermatozoa of the Rabbit, J. Reprod. Fert. 7,221 (1964).

26. Bedford, J.M., Morphological Changes in Rabbit Spermatozoa During Passage Through the Epldidymis, J. Reprod. Fert. 5, 169 (1963).

27. Bedford, J.M., Non-Specific Ta11-Tall Agglutination of Mammalian Spermatozoa, Exp. Cell. Res. 38, 654 (1965).

28. Bedford, J.M., The Importance of Capacitation for Establishing Contact Between Eggs and Sperm in the Rabb1t, J. Reprod. Fert. 13, 365 (1967).

29. Bedford, J.M. and A.M. Bibeau, Fallure of Sperm Sedimentation to Influence the Sex Ratio of Rabbits, J. Reprod. Fert. 14, 167 (1967).

30. Bedford, J.M. and M.C. Chang, Fertilization of Rabbit Ova IN VITRO, Nature 193 (4818), 898 (1962). 
31. Bedford, J.M. and R. Shalkovsky, Species-Spectficity of Sperm Capacitation in the Rabbit, J. Reprod. Fert: 13, 361 (1967).

32: Benedict, R.C., V.N. Schumaker and R.E. Davies; The Buoyant Density of Bovine and Rabbit Spermatozoa, J. Reprod. Fert. 13, 237 (1967).

33. Bhargava, P.M., M.W.H. Blohop and T.S. Work, The Chemlcal Composition of Bull Semen with Spectal Reference to Nucleic Actds, Free NucleotIdes, and Free Amino Aclds, Blochem. J. 73, 242 (1959).

34. Bhannasir1, T., R. Bogart and H. Krueger, The Effect of Antibiotics on Semen Production in Rabbits, Am. J. Vet. Res. 20, 756 (1959).

35. Bhattacharya, B.C., Die Verschiedene Sedimentationsgeschwindigke1t der X- und Y-Spermien und die Frage der Willkilrlichen Geschlechtsbestimung, Ze1tschrift für wissenschaftliche Zoologie Bd 166, 2C3 (1962).

36. Bhattacharya, B.C., Sex Control in Mammals, Zeitschr. für Tierzüchtung und Züchtungsbiologie 72 (3), 250. (1958).

37. Bhattacharya, B.C., A.D. Bangham, R.J. Cro, R.D. Keynes and L.E.A. Rowson, An Attempt to Predetermine the Sex of Calves by Artificial Insemination with Spermatozoa Separated by Sedimentation, Nature 211, (5051), 863 (1966).

38. Bler, M., Electrophores18. Theory, Methods and Applications, p 438, Academic Press, Inc., New York (1959).

39. B1ggers, J.D., R.B.L. Gwatkin and R.L. Brinster, Development of Mouse Embryos in Organ Cultures of Fallopian Tubes on a Chemically Defined Medium, Nature 194, 747 (1962).

40. Blshop, D.W. (Ed), Spermatozoan Mot1lity, Amertcan Assn. for the Advancement of Science, Washington, D.C. (1962).

41. Bishop, D.W., X and Y Spermatozoa, Nature 187 (4733), 255 (1960).

42. B1shop, M.W.H. and A. Walton, Spermatogenesis and the Structure of Mammalian Spermatozoa, Markhall's Physlology of Reproduction, Chap. 7,. Ed. A.S. Parkes, Longmans, Green, London (1960).

43. Blandau, R.J. and R.E. Rumery, The Relationships of Swimming Movements of Epldidymal Spermatozoa to Their fertilizing Capacity, Fertility and Sterility 15 (6), 571 (1964).

44. Boettcher, B., Correlation Between Human ABO Blood Group Antigens In Seminal Plasma and on Seminal Spermatozoa, J. Reprod. Fert. 16 (1), 49 (1968).

45. Bouters, R., C. Esnault, G.W. Sallsbury and R. Ortavant, Discrepancies In Analyses of Deoxyribonucleic Acid in Rabbit Spermatozoa, Involving Feulgen Staining (Feulgen-DNA) and Ultravlolet Light Absorption (UV-DNA) Measurement8, J. Reprod. Fert: 14, 355 (1967). 
46. Bredderman, P.J., R.H. Foote and A.M. Yassen, An Improved Artiflclal Vaglna for Collecting Rabb1t Semen, J. Reprod. Fert. I, 401 (1964).

47. Burfening, P.J. and L.C. Ulberg, Embryontc Survival Subsequent to Culture of Rabbit Spermatozoa at $38^{\circ}$ and $40^{\circ} \mathrm{C}$, J. Reprod. Fert. 15, 87 (1968).

48. Carady, R.B., P.B. Sawln and J. Van Dam, Commerclal Rabbit Raising, Agriculture Handbook No. 309, U.S.D. Agric. (1966).

49. Chang, M.C., A Detximental Effect of Seminal Plasma on the Fertil1zing Capactiy of Sperm, Nature 179, 258 (1957).

50. Chang, M.C., Development and Fate of Transferred Rabbit Ova or Blastocysts in Relation to the Ovulation Time of Reciplents, J. Exp. Zool. 114, 197 (1950).

51. Chang, M.C., Fert1lization of Rabbit Ova IN VITRO, Nature 184 (4684), 466 (1959).

52. Chang, M.C., The Effects of Serum on Spermatozoa, J. Gen. Phystol. $30(4), 321(1947)$.

53. Chang, M.C., The Maturation of Rabbit Oocytes in Culture and Their Maturation, Activation, Fertilization, and Subsequent Development in the Fallopian Tubes, J. Exp. Zool. 128 (2), 379 (1955).

54. Chang, M.C. and G. P1ncus, Physiology of Fertilization in Mamals, Physlological Reviews, 31 (I), 1 (1951).

55. Chang, M.C. and J.M. Bedford, Fertilizability of Rabbit Ova after Removal of the Corone Radiata. Fertility and Sterlilty 13 (5), 421 (1962).

56. Cheng, P. and L.E. Casida, Fertility in Rabbit as Affected by the Dilution of Semen and the Number of Spermatozoa, Proc. Soc. Exper. B101. and Med. 69,36 (1948).

57. Chiarell1, B., L. DeCarl1 and F. Nusso, Analysi Morfometrica de1 Cromosomf del Confglio (Oryctolagus Cuniculus L.), Caryologia 15 (3), 565 (1962).

58. Crooke, A.C., A Rapid Supravital Staining Method for Assessing the Viab1lity of Human Spermatozoa, Nature 159, 749 (1947).

59. Dantel, J.C., Jr. and J.D. Olson, Amino Acld Requirements for Cleavage of the Rabbit Ovum, J. Reprod. Fert. 15, 453 (1968).

60. Dav18, I.S., R.W. Bratton and R.H. Foote, Livability of Bovine Spermatozoa at $5^{\circ} \mathrm{C}$ in Trie-Buffered and C1trate-Buffered Yolk-Glycerol Extender , J. Dairy Sc1. 46 (1), 57 (1968).

61. Dav1s, I.S., R.W. Bratton, and R.H. Foote, Livability of Bovine Spermatozoa at $5,-25$, and $-85^{\circ} \mathrm{C}$ in Tris-Buffered and C1trate-Buffered Yolk-Gylcerol Extenders, J. Dairy Sc1. 46 (4), 333 (1963). 
62. Dawson, R.M.C. and T.W. Scott, Phospholipid Composition of Epididymal Spermatozoa Prepared by Density Gradient Centrifugation, Nature 202, 292 (1964).

63. Degerman, G. and J.E. Klhlström, Cyclic Varlation in the Body Temperature of the Male Rabbit, Acta Physiol. Scand. 62, 46 (1964).

64. Desjardins, C., K.T. K1rton, and H.D. Hafs, Sperm Output of Rabbits at Various Ejaculation Frequencies and Their Use in the Design of Experiments, J. Reprod. Fert. 15, 27 (1968).

65. Dickmann, Z., Chemotoxis of Rabbit Spermatozoa, J. Exp. B1o1. 40 (1), 1 (1963).

66. Dickmann, Z., The Zona Pellucida of the Rabbit Egg, Fert1lity and Sterflity 14 (5), 490 (1963).

67. Dickmann, Z. and P.J. Dziuk, Sperm Penetration of the Zona Pellucida of the P1g Egg, Anat. Rec. 148 (2), 276 (1964).

68. Doggett, V.C., Perlodicity in the Fecundity of Male Rabbits, Am. J. Physiol. 187, 445 (1956).

69. Dukelow, W.R., Effects of Extracted Wheat Germ Oil in Sheep and Cattle, J. Reprod. Fert. 11, 465 (1966).

70. Dukelow, W.R., H.N. Chernoff and W.L. Williams, Properties of Decapacitation Factor and Presence in Various Species, J. Reprod. Fert. 14, 393 (1967).

71. Dukelow, W.R. and W.L. Williams, Survival of Capacitated Spermatozoa in the Oviduct of the Rabb1t, J. Reprod. Fert. 14, 477 (1967).

72. Dziuk, P.J., Double Mating of Rabbits to Determine Capacitation Time, J. Reprod. Fert. 10, 389 (1965).

73. Edwards, R.G., Antigenicity of Rabbit Semen, Bull Semen and Egg Yolk After Intravaginal or Intramuscular Injections into Female Rabbits, J. Reprod. Fert. 1, 385 (1960).

74. Edwards, R.G., Cleavage of One- and Two-Celled Rabbit Eggs IN VITRO After Removal of the Zona Pellucida, J. Reprod. Fert. 7, 413 (1964).

75. Edwards, R.G., L.C. Ferguson and R.R.A. Coombs, Blood Group Antigens on Human Spermatozoa, J. Reprod. Fert. 7, 153 (1964).

76. Eliasson, R., Effect of Frequent Ejaculations on the Composition of Human Seminal Plásma, J. Reprod. Fert. $\underline{9}, 331$ (1965).

77. E1 Jack, M.H. and P.E. Lake, The Effect of Resting Roosters from Ejaculation on the Quality of Spermatozoa in Semen, J. Reprod. Fert. 11 ; 489 (1966).

78. Emmens, C.W., The Effect of Variations in Osmotic Pressure and Electrolyte Concentration on the Motility of Rabbit Spermatozoa at Different 
Hydrogen-Ion Concentrations, J. Physiol. 107, 129 (1948).

79. Emmens, C.W.; The Motility and Viability of Rabbit Spermatozoa at Different Hydrogen-Ion Concentrations, J. Physiol. 106, 471 (1947).

80. Emmens, C.W. and G.I.M. Swyer, Observations on the Motility of Rabbit Spermatozoa in Dilute Suspension, J. of Gen. Physiol. 32(2), 121 (1948).

81. Foote, R.H., Effect of Sperm Losses in Semen Collection Equipment on Estimated Sperm Output by Bulls, J. Dairy Sci. 46 (3), 242 (1963).

82. Foote, R.H., H.D. Hafs, R.E. Staples, A.T. Gregoire and R.W. Bratton, Ovulation Rates and Litter Sizes in Sexually Receptive and Nonreceptive Artificially Inseminated Rabbits Given Varying Dosages of Luteinizing Hormone, J. Reprod. Fert. $\underline{5}, 59$ (1963).

83. Foote, R.H., R.W. Bratton, C.R. Henderson, E.M. Shantz and J.K. Pol- . lard, Survival of Bovine Spermatozoa at Room Temperature in Citrate and Cornell University and Tris Extenders Containing Whole and Fractionated Coconut Milk, J. Dairy Sci. 45 (11), 1383 (1962).

84. Fox, R.R., Preservation of Rabb1t Spermatozoa: Fertility Results from Frozen Semen, Proc. Soc. Biol. and Med. 108 (3), 663 (1961).

85. Fox, R.R. and J.F. Burdick, Preservation of Rabbit Spermatozoa, Ethylene Glycol vs. Glycerol for Frozen Semen, Proc. Soc. Exp. B101. and Med. 113 (4), 853 (1963).

86. Freund, M. and B. Carol, Factors Affecting Haemocytometer Counts of Sperm Concentration in Human Semen, J. Reprod. Fert. 8,149 (1964).

87. Freund, M. and B. Carol, Varlance Aooociated with Dotormination of Sperm Concentration of Human Semen by Hemocytometer Count, Federation Proceedings 22 (2 Part I), 569 (1963).

88. Freund, M: and J. Wiederman, Factors Affecting the Dilution, Freezing and Storage of Human Semen, J. Reprod. Fert. 11, 1 (1966).

89. Gardner, R.L. and R.G. Edwards, Control of the Sex Ratio at Full Term in the Rabbit by Transferring Sexed Blastocysts, Nature 218, 346 (1968).

90. Goldstein, Avram, Blostatistics: An Introductory Text, The Macmillan Company, New York, (1964).

91. Gordon, M.J., Control of Sex Ratio in Rabbits by Electrophoresis of Spermatozoa, Proc. Nat. Acad. Sci. 43, 913 (1957).

92. Gordon, M.J., Physico-Chemical Investigations on Rabbit Spermatozoa with Special Reference to Electrophoretic Separation of X-and YChromosome-Bearing Spermatozoa ( $\mathrm{PhD}$ Thesis), University of California, Berkeley (G66382), 1955.

93. Graves, C.N. and G.W. Salisbury, The Metabolism of Carbohydrates and Other Compounds by Epididymal-Like Bovine Spermatozoa, J. Reprod.

Fert. 12, 213 (1966). 
94. Gregolre, A.T., R.W. Bratton and R.H. Foote, Sperm Output and Fertllity of Rabbits Ejaculated Either Once a Week or Once a Day for Forty-Three Weeks, J. of Animal Sc1. 17, 243 (1958).

95. Gulyas, Bela J., IN VITRO Maintenance of Rabbit Sperm at Room Temperature, Nature 211 (5052), 987 (1966).

96. Gustavsson, Ingemar, Chromosome Abnormality in Cattle, Nature 211 $(5051), 865$ (1966).

97. Gwatkin, R.B.L. and J.D. Biggers, Histology of Mouse Fallopian Tubes Malntained as Organ Cultures on a Chemically Defined Medium, Int. J. Fert. $\underline{8}$ (1), 453 (1963).

98. Hadeck, R., Study of the Sperm Capacitation Factor in the Genftal Tract of the Female Rabbit, Am. J. Vet. Res. 20, 753 (1959).

99. Hafez, E.S.E., Effects of Antibiotics on Viability of Fertilized Rabbit Ova IN VITRO, Fertility and Sterllity 13 (6), 583 (1962).

100. Hafez, E.S.E., Growth and Survival of Blastocysts in the Domestic Rabbit. IJ. Quantitative Effects of Exogenous Progesterone FollowIng Ovariectomy, J. Reprod. Fert. 7.241 (1964).

101. Hafez, E.S.E., Storage of Fertilized Ova, Int. J. Fert. $\underline{8}$ (1), 459 (1963).

102. Hafez, E.S.E., Storage of Rabbit Ova in Gelled Media at $10^{\circ} \mathrm{C}$, J. Reprod. Fert. 2, 163 (1961).

103. Hafez, E.S.E. and E. Rajakosk1, Growth and Survival of Blastocysts In the Domestic Rabbit. I. Effect of Maternal Factors, J. Reprod. Fert. 7, 229 (1964).

104. Hamilton, W.D., Extraordinary Sex Ratios, Science 156, 477 (1967).

105. Hammond, J. and F.H.A. Marshal1, Reproduction in the Rabbit, London: Ollver and Boyd (1925).

106. Hammond, M., M.A. Boone, B.D. Barnett and D.E. Turk, Studies of the Composition of the Deoxyribonucleic Acid of the Fowl Spermatozoon, J. Reprod. Fert. 9 , 347 (1965).

107. Hamner, C.E. and W.L. Williams, Effect of the Female Reproductive Tract on Sperm Metabolism in the Rabbit and Fowl, J. Reprod. Fert. 5, 143 (1963).

108. Hamner, C.E. and W.L. Williams, Identification of Sperm Stimulating Factor of Rabbit Oviduct Fluld, Proc. Soc. Exptl. Biol. Med. 117 (1), 240 (1964).

109. Hancock, J.L., A Staining Technique for the Study of TemperatureShock in Semen, Nature 167, 323 (1951).

110. Hancock, J.L., The Morphology of Bull Spermatozoa, J. Exp. Biol. 29, 445 (1952). 
111. Hartree, E.F. and P.N. Srivastava, Chemical Composition of the Acrosomes of Ram Spermatozoa, J. Reprod. Fert. 9, 47 (1965).

112. Harvey, E.N., Can the Sex of Mammallan Offspring Be Controlled? J. Hered. 37,71 (1946).

113. Hathaway, R.R. and E.F. Hartree, Observations on the Mammalian Acrosome: Experimental Removal of Acrosomes from Ram and Bull Spermatozoa, J. Reprod. Fert. 5, 225 (1963).

114. Hayes, T.L., R.F.W. Pease and A.S. Camp, Stereoscopic Scanning Electron Microscopy of Living Tribolium confusum, J. Insect Physiol. 13, 1143 (1967).

115. Holmberg, B., on the Permeability to Lissamine Green and other Dyes in the Course of Cell Injury and Cell Death, Exp. Cell Res, 22, 406 (1961).

116. Howarth, B., Jr., C.W. Alliston and L.C. Ulberg, Importance of UterIne Environment in Rabbit Sperm Prior to Fertilization, J. An1m. Sc1. 24, 1027 (1965).

117. Hunter, A.G. and H.D. Hafs, Antigenicity and Cross-Reactions of Bovine Spermatozoa, J. Reprod. Fert. 7, 357 (1964).

118. Hunter, R.H.F., C. Polge and L.E.A. Rowson, The Recovery, Transfer and Survival of Blastocysts in P1gs, J. Reprod. Feri. 14, 501 (1967).

119. Hunter, R.H.F. and P.J. Dzluk, Sperm Penetration of Pig Eggs in Relation to the Timing of Ovulation and Insemination, J. Reprod. Fert. 15, $199(1968)$.

120. Inouye, N., Two X-ray Induced Translocations in the Domestic Fowl, (M.S. Thesis), University of California (Berkeley), (1952).

121. Iversen, S., Surface Reflexion Interference Microscopy of Bull Spermatozoa, Q.J. Micr. Sci. 105, 245 (1964).

122. Joël, C.A., A. Katchalsky, O. Kedem and N. Sternberg, Electrophoretic Measurements of Human Spermia, Experient1a I, 274 (1951).

123. Kihlström, J.E., On Some Effects of Light Upon the Activity of the Male Accessory Glands in Rabbits, Arkiv För Zoolog1 11 (21), $379(1958)$.

124. Kth1ström, J.E., Spectfic Gravity of Different Parts of Bull Spermatozoa, Arkiv För Zoologi 11 (35), 569 (1958).

125. Kok, J.C.N., Some Factors Influencing the Longevity of Bull Sperm Celis IN VITRO, in Mammalian Germ Cells (G.E.W. Wolstenholme, Ed.), p 82, Little, Brown and Company, Boston (1953).

126. Kormano, M., Development of the Rectum-Testis Temperature Difference In the Post-Natal Rat, J. Reprod. Fert. 14, 427 (1967). 
127. Kousaku, T. and S. Okamoto, Temperature Shock in Chicken Spermatozoa, Japan. Poultry Sci. 4 (3), 116 (1967).

128. Krehblel, R.H. and H.P. Carstens, Roentgen Studies of the Mechanism Involved in Sperm Transportation in the Female Rabbit, Am. J. Physiol. 125,571 (1939).

129. Lalrd, C.W., Dimorphisms Observed in Bovine Sperm Cells and Electrophoretic Separation of Types, Fertility and Sterility 15, (6), 675 (1964).

130. Lake, P.E., Artificlal Insemination in Poultry and the Storage of Semen - A Re-appralsal, World's Poult. Sc1. J. 23 (2), 111 (1967).

131. Lasley, J.F., G.T. Easley and F.F. McKenzie, A Staining Method for the Differentiation of Live and Dead Spermatozoa, Anat. Rec. $\underline{82}$, 167 (1942).

132. Lavon, U., R. Volcan1, D. Am1r and D. Danon, The Specific Cravity of Bull Spermatozoa and Seminal Plasma, J. Reprod. Fert. 11, 447 (1966).

133. Lavon, U., R. Volcani, D. Amir and D. Danon, The Speciflc Gravity of Bull Spermatozoa from Different Parts of the Reproductive Tract, J. Reprod. Fert. 12, 597 (1966).

134. Leif, R.C. and J. Vinograd, The Distribution of Buoyant Density of Human Erythrocytes in Bovine Serum Albumin Solutions, Proc. Natn. Acad. Sci., U.S.A. 51, 520 (1964).

135. Lewin, S., Artificlal Sex Regulation of Mammalian Offpsring, Brit. Vet. J. 112,549 (1956).

136. Lewin, S., Studies in the Reaction of Amino and Imino Compounds with Sugars: The Reaction of Histidine with Glucose, Biochem. J. 63, 14 (1956).

137. Lindah1, P.E., Separation of Bull Spermatozoa Carrying X- and YChromosomes by Counter-Streaming-Centrifugation, Acta Agriculturae Scandinavica 8 (3), 226 (1958).

138. Lındah1, P.E. and J.E. Kihlström, Alterations in Specific Gravity During the Ripening of Bull Spermatozoa, J. Dairy Sc1. 35, 393 (1952).

139. Lindah1, P.E. and L.0. Thunqvist, Specific Gravity of Epididymal and Ejaculated Bull Spermatozoa and of I'heir Parts, Experientia 21 (2), 94 (1965).

140. Lorenz, F.W., Reproduction in the Domestic Fowl: Physiology of the Male, in Reproduction in Domestic Animals, Ed. H.H. Cole and P.T. Cupps, Vol. 2, 343, Academlc Press, Inc., New York (1959).

141. Lovelock, J.E., Comment on the Possibility of Controlling Sex Ratio and Development, in Sex Differentiation and Development, Mem. Soc, Endocr. I, 113 (1960). 
142. Lucas, J.J., B.W. Pickett and R.J. Komarek, Variance Component Estimates of Some Boar Semen Characteristics and Their Use in Designing Experiments, J. Reprod. Fert. 15, 423 (1968).

143. Lush, J.L., The Possibility of Sex Control by Artificial. Insemination with Centrifuged Spermatozoa, J. Agric. Res. 30, 893 (1925).

144. Lyster, W.R. and M.W.H. Bishop, An Association Between Rainfall and Sex Ratio in Man, J. Reprod. Fert. 10, 35 (1965).

145. MacLeod, J., Human Spermatozoan Production in Health and Disease, In Mammalian Germ Cells (G.E.W. Wolstenholme, Ed.) p 134. Little, Brown and Company, Boston (1953).

146. Macpherson, J.W. and S.D. Vesselinovitch, Electrophoresis of Bovine Semen. IV. Sex Ratio in Cattle Resulting from Electrophoretically Treated Semen, Canad. J. Compar. Med. and Vet. Sc1. 23, 375 (1959).

147. Masaki, J. and E.F. Hartree, Distribution of Metabolic Activity, Phospholipid and Hyaluronidase Between the Heads and Talls of Bull syeimatozoa, Biochem. J. 84, 347 (1962).

148. Mastroianni, I. Jr. and J. Ehteshamzadeh, Corona Cell Dispersing Properties of Rabbit Tubal Fluid, J. Reprod. Fert. 8 , 145 (1964).

149. Mastroianni, L. Jr. and R. Jones, Oxygen Tension Within the Rabbit rallopiari lube, J. Reprod. Fert. 9, 99 (1965).

150. Maude, A.D., Non-random Distribution of Bull Spermatozoa in a Drop of Sperm Suspension, Nature, 200 (4904), 381 (1963).

151. Maurer, R.R., W.L. Hunt and R.H. Foote, Repeated Superovulation Following Administration of Exogenous Gonadotrophins in Dutch-Bel,ted Rabb1ts, J. Reprod. Fert. 15, 93 (1968).

152. Mayer, D.T., C.D. Squiers, R. Bogart and M.M. Oloufa, The Technique. for Characterizing Mammalian Spermatozoa as Dead or Living by Differentlal Staining, J. Animal Sci. 10, 226 (1951).

153. Mc Gaughey, R.W. and J.C. Daniel, Jr., Effect of Oestrad1o1-17B on Fertilized Rabb1t Eggs IN VITRO, J. Reprod. Fert. 11, 325 (1966).

154. Mc Gaughey, R.W., J.H. Marston and M.C. Chang, Fertilizing Life of Mouse Spermatozoa in the Female Tract, J. Reprod. Fert. 16 (1), 147 (1968).

155. Mc Laren, A., Immunological Control of Fertility in Female Mice, Nature, $201,582(1964)$.

156. Mel, H.C., Stable-Flow Free-Boundary Migration and Fractionation of Cell Mixtures. I. Apparatus and Hydrodynamic Feedback Principles, J. Theor. Biol. 6, 159 (1964).

157. Mel, H.C., Stable-Flow Free-Boundary Migration and Fractionation of Cell Mixtures. II. Laminar Flow and Density Gradient Stability, J. 
Theor. Biol. 6, 181 (1964).

158. Mel, H.C., Stable-Flow Free-Boundary (STAFLO) Migration and Fractionation of Cell Mixtures. III. Migration Principles - Sedimentation and Electrophoresis, J. Theor. B101, 6, 307 (1964).

159. Mel, H.C., L.T. Mitchel1 and B. Thore11, Continuous Free-Flow Fractionation of Cellular Constituents in Rat Bone Marrow, Blood 25 (1), 63 (1965).

160. Mischler, T.W. and E.P. Reineke, Some Electrophoretic and Immunological Properties of Human Semen, J. Reprod. Fert. 12, 125 (1966).

161. Moghiss1, K.S., D. Dabich, J. Levine and O.W. Neuhaus, Mechanism of Sperm Migration, Fertility and Sterility 15, (1), 15 (1964).

162. More O'Ferra11, G.J., T.N. Meacham and W.E. Foreman, Attempts to Separate Rabbit Spermatozoa by Means of Froth Flotation and The Sex Ratio of Offspring Born, J. Reprod. Fert. 16 (2), 243 (1968).

163. Morlcard, R., Observation of IN VITRO Fertilization in the Pabbit, Nature 173 , 1140 (1954).

164. Morton, B.E., A D1sruption and Fractionation of Bovine Epididyma1 Spermatozoa, J. Reprod. Fert. 15, 113 (1968).

165. Munro, I.B., Bovine Semen Characteristics and Fertility, J. Reprod. Fert: 2, 513 (1961).

166. Murdock, R.N. and I.G. White, The Metabolism of Glucose, Fructose, Acetate, Lactate and Pyruvate by Ram, Bull, Dog and Rabbit Spermatozoa, J. Reprod. Fert. 12, 271 (1966).

167. Murdoch, R.N. and I.G. White, The Metabolism of Labelled Glucose by Rabbit Spermatozoa after Incubation IN UTERO, J. Reprod. Fert. 14, 213 (1967).

168. Nalbandov, A.V., Reproductive Physiology (2nd Ed.), W.H. Freeman and Company, San Francisco (1964).

169. Napler, R.A.N., Fertility in the Male Rabbit. I. Sensitivity of Spermatozoa to Handling Techniques, J. Reprod. Fert. 2 , 246 (1961).

170. Napier, R.A.N., Fertllity in the Male Rabbit. II. Variation in the Percentage of Eggs Fert1lized, J. Reprod. Fert. 2, 260 (1961).

171. Napier, R.A.N., Fertility in the Male Rabbit. III. Estimation of Spermatozoan Quality by Mixed Insemination, and the Inheritance of Spermatozoan Characters, J. Reprod. Fert. 2, 273 (1961).

172. Napier, R.A.N., Rabbits, Ch. II in Animals for Research, Principles of Breeding and Management, W. Lane-Petter, Ed., Academic Press, New York (1963)

173. Nevo, A.C., Dependence of Sperm Motility and Respiration on Oxygen 
Concentration, J. Reprod. Fert. 9, 103 (1965).

174. Nevo, A.C., I. Michaeli and H. Schindler, Electrophoretic Properties of Bull and of Rabbit Spermatozoa, Exp. Cell Res. 23, 69 (1961).

175. Nicander, L., On the Reglonal Histology and Cytochemistry of the Ductus Epididymis in Rabbits, Acta Morphol. Neerlando-Scandin. 1 , 99 (1957).

176. Norman, C. and E. Goldberg, The Effect of Visible Light on Spermatozoa, J. Reprod. and Fert. 2, 511 (1961).

177. Noyes, R.W., A. Walton and C.E. Adams, Capacitation of Rabbit Spermatozoa, Nature 181 (4617), 1209 (1958).

178. Noyes, R.W., C.E. Adams and W. Walton, Transport of Spermatozoa into the Uterus of the Rabbit, Fertility and Sterility $\underline{9}, 288$ (1958).

179. Ogasawara, F.X., F.W. Lorenz and L.W. Bobr, Distribution of Spermatozoa In the Oviduct and Fertility in Domestic Birds. 111. IntraUterine Insemination of Semen from Low-Fecundity Cocks, J. Reprod. Fert. 11, 33 (1966).

180. Orgebin-Crist, M.C., Fertility in Does Inseminated with Epididymal Spermatozoa, J. Reprod. Fert. 14, 346 (1967).

181. Orgebin-Crist, M.C., Gonadal and Epididymal Speim Keserves in the Rabbit: Estimation of the Dafly Sperm Production, J. Reprod. Fert. $15,15(1.968)$.

182. Oigelin-Crist, M.C., Maturation of Spermatozoa in the Rahhit. Fipldidymis: Delayed Fertilization in Does Inseminated with Epididymal Spermatozoa, J. Reprod. Fert. 16 (1), 29 (1968).

183. Ortavant, R., Spermatogenesis and Morphology of the Spermatozoon, in Reproduction in Domestic Animals, Vol. 2, P 1, Ed. H.H. Cole and P.T. Cupps, Academic Press, New York (1959).

184. Pavlok, A., Development of Mouse Ova in Explanted Oviducts: Ferti11zation, Cultivation and Transplantation, Sclence 157, 1457 (1967).

185. Peacock, W.J. and J. Erickson, Segregation-Distortion and Regularly Nonfunctional Products of Spermatogenesis in Drosophila melanogaster, Genetics 51, 313 (1965).

186. Phadke, A.M. and K. Padukone, Presence and Significance of Autoantibodles Against Spermatozoa in the Blood of Men with Obstructed Vas Deferens, J. Reprod. Fert. 7, 163 (1964).

187. Pincus, G., The Control of Fertility, Academlc Press, New York (1965).

188. Polge, C., The Preservation of Spermatozoa at Low Temperatures, in Mammalian Germ Cells (G.E.W. Wolstenholme, Ed.) p 108, Little, Brown and Company, Boston (1953). 
189. Proudfood, F.G. and D.K.R. Stewart, The Effect of Oxygen and Type of Container on the Retention of the Fertilizing Capacity of Fowl Spermatozoa Stored IN VITRO, J. Reprod. Fert. 13, 251 (1967).

190. Psychoyos, Alexandre, Influence of Oestrogen on the Loss of the Zona Pellucida in the Rat, Nature 211 (5051), 864 (1966).

191. Quinn, P.J. and I.G. White, Distribution of Adenosinetriphosphatase Actlvity in Ram and Bull Spermatozoa, J. Reprod. Fert. 15, 449 (1968).

192. Quinn, P.J. and I.G. White, The Effect of Cold Shock and Deep-FreezIng on the Concentration of Major Cations in Spermatozoa, J. Reprod. Fert. 12, 263 (1966).

193. Quinn, P.J., I.G. White and B.R. Wirrick, The Effect of Dilution on the Concentration of Sodium, Potassium, Calcium and Magnesium in Ram and Bull Spermatozoa, J. Reprod. Fert. 12, 131 (1966).

194. Rikmenspoel, R., An Optically Clear Egg-Yolk Diluent for Bull Spermatozoa, Experientia 13, 124 (1957).

195. Roche, J.F. P.J. Dzluk and J.R. Lodge, Competition Between Fresh and Aged Spermatozoa in Fertilizing Rabbit Eggs, J. Reprod. Fert. 16, (1), 155 (1968).

196. Rock, J., Sex, Science and Survival, J. Reprod. Fert. $\underline{8}, 397$ (1964).

197. Rothschild, Lord , Non-Random Distribution of Bull Spermatozoa in a Drop of Sperm Suspension, Nature 198 (4886), 1221 (1963).

198. Rothschild, Lord , X and Y Spermatozoa, Nature 187 (4733), 253 (1960).

199. Rousse1, J.D. and C.R. Austin, Preservation of Primate Spermatozoa by Freezing, J. Reprod. Fert. 13, 333 (1967).

200. Rowe11, J.G. and D.M. Cooper, Some Effects of Diluting Cock Semen, Poultry Science XXXIX (6), 1381 (1960).

201. Salisbury, G.W., Ionic and Osmotic Conditions in Relation to Metabo11c Control, in Spermatozoan Motility (D.W, Bishop, Ed.), p 59, Am. Assn. for the Adv. of Sci., Washington, D.C. (1962).

202. Salisbury, G.W. and F.N. Baker, Frequency of Occurrence of Diploid Bovine Spermatozoa, J. Reprod. Fert. 11, 477 (1966).

203. Sawada,Y. and M.C. Chang, Mot1lity and Fertllizing Capacity of Rabbit Spermatozoa after Freezing in a Medium Containing Dimethy 1 Sulfoxide, Fertility and Sterility 15 (2), 222 (1964).

204. Schachman, H.K., Ultracentrifugation in Biochemistry, Academic Press, New York (1959).

205. Schilling, E., Experiments in Sedimentation and Centrifugation of Bull Spermatozoa and the Sex Ratio of Born Calves, J. Reprod. Fert. 11, 469 (1966). 
206. Schröder, V., Künstliche Geschlechtsregulation der Nachkommenschaft der Säugetiere und thre Blologische Kontrolle, Z.F. Tierzüchtg. u. Züchtgsbiol. 50, 1 (1941).

207. Schröder, V., Über die Biochemischen und Physiologischen Eigentümllchkelten der X- und Y-Spermien, Z. f. Tlerzüchtg. u. Züchtgsbiol. 50, 16 (1941).

208. Schröder, V., Über die Biochemischen und Physiologischen Eigentümlichkeiten der X- und Y-Spermien, Z. f. Tierzüchtg. U. Züchtgsbiol. 50, 16 (1941).

209. Segal, S.J. and K.A. Laurence, Automatic Analysis of Particulate Matter in Human Semen, Annals New York Academy of Sciences 99, 271 (1962).

210. Sevinf, A., Experiments on Sex Control by Electrophoretic Separation of Spermatozoa in the Rabbit, J. Reprod. Fert. 16 (1), (1968).

211. Sharma, S.C., S. Singh and R.N. Desa1, The Ut1lity of the Varlous Antiblotics and Sulfa Drugs in Bovine Semen, Indian Vet. J. 39 (12), 648 (1962).

212. Shaver, E.L. and D.H. Carr, Chromosome Abnormalities in Rabbit Blastocysts Following Delayed Fertilization, J. Reprod. Fert. 14, 415 (1967).

213. Shettles, L.B., Nuclear Morphology of Human Spermatozoa, Nature 186 $(4725), 648$ (1960).

214. Shettles, L.B., X and Y Spermatozoa, Nature 187 (4733), 254 (1.960).

215. Short, D.J. and D.P. Woodnott, The A.T.A. Manual of Laboratory Animal Practice and Techniques, Charles C. Thomas, Springfield, I11. (1964).

216. Sittmann, D.B., W.C. Rollins, K. Sittmann, and R.B. Casady, Seasonal Variation in Reproductive Traits of New Zealand White Rabbits, J, Reprod. Fert. $\underline{8}, 29$ (1964).

217. Soupart, P., Studies on the Hormonal Control of Rabbit Sperm Capacitation, J. Reprod. Fert. Supp. 2, 49 (1967).

218. Staples, R.E. and D.E. Holtkamp, Influence of Body Weight upon Corpus Luteum Formation and Malntenance of Pregnancy in the Rabbit, J. Reprod. Fert. 12; 221 (1966).

219. Stevens, K.R., H.D. Hafs, and K.T. Kirton, Volume, pH and Protein Content of Flulds from Ligated Uteri of Oestrous Rabbits, J. Reprod. Fert. I, 331 (1964).

220. Sturkie, P.D., Avlan Physiology (2nd Ed.), Cornell University Press, (Comstock Publishing Associates, a division of Cornell University Press), Ithaca, New York (1965).

221. Suzuk1, S., IN VITRO Cultivation of Rabbit Ova Following IN VITRO Fertilization in Tubal Fluid, Cytologia 31, (4), 416 (1966). 
222. Swlerstra, E.E. and R.H. Foote, Cytology and Kinetics of Spermatogenesis in the Rabbit, J. Reprod. Fert. 5, 309 (1963).

223. Swierstra, E.E. and R.H. Foote, Duration of Spermatogenesis and Spermatozoan Transport in the Rabbit Based on Cytological Changes, DNA Synthesis and Labelling with Tritiated Thymidine, Am. J. Anat. 116, 401 (1965).

224. Szollosi, D.G. and H. RIs, Observations on Sperm Penetration in the Rat, J. of Biophysical and Biochemical Cytology 10 (2), 275 (1961).

225. Takeda, A., C. Lutwak-Mann and T. Mann, Labelling of Semen with Radioactive Phosphate, J. Reprod. Fert. 16 (1), 141 (1968).

226. Tampion, D., Fluctuations in the Swimming Rate of Individual Bull Spermatozoa, J. Reprod. Fert. 11,371 (1966).

227. Tampion, D.,' The Effect of Some Drugs and Hormones on the Swimming Performance of Bull Spermatozoa, J. Reprod. Fert. 16 (2), 319 (1968).

228. Tesh, J.M. and T.D. Glover, The Influence of Aging of Rabbit Spermatozoa on Fertilization and Prenatal Development, J. Reprod. Fert. 12,414 (1966).

229. Tippetts, R.D., H.C. Mel and A.V. Nichols, Stable-Flow Free Boundary (STAFLO) Electrophores1s: Three Dimensional Fluid Flow Properties and Applications to Lipoprotein Studies. In: Chemical Engineering in Medicine and Biology, D. Hersey, Ed., Plenum Press, New York (1967).

230. Van Duijn, C., Relationship Between Spermatozoan Numbers and Fertility, Internat. J. Fert. 9, 609 (1964).

231. Van Wambeke, F., The Storage of Fow I Spermatozoa. I. Preliminary Results with New Diluents, J. Reprod. Fert. 13, 571 (1967).

232. Varian, N.B., R.R. Maurer and R.H. Foote, Ovarian Response and Cleavage Rate of Ova in Control and FSH-Primed Rabbits Receiving Varying Levels of Luteinizing Hormone, J. Reprod. Fert. 13, 67 (1967).

233. Vesselinovitch, S.D., Electrophoresis of Spermatozoa and Sex Control, Cornell Vet. 50, 326 (1960).

234. Vickers, A.D., A Direct Measurement of the Sex Ratio in Mouse Blastocysts, J. Reprod. Fert. 13, 375 (1967).

235. Vickers, A.D., Amniotic Sex-Chromatin and Foetal Sexing in the Mouse, J. Reprod. Fert. 14, 503 (1967).

236. Wales, R.G. and J.C. Wallace, Effects of Diluent Composition on the Metabolism of Bull, Dog, Rabbit and Fowl Spermatozoa, J. Reprod. Fert. 8, 361 (1964).

237. Wales, R.G. and T. O'Shea, Effects of Low Levels of Carbon Dioxide on the Metabolism of Ram and Bull Spermatozoa, J. Reprod. Fert. 11, 171 (1966). 
238. Wales, R.G., J.C. Wallace and I.G. White, Composition of Bull Epididymal and Testicular Fluid, J. Reprod. Fert. 12, 139 (1966).

239. Walton, A., Improvement in the Design of an Artificlal Vagina for the Rabbit, J. of Physiol. 143, 26P (1958).

240. We11, A.J. and C.0. Roberts, Fertillty of Female Rabbits After Isoimmunization with Seminal Plasma, Fertility and Sterility 16 (3), 356 (1965).

241. Welch, R.M. and E.W. Hanly, The Experimental Error of Feulgen Cytophotometry in the Analysis of Bull Spermatozoa over an Extended Period of Time; J. Histochem. and Cytochem. 9 (3), 251 (1961).

242. Welch, R.M., E.W. Hanly and W. Guest, The Deoxyribonuclelc Acid (DNA) Deviation in the Semen Spermatozoa of Bulls of Unknown Fertility Under Two Years of Age and Its Relationship to Motflity, Count and Morphology, J. of Morphl. 108. (2), 145 (1961).

243. Williams, W.L. and C.E. Hamer, Effect of Light on Sperm Metabolism, Fed. Proc: 21 (2), 437 (1962).

244. Wilson, L., Sperm Agglutinins in Human Semen and Blood, Proc. Soc. Expt1. B1ol. Med. 85, 652 (1954).

245. Yanagimachi, R., Time and Process of Sperm Penetration into Hamster Ova IN VIVO and IN VITRO, J. Reprod. Fert. 11, 359 (1966).

246. Yanagimachi, R. and M.C. Chang, Fertilization of Hamster Eggs IN VITRO, Nature 200, 281 (1963).

247. Yanagimachi, R. and M.C. Chang, Sperm Ascent Through the Oviduct of the Hamster and Rabbit in Relation to the Time of Ovulation, J. Reprod. Fert. 6 (3), 413 (1963).

248. Yoshlak1, S. and M.C. Chang, Motility and Fertilizing Capacity of Rabbit Spermatozoa After Freezing in a Medium Containing Dimethyl Sulfoxide, Fertility and Sterility 15 (2), 222 (1964).

249. Adams, C.E., Intraperitoneal Insemination in the Rabb1t, J. Reprod. Fert. 18, 333 (1969).

250. Bell, E.B., Immunological Control of Fertility in the Mouse: A Comparison of Systemic and Intravaginal Immunization, J. Reprod. Fert. 18,183 (1969).

251. Bey, E., The Electrophoretic Mobility of Sperm Cells, in Cell Electrophorests, Ed. E.J. Ambrose, Little, Brown and Co., Boston (1965).

252. Branham, J.M., Movements of Free-Swimming Rabbit Spermatozoa, J. Reprod. Fert. 18,96 (1969).

253. Etzion1, A., Sex Control, Sclence and Society, Science 161, 1107 (1968). 
254. Folstad, L., J.P. Bennett and R.I. Dorfman, The IN VITRO Cu1ture of Rat Ova, J.' Reprod. Fert. 18, 145 (1969).

255. Kiefer, D.M., Population, Technology's Desperate Race with Fertility, C \& EN, 118 (Oct. 7, 1968).

256. Lindsay, D.R., Sexual Activity and Semen Production of Rams at High Temperatures, J. Reprod. Fert. 18, 1 (1969).

257. Mc Cosker, P.J., Abnormal Spermatozoan Chromatin in Infertile Bulls, J. Reprod. Fert. 18, 363 (1969).

258. Nevo, A.C. and R. Mohan, Migration of Motile Spermatozoa into SpermFree Medium and the "Dilution Effect", J. Reprod. Fert. 18, 379 (1969).

259. QuInn, P.J. and I.G. White, Osmotic Shock of Ram Spermatozoa, J. Reprod. Fert. 18, 375 (1969).

260. Quina, P.J., I.G. White and K.W. Cleland, Chemical and U1trastructure Changes in Ram Spermatozoa after Washing, Cold Shock and Freezing, J. Reprod. Fert. 18, 209 (1969).

261. Robinson, D., J. Rock and M.F. Menkin, Control of Human Spermatogenesis by Induced Changes of Intrascrotal Temperature, JAMA 204 (4), 80 (1968).

262. Yanagimachi, R., IN VITRO Capacitation of Hamster Spermatozoa by Follicular Fluid, J. Reprod. Fert. 18, 275 (1969).

263. Tippetts, R.D., Stable-Flow Free-Boundary Electrophorests Theory and Practice as Applied to the Study of Human Serum Lipoproteins, PhD Thesis, University of California, Berkeley, UCRL 16290 (1965).

264. Maurer, R.R., W.L. Hunt, L.D. Van Vleck and R.H. Foote, Developmental Potential of Syperovulated Rabbit Ova, J. Reprod. Fert. 15, 171 (1968).

265. Brackett, B.G., D.E. Weinman and W.L. Will1ams, ATP Content and Effect of Light on Sperm Respiration, Fed. Proc. 22, 569 (1963).

266. Burrows, W.H. and J.P. Quinn, Artificial Insemination of Chickens and Turkeys, C1rc. U.S. Dept. Agr1c. 525 (1939).

267. Ruffer, E., N1tabell Rabbitry, Hayward, California, Personal CommunIcation (1967).

268. Baker, F.N. and G.W. Salisbury, Distribution of Feulgen-DNA In Sperm Populations, Jour. Animal Sc1. 22, 815 (1963).

269. Tanaka, K. and S. Okamoto, Temperature Shock in Chicken Spermatozoa, Japan. Poultry Sc1. 4 , 116 (1967).

270. Albertsson, P., Partition of Cell Particles and Macromolecules, John Wiley and Sons, New York (1960).

271. Baker, F.N. and G.W. Salisbury, Nuclear Size of Live and Dead Bovine 
Spermatozoa, Nature 197, 820 (1963).

272. Rosenfeld, A., Science, Sex and Tomorrow's Morality, Life 66 (23), 37 (1969).

273. Hancock, J.L., Evidence of an Inherited Seminal Character Associated with Infertility of Frlesian Bulls, Vet. Rec. 61, 308 (1949).

274. Rorvik, D.M. and L.B. Shettles, Your Baby's Sex: Now You Can Choose It, Dodd, Mead and Company, New York (1970). 


\section{LEGAL NOTICE}

This report was prepared as an account of Government sponsored work. Neither the United States, nor the Commission, nor any person acting on behalf of the Commission:

A. Makes any warranty or representation, expressed or implied, with respect to the accuracy, completeness, or usefulness of the information contained in this report, or that the use of any information, apparatus, method, or process disclosed in this report may not infringe privately owned rights; or

B. Assumes any liabilities with respect to the use of, or for damages resulting from the use of any information, apparatus, method, or process disclosed in this report.

As used in the above, "person acting on behalf of the Commission" includes any employee or contractor of the Commission, or employee of such contractor, to the extent that such employee or contractor of the Commission, or employee of such contractor prepares, disseminates, or provides access to, any information pursuant to his employment or contract with the Commission, or his employment with such contractor. 
TECHIYAOAL INFORMATION DIVISION

LAWRENCE RADIATION LABORATORY

UNIVERSITY OF CALIFORNIA

BERKELEY, CALIFORNIA 94720 